\title{
Schwingungsspektroskopische Untersuchung der Aggregation und Isomerisierung von Modellpeptiden in der Jet-Expansion am Beispiel kleiner sekundärer Amide
}

\author{
DISSERTATION
}

zur Erlangung des mathematisch-naturwissenschaftlichen Doktorgrades "Doctor rerum naturalium" der Georg-August-Universität Göttingen

im Promotionsprogramm Chemie der Georg-August University School of Science (GAUSS)

vorgelegt von

Thomas Forsting

aus Nordhorn

Göttingen, 2020 


\section{Betreuungsausschuss}

Prof. Dr. Martin A. Suhm

Georg-August-Universität Göttingen, Institut für Physikalische Chemie apl. Prof. Dr. Burkhard Geil

Georg-August-Universität Göttingen, Institut für Physikalische Chemie

\section{Mitglieder der Prüfungskommission}

Referent: Prof. Dr. Martin A. Suhm

Georg-August-Universität Göttingen, Institut für Physikalische Chemie Koreferent: apl. Prof. Dr. Burkhard Geil

Georg-August-Universität Göttingen, Institut für Physikalische Chemie

\section{Weitere Mitglieder der Prüfungskommission}

Dr. Sebastian Kruss

Georg-August-Universität Göttingen, Institut für Physikalische Chemie Prof. Dr. Ricardo Mata

Georg-August-Universität Göttingen, Institut für Physikalische Chemie Prof. Dr. Dirk Schwarzer

Max-Planck-Institut für Biophysikalische Chemie

Prof. Dr. Dietmar Stalke

Georg-August-Universität Göttingen, Institut für Anorganische Chemie

Tag der mündlichen Prüfung: 12.08.2020 
The first principle is that you must not fool yourself

—and you are the easiest person to fool.

R. P. FEYNMAN 



\section{Danksagung}

Die Erstellung dieser Arbeit wurde durch vielfältige Hilfe und Unterstützung begleitet.

An erster Stelle danke ich Prof. Dr. Martin Suhm für die exzellente Betreuung dieser Arbeit. Viele Stunden der Diskussion von Messergebnissen, Troubleshooting am curry-Jet oder dem Erklären spektroskopischer und thermodynamischer Zusammenhänge waren immer getragen von einer gefühlt unerschöpflichen Expertise, den richtigen Fragen, einem feinen Humor, viel Geduld und einer unermüdlichen Begeisterung für Naturwissenschaften.

Ich danke auch Prof. Dr. Burkhard Geil für die Übernahme der Zweitbetreuung und seine gute Hinführung in die Programmierung mit Python, von der ich an vielen Stellen während der Arbeit sehr profitiert habe. Den Mitgliedern der Prüfungskommission danke ich für die Begutachtung dieser Arbeit.

In der langen Zeit in diesem Arbeitskreis wurde es nie langweilig und für eine stets kollegiale, sehr angenehme Arbeitsatmosphäre bin ich allen Mitdoktorandinnen und -doktoranden des AK Suhm dankbar.

Mit Kathi Meyer ließ sich bei Tee und Wochenendarbeit sowohl Fachliches als auch Popmusikalisches bestens diskutieren. Unzählbare Kicker-Runden und gemütlichen Spieleabende wären ohne Kathi, Hannes, Sönke, Anja, Sebastian, Christoph und Philip nicht denkbar gewesen.

Ich danke Petra Lawecki für eine immer offene Tür und die Hilfe bei der Erledigung aller Verwaltungsangelegenheiten.

Ich danke Sönke Oswald (Masterarbeit), Beppo Hartwig (Bachelorarbeit) sowie Sven Meyer und Niklas Henning (Abteilungspraktikanten) für viele gute Ideen und wertvolle Erkenntnisse.

Eine experimentelle Arbeit mit apparativen Weiterentwicklungen wie diese benötigt technische Expertise, die für jedes Problem eine passende Lösung anbietet. Stellvertretend für die Arbeit der feinmechanischen und elektronischen Werkstätten des Instituts danke ich Herrn Hildebrandt, Herrn Meyer und Herrn Knorr.

Für das Korrekturlesen danke ich Sönke Oswald, Johanna Forsting, Katharina Meyer, Anja Poblotzki und meiner Mutter Elisabeth.

Ich danke meinen Schwiegereltern für die Unterstützung durch Kinderbetreuung, den ständig freien Schreibtisch und kulinarische Großtaten. In ganz besonderem Maße möchte ich mich für das Tragen durch die und Ertragen der Erstellung dieser Arbeit bei meiner Familie bedanken. Die ununterbrochene Unterstützung und beständige Ermutigung durch meine Frau Johanna hat die Fertigstellung neben dem Referendariat und dem Familienleben überhaupt erst möglich gemacht.

Für immer dankbar bin ich meinen Eltern, die mir das Studium der Chemie und damit auch die Promotion ermöglicht haben. 



\section{Inhaltsverzeichnis}

\begin{tabular}{lll}
\hline & Einleitung & 1
\end{tabular}

$\begin{array}{lll}2 & \text { Grundlagen } & 7\end{array}$

2.1 Nomenklatur von Amiden . . . . . . . . . . . . . . . . . . . . . 7

2.2 Charakteristische Normalschwingungen sekundärer Amide . . . . . . . . . . . 8

2.3 Dichtefunktionaltheorie: Das B3LYP-Funktional . . . . . . . . . . . . . . . . 11

2.4 Thermodynamik der Konformationsisomerie . . . . . . . . . . . . . . . . . 12

3 Experimentelle und theoretische Methoden 15

3.1 Raman-Spektroskopie: Der curry-Jet . . . . . . . . . . . . . . . . . . . . . 15

3.1 .1 Aufbau und Funktionsweise . . . . . . . . . . . . . . . . . . 15

$3.1 .2 \quad$ Heizbare Substanzzuführung $\ldots \ldots \ldots \ldots \ldots$

3.1 .3 Anregungsoptik . . . . . . . . . . . . . . . . . . . 21

3.1 .4 Detektionsoptik und Datenaufnahme . . . . . . . . . . . . . 24

3.1 .5 Datenauswertung . . . . . . . . . . . . . . . . 26

3.1 .6 Kalibrierung . . . . . . . . . . . . . . . . . . . . . . 29

3.2 FTIR-Spektroskopie: Der filet-Jet $\ldots \ldots \ldots \ldots$. . . . . . . . . . 30

3.3 Quantenchemische Rechnungen . . . . . . . . . . . . . . . . . . 30

3.4 Frequenzrechnungen und Raman-Aktivitäten $\ldots \ldots \ldots$. . . . . . . . . . . 31

3.5 Verwendete Substanzen $\ldots \ldots \ldots \ldots \ldots \ldots$. . . . . . . . . . . . 33

\begin{tabular}{|lll}
\hline 4 & N-Methylacetamid & 35
\end{tabular}

4.1 Schwingungsspektroskopie des Monomers . . . . . . . . . . . . . . . 36

4.2 cis-/trans-Isomerisierung . . . . . . . . . . . . . . . . . . 47

4.3 Dimerisierung . . . . . . . . . . . . . . . . . . . . . . 52

4.4 Trimere und höhere Aggregate $\ldots \ldots \ldots \ldots \ldots$. . . . . . . . . . 65

4.5 Zusammenfassung und Diskussion $\ldots \ldots \ldots \ldots \ldots$. . . . . . . . 70

5 N-Methylformamid

5.1 Schwingungsspektroskopie des Monomers . . . . . . . . . . . . . . 74

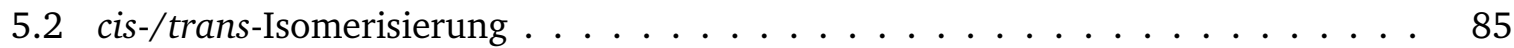

5.3 Dimerisierung . . . . . . . . . . . . . . . . . . . . 89

5.4 Trimere und höhere Aggregate $\ldots \ldots \ldots$. . . . . . . . . . . . . 99

5.5 Zusammenfassung und Diskussion . . . . . . . . . . . . . . 106 
$\begin{array}{lll}6 & N \text {-Phenylformamid } & 109\end{array}$

6.1 Struktur und Schwingungsspektroskopie des Monomers . . . . . . . . . . . . . 109

6.2 cis-/trans-Isomerisierung . . . . . . . . . . . . . . . . . . . . . . . . 114

6.3 Dimerisierung . . . . . . . . . . . . . . . . . . . . . 118

6.4 Trimerbildung . . . . . . . . . . . . . . . . . . . . . . . . 126

6.5 Zusammenfassung und Diskussion $\ldots \ldots \ldots \ldots \ldots \ldots$. . . . . . . . . 128

7 Längerkettige Alkylamide 133

8 gamma-Butyrolactam 139

8.1 Struktur und Isomerisierung . . . . . . . . . . . . . . . . . . . . 139

8.2 Schwingungsspektroskopie . . . . . . . . . . . . . . . . . . . . 142

8.3 Zusammenfassung und Diskussion $\ldots \ldots \ldots \ldots \ldots \ldots$. . . . . . . . . 148

9 Zusammenfassung und Ausblick $\quad 151$

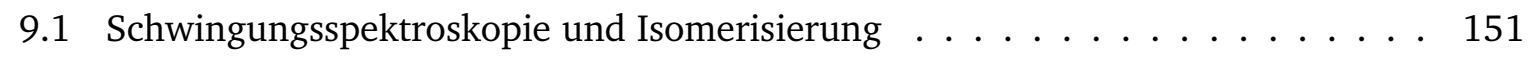

9.2 Molekülaggregate . . . . . . . . . . . . . . . . . . . . . . . 154

9.3 Apparative Entwicklungen des curry-Jets . . . . . . . . . . . . . . . . 157

\begin{tabular}{ll}
\hline A Anhang & 159
\end{tabular}

A.1 Publikationen . . . . . . . . . . . . . . . . . . . . . . . . . . . . . . . . 159

A.2 Verwendete Chemikalien . . . . . . . . . . . . . . . . . . . . . . . 159

A.3 Imidazol . . . . . . . . . . . . . . . . . . . . . . . . . 160

A.4 Komponenten des curry-Jets . . . . . . . . . . . . . . . . . . . . . . 163

A.5 Input-Zeilen der GAUSSIAN-Rechnungen . . . . . . . . . . . . . . . . . . 165

A.6 Spektrenverzeichnis . . . . . . . . . . . . . . . . . . . . . . 165

A.6.1 Raman-Spektren . . . . . . . . . . . . . . . . . . . . . 165

A.6.2 $\quad$ FTIR-Spektren . . . . . . . . . . . . . . . . . . . . . . . . 171

A.6.3 NMR-Spektren . . . . . . . . . . . . . . . . . . . . . . . . 174

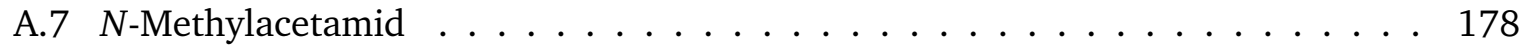

A.7.1 Ergänzende Abbildungen . . . . . . . . . . . . . . . . . 178

A.7.2 Tabellenwerk . . . . . . . . . . . . . . . . . . . . . . . . 181

A.8 $\quad N$-Methylformamid . . . . . . . . . . . . . . . . . . . . . . . . . . . . . 191

A.9 $\quad N$-Phenylformamid $\ldots \ldots \ldots \ldots \ldots \ldots \ldots \ldots$

A.9.1 Ergänzende Abbildungen . . . . . . . . . . . . . . . . . . . 196

A.9.2 Tabellenwerk . . . . . . . . . . . . . . . . . . . 196

A.10 gamma-Butyrolactam . . . . . . . . . . . . . . . . . . . 198

A.10.1 Ergänzende Abbildungen . . . . . . . . . . . . . . . . . . 198

A.10.2 Tabellenwerk . . . . . . . . . . . . . . . . . . . . . . . 198

A.11 Längerkettige Amide . . . . . . . . . . . . . . . . . . . . . . . . 202

A.12 Auswertskripte . . . . . . . . . . . . . . . . . . . . 204

\begin{tabular}{ll}
\hline Literaturverzeichnis & 227
\end{tabular} 


\section{Einleitung}

Die Stoffklasse der Amide spielt in der chemischen Industrie eine wichtige Rolle, zum Beispiel als Lösungsmittel für Farben und Lacke (Formamid) sowie Polymere ( $N, N$-Dimethylformamid) und als Edukt in der Herstellung von Pharmazeutika ( $N$-Phenylformamid) und Agrochemikalien ( $N$-Methylacetamid) [1]. Eine besondere Bedeutung kommt den industriell gefertigten Polyamiden zu, die mit einer Jahresproduktion von 1.2 Mio. Tonnen ${ }^{1)}$ zu Kunstfasern wie z. B. Nylon, Perlon und Kevlar weiterverarbeitet werden.

Essentiell für alle Lebensformen sind Polyamide jedoch in ihrer natürlichen Form als sogenannte Polypeptide (amidisch verknüpfte Aminosäuren), die der Grundbaustein aller Proteine sind. Hier ist die namensgebende Peptidbindung die kovalente Verbindung zweier benachbarter Aminosäuren und eine von drei typischen Bindungen im Proteingerüst (backbone) [3, S. 285295].

Die funktionelle Gruppe der Amide besteht aus zwei stark polaren Teilgruppen ( $\mathrm{C}=\mathrm{O}$ und $\mathrm{N}-\mathrm{H}$ ), die sowohl intra- als auch intermolekulare Wechselwirkungen in Form von Wasserstoffbrückenbindungen ermöglichen. Hierbei fungiert die Carbonyl-Teilgruppe als Wasserstoffbrücken-Akzeptor und die Amino-Teilgruppe als Wasserstoffbrückendonor. Darüber hinaus weist die zentrale C-N-Bindung einen partiellen Doppelbindungscharakter von ca. $40 \%$ auf [4, S. 81]. Dieser ist qualitativ leicht aus den mesomeren Grenzstrukturen ersichtlich (siehe Abb. 1-1). Die über die drei Zentren $\mathrm{O}-\mathrm{C}-\mathrm{N}$ delokalisierten $\pi$-Elektronen führen zu einer Bindungsordnung von 1.5 je Bindung und folglich zu einer planaren Struktur der gesamten Amidgruppe [5].

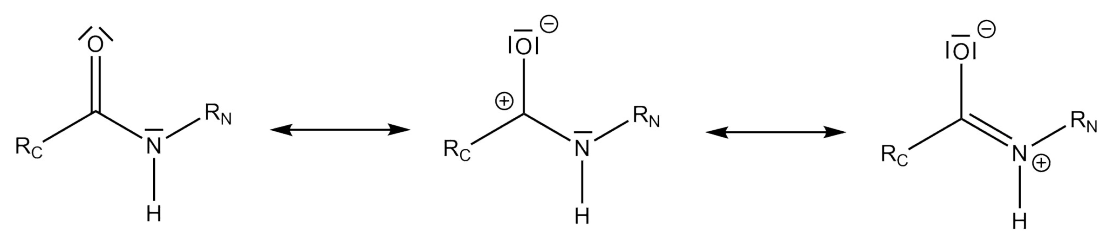

Abb. 1-1. Mesomere Grenzstrukturen der Amidbindung [5]. Die Indizes der Reste beziehen sich auf den C- und NTerminus der Amidgruppe.

Die daraus resultierende gehinderte Drehung um die C-N-Bindung (Amid- oder Peptidbindung) führt bei monosubstituierten (sekundären) und unterschiedlich disubstituierten (tertiären) Amiden zu zwei unterscheidbaren Konformationsisomeren, die aufgrund der Planarität der Amidgruppe üblicherweise mit den C-C-N-C-Diederwinkeln von $0^{\circ}$ (cis) und $180^{\circ}$ (trans) vorliegen (Details zur Nomenklatur von Amiden finden sich in Abschnitt 2.1).

\footnotetext{
${ }^{1)}$ Primärformen in Deutschland, 2018 [2].
} 
Diese gehinderte Drehung spielt eine wichtige Rolle in der Faltung von Proteinen. Dabei ist die trans-Konformation aus sterischen Gründen energetisch bevorzugt. Sie macht ca. 99.7\% der Peptidbindungen (ohne Prolinbeteiligung) in Proteinen aus [6]. Die dennoch vorkommende cis-Konformation findet sich vor allem (ca. 90\%) am $N$-Terminus von Prolin2), welche als einzige proteinogene Aminosäure eine Ringstruktur unter Beteiligung des Stickstoffatoms der Aminogruppe und des $\mathrm{C}_{\alpha}$-Atoms besitzt. Dadurch ist weder eine Drehung um die $\mathrm{C}_{\alpha}-\mathrm{N}$-Bindung (Diederwinkel $\varphi$, siehe Abschnitt 2.1) möglich, noch ein Wasserstoffatom am N-Atom vorhanden. Prolin bildet also $N$-seitig tertiäre Peptide [7], deren trans-Konformation aufgrund der konstitutionellen Besonderheiten geringere sterische Vorteile bietet und somit der cis-Anteil gegenüber den anderen Peptidbindungen in Proteinen deutlich erhöht ist [8].

Die Kinetik der cis-/trans-Isomerisierung in Polypeptiden hat erheblichen Einfluss auf die Faltung von Proteinen [9] und die Wiederfaltung fehlerhaft gefalteter und damit potentiell pathogener Proteine [10]. Es sind inzwischen sogar eine Reihe von Enzymen bekannt, die die cis/trans-Isomerisierung katalysieren, so z. B. das Chaperon DnaK [11]. Ebenfalls einen bedeutenden Einfluss auf die Proteinfaltung haben zwischenmolekulare Wechselwirkungen, vornehmlich in Form von Wasserstoffbrückenbindungen zwischen den Peptidgruppen des Backbones, den Aminosäureresten und der wässrigen Umgebung [12-14].

Die Isomerisierung und die Möglichkeit zur Ausbildung von Wasserstoffbrückenbindungen eines Polypeptids sind also von großer biologischer Bedeutung. Chemisch gesehen führen Wasserstoffbrücken unter Beteiligung des Amino-Wasserstoffatoms zu einer Schwächung der kovalenten $\mathrm{N}-\mathrm{H}$-Bindung. Diese ist über eine typische Rotverschiebung und Intensitätszunahme der N-H-Bande im Schwingungsspektrum direkt erkennbar und eines von mehreren von der IUPAC ${ }^{33}$ festgelegten Kriterien zur Validierung einer Wasserstoffbrückenbindung [15, 16]. Aber auch eine Veränderung der direkten chemischen Umgebung z. B. durch eine Konformationsänderung und eine damit einhergehende Änderung intramolekularer Wechselwirkungen wird durch eine veränderte Bandenposition im Schwingungsspektrum sichtbar (wie auch bei transund gauche-Ethanol [17, 18]). Daher sind schwingungsspektroskopische Methoden, wie Infrarot- und Ramanspektroskopie, geeignete Verfahren, um diese Art von Wechselwirkungen zu untersuchen.

Allerdings ist eine spektroskopische Untersuchung von Oligopeptiden und insbesondere von Proteinen auf Grund ihrer Größe und Empfindlichkeit gegenüber Temperaturen und Umgebung (z. B. Lösungsmittel) schwierig. Daher haben sich kleine sekundäre Amide wie $N$-Methylacetamid (NMA) als kleinstmögliches Molekül mit Peptidbindung und $N$-Methylformamid (NMF) als kleinstes sekundäres Amid als Verbindungen zur Modellierung der Peptidbindung etabliert [19]. Sie sind unterschiedlichen spektroskopischen Methoden und rechenintensiven quantenmechanischen $a b$ initio-Methoden zugänglicher. Die experimentellen Ergebnisse zu solchen Modellverbindungen können wieder zur Parametrisierung empirischer Rechenmethoden herangezogen werden, mit denen, aufgrund ihres deutlich geringeren Rechenaufwands, wiederum die

\footnotetext{
${ }^{2)}$ Diese Zahlen sind mit einer gewissen Vorsicht zu genießen, da diese Analysen automatisiert durchgeführt werden und solche „Refinement-Programme“ z. B. alle nicht-prolyl cis-Konformere automatisch in ihre trans-Form überführen [7, S. 181].

${ }^{3)}$ International Union of Pure and Applied Chemistry
} 
biologisch relevanten Peptide charakterisiert werden können.

Die Thermodynamik der cis-/trans-Isomerisierung und die intermolekularen Wechselwirkungen in Form von Clusterbildung für kleine sekundäre Amide als Peptidmodellverbindungen sind Gegenstand der aktuellen Forschung [20,22] und stehen in Form einer experimentellen Abschätzung der Enthalpiedifferenz zwischen cis- und trans-Konformeren für verschiedene sekundäre Amide und dem spektroskopischen Nachweis kleinster Molekülaggregate, wie Dimer und Trimer, im Zentrum dieser Arbeit.

Für einen schwingungsspektroskopischen Nachweis solcher Cluster bedarf es Bedingungen, unter denen die Bildung nennenswerter Mengen schwach gebundener Aggregate stattfindet. Außerdem müssen die, durch Druck und Temperatur in der Gasphase unter Standardbedingungen ${ }^{4)}$ hervorgerufenen, starken Signalverbreiterungen ebenso wie die, durch Umgebungseinflüsse verursachte, Verschiebung der Bandenposition im Schwingungsspektrum minimiert werden. Idealerweise wird daher bei niedrigen Drücken und tiefen Temperaturen gemessen. Damit die Substanz nicht kondensiert, sondern möglichst kleine Aggregate entstehen, wird bevorzugt eine der folgenden Nichtgleichgewichtsmethoden verwendet.

Etablierte Methoden für eine schwingungsspektroskopische Analyse kalter Moleküle und Cluster sind die Techniken Matrix-Isolation [23], Helium-Nanotröpfchen [24] und freie JetExpansion [25]. Sowohl die Methode der Helium-Nanotröpfchen als auch die der Matrix-Isolation leidet unter einer methodenbedingten Verschiebung der Banden im Vergleich zum Gasphasenspektrum, was durch unvermeidbare Wechselwirkungen zwischen den zu untersuchenden Molekülen und ihrer Umgebung (Inertgas-Matrix, überkritisches Helium) hervorgerufen wird. Dieser Effekt fällt für Helium-Nanotröpfchen-Experimente schwächer aus und beträgt nur einige $\mathrm{cm}^{-1}$ [26], vergrößert sich aber für wasserstoffbrückengebundene Molekülaggregate, was als Helium solvent shift bekannt ist [27]. Die deutlich größeren Verschiebungen in der Matrix [28, 29], die in beide Richtungen ausfallen können, führen dazu, dass diese Technik für Untersuchungen, in denen eine gute Vergleichbarkeit von Theorie und Experiment benötigt wird (z. B. Benchmarking), nicht in Frage kommt [30].

Eine weitere Methode ist die freie Jet-Expansion, bei der die zu untersuchenden Substanz in einem großen Überschuss eines Trägergases (vornehmlich Helium) durch eine Düse in eine Vakuumkammer expandiert wird. Dies führt zu Beginn der Expansion zu einer schnellen adiabatischen Abkühlung der Substanz und somit zu kalten, isolierten Probemolekülen und Probemolekülaggregaten in der Trägergasatmosphäre. Die einzelnen Freiheitsgrade werden allerdings unterschiedlich effektiv gekühlt. So hat eine Messung an Stickstoff in Helium Translationstemperaturen von ca. $6 \mathrm{~K}$ und Rotationstemperaturen von ca. $20 \mathrm{~K}$ ergeben [31, 32]. Konformationstemperaturen von 100-120 K ergaben Messungen an Ethanol und Alkanen an derselben Apparatur [31, 33, 5) Dabei sind die Wechselwirkungen mit dem Trägergas stark

\footnotetext{
4) vgl. Eintrag in IUPAC - Compendium of Chemical Terminology, 2. Auflage (the "Gold Book"). Zusammengestellt von A. D. McNaught und A. Wilkinson. Blackwell Scientific Publications, Oxford (1997). Online Version (2019-) erstellt von S. J. Chalk. https://doi.org/10.1351/goldbook.S05910 (abgerufen: 02.04.2020).

${ }^{5)} \mathrm{Da}$ in der freien Jet-Expansion Bedingungen weit jenseits eines thermodynamischen Gleichgewichts herrschen, können die Temperaturen der einzelnen Freiheitsgrade nur separat abgeschätzt werden und es kann keine thermodynamische Temperatur angegeben werden.
} 
abhängig von der Wahl des Gases und für Helium minimal, aber z. B. für Argon nicht mehr vernachlässigbar, sobald es auf den Clustern zu kondensieren beginnt [33].

Durch die Bedingungen in einer solchen Jet-Expansion ist es bei einer hinreichend hohen Isomerisierungsbarriere (z. B. ca. $60 \mathrm{~kJ} \cdot \mathrm{mol}^{-1}$ für NMA [34, 35]) möglich, das PräexpansionsIsomerenverhältnis einzufrieren und so mit einer Konformationsanalyse mittels Van-'t-Hoff-Plot den Enthalpieunterschied zwischen den Isomeren zu bestimmen.

Somit ist die Kombination aus freier Jet-Expansion [25] mit schwingungsspektroskopischen Messmethoden [36-38] eine gute Möglichkeit, sowohl die cis-/trans-Isomerisierung als auch die Clusterbildung zu untersuchen und bildet die Grundlage für alle experimentellen Ergebnisse dieser Arbeit.

Insbesondere der Einsatz von IR-Spektroskopie zur Untersuchung von Peptiden ist schon seit den 50er Jahren des letzten Jahrhunderts etabliert [39-42] und auch die Verwendung der Raman-Spektroskopie ist in der Analytik von Biomolekülen eine bekannte Methode [43]. Für eine Zuordnung der Banden in den gemessenen Spektren ist, neben vorhandenen Literaturwerten für ähnliche Verbindungen, in der Regel ein Vergleich mit quantenchemischen Frequenzrechnungen unabdingbar. Hierzu werden den experimentellen Ergebnissen dispersionskorrigierte quantenchemische DFT-Rechnungen mit dem B3LYP-Hybridfunktional gegenübergestellt.

Eine besondere Herausforderung liegt für sekundäre Amide im Nachweis eines cis-cis-Dimers in der Gasphase. Hierbei ist eine Clustergeometrie mit zwei intermolekularen $\mathrm{N}-\mathrm{H} \cdots \mathrm{O}=\mathrm{C}$-Wasserstoffbrückenbindungen möglich und wurde in der Form in quantenchemischen Rechnungen für verschiedene Amide als stabilstes Dimer bestimmt [44, 45]. Es konnte jedoch für die genannten Modellverbindungen experimentell bisher nicht nachgewiesen werden. Da jedoch die zugrundeliegenden cis-Monomere im Allgemeinen weniger stabil als die entsprechenden transKonformere sind [46, S. 145] und damit das cis-/trans-Monomerverhältnis im Gleichgewicht weit auf der Seite der trans-Konformere liegt, ist der bisherige Nachweis vermutlich an der statistisch geringen Bildungswahrscheinlichkeit eines solchen Dimers gescheitert.

Zur Erhöhung des cis-Anteils sind verschiedene experimentelle Strategien bekannt. Dabei sind Techniken, wie z. B. der Einsatz von Lösungsmitteln [46, S. 151] oder die Veränderung des pH-Wertes [46, S. 152] für Gasphasenexperimente, ungeeignet. Andere Methoden, wie eine induzierte Isomerisierung mit UV-Laserpulsen [46, S. 161], sind mit einem apparativen Mehraufwand verbunden, der im Rahmen dieser Arbeit nicht realisierbar ist. Eine weitere experimentelle Strategie zur Erhöhung des cis-Anteils ist die Verschiebung des Gleichgewichts über die Temperatur des Substanz-Trägergas-Gemisches vor der Expansion. Die Vor- und Nachteile dieses Ansatzes werden in dieser Arbeit diskutiert (siehe Abschnitt 3.1.2).

Allerdings zeigen Untersuchungen zu sterisch anspruchsvoll substituierten sekundären Amiden wie $\mathrm{N}$-Phenylformamid (NPhF), dass der Anteil des cis-Konformers im Gleichgewicht bei Raumtemperatur hier deutlich höher liegt [47]. Die Substituenten der Amidgruppe haben also über sterische Effekte und intramolekulare Wechselwirkungen Einfluss auf den cis-/transEnergieunterschied. Dieser Substituenteneinfluss auf den Energieunterschied und damit auf das Verhältnis zwischen cis- und trans-Konformer soll ebenfalls in dieser Arbeit experimentell und theoretisch bestimmt werden. 
Einen weiteren Schwerpunkt bildet die Untersuchung höherer Amid-Cluster bis hin zum Tetramer, deren energetisch bevorzugte Konformation in der Gasphase bisher aus Kristallstrukturexperimenten [48] und quantenchemischen Rechnungen [49, 50] als kettenförmig abgeleitet wurde. Mit dieser Annahme zur Struktur ergeben sich jedoch z. B. bei der Interpretation spektroskopischer Clustermessungen von NMA Widersprüche [51]. Zur Klärung der Inkonsistenzen zwischen Theorie und Experiment bedarf es einer systematischen Untersuchung beider Seiten. Denn neben den hinlänglich bekannten Fehlerquellen in Durchführung und Auswertung von Experimenten, können die gewählten Vereinfachungen in den quantenchemischen Rechnungen ebenfalls zu falschen Ergebnissen führen. Es muss also sowohl die Theorie das experimentelle Ergebnis korrekt vorhersagen als auch das Experiment den Rechnungen genügen [52]. Um zu überprüfen, ob eine Vorhersage zutrifft, bedarf es genauer und vor allem vergleichbarer Daten aus dem Experiment. Daher wird in dieser Arbeit durch ergänzende Messungen und quantenchemische Rechnungen nach weiteren Dimer-, Trimer- und in Einzelfällen auch Tetramerstrukturen gesucht, die eine konsistente Dateninterpretation ermöglichen. Hierbei soll vor allem die Möglichkeit einer Bildung von zyklischen Clustern überprüft werden, wie sie bereits als intramolekularer Wasserstoffbrückenring bei $\gamma$-Peptiden mit drei Amidgruppen gefunden wurden [53].

Auch für das Amin Imidazol (Im) wird ein zyklisches Trimer mit drei N-H $\cdots \mathrm{N}$-Wasserstoffbrücken als stabilstes Trimer vorhergesagt [54]. Diese Vorhersage wurde in Folgestudien nicht weiter verfolgt [55]. Die inkonsistente Behandlung in der Literatur sowie die nicht eindeutig interpretierbaren FTIR-Spektren von Zischang [56] geben Anlass für eine ergänzende Ramanspektroskopische Untersuchung 6 (6)

Zuerst wird in dieser Arbeit die Isomerisierung und Aggregation für die Modellverbindungen NMA (siehe Kapitel 4) und NMF (siehe Kapitel 5) untersucht. Inwieweit der cis-Monomeranteil in der Jet-Expansion in Zusammenhang mit der spektroskopischen Nachweisbarkeit des cis-cisDimers steht sowie sterisch anspruchsvolle Substituenten zur Bildung signifikanter Mengen dieses Dimers führen, wird mit der Erweiterung der Experimente auf $N$-Phenylformamid (NPhF) untersucht. Der energetische Unterschied zwischen cis- und trans-Isomer wird für NMA, NMF und $\mathrm{NPhF}$ aus den Raman-Spektren einer Temperaturmessreihe mittels van 't-Hoff-Plot bestimmt. 2-Pyrrolidinon ( $\gamma$-Butyrolactam, gBL) ist ein zyklisches Amid, das aufgrund des Fünfrings einerseits wenig Spannung aufweist und damit stabil ist und andererseits die Konformationseinschränkung so groß ist, dass ausschließlich cis-cis-Dimere gebildet werden können. Es kann daher als Anhaltspunkt für die gesuchte experimentelle Dimerbandenposition dienen. Ein Trend für größere sekundäre Amide bis hin zu N-Propylbutanamid (NPB) wird mit DFT-Rechnungen abgeschätzt. Darüber hinaus wird als Alternative zu kettenförmigen Clustern aus transMonomeren die Möglichkeit der Bildung zyklischer Trimere und Tetramere für NMA, NMF und NPhF untersucht. Hierfür werden ebenfalls Raman- und FTIR-Jet-Messungen sowie DFT-Rechnungen durchgeführt. Zudem wird jenseits der Stoffklasse der Amide auch die Clusterbildung des zyklischen Amins Imidazol (Im) in die Untersuchung [57] und die abschließende Diskussion mit einbezogen (siehe Kapitel9).

\footnotetext{
6) Durchgeführt als ergänzendes Projekt zu dieser Arbeit: Forsting et al. [57].
} 
Die Ergebnisse dieser Arbeit wurden zum Teil bereits im Vorfeld publiziert (siehe Abschnitt A.1 im Anhang). 


\section{Grundlagen}

\subsection{Nomenklatur von Amiden}

Die Nomenklatur der Konformationsisomere sekundärer Amide erfolgt häufig gemäß der historischen cis/trans-Bezeichnung analog der IUPAC-IUB-Empfehlung zur Benennung von Peptiden [58]. Die Verwendung dieser Relativnomenklatur wird erst eindeutig durch den Bezug auf die Backbone-Reste R und $\mathrm{R}^{\prime}$ der Peptidkette. cis bezieht sich hierbei auf einen $\mathrm{R}-\mathrm{C}-\mathrm{N}-\mathrm{R}^{\prime}$ Diederwinkel $\omega$ von $0^{\circ}$, trans auf $\omega=180^{\circ}$. Dennoch bleibt die cis/trans-Nomenklatur für Amide ohne zusätzliche Konventionen nicht eindeutig bei unterschiedlichen Substituenten.

Eine allgemein anwendbare und den aktuellen IUPAC-Empfehlungen entsprechende Variante ist die $E / Z$-Nomenklatur. Hierbei werden die vier Substituenten der zu betrachtenden Bindung nach den CIP-Regeln priorisiert (eine detaillierte Beschreibung findet sich bei Dugave [59]) und es folgt eine eindeutige Nomenklatur, bei der die jeweils höchstpriorisierten Substituenten entweder „entgegen“-gesetzt $(E)$ positioniert sind, oder sich „zusammen“ $(Z)$ auf einer Seite der Diederachse befinden.

Die ansonsten fehlende direkte Entsprechung zwischen beiden Nomenklaturen ist im Falle der Benennung von Peptidbindungen in Proteinen und sekundären Alkyl- und Arylamiden gegeben: cis $\widehat{=} E$ und trans $\widehat{=} Z$. Dennoch finden sich in der Literatur auch Fälle, in denen die Bezeichnung der Isomere genau entgegen gesetzt erfolgt [45, 60].

Die verschiedenen Nomenklaturen sind in Abb. 2-1 den entsprechenden Konformeren zugeordnet. In dieser Arbeit wird durchgehend die cis/trans-Nomenklatur für Peptide gemäß IUPACIUB verwendet.

Wenn es um die Stellung der C- und N-seitigen Alkylreste von Amiden geht, greift die syn/anti-Nomenklatur der IUPAC. Während für die terminalen Methyltorsionen jeder Alkylkette nur zwei unterscheidbare Diederwinkel möglich sind: $0^{\circ} \widehat{=} \operatorname{syn}(\mathrm{s})$ und $180^{\circ}\left(=60^{\circ}\right) \widehat{=}$ anti (a), muss für alle weiteren Torsionen der Alkylkette zusätzlich die Bezeichnung gauche ( $\mathrm{g}$ ) für den Diederwinkel $60^{\circ}$ eingeführt werden. Als besonders relevant erweisen sich die zu $\omega$ benachbarten Diederwinkel, die in dieser Arbeit analog der Benennung der Winkel in Peptidketten und Proteinen gemäß IUPAC-IUB-Empfehlung [58] als $\varphi$ (N-seitig) und $\psi$ (C-seitig) bezeichnet werden. Hierbei wird sich immer auf die $\mathrm{C}-\mathrm{N}$-Amidbindung und den größten Alkylrest bezogen (siehe Abb. 2-1). Für längerkettige Alkylamide (wie z. B. in Abschnitt 7) werden die entlang der Kette auf $\varphi$ und $\psi$ folgenden Diederwinkel entsprechend mit $\varphi^{\prime}, \varphi^{\prime \prime}, \ldots$ und $\psi^{\prime}, \psi^{\prime \prime}, \ldots$ bezeichnet.

Die in dieser Arbeit verwendeten Abkürzungen für die Benennung der unterschiedlichen Konformere eines Amids lauten $t$ für jedes trans- und $c$ für jedes cis-Monomer, für die Bezeichnung 


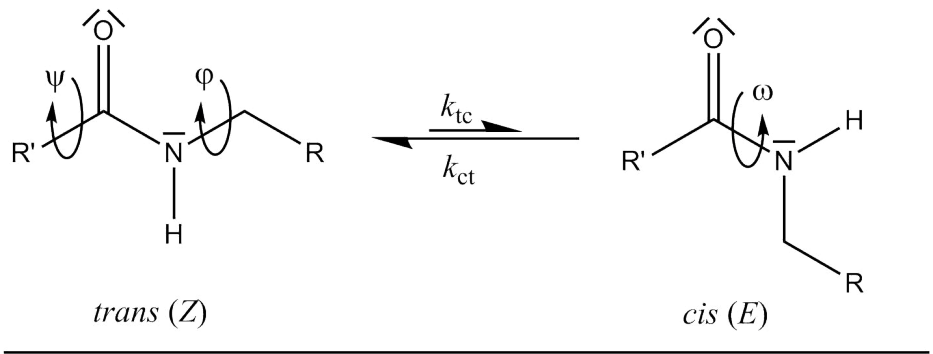

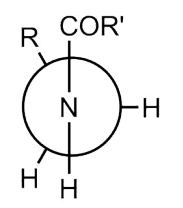

$\operatorname{syn}(s): \varphi=0^{\circ}$

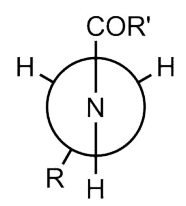

$\operatorname{anti}(a): \varphi=180^{\circ}$

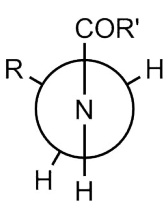

gauche $(g): \varphi=60^{\circ}$

Abb. 2-1. Mögliche Konformere sekundärer Amide und deren Nomenklatur. Der Zusammenhang zwischen cis-/transund $E / Z$-Nomenklatur wird ebenso gezeigt, wie die drei wichtigsten Diederwinkel $\omega, \varphi$ und $\psi$. Die syn-/anti- und gaucheStellungen von Alkylresten sind stellvertretend für $\varphi$ als Newman-Projektionen dargestellt (es gilt: $g \widehat{=} a$ für $R=H$ ).

von Clustern werden die Monomerbezeichnungen in Donor-Akzeptor-Richtung der Wasserstoffbrückenbindungen zusammengesetzt, so dass zum Beispiel ein cis-trans-Heterodimer mit dem trans-Monomer als Wasserstoffbrückendonor die Abkürzung tc erhält. Typischer H-Brückendonor ist die N-H-Gruppe und als zwei mögliche Akzeptorstellen dienen in der Regel die freien Elektronenpaare des Carbonyl-Sauerstoffs.

Zur näheren Bezeichnung der Alkylrestkonformation werden als Indizes die Abkürzungen $s, g$ und $a$ der Diederwinkel in der Reihenfolge $\varphi^{\prime}, \varphi^{\prime \prime}, \ldots, \psi^{\prime}, \psi^{\prime \prime}, \ldots$ verwendet. Wenn in bestimmten Fällen eine ergänzende oder abweichende Bezeichnung benötigt wird, ist dies ebenda vermerkt.

\subsection{Charakteristische Normalschwingungen sekundärer Amide}

In den Schwingungsspektren von Amiden und Peptiden finden sich eine Reihe von charakteristischen Banden, deren zugehörige Normalmoden sich hauptsächlich aus den unterschiedlichen lokalen Moden der Amidgruppe zusammensetzen. Diese insgesamt neun Amidbanden werden in Schwingungen in der Ebene der NHCO-Gruppe (Amid A, B, I-IV) und aus der Ebene heraus (Amid V-VII) unterteilt [4]. Die ursprüngliche Beschreibung geht auf Randall et al. [61] zurück und wurde im Rahmen einer IR-Studie [62] gepaart mit einer einfachen Normalmodenanalyse [63] erstmalig für das Modellpeptid $N$-Methylacetamid zugeordnet und später durch weitere Normalmodenanalysen [40, 64, 65] bis hin zu ab initio-Rechnungen für das vollständige Molekül [66] grundsätzlich bestätigt [67].

Die Lage insbesondere der niederfrequenten Banden kann abhängig vom untersuchten Molekül, dessen Aggregatzustand und der verwendeten Messmethode deutlich variieren.

Amid A/B Die Amid A-Bande entspricht zu nahezu 100\% lokalisierten N-H-Streckschwingung. Sie liegt als einzige Amidschwingung deutlich oberhalb von $3000 \mathrm{~cm}^{-1}$ und ist ein guter 
Indikator für Wasserstoffbrücken-gebundene Aggregate und Lösungsmitteleffekte [67]. Diese intermolekularen Wechselwirkungen führen zur Schwächung der N-H-Bindungsstärke und damit zu der typischen Verschiebung hin zu niedrigeren Wellenzahlen im Spektrum. Daher reicht die Amid A-Bande von ca. $3500 \mathrm{~cm}^{-1}$ für kleine isolierte Amide (z. B. $N$-Methylacetamid) im Jet [51] oder in der Argon- und Stickstoff-Matrix [68] bis $3300 \mathrm{~cm}^{-1}$ bei Peptiden in wässriger Lösung [4, 40].

Die N-H-Bindung ist typischerweise besonders IR-aktiv und daher schwingungsspektroskopisch gut zugänglich. Die Amid A-Bande ist zudem sehr sensitiv für die Molekülkonformation und liegt für sekundäre cis-Amide bei deutlich niedrigeren Wellenzahlen als das trans-Konformer (für NMA in der $\mathrm{N}_{2}$-Matrix [68]: $v_{\mathrm{NH}}^{c}=v_{\mathrm{NH}}^{t}-40 \mathrm{~cm}^{-1}$ ).

Für flüssige Amide sowie Amide und Peptide in Lösung findet sich bei ca. $3100 \mathrm{~cm}^{-1}$ die sogenannte Amid B-Bande [69]. Diese ist eine durch Fermi-Resonanz mit der N-H-Streckschwingung verstärkte Bande aus Oberton- oder Kombinationsbanden im Amid I-III-Bereich, deren genauer Ursprung kontrovers diskutiert wird [19, 70, 71]. Für kleine Amide in der Gasphase wird die Amid A zu höheren Wellenzahlen verschoben und die Resonanz nimmt ab [72], so dass für Gasphasen- und Jet-Spektren die Amid B schwache [69] bis keine [51, 68, 72,74] Intensität zeigt.

Amid I Die Amid I-Bande ist die fast vollständig lokalisierte C=O-Streckschwingung mit einem geringen Anteil CN-Streck- und je nach Normalmodenanalyse einer NCO- [19] oder CCN-Deformationsschwingung [40]. Die Carbonylgruppe ist als Wasserstoffbrückenakzeptor mit den beiden freien Elektronenpaaren als mögliche Akzeptorstellen ursächlich für die hohe Sensitivität der C=O-Streckschwingung für zwischenmolekulare Wechselwirkungen [41, 42] bei gleichzeitig hoher IR-Aktvität. Die Bandenposition schwankt daher zwischen 1740 und $1600 \mathrm{~cm}^{-1}$, abhängig vom Lösungsmittel und der erweiterten molekularen Umgebung und macht die Amid I zur Standardbande für die schwingungsspektroskopische Untersuchung von Peptiden [41, 42, 75]. Eine quantitative Analyse der Amid I-Bandenform erlaubt sogar Vorhersagen über die Sekundärstruktur von kleinen Peptiden und Proteinen [40], wobei die Methode ihre Grenzen hat [76].

Auch wenn Studien zur Bandenposition und -intensität der Amid I in Abhängigkeit von den Aminosäureresten in Peptiden eine Konformationsempfindlichkeit feststellen [77], lassen sich cis-Konformere von trans-Isomeren mit der Amid I-Bande üblicherweise nicht unterscheiden, da die Verschiebung im Spektrum zu gering ist [4, S. 79].

Amid II Die Amid II-Bande setzt sich hauptsächlich aus der gegenphasigen Kombination von $\mathrm{N}-\mathrm{H}$-Deformations- und C-N-Streckschwingung zusammen [40] und liegt zwischen 1580 und $1500 \mathrm{~cm}^{-1}$ [42, 78, 1) Sie ist deutlich weniger konformationssensitiv als Amid I [42], erfährt aber durch den NH-Knickschwingungsanteil eine moderate Erhöhung der Frequenz (Blauverschiebung) bei der Ausbildung von Wasserstoffbrückenbindungen [19]. Die Amid II ist in Ra-

\footnotetext{
1) Der genaue Ursprung der Amid II-Bande wurde kontrovers diskutiert. Eine gute Zusammenfassung der frühen Diskussion liefert Bellamy [79].
} 
manspektren von sekundären Amiden (mit der Ausnahme von $N$-Methylformamid) schwach bis gar nicht vorhanden [62, 69], kann aber mit UV-angeregter Präresonanz- und ResonanzRaman-Spektroskopie verstärkt werden [80, 81]. Die grundsätzliche Beobachtung, dass diese Bande nicht bei sekundären Amiden in der cis-Konformation vorhanden ist [62, 70], konnte zumindest für $N$-Methylacetamid in wässriger Lösung widerlegt werden [82] (siehe hierzu auch Abschnitt 4).

Amid III Bei der Amid III-Bande handelt es sich vereinfacht um die Kombination von $\mathrm{N}-\mathrm{H}-$ Deformations- und C-N-Streckschwingung in Phase [40]. Bei genauerer Betrachtung hat diese Bande eine durch die Kombination mit anderen lokalen Moden hervorgerufene komplizierte Substruktur aus mindestens drei Teilbanden [19, 42]. Die Position der Amid III ist stark struktur- und umgebungsbedingt und liegt zwischen 1340 und $1200 \mathrm{~cm}^{-1}$ [42], dabei zeigt sich eine deutliche Beeinflussung durch den Diederwinkel $\psi$ bei gleichzeitiger Unabhängigkeit von $\varphi[43,83]$. Durch die gleichphasige Schwingung ist die Änderung des Dipolmoments nur gering und daher ist die Amid III-Bande nur schwach IR-aktiv, allerdings im Ramanspektrum gut sichtbar [41, 42].

Amid IV Die Amid IV-Bande ist mit einer Frequenz um die $600 \mathrm{~cm}^{-1}$ die niederfrequenteste Amidbande in der Ebene. Sie setzt sich hauptsächlich aus der NCO-Knickschwingung $\left(\delta_{\mathrm{NCO}}\right)$ und der C-C-Streckschwingung $\left(v_{\mathrm{CC}}\right.$ ) zusammen [40]. In der Arbeit zu N-Methylacetamid von Herrebout et al. [19] wird die Bande bei ca. $800 \mathrm{~cm}^{-1}$, die bisher als $v_{\mathrm{CC}}$ bezeichnet wurde, ebenfalls als Amidbande eingeführt, da hier die C-N-Streckschwingung $\left(v_{\mathrm{CN}}\right)$ und auch $\delta_{\mathrm{NCO}}$ beteiligt sind. Aufgrund der Ähnlichkeit werden die beiden Banden als Amid IVa (für die $800 \mathrm{~cm}^{-1}$ ) und Amid IVb (für die $600 \mathrm{~cm}^{-1}$ ) bezeichnet.

Amid V-VII Diese drei Banden sind die klassischen Amidbanden, deren zugehörigen Schwingungen aus der Ebene herausführen [4]. Sie sind sehr variabel in Position und Intensität mit Frequenzen zwischen 900 und $400 \mathrm{~cm}^{-1}$ [4, 67, 84]. Die Amid V-Bande setzt sich hauptsächlich aus der $\mathrm{N}-\mathrm{H}$-Knickschwingung aus der Ebene $\left(\pi_{\mathrm{NH}}\right)$ und der $\mathrm{C}-\mathrm{N}$-Torsionsschwingung $\left(\tau_{\mathrm{CN}}\right)$ zusammen und ist daher stark durch Wasserstoffbrückenbindungen und demnach durch die Peptid-Sekundärstruktur, genauso wie durch die Molekülkonformation in direkter Umgebung beeinflusst [40]. Damit lassen sich Bandenpositionen zwischen $725 \mathrm{~cm}^{-1}$ für flüssiges [40] und $439 \mathrm{~cm}^{-1}$ [68] für NMA in der Stickstoff-Matrix genauso erklären, wie die Einschätzung dieser Bande als wenig hilfreich zur Strukturaufklärung von Peptiden [67]. Die Amid V ist außerdem nur schwach Raman aktiv [62, 69] und wird daher für die Amide in dieser Arbeit nicht genauer diskutiert.

Gleiches gilt für Amid VI, die neben der C=O-Knickschwingung aus der Ebene $\left(\pi_{\mathrm{CO}}\right)$ ebenfalls anteilig $\left(\tau_{\mathrm{CN}}\right)$ enthält und Amid VII, deren Zusammensetzung aus lokalen Moden in den verschiedenen PED-Rechnungen (Potential Energy Distribution) ganz unterschiedlich ausfallen [19, 40]. 
Weitere Amidbanden Neben den klassischen neun Amidbanden wurden eine Reihe weiterer Banden beschrieben, die hier nur der Vollständigkeit halber Erwähnung finden.

In UV-Resonanz-Raman-Spektren (UVRR) von sekundären Amiden wurde eine neue Bande bei ca. $1390 \mathrm{~cm}^{-1}$ nachgewiesen, die als Amid $S$ bezeichnet wird [85, 86]. Der Ursprung dieser Bande ist nicht endgültig geklärt und unterliegt unterschiedlichen - teils kontroversen - Interpretationen [19, 42, 85, 90]. Weiterhin werden von Herrebout et al. [19] die niederfrequenten Banden in $N$-Methylacetamid bei $276 \mathrm{~cm}^{-1}$ und $170 \mathrm{~cm}^{-1}$ als Amid VIII und Amid IX eingeführt, da beide Schwingungen in der sekundären Amidgruppe lokalisiert sind.

\subsection{Dichtefunktionaltheorie: Das B3LYP-Funktional}

Dichtefunktionaltheoretische (DFT) Methoden im Rahmen des Kohn-Sham-Formalismus [91, 92] liefern eine exakte Beschreibung des Elektronensystems unter Berücksichtigung der Elektronendichte. Hierbei werden alle nicht direkt-zugänglichen Teile der Gesamtenergie in einen Term namens Austausch-Korrelations-Energie $E_{X C}$ verschoben, für den es theoretisch ein eindeutiges Funktional für alle Systeme gibt.

Bei der in dieser Arbeit hauptsächlich verwendeten DFT-Methode B3LYP (Becke 3-Parameter, Lee, Young, Parr) handelt es sich um eine Kombination des Becke 3-Parameter-Hybridfunktionals [93] mit dem GGA-Korrelationsfunktional nach Lee, Yang und Parr [94]. B3LYP ist ein Austausch-Korrelationsfunktional auf Basis der Local Spin Density Approximation (LSDA), in dem $20 \%$ (Parameter $a_{0}$ ) der LSDA-Austauschenergie $E_{\mathrm{X}}^{\mathrm{LSDA}}$ durch die exakte Austauschenergie einer Hartree-Fock-Rechnung $E_{\mathrm{X}}^{\mathrm{HF}}$ ersetzt und mit dem Korrekturterm des Austauschfunktionals im Rahmen der General Gradient Approximation (GGA) nach Becke $\Delta E_{\mathrm{X}}^{\mathrm{B} 88}$ [95] teilweise (Parameter $a_{\mathrm{X}}$ ) ergänzt werden. Die LSDA-Korrelationsenergie $E_{\mathrm{C}}^{\mathrm{LSDA}}$ wird anteilig (Parameter $a_{\mathrm{C}}$ ) durch die Korrelationsenergie nach Lee, Yang und Parr [94] $E_{\mathrm{C}}^{\mathrm{LYP}}$ ersetzt (siehe Gleichung 2-1] [96].

$$
\begin{aligned}
& E_{\mathrm{XC}}=\left(1-a_{0}\right) E_{\mathrm{X}}^{\mathrm{LSDA}}+a_{0} E_{\mathrm{X}}^{\mathrm{HF}}+a_{\mathrm{X}} \Delta E_{\mathrm{X}}^{\mathrm{B} 88}+\left(1-a_{\mathrm{C}}\right) E_{\mathrm{C}}^{\mathrm{LSDA}}+a_{\mathrm{C}} E_{\mathrm{C}}^{\mathrm{LYP}} \\
& \text { mit: } a_{0}=0.20, \quad a_{\mathrm{X}}=0.72, \quad a_{\mathrm{C}}=0.81
\end{aligned}
$$

B3LYP basiert nicht auf einer eindeutigen Beschreibung durch eine bestimmte Veröffentlichung, sondern ist vielmehr aufgrund der guten Ergebnisse für eine Vielzahl von Fragestellungen eine beliebte Kombination von Austausch- und Korrelationsfunktional geworden, die dadurch Einzug in alle gängigen QM-Programmpakete in Form eines explizit definierten Funktionals erhielt [97]. Dies führt zu einigen Besonderheiten: So existiert keine Parametrisierung für B3LYP, sondern die ursprünglich für das Becke, 3-Parameter-Hybridfunktional mit der Korrelationsenergie nach Perdew und Wang [98] (B3PW91) gefitteten Parameter wurden für B3LYP direkt übernommen. Außerdem ist die LSDA-Austauschenergie je nach Programmpaket unterschiedlich implementiert. Dabei basiert diese immer auf Vosko, Wilk und Nusair (VWM) [99], jedoch liefern diese in ihrer Arbeit unterschiedliche Beschreibungen für $E_{\mathrm{C}}^{\mathrm{LSDA}}$, so dass die Be- 
schreibung der Korrelationsenergie von B3LYP-Rechnungen insbesondere in Gaussian nicht mit denen anderer Programme übereinstimmt.

Nahezu alle DFT-Methoden scheitern an der Beschreibung der $r^{-6}$-Abhängigkeit der Dispersionswechselwirkung vom Abstand $r$ zweier Atome und neigen entweder zu Überschätzung der Bindungsstärke (LSDA) oder sind vollständig repulsiv (GGA) [100, 101]. Zur richtigen Beschreibung von großen Molekülen und Molekülaggregaten, bei denen van-der-Waals-Wechselwirkungen eine nennenswerte Rolle spielen, wurden daher verschiedene Ansätze zur Dispersionskorrektur vorgestellt [102, 103], von denen sich der empirische DFT-D-Ansatz von Grimme [104] in der dritten Version DFT-D3 als besonders vorteilhaft erwiesen hat [105]. Dabei wird die DFT-Energie um eine empirische Dispersionsenergie korrigiert. Diese Korrekturenergie beinhaltet die mit dem Atomabstand gewichteten Dispersionskoeffizienten für jedes Atompaar und eine Dämpfungsfunktion (Becke-Johnson-Damping, kurz: BJ), die für sehr kleine Atomabstände Singularitäten umgeht und mit einem Cut-Off-Radius eine Mehrfacheinbeziehung von Korrelationseffekten vermeidet [106].

Auch wenn das B3LYP-D3(BJ)-Funktional in einem frühen universellen Bechmarking mit experimentellen und theoretischen Datensätzen nur mittelmäßig abschneidet [107], haben andere Untersuchungen gezeigt, dass es generell gut geeignet ist, zwischenmolekulare Wechselwirkungen und die zugehörigen Schwingungsspektren zufriedenstellend zu beschreiben [108110].

\subsection{Thermodynamik der Konformationsisomerie}

Die Konformationsisomerie behandelt die Umwandlung eines Konformers durch Drehung um eine Bindung in ein anderes. Bei den beschriebenen Konformeren handelt es sich in der Regel um lokale Minima auf der Potentialhyperfläche des Moleküls, die durch die Veränderung von Diederwinkeln ineinander überführt werden können. Im Vordergrund dieser Arbeit steht die Isomerisierung der Amidbindung, also die Drehung um den Diederwinkel $\omega$ mit den beiden, typischerweise als cis- und trans-Form bezeichneten, Konformeren des Amids.

Wenn die allgemeine Gleichgewichtsreaktion der Isomerisierung trans-Amid $\rightleftharpoons$ cis-Amid lautet, dann lässt sich die freie molare Standardreaktionsenthalpie $\Delta_{\mathrm{r}} G^{\ominus}$ über die Gleichgewichtskonstante $K$ der Isomerisierungsreaktion ausdrücken:

$$
\Delta_{\mathrm{r}} G^{\ominus}=-R T \ln K
$$

Durch die unterschiedliche Molekülgeometrie haben zwei Konformere eines Moleküls häufig sich voneinander unterscheidende Schwingungsspektren, so dass im Gleichgewicht das Peakflächenverhältnis $F$ gut separierter Banden bestimmt werden kann:

$$
F=\frac{F_{\text {cis }}}{F_{\text {trans }}}
$$

Dieses Verhältnis muss noch um die jeweiligen differentiellen Streuquerschnitte $\sigma^{\prime}$ korrigiert werden, um das Besetzungverhältnis $N_{\text {cis }} / N_{\text {trans }}$ zu erhalten, welches $K$ entspricht [35]: 


$$
K=\frac{\sigma_{\text {trans }}^{\prime}}{\sigma_{\text {cis }}^{\prime}} \cdot F=\exp \left[-\frac{\Delta_{\mathrm{r}} G^{\ominus}}{R T_{\mathrm{K}}}\right]
$$

Die linearisierte Form von Gleichung 2-5 lautet:

$$
\ln K=\ln F+\ln \left[\frac{\sigma_{\text {trans }}^{\prime}}{\sigma_{\text {cis }}^{\prime}}\right]=-\frac{\Delta_{\mathrm{r}} G^{\ominus}}{R} \cdot \frac{1}{T_{\mathrm{K}}}
$$

Unter der Annahme, dass die Standardreaktionsenthalpie $\Delta_{\mathrm{r}} H^{\ominus}$ und die Standardentropie $S^{\ominus}$ im untersuchten Temperaturbereich konstant sind, kann $\Delta_{\mathrm{r}} H^{\ominus}$ mit Hilfe der Van-'t-HoffBeziehung in der ersten Ulich'schen Näherung (Gleichung 2-7) abgeschätzt werden [111]. Sie ergibt sich aus der partiellen ersten Ableitung von Gleichung 2-6.

$$
\left(\frac{\partial \ln K}{\partial T}\right)_{p}=\frac{\Delta_{\mathrm{r}} H^{\ominus}}{R T^{2}} \Leftrightarrow\left(\frac{\partial \ln K}{\partial 1 / T}\right)_{p}=-\frac{\Delta_{\mathrm{r}} H^{\ominus}}{R}
$$

Anschaulich handelt sich hierbei um die Steigung einer linearen Auftragung von $\ln K$ über $1 / T$. Aus Gleichung 2-6 wird offensichtlich, dass die Streuquerschnitte nicht die Steigung und somit die Enthalpiebestimmung beeinflussen, sondern sich nur im Y-Achsenabschnitt der Auftragung niederschlagen. Daher kann zur Enthalpiebestimmung mittels Van 't-Hoff-Plots direkt das Peakflächenverhältnis $F$ als Auftragung von $\ln F$ über $1 / T$ verwendet werden. Folglich beruhen die bestimmten Enthalpien auf rein experimentellen Ergebnissen.

Für die Auftragung des Van' t-Hoff-Plots ergibt sich der Fehlerbalken in $y$-Richtung, $\Delta \ln K$, unter Vernachlässigung möglicher Unsicherheiten in der Streuquerschnittsbestimmung nur aus der Abschätzung der Fehlergrenzen in der Bestimmung der Peakflächen $F_{c i s}$ und $F_{\text {trans }}$ und ist somit äquivalent zu $\Delta \ln F$ :

$$
\Delta \ln K \equiv \Delta \ln F=\left|\frac{\partial \ln F}{\partial F} \cdot \Delta F\right|=\frac{\Delta F}{F}
$$

mit:

$$
\Delta K \equiv \Delta F=\left|\frac{\partial F}{\partial F_{\text {cis }}} \cdot \Delta F_{\text {cis }}\right|+\left|\frac{\partial F}{\partial F_{\text {trans }}} \cdot \Delta F_{\text {trans }}\right|=\left|\frac{\Delta F_{\text {cis }}}{F_{\text {trans }}}\right|+\left|\frac{\Delta F_{\text {trans }} \cdot F_{\text {cis }}}{F_{\text {trans }}^{2}}\right|
$$





\section{Experimentelle und theoretische Methoden}

\subsection{Raman-Spektroskopie: Der curry-Jet}

\subsubsection{Aufbau und Funktionsweise}

Der Prototyp des curry-Jets wurde ursprünglich von P. Zielke [18] entworfen und gebaut und von T. Wassermann maßgeblich weiterentwickelt [31]. Weitere Ergänzungen stammen von N. Lüttschwager (erste heizbare Substanzzuführung) [32], K. Otto (neue Kammer aus Edelstahl, Prototyp eines verbesserten heizbaren Sättigers, erste IR-Raman-Doppelresonanz-Experimente mit Optisch-Parametrischem Oszillator (OPO)) [112] und S. Bocklitz (OPO-Optimierung, Käfighalterung für Optiken) [113].

Der Gesamtaufbau des curry-Jets ist in Abb. 3-1 dargestellt.1)

In der Apparatur wird eine Substanz in einem inerten Trägergas durch eine Düse kontinuierlich expandiert. Diese Expansion wird in definiertem Abstand $d_{\mathrm{D}}$ mittels Dauerstrichlaser $(532 \mathrm{~nm}, 20 \mathrm{~W}$ oder $25 \mathrm{~W})$ bestrahlt, das Streulicht in $90^{\circ}$-Streugeometrie mit einem KameraNormalobjektiv ( $f=50 \mathrm{~mm}, f / 1.2$ ) kollimiert, nach Verlassen der Kammer mit einer achromatischen Sammellinse $(f=350 \mathrm{~mm}, \varnothing=50 \mathrm{~mm})$ über einen Monochromator $(f=1000 \mathrm{~mm}$; f/8.7, 1200 Linien/mm Gitter) spektral getrennt und mit einer CCD-Kamera $(1340 \times 400 \mathrm{px}$; $-120^{\circ} \mathrm{C}$ ) detektiert.

Der Stoffmengenanteil $x_{\text {sub }}$ in der expandierten Gasmischung wird über das Partialdruckverhältnis von Substanz und Trägergas in einem thermostatisierten Sättigersystem gesteuert. Als Einflussparameter dienen hier der Druck des Trägergases $p_{\text {sat }}$ und der Dampfdruck der Substanz $p_{\text {sub }}$, welcher über die Sättigertemperatur $\vartheta_{\text {sat }}$ eingestellt wird.

Das Gasgemisch wird in ein Edelstahlreservoir (4.7 L, max. $25^{\circ} \mathrm{C}$ ) geleitet und von dort durch eine Schlitzdüse $\left(4.0 \times 0.15 \mathrm{~mm}^{2}\right)$ in die Kammer expandiert. Der Stagnationsdruck $p_{\mathrm{s}}$ im Reservoir wird mit einem über einen Druckmesskopf gesteuertes Magnetventil eingestellt. Dies führt zu einem quasi-kontinuierlichen Betrieb des Sättigers, so dass der tatsächliche Partialdruck der Substanz unterhalb ihres Dampfdrucks liegt, da sich bei annährend kontinuierlicher Durchströmung mit dem Trägergas der Dampfdruck nicht vollständig einstellen kann.

Für einen konstanten Hintergrunddruck $p_{\mathrm{BG}}$ in der Vakuumkammer von ca. $10^{-2} \mathrm{mbar}$ (ca. 1 mbar während der Expansion) sorgt ein Pumpstand mit zwei Wälzkolbenpumpen (max. $560 \mathrm{~m}^{3} / \mathrm{h}$ und max. $270 \mathrm{~m}^{3} / \mathrm{h}$ ) und einer Drehschieberpumpe (max. $100 \mathrm{~m}^{3} / \mathrm{h}$ ) als Vorpumpe.

\footnotetext{
${ }^{1)}$ Teile des Setups wurden im Rahmen dieser Arbeit modifiziert oder ersetzt. Für eine genaue Rekonstruktion, welche Daten mit welchen Konfigurationen aufgenommen wurden, siehe Tab. A-6 in Anhang A.6.1
} 


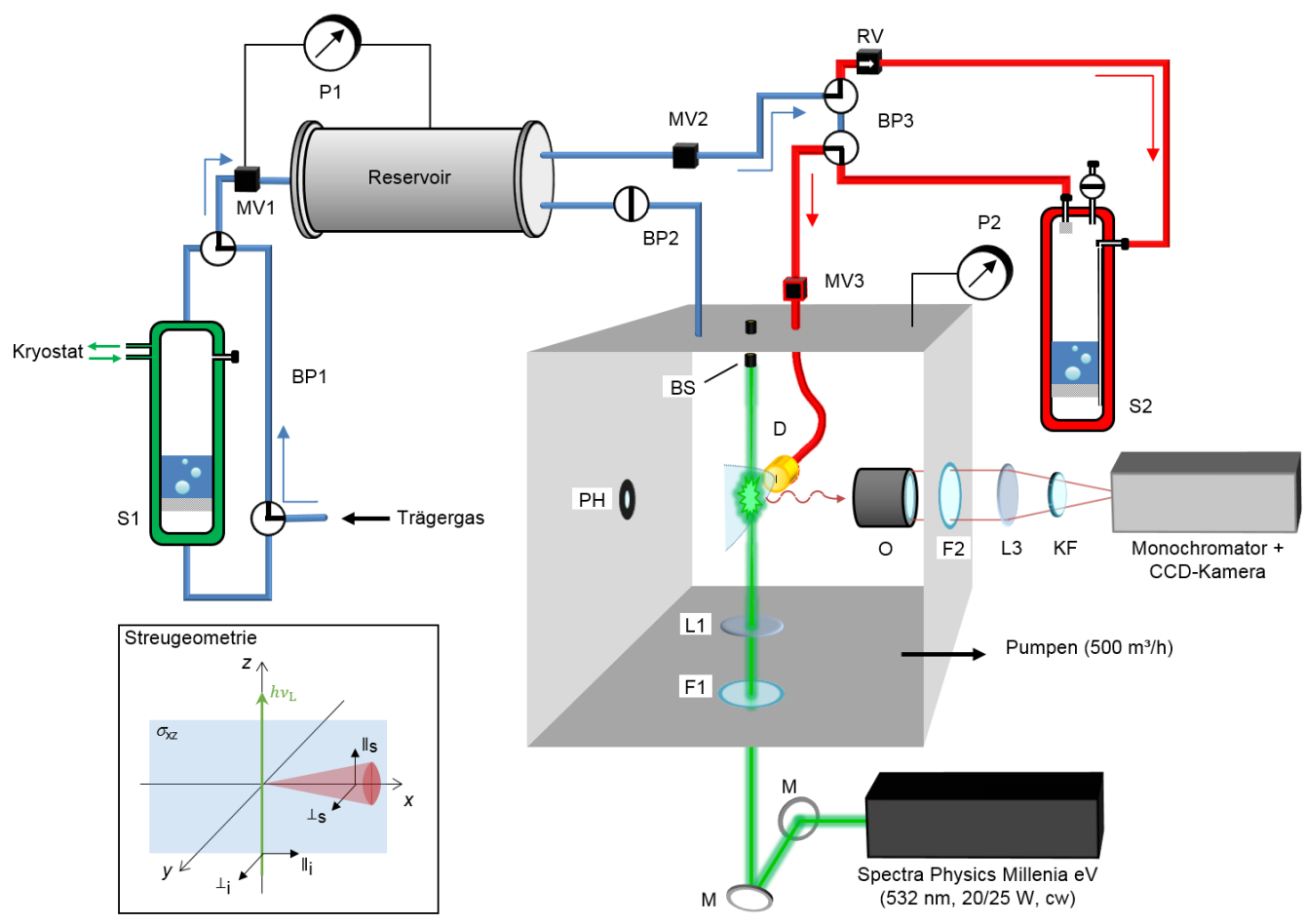

Abb. 3-1. Schematischer Aufbau des curry-Jets (BP: Bypass, BS: Strahlfänger, D: Düse, F: Fenster, KF: Kantenfilter, L: Linse, M: Laserspiegel, MV: Magnetventil, P: Druckmesskopf, O: Objektiv, S: Sättiger, PH: Pinhole).

Teilabbildung: Streugeometrie mit den Richtungspfeilen der senkrechten $(\perp)$ und parallelen $(\|)$ Polarisation zur Streuebene $\sigma_{\mathrm{xz}}$ vom eingestrahlten Laser (Index i) und dem Streulicht (Index s). (veröffentlicht unter: Forsting, Thomas; Suhm, Martin (2019): Curry-Jet SETUP. figshare. Dataset. (DOI: 10.6084/m9.figshare.6395840.v1), publiziert unter der CC BY 4.0 Lizenz [https://creativecommons.org/licenses/by/4.0/]) 
Für eine Überschall-Jet-Expansion wird eine Düse mit einer Öffnung größer als die mittlere freie Weglänge der Trägergasmoleküle zur effektiven Kühlung der Expansion durch anfängliche Kollisionen benötigt und es muss der Hintergrunddruck der Kammer mindestens halb so so groß wie der Stagnationsdruck in der Düse sein, um die Dichte der Expansion und damit die lokale Schallgeschwindigkeit unter die Expansionsgeschwindigkeit (Zone of Silence) abzusenken $[32,114]$. Im Überschall befinden sich die Moleküle nicht mehr im thermischen Gleichgewicht und haben somit auch keine allgemeine Temperatur mehr. Lokale Boltzmann-Verteilungen in der Besetzung von Schwingungs- und Rotationsniveaus sowie Maxwell-Boltzmann-Geschwindigkeitsverteilungen parallel und quer zur Expansionsrichtung erlauben die Definition von unabhängigen Temperaturen für die Translation, die Vibration und die Rotation des Moleküls [115].

Die Verwendung einer mit der zu untersuchenden Substanz dotierten Edelgasexpansion führt zu einer sehr effektiven Kühlung dieser Jet-Expansion, da auf Grund des Überschusses und der nicht vorhandenen inneren Freiheitsgrade der Edelgasatome nahezu jede Kollision von Analytmolekülen und Trägergas zur Kühlung insbesondere der Translation führt, die im Idealfall in der Zone of Silence bei $0 \mathrm{~K}$ liegt und einer uniformen Molekülbewegung ohne zwischenmolekulare Wechselwirkung entspricht. Tatsächlich wurde für einen mit $\mathrm{N}_{2}$-dotierten (1\%) He-Molekularstrahl im curry-Jet die Translationstemperatur zu ca. $6 \mathrm{~K}$ bestimmt, während die Rotationstemperatur mit ca. $20 \mathrm{~K}$ leicht darüber liegt [31]. Die Kühlung von Schwingungsfreiheitsgraden im Überschall-Jet ist deutlich weniger effektiv und liefert Temperaturen zwischen $120 \mathrm{~K}$ [31, 33] bis hin zur Temperatur der Düse. Für Konformationsänderungen ist eine Relaxation der Zustände abhängig von der Höhe der Isomerisierungsbarriere, so dass für hohe Barrierenenergien das Besetzungsverhältnis unter Expansionsbedingungen „eingefroren“ werden kann [116].

Für alle curry-Jet-Messungen in dieser Arbeit wurde Helium als Trägergas verwendet. Schwere Edelgase führen zwar zu einer besseren Kühlung, allerdings ist Neon auf Grund der Weltmarktpreise nicht realistisch einsetzbar und Argon sowie schwerere Edelgase neigen zur Clusterbildung mit der zu untersuchenden Substanz [33].

Der optimale Abstand des Lasers zur Düse im curry-Jet hängt von den gewählten Expansionsbedingungen ab. Je größer der gewählte Düsenabstand bei ansonsten gleichen Messbedingungen ist, desto mehr größere Cluster sind in der Expansion gebildet und können gemessen werden. Gleichzeitig sinkt die absolute Konzentration im Messbereich schnell ab. Die ÜberschallExpansion ist in Propagationsrichtung durch die normale Stoßfront mit warmem Hintergrundgas begrenzt. Für die vorliegende Düsenkonfiguration wurde von Wassermann et al. [108] die Lage der normalen Stoßfront für die Expansion mit Helium auf einen Düsenabstand von $d_{\mathrm{D}} \geq$ $5 \mathrm{~mm}$ bestimmt. Der tatsächlich sinnvolle Düsenabstand liegt allerdings im Bereich von $d_{\mathrm{D}}=$ 1-3 mm, da für kleinere Abstände des Lasers zur Düse das durch die Düsenspitze hervorgerufene Streulicht zu groß wird und für über $3 \mathrm{~mm}$ die Dichte der Expansion zu gering für den Raman-spektroskopischen Nachweis ist.

Für optimale Messbedingungen müssen die Anregungsoptik, die Düse und die Streulichtsammeloptik justiert werden. Dies geschieht über Mikroaktuatoren (Mindestschrittweite: < 
$0.5 \mu \mathrm{m}$ ), mit denen die Düse in $x / z$-Richtung und das Objektiv in alle drei Raumrichtungen verstellt werden können. Zur Bestimmung des Düsenabstandes $d_{\mathrm{D}}$ zum Laser wird die absolute $y$-Position der Düse mit einem hochpräzisen Aktuator (Mindestschrittweite: $0.1 \mu \mathrm{m}$, Auflösung: $0.035 \mu \mathrm{m}$ ) eingestellt und über eine Neon-Emissionslampe am Pinhole (PH) auf das Objektiv justiert [31].

Details zu den Spezifikationen der verwendeten Geräte sowie technischen und optischen Bauteile finden sich im Anhang in Tab. A-3.

\subsubsection{Heizbare Substanzzuführung}

Eine große Beschränkung des curry-Jets in seiner oben beschriebenen Standardausführung liegt in der Limitierung der Sättigertemperatur. Da alle Komponenten nach dem Sättigersystem nicht temperierbar sind, muss der Sättiger unterhalb der Raumtemperatur betrieben werden, um ungewollte Kondensation der Substanz in Schläuchen, im Reservoir und vor allem an der Düse zu vermeiden. Dies schließt schwerflüchtige Substanzen mit niedrigem Dampfdruck von der Untersuchung aus.

Daher wurde zur Raman-spektroskopischen Untersuchung von Alkanen [117] von N. Lüttschwager ein erster passiv geheizter Prototyp eines heizbaren Sättigersystems mit heizbarer Substanzführung und Düse entwickelt und durch eine zweite aktiv-geheizte Version ersetzt [32]. Die damit einhergehenden Probleme und eine Weiterentwicklung des Systems beschreibt K. Otto [112]. Die von ihr entwickelte dritte Version brachte viele Vorteile, erwies sich jedoch als weiterhin problematisch: Es war nur schwer möglich die Substanzkonzentration während einer Messung konstant zu halten. Insbesondere in der Nähe des Schmelzpunkts gab es immer wieder Rekristallisierung an lokal kälteren Stellen (vor allem unterhalb der Fritte). Außerdem griffen reaktivere Stoffe wie z. B. Amide den Messingkern des Sättigers an. Zudem war die Temperatur der Düsenzuleitung auf Grund des verwendeten 4/2-X-Kugelhahns auf maximal $80^{\circ} \mathrm{C}$ beschränkt, was unterhalb der Möglichkeiten des Sättigers liegt und für einige der in dieser Arbeit untersuchten Substanzen zu gering ist.

Die in diesem Kapitel vorgestellte Weiterentwicklung des heizbaren Sättigersystems löst die oben beschriebenen Probleme zufriedenstellend, erfolgte allerdings graduell, so dass nicht alle hier vorgestellten Ergebnisse mit den gleichen Systemkomponenten gemessen wurden (siehe Tab. A-6 in Anhang A.6.1). Die konstantesten Messergebnisse für verschiedene Sättigertemperaturen wurden erreicht, wenn der curry-Jet auch in den Heizphasen kontinuierlich weiterbetrieben wurde und die Scans aus dem Übergangsbereich zwischen Start- und Zieltemperatur in der Auswertung einzeln selektiert wurden.

Diese vierte Version des heizbaren Systems übernimmt grundlegende Elemente seiner Vorgängerversionen, wie die heizbare Düse, die Sättigergeometrie der Version 3 und die Positionierung im Gesamtsystem. Hauptveränderung stellt der neue Sättiger (siehe Abb. 3-2) dar, der nun komplett aus Edelstahl gefertigt ist. Er verfügt zusätzlich über einen Einlassstutzen zur Substanzzuführung (B), einen Auslass- (C) sowie einen Belüftungssstutzen (A). Außerdem sind alle Ein- und Auslässe nun mit Kugelhähnen verschließbar. 


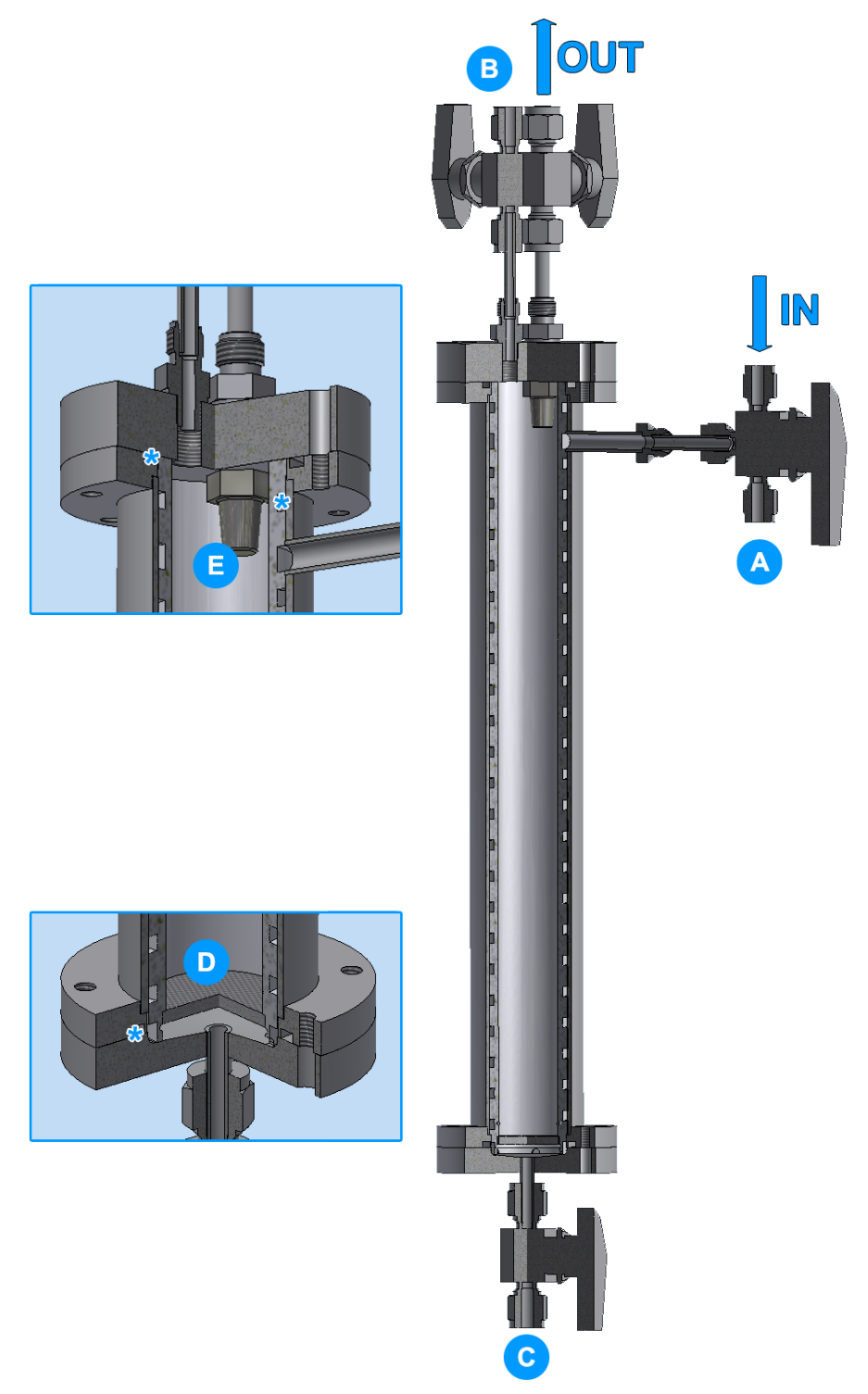

Abb. 3-2. Skizze des heizbaren Sättigers (Version 4). Edelstahlgehäuse mit zylindrischem Edelstahlkern. (Trägergaszuleitung (IN), Ausgang zur Düse (OUT), Belüftungsstutzen (A), Einfüllstutzen für Substanzen (B), Ablasshahn (C), Edelstahlfritten unter der Substanz (D) und am Ausgang (E), Viton ${ }^{\circledR}$-Ringe $\left(^{*}\right)$ ) 
Das Totvolumen unterhalb der Fritte wurde reduziert und die Heizleistung deutlich gedrosselt, um ein lokales Überheizen auszuschließen, denn das Thermoelement des PID-Reglers wurde nun im Sättigerboden befestigt, so dass auf den nominell kältesten Punkt des Sättigers geregelt wird. Der Substanzauslass im Deckel des neuen Sättigers wurde mit einer Edelstahlfritte (E) ausgestattet, um eine Mitnahme von Substanztröpfchen oder -partikeln im Gasstrom zu vermeiden. Damit die Substanz nicht am Deckel kondensiert, wurde hier eine Heizpatrone mit gesonderter PID-Regelung eingelassen. Zusätzlich zu den Veränderungen am Sättiger wurde der verbaute x-Hahn (siehe K.Otto [112]) am Verteiler des Bypasses BP3 durch zwei 3/2-Kugelhähne (Swagelok) ersetzt. Ein durch Fehlbedienung der Hähne nun möglicher ungewollter Rückschlag der Substanz in Richtung Reservoir könnte das Magnetventil MV2 beschädigen. Daher wurde zur Sicherheit ein Rückstoßventil RV (SS-6C-MM-1/3, Swagelok) dazwischen eingebaut.

Mit diesen Änderungen ist das Temperaturlimit des Sättigersystems nun von den verwendeten Gummidichtungen (Viton Extreme ETP (Vi982), Dupont/ C. O. Gehrkens) im Sättiger und der Düse abhängig und erhöht sich dadurch auf $230^{\circ} \mathrm{C}$. Es wurde zudem die Nutzung von PID-Reglern zur Temperaturregelung auf die gesamte Substanzzuführung ausgeweitet. Die Flexschläuche am Sättiger wurden durch eine Edelstahlverrohrung ersetzt, um die Ablagerung sehr schwerflüchtiger Substanzen in den Schlauchfalten zu vermeiden.

Alle Varianten des heizbaren Sättigers platzieren diesen hinter dem Reservoir, da dieses nicht heizbar ist. Das führt dazu, dass der Trägergas-Partialdruck gleich dem Stagnationsdruck ist $\left(p_{\text {sat }}=p_{\mathrm{s}}\right.$ ). Damit geht ein unabhängiger Messparameter verloren, da nun eine Veränderung des Stagnationsdrucks immer auch eine Änderung der Substanzkonzentration $c_{\text {sub }}$ nach sich zieht (dabei führt eine Absenkung von $p_{\mathrm{s}}$ zu einer Erhöhung von $c_{\text {sub }}$ ).

Das heizbare System besteht aus fünf Segmenten und kann in zwei Teile unterteilt werden (siehe Abb. 3-3):

a) Die Zuleitung verläuft vom Verteiler zum Sättiger $(\mathbf{I}, 60 \mathrm{~cm})$. Sie dient zum Vorheizen des Trägergases und entspricht zusammen mit der inneren Wendel des Sättigers $(256 \mathrm{~cm})$ einer Vorheizstrecke von ca. $316 \mathrm{~cm}$.

b) Die Rückleitung verläuft vom Sättiger zum Verteiler (II, $43 \mathrm{~cm}$ ), weiter über die Verbindung vom Verteiler zum heizbaren Magnetventil MV3 (III) und vom Magnetventil bis zur Kammerdurchführung DF (IV, $91 \mathrm{~cm}$ inklusive III und MV3), sowie innerhalb der Kammer über die Verbindung von der Durchführung zur Düse $(\mathrm{V}, 81 \mathrm{~cm})$. Zusammen mit dem Sättiger $(29 \mathrm{~cm})$, der Durchführung $(11 \mathrm{~cm})$ und dem Düsenkörper $(10 \mathrm{~cm})$ ergibt dies eine Heizstrecke von $265 \mathrm{~cm}$.

Jedes Segment I-V verfügt über ein eigenes Thermoelement mit PID-Regler (Eurotherm 3216, Schneider Electric oder E6C, RS Components). Im regulären Messbetrieb wurde die Temperatur der Vorheizstrecke und des Sättigers auf die gewünschte Sättigertemperatur $\vartheta_{\text {sat }}$ und die Temperaturen des Sättigerdeckels $\vartheta_{\mathrm{D}}$ und der Heizstrecke samt Düse $\vartheta_{\mathrm{H}}$ mindestens $10^{\circ} \mathrm{C}$ über der des Sättigers eingestellt, um eine Kondensation der Substanz in der Apparatur zu verhindern. 


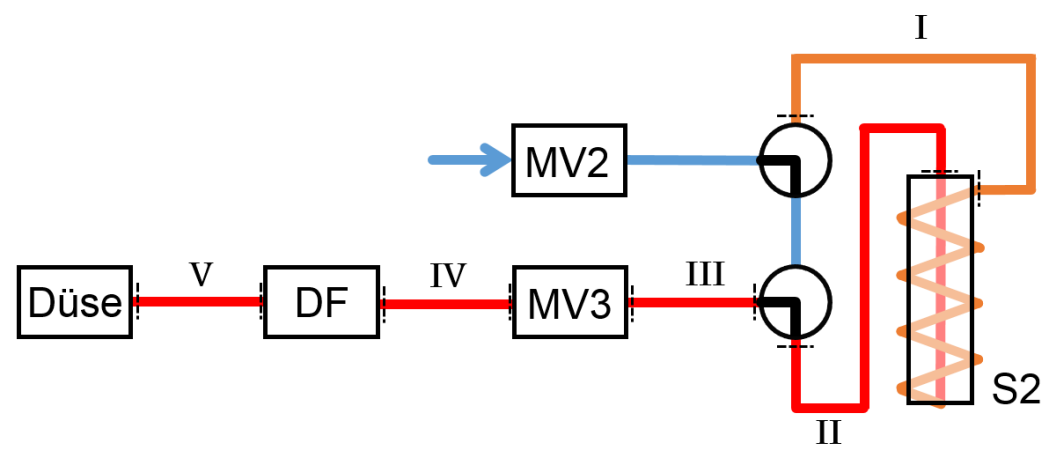

Abb. 3-3. Schematische Darstellung der heizbaren Zuleitung des curry-Jets. Die Segmente $\mathbf{I}-\mathbf{V}$ sind separat heizbar. (MV: Magnetventil; S2: heizbarer Sättiger; DF: Kammerdurchführung; blau: nicht heizbar; orange: Vorheizstrecke; rot: Heizstrecke)

\subsubsection{Anregungsoptik}

Zur Anregung wurde ein frequenz-verdoppelter Nd:YVO mit einer maximalen Leistung $P_{\mathrm{L}} \leq 25 \mathrm{~W}$ eingesetzt. ${ }^{2)}$ Dieser wurde im Rahmen der vorliegenden Arbeit und der Arbeit von S. Bocklitz [113] neu beschafft und wird daher im Folgenden genauer beschrieben und charakterisiert.

Grundsätzlich kann kohärente Laserstrahlung in sehr guter Näherung mit der Gaußschen Strahlenoptik beschrieben werden. Ein Gaußstrahl ist eine Lösung der paraxialen Helmholtzgleichung, dessen optische Intensität $I$ dem Quadrat seiner komplexen Amplitude entspricht. Diese Intensität $I(\rho, z)$ (mit der radialen Position $\rho=\sqrt{x^{2}+y^{2}}$ ) ist senkrecht zur Ausbreitungsrichtung $z$ normalverteilt (Gl. 3-1) [118].

$$
I(\rho, z)=I_{0}\left[\frac{w_{0}}{w(z)}\right]^{2} \cdot \exp \left[-\frac{2 \rho^{2}}{w^{2}(z)}\right]
$$

Für $\rho=w(z)$ nimmt die radiale Intensität auf das $1 / \mathrm{e}^{2}$-fache $\mathrm{ab}$, was als Strahlradius definiert ist. An der Strahltaille bei $z=0$ wird der Taillenradius als $w_{0} \equiv w(z=0)$ definiert.

In Ausbreitungsrichtung verbreitert sich der Strahl ausgehend von $z=0$ mit dem Divergenzwinkel $\theta_{0}$, so dass entlang der $z$-Achse ein Lorentz-verteiltes Intensitätsprofil mit einem Maximum $I_{0}$ in der Strahltaille bei $z=0$ erhalten wird. Als Rayleighlänge $z_{0}$ eines Gaußstrahls wird genau die Entfernung von der Strahltaille bezeichnet, bei der die Intensität entlang der $z$ Achse auf $\frac{1}{2} \cdot I_{0}$ abgefallen ist. Gleichzeitig hat sich der Strahl auf das $\sqrt{2}$-fache des Taillenradius aufgeweitet.

Der Radius eines Gaußstrahls an beliebiger $z$-Position $w(z)$ ist abhängig von seinem Taillenradius $w_{0}$, seiner Rayleighlänge $z_{0}$ und der Wellenlänge $\lambda$ gemäß Gleichung 3-2 [118].

\footnotetext{
${ }^{2)}$ Es wurden tatsächlich zwei baugleiche Spectra-Physics Milennia eV Laser mit Maximalleistungen von $20 \mathrm{~W}$ und $25 \mathrm{~W}$ verwendet. Siehe dazu auch Tabellen A-3 und A-6 im Anhang.
} 


$$
\begin{aligned}
w(z) & =w_{0} \sqrt{1+\left(\frac{z}{z_{0}}\right)^{2}} \\
z_{0} & =\frac{w_{0}^{2} \pi}{\lambda}
\end{aligned}
$$

Der emittierte Laserstrahl weist laut Hersteller einen Durchmesser von $2 w_{0}=2.3 \mathrm{~mm} \pm 10 \%$ bei einer Divergenz von $2 \theta_{0}<0.5 \mathrm{mrad} \pm 10 \%$ auf. Dies entspricht gemäß Gleichung $3-3$ einer Rayleighlänge von $z_{0}=7.81 \mathrm{~m}$. Im curry-Jet wird der Laser mit zwei Spiegeln $\mathbf{M}$ umgelenkt und tritt durch das Fenster F1 in die Messkammer ein (vgl. 3-1). An der Stelle $z=1 \mathrm{~m}$, und damit innerhalb der Rayleighlänge $\left(z<z_{0}\right)$, wird der Strahl mit einer Linse (L1, $f=50 \mathrm{~mm}$ ) fokussiert. Die resultierende bildseitige Brennweite $f^{\prime}$ einer Linse ist wellenlängenabhängig und wird vom Hersteller mit $f^{\prime}=54.6 \mathrm{~mm}$ für $\lambda=546 \mathrm{~nm}$ angegeben und hier in Gleichung 3-5 näherungsweise eingesetzt.

Der Strahlradius im bildseitigen Fokus einer dünnen Linse, $w_{0}^{\prime}$, hängt im Allgemeinen über den Vergrößerungsfaktor $M$ mit $w_{0}$ zusammen [118]:

$$
\begin{aligned}
& w_{0}^{\prime}=M \cdot w_{0} \\
& M=\frac{f^{\prime}}{\sqrt{\left(z-f^{\prime}\right)^{2}+z_{0}^{2}}}
\end{aligned}
$$

Der Vergrößerungsfaktor ist über Gleichung 3-5 definiert und beträgt $M=6.9 \cdot 10^{-3}$. Daraus ergibt sich ein Strahldurchmesser im fokussierten Laserstrahl von $2 w_{0}^{\prime}=16 \mu \mathrm{m}$.

Die Leistung eines Laserstrahls $P$ ist die Integration der Intensität über eine Kreisfläche in der $(x, y)$-Ebene mit unendlich großem Radius $\rho \rightarrow \infty$ :

$$
P=\int_{0}^{\infty} I(\rho, z) 2 \pi \rho \mathrm{d} \rho=\frac{I_{0}}{2} \cdot \pi w_{0}^{2}
$$

Die Laserspiegel haben laut Hersteller jeweils eine Reflektivität $R=99.8 \%$, das Einkoppelfenster und die Fokuslinse jeweils $R<0.5 \%$. Unter der näherungsweisen Annahme, dass für die Transmission $T=1-R$ gilt, ergibt sich nach dem Passieren aller vier Komponenten eine reduzierte Laserleistung $P_{\text {red }}$ von $98.6 \% \cdot P_{\mathrm{L}}=24.65 \mathrm{~W}$. Gleichung 3-6 ergibt für die Intensität im Laserfokus: $I_{0}^{\prime}=245 \mathrm{GW} \cdot \mathrm{m}^{-2}$ und für den unfokussierten Strahl mit voller Laserleistung $P_{\mathrm{L}}$ $=25 \mathrm{~W}: I_{0}=12.0 \mathrm{MW} \cdot \mathrm{m}^{-2}$ und ist somit mehrere Größenordnungen unterhalb der Zerstörschwelle der Beschichtungen auf den optischen Komponenten (vgl. hierzu die entsprechenden Angaben in Tab. A-3). Somit können die bisherigen optischen Komponenten ohne Einschränkung auch mit dem neuen Laser verwendet werden.

Die Emissionswellenlänge $\lambda_{\mathrm{L}}$ des Millennia $\mathrm{eV}$-Lasers ist von Herstellerseite mit $532 \mathrm{~nm}$ eher ungenau ${ }^{3)}$ angegeben und muss daher gesondert kalibriert werden (siehe Abschnitt 3.1.6). Ab-

\footnotetext{
${ }^{3)}$ Bei einem Größtfehlerannahme von $\pm 0.5 \mathrm{~nm}$ entspräche dies einer Ungenauigkeit von $\pm\left(2 \cdot 10^{1}\right) \mathrm{cm}^{-1}$
} 
bildung 3-4 zeigt die Laserlinie als Rayleighlinie in Luft bei $P_{\mathrm{L}}=25 \mathrm{~W}$, die im curry-Jet bei unterschiedlichen Eintrittspaltöffnungen des Monochromators gemessen wurde und ergibt $\lambda_{\mathrm{L}}=$ $532.259 \mathrm{~nm}\left(10 \mu \mathrm{m}\right.$ Eintrittsspalt, entspricht $\left.18787.8 \mathrm{~cm}^{-1}\right)$. Die Messungenauigkeit entspricht dabei mindestens der spektralen Breite eines Pixels von $d_{\mathrm{px}}=0.53 \mathrm{~cm}^{-1} \widehat{=} 0.015 \mathrm{~nm}$.

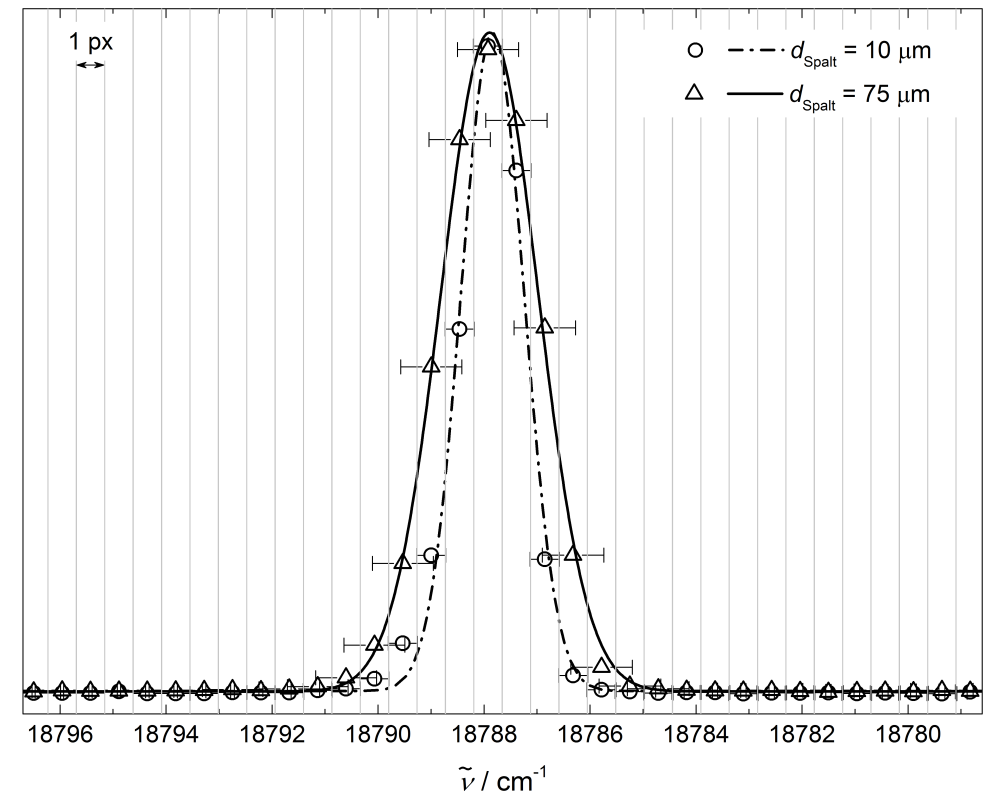

Abb. 3-4. Spektrum der Rayleighstreuung an Luft bei $25 \mathrm{~W}$ Laserleistung und unterschiedlichen Öffnungsweiten $d_{\text {Spalt }}$ des Monochromators mit instrumentell gewichteten Fits an die Gaußfunktion $y=y_{0}+A \exp \left[\frac{-\left(x-x_{\mathrm{c}}\right)^{2}}{2 w^{2}}\right]$. Die vertikalen Linien entsprechen der Breite eines CCD-Pixels von $d_{\mathrm{px}}=0.53 \mathrm{~cm}^{-1} \widehat{=} 0.015 \mathrm{~nm}$.

Die Emissionswellenlänge ist dabei abhängig von Temperaturschwankungen und der eingestellten Laserleistung $P_{\mathrm{L}}$. Daher ist eine konstante Kühlung des Laserkopfes mittels externem Kühler (Thermorack 401, Solid State) auf $20^{\circ} \mathrm{C}$ ebenso wichtig, wie eine Bestimmung von $\lambda_{\mathrm{L}}$ bei der gleichen Laserleistung, wie die der zugehörigen Messungen. Der Zusammenhang zwischen $P_{\mathrm{L}}$ und $\lambda_{\mathrm{L}}$ ist in Abbildung 3-5 dargestellt und zeigt, dass die Wellenlänge des emittierten Strahls für $P_{\mathrm{L}}<10 \mathrm{~W}$ abnimmt.

Auch wenn die Laserwellenlänge über einen kurzen Messzeitraum konstant bleibt, wurden langfristige Schwankungen in einem Bereich von $532.242-532.285 \mathrm{~nm}\left(\Delta \lambda_{\mathrm{L}}=0.043 \mathrm{~nm}\right.$, entspricht $\Delta \tilde{v}=1.4 \mathrm{~cm}^{-1}$ ) festgestellt, so dass für die einzelnen Messserien diese jeweils neu bestimmt wurde.

Für die Bewertung der Bandenintensitäten im Raman-Spektrum spielt die Polarisationsrichtung des Lasers in Zusammenhang mit der Streugeometrie des Setups (siehe Teilabbildung in Abb. 3-1) und dem Depolarisationsgrad einer Raman-aktiven Normalschwingung eine große Rolle. Der Strahl des Millennia eV-Lasers ist vertikal polarisiert $\left(\perp_{1}, 100: 1\right.$ laut Hersteller) und verläuft entlang der z-Achse des curry-Jets. Die Aufnahme der Streulichtes erfolgt in $90^{\circ}$ Streugeometrie entlang der $x$-Achse. Daraus ergibt sich die $x z$-Ebene als Streuebene und die Definition für die senkrechte $(\perp)$ und parallele $(\|)$ Polarisation des Lasers (Index 1) und der Streustrahlung (Index s). Details hierzu und zum Raman-Streuquerschnitt im curry-Jet finden 


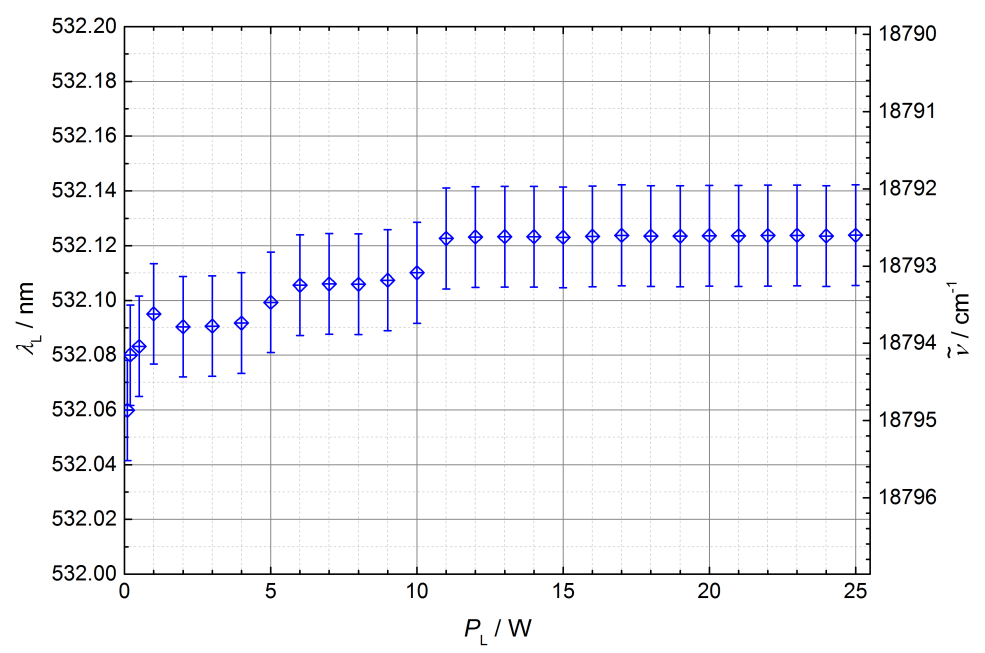

Abb. 3-5. Abhängigkeit der Emissionswellenlänge $\lambda_{\mathrm{L}}$ des Millennia eV $25 \mathrm{~W}$-Lasers von der eingestellten Laserleistung $P_{\mathrm{L}}$ (mit entsprechender Wellenzahlskala).

sich im Abschnitt 3.4 .

\subsubsection{Detektionsoptik und Datenaufnahme}

Die Datenaufnahme erfolgte für Molekülschwingungen im Stokes-Bereich, also in einem Gesamtmessbereich zwischen der Laserwellenlänge $\lambda_{\mathrm{L}} \approx 532 \mathrm{~nm}$ und $\approx 654 \mathrm{~nm}$, was Molekülschwingungen bis hin zu $3550 \mathrm{~cm}^{-1}(\mathrm{~N}-\mathrm{H}$-Streckschwingungen) entspricht. Dabei ist der Messbereich eines Spektrums durch die Breite des Detektors und die Auflösung des Monochromators begrenzt und beträgt ca. $20 \mathrm{~nm}$. Für den gesamten Messbereich weist das verwendete CCD-Array laut Hersteller eine Quantenausbeute von 95-97\% (bei $25^{\circ} \mathrm{C}$ ) auf und fällt bei einer Betriebstemperatur von $-120^{\circ}$ deutlich geringer aus. Die Quantenausbeute des CCD-Arrays kann jedoch für das Messfenster eines Spektrums als annähernd konstant angenommen werden.

Durch die Verwendung eines Langpass-Kantenfilters KF (REFUS532-25 USLR, LOT-Quantum Design) zur effektiven Unterdrückung der Rayleighstreuung beginnt aufgrund der endlichen Flanke der tatsächliche Messbereich erst bei $\approx 534 \mathrm{~nm}(T>90 \%)$, was einer Stokes-Verschiebung von $\approx 70 \mathrm{~cm}^{-1}$ entspricht.

Die spektrale Auflösung wird von apparativer Seite durch das Auflösungsvermögen und die Größe des Eintrittspalts $d_{\text {Spalt }}$ des Monochromators, die Pixelgröße des Detektors und die Linienbreite des Lasers begrenzt. Sie hängt außerdem vom Grad der Ausleuchtung des verwendeten Gitters ab. Dieser ist maximal, wenn die Einkoppeloptik der $f$-Zahl des Monochromators entspricht. Bei der Verwendung eines Objektivs mit einer Brennweite von $f=50 \mathrm{~mm}$ bei einer Blendenzahl von $f / 1.2$ hat der kollimierte Strahl einen Durchmesser von $42 \mathrm{~mm}$. Die verwendete Fokuslinse entspricht mit $f=350 \mathrm{~mm}$ einer Blendenzahl von $f / 8.3$, was dem Monochromator mit $f / 8.7$ nahe kommt. 
Die Auflösung eines Gitter-Spektrometers ist über das Rayleigh-Kriterium definiert [119], wird hier jedoch als die minimal-darstellbare Halbwertsbreite (FWHM) einer natürlich schmalen Spektrallinie festgelegt. Dessen theoretische Auflösung von $0.01 \mathrm{~nm}$ (bei einem Eintrittsspalt von $10 \mu \mathrm{m}$ ) wird jedoch nicht erreicht. Der Monochromator wurde im Rahmen dieser Arbeit durchgehend mit einem Eintrittspalt $d_{\text {Spalt }}=75 \mu \mathrm{m}$ betrieben, was erfahrungsgemäß einen guten Kompromiss zwischen Auflösung und Lichtmenge darstellt [112].

Die spektrale Breite $d \tilde{v}$ eines Detektorpixels ergibt sich zu $d \tilde{v}=\frac{d \lambda}{\lambda^{2}}=0.53 \mathrm{~cm}^{-1}$ bei einer Wellenlänge $\lambda_{\mathrm{MC}}=532 \mathrm{~nm}$ (4) Mit einer Halbwertsbreite (FWHM) von 2.16(2) $\mathrm{cm}^{-1}$ bei einer Spaltbreite von $75 \mu \mathrm{m}$ im Vergleich zu 1.40 (3) $\mathrm{cm}^{-1}$ bei $10 \mu \mathrm{m}$ (siehe Abb. 3-4) ist die Auflösung der curry-Jet-Spektren durch den Eintrittsspalt des Monochromators begrenzt und es wird für die Stokes-Verschiebungen $\Delta \tilde{v}_{s}$ aller Ergebnisse dieser Arbeit eine Standardabweichung von:

$$
\sigma\left(\Delta \tilde{v}_{\mathrm{s}}\right)=\frac{\mathrm{FWHM}}{2 \sqrt{2 \ln 2}}=0,917 \mathrm{~cm}^{-1} \approx 1 \mathrm{~cm}^{-1}
$$

angenommen.

Für die Datenaufnahme wurde eine „PyLoN 400 B“-Kamera (Princeton Instruments) mit 1340 Pixel breitem und 400 Pixel hohem rückseitig-beleuchtetem CCD-Array verwendet. Mit einer Pixelgröße von $20 \mu \mathrm{m}$ ergibt sich so eine absolute Arrayfläche von $26.8 \times 8.0 \mathrm{~mm}$. CCDDetektoren haben typischerweise drei Rausch-Quellen: Das Ausleserauschen, das abhängig von der Ausleserate ist und hier mit $3 e^{-} @ 100 \mathrm{kHz}$ angegeben wird, das Dunkelrauschen, welches laut Hersteller $0.3 e^{-} / \mathrm{px} / \mathrm{h}$ bei einer Detektortemperatur von $-120^{\circ} \mathrm{C}$ beträgt und das natürliche Schrotrauschen was der durch die Quantisierung hervorgerufene statistische Leistungsschwankung des Photonenflusses und der unkorrelierten Erzeugung von Photoelektronen im Detektor Rechnung trägt [120]. Bei gegebener Messzeit ist dabei das Rauschen für niedrige Detektortemperaturen und kleine Ausleseraten minimal.

Für das Spektrum sind nur die Intensitäten entlang der horizontalen Detektordimension relevant, aber durch die vollständige vertikale Ausleuchtung des Detektors kann durch die Summation des gesamten Signals jeder Pixelspalte (Full-Vertical-Binning, FVB) die Intensität des Spektrums deutlich erhöht werden ohne die Anzahl der Auslesevorgänge zu verändern, so dass das Signal-zu-Rausch-Verhältnis ebenfalls deutlich verbessert wird. Der 16 bit Analog-DigitalWandler (ADC) der Kamera wird mit einer Ausleserate von $100 \mathrm{kHz}$ bei einem Signalverstärkungsfaktor (Gain) von 3 betrieben und die Software WinSpec/32 in der Version 2.6.23.0 zur Datenaufnahme verwendet.

Die Intensität des gemessenen Signals (Zählereignisse pro Pixelspalte) unterliegt systematischen Schwankungen, die durch die Justage der Strahlengänge sowie den Verschmutzungsgrad und die Wellenlängenabhängigkeit verschiedener Komponenten hervorgerufenen werden. Die Leistungsschwankung und damit auch die Schwankung der auf die Probe eingestrahlten Intensität $I_{\mathrm{i}}$ des $25 \mathrm{~W}$-Lasers ist von Herstellerseite mit $\pm 1 \%$ (aufgewärmt, über $2 \mathrm{~h}$ gemessen) angegeben. Dieser Effekt betrifft alle Intensitäten eines Spektrums gleichermaßen und reduziert sich mit längerer Integrationsdauer pro Spektrum.

\footnotetext{
4) Die Abschätzung erfolgt aus der linearen Regression eines exemplarischen Kr-Kalibrierspektrums zur Rayleighlinien-Bestimmung.
} 
Für die Intensität des gestreuten Signals $I_{\mathrm{S}}$ ist außerdem eine Wellenlängenabhängigkeit der Quantenausbeute des CCD-Detektors genauso wie die aller optischen Bauteile im Strahlengang (Gitter und Spiegel des Monochromators, achromatische Linse, Fenster und Objektiv) zu betrachten. Im Messbereich von $532 \mathrm{~nm}$ bis ca. $654 \mathrm{~nm}$ hat diese für den Detektor laut Datenblatt des Herstellers einen Effekt von einigen Prozent, so dass Peakintensitäten über einen weiten Spektralbereich nicht quantitativ vergleichbar sind, da keine Eichmessungen vorliegen. Für die Breite des Messbereichs einer Messung von ca. 20 nm kann diese Abhängigkeit aber vernachlässigt werden.

Ebenfalls abhängig von der Wellenlänge ist die polarisationsabhängige Sensitivität des Monochromatorgitters. Das Verhältnis von senkrecht zu parallel zur Streuebene polarisierten Lichtes $\left(I\left(\perp_{s}\right) / I\left(\|_{s}\right)\right)$ wurde für das gleiche Setup mit dem Kamera-Vorgängermodell Spec-10 von Lüttschwager [32] $\mathrm{zu} \approx 1.5$ für eine Stokes-Verschiebung $\Delta \tilde{v}_{\mathrm{s}}$ von $0 \mathrm{~cm}^{-1}$ bis $\mathrm{zu} \approx 2.25$ für $\Delta \tilde{v}_{\mathrm{s}} \approx$ $3500 \mathrm{~cm}^{-1}$ bestimmt und kann für die $k$-te Normalmode näherungsweise mit folgender Geradengleichung angenähert werden [113]:

$$
\Theta\left(\tilde{v}_{k}\right)=\frac{I\left(\perp_{s}\right)}{I\left(\|_{s}\right)}=1.5+\left(2 \cdot 10^{-4}\right) \mathrm{cm} \cdot \tilde{v}_{k}
$$

Die Kompensation dieses Effektes erfolgt durch die Anpassung der theoretischen RamanStreuquerschnitte $\sigma$ (siehe Abschnitt 3.4).

Die genannten Fehlerquellen sind klein, verglichen mit dem Intensitätsverlust durch die über eine längere Messdauer sukzessive Verschmutzung der optischen Komponenten in der Messkammer ${ }^{5)}$ und die regelmäßig notwendige Justage der Komponenten (bedingt durch Reinigung der Optiken und Anpassung an eine thermische Dejustierung durch das Heizsystem).

Auch wenn aus den absoluten Signalintensitäten, anders als in der FTIR-Spektroskopie (siehe Abschnitt 3.2), keine physikalisch sinnvolle Größe abgeleitet werden kann, so liefert der Vergleich relativer Intensitäten von Banden (unter Berücksichtigung des jeweiligen Streuquerschnitts) innerhalb eines Spektrums und auf eine Bande normierte Intensitäten unterschiedlicher Spektren durchaus sinnvolle Ergebnisse.

\subsubsection{Datenauswertung}

CCD-Detektoren sind sehr sensitiv für hochenergetische Strahlung, die entweder aus lokalen radioaktiven Zerfällen oder Elementarteilchen (größtenteils Myonen [121]) kosmischen Ursprungs besteht und daher auch als „Cosmic Rays“ bezeichnet wird [122]. Diese erzeugt zeitlich und räumlich zufällig verteilte sehr schmalbandige, intensive Signale, sogenannte Spikes. Für eine sinnvolle Datenauswertung müssen diese entweder verhindert werden, was allerdings nur bedingt möglich ist, oder nachträglich eliminiert werden. Dazu gibt es unterschiedliche Ansätze, wobei in der Raman-Spektroskopie insbesondere räumliche und zeitliche Filtermethoden verbreitet sind. Erstere beruhen auf der Annahme, dass Spikes schmal gegenüber Messsignalen sind und daher mit Glättungsalgorithmen [123-125] entfernt werden können.

\footnotetext{
${ }^{5)}$ Die Zeitskala der Verschmutzung hängt von der zu untersuchenden Substanz ab und schwankt zwischen $1-2 \mathrm{~h}$ für schwerflüchtige Substanzen bis hin zur Vernachlässigbarkeit für Leichtflüchtiges.
} 
Die zeitliche Filterung vergleicht mehrere Einzelspektren und detektiert zufällig auftretende Spikes als Werte, die einem oberen Grenzwert aus statistischem Rauschen und konstant auftretendem Messsignal überschreiten. Dies wird z. B. in den Algorithmen der Robusten Summation (RS) [126] und des Upper-Bound Spectrum (UBS) [127] und Varianten für große Datensätze unterschiedlicher Daten [128] und Einzelspektren [129, 130] (,Multitrack-Comparison“: jede Pixelreihe des Einzelspektrums wird als gesondertes Spektrum behandelt) angewendet.

Beide Ansätze haben Vor- und Nachteile, die im Detail bei Hill [131], Cappel et al. [132] und Li und Dai [133] diskutiert werden.

An der curry-Jet-Apparatur enthält bei Belichtungszeiten oberhalb ca. einer Minute jedes Spektrum mindestens einen Spike, bei einer typischen Messzeit von $300 \mathrm{~s}$ sind im Schnitt $17 \pm 6$ Spikes mit einer Breite von 2-4 Pixeln pro Einzelspektrum enthalten, was bei 1340 Pixeln bedeutet, dass ungefähr 3.8\% der Pixelspalten mit Spikes belegt sind. Für Spikes auf demselben Pixel zweier aufeinanderfolgenden Einzelspektren liegt die Wahrscheinlichkeit damit bei $0.14 \%$. Für Messzeiten jenseits der 10 Minuten sind Spektren, die zum größten Teil Spikes enthalten, wahrscheinlich.

Die vom Kamerahersteller zur Verfügung gestellte Auswertesoftware WinSpec bietet die nicht näher erläuterte Möglichkeiten des „Spatial-“ und „Temporal Cosmic Ray Removal“. Tests dieser implementierten Spike-Entfernung ergaben aber kein zufriedenstellendes Ergebnis. Es wurde daher auf das Matlab-Skript „gui_despike“ [32] von N. Lüttschwager zurückgegriffen. Der Algorithmus vergleicht dabei die Werte eines Pixels über den Verlauf mehrerer Einzelmessungen. Spikes werden hierbei durch einen Grenzwert oberhalb vom Mittelwert einer Pixelreihe definiert und sukzessive reduziert. Die Nachteile dieser Methode sind die willkürlich wählbaren Werte für den Grenzwert und die Anzahl der Iterationen, und somit die Beurteilung des Endergebnisses durch visuelle Einschätzung des Experimentators.

Als Alternative wird hier ein Algorithmus namens finning vorgestellt, der zusammen mit weiteren Funktionen ${ }^{6)}$ Bestandteil der Curry()-Klasse des Python-Moduls madras ist (für den vollständigen Quelltext und weitere Erklärungen, siehe Anhang A.12).

Für die Spektren des curry-Jets können einzelne Signale (insbesondere der Edelgas-Emissionslinien in den Kalibriermessungen) in Intensität und Bandbreite nicht von Spikes unterschieden werden, so dass die räumlichen Filtermethoden nicht in Frage kommen. Die (innerhalb der in Abschnitt 3.1.4 diskutierten Schwankungen) stationären Messbedingungen der kontinuierlichen Expansion erlauben eine Verwendung des Prinzips des Pixel-weisen Vergleichs der Einzelspektren.

Bei der finning-Methode handelt es sich um eine Vereinfachung der RS-Methode von Takeuchi et al. [126] und der UBS-Methode von Zhang et al. [127], wobei auf die Vorskalierung der Spektren untereinander genauso verzichtet wird wie auf die explizite Definition des Ausleserauschens. Außerdem werden in Anbetracht der finalen Mittelung aller Spektren die mit Spikes kontaminierten Datenpunkte nicht durch Nachbar- oder Mittelwerte ersetzt.

\footnotetext{
6) Als weitere Funktionen stehen neben der Edelgas-Kalibrierung (siehe Abschnitt 3.1.6) auch der bisherige DespikeAlgorithmus zur Verfügung. Weiterhin ist ein direkter Import der binären *.SPE-Dateien von WinSpec möglich. Details finden sich in Anhang A.12.
} 
Es gibt bei dieser Methode also für jede Pixelreihe einen einzigen Grenzwert $g w$, der die Signalintensität eines Pixels, sobald er oberhalb von $g w$ liegt, als Spike einschätzt und sich selbst optimiert.

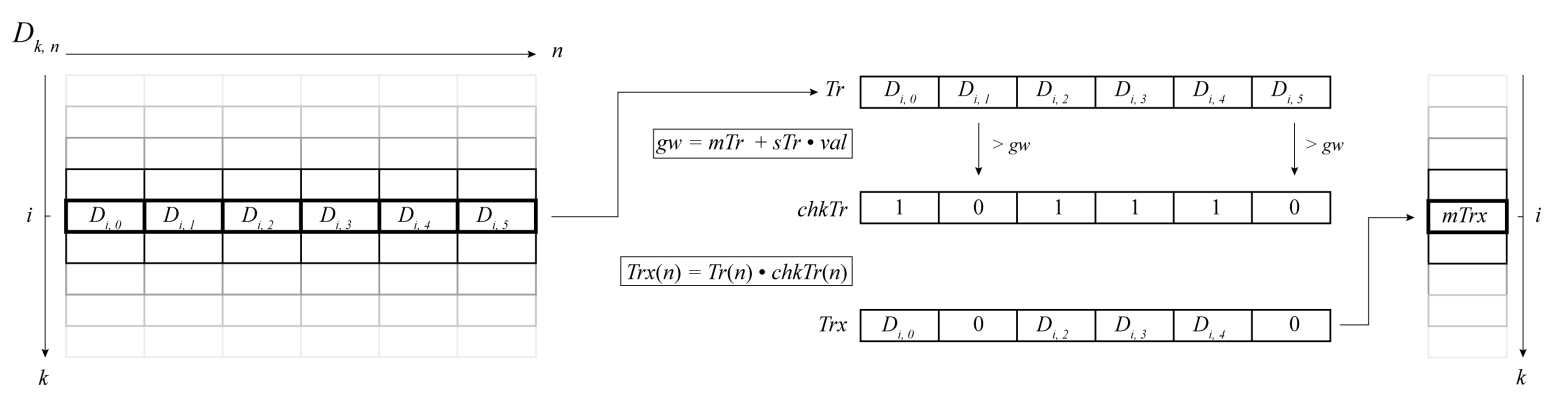

Abb. 3-6. Schema des Curry.finning()-Algorithmus. Jede Zeile $i$ der Datenmatrix $D_{k, n}$ aus $n$ Einzelspektren eines Detektors mit $k$ horizontalen Pixeln wird als $\operatorname{Tr}$ nach Spikes in Form von Signalen größer dem Grenzwert $g w$ durchsucht. Jeder Treffer wird durch den aktuellen Median der Zeile $(m T r)$ ersetzt und das entsprechende Element in einem Kontrollvektor durch 0 gekennzeichnet. Anschließend werden alle Spikes eliminiert $(\operatorname{Tr} x)$ und der gewichtete Mittelwert $(m \operatorname{Tr} x$, siehe GI. 3-8 ist $i$-tes Element der $(k \times 1)$-Ergebnismatrix.

Die Curry.finning()-Methode ist in Abbildung 3-6 schematisch dargestellt. Dabei wird die Datenmatrix $D_{k, n}$ einer Messung mit einem einzeiligen Detektor (durch FVB) mit $k$ horizontalen Pixeln und $n$ Einzelspektren eingelesen. Diese Matrix wird nun Reihe für Reihe $(i)$ nach Spikes durchsucht. Der aktuelle Höchstwert der Reihe $\operatorname{Tr}$ wird jetzt so lange durch den aktuellen Median von $\operatorname{Tr}(m T r)$ ersetzt, bis kein Wert von $\operatorname{Tr}$ mehr oberhalb des Grenzwertes $g w$ liegt. Dieser definiert sich als Summe aus dem Median7) $(m T r)$ und der Standardabweichung $(s T r)$ von $T r$. Die Standardabweichung kann vom Nutzer um einen Faktor var korrigiert werden, um unterschiedliche Konfidenzintervalle abzudecken (Standard: var $=2.0 \Rightarrow 8$ ) Konfidenzintervall: $\approx 95 \%$ bei weißem Rauschen). Für jeden eliminierten Wert in $\operatorname{Tr}$, also jeden gefundenen Spike, wird in einem Kontrollvektor chkTr das entsprechene Element von 1 auf 0 geändert. Durch abschließende elementweise Multiplikation von $\operatorname{Tr}$ mit $c h k T r$ ergibt sich der korrigierte Reihenvektor Trx, dessen Mittelwert nach Gleichung 3-8 das $i$-te Element der $(k \times 1)$-Ergebnismatrix ergibt.

$$
\frac{\sum_{n} \operatorname{Tr} x}{\sum_{n} \operatorname{chkTr}}
$$

Der Algorithmus erzeugt also für jeden Wert $m \operatorname{Tr} x(k)$ der Ergebnismatrix abhängig von der Anzahl der gefundenen Spikes einen Mittelwert aus unterschiedlich vielen Einzelspektren. Dies ist aber mindestens so legitim wie der Ersatz eines Spikes durch den Mittelwert der beiden Werte der benachbarten Einzelspektren in den Methoden RS und UBS sowie im Vorläuferalgorithmus despike. Hier erfolgt jede Mittelwertbildung zwar formal aus der gleichen Zahl an Einzelspektren, aber einzelne Werte erfahren eine erhöhte Gewichtung nur auf Grund der Nach-

\footnotetext{
${ }^{7)}$ Die Summe von Median und Standardabweichung ist statistisch wenig sinnvoll, allerdings konvergiert der Algorithmus unter Verwendung des Medians schneller und ist robuster im Fall von Spikes an der gleichen Position in zwei oder mehr aufeinander folgenden Einzelspektren.

${ }^{8)}$ Für kritische Fälle (z. B. wenige Einzelspektren) hat sich var $=1.0$ bewährt.
} 
barschaft zu einem Spike.

Für eine erfolgreiche Verwendung von Curry.finning() für die Auswertung von curry-JetSpektren hat sich eine Mindestzahl von 6 Einzelspektren und eine Höchstmesszeit von 10 Minuten pro Spektrum als sinnvoll erwiesen.

\subsubsection{Kalibrierung der Messungen}

Für die Umrechnung der relativen Signalposition (als Spaltennummer des Detektors in Pixel $(\mathrm{px})$ ) in die entsprechende Raman-Verschiebung ist eine Kalibrierung der gegenwärtigen Monochromatorstellung ebenso notwendig wie die genaue Bestimmung der Rayleighwellenlänge $\lambda_{R}$. Der Monochromator zeigt zwar eine Wellenlänge $\lambda_{M C}$ an, welche sich auf den Austrittsspalt des Monochromators bezieht und in der Bedienungsanleitung mit einer Genauigkeit von $0.05 \mathrm{~nm}$ mit einer Reproduzierbarkeit von $0.01 \mathrm{~nm}$ angegeben wird. Durch die Benutzung eines CCD-Flächendetektors statt des Austrittsspalts lässt $\lambda_{\mathrm{MC}}$ jedoch keine Aussage über die spektrale Zuordnung der Kamerapixel zu und hat als Zentralwellenlänge (ZWL) im Experiment lediglich orientierenden Charakter. Daher wird mittels Edelgas-Emissionslampen (Neon und Krypton, Oriel Instruments) kalibriert, die einen Satz genau definierter und scharfer Emissionslinien ${ }^{97}$ im gesamten Messbereich haben. Die Zuordnung der Linien erfolgt an den tabellierten Werten der NIST Atomic Spectra Database [134]. Der Zusammenhang zwischen der Detektorposition und der Wellenlänge $\lambda$ ist nicht linear und kann sehr gut mit einem Polynom 2 . Ordnung angenähert werden. Für diesen Fit sind in jedem Messbereichsausschnitt ausreichend viele Linien vorhanden.

Zur Berechnung der Raman-Verschiebung (in dieser Arbeit ausschließlich die Stokes-Verschiebung $\Delta \tilde{v}_{s}$ ) wird zusätzlich zur Wellenzahl $\tilde{v}$ der Signale im Spektrum auch die Energie des Anregungslasers benötigt. Da alle Messungen unter atmosphärischen Bedingungen im Monochromator stattfinden, muss bei der Berechnung der Energie die Dispersion der Luft $n(\lambda)$ berücksichtigt werden (Gl. 3-9). Diese ist allerdings nicht-linear von der Wellenlänge abhängig und somit in jedem Messbereich anders. Die in der NIST Atomic Spectra Database dokumentierten Kalibrierlinien in Luft sind aus den entsprechenden Vakuumlinien mit einer empirischen Fünfparameter-Gleichung [135] berechnet. Da in der Raman-Spektroskopie nur Energien relativ zum Anregungslaser gemessen werden, kann also unter konsequenter Verwendung der Vakuumlinien für die Kalibrierung des Messbereichs als auch der Laserwellenlänge auf eine Dispersionskorrektur verzichtet werden.

$$
\lambda=\frac{c_{0}}{n(\lambda)} \frac{1}{\tilde{v}}
$$

mit: $c_{0}$ : Lichtgeschwindigkeit im Vakuum; $n$ : Brechungsindex des Mediums

Die Kalibrierung erfolgt an jedem Messtag und nach jeder Modifikation am Laser und am Monochromator.

\footnotetext{
9) Die spektrale Breite der Emissionslinien werden bei Otto [112] ausführlich diskutiert.
} 


\subsection{FTIR-Spektroskopie: Der filet-Jet}

Neben den schwerpunktmäßig durchgeführten Raman-Messungen wurden in einigen Fällen (siehe 4.3) auch Infrarot-Spektren aufgenommen. Dazu wurde der filet-Jet (,fine but lengthy") verwendet, ein FTIR-Jet-Spektrometer, dessen besonders gutes Signal-zu-Rauschen-Verhältnis aus dem langen Absorptionsweg und den eingesetzten Filtern resultiert. Die Substanzzuführung ist am filet-Jet nur über kühlbare Sättiger möglich, wodurch die Sättigertemperatur nach oben hin durch die Raumtemperatur limitiert ist, um Substanzkondensation in den Zuleitungen und im Reservoir zu vermeiden. Eine ausführliche Beschreibung des Spektrometers und dessen Spezifikationen findet sich bei Suhm und Kollipost [37], Kollipost [136] und Heger [137].

Die FTIR-Spektren liefern mit der Absorbanz eine Größe die nach Lambert-Beer proportional zur Substanzkonzentration ist. Daher sind die Peakintensitäten der Spektren ein direktes Maß für die Substanzkonzentration.

Alle Messungen wurden mit Helium als Trägergas durchgeführt.

Für die Aufnahme von Gasphasenspektren lässt sich die Messkammer über einen Bypass mit dem Substanzgemisch bis zu einem bestimmten Hintergrunddruck befüllen und die Puffervolumina mitsamt der Vakuumpumpen von der Kammer trennen.

Die Datenauswertung erfolgte mit der Software OPUS (Vers. 7.0.129, Bruker Optik GmbH).

\subsection{Quantenchemische Rechnungen}

Die Auswertung der gemessenen Spektren wird durch die Ergebnisse quantenchemischer Rechnungen unterstützt. Im Rahmen dieser Arbeit wurden für alle Moleküle und deren Aggregate bis hin zum Tetramer Geometrieoptimierungen und Frequenzrechnungen auf unterschiedlichen quantenchemischen Rechenniveaus durchgeführt. Die Auswahl der Methode erfolgte unter Abwägung von rechnerischer Genauigkeit und zeitlichem Rechenaufwand. Maßgebend war hier immer das größte System, zu dem Vergleiche hergestellt werden sollen.

Für alle Systeme wurden mindestens Geometrieoptimierungen und Frequenzrechnungen in doppelt-harmonischer Näherung mit dem B3LYP-Hybridfunktional durchgeführt. Diese wurden im Einzelfall durch störungstheoretische MP2-Rechnungen [138, 139] und anharmonische VPT2-Rechnungen [140-142] ergänzt. Die B3LYP-Rechnungen wurden sowohl mit als auch ohne Dispersionskorrektur mit der DFT-D3-Methode nach Grimme et al. [105] mit der Dämpfungsfunktion von Becke und Johnson [106] durchgeführt. Der Unterschied ist in Abschnitt 2.3 erläutert und die Auswirkungen der Dispersionskorrektur werden ausführlich in Abschnitt 4.4 diskutiert.

Als Basissätze dienen zum einen die Karlsruher def2XZVP (mit $X=\mathrm{T}$, Q) der Ahlrichs-Gruppe [143] und die korrelations-konsistenten Dunning-Basissätze [144, 145] cc-pVXZ (mit $X=\mathrm{T}$, Q, 5) mit optionalen diffusen Funktionen (aug) [146].

Alle Rechnungen in dieser Arbeit wurden mit dem Programm Gaussian 09, Revision E.01 [147] durchgeführt. Geometrieoptimierungen erfolgten mit den Konvergenzkriterien der Option „tight“ (in einzelnen Fällen wurden die Optionen „fopt“ oder „verytight“ verwendet) und für 
alle DFT-Rechnungen wurde das Integrationsgitter „SuperFine“ gewählt. Die Suche nach möglichen stabilen Konformationsisomeren erfolgte durch relaxierte Scans („modredundant") der Potentialhyperfläche entlang der Drehung um die entsprechenden Diederwinkel des Moleküls.

Frequenzen für deuterierte Moleküle und freie Enthalpien wurden mit dem GAUSSIAN-eigenen Skript freqchk bestimmt. In GAUSSIAN ist die Grimme'sche Dispersionskorrektur als Option „GD3BJ“ für den Parameter empiricaldispersion implementiert und wird im Folgenden als DFTD3(BJ) bezeichnet.

Bei jeder quantenchemischen Rechnung, die nicht im vollständigen Basissatz (CBS) durchgeführt wird, tritt der sogenannte Basissatz-Überlagerungsfehler (BSSE) auf, der durch die Mitnutzung der Basisfunktionen benachbarter Atome den eigenen Basissatz vergrößert und dadurch zu einer Verzerrung der Potentialhyperfläche führt [148]. Für DFT-Methoden ist der BSSE nicht so groß wie für MP2-Methoden und in verschiedenen wasserstoffbrückengebundenen Komplexen beträgt dessen Anteil an der auf B3LYP/double- $\zeta$-Niveau berechneten Bindungsenergie weniger als $1 \mathrm{~kJ} \cdot \mathrm{mol}^{-1}$ [149]. Da Methoden zur BSSE-Korrektur wie die häufig verwendete Counterpoise-Korrektur (CP) nach Boys und Bernardi [150] unterschiedlich bewertet werden [148, 149] und der BSSE kleiner als die anzunehmende Unsicherheit der DFTRechnung ist, wird auf eine BSSE-Korrektur in dieser Arbeit verzichtet (siehe auch [151]).

In dieser Arbeit wird zur Dispersionskorrektur durchgehend die DFT-D3-Methode von Grimme etal. [105] mit der Dämpfungsfunktion von Becke und Johnson [106] verwendet, die in GAUSSIAN als Option „GD3BJ“ für den Parameter empiricaldispersion implementiert ist und im Folgenden als DFT-D3(BJ) bezeichnet wird. Die verwendeten input-Zeilen in den GAUSSIAN job files finden sich im Anhang A.5 in Tab. A-4.

\subsection{Von der Raman-Aktivität zum Streuquerschnitt}

Für die Zuordnung von Banden in Schwingungsspektren sind theoretische Frequenzen auf der Basis quantenchemischer Rechnungen ein gutes Hilfsmittel. In GAUSSIAN ist einerseits die Berechnung von Kraftkonstanten (und über die reduzierte Masse Frequenzen), Infrarot-Intensitäten und Raman-Aktivitäten auf Basis einer doppelt-harmonischen Näherung möglich. Diese beinhaltet sowohl die mechanische Harmonizität als linearen Zusammenhang zwischen Kraftkonstante und Auslenkung (Hooke'sches Gesetz) als auch die elektronische Harmonizität als lineare Abhängigkeit der Änderung des Dipolmoments mit dem elektrischen Feld. Andererseits gibt es die Möglichkeit anharmonische Frequenzen und IR-Intensitäten mit dem störungstheoretischen VPT2-Ansatz zu berechnen.

Die theoretische Raman-Aktivität $A_{k}$ der $k$-ten Normalmode berechnet GAUSSIAN aus den Invarianten $\alpha_{k}^{\prime}$ (Änderung der mittleren Polarisierbarkeit) und $\gamma_{k}^{\prime}$ (anisotrope Änderung) des Polarisierbarkeitstensors gemäß Gleichung 3-10.

$$
A_{k}=\underbrace{45 \alpha_{k}^{\prime 2}+4 \gamma_{k}^{2}}_{\perp_{s}}+\underbrace{3 \gamma_{k}^{2}}_{\|_{s}}
$$


Die tatsächliche Intensität $I_{k}^{\prime}$ der differentiellen Streustrahlung ${ }^{10)}$ der Bande $k$ im RamanSpektrum ist unter nicht-resonanten Bedingungen linear abhängig von der Konzentration $c_{k}$, der Intensität des Anregungslasers $I_{0}$, dem differentiellen Streuquerschnitt $\sigma_{k}^{\prime}$ und einer instrumentellen Proportionalitätskonstante $a:[18,31]$

$$
I_{k}^{\prime}=a^{\prime} \cdot \sigma_{k}^{\prime} \cdot c_{k} \cdot I_{0}
$$

Für den curry-Jet ist $a^{\prime}$ nicht bekannt, so dass eine absolute Bandenintensität nicht berechnet werden kann. Dennoch ist aus Gleichung 3-11 ersichtlich, dass das Verhältnis der Intensitäten zweier isolierter Banden in einem Spektrum um die jeweiligen Streuquerschnitte korrigiert über die Konzentration ein Maß für das Besetzungsverhältnis der zugrundeliegenden Schwingungszustände ist.

Der differentielle Streuquerschnitt ist proportional zur theoretischen Raman-Aktivität und abhängig von der Streugeometrie des Aufbaus (siehe Abb. 3-1) und für den curry-Jet bei Wassermann [31] ausführlich hergeleitet. Für jede nicht-entartete Normalschwingung $k$ gilt für $\sigma_{k}^{\prime}$ :

$$
\sigma_{k}^{\prime}=\frac{2 \pi^{2} h}{45 c \tilde{v}_{k}} \cdot \frac{\left(\tilde{v}_{0}-\tilde{v}_{k}\right)^{3} \tilde{v}_{0}}{1-\exp \left(-\frac{h c \tilde{v}_{k}}{k T}\right)} \cdot A
$$

Für die im Rahmen der hier durchgeführten Messungen vorliegenden hohen Wellenzahlen bei niedrigen Temperaturen der einzelnen Schwingungsfreiheitsgrade in der Expansion $\left(h c \tilde{v}_{k} \gg k T\right)$ gilt näherungsweise $1-\exp \left(-\frac{h c \tilde{v}_{k}}{k T}\right) \approx 1$, weswegen Gleichung 3-12 in folgender Vereinfachung angewendet wird (vgl. [31]):

$$
\sigma_{k}^{\prime}=\frac{2 \pi^{2} h}{45 c \tilde{v}_{k}} \cdot\left(\tilde{v_{0}}-\tilde{v}_{k}\right)^{3} \tilde{v}_{0} \cdot A
$$

Außerdem wird das Depolarisationsverhältnis $\delta_{k}$ für linear polarisiertes Licht angegeben, was für die Streugeometrie des curry-Jets entsprechend Gleichung 3-14 mit den Tensorinvarianten zusammenhängt.

$$
\delta_{k}=\frac{I\left(\|_{s}\right)}{I\left(\perp_{s}\right)}=\frac{\frac{3 \gamma_{k}^{\prime 2}}{45}}{\frac{45 \alpha_{k}^{\prime 2}+4 \gamma_{k}^{\prime 2}}{45}}=\frac{3 \gamma_{k}^{\prime 2}}{45 \alpha_{k}^{\prime 2}+4 \gamma_{k}^{\prime 2}} \leq 0.75
$$

Wie in Abschnitt 3.1.4 erwähnt, ist das Gitter des Monochromators gemäß Gleichung 3-7 um $\Theta(\tilde{v})$ sensitiver für $\perp_{s}$ als für $\|_{s}$. Für eine Vergleichbarkeit der theoretischen Streuquerschnitte mit den gemessenen Bandenintensitäten wird eine korrigierte Ramanaktivität $A_{k}^{\text {corr }}$ wie folgt berechnet:[113]

$$
A_{k}^{\mathrm{corr}}=45 \alpha_{k}^{\prime 2}+4 \gamma_{k}^{2}+\frac{3 \gamma_{k}^{\prime 2}}{\Theta\left(\tilde{v}_{k}\right)}
$$

Da GAUSSIAN nicht die Tensorinvarianten ausgibt, wird $A_{k}^{\text {corr }}$ mit den Gleichungen 3-15 3-10 und 3-14 nur in Abhängigkeit von $A_{k}, \delta_{k}$ und $\Theta\left(\tilde{v}_{k}\right)$ berechnet:

10) „Differentiell“ bedeutet hier, dass die Größe auf den Einheitsraumwinkel bezogen ist. 


$$
A_{k}^{\text {corr }}=A+\frac{\delta_{k}}{1+\delta_{k}}\left(\frac{1}{\Theta\left(\tilde{v}_{k}\right)}-1\right) \cdot A \quad \leq A
$$

Die korrigierte Raman-Aktivität ist somit immer kleiner als $A$ und nur für total-symmetrische Schwingungen mit $\delta_{k}=0$ gilt: $A_{k}^{\text {corr }}=A_{k}$.

\subsection{Verwendete Substanzen}

In dieser Arbeit wurden Messungen mit den aliphatischen sekundären Amiden N-Methylformamid (NMF) und $N$-Methylacetamid (NMA), sowie dem zyklischen $\gamma$-Butyrolactam (gBL, auch: 2-Pyrrolidon) und dem aromatischen $N$-Phenylformamid (NPhF, auch: Formanilid) durchgeführt. Zudem wurde das aromatische, heterocyclische Amin Imidazol (Im) untersucht. Die grundlegenden Daten und einige physikalische Eigenschaften der verwendeten Substanzen sind in Tab. 3-1 aufgeführt.

Tab. 3-1. Grundlegende Daten und physikalische Eigenschaften der verwendeten Substanzen N-Methylformamid (NMF), $N$-Methylacetamid (NMA) , $\gamma$-Butyrolactam (gBL, auch: 2-Pyrrolidon) und $N$-Phenylformamid (NPhF, auch: Formanilid). Mit $M$ : Molmasse, $\vartheta_{\mathrm{m}}$ : Schmelzpunkt, $\vartheta_{\mathrm{b}}$ : Siedepunkt, $p_{\mathrm{vap}}$ : Dampfdruck und $H_{\mathrm{vap}}$ : Verdampfungsenthalpie

\begin{tabular}{llcllll}
\hline Substanz & Summenformel & $M^{\mathrm{a}} / \mathrm{g} \mathrm{mol}^{-1}$ & $\vartheta_{\mathrm{m}}{ }^{\mathrm{a}} /{ }^{\circ} \mathrm{C}$ & $\vartheta_{\mathrm{b}}{ }^{\mathrm{a}} /{ }^{\circ} \mathrm{C}$ & $p_{\text {vap }} / \mathrm{mmHg}$ & $H_{\text {vap }} / \mathrm{kJ} \mathrm{mol}^{-1}$ \\
\hline NMF & $\mathrm{C}_{2} \mathrm{H}_{5} \mathrm{NO}$ & 59.07 & -3.8 & 199.5 & $0.253\left(25^{\circ} \mathrm{C}\right)^{\mathrm{b}}$ & 56.19 \\
$\mathrm{NMA}$ & $\mathrm{C}_{3} \mathrm{H}_{7} \mathrm{NO}$ & 73.09 & 28 & 205 & $0.44\left(23^{\circ} \mathrm{C}\right)^{\mathrm{c}}$ & $59.4^{\mathrm{d}}$ \\
$\mathrm{gBL}$ & $\mathrm{C}_{4} \mathrm{H}_{7} \mathrm{NO}$ & 85.11 & 25 & 251 & $0.00949\left(25^{\circ} \mathrm{C}\right)^{\mathrm{b}}$ & $56.7^{\mathrm{e}}$ \\
$\mathrm{NPhF}$ & $\mathrm{C}_{7} \mathrm{H}_{7} \mathrm{NO}$ & 121.14 & 46 & 271 & $0.0035\left(25^{\circ} \mathrm{C}\right)^{\mathrm{b}}$ & - \\
$\mathrm{Im}$ & $\mathrm{C}_{3} \mathrm{H}_{4} \mathrm{~N}_{2}$ & 68.08 & 90.5 & 257 & $0.002\left(20^{\circ} \mathrm{C}\right)^{f}$ & - \\
\hline
\end{tabular}

\footnotetext{
a Lide [152.; ${ }^{\mathrm{b}}$ Daubert und Danner $153, \overline{{ }^{\mathrm{C}} \text { Yaws }[154}$ S. 186]; ${ }^{\mathrm{d}}$ Riddick et al. 155$] ;{ }^{\mathrm{e}}$ Ullmann et al. $[156$ Bd. A22, S. 437];

${ }^{f}$ Kim etal. 157] (https://pubchem.ncbi.nlm.nih.gov/compound/795; 28.06.2018)
}

Die genannten Chemikalien wurden nach der Lieferung ohne weitere Aufreinigung verwendet. Weitere Details zu den Chemikalien befinden sich in Anhang A.2 in Tab. A-1. Die angegebenen Reinheiten entsprechen den Herstellerangaben. Da einige der Substanzen schon seit längerer Zeit im Chemikalienbestand des Arbeitskreises vorhanden sind, wurde die Reinheit dieser Substanzen mittels H-NMR-Spektroskopie überprüft. Die Ergebnisse sind in Anhang A.6.3 zusammengefasst. 



\section{$4 \mathrm{~N}$-Methylacetamid}

Die Ergebnisse dieses Kapitels wurden teilweise bereits veröffentlicht in:

T. Forsting, H. C. Gottschalk, B. Hartwig, M. Mons, M. A. Suhm, Correcting the record: the dimers and trimers of trans-N-methylacetamide, Phys. Chem. Chem. Phys., 2017, 19 (17), 10727-10737.

$N$-Methylacetamid ist mit je einer Methylgruppe am Stickstoff als auch am Kohlenstoff der $\mathrm{N}(\mathrm{H})-\mathrm{C}=\mathrm{O}$-Amidgruppe das kleinste Molekül mit einer Peptideinheit, wenn die beiden MethylC-Atome als $\mathrm{C}_{\alpha}$ interpretiert werden und gilt in der Forschung als die Standard-Modellverbindung für die Peptidbindung[84]. Dabei stehen intermolekulare Wechselwirkungen, Lösungsmitteleinflüsse und die Isomerisierung entlang der partiellen $\mathrm{C}-\mathrm{N}$-Doppelbindung im Fokus.

Es wurde, analog zu den Peptidketten in Proteinen [158], von den zwei möglichen Konformationen durch die gehinderte Isomerisierung trans-NMA als die energetisch günstigste angenommen [69]. Dies wurde später sowohl experimentell durch die Strukturaufklärung mittels NMR-Spektroskopie [159 163], Elektronenbeugung in der Gasphase [164, 165] als auch mittels Röntgenstrukturanalyse [166, 167] und Neutronenbeugungsexperimenten [48] am Kristall bestätigt.

Die Konformation des gesamten Moleküls wird neben dem Diederwinkel $\omega$ auch durch die beiden Ramachandranwinkel $\varphi$ und $\psi$, also die Stellung der Wasserstoffatome der beiden endständigen Methylgruppen festgelegt. Diese können dabei jeweils in anti- oder syn-Stellung zur $\mathrm{C}=\mathrm{N}$-Amidbindung sein, so dass insgesamt die acht Konformere $t_{\mathrm{aa}}, t_{\mathrm{as}}, t_{\mathrm{sa}}, t_{\mathrm{ss}}$, sowie $c_{\mathrm{aa}}, c_{\mathrm{as}}$, $c_{\mathrm{sa}}, c_{\mathrm{ss}}$ möglich sind (siehe Abschnitt 2.1 für die Nomenklatur).

Unter der Annahme, dass syn- und anti-Stellung Minima darstellen, ist der Winkel zwischen zwei benachbarten Minima $60^{\circ}$ und das Potential der beiden Methylrotation sechszählig. Die Methylrotation ist dabei, anders als für die cis-/trans-Isomerisierung, nicht durch eine partielle Doppelbindung gehindert, so dass diese mit Barrieren ${ }^{10}$ von $0.95 \mathrm{~kJ} \cdot \mathrm{mol}^{-1}$ (N-seitig) und $0.88 \mathrm{~kJ} \cdot \mathrm{mol}^{-1}$ (C-seitig) [169] für trans-NMA um zwei Größenordnungen niedriger liegen. Für c-NMA ist keine entsprechende Barriere bekannt. Experimentelle Aussagen zur Methylgruppenstellung in der Minimumstruktur liefern lediglich die Elektronenbeugungsexperimente von Kitano etal. [165], deren Daten sich am besten an das $t_{\mathrm{aa}}$-Konformer fitten lassen und die Kristallstrukturmessungen von Hagler et al. [167], die das $t_{\text {as }}$-Konformer finden.

Die Frage nach der Methylgruppenorientierung ist hauptsächlich Gegenstand in der theoretischen Literatur und für trans-NMA finden sich quer durch alle gängigen theoretischen Metho-

\footnotetext{
${ }^{1)}$ Sehr hohe Barrieren von 7-8 kJ.mol ${ }^{-1}$ mitsamt falscher Zuordnung der Methyltorsion als Amid VII-Schwingung finden sich bei Fillaux und Tomkinson [168].
} 
den alle vier Isomere wieder, wobei $t_{\mathrm{as}}$ das gemeinhin favorisierte Konformer ist. Anders bei cisNMA, für das nahezu einhellig $c_{\mathrm{aa}}$ als das energetisch günstigste Konformer gefunden wurde, ${ }^{2)}$

Die in dieser Arbeit durchgeführten DFT-Rechnungen zur Struktur von NMA auf B3LYPD3(BJ)/augccpVTZNiveau ${ }^{3)}$ ergeben $t_{\mathrm{as}}$ und $t_{\mathrm{ss}}$ mit einem vernachlässigbaren Energieunterschied von $0.05 \mathrm{~kJ} \cdot \mathrm{mol}^{-1}$ (elektronisch; $0.49 \mathrm{~kJ} \cdot \mathrm{mol}^{-1}$ nullpunktskorrigiert) für trans-NMA und $c_{\mathrm{aa}}$ als einzige Struktur ohne imaginäre Frequenzen in der harmonischen Frequenzrechnung für cis-NMA (für Molekülstrukturen, siehe Abb. 4-1; Details zu den Rechnungen finden sich in Tab. A-11 im Anhang A.7.2).
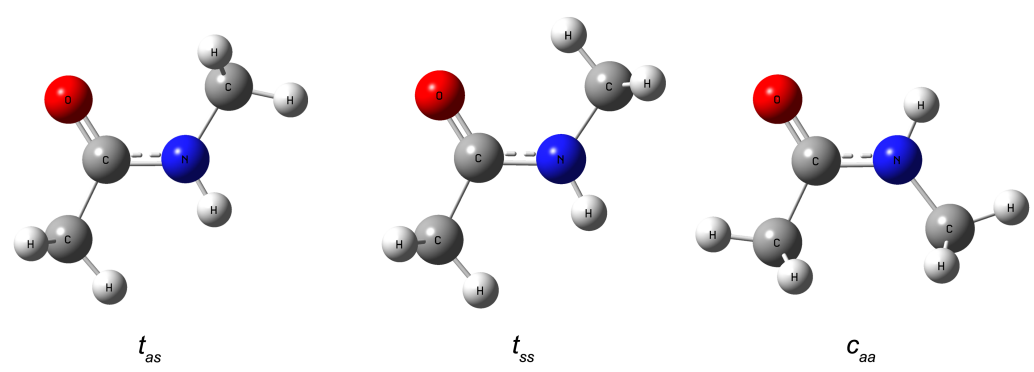

Abb. 4-1. Die drei Minimumstrukturen von NMA nach Geometrieoptimierung und Punktrechnung auf B3LYP$\mathrm{D} 3(\mathrm{BJ}) / \mathrm{aVTZ}-$ Niveau $\left(E_{\mathrm{el}}\left(t_{\mathrm{as}}\right)=E_{\mathrm{el}}\left(t_{\mathrm{ss}}\right)-0.05 \mathrm{~kJ} \cdot \mathrm{mol}^{-1}=E_{\mathrm{el}}\left(c_{\mathrm{aa}}\right)-8.80 \mathrm{~kJ} \cdot \mathrm{mol}^{-1}\right)$

Durch das sehr flache Torsionspotential der Methylgruppen können zugrunde gelegte Näherungen der gewählten Methode oder der intramolekulare BSSE so groß sein, dass das gefundene Minimum darauf zurückzuführen ist und keine physikalische Ursache hat [170]. Die Frage nach den echten cis- und trans-Minimumstrukturen von NMA unter Einbeziehung der Methylgruppenstellung lässt sich also nur mit hinreichend genauen und damit aufwendigen Rechnungen klären und ist nicht Gegenstand dieser Arbeit.

\subsection{Schwingungsspektroskopie des Monomers}

NMA wird seit den ersten IR-Experimenten von Mizushima et al. [69] schwingungsspektroskopisch erforscht. Dabei ist es nicht nur zur experimentellen Bestimmung des cis-/trans-Enthalpieunterschieds interessant, neben dem vorherrschenden trans-NMA auch das cis-Konformer spektroskopisch nachzuweisen.

Dies gelang in Lösung bereits 1956 durch Russell und Thompson [171]. Sie fanden in IRSpektren von NMA in Tetrachlormethan $\left(\mathrm{CCl}_{4}\right)$ zwei Banden im Amid A-Bereich, die sie transund cis-NMA zugeordnet haben, was einem cis-Anteil von $5 \%$ entspricht. Die ersten ${ }^{1} \mathrm{H}-\mathrm{NMR}$ Spektren [159] und ${ }^{15} \mathrm{~N}-\mathrm{NMR}-S p e k t r e n$ [172] zeigen, genauso wie Röntgen-Messungen im Kristall [48, 166], einen Anteil von 0\% cis-NMA. Spätere Arbeiten weisen cis-Anteile von 1.5$7 \%$ in Wasser [160, 162, 173] und 2.1-2.9\% in $\mathrm{CDCl}_{3}$ [162, 163, 173] auf. In der Stick-

\footnotetext{
${ }^{2)}$ Anstatt an dieser Stelle alle theoretischen Arbeiten zu NMA zu zitieren, sind diese mit den jeweiligen Konformeren in der Spalte „Konf.“ in den Tabellen 4-2, 4-5, 4-7 der folgenden Abschnitte und A-14iim Anhang zu finden.

${ }^{3)}$ Vorteile und Limitierungen der Methode werden in Abschnitt 2.3 diskutiert.
} 
stoffmatrix [68] zeigen sich für eine Düsentemperatur von $770 \mathrm{~K}$ ca. $22 \%$ cis-NMA.4) Jüngere Untersuchungen in FTIR-Jet-Experimenten zeigen jedoch keine Spuren von cis-NMA [51], was aber auch am schlechten Signal-zu-Rauschen-Verhältnis der Spektren liegen kann.

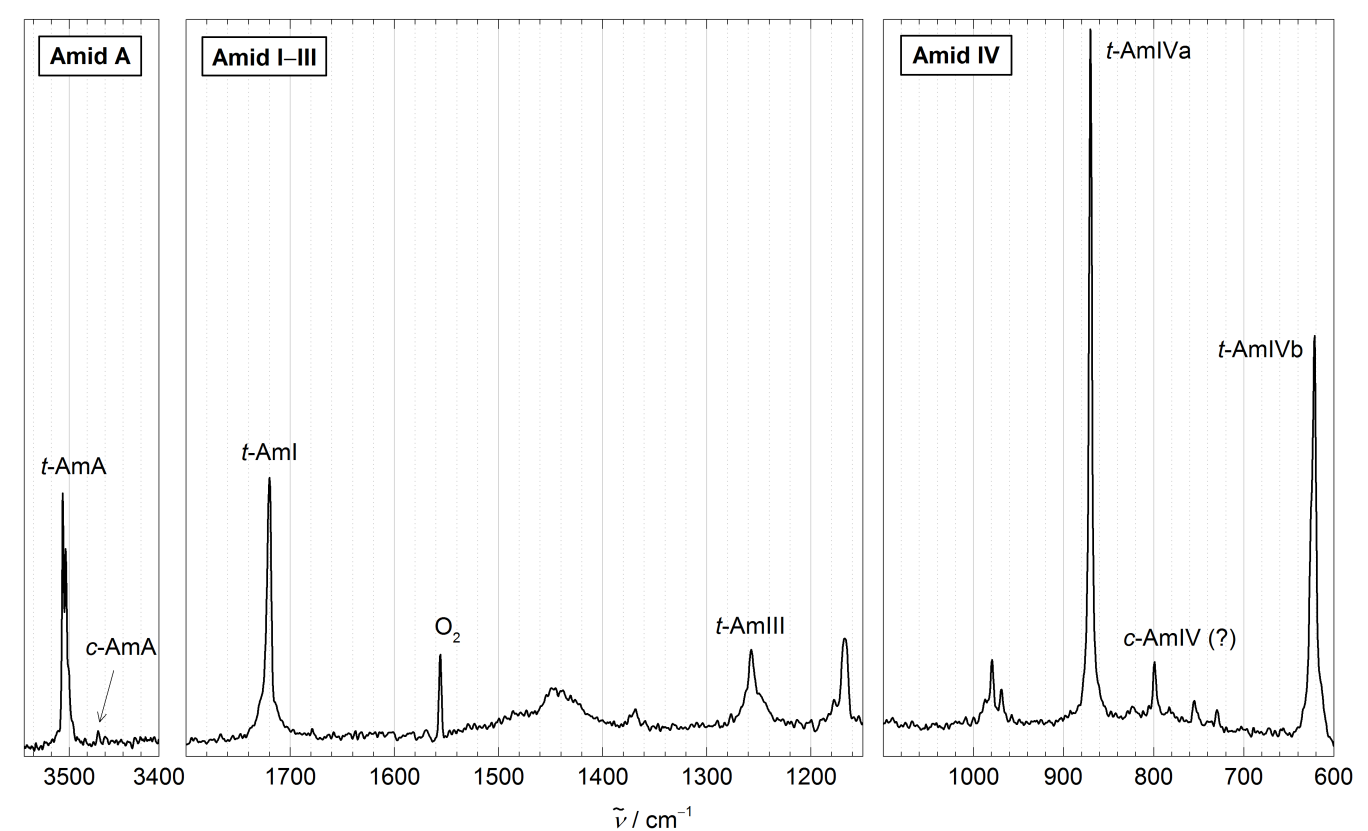

Abb. 4-2. Jet-Raman-Übersichtsspektrum vom NMA-Monomer mit Amidbandenzuordnung. Die Spektren in jeder Teilabbildung stammen aus unabhängig voneinander aufgenommenen Messungen der gleichen Messreihe mit vergleichbar guter Justage, so dass die Signalintensität zwischen den Abbildungen grundsätzlich vergleichbar ist. (Details zu den Messungen befinden sich im Raman-Spektrenverzeichnis in Tab. A-6in Anhang A.6.1)

Die erstmalig im Rahmen dieser Arbeit durchgeführten Raman-Jet-Messungen von NMA liefern unter „clusterfreien“ Messbedingungen ${ }^{5)}$ das Übersichtsspektrum in Abb. 4-2. Es zeigt die drei für die Amidbanden A, I-III und IV relevanten Spektralbereiche.

Amid A Der Amid A-Bereich reicht für NMA von etwas über $3500 \mathrm{~cm}^{-1}$ im Jet und in der Gasphase bis $3300 \mathrm{~cm}^{-1}$ für die Flüssigkeit (siehe Tab.4-1). In diesem Bereich zeigt das RamanSpektrum eine starke Bande bei $3508 \mathrm{~cm}^{-1}$ und eine sehr schwache Bande bei $3469 \mathrm{~cm}^{-1}$. Die in Tab. 4-1 aufgeführten Literaturwerte für die trans-Amid A-Bande ( $t$-AmA) von $3510 \mathrm{~cm}^{-1}$ im Jet und $3509 \mathrm{~cm}^{-1}$ in der Gasphase sind in sehr guter Übereinstimmung mit der intensiven Bande. Auch IR-Messungen in unterschiedlichen Matrizen von $3495-3507 \mathrm{~cm}^{-1}$ stützen diese Zuordnung. Für cis-NMA wurden in der $\mathrm{N}_{2}$-Matrix $3458 \mathrm{~cm}^{-1}$ gemessen. In den Daten von Fillaux und Lozé [74] finden sich Banden bei 3455 und $3460 \mathrm{~cm}^{-1}$, die die Autoren allerdings nicht dem cis-Konformer zuordnen. Der in der Gasphase gemessene Wert von $3435 \mathrm{~cm}^{-1}$ [72] sollte kritisch betrachtet werden, da die zugrunde liegenden Spektren zwei sehr breite und

\footnotetext{
4) Die Zuordnung einiger Peaks in $\mathrm{N}_{2}$ - und Ar-Matrixmessungen zum cis-Konformer von Fillaux und Lozé [74] sind im groben Widerspruch zu Ataka et al. [68] und theoretischen Vorhersagen[174] und wohl eher auf Matrixeffekte zurückzuführen.

${ }^{5)}$ Clusterfreie (bzw. clusterarme) Spektren werden am curry-Jet u. a. mit kleinen Düsenabständen und hohen Düsentemperaturen erreicht (siehe Abschnitt 3.1.1).
} 
überlappende Signale für die $t$-AmA- und c-AmA-Bande aufweisen.

Der Stickstoff-Matrix-Wert von Ataka et. al. liegt mit $11 \mathrm{~cm}^{-1}$ unterhalb dem hier gemessenen Wert der sehr schwachen Bande, was eine nahezu gleiche Verschiebung wie bei der trans-Amid A ist. Diese konstante Rotverschiebung kann auf Matrixeffekte [28] zurückgeführt werden und wird ausführlich von Albrecht [175] besprochen. Somit lässt sich die Bande bei $3469 \mathrm{~cm}^{-1}$ als Amid A des cis-Konformers zuordnen.

Tab. 4-1. Vergleich experimenteller Positionen der Amid A-Bande von trans- $\left(\tilde{v}_{A}^{t}\right)$ und cis-NMA $\left(\tilde{v}_{A}^{c}\right)$ und weitere Banden oberhalb von $3050 \mathrm{~cm}^{-1}$ (Alle Angaben in $\mathrm{cm}^{-1}$; zusätzliche Bandenzuweisungen in runden Klammern; vorläufige Zuordnungen in eckigen Klammern).

\begin{tabular}{|c|c|c|c|}
\hline Methode & $\tilde{v}_{\mathrm{A}}^{t}$ & $\tilde{v}_{\mathrm{A}}^{c}$ & weitere Banden \\
\hline \multicolumn{4}{|l|}{ Jet-Expansion: } \\
\hline Raman $^{\mathrm{a}}$ & 3508 & 3469 & $3504,3501,[3486]$ \\
\hline FTIR $^{\mathrm{a}}$ & 3508 & [3470] & $3425\left(2 v_{\mathrm{CO}}\right)$ \\
\hline FTIR 51 & 3510 & - & - \\
\hline \multicolumn{4}{|l|}{ Matrix: } \\
\hline$N_{2}-\operatorname{IR} 68$ & 3498 & 3458 & - \\
\hline $\mathrm{N}_{2}-\mathrm{IR} 74$ & 3495 & $3460^{\mathrm{b}}$ & 3490,3472 \\
\hline $\mathrm{Ar}-\mathrm{IR}$ & 3507 & $3455^{b}$ & 3503,3500 \\
\hline para- $\mathrm{H}_{2}-\mathrm{IR}$ 176 & 3501 & - & - \\
\hline \multicolumn{4}{|l|}{ Gasphase: } \\
\hline $\mathrm{FTIR}^{\mathrm{a}}$ & 3509,3494 & - & $3425\left(2 v_{\mathrm{CO}}\right)$ \\
\hline IR 73 & 3501,3489 & - & - \\
\hline FTIR & 3490 & 3435 & - \\
\hline Resonanz-Raman 177 & - & - & $3440\left(2 v_{\mathrm{CO}}\right)$ \\
\hline \multicolumn{4}{|l|}{ Lösung: } \\
\hline $\mathrm{CCl}_{4}-\mathrm{IR} 171$ & 3472 & 3440 & - \\
\hline $\mathrm{CCl}_{4}-\mathrm{IR} 178$ & 3470 & - & - \\
\hline $\mathrm{CCl}_{4}-\mathrm{IR} 179$ & 3471 & - & - \\
\hline $\mathrm{CCl}_{4}-\mathrm{IR}[180$ & 3480 & - & - \\
\hline $\mathrm{H}_{2} \mathrm{O}+\mathrm{CCl}_{4}-\mathrm{FTIR} 181$ & 3475 & 3431 & - \\
\hline DMSO - ATR-FTIR [182. & 3290 & - & 3099 (Amid B) \\
\hline \multicolumn{4}{|l|}{ Flüssigkeit: } \\
\hline IR 69 & 3300 & - & 3100 (Amid B) \\
\hline FTIR [183] & 3308 & - & - \\
\hline Raman 69 & 3308 & - & - \\
\hline Raman 19 & 3323 & - & 3080 (Amid B) \\
\hline
\end{tabular}

a: aus dieser Arbeit; ${ }^{b}:$ In der Originalveröffentlichung wurde fälschlicherweise eine andere Bande dem cis-Konformer zugeordnet

Das Detailspektrum des Amid A-Bereichs in Abb. 4-3 stellt den Spektren der Jet-Raman-Messungen bei zwei unterschiedlichen Düsentemperaturen die berechneten Bandenpositionen aus einer doppelt-harmonischen Frequenzrechnung auf B3LYP-D3(BJ)/aVTZ-Niveau gegenüber. Es offenbart eine Substruktur der trans-Amid A-Bande ( $t$-AmA) aus drei Peaks bei 3508, 3504 und $3501 \mathrm{~cm}^{-1}$ und die harmonischen Wellenzahlen sind so skaliert, dass die höchstfrequente Normalmode des $t_{\mathrm{as}}$-Konformers auf die $t$-AmA-Fundamentale bei $3508 \mathrm{~cm}^{-1}$ fällt. Die Rechnung zeigt, dass die Mode zu $100 \%$ in der N-H-Streckschwingung lokalisiert ist (siehe auch die graphische Darstellung der Normalmoden in den Molekülstrukturen in Abb. 4-3), weswegen sie auch als $v_{\mathrm{NH}}^{t}$ bezeichnet wird.

Mit dieser Skalierung ergibt sich für das $c_{a a}$-Konformer eine sehr gute Übereinstimmung 


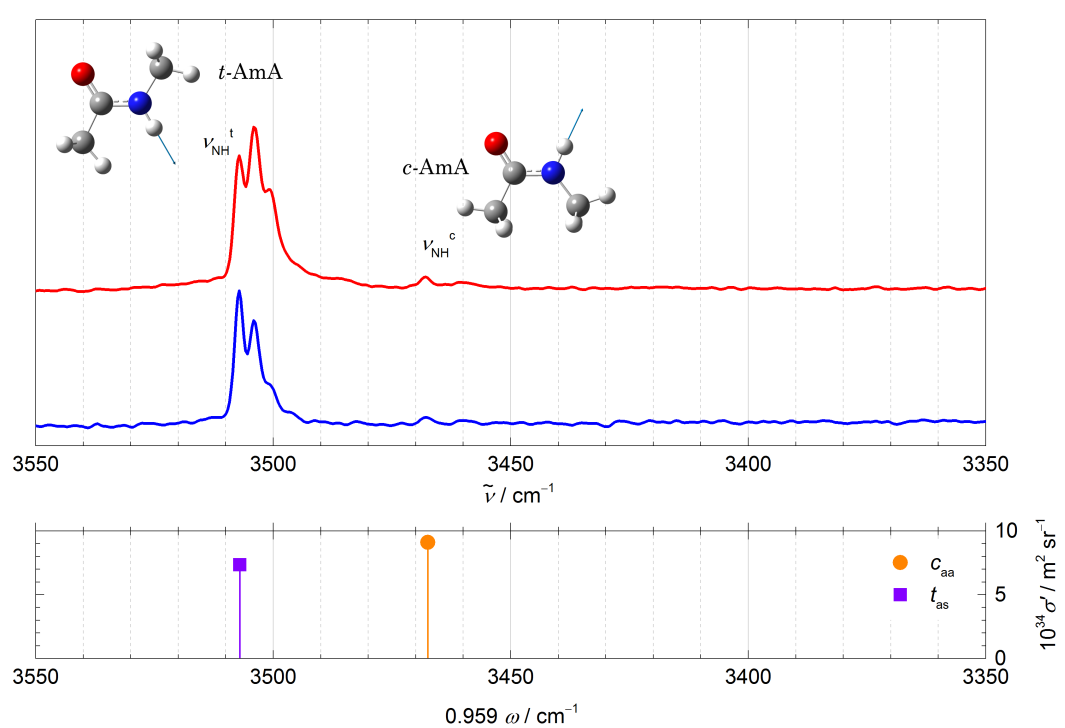

Abb. 4-3. curry-Jet-Detailspektrum vom NMA-Monomer im Amid A-Bereich bei $\vartheta_{\mathrm{H}}=130^{\circ} \mathrm{C}$ (unten, blau) und $190^{\circ} \mathrm{C}$ (oben, rot), normiert auf die trans-Monomerfundamentale. Darunter befinden sich Strichspektren des jeweils stabilsten trans- (Rechteck, violett) und cis-Konformers (rund, orange) auf B3LYP-D3(BJ)/aVTZ-Niveau und an den jeweiligen Molekülstrukturen illustrierte Normalmoden. Eine Skalierung der harmonischen Wellenzahl um 0.959 bezieht die theoretische Linie von $t$-NMA auf $v_{\mathrm{NH}}^{t}$. (Details zu den Messungen befinden sich im FTIR-Spektrenverzeichnis in Tab. A-8 in Anhang A.6.2, Details zu den Frequenzrechnungen befinden sich in Tab. A-15in Anhang A.7.2)

der gerechneten und skalierten Bandenposition der $v_{\mathrm{NH}}^{c}$ von $3467 \mathrm{~cm}^{-1}$ mit der vermuteten $c$ AmA-Bande im Raman-Spektrum. Der berechnete Streuquerschnitt ist dabei 1.25 mal so groß wie der der trans-Bande, was die spektroskopische Sichtbarkeit begünstigt (siehe Tab. A-15 in Anhang A.7.2).

Der Blick auf quantenchemische Frequenzrechnungen für das isolierte NMA-Molekül in der Literatur (siehe Tab. 4-2) zeigt ein uneinheitliches Bild. Erwartungsgemäß liegen unskalierte Frequenzen aller doppelt-harmonischen Methoden oberhalb der gemessenen Werte und sollten in erster Näherung um das doppelte der diagonalen Anharmonizität $\left(-2 x_{1,1}\right)$ bezüglich der experimentellen Bandenposition verschoben sein. Eine grobe Abschätzung der Anharmonizität der NH-Streckschwingung für trans-NMA liefert der Vergleich von den Bandenpositionen des ersten Obertons bei $6800 \mathrm{~cm}^{-1}$ [184] und der Fundamentalen bei $3471 \mathrm{~cm}^{-1}$ [171, 178] in verdünnter $\mathrm{CCl}_{4}$-Lösung. Dies ergibt für die anharmonische Verschiebung: $2 x_{1,1}=-142 \mathrm{~cm}^{-1}$. Die Spanne der Abweichungen der harmonischen Rechnungen vom experimentellen Wert ist jedoch sehr groß und reicht von $+(35-182) \mathrm{cm}^{-1}$ für die $v_{\mathrm{NH}}^{t}$ und $+(50-169) \mathrm{cm}^{-1}$ für die $v_{\mathrm{NH}}^{c}$. Dabei reproduzieren die B3LYP-Methoden mit einer Verschiebung von $130-149 \mathrm{~cm}^{-1}$ die abgeschätzte experimentelle Anharmonizität am besten. Die DFT-Frequenzrechnungen mit PW91Anteil liegen am nächsten an den experimentellen Werten, was ein Indiz für eine schlechte harmonische Methode ist. Dies bestätigt sich beim Blick auf die Differenz zwischen trans- und cis-Frequenzen $\left(\Delta_{c-t}\right.$, in Tab. 4-2), wo der experimentelle Unterschied von $39 \mathrm{~cm}^{-1}$ im RamanJet-Spektrum nur um maximal $15 \mathrm{~cm}^{-1}$ verfehlt wird, dies allerdings eben mit der $\mathrm{PW} 91_{\mathrm{XC}} / 6$ $31+G^{*}$-Rechnung.

Die mit anharmonischen Methoden berechneten Frequenzen ergeben ein zweigeteiltes Bild. 
Während jene auf MP2-Basis nach wie vor zu hohe Frequenzen für $v_{\mathrm{NH}}^{t}$ liefern (allerdings mit maximal $36 \mathrm{~cm}^{-1}$ deutlich näher am Experiment liegend), liegen die Ergebnisse der DFTbasierten Methoden 29-42 $\mathrm{cm}^{-1}$ unterhalb des experimentellen Wertes von $3508 \mathrm{~cm}^{-1}$. Prinzipiell liegt mit der cc-VSCF auf MP2-Grundlage [185] die beste anharmonische Rechnung mit einer Differenz von $15 \mathrm{~cm}^{-1}$ immer noch deutlich oberhalb der $t$-AmA-Bande. Es sind keine anharmonischen Werte für cis-NMA bekannt, was zumindest für die eigenen VPT2-Rechnungen auf imaginäre Frequenzen für alle möglichen cis-Konformere zurückzuführen ist.

Tab. 4-2. Harmonische und anharmonische Wellenzahlen der NH-Streckschwingung von trans- $\left(\tilde{v}_{N H}^{t}\right)$ und cis-NMA $\left(\tilde{v}_{\mathrm{NH}}^{c}\right)$ mit unterschiedlichen quantenmechanischen Methoden (Auswahl - Eine umfassende Übersicht liefert Tab. A-12 im Tabellenwerk A.7.2 im Anhang). Die den Berechnungen zugrunde liegenden Konformationen (Konf.) sind angegeben, soweit sie aus den jeweiligen Veröffentlichungen hervorgehen. Zum Vergleich sind zusätzlich die experimentellen Werte der Raman- und FTIR-Jet-Messung angegeben. Alle Angaben in $\mathrm{cm}^{-1}$.

\begin{tabular}{|c|c|c|c|c|c|}
\hline Methode & $\tilde{v}_{\mathrm{NH}}^{t}$ & $\tilde{v}_{\mathrm{NH}}^{c}$ & $\Delta_{c-t}$ & weitere Banden & Konf. \\
\hline \multicolumn{6}{|l|}{ doppelt-harmonisch: } \\
\hline $\mathrm{HF} / 6-31 \mathrm{G}^{* \mathrm{~b}}, 66,174$ & 3510 & 3471 & -39 & - & $t_{\mathrm{as}} / c_{\mathrm{aa}}$ \\
\hline MP2/aVTZ [186] & 3690 & 3638 & -52 & - & $t_{\mathrm{ss}} / c_{\mathrm{aa}}$ \\
\hline $\mathrm{B} 3 \mathrm{LYP} / 6-31+\mathrm{G}^{\star}[181]$ & 3643 & 3603 & -40 & - & $t_{\mathrm{sa}} / c_{\mathrm{ss}}$ \\
\hline B3LYP/6-311++G & 3644 & - & - & - & $t_{\mathrm{ss}} / c_{\mathrm{aa}}$ \\
\hline B3LYP-D3(BJ)/def2TZVPa & 3638 & 3605 & -33 & - & $t_{\mathrm{ss}} / c_{\mathrm{aa}}$ \\
\hline B3LYP-D3(BJ)/aVTZa & 3657 & 3616 & -41 & - & $t_{\mathrm{as}} / c_{\mathrm{aa}}$ \\
\hline BPW91/6-31G(d,p) [187] & 3576 & 3544 & -32 & - & $t_{\mathrm{ss}} / c_{\mathrm{aa}}$ \\
\hline$P W 91 \times C / 6-31+G^{*}$ & 3543 & 3519 & -24 & - & $t_{\mathrm{ss}} / c_{\mathrm{aa}}$ \\
\hline \multicolumn{6}{|l|}{ anharmonisch: ${ }^{d}$} \\
\hline B3LYP/6-31+G*(3MR-PES) + VCI [189] & 3479 [3643] & - & - & - & $t_{\mathrm{as}} /-$ \\
\hline B3LYP/6-31+G*(3MR-PES) + VPT2 189 & 3466 [3643] & - & - & - & $t_{\mathrm{as}} /-$ \\
\hline B3LYP-D3(BJ)/VTZ + VPT2 ${ }^{\mathrm{a}}$ & $3478[3650]$ & - & - & $3436\left(2 v_{\mathrm{CO}}\right)$ & $t_{\mathrm{ss}} /-$ \\
\hline MP2/DZP(2MR-PES) + cc-VSCF 185 & 3523 [3751] & - & - & - & $t_{\mathrm{as}} /-$ \\
\hline MP2/DZP(DZP pair potential) + cc-VSCF 190 & $3542[3752]$ & - & - & - & $-1-$ \\
\hline MP2/aVTZ(part. 3MR-PES) + VCI 191] & $3544[3705]$ & - & - & - & $t_{\mathrm{as}} /-$ \\
\hline \multicolumn{6}{|l|}{ experimentell: ${ }^{\mathrm{e}}$} \\
\hline Jet-Messungen $^{a}$ & 3508 & 3469 & -39 & $3425\left(2 v_{\mathrm{CO}}\right)$ & $-1-$ \\
\hline
\end{tabular}

Bei genauerer Betrachtung der Substruktur der trans-Amid A-Bande fällt auf, dass die Signale bei 3504 und $3501 \mathrm{~cm}^{-1}$ mit zunehmender Temperatur (vom unteren zum oberen Spektrum) gegenüber dem Peak bei $3508 \mathrm{~cm}^{-1}$ an Intensität zunehmen und der mittlere Peak den höchstfrequenten sogar übersteigt. Dabei kann es sich um heiße Banden handeln, bei denen die Anregung der N-H-Strecknormalmode in Kombination mit thermisch angeregten niederfrequenten Moden erfolgt. Damit diese im Spektrum separat erkennbar sind, müssen sie von der Fundamentalen eine hinreichende Verschiebung aufweisen. Diese Verschiebung ist ein Ausdruck der Anharmonizität und entspricht dem nicht-diagonalen Kopplungselement $x_{i j}$ der zugrunde liegenden Normalmoden $v_{i}$ (Fundamentale) und $v_{j}$ (angeregte niederfrequente Mode) [192]. Durch anharmonische Frequenzrechnungen lässt sich eine solche Anharmonizität abschätzen.

Die in Tabelle 4-3 aufgeführten Ergebnisse einer anharmonischen VPT2-Rechnung (siehe Abschnitt 3.3. auf B3LYP-D3(BJ)/VTZ-Niveau für das $t_{\mathrm{ss}}$-Konformer ${ }^{6}$ liefert eine konsistente Er-

${ }^{6)}$ Wie aus Tab. A-11 im Anhang A.7.2 ersichtlich, ist dies das einzige trans-Konformer, was ausschließlich nicht- 
klärung für die Substruktur der Amid A-Bande. Demnach handelt es sich bei dem $3508 \mathrm{~cm}^{-1}$ Signal um die N-H-Streckfundamentale $\left(v_{\mathrm{NH}}^{t}\right.$ oder $v_{1}$ ). Der Peak bei $3504 \mathrm{~cm}^{-1}$ liegt um $4 \mathrm{~cm}^{-1}$ rotverschoben zur Fundamentalen, was in guter Übereinstimmung mit den Kopplungskonstanten $x_{1,30}$ und $x_{1,28}$ mit -4.8 respektive $-4.5 \mathrm{~cm}^{-1}$ ist und einer Kombination der $v_{1}$ mit der thermisch angeregten niedrigsten $\left(v_{30}\right)$ bzw. drittniedrigsten Mode $\left(v_{28}\right)$ entspricht. Die gleiche Verschiebung für die zwei resultierenden unabhängigen heißen Banden $\left(v_{1}+v_{30}\right)-v_{30}$ und $\left(v_{1}+v_{28}\right)-v_{28}$ erklärt auch die Möglichkeit, dass der Peak bei $3504 \mathrm{~cm}^{-1}$ die Intensität der $\mathrm{N}-\mathrm{H}$-Streck-Fundamentalen übersteigt.

Tab. 4-3. Experimentelle und theoretische anharmonische Konstanten $x_{i, j}^{\exp }$ und $x_{i, j}^{\mathrm{VPT} 2}$ für die Amid A-Bande von trans$\mathrm{N}$-Methylacetamid aus dem Jet-Ramanspektrum in Abb. 4-3 und ausgewählten niederfrequenten Normalmoden $j$ basierend auf dem Vergleich mit VPT2-Rechnungen des $t_{\mathrm{ss}}$-Konformers (B3LYP-D3(BJ)/VTZ). Zusätzlich sind die berechneten harmonischen und anharmonischen Wellenzahlen $\tilde{v}_{j}^{\text {harm }}$ bzw. $\tilde{v}_{j}^{\mathrm{VPT} 2}$ der niederfrequenten Normalmode $j$ angegeben. Die Auswahl beinhaltet nur die niederfrequentesten Normalmoden $j$ mit $x_{1, j}^{\mathrm{VPT} 2}>1 \mathrm{~cm}^{-1}$. Die Symmetrierassen aller angegebenen $j$ bezüglich einer $C_{\mathrm{s}}$-Molekülsymmetrie ist $A^{\prime \prime}$ (aus der Molekülebene), während $i=1 A^{\prime}$-symmetrisch ist (in der Ebene).

\begin{tabular}{cccccc}
\hline$i$ & $j$ (Sym.) & $\tilde{v}_{j}^{\text {harm }} / \mathrm{cm}^{-1}$ & $\tilde{v}_{j}^{\mathrm{VPT} 2} / \mathrm{cm}^{-1}$ & $x_{i, j}^{\mathrm{VPT} 2} / \mathrm{cm}^{-1}$ & $x_{i, j}^{\mathrm{exp}} / \mathrm{cm}^{-1}$ \\
\hline 1 & $30\left(A^{\prime \prime}\right)$ & 56 & 23 & -4.8 & -4 \\
1 & $28\left(A^{\prime \prime}\right)$ & 166 & 165 & -4.5 & -4 \\
1 & 25 & 446 & 433 & -18.7 & -22 \\
1 & $2 \times 30\left(A^{\prime \prime}\right)$ & 112 & 42 & -9.6 & -7 \\
1 & $2 \times 28\left(A^{\prime \prime}\right)$ & 332 & 331 & -9.0 & -7 \\
\hline
\end{tabular}

Der $3501 \mathrm{~cm}^{-1}$-Peak weist mit $-7 \mathrm{~cm}^{-1}$ eine ungefähr doppelt so große Verschiebung zur Fundamentalen auf wie das $3504 \mathrm{~cm}^{-1}$-Signal, was eine Interpretation als heiße Bande mit den Obertönen der in der ersten heißen Bande involvierten niederfrequenten Moden nahelegt. Die theoretische Verschiebung für Oberton-Kombinationsbanden liegt bei $2 x_{1, j}^{\mathrm{VPT} 2}$, also bei -9.6 respektive $-9.0 \mathrm{~cm}^{-1}$. Damit wird die Verschiebung in der Rechnung leicht überschätzt kann aber aufgrund der involvierten Näherungen in der anharmonischen Rechnung und der Messunsicherheit des Experiments (siehe 3.1.4) generell als gute Übereinstimmung gesehen werden. Auch hier ist der im Spektrum sichtbare Peak eine Überlappung der Signale beider Übergänge. Eine gute Möglichkeit zur Abschätzung der Validität der Zuordnung ist ein Vergleich der Düsentemperatur mit der aus dem Bandenintensitätsverhältnis berechneten Boltzmann-Temperatur. Auf Grund der besprochenen Überlappung in beiden heißen Banden lässt sich in diesem Fall die Intensität nicht sinnvoll abschätzen.

Die bisher nicht erwähnten niederfrequenten Moden $v_{26}, v_{27}$ und $v_{29}$ sind bei den experimentellen Bedingungen nach Boltzmann ebenfalls thermisch angeregt und sollten ebenso zu sichtbaren heißen Banden führen. Die Kopplungskonstanten $2 x_{1, j}^{\mathrm{VPT} 2}$ aus der VPT2-Rechnung ergeben aber eine maximale Verschiebung von $+0.3 \mathrm{~cm}^{-1}$, weswegen sie im Spektrum nicht als separate Banden aufgelöst werden können.

Eine andere mögliche Ursache für die Bandenstruktur ist eine Tunnelaufspaltung, die durch die niedrige Höhe der $V_{3}$-Potentiale der Methylrotoren hervorgerufen wird. Dies ergibt für jedes Potential eine Aufspaltung in ein A-Energieniveau und zwei entartete E-Niveaus, die durch Kopplung weiter aufspalten [169]. Die Energieunterschiede der Niveaus sind bisher noch nicht

imaginäre Frequenzen liefert. 
bekannt, allerdings liefert die Entartung des E-Niveaus ebenfalls eine gute Erklärung für die Übersteigung der Intensität der Fundamentalen durch die Bande bei $3504 \mathrm{~cm}^{-1}$ im heißen Spektrum.

Amid I-III Im mittleren Bildteil des Übersichtsspektrums (Abb. 4-2) ist mit dem Bereich von $1800-1150 \mathrm{~cm}^{-1}$ die Amid I-III-Region abgebildet. Sie weist neben einigen kleinen Banden und einer sehr breiten Bande bei $1440 \mathrm{~cm}^{-1}$ vor allem eine starke und vergleichsweise schmale Bande bei $1720 \mathrm{~cm}^{-1}$ und zwei kleinere Banden bei $1258 \mathrm{~cm}^{-1}$ und $1168 \mathrm{~cm}^{-1}$ auf. Außerdem ist die sehr Raman-aktive Sauerstoffschwingung7) bei $1556 \mathrm{~cm}^{-1} \mathrm{zu}$ erkennen, was eine unabhängige Bestätigung der Kalibrierung ermöglicht [193].

Tab. 4-4. Vergleich experimenteller Werte $\tilde{v}_{\mid}, \tilde{v}_{\mid l}, \tilde{v}_{|I|}$ der Amid I-III-Banden von trans-NMA und cis-NMA (in Klammern) und $\mathrm{CH}$-Gerüstschwingungen zwischen 1800 und $1100 \mathrm{~cm}^{-1}$. Alle Angaben in $\mathrm{cm}^{-1}$. (Eine umfassende Liste findet sich in Tab. A-9 im Tabellenwerk A.7.2 im Anhang.)

\begin{tabular}{|c|c|c|c|c|}
\hline Methode & $\tilde{v}_{1}$ & $\tilde{v}_{\|}$ & $\tilde{v}_{\mid I I}$ & weitere Banden \\
\hline \multicolumn{5}{|l|}{ Jet-Expansion: } \\
\hline Raman $^{\mathrm{a}}$ & 1720 & - & 1258 & 1440vb, 1369, 1178, 1168 \\
\hline FTIR $^{\mathrm{a}}$ & 1720 & 1534 & 1258 & $1490,1475,1451,1423,1370$ \\
\hline FTIR $[51]$ & 1722 & - & - & - \\
\hline IRMPD + VUVC 194$]$ & 1707 & 1519 & 1248 & $1478,1415,1364$ \\
\hline \multicolumn{5}{|l|}{ Matrix: } \\
\hline $\mathrm{N}_{2}-\mathrm{IR} 68$ & 1707 & $1511(1485)$ & $1266(1325)$ & $1445,1432,1419,1370,1168$ \\
\hline $\mathrm{N}_{2}-\mathrm{IR} 74$ & 1706195 & 1524,1511 & 1265 & - \\
\hline $\mathrm{Ar}-\mathrm{IR} \mid 74$ & 1708 195 & 1523 & 1264 & - \\
\hline para- $\mathrm{H}_{2}-\mathrm{IR}$ 176 & 1710 & 1513 & 1261 & $1473,1449,1444,1430,1420,1369$ \\
\hline \multicolumn{5}{|l|}{ Gasphase: } \\
\hline $\mathrm{FTIR}^{\mathrm{a}}$ & 1730,1715 & - & 1254 & $1534,1493,1372$ \\
\hline FTIR [73] & 1731,1713 & 1497 & 1257 & 1426,1377 \\
\hline FTIR 72 & 1731,1713 & 1535,1500 & 1256,1247 & - \\
\hline FTIR & 1731,1714 & 1499 & 1255 & - \\
\hline UVRR [177] & 1728 & 1500 & 1259 & - \\
\hline
\end{tabular}

Der Vergleich mit den Literaturwerten in Tab. 4-4 erklärt die $1720 \mathrm{~cm}^{-1}$-Bande als Amid I, mit $-2 \mathrm{~cm}^{-1}$ Differenz zum FTIR-Jet und den bereits in gleicher Höhe für die Amid A gefundenen Unterschied von $+10-14 \mathrm{~cm}^{-1}$ zu den Matrix-Daten. Die leichte Abweichung kann ihre Ursache sowohl in der um $80^{\circ} \mathrm{C}$ niedrigeren Düsentemperatur oder dem schlechten Signal-zuRauschen-Verhältnis der popcorn-Jet-Spektren in [51] haben. In der Gasphase zeigt die Amid I eine Rotations-Substruktur mit P- und R-Zweig, was in manchen Quellen als Doppelpeak mit zwei Wellenzahlen angegeben wird (siehe Tab. 4-4). Die Gasphasen-IR-Daten in der Literatur sind somit ebenfalls in guter Übereinstimmung mit der Raman-Jet-Messung.

Im Bereich knapp oberhalb von $1500 \mathrm{~cm}^{-1}$, wo sich in den IR-Spektren im Jet und in der Matrix die Amid II-Bande befindet, ist im Ramanspektrum keine Bande vorhanden, was zur generell schwachen Raman-Intensität der Amid II Übergänge passt (siehe dazu auch Abschnitt 2.2). Lediglich im elektronisch angeregten Zustand von UV-Resonanz-Raman-Experimenten (UVRR) ist der Nachweis der Amid II möglich [177].

\footnotetext{
${ }^{7)}$ Hervorgerufen durch den Restluftanteil in der Vakuumkammer und dem expandierten Gasgemisch.
} 
Die Amid III befindet sich bei $1258 \mathrm{~cm}^{-1}$ mit einer Schulter auf der niederfrequenten Seite. Die Position stimmt mit den Gasphasen- und Matrixdaten in der Literatur (siehe Tab. 4-4) gut überein. Dabei liegen die Matrixwerte $3-8 \mathrm{~cm}^{-1}$ oberhalb und die Gasphasenwerte zwischen +1 und $-4 \mathrm{~cm}^{-1}$ bezogen auf die Raman-Jet-Position.

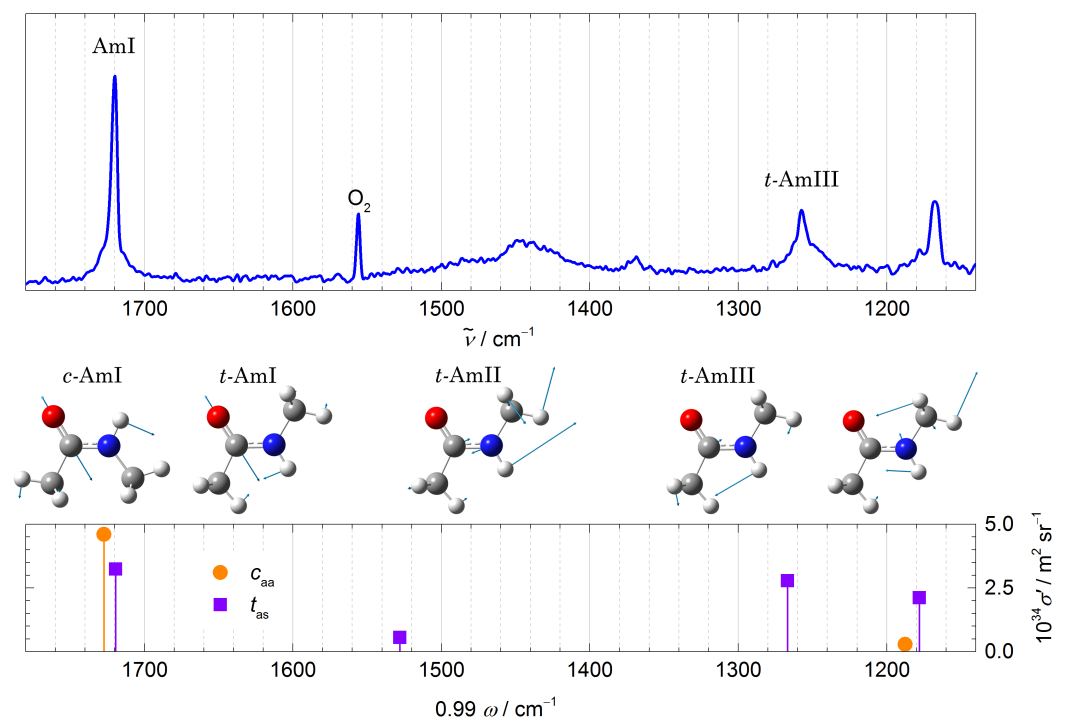

Abb. 4-4. curry-Jet-Spektrum vom NMA-Monomer im Amid I-III-Bereich bei $130^{\circ} \mathrm{C}$. Darunter Strichspektren ausgewählter Normalmoden des jeweils stabilsten trans- (Rechteck, violett) und cis-Konformers (rund, orange) auf B3LYPD3(BJ)/aVTZ-Niveau und an den jeweiligen Molekülstrukturen illustrierte Normalmoden. Eine Skalierung der harmonischen Wellenzahl um 0.99 bezieht die berechnete $v_{\mathrm{CO}}^{t}$-Bandenposition auf das Maximum der experimentellen AmlBande. (Details zu den Messungen befinden sich im FTIR-Spektrenverzeichnis in Tab. A-8 in Anhang A.6.2, Details zu den Frequenzrechnungen befinden sich in Tab. A-15 in Anhang A.7.2)

Das Detailspektrum in Abb. 4-4 liefert wieder den Vergleich mit den skalierten Daten der B3LYP-D3(BJ)/aVTZ-Frequenzrechnung. Hier wird eine leichte Schulter der AmI-Bande auf der hochfrequenten Seite erkennbar, die sich mit einem Blick auf die theoretischen Bandenposition als c-AmI-Bande erklären ließe. Es ist in der experimentellen Literatur aber keine Messung zur c-AmI bekannt, weswegen die Zuordnung hier nicht experimentell bestätigt werden kann. Die theoretische Literatur (siehe Tab. 4-5) findet die AmI des cis-Konformers auch immer zwischen 3-7 $\mathrm{cm}^{-1}$ oberhalb vom trans-Konformer (nur die skalierten HF-Daten von Mirkin et. al. zeigen einen viel größeren Unterschied). Für die Bewertung der harmonischen $t$-AmI-Frequenzen hilft, wie bereits bei der Amid A-Bande, der Blick auf die Anharmonizität der zugrundeliegenden Schwingung. Mit Hilfe des Obertons wurde die diagonale Kopplungskonstante der COStreckschwingung von $t$-NMA in der Wasserstoff-Matrix zu $-8.5(2) \mathrm{cm}^{-1}$ bestimmt [176]. Eine gute harmonische Rechnung würde folglich eine Wellenzahl für die $v_{\mathrm{CO}}^{t} \mathrm{ca} .17 \mathrm{~cm}^{-1}$ oberhalb der gemessenen Bandenposition bei $1720 \mathrm{~cm}^{-1}$ vorhersagen, was exakt dem Wert der B3LYPD3(BJ)/aVTZ-Rechnung dieser Arbeit entspricht. Außerdem liefern auch die MP2/aVTZ- [186] und der BPW91-Rechnungen [187] sehr gute Werte. Wie bereits für die Amid A, ist die PW91 XC- $^{-}$ Rechnung [188], hier gemeinsam mit einer MP2/VTZ-Rechnung [186], die am stärksten abweichende Methode (siehe Tab.4-5).

Allgemein liegen die absoluten Bandenposition der $t$-AmI aus anharmonischen VCI- und 
Tab. 4-5. Harmonische und anharmonische Wellenzahlen $\tilde{v}_{1}, \tilde{v}_{I I}, \tilde{v}_{I I I}$ der Amid I-III Normalmoden von trans-NMA und cisNMA (in Klammern), berechnet mit unterschiedlichen Methoden (Auswahl - Eine umfassende Übersicht liefert Tab. A-13 im Tabellenwerk A.7.2 im Anhang.). Zum Vergleich sind zusätzlich die experimentellen Werte der Raman- und FTIR-JetMessung angegeben. Die den Berechnungen zugrundeliegenden Konformationen (Konf.) sind angegeben, soweit sie aus den jeweiligen Veröffentlichungen hervorgehen. Alle Angaben in $\mathrm{cm}^{-1}$; cis-Konformere in runden Klammern.

\begin{tabular}{|c|c|c|c|c|}
\hline Methode / Basissatz & $\tilde{v}_{1}$ & $\tilde{v}_{\| I}$ & $\tilde{v}_{\mid I I}$ & Konf. \\
\hline \multicolumn{5}{|l|}{ doppelt-harmonisch: } \\
\hline $\mathrm{HF} / 6-31 \mathrm{G}^{* \mathrm{~d}} 66 \quad 174$ & $1700(1717)$ & $1512(1481)$ & 1266 & $t_{\mathrm{as}} / c_{\mathrm{aa}}$ \\
\hline MP2/VTZ 186] & $1770(1777)$ & $1578(1545)$ & 1284 & $t_{\mathrm{ss}} / c_{\mathrm{aa}}$ \\
\hline MP2/aVTZ 186] & $1740(1750)$ & $1559(1544)$ & 1289 & $t_{\mathrm{sS}} / c_{\mathrm{aa}}$ \\
\hline B3LYP-D3(BJ)/def2TZVPa & $1749(1755)$ & $1563(1523)$ & $1273(1349)$ & $t_{\mathrm{ss}} / c_{\mathrm{aa}}$ \\
\hline B3LYP-D3(BJ)/aVTZa & $1737(1744)$ & $1543(1523)$ & $1280(1347)$ & $t_{\mathrm{as}} / c_{\mathrm{aa}}$ \\
\hline BPW91/6-31G(d,p) [187] & $1736(1741)$ & $1522(1493)$ & $1231(1308 ?)$ & $t_{\mathrm{ss}} / c_{\mathrm{aa}}$ \\
\hline$P W 91 \times C / 6-31+G^{*} 188$ & $1706(1709)$ & $1528(1496)$ & $1244(1322)$ & $t_{\mathrm{ss}} / c_{\mathrm{aa}}$ \\
\hline \multicolumn{5}{|l|}{ anharmonisch: ${ }^{\mathrm{e}}$} \\
\hline B3LYP/6-31+G*(3MR-PES) + VCI 189 & $1725[1751]$ & $1537[1560]$ & $1272[1292]$ & $t_{\text {as }}$ \\
\hline B3LYP/6-31+G*(3MR-PES) + VPT2 189] & $1725[1751]$ & $1505[1560]$ & 1256 [1292] & $t_{\text {as }}$ \\
\hline B3LYP-D3(BJ)/VTZ + VPT2 ${ }^{\mathrm{a}}$ & $1727[1755]$ & $1511[1563]$ & $1245[1272]$ & $t_{\mathrm{sS}}$ \\
\hline MP2/DZP(2MR-PES) + cc-VSCF $185^{c}$ & $1751[1780]$ & $1547[1584]$ & $1283[1309]$ & $t_{\text {as }}$ \\
\hline MP2/DZP(DZP pair potential) + cC-VSCF 190 & 1749 [1749] & $1536[1585]$ & $1284[1310]$ & \\
\hline MP2/aVTZ(part. 3MR-PES) + VCI [191] & 1727 & 1519 [1561] & $1253[1290]$ & $t_{\text {as }}$ \\
\hline \multicolumn{5}{|l|}{ experimentell: ${ }^{\dagger}$} \\
\hline Jet-Messungen $^{a}$ & 1720 & 1534 & 1258 & $-1-$ \\
\hline
\end{tabular}

VPT2-Rechnungen nur 5-7 $\mathrm{cm}^{-1}$ über dem experimentellen Wert von $1720 \mathrm{~cm}^{-1}$, was einer sehr guten Übereinstimmung entspricht. Dahingegen liefern die cc-VSCF-Rechnungen Werte, die genauso stark vom Messwert abweichen, wie die Rechnungen in doppelt-harmonischer Näherung. Letztendlich findet sich im Detailspektrum auch unter Einbeziehung der Theorie kein Signal, das sich alleine dem cis-Konformer zuordnen lässt.

Die Theorie liefert hier zumindest die Erklärung für das Nichtvorhandensein der Amid IIBande im Raman-Spektrum, denn die zugrunde liegende Normalmode besteht hauptsächlich aus der N-H-Deformationsschwingung, die die Polarisierbarkeit kaum verändert und zu einem minimalen Streuquerschnitt führt (siehe Modenbild und Strichspektrum in Abb. 4-4 und Tab. A-15 in Anhang A.7.2.

Bei allen anderen Banden handelt es sich um Gerüstschwingungen insbesondere der Methylgruppen, die hier nicht näher betrachtet werden.

Amid IV Unterhalb von $1000 \mathrm{~cm}^{-1}$, in dem häufig als „Fingerprint“ bezeichneten Bereich eines Schwingungsspektrums, finden sich die verbliebenen Amid-Banden IV-IX [19], wobei das vorliegende Detailspektrum zweier Raman-Jet-Messungen bei unterschiedlichen Düsentemperaturen (Abb. 4-5) nur bis $500 \mathrm{~cm}^{-1}$ reicht und hauptsächlich die Amid IV-Banden erkennen lässt. Es finden sich neben einem schwachen Multiplett bei ca. $980 \mathrm{~cm}^{-1}$ und weiteren kleineren Peaks eine sehr intensive Bande bei $870 \mathrm{~cm}^{-1}$ und eine etwas breitere ebenfalls starke Bande bei $622 \mathrm{~cm}^{-1}$. Die drittstärkste Bande ist ein schwaches Signal bei $799 \mathrm{~cm}^{-1}$.

Die Zuordnung der Banden erfolgt vor dem Hintergrund, dass den beiden verwandten Nor- 


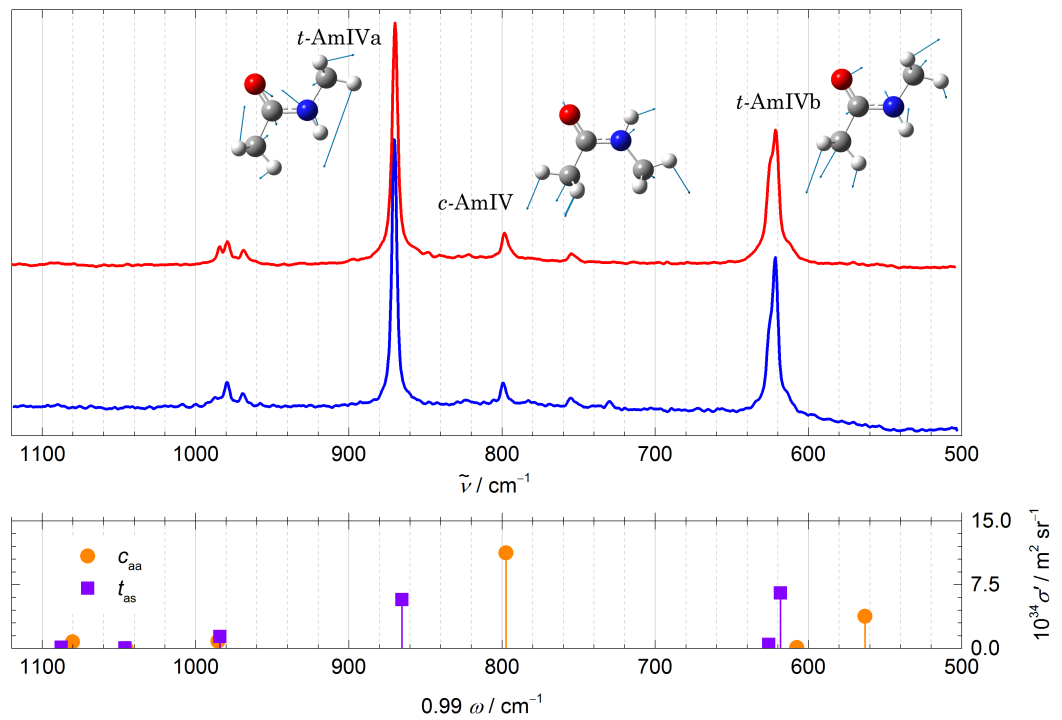

Abb. 4-5. curry-Jet-Spektren vom NMA-Monomer im sogenannten Fingerprintbereich bei $130^{\circ} \mathrm{C}$ (unten, blau) und $190^{\circ} \mathrm{C}$ (oben, rot), normiert auf die $t$-AmIVa-Bande bei $870 \mathrm{~cm}^{-1}$. Darunter Strichspektren des jeweils stabilsten trans(Rechteck, violett) und cis-Konformers (rund, orange) auf B3LYP-D3(BJ)/aVTZ-Niveau und an den jeweiligen Molekülstrukturen illustrierten Normalmoden. Die Wellenzahlskalierung ist die gleiche wie in Abb. 4-4. (Details zu den Messungen befinden sich im FTIR-Spektrenverzeichnis in Tab. A-8 in Anhang A.6.2, Details zu den Frequenzrechnungen befinden sich in Tab. A-15 in Anhang A.7.2)

malmoden Amid IVa und IVb im trans-Konformer nur eine Amid IV-Mode im cis-NMA gegenüber steht [19] und beruht hauptsächlich auf dem Vergleich mit der experimentellen Literatur in Tab. 4-6. Dabei entspricht die intensivste Bande der Amid IVa, was mit der bereits erwähnten Differenz in guter Übereinstimmung mit den $\mathrm{N}_{2}$-Matrixdaten [68] ist. Die Gasphasenmessungen ergeben allerdings deutlich kleinere Werte. In Lösung ist die AmIVa generell um $11-16 \mathrm{~cm}^{-1}$ blauverschoben.

Für die zweitstärkste Bande im Raman-Spektrum gibt es eine breite Übereinstimmung mit der Amid IVb bei einer maximalen Abweichung von $4 \mathrm{~cm}^{-1}$ sowohl in der Gasphase als auch in der Matrix. Selbst in Lösung ist der maximale Unterschied bei $+10 \mathrm{~cm}^{-1}$.

Die UVRR-Messung in wässriger Lösung [82] ist die einzig bekannte Arbeit mit einer Bandenposition für die AmIV-Bande von cis-NMA. Deren Peak bei $822 \mathrm{~cm}^{-1}$ wäre eine mögliche Erklärung für die drittstärkste Bande bei $799 \mathrm{~cm}^{-1}$ im Raman-Spektrum.

Tab. 4-6. Vergleich ausgewählter experimenteller Werte $\tilde{v}_{I V}$ der Amid IV-Banden von trans- und cis-NMA. Alle Angaben in $\mathrm{cm}^{-1}$. (Eine umfassende Liste findet sich in Tab. A-10 im Tabellenwerk A.7.2 im Anhang.)

\begin{tabular}{|c|c|c|c|}
\hline Methode & $\tilde{v}_{\text {IVa }}^{t}$ & $\tilde{v}_{\mathrm{IV}}^{c}$ & $\tilde{v}_{\mathrm{IVb}}^{t}$ \\
\hline Jet - Raman ${ }^{\mathrm{a}}$ & 870 & 799 & 622 \\
\hline $\mathrm{N}_{2}$-Matrix $-\mathrm{IR}^{\mathrm{b}}[68]$ & 857 & - & 619 \\
\hline Gasphase - FTIR & $81273,805[62$ & - & $626[73$ \\
\hline Gasphase - Resonanz-Raman [177] & $-\square$ & - & 626 \\
\hline Lösung (in $\mathrm{CCl}_{4}$ ) - IR 62 & 881 & - & 627 \\
\hline Lösung (in $\mathrm{H}_{2} \mathrm{O}$ ) - UVRR 81 & 881 & - & 628 \\
\hline Lösung (in $\mathrm{H}_{2} \mathrm{O}$ ) - UVRR & 88682 & 822 82] & $6 3 2 \longdiv { 3 5 }$ \\
\hline Lösung (in $\mathrm{H}_{2} \mathrm{O}$ ) - IR-Raman [88] & 883 & - & 632 \\
\hline
\end{tabular}


Die knappe experimentelle Datenlage macht die Einbeziehung der Theorie zur Interpretation der Bande notwendig. Dabei bestätigen sowohl die B3LYP-D3(BJ)/aVTZ-Rechnungen (siehe Strichspektrum in Abb. 4-5 und Daten in Tab. 4-7) mit einer c-AmIV-Bandenposition bei $805 \mathrm{~cm}^{-1}$ (skaliert: $797 \mathrm{~cm}^{-1}$ ) als auch die HF-Rechnungen von Mirkin et. al. [66, 174] mit $798 \mathrm{~cm}^{-1}$ diese Zuordnung.

Damit wäre dies die erste Messung der c-AmIV-Bande im Jet und die AmIV mit den deutlich separierten trans- und cis-Banden nach der AmA ein zweiter möglicher Kandidat für eine Bestimmung der Enthalpiedifferenz (siehe Abschnitt 4.2).

Tab. 4-7. Vergleich harmonischer und anharmonischer Wellenzahlen $\tilde{v}_{\text {IV }}$ der Amid IV Normalmoden von trans-NMA $\left(\tilde{v}^{t}\right)$ und cis-NMA $\left(\tilde{v}^{c}\right)$, berechnet mit unterschiedlichen Methoden. Die den Berechnungen zugrunde liegenden Konformationen (Konf.) sind angegeben, soweit sie aus den jeweiligen Veröffentlichungen hervorgehen. (Eine umfassende Übersicht liefert Tab. A-10 im Tabellenwerk A.7.2 im Anhang.)

\begin{tabular}{|c|c|c|c|c|}
\hline Methode & $\tilde{v}_{\mathrm{IVa}}^{t}$ & $\tilde{v}_{\mathrm{IV}}^{c}$ & $\tilde{v}_{\mathrm{IVb}}^{t}$ & Konf. \\
\hline \multicolumn{5}{|l|}{ doppelt-harmonisch: } \\
\hline $\mathrm{HF} / 6-31 \mathrm{G}^{*}[66,174$ & $861^{\mathrm{c}}$ & $798^{\mathrm{C}}$ & $649,639^{c, d}$ & $t_{\mathrm{as}} / c_{\mathrm{aa}}$ \\
\hline MP2/aVTZ [186] & 881 & 823 & 629 & $t_{\mathrm{ss}} / c_{\mathrm{aa}}$ \\
\hline B3LYP/6-311++G** [183] & 867 & - & 622 & $t_{\mathrm{ss}} / c_{\mathrm{aa}}$ \\
\hline B3LYP-D3(BJ)/def2TZVPa & 869 & 806 & 624 & $t_{\mathrm{ss}} / c_{\mathrm{aa}}$ \\
\hline B3LYP-D3(BJ)/aVTZa & 874 & 805 & 625 & $t_{\mathrm{as}} / c_{\mathrm{aa}}$ \\
\hline$P W 91 \times C / 6-31+G^{*} 188$ & 849 & - & 607 & $t_{\mathrm{ss}} / c_{\mathrm{aa}}$ \\
\hline \multicolumn{5}{|l|}{ anharmonisch: ${ }^{\dagger}$} \\
\hline B3LYP/6-31+G*(3MR-PES) + VCI [189] & 869 [879] & - & 619 [623] & $t_{\mathrm{as}} /-$ \\
\hline B3LYP/6-31+G*(3MR-PES) + VPT2 189] & $861[879]$ & - & 614 [623] & $t_{\mathrm{as}} /-$ \\
\hline B3LYP-D3(BJ)/VTZ + VPT2 ${ }^{a}$ & 847 & - & 618 & $t_{\mathrm{ss}} /-$ \\
\hline MP2/DZP(2MR-PES) + cc-VSCF [185 $]^{\mathrm{d}}$ & $891[890]$ & - & 636 [624] & $t_{\mathrm{as}} /-$ \\
\hline MP2/DZP(DZP pair potential) + cc-VSCF [190 & $883[890]$ & - & 715 [624] & $-1-$ \\
\hline MP2/aVTZ(part. 2MR-PES) + VCI 191] & $886[883]$ & - & $633[630]$ & $t_{\mathrm{as}} /-$ \\
\hline \multicolumn{5}{|l|}{ experimentell: 9} \\
\hline Jet - Ramana & 870 & 799 & 622 & $-1-$ \\
\hline
\end{tabular}

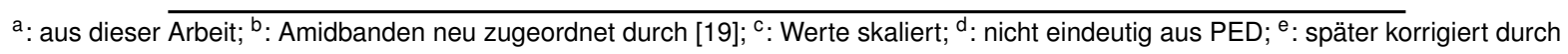
190;; : harmonische Werte in eckigen Klammern; g: siehe auch Tab. 4-6 


\section{2 cis-/trans-Isomerisierung}

Der Enthalpieunterschied von cis- und trans-NMA wird je nach experimenteller Technik auf 7.1 bis $15.3 \mathrm{~kJ} \cdot \mathrm{mol}^{-1}$ in wässriger Lösung [82, 161, 196] und auf $9.6 \mathrm{~kJ} \cdot \mathrm{mol}^{-1}$ in einer Düsenexpansion mit $\mathrm{N}_{2}$ als Trägergas und Düsentemperaturen zwischen Raumtemperatur und $770 \mathrm{~K}$ [68] bestimmt. Theoretisch berechnete Enthalpieunterschiede liegen je nach Methode und Basissatz zwischen 8.2 und $12.9 \mathrm{~kJ} \cdot \mathrm{mol}^{-1}$, was in sehr guter Übereinstimmung mit dem Matrixwert ist. Eine Übersicht der diskutierten Werte findet sich in Tabelle A-14.

Aufgrund der partiellen Doppelbindung unterliegt die cis-/trans-Isomerisierung einer sehr hohen Aktivierungsbarriere, die für flüssiges NMA auf $59 \mathrm{~kJ} \cdot \mathrm{mol}^{-1}$ [34] und NMA in wässriger Lösung auf 58(4) kJ.mol ${ }^{-1}$ [35] in Raman-Experimenten oder in Lösung mit verschiedenen NMR-Techniken [161, 196] zu 95-98 kJ. $\mathrm{mol}^{-1}$ bestimmt wurde. Die vereinfachte Beschreibung der cis-/trans-Isomerisierung als zweizähliges $\mathrm{C}-\mathrm{N}$-Torsionspotentia/8) wird in verschiedenen theoretischen Arbeiten auf die Analyse der vollständigen Potentialhyperfläche ausgeweitet und es werden zwei Reaktionspfade mit je einem Übergangszustand beschrieben, deren Energieunterschiede zum globalen Minimum zuerst auf MP2/6-31G*(MP4\#/6-31G*)-Niveau [198] zu 70.4(69.7) $\mathrm{kJ} \cdot \mathrm{mol}^{-1}$ und 88.7(87.4) $\mathrm{kJ} \cdot \mathrm{mol}^{-1}$ berechnet und später durch MP2/6-31G*-Rechnungen [199] mit leicht veränderten Startkonformationen und B3LYP/6-331+ + G-Rechnungen [20] bestätigt wurden.9) (siehe Tabelle A-14).

Durch die hohe Isomerisierungsbarriere kann das cis-Konformer sowohl im Jet als auch in der Matrix fast gar nicht relaxieren und das Konformerenverhältnis im thermodynamischen Gleichgewicht bei Expansionsbedingungen kann nährungsweise als eingefroren betrachtet werden [116, 204]. Dies erklärt einerseits die Sichtbarkeit im Jet-Spektrum und ermöglicht andererseits durch die Veränderung der Düsentemperatur im curry-Jet eine Bestimmung der cis-/transEnthalpiedifferenz mittels Van-'t-Hoff-Plot ohne störende Einflüsse wie z. B. Lösungsmittel oder Aggregation.

Zur Bestimmung der Enthalpiedifferenz von cis- und trans-NMA wurden curry-Jet-Spektren im NH-Streckbereich bei unterschiedlichen $T_{\mathrm{H}}$ aufgenommen. Die Temperatur vor der Expansion, $T_{\mathrm{H}}{ }^{10)}$ kann aus den genannten Gründen als Konformationstemperatur $T_{\mathrm{K}}$ in Gleichung 2-5 eingesetzt werden. In Abbildung 4-6 sind Raman-Jetspektren mit von (a) nach (e) zunehmenden Temperaturen dargestellt und eine Abnahme des trans-Bandenintegrals mit zunehmender Temperatur bedeutet eine Verschiebung des cis-/trans-Verhältnisses hin zum cis-Konformer. Dabei wurden Expansionsbedingungen gewählt, die die Bildung von Clustern ausschließen, da die Clusterbildung die Monomerpopulationen möglicherweise unterschiedlich stark entvölkert (siehe Abschnitt 3.1.1) und somit $K$ verändert. Die cis- und trans-Banden der Spektren werden im Anschluss integriert, wobei die bereits diskutierten heißen Banden mit berücksichtigt werden.

Die lineare Anpassung der Gerade $y=a+b \cdot x$ an eine Auftragung von $\ln K$ über $1 / T$ (Van-'t-

\footnotetext{
${ }^{8)}$ siehe Miyazawa [4]. Einen umfassenden Überblick zu den frühen Arbeiten zur cis-/trans-Isomerisierung von NMA liefern Hallam und Jones [197].

${ }^{9)}$ siehe hierzu auch: [200, 201]

${ }^{10)} T_{\mathrm{H}}$ ist die eingestellte Temperatur der heizbaren Zuleitungen und der Düse (siehe hierzu auch Abschnitt 3.1.2.
} 
Tab. 4-8. Experimentelle und theoretische Energieunterschiede zwischen cis- und trans-NMA, sowie Isomerisierungsbarrieren. (Eine umfassende Übersicht liefert Tab. A-14 im Tabellenwerk A.7.2 im Anhang.)

\begin{tabular}{|c|c|c|c|c|}
\hline \multicolumn{5}{|l|}{ experimentell } \\
\hline Methode & Bedingungen & $\Delta G / \mathrm{kJ} \cdot \mathrm{mol}^{-1 \mathrm{a}}$ & $\Delta H / \mathrm{kJ} \cdot \mathrm{mol}^{-1}$ & $\Delta E_{\mathrm{A}} / \mathrm{kJ} \cdot \mathrm{mol}^{-1 \mathrm{f}}$ \\
\hline Raman - Jet ${ }^{b}$ & $\theta_{\mathrm{H}}=388.15-483.15 \mathrm{~K}$ & $12.7(388.15)$ & $8 \pm 2$ & - \\
\hline Raman 34 & flüssig & - & - & 58.6 \\
\hline NMR 161 & in 1,2-Dichlorethan & $11.7 \pm 1.5(333.15)$ & $12 \pm 15$ & 95 \\
\hline NMR 161 & in $\mathrm{H}_{2} \mathrm{O}$ & $10.4 \pm 2.5(333.15)$ & $14 \pm 15$ & 97 \\
\hline 2D-NMR 196 & in $\mathrm{H}_{2} \mathrm{O} / 293-313 \mathrm{~K}$ & $10.2(293)$ & 15.3 & - \\
\hline 2D-NMR 196 & in DMSO / 293-313 K & $11.4(293)$ & 1.8 & $98 \pm 11$ \\
\hline 2D-NMR 196. & in Aceton / 293-313 K & $7.1(293)$ & 7.1 & - \\
\hline IR-Matrix 68, & $\mathrm{N}_{2} / T_{\text {Düse }}=298-770 \mathrm{~K}$ & - & 9.6 & - \\
\hline UVRR 35 & in $\mathrm{H}_{2} \mathrm{O}$ & $10.9 \pm 1.7$ (n. a.) & - & $57.7 \pm 3.3$ \\
\hline UVRR 82. & in $\mathrm{H}_{2} \mathrm{O}$ & - & $7.1 \pm 0.8$ & - \\
\hline
\end{tabular}

\begin{tabular}{|c|c|c|c|c|c|}
\hline \multicolumn{6}{|l|}{ theoretisch } \\
\hline Methode & $\Delta E / \mathrm{kJ} \cdot \mathrm{mol}^{-1} \mathrm{~g}$ & $\Delta G / \mathrm{kJ} \cdot \mathrm{mol}^{-1 \mathrm{a}}$ & $\Delta H / \mathrm{kJ} \cdot \mathrm{mol}^{-1}$ & $\Delta E_{\mathrm{A}} / \mathrm{kJ} \cdot \mathrm{mol}^{-1 \mathrm{f}}$ & Konf. \\
\hline $\mathrm{HF} / 4-31 \mathrm{G}^{*} 174$ & 10.3 & - & - & - & $t_{\mathrm{as}}^{\mathrm{j}} / c_{\mathrm{aa}}$ \\
\hline $\mathrm{HF} / 6-31 \mathrm{G}(\mathrm{d}, \mathrm{p}) 202$ 203 & 10.6 & $9.4(298.15)$ & 12.9 & $68.4 / 87.2^{\mathrm{e}}$ & n.a. \\
\hline $\mathrm{HF} / 6-31+\mathrm{G}(\mathrm{d}) 202203$ & 11.0 & $17.2(298.15)$ & 8.2 & $70.0 / 88.3^{e}$ & n. a. \\
\hline $\mathrm{HF} / 6-311++\mathrm{G}^{\star \mathrm{k}} 202$ & 11.8 & $18.8(298.15)$ & 9.0 & - & $t_{\mathrm{as}}$ \\
\hline MP2/6-31G* 198 & 9.3 & $10.6(298)$ & 8.9 & $70.4 / 88.7^{e}$ & $t_{\mathrm{sa}} / c_{\mathrm{aa}}$ \\
\hline MP2/6-31G* $\overline{199}$ & $9.4[10.4]$ & $15.4(298)$ & 9.3 & $69.5 / 87.8^{\mathrm{e}}$ & $t_{\mathrm{as}} / \mathrm{c}_{\mathrm{aa}}$ \\
\hline$M P 2 / 6-311++G^{* *} 202$ & 9.5 & $16.0(298.15)$ & 12.6 & - & $t_{\mathrm{as}}$ \\
\hline MP4\#/6-31G* 198 & 9.3 & $10.6(298)$ & 9.0 & $69.7 / 87.4^{e}$ & $t_{\mathrm{sa}} / \mathrm{c}_{\mathrm{aa}}$ \\
\hline $\mathrm{B} 3 \mathrm{LYP} / 6-311++\mathrm{G}^{* \star}$ & 10.4 & $9.2(298.15)$ & 12.6 & - & $t_{\mathrm{as}}$ \\
\hline B3LYP/6-311++G(3dt,3pd) 20 & 9.6 & - & - & $78.2 / 91.6^{\mathrm{e}}$ & $t_{\mathrm{as}}$ \\
\hline B3LYP-D3(BJ)/def2TZVP ${ }^{b}$ & $8.7[9.2]$ & $10.9(388.15)$ & $8.4^{\mathrm{h}}$ & - & $t_{\mathrm{ss}} / c_{\mathrm{aa}}$ \\
\hline B3LYP-D3(BJ)/aVTZ b & $8.8[9.0]$ & $6.4(388.15)$ & $8.5^{k}$ & - & $t_{\mathrm{as}} / \mathrm{c}_{\mathrm{aa}}$ \\
\hline LC-BLYP/6-311++G(d,p) d 21 & {$[10.7]$} & - & - & - & $t_{\mathrm{as}}$ \\
\hline
\end{tabular}

n. a.: nicht angegeben; UVRR: UV-Resonanz-Raman-Spektroskopie; ${ }^{\mathrm{a}}$ : (Temperatur in $\left.\mathrm{K}\right)$; $^{\mathrm{b}}$ : aus dieser Arbeit; ${ }^{\mathrm{c}}$ : freie Enthalpie $\Delta G$ (Temperatur in K)); ${ }^{d}$ : Counterpoise-Korrektur (CP) 150 ; $e^{e}$ : zwei mögliche Übergangszustände; ${ }^{\dagger}$ : Barriere bezogen auf trans-NMA; $g$ : elektronische [nullpunktskorrigierte] Energieunterschiede; ${ }^{\text {h: }}$ Mittelwert der $\Delta H$-Werte der Frequenzrechnungen im Temperaturbereich 388.15-483.15 K; ${ }^{i}$ : Geometrie MP2/aVDZ optimiert; ${ }^{\mathrm{j}}$ : trans-Konformer in [66]; ${ }^{\mathrm{k}}$ : Aus Van 't-Hoff-Plot auf Basis der berechneten $\Delta G$ Werte im Temperaturbereich 388.15-483.15K.

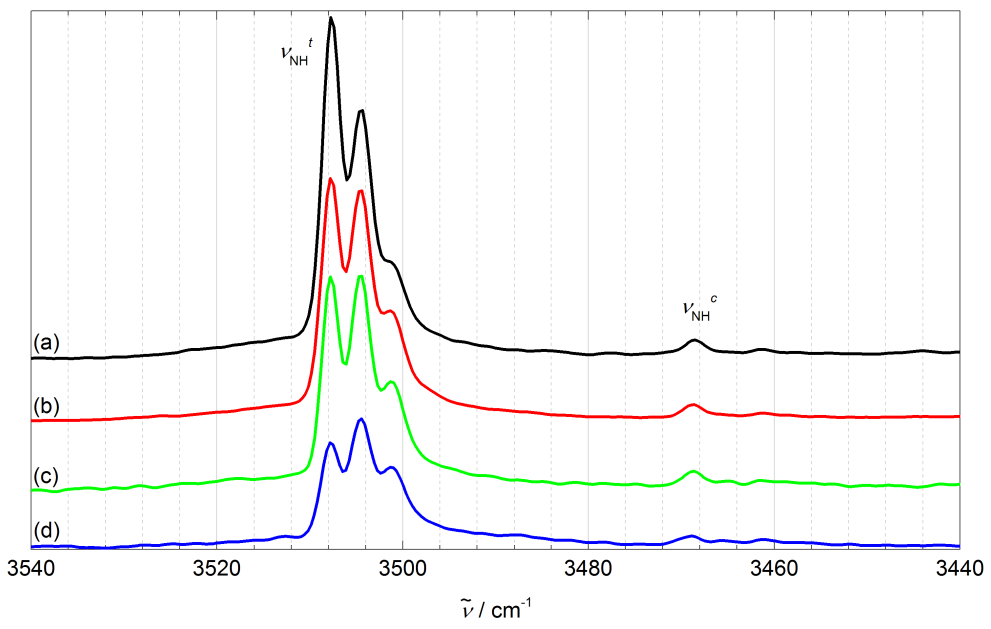

Abb. 4-6. Temperaturmessreihe von NMA im NH-Streckbereich. Die Temperaturen vor der Expansion, $\vartheta_{\mathrm{H}} /{ }^{\circ} \mathrm{C}$, betragen von (a) nach (d): 115, 150, 170, 210. Die Spektren sind auf $v_{\mathrm{NH}}^{c}$ bei $3469 \mathrm{~cm}^{-1}$ normiert (Details zu den Messungen finden sich im Raman-Spektrenverzeichnis in Tab. A-6 in Anhang A.6.1. eine auf $v_{\mathrm{NH}}^{t}$ normierte Darstellung der gleichen Spektren befindet sich in Abb. A-8 im Anhang A.6.1. 
Hoff-Plot) ist in Abbildung 4-7 dargestellt und liefert gemäß Gleichung 2-7 als Steigung:

$$
b=-\frac{\Delta_{\mathrm{r}} H_{\mathrm{exp}}^{\ominus}}{R}=(9.2 \pm 1.6) \cdot 10^{2} \mathrm{~K}
$$

Daraus ergibt sich ein Enthalpieunterschied von:

$$
\Delta_{\mathrm{r}} H_{\mathrm{exp}}^{\ominus}=8(2) \frac{\mathrm{kJ}}{\mathrm{mol}}
$$

Aus Gleichung 2-5 ist offensichtlich, dass die Steigung einer Auftragung von $\ln K$ gegen $1 / T$ unabhängig vom Streuquerschnittsverhältnis ist, so dass die ermittelten Enthalpien aus dem Van-'t-Hoff-Plot rein experimentelle Werte sind. Zum Vergleich beträgt die theoretische Standardenthalpiedifferenz der Konformere (siehe Abb.4-7):

$$
\Delta_{\mathrm{r}} H_{\text {theo }}^{\ominus}=8.506(7) \frac{\mathrm{kJ}}{\mathrm{mol}}
$$

Die Berechnung erfolgte mit Gleichung 2-3 und den mit Hilfe des Gaussian-Tools „freqchk“ aus harmonischen Rechnungen auf B3LYP-D3(BJ)/aVTZ-Niveau erhaltenen freien Enthalpien für die jeweiligen Temperaturen.

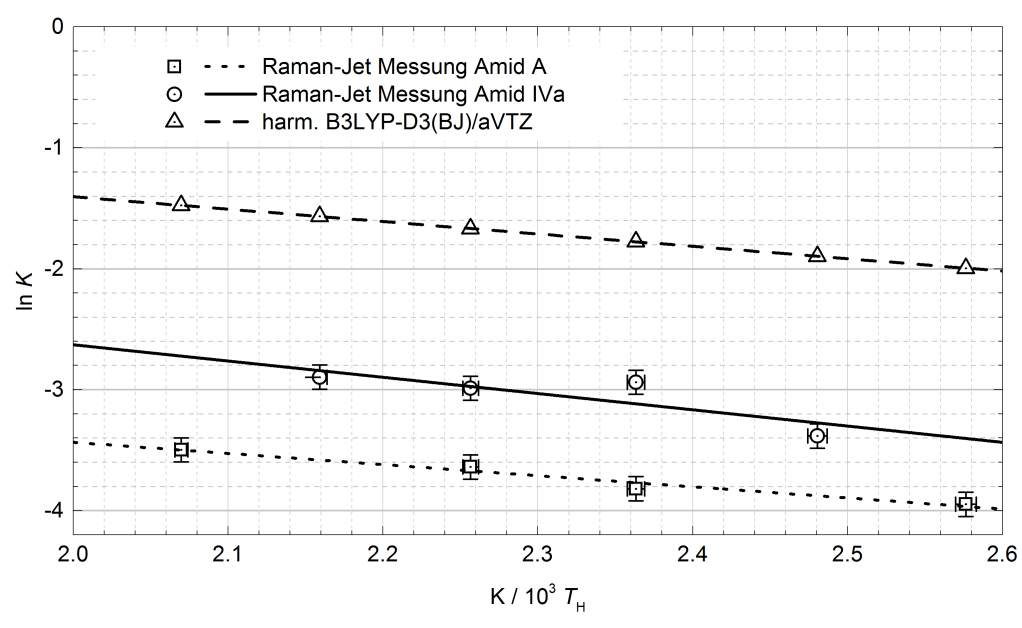

Abb. 4-7. Van-'t-Hoff-Plots für die cis-/trans-Isomerisierung von NMA. Unten: Auftragung nach Gleichung 2-7 aus den Intensitätsverhältnissen $F$ der nicht-normierten Amid A-Banden in Abb. A-8 und den aus B3LYP-D3(BJ)/aVTZ berechneten Streuquerschnitten $\sigma^{\prime}$ (siehe auch GI. 2-5. Die Fehler-gewichtete lineare Anpassung ergibt für die Steigung $b=-(9.2 \pm 1.6) \cdot 10^{2} \mathrm{~K}$.

Mitte: Analoge Auftragung mit den Intensitätsverhältnissen der Amid IVa-Banden in Abb. 4-8, Die Fehler-gewichtete lineare Anpassung ergibt für die Steigung $b=-(1.34 \pm 0.84) \cdot 10^{3} \mathrm{~K}$.

Die Fehlerbalken entsprechen einer angenommenen Messunsicherheit von $\pm 1 \mathrm{~K}$ für $T_{\mathrm{H}}$ und $10 \%$ für die integrierten Bandenintensitäten.

Oben: Auf Grundlage der mit dem Gaussiantool "freqchk“ für die jeweiligen Temperaturen bestimmten freien Enthalpien aus harmonischen Frequenzrechnungen auf B3LYP-D3(BJ)/aVTZ-Niveau. Steigung der linearen Anpassung: $b=-1.0230(8) \cdot 10^{3} \mathrm{~K}$.

Für diese Art der Bestimmung der Enthalpiedifferenz der beiden Konformere kommen prinzipiell alle intensiven, vollständig separierten cis/trans-Bandenpaare in Frage, so lange diese in einem Spektrum aufgenommen wurden, um die Auswirkung einer unterschiedlichen Justage 
auf die relative Signalstärke auszuschließen (siehe Abschnitt 3.1.1). Auch wenn die theoretischen Streuquerschnitte um die Polarisationssensitivität der Detektion (vgl. Abschnitt 3.4) korrigiert sind, ist diese sehr rudimentär und das Depolarisationsverhältnis der verglichenen Banden sollte daher möglichst ähnlich sein. Dies ist nach den B3LYP-Rechnungen sowohl für die Amid A- als auch für die Amid IV-Bande von cis- und trans-NMA gegeben (siehe Tabellen A-15 und A-19]. Die Auswertung für die Amid A-Banden ist durch die hohe Population niedriger Energieniveaus und die damit verbundenen heißen Banden schwierig, so dass als Alternative $\Delta_{\mathrm{r}} H^{\ominus}$ mit Hilfe der Amid IVa-Bande bestimmt werden soll. Diese ist ebenfalls sehr intensiv, gut separiert und zusätzlich frei von spektral verschobenen heißen Übergängen.

Hierfür wurden curry-Jet-Spektren bei vier unterschiedlichen Temperaturen aufgenommen und analog ausgewertet. Die Spektren der Temperaturreihe sind in Abbildung 4-8 dargestellt. Der zugehörige Van-'t-Hoff-Plot findet sich in Abbildung 4-7. Er liefert für die Standardreaktionsenthalpie der Isomerisierung:

$$
\Delta_{\mathrm{r}} H^{\ominus}=(11 \pm 7) \mathrm{kJ} \mathrm{mol}^{-1}
$$

Damit weichen die aus den Raman-Spektren ermittelten Enthalpieunterschiede innerhalb ihrer sehr großen Fehlerintervalle voneinander ab. Hier ist vor allem die Integration der cisBanden problematisch, da sich diese kaum aus dem Rauschen der Basislinie hervorheben. Der Vergleich mit den Literaturwerten in Tab. A-14 zeigt allerdings, dass sich beide Werte um ca. $2 \mathrm{~kJ} \cdot \mathrm{mol}^{-1}$ von dem $\mathrm{N}_{2}$-Matrixwert [68] unterscheiden. Alle weiteren Experimente sind mit flüssigem NMA oder NMA in Lösung durchgeführt worden und der gemessene Enthalpieunterschied variiert zwischen 7.1 und $15.3 \mathrm{~kJ} \cdot \mathrm{mol}^{-1}$ 11) Damit liegen die Werte dieser Arbeit innerhalb der experimentellen Literaturwerte. Die theoretischen Studien in Tab. A-14liefern elektronische Energieunterschiede im Bereich von 8-12 kJ.mol ${ }^{-1}$ und Enthalpiedifferenzen zwischen 9 und $13 \mathrm{~kJ} \cdot \mathrm{mol}^{-1}$, wobei die in dieser Arbeit verwendete B3LYP-D3(BJ)/aVTZ-Rechnung mit $8.5 \mathrm{~kJ} \cdot \mathrm{mol}^{-1}$ am unteren Ende des Bereichs liegt. Es handelt sich allerdings auch um die einzige Rechnung mit Dispersionskorrektur.

$\overline{{ }^{11)} \text { Der Enthalpieunterschied von } 1.8 \mathrm{~kJ} \cdot \mathrm{mol}^{-1}}$ in DMSO aus [196] ist ungewöhnlich niedrig und sollte nur unter Vorbehalt verwendet werden. 


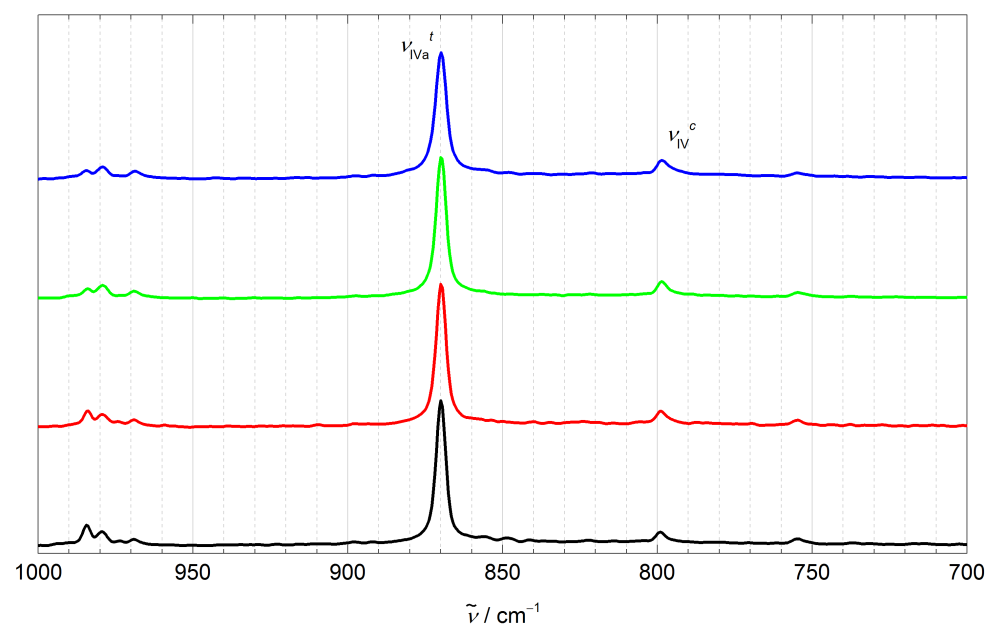

Abb. 4-8. Temperaturmessreihe von NMA im Bereich 1000-700 $\mathrm{cm}^{-1}$. Die Temperaturen vor der Expansion, $\vartheta_{\mathrm{H}} /{ }^{\circ} \mathrm{C}$, betragen von (a) nach (d): 130, 150, 170, 190. Die Spektren sind normiert auf $v_{\mathrm{IVa}}^{c}$ bei $799 \mathrm{~cm}^{-1}$ (Details zu den Messungen befinden sich im Raman-Spektrenverzeichnis in Tab. A-6 in Anhang A.6.1. 


\subsection{Dimerisierung}

Die Aggregation sekundärer Amide ist ein verbreitetes Modell zur Untersuchung von Wasserstoffbrückenbindungen des Typs $\mathrm{N}-\mathrm{H} \cdots \mathrm{O}=\mathrm{C}$, die in der Proteinfaltung hauptverantwortlich für die Ausbildung der Sekundärstruktur sind [76]. Eine solche Wasserstoffbrückenbindung zwischen Amidgruppen verlängert auf der Donorseite die N-H-Bindung und auf der Akzeptorseite die $\mathrm{C}=\mathrm{O}$-Bindung im Molekül. Dies hat Auswirkungen auf die Schwingungsfrequenz der entsprechenden lokalen Moden, wobei eine Wasserstoffbrücke in der Regel $\mathrm{N}-\mathrm{H}$ - und $\mathrm{C}=\mathrm{O}-$ Streckschwingungen zu niedrigeren Frequenzen verschiebt (Rotverschiebung) und für Normalmoden mit $\mathrm{N}-\mathrm{H}$ - oder $\mathrm{C}=\mathrm{O}$-Knickschwingungsanteil zu einer Verschiebung zu höheren Frequenzen (Blauverschiebung) auftritt [205]. Daher ist Schwingungsspektroskopie grundsätzlich sehr sensitiv für Wasserstoffbrückenbindungen, und es gibt schon eine Reihe von experimentellen Arbeiten zur Aggregation von NMA, wie z. B. IR-Spektren der Amid I-Bande in Argonund $\mathrm{N}_{2}$-Matrizen [195], Raman- und FTIR-Daten von NMA als Flüssigkeit und in Acetonitril$d_{3}$-Lösung [206], IR-Experimente im NH-Streck-Obertonbereich in Lösung [184], sowie eine umfangreiche Studie mit FTIR- und Ramanspektren in der Flüssigkeit und im Festkörper [19].

Die verwendeten Techniken unterliegen allerdings ihren ganz eigenen Limitierungen, so sind inerte Matrizen von Matrix-Effekten [28] betroffen und Gasphasenspektroskopie leidet unter thermischer Verbreiterung und niedrigen Dimerkonzentrationen, während in Lösung Lösemittel und thermische Effekte überwiegen, die in kondensierter Phase dann vollständig dominieren [180, 207-210].

Eine gute Alternative stellt die Jet-Spektroskopie dar [211]. Von den zwei bisher bekannten Arbeiten im Jet ist die erste eine FTIR-Studie der Amid A- und Amid I-Bande [51], während die zweite IR-Multiphotonen-Dissoziation mit VUV-Ionisierung (IRMPD) im Amid I-III-Bereich kombiniert [194]. Beide Arbeiten liefern Bandenpositionen für das Dimer und das Trimer (siehe hierzu Abschnitt 4.4), unterliegen aber auch Einschränkungen. Die FTIR-Studie leidet unter einem schlechten Signal-zu-Rauschen-Verhältnis (SRV), weswegen die Bandenzuweisung insbesondere für das Trimer unter Vorbehalt erfolgte und auf der Basis von theoretischen Arbeiten von M. Mons et. al. in Frage gestellt wurde. ${ }^{12)}$ Die IRMPD-Studie ist an sich universell einsetzbar und hat ein besseres SRV und eine höhere Auflösung [194, 212]. Sie weist allerdings eine Reihe von Schwächen auf, wobei die größte Unzulänglichkeit in einer finalen Spektreninterpretation liegt, die ohne den Ausschluss möglicher Fehlerquellen oder zumindest deren Diskussion auskommt (siehe unten).

Die beschriebenen Nachteile vorhandener Jet-Experimente sind die Motivation für eine erneute schwingungsspektroskopische Untersuchung der NMA-Aggregation im Jet, wobei dieses Mal mit dem filet-Jet eine FTIR-Jet-Apparatur mit deutlich besserem SRV (siehe Abschnitt 3.2) und mit dem curry-Jet eine komplementäre Messmethode zur Verfügung stehen.

Abbildung 4-9 zeigt die neu mit dem filet-Jet aufgenommenen Spektren in der Amid I-III-Region mit einem Gasphasenspektrum (oben) und einem Jet-Spektrum (unten). Die Amid I-Bande in der Gasphase hat die bereits diskutierte Rotationsstruktur mit P- und R-Zweig und die Maxi-

12) Durch persönliche Mitteilung an M. A. Suhm, weitere Ausführungen hierzu in [212]. 


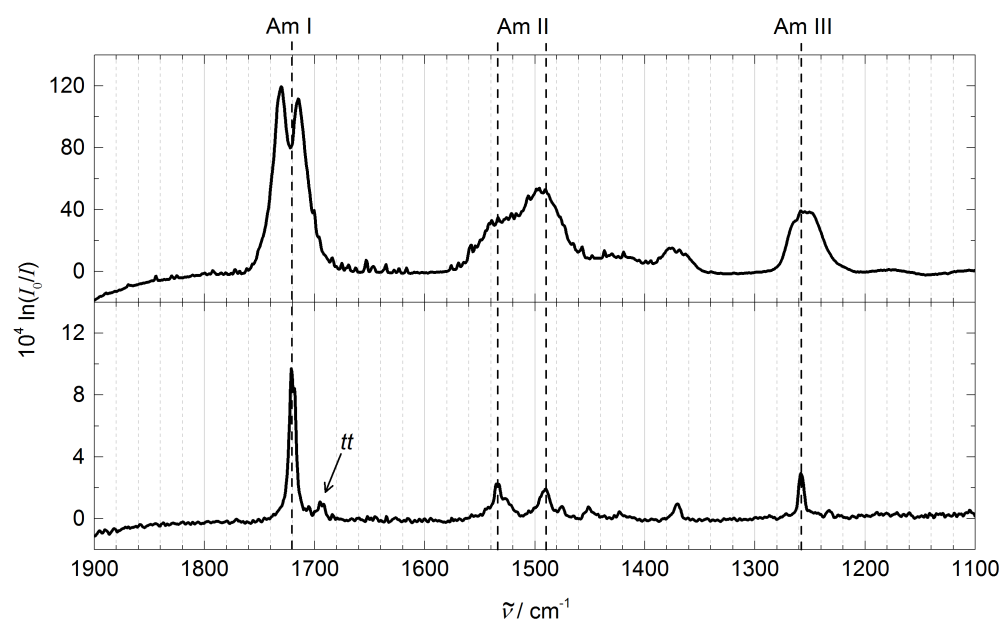

Abb. 4-9. FTIR-Spektren von NMA in der Gasphase (oben) und im Jet (unten) in der Amid I-III-Region. (adaptiert aus: Forsting et al. [212] publiziert unter der CC BY 3.0 Lizenz [https://creativecommons.org/licenses/by/3.0/]; Die zugrunde liegenden Messbedingungen der beiden Spektren befinden sich als Datensätze A und B im FTIR-Spektrenverzeichnis in Tab. A-8 in Anhang A.6.2

ma der beiden Zweige stimmen bis auf $1 \mathrm{~cm}^{-1}$ für den R-Zweig und $2 \mathrm{~cm}^{-1}$ für den P-Zweig mit der Literatur überein (siehe Tabelle 4-4 im vorangegangenen Abschnitt). Der Vergleich mit dem Jet-Spektrum zeigt, dass hier die Rotationsstruktur zusammenfällt, was in Einklang mit einer im Jet typischerweise sehr niedrigen Rotationstemperatur ist (ca. $10 \mathrm{~K}$ [136]). Die Position der $v_{\mathrm{I}}^{t}$ im Jet liegt mit $1720 \mathrm{~cm}^{-1}$ um $2 \mathrm{~cm}^{-1}$ niedriger als in der alten Jet-Studie. In Anbetracht der nicht ganz symmetrischen Struktur (vergleiche hierzu auch die Diskussion der Amid A-Bande in Abschnitt 4.1] und einer Halbwertsbreite von ca. $7 \mathrm{~cm}^{-1}$ [212] ist die Übereinstimmung der beiden FTIR-Jetspektren im Rahmen der Messgenauigkeit gegeben. Der Vergleich mit der Amid IBandenposition der IRMPD-Arbeit zeigt, dass diese mit $1707 \mathrm{~cm}^{-1}$ um $13 \mathrm{~cm}^{-1}(\approx 1 \%)$ niedriger liegt [194]. Die Ursache liegt wahrscheinlich in einem Kalibrierungsfehler (ausführlich diskutiert in [212]).

Für alle weiteren Banden werden diese von der Gasphasenmessung zum Jet-Spektrum hin ebenfalls deutlich schmaler. Dies legt sogar eine Substruktur der Amid II-Bande offen, deren sehr breites Signal sich zu einem Peakpaar bei $1534 \mathrm{~cm}^{-1}$ und $1490 \mathrm{~cm}^{-1}$ entwickelt: eine Aufspaltung, die bisher weder experimentell beobachtet und explizit diskutiert wird ${ }^{13)}$ noch im Rahmen anharmonischer Frequenzrechnungen [185, 189,-191] erfasst wurde. Die Ausbildung einer Fermi-Resonanz wäre durchaus eine mögliche Ursache. Hierfür sprechen sowohl die Arbeit von Dellepiane et al. [213], die im Amid II-Bereich von festem NMA eine Fermi-Resonanz findet, sowie die anharmonische VPT2-Rechnung zum $t_{\mathrm{ss}}$-NMA (siehe Abschnitt4.1), in der eine ganze Reihe von möglichen Resonanzpartnern gefunden werden, die diese Theorie bestätigen. Allerdings lassen sich die Moleküleigenschaften im Festkörper nicht eins zu eins auf die in der Jet-Expansion übertragen und die Aussagekraft der Rechnung ist aufgrund der niederfrequen-

\footnotetext{
13)Yatsyna et al. [194] finden eine Bande bei $1478 \mathrm{~cm}^{-1}$, die aber den Methyldeformationsschwingungen zugeordnet wird, was das hohe Bandenintegral im IR-Spektrum nur durch eine Fermi-Resonanz mit der Amid II erklären könnte.
} 
ten Methyltorsionen, die grundsätzlich problematisch für VPT2-Rechnungen sind [29, 214], ist begrenzt. Daher steht die Zuordnung als Fermi-Paar trotz guter Indizien unter Vorbehalt.

Die kleine Bande im Jet-Spektrum bei $1695 \mathrm{~cm}^{-1}$ ist um $-25 \mathrm{~cm}^{-1}$ bezüglich des Monomers verschoben und es könnte sich hierbei um eine Dimerbande handeln. Diese Annahme wird durch Matrixmessungen gestützt, die in $\mathrm{N}_{2}$ genau die gleiche und in Argon mit $-22 \mathrm{~cm}^{-1}$ nahezu die gleiche Verschiebung aufweisen. Zudem liegt diese Bande in der Nähe einer breiteren Bande bei $1690 \mathrm{~cm}^{-1}$ in den wesentlich höher konzentrierten Jet-Spektren von Albrecht et al. [51], die neben dem Dimer wahrscheinlich signifikante Beiträge des Trimers enthält.

Eine genauere Zuordnung ermöglicht ein direkter Vergleich mit Raman-Jet-Spektren (Abb.4-10). Die abgebildeten Spektren sind aus einer Messreihe mit nach unten hin zunehmender Düsentemperatur bei möglichst konstanter Konzentration im Trägergas und auf die trans-Monomerbande bei $1720 \mathrm{~cm}^{-1}$ skaliert.

Eine zunehmende Düsentemperatur reduziert die Cluster in der Expansion, so dass der Anteil der Aggregate von unten nach oben zunimmt. Da die Methode nicht größenselektiv ist, sind neben dem Dimer auch immer höhere Cluster zu finden. Eine erste Zuordnung erfolgt über die relative Zunahme, wobei in einem auf das Monomersignal normierten Spektrum die zuerst gebildeten, wachsenden Banden dem Dimer zuzuordnen sind.

Auf der niederfrequenten Seite der Amid I entstehen eine ganze Reihe von Banden im Raman-Spektrum, wobei die zwei nächsten bei $1705 \mathrm{~cm}^{-1}$ und $1695 \mathrm{~cm}^{-1}$ vergleichsweise schmal sind und die zweite ihre Entsprechung im FTIR-Jet-Spektrum in der vermuteten Dimerbande hat. Die andere Bande hat ein ähnliches Wachstumsverhalten mit sinkender Düsentemperatur, so dass es sich hierbei um die zweite (ungebundene) $\mathrm{C}=\mathrm{O}$-Streckschwingung handeln könnte. Allerdings fehlt eine Erklärung für die asymmetrische Bandenform der $1695 \mathrm{~cm}^{-1}$-Bande, die mit abnehmender Düsentemperatur auf der niederfrequenten Seite stärker zunimmt als an der höherfrequenten. Auch der kleine Peak bei $1683 \mathrm{~cm}^{-1}$ zeigt das gleiche Wachstum wie die anderen beiden und müsste daher ebenfalls ein Dimersignal sein.

Da die Amid II-Bande erwartungsgemäß fehlt (siehe Abschnitte 2.2 und 4.1), liegt der Blick nun auf der Amid III-Bande, für die sich ein komplett umgekehrtes Bild im Vergleich zur Amid I ergibt. Hier liegt ebenfalls eine clusterbedingte Bandenentstehung mit sinkender Düsentemperatur vor, allerdings sind hier alle Banden zu höheren Frequenzen verschoben. Wenn man beachtet, welche lokalen Moden der Amid III-Normalmode zugrunde liegen (siehe Abschnitt2.2), dann ist diese Blauverschiebung einleuchtend. Auch hier würde der Blick auf die Entwicklung der Bandengröße eine Dimerzuordnung für die vom Monomer aus gesehen nächst höhere neue Bande bei $1271 \mathrm{~cm}^{-1}$ ermöglichen.

Für eine sichere Zuordnung ist das Raman-Spektrum der Amid I-III-Region zu unübersichtlich, so dass im Folgenden erst einmal der Amid A-Bereich ausgewertet wird. In Abbildung 4-11 sind daher drei FTIR-Jet-Spektren mit von unten nach oben zunehmender NMA-Konzentration (oberstes Panel) einer, auf die $t$-AmA-Bande skalierte, Raman-Jet-Spektrenserie mit der angesprochenen Variation der Düsentemperatur gegenüber gestellt.

Die FTIR-Spektren zeigen bei etwas über $3500 \mathrm{~cm}^{-1}$ eine starke Bande mit untypischer Form. Hier lassen sich zwei Einzelpeaks bei 3508 und $3506 \mathrm{~cm}^{-1}$ erkennen. Ersterer lässt sich im Ver- 


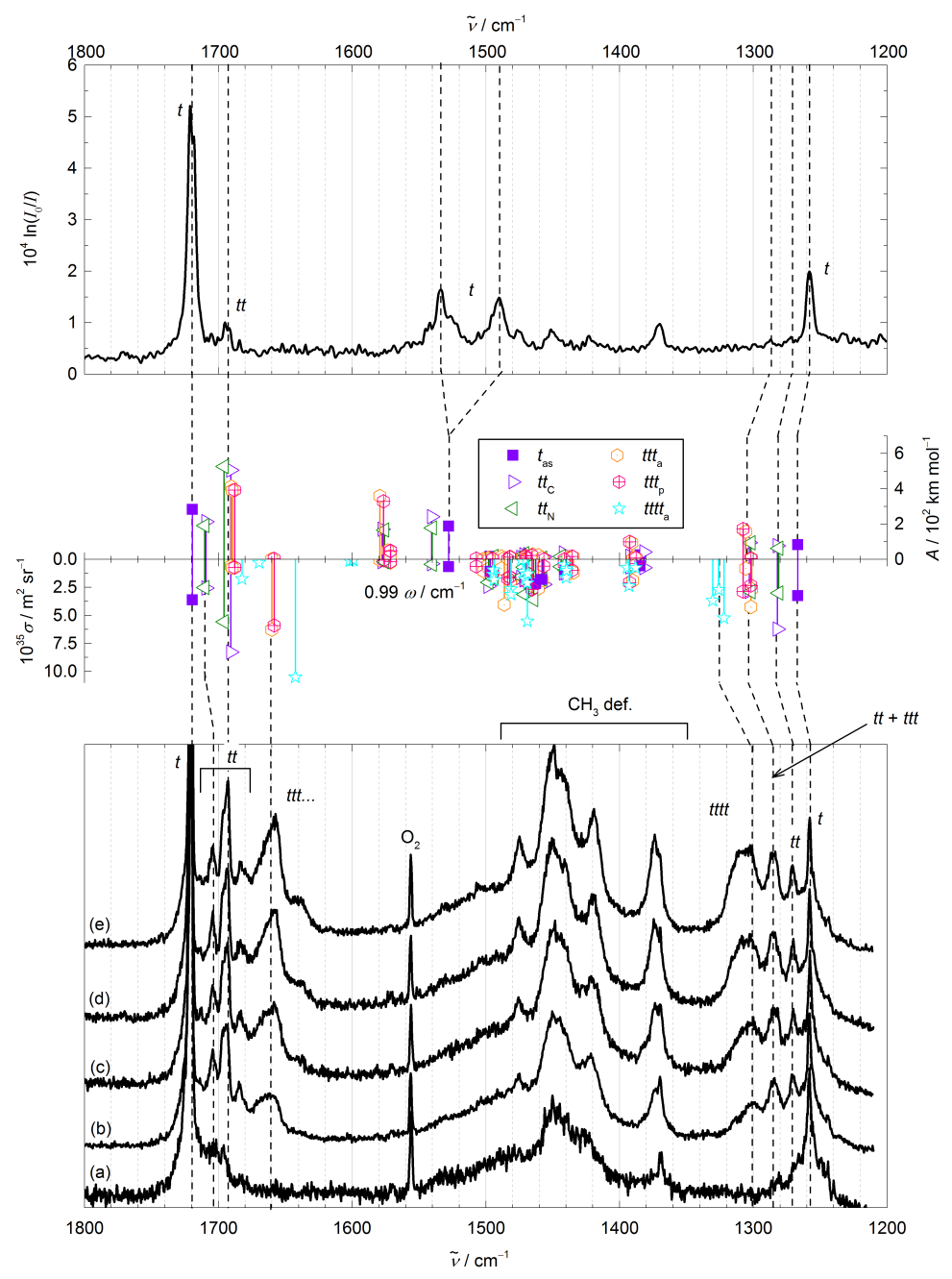

Abb. 4-10. Vergleich von Raman- (unten, von (a) bis (e) mit abnehmender Düsentemperatur) und FTIR-Jet-Spektren(oben) der Amid I-III-Region von NMA in He mit Strichspektren skalierter harmonischer Frequenzen auf B3LYPD3(BJ)/aVTZ-Niveau. Die gestrichelten Linien setzten ausgewählte Banden in Bezug zu den Linien im Strichspektrum. Einige Banden werden dem Monomer $(t)$, Dimer ( $t t)$, Trimer $(t t t)$ oder Tetramer (tttt) von trans-NMA zugeordnet. (adaptiert aus: Forsting et al. [212] publiziert unter der CC BY 3.0 Lizenz [https://creativecommons.org/licenses/by/3.0/]; Details zu den Messungen der Spektren (a) bis (e) befinden sich im Raman-Spektrenverzeichnis in Tab. A-6 in Anhang A.6.1 und das FTIR-Spektrum entspricht dem Datensatz B1 in Tab. A-8 in Anhang A.6.2 Details zu den Frequenzrechnungen finden sich in Tab. A-16 in Anhang A.7.2) 


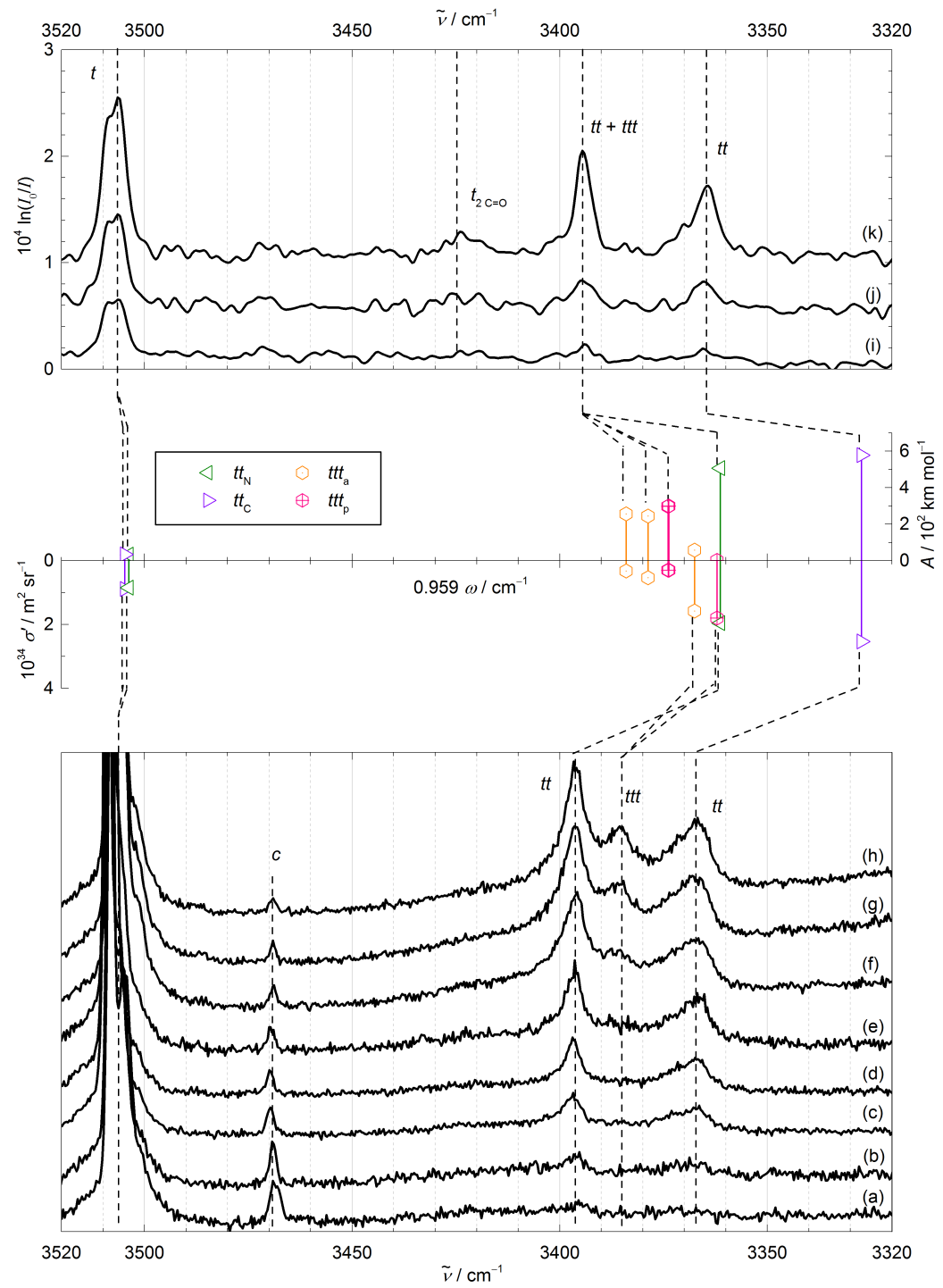

Abb. 4-11. Vergleich von Raman- (unten, von (a) nach (h) mit abnehmender Düsentemperatur) und FTIR-Jet-Spektren (oben, mit von (i) nach (k) zunehmender Konzentration) der Amid A-Region von NMA in He mit Strichspektren skalierter harmonischer Frequenzen auf B3LYP-D3(BJ)/aVTZ-Niveau. Die gestrichelten Linien setzten ausgewählte Banden in Bezug zu den Linien im Strichspektrum. Einige Banden werden dem Monomer $(t)$, Dimer (tt) und Trimer ( $t t t)$ von trans-NMA und dem Monomer $(c)$ von cis-NMA zugeordnet. Weiterhin ist der Oberton der $\mathrm{C}=\mathrm{O}-\mathrm{Streck}-$ schwingung des trans-Monomers $\left(t_{2 \mathrm{C}=0}\right)$ markiert. (adaptiert aus: Forsting et al. 212] publiziert unter CC BY 3.0 [https://creativecommons.org/licenses/by/3.0/]; Details zu den Messungen der Spektren (a) bis (h) befinden sich im RamanSpektrenverzeichnis in Tab. A-6 in Anhang A.6.1 und die Spektren (i), (j) und (k) entsprechen den Datensätzen C, D bzw. $\mathrm{E}$ in Tab. A-8 in Anhang A.6.2 Details zu den Frequenzrechnungen befinden sich in Tab. A-15 in Anhang A.7.2) 
gleich mit dem in Abschnitt 4.1 diskutierten Ramanspektrum der trans-Amid A des Monomers zuordnen. Die Intensität der niedrigeren übersteigt mit zunehmender Temperatur ${ }^{14)}$ die der Fundamentalen; ein Verhalten, was bereits im Monomer für das als heiße Bande bezeichnete Signal bei $3506 \mathrm{~cm}^{-1}$ beobachtet wurde. Dieser Unterschied kann seine Ursache in der freien NH-Streckschwingung aufkommender Cluster haben.

Der Blick zu niedrigeren Frequenzen offenbart zwei Peaks bei 3395 und $3365 \mathrm{~cm}^{-1}$, die beide gleichzeitig mit zunehmender Konzentration größer werden, wobei die höherfrequente Bande schneller wächst. In den Raman-Spektren finden sich beide Banden an nahezu der gleichen Position wieder (1-2 $\mathrm{cm}^{-1}$ Unterschied, siehe Tab. 4-1). Beide Banden liegen auch im FTIR-Jet bei Albrecht et. al. vor und werden dort allerdings dem Dimer bzw. Trimer zugeordnet. Dagegen spricht die simultane Entstehung, die im Ramanspektrum noch offensichtlicher ist und eine Interpretation der $3365 \mathrm{~cm}^{-1}$-Bande als Trimersignal ausschließt. Beide Banden müssten daher ihren Ursprung zumindest teilweise im Dimer haben.

Zunächst kann eine Beteiligung von cis-NMA in Form von gemischten cis-trans-Dimeren oder eines cis-cis-Dimers als Ursache für die Dimerbanden von vornherein statistisch ausgeschlossen werden, wenn die sehr schwache cis-Amid A im Ramanspektrum und das Fehlen im FTIRSpektrum betrachtet wird 15) An dieser Stelle sei angemerkt, dass die scheinbare Abnahme des cis-Monomerpeaks in den Raman-Spektren mit zunehmender Clusterbildung in erster Linie nicht auf die Bildung von Clustern zurückzuführen ist, sondern es sich hierbei vorwiegend um die thermodynamische Verschiebung des Konformerenverhältnisses durch die Änderung der Düsentemperatur handelt, was bereits in Abschnitt 4.2 zur Bestimmung des cis-/trans-Energieunterschieds verwendet wurde.

Eine weitere mögliche Interpretation dieser Banden wäre eine Fermi-Resonanz mit dem Oberton der $\mathrm{C}=\mathrm{O}$-Streckschwingung, der in der Gasphase bei $3440 \mathrm{~cm}^{-1}$ gemessen wurde [177] und damit in der Nähe der Banden liegt, was eine genaue Überprüfung der Oberton-Bandenposition im Jet nötig macht. Die Lage des Obertons kann über die Fundamentale bei $1720(3) \mathrm{cm}^{-1}$ in Kombination mit einer diagonalen Anharmonizitätskonstante [176] von $-8.5(2) \mathrm{cm}^{-1}$ zu 3423(10) $\mathrm{cm}^{-1}$ berechnet werden. Das höchstkonzentrierte FTIR-Spektrum in Abb. 4-11 zeigt eine sehr schwache und breite Bande bei $3425 \mathrm{~cm}^{-1}$, die sich mit der berechneten Position deckt und daher dem CO-Streck-Oberton des trans-Monomers zugewiesen wird. Diese Wellenzahl dürfte eine gute Abschätzung für den Oberton der freien CO-Streckschwingung im trans-trans-Dimer ( $t t$ ) sein und liegt zu hoch, um für eine Fermi-Resonanz mit der N-H-Streckschwingung des H-Brückendonors in Frage zu kommen.

Es gibt allerdings auch die Möglichkeit einer Fermi-Resonanz durch die Wasserstoffbrückenbindung hindurch [215], also zwischen der N-H-Streckschwingung des Donors und dem Oberton der CO-Streckschwingung des Akzeptors. Die ungestörte Bandenposition dieses DimerObertons ergibt sich aus dem Monomer-Oberton zuzüglich der doppelten Verschiebung der

\footnotetext{
14) filet-Jet-Messungen werden bei Raumtemperatur durchgeführt, die Düse ist allerdings nicht thermostatisiert, so dass Schwankungen der Raumtemperatur und die zur Einstellung der Substanzkonzentration verwendete Sättigertemperatur zu unterschiedlichen Temperaturen in Reservoir und Düse führen können (siehe auch Abschnitt 3.2).

${ }^{15)}$ Dies wird am Ende dieses Kapitels noch einmal genauer ausgeführt.
} 
Dimerfundamentalen von $-25 \mathrm{~cm}^{-1}$ (siehe Tab. 4-9), was einen Wert von $3375 \mathrm{~cm}^{-1}$ ergibt. Dieser Wert liegt ziemlich genau in der Mitte der beiden beobachteten Banden und daher kann eine Fermi-Resonanz nicht ausgeschlossen werden.

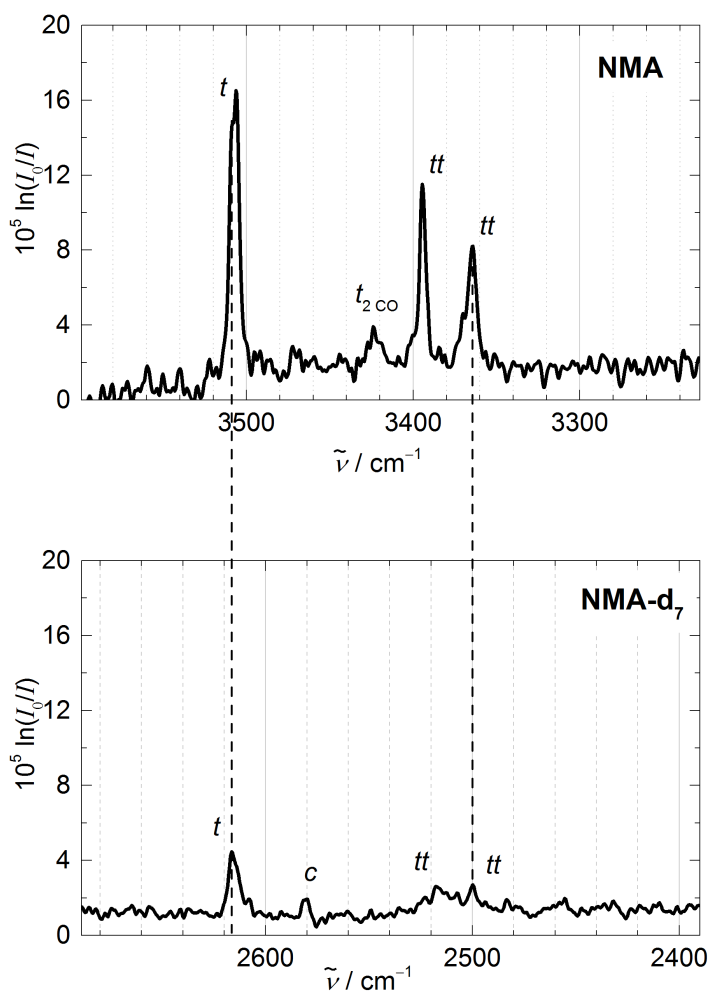

Abb. 4-12. Vergleich von FTIR-Jet-Spektren der Amid A-Region von NMA und NMA-d7. Die gestrichelten Linien zeigen die Bezugspunkte für die Skalierung (Faktor 1.235). Einige Banden werden dem Monomer $(t)$ und Dimer $(t t)$ von trans-NMA und dem Monomer $(c)$ von cis-NMA zugeordnet. Weiterhin ist der Oberton der $\mathrm{C}=\mathrm{O}-\mathrm{Streckschwingung}$ des trans-Monomers $\left(t_{2 \mathrm{C}=0}\right)$ markiert. (adaptiert aus: Forsting et al. [212] publiziert unter der CC BY 3.0 Lizenz [https://creativecommons.org/licenses/by/3.0/]; Details zu den Messungen befinden sich im FTIR-Spektrenverzeichnis in Tab. A-8 in Anhang A.6.2,

Um dies genauer zu überprüfen, wurden FTIR-Jet-Spektren von vollständig deuteriertem NMA (NMA- $d_{7}$ ) im N-D-Streckbereich aufgenommen. Die Deuterierung bewirkt über die Veränderung der reduzierten Masse eine Rotverschiebung der Amid A-Bande um ungefähr den Faktor $1 / \sqrt{2}$ ohne die $\mathrm{C}=\mathrm{O}$-Streckschwingung nennenswert zu beeinflussen. Damit wäre eine mögliche Fermi-Resonanz aufgehoben und die Doppelbande sollte sich im deuterierten Spektrum zu einer ungestörten Bande reduzieren. In Abbildung 4-12 sind die Amid A-Spektren von NMA und NMA- $d_{7}$ gegenübergestellt, wobei die $x$-Achse so skaliert wurde, dass die $t$-AmA und die niederfrequentere Dimerbande übereinander liegen. Dabei zeigt sich im Spektrum der deuterierten Substanz ein sehr ähnliches Bandenmuster mit der cis-Monomerbande und zwei Dimerbanden, die allerdings aufgrund der viel geringeren Konzentration im NMA-d $\mathrm{d}_{7}$-Spektrum deutlich schwächer ausfallen. Damit kann eine Fermi-Resonanz nicht die Ursache für die Doppelbande sein.16)

Da auch Tunneleffekte ausgeschlossen werden können [212], kann die Ursache nur in der

16) Ergänzende Informationen zu den Bandenpositionen von NMA- $d_{7}$ und ein Vergleich mit dem Gasphasenspektrum findet sich in Abb. A-10 in Anhang A.7.2 
Tab. 4-9. Experimentelle Bandenpositionen des $t t$-Dimers von NMA. Angegeben sind die Werte (in $\mathrm{cm}^{-1}$ ) für die freie $\left(v_{\mathrm{NH}}^{a}\right)$ und die Wasserstoffbrücken-gebundene $\mathrm{N}-\mathrm{H}$-Streckschwingung $\left(v_{\mathrm{NH}}^{d}\right)$, die freie $\mathrm{C}=\mathrm{O}-\mathrm{Streckschwingung}$ des Akzeptors $\left(v_{\mathrm{CO}}^{a}\right)$ und des Donors $\left(v_{\mathrm{NH}}^{d}\right)$ genauso wie Akzeptor- und Donorbanden der Amid III-Mode (Index: III). In Klammern dahinter die relative Verschiebung zum Monomer (vorläufige Zuordnungen sind mit einem Fragezeichen markiert). Alle Angaben in $\mathrm{cm}^{-1}$.

\begin{tabular}{|c|c|c|c|c|c|c|}
\hline Methode & $v_{\mathrm{NH}}^{d}$ & $v_{\mathrm{NH}}^{a}$ & $v_{\mathrm{CO}}^{d}$ & $v_{\mathrm{CO}}^{a}$ & $v_{\mathrm{III}}^{d}$ & $v_{\text {III }}^{a}$ \\
\hline \multicolumn{7}{|l|}{ Jet-Expansion: } \\
\hline \multirow[t]{2}{*}{ Raman $^{\mathrm{a}}$} & $3396(-112)$ & $3505(-3) ?$ & $1705(-15)$ & $1695(-25)$ & $1285(+27)$ & $1271(+13)$ \\
\hline & $3367(-141)$ & - & & & & \\
\hline \multirow[t]{2}{*}{ FTIR $^{a}$} & $3395(-113)$ & $3506(-2) ?$ & - & $1695(-25)$ & - & - \\
\hline & $3365(-143)$ & - & & & & \\
\hline \multirow[t]{2}{*}{ FTIR 51} & $3395(-115)$ & - & - & $1690(-32)$ & - & - \\
\hline & $3365(-145)^{d}$ & - & & & & \\
\hline IRMPD + VUVb [194] & - & - & $1702(-5)$ & $1681(-26)$ & $1277(+29)$ & - \\
\hline \multicolumn{7}{|l|}{ Matrix-Isolation: } \\
\hline $\mathrm{N}_{2}-\mathrm{FTIR}$ & - & - & $1693(-13)^{d}$ & $1681(-25)$ & - & - \\
\hline $\mathrm{Ar}-\mathrm{FTIR}$ 195 & - & - & $1695(-13)^{d}$ & $1686(-22)$ & - & - \\
\hline \multicolumn{7}{|l|}{ Lösung: } \\
\hline $\mathrm{CCl}_{4}-2 \mathrm{C}-\mathrm{fs} V S[210]^{\mathrm{c}}$ & - & - & - & $1670(-20)$ & & \\
\hline $\mathrm{H}_{2} \mathrm{O}+\mathrm{CCl}_{4}-\mathrm{FTIR} 181$ & $3391(-84)$ & $3470(-5)$ & - & - & - & - \\
\hline
\end{tabular}

Existenz zweier $t t$-Dimere liegen, deren NH-Streckschwingungen in der Wasserstoffbrücke sich mit einer Differenz von $30 \mathrm{~cm}^{-1}$ stark unterscheiden, aber die Strukturen gleichzeitig energetisch so ähnlich sind, dass beide Isomere hinreichend populiert sind. Hierzu wurde mit quantenchemischen Rechnungen auf B3LYP-D3(BJ)/aug-cc-pVTZ-Niveau die Potentialhyperfläche des trans-trans-Dimers nach Geometrien minimaler Energie durchsucht und deren Normalmoden in doppelt-harmonischer Näherung berechnet. ${ }^{17)}$ Die Rechnungen ergeben als Minimumstrukturen zwei elektronisch isoenergetische Isomere (nullpunktskorrigierte relative Energiedifferenz: $\Delta E_{\mathrm{el}}=0.1 \mathrm{~kJ} \cdot \mathrm{mol}^{-1}$ ), in denen die Molekülebenen der über eine Wasserstoffbrücke verbundenen Monomereinheiten um ca. $90^{\circ}$ gegeneinander verdreht sind. Es handelt sich dabei um die beiden Strukturen $t t_{\mathrm{N}}$ und $t t_{\mathrm{C}}$, bei denen entweder das Amin-seitige $\left(t t_{\mathrm{N}}\right)$ oder das Acetyl-seitige $\left(t t_{C}\right)$ freie Elektronenpaar des Carbonyl-Sauerstoffs als Akzeptor der Wasserstoffbrückenbindung fungiert (siehe Abb. 4-13).

In der Literatur finden sich eine Reihe von theoretischen Arbeiten zur Molekülstruktur des $t t$-Dimers. Es wurden unter anderem zwei coplanare, $C_{\mathrm{s}}$-symmetrische Strukturen mit einer $\mathrm{N}-\mathrm{H} \cdots \mathrm{O}=\mathrm{C}$-Wasserstoffbrücke und paralleler oder antiparalleler Ausrichtung der Monomereinheiten auf HF/3-21G- (nur parallel) [207], HF/6-31++G** [195] und mit dem empirischen Dichtefunktional EDF1 [151] diskutiert. Diese wurden später in MP2/6-31++G(d,p)[216] und HF/6-311++G**-Rechnungen [217] um eine dritte, nicht-planare (als $\alpha$-helical beschriebene) Struktur ergänzt, deren Orientierung der Monomereinheiten der $t t_{\mathrm{N}}-\mathrm{Geometrie}$ entspricht und von beiden unbeachtet bereits Mitte der 90er Jahre mittels Hartree-Fock und

\footnotetext{
17) Die im vorherigen Abschnitt diskutierte Methylgruppenkonformation wird auf Grund der niedrigen Barriere und der im Prinzip freien Drehbarkeit selbst bei Jet-Messbedingungen nicht näher betrachtet. Allen gerechneten Cluster-Molekülstrukturen liegt das $t_{\text {as }}$-Konformer zugrunde.
} 

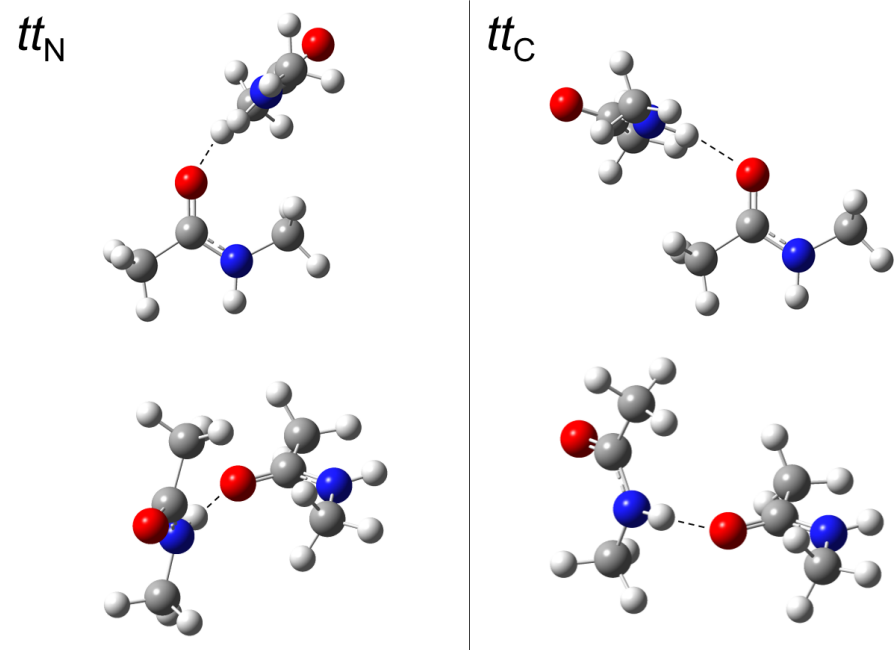

Abb. 4-13. Molekülgeometrien der beiden trans-trans-NMA Minimumstrukturen auf B3LYP-D3(BJ)/aVTZ-Niveau. (aus: Forsting etal. 212] publiziert unter der CC BY 3.0 Lizenz [https://creativecommons.org/licenses/by/3.0/])

MP2-Rechnungen und unterschiedlichen double- $\zeta$-Basissätzen gefunden wurde [218]. Die coplanaren Strukturen halten einer Überprüfung auf B3LYP/6-31+ $G^{*}$-Niveau [181, 188] und mit dem PW91 ${ }_{\mathrm{XC}}$-Funktional [188] nicht stand und liefern stattdessen das $t t_{\mathrm{C}}$-Konformer.

Vargas et al. [44] findet auf MP2/aVTZ-Niveau ein Dimer mit zusätzlicher C-H ‥ O-Wechselwirkung, welches aber bei einer Neuberechnung auf B3LYP-D3(BJ)/aVTZ-Niveau zu $t_{C}$ konvergiert. Parallel zu dieser Arbeit haben Maeyama et al. [21] unabhängig davon ebenfalls $t t_{\mathrm{C}}$ und $t t_{\mathrm{N}}$ als Dimerstrukturen gefunden und mit einer Vielzahl von Methoden berechnet (MP2, B3LYP, LC-BLYP, B97D, $\omega$ B97x-D, alle mit 6-311++G(d,p) Basis). Dabei variieren die nullpunktskorrigierten Energieunterschiede zwischen 0.2 und $0.7 \mathrm{~kJ} \cdot \mathrm{mol}^{-1}$ und die beiden Dimere können auch hier als isoenergetisch betrachtet werden. Vergleichbare Strukturen wurden auch für gemischte Indol-NMA-Cluster in konformationsselektiver IR-Spektroskopie ermittelt [219].

Tab. 4-10. Berechnete Eigenschaften der NMA-Dimere $t t_{C}$ und $t t_{N}$ auf B3LYP/aVTZ-Niveau mit und ohne D3(BJ)-Dispersionskorrektur. Angegeben sind die relativen elektronischen und nullpunktskorrigierten Energien $\Delta E_{\text {el }}$ bzw. $\Delta E_{0}$. Länge der Wasserstoffbrückenbindung $d(\mathrm{H} \cdots \mathrm{O})$, der Bindungswinkel der Wasserstoffbrückenbindung mit der $\mathrm{C}=\mathrm{O}$-Bindung $\alpha(\mathrm{H} \cdots \mathrm{O}=\mathrm{C})$, die niedrigste Frequenz in doppelt-harmonischer Näherung $\tilde{v}_{\min }$ und die Dipolmomente $\mu / \mathrm{D}$. [212]

\begin{tabular}{lllllll}
\hline & \multicolumn{3}{c}{$t_{\mathrm{C}}$} & & \multicolumn{2}{c}{$t_{\mathrm{N}}$} \\
\cline { 2 - 3 } \cline { 6 - 6 } \cline { 6 - 6 } & B3LYP-D3(BJ) & B3LYP & & B3LYP-D3(BJ) & B3LYP \\
\hline$\Delta E_{\mathrm{el}} / \mathrm{kJ} \cdot \mathrm{mol}^{-1}$ & 0.0 & 0.0 & & 0.0 & 1.0 \\
$\Delta E_{0} / \mathrm{kJ} \cdot \mathrm{mol}^{-1}$ & 0.0 & 0.0 & & 0.1 & 0.8 \\
$\alpha(\mathrm{H} \cdots \mathrm{O}=\mathrm{C}) /{ }^{\circ}$ & 119 & 139 & & 139 & 157 \\
$d(\mathrm{H} \cdots \mathrm{O}) / \mathrm{pm}$ & 192 & 199 & & 193 & 200 \\
$\tilde{v}_{\min } / \mathrm{cm}^{-1}$ & 12 & 7 & & 12 & 8 \\
$\mu / \mathrm{D}$ & 8 & 9 & & 8 & 9 \\
\hline
\end{tabular}

Die Isomerisierungsbarriere zwischen den beiden Dimeren kann hier durch vorläufige Übergangszustandsberechnungen auf $<4 \mathrm{~kJ} \cdot \mathrm{mol}^{-1}$ abgeschätzt werden. Damit ist sie nicht hoch genug, um die beiden Konformere im Jet bereits zu einem frühen Zeitpunkt in der Expansion 
einzufrieren und daher ist eine Verwendung der berechneten Energie anstatt der freien Enthalpie gerechtfertigt, um die relative Stabilität abzuschätzen (siehe Tab. 4-10). Der berechnete Energieunterschied ist auf dem Rechenniveau nicht signifikant und eine äquivalente Besetzung beider Konformere im Jet kann angenommen werden.

Die Rechnungen ergeben für die N-H-Donorbande $v_{\mathrm{NH}}^{d}$ von $t t_{\mathrm{C}}$ mit Dispersionskorrektur eine skalierte Rotverschiebung von $34 \mathrm{~cm}^{-1}$ gegenüber der entsprechenden Bande von $t t_{\mathrm{N}}$, bei gleichzeitig höheren IR-Bandenintensitäten und Raman-Streuquerschnitten (siehe Tab. 4-11). Dies bedeutet eine schwächere und schlechter sichtbare $v_{\mathrm{NH}}^{d}$ von $t t_{\mathrm{N}}$, was sich mit einer stärker gespannten Wasserstoffbrückenbindung erklären ließe. Der ideale Bindungswinkel $\alpha(\mathrm{H} \cdots \mathrm{O}=\mathrm{C})$ liegt bei $120^{\circ}$ und ist mit Dispersionskorrektur für $t t_{\mathrm{C}}$ fast ideal, während er für $t t_{\mathrm{N}}$ um ca. $20^{\circ}$ größer ist.

Der Vergleich mit quantenchemischen Rechnungen auf dem gleichen Niveau nur ohne Dispersionskorrektur (in Tab. 4-10) ergibt einen leicht erhöhten energetischen Vorteil von ca. $1 \mathrm{~kJ} \cdot \mathrm{mol}^{-1}$, was im Rahmen der Genauigkeit der Methode eine gewisse Präferenz für eine Relaxation in die stabilere Form erwarten lässt. Die Länge der Wasserstoffbrückenbindung nimmt für beide Isomere um $7 \mathrm{pm}$ zu, während der Bindungswinkel $\alpha$ um ca. $20^{\circ}$ zunimmt. Daraus folgt, dass dispersive Wechselwirkungen beide Isomere gleichermaßen stabilisieren und daher die verschiedenen Bindungswinkel und damit die unterschiedlich stark gespannte Wasserstoffbrücke von $t t_{\mathrm{C}}$ und $t t_{\mathrm{N}}$ ihren Ursprung in einer sterischen Hinderung durch die N-seitige Methylgruppe haben muss.

Damit lassen sich die Dimer-Banden im FTIR-Spektrum der Amid A-Region sehr gut einer der beiden Strukturen zuordnen. Auch wenn für beide Banden die für B3LYP (und auch andere DFT-Methoden) bekannte Überschätzung der Verschiebung relativ zum Monomer ${ }^{18)} \mathrm{zu}$ sehen ist, entspricht der experimentelle Abstand der beiden Dimerbanden mit $30 \mathrm{~cm}^{-1} \mathrm{sehr}$ gut der theoretischen Vorhersage. Die Bande bei $3395 \mathrm{~cm}^{-1}$ entspricht $t t_{\mathrm{N}}$ und $t t_{\mathrm{C}}$ lässt sich der Bande bei $3365 \mathrm{~cm}^{-1}$ zuordnen, was auch grafisch durch die Strichspektren in Abbildung 4-11 dargestellt ist. Auch die vorläufige Zuordnung des niederfrequenten Peaks der trans-Amid AMonomerbande bei $3506 \mathrm{~cm}^{-1}$ als Akzeptorbande des Dimers wird durch die Rechnung bestätigt, die mit 3504 und $3503 \mathrm{~cm}^{-1}$ für beide $t t$-Isomere eine fast identische Bandenposition vorhersagt. Dies ist auch nachvollziehbar, da die freie N-H-Streckschwingung keinerlei sterische Einschränkung durch eine C- oder N-seitige Wasserstoffbrückenbindung im Dimer erfährt. Aus der Gegenüberstellung in Abbildung 4-11 wird ersichtlich, dass die getroffene Zuordnung von Theorie und FTIR auch mit den Ramanspektren übereinstimmt.

Mit den Ergebnissen der Frequenzrechnung soll nun erneut die Amid I-III-Region ausgewertet werden. Die Werte in Tabelle 4-11 und die dazugehörigen Strichspektren in Abbildung 4-10 bestätigen die Zuordnung der Bande bei $1695 \mathrm{~cm}^{-1}$ im FTIR-Spetrum als Akzeptorbande des Dimers. Die beiden Isomere unterscheiden sich nur um $5 \mathrm{~cm}^{-1}$, was bedeutet, dass die Stärke der Wasserstoffbrückenbindung die $\mathrm{C}=\mathrm{O}$-Streckschwingung erwartungsgemäß nicht so stark beeinflusst wie die N-H-Streckschwingung. Die experimentelle Bandenposition liegt dabei genau zwischen den beiden theoretisch vorhergesagten. Bei ganz genauer Betrachtung lässt die

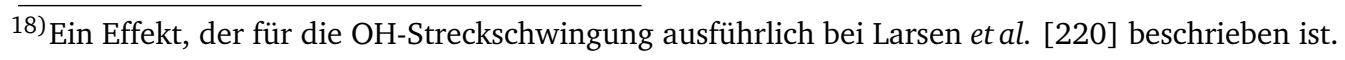


Tab. 4-11. Berechnete Normalmoden der tt-Dimere von NMA für die $\mathrm{N}-\mathrm{H}$ - und $\mathrm{C}=\mathrm{O}$-Streckschwingung sowie die Amid III-Mode des Donor- und Akzeptormoleküls auf B3LYP-D3(BJ)/aVTZ-Niveau und Zuordnung zu den experimentellen Raman-Banden ( $\tilde{v}_{\mathrm{Ra}}$ ) und FTIR-Banden $\tilde{v}_{\mathrm{IR}}$ mit samt relativer Verschiebung zur trans-Monomerbande in Klammern. Angegeben sind zudem die skalierten harmonischen Wellenzahlen $\omega$ (Skalierungsfaktor $x$ ist 0.959 oberhalb von $3000 \mathrm{~cm}^{-1}$ und 0.99 unterhalb), die IR-Intensität $A$ und der Raman-Streuquerschnitt $\sigma^{\prime}$ bei $532 \mathrm{~nm}$ Anregungswellenlänge und $90^{\circ}$ Streugeometrie. In Klammern ist die relative Verschiebung zur skalierten Wellenzahl des $t_{\mathrm{as}}$-Monomers angegeben (Weitere Daten der Frequenzrechnungen befinden sich in den Tab. A-15, A-16 und A-18 im Tabellenwerk in Anhang A.7.2).

\begin{tabular}{|c|c|c|c|c|c|}
\hline Konformer & $x \cdot \omega / \mathrm{cm}^{-1}$ & $A / \mathrm{km} \cdot \mathrm{mol}^{-1}$ & $10^{35} \sigma^{\prime} / \mathrm{m}^{2} \mathrm{sr}^{-1}$ & $\tilde{v}_{\mathrm{Ra}} / \mathrm{cm}^{-1}$ & $\tilde{v}_{\mathrm{IR}} / \mathrm{cm}^{-1}$ \\
\hline \multicolumn{6}{|c|}{ NH-Streckschwingungen: } \\
\hline \multirow[t]{2}{*}{$v_{\mathrm{NH}}^{a}$} & $3504(-3)$ & 34 & 0.89 & \multirow{2}{*}{$3505(-3)$} & \multirow{2}{*}{$3506(-2)$} \\
\hline & $3503(-4)$ & 33 & 0.85 & & \\
\hline \multirow[t]{2}{*}{$v_{\mathrm{NH}}^{d}$} & $3326(-181)$ & 578 & 2.54 & $3367(-141)$ & $3365(-143)$ \\
\hline & $3360(-147)$ & 508 & 1.96 & $3396(-112)$ & $3395(-113)$ \\
\hline \multicolumn{6}{|c|}{ CO-Streckschwingungen: } \\
\hline$v_{\mathrm{CO}}^{d} \quad t t_{\mathrm{C}}$ & $1709(-10)$ & 213 & 0.26 & \multirow{2}{*}{$1705(-15)$} & \multirow{2}{*}{-} \\
\hline$t t_{\mathrm{N}}$ & $1710(-9)$ & 191 & 0.25 & & \\
\hline \multirow{2}{*}{$v_{\mathrm{CO}}^{a}$} & $1691(-28)$ & 505 & 0.83 & \multirow{2}{*}{$1695(-25)$} & \multirow{2}{*}{$1695(-25)$} \\
\hline & $1696(-23)$ & 526 & 0.56 & & \\
\hline \multicolumn{6}{|c|}{ Amid II-Schwingungen: } \\
\hline \multirow[t]{2}{*}{$v_{\mathrm{II}}^{d}$} & $1578(+50)$ & 171 & 0.02 & \multirow{2}{*}{-} & \multirow{2}{*}{-} \\
\hline & $1576(+48)$ & 167 & 0.02 & & \\
\hline \multirow[t]{2}{*}{$v_{\mathrm{II}}^{a}$} & $1541(+13)$ & 242 & 0.04 & \multirow{2}{*}{-} & \multirow{2}{*}{-} \\
\hline & $1540(+12)$ & 178 & 0.04 & & \\
\hline \multicolumn{6}{|c|}{ Amid III-Schwingungen: } \\
\hline \multirow{2}{*}{$\begin{array}{ll}v_{\mathrm{III}}^{d} \quad t t_{\mathrm{C}} & t t_{\mathrm{N}}\end{array}$} & $1303(+36)$ & 95 & 0.29 & \multirow{2}{*}{$1285(+27)$} & \multirow{2}{*}{-} \\
\hline & $1302(+35)$ & 93 & 0.29 & & \\
\hline \multirow[t]{2}{*}{$v_{\mathrm{III}}^{a}$} & $1282(+15)$ & 77 & 0.62 & \multirow{2}{*}{$1271(+13)$} & \multirow{2}{*}{-} \\
\hline & $1281(+15)$ & 62 & 0.30 & & \\
\hline
\end{tabular}


Bande sogar einen Doppelpeak erahnen, was nicht nur bezüglich der berechneten Bandenposition sondern auch mit der ungefähr gleich großen IR-Intensität beider Isomere sehr gut mit der Theorie übereinstimmt. Die freie Carbonylschwingung des Donors wird mit einer Verschiebung von -(9-10) $\mathrm{cm}^{-1}$ berechnet. Die beiden Isomere liegen hier, wie bei der freien NH-Bande, aus den gleichen Gründen nahe beieinander und könnten unter Vorbehalt der niederfrequenten Schulter der Amid I-Bande im FTIR-Spektrum zugeordnet werden.

Im Raman-Spektrum ist die oben getroffene, vorläufige Zuordnung der beiden gut separierten Peaks unterhalb der t-Amid I bei 1705 und $1695 \mathrm{~cm}^{-1}$ in sehr guter Übereinstimmung mit der Theorie. Allerdings liefert dies nach wie vor keine Erklärung für die asymmetrische Bandenform der $1695 \mathrm{~cm}^{-1}$-Bande und für den kleinen Peak bei $1683 \mathrm{~cm}^{-1}$.

Der Vergleich der skalierten Amid II-Bandenpositionen der tt-Dimere in Tabelle 4-11 zeigt auch hier sowohl für die Donor als auch für die Akzeptorbande jeweils nur einen Unterschied von $1 \mathrm{~cm}^{-1}$, so dass von der Theorie her eine Unterscheidbarkeit von $t t_{\mathrm{C}}$ und $t t_{\mathrm{N}}$ im Spektrum nicht zu erwarten ist. Beide Dimer-Banden werden blauverschoben vorhergesagt, wobei die Donorbande um fast $40 \mathrm{~cm}^{-1}$ stärker verschoben wird als das Akzeptorsignal. Da es sich hier hauptsächlich um eine N-H-Knickschwingung in der Molekülebene handelt, ist dies zu erwarten (siehe dazu auch Abschnitt 2.2). Im FTIR-Spektrum sind oberhalb der beiden Monomerbanden (siehe oben) keine Signale zu erkennen, die auf eine Dimerbande schließen lassen. Die berechneten IR-Intensitäten sind auch nur ca. halb so groß wie die der Amid I-Akzeptorbande, so dass, wenn überhaupt, nur zwei sehr kleine Peaks zu erwarten wären. Die höherfrequente Amid II-Monomerbande hat allerdings beidseitig ausgeprägte Schultern, die eventuell auf ein Dimersignal zurückzuführen sind. Da hier aber mit hoher Wahrscheinlichkeit eine Resonanz vorliegt, ist hier eine genaue Zuweisung auch unter Zuhilfenahme der Ergebnisse aus der Frequenzrechnung nicht möglich.

Für die Amid III-Bande sind theoretisch ebenfalls zwei zu höheren Wellenzahlen verschobene Dimerbanden vorhergesagt, welche sich für die beiden Isomere erneut kaum unterscheiden (siehe Tab. 4-11). Die $v_{\mathrm{III}}^{a}$ liegt mit einer theoretischen Verschiebung von $+15 \mathrm{~cm}^{-1}$ in sehr guter Übereinstimmung mit der Bande bei $1271 \mathrm{~cm}^{-1}$ im Raman-Spektrum, welche um $+13 \mathrm{~cm}^{-1}$ zum Monomer verschoben ist. Die skalierte Donorbandenposition aus der Frequenzrechnung liefert nur eine moderate Übereinstimmung mit der Bande bei $1285 \mathrm{~cm}^{-1}$. Dabei wird die Verschiebung von der Theorie um ca. 30 \% überschätzt, weswegen die Zuordnung als Dimerbande vor allem in Anbetracht des bereits angesprochenen Wachstumsverhaltens nur unter Vorbehalt bestehen bleiben kann.

Bezüglich der Aufklärung der Dimerbandenzuordnung der Amid III-Bande liefert das FTIRSpektrum keine zusätzlichen Informationen, da sich hier an den entsprechenden Wellenzahlen des Raman-Spektrums nur Banden im Rauschen erahnen lassen.

Zum Abschluss dieses Kapitels soll noch einmal das weiter oben bereits kurz erwähnte ciscis-Dimer (cc) ausführlicher diskutiert werden. Einfache Überlegungen zur Struktur haben spätestens in den 50er Jahren ein zyklisches NMA-Dimer aus zwei cis-Monomereinheiten (Punktgruppe: $C_{2 h}$ ) (siehe Abb. 4-14) auf Grund der Möglichkeit zur Ausbildung von zwei Wasserstoffbrückenbindungen als stabilstes Dimer vorhergesagt [221]. Eine erste Rechnung von Di- 
xon et al. [218] liefert für das zyklische Dimer eine Dissoziationsenergie von $D_{\mathrm{e}}=-59.6 \mathrm{kmol}$ (MP2(FC)/aVDZ-CP) und ist damit ca. doppelt so groß wie für das $t t_{\mathrm{N}}$-Dimer. In einer Vielzahl von späteren Arbeiten [44, 151, 181, 188, 222] ist das höchste Level eine MP2- und eine CCSD(T)-Rechnung am Basissatzlimit (CBS) mit $D_{\mathrm{e}}=-72.8 \mathrm{kmo} 1{ }^{19)}$.

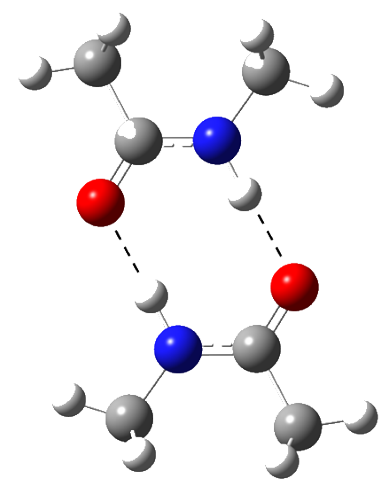

Abb. 4-14. Molekülstruktur des cc-NMA Dimers auf B3LYP-D3(BJ)/aVTZ-Niveau.

Experimentell gibt es bisher erst einen Hinweis auf das $c c$-Dimer: In $\mathrm{N}_{2}$ - und Ar-Matrixspektren [195] wurde eine Bande der c-AmI zugeordnet $\left(\mathrm{N}_{2}: 1693 \mathrm{~cm}^{-1}\right.$; Ar: $\left.1695 \mathrm{~cm}^{-1}\right)$, allerdings ist gleichzeitig kein Monomer sichtbar, weswegen der Verdacht nahe liegt, dass die gemessenen Banden ihren Ursprung im trans-NMA haben oder auf Matrixeffekte zurückzuführen sind. Die B3LYP-D3(BJ)/aVTZ-Frequenzrechnung zeigt, dass die Amid I-Region aufgrund von zu erwartender Überlappung mit den $t t$-Dimerbanden und höheren Clusterbanden eher nicht für einen Nachweis des cc-Dimers geeignet ist (siehe Tab. A-16), auch wenn eine Kombination von FTIR- und Ramanspektroskopie aufgrund der Inversionssymmetrie des Clusters und dem damit einhergehenden Alternativverbot grundsätzlich eine passende Methode darstellt.

Sowohl die symmetrische als auch die antisymmetrische NH-Streckschwingung unterliegen dem Alternativverbot. Sie haben allerdings eine theoretisch vorhergesagte (skalierte) Verschiebung von $-324 \mathrm{~cm}^{-1}$ (IR) und $-374 \mathrm{~cm}^{-1}$ (Raman) gegenüber dem $c$-Monomer. Damit sollte es hinreichend separiert von anderen Clusterbanden erscheinen, wäre aber außerhalb der Spektren in Abbildung 4-11.

Doch auch wenn eine Sichtbarkeit im Schwingungsspektrum aufgrund der Verschiebung gegeben ist, erklärt eine statistische Überlegung, warum im Rahmen der vorliegenden Ergebnisse kein Zuordnungsversuch erfolgt: Damit sich in der Jet-Expansion ein cc-Dimer bilden kann, müssen zwei c-Monomere miteinander kollidieren, da bei der berechneten Barriere (siehe Tab. A-14 keine Isomerisierung zu erwarten ist. Bei einem $c$-Monomeranteil von ca. 1.6\% (siehe hierzu Abb. A-8 und die berechneten Streuquerschnitte in Tab. A-15) liegt der zu erwartende $c c$-Dimeranteil dem Quadrat des Stoffmengenanteils des c-Monomers also bei ca. $0.03 \%$ und wäre damit nicht im Rauschen der Basislinie erkennbar.

Für einen spektroskopischen Nachweis des cc-Dimers von NMA bedarf es also einer Erhöhung des Anteils an cis-Monomer, worauf in Abschnitt 9 näher eingegangen wird.

19) in: Zeng et al. [223]; Geometrie: $\omega$ B97X-D/aVDZ, allerdings Integrationsgitter entgegen der Gaussian-Empfehlung zu klein gewählt. 


\subsection{Trimere und höhere Aggregate}

Die Jet-FTIR-Studie von Albrecht et al. [51] ordnet den Peak bei $3365 \mathrm{~cm}^{-1}$ unter Vorbehalt dem NMA-Trimer zu. Es wird ein lineares Trimer mit zwei Wassersoffbrückenbindungen ähnlich dem in Abbildung 4-15 angenommen und mit der Kooperativität der Wasserstoffbrückenbindung [224-226] die Rotverschiebung zum Dimer erklärt. Für das Fehlen einer zweiten NHStreck-Donorbande und die vergleichsweise schwache Rotverschiebung werden allerdings keine plausiblen Argumente geliefert. Diese Zuordnung wurde schließlich von M. Mons aufgrund eigener Frequenzrechnungen mit dem B97-D-Funktional und einer speziell auf Peptide abgestimmten Skalierung [212, 227] angezweifelt und die Bande lässt sich mit den Erkenntnissen zu den zwei energetisch äquivalenten Dimeren aus dem letzten Abschnitt schlüssiger erklären. Dies war der Anstoß zu der erneuten Jet-spektroskopischen Untersuchung der Cluster von NMA im Rahmen dieser Arbeit.

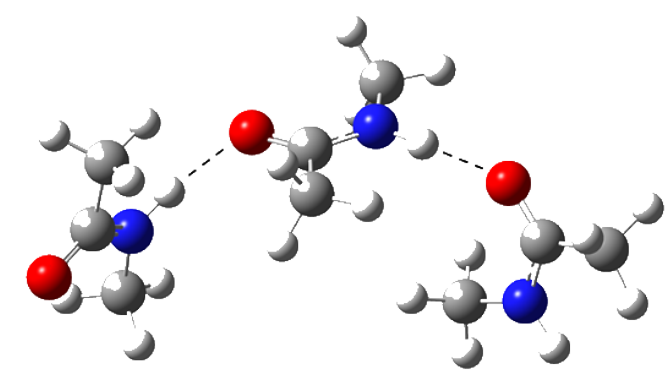

Abb. 4-15. Molekülstruktur eines offenkettigen (linearen) all-trans-NMA-Trimers auf B3LYP-D3(BJ)/aVTZ-Niveau.

Die beiden eingesetzten Jet-spektroskopischen Methoden sind nicht größenselektiv, so dass bei Messbedingungen zum Nachweis von Dimeren Banden höherer Cluster nur schwer auszuschließen sind. Aus stoßtheoretischen Überlegungen ist es klar, dass Trimere am wahrscheinlichsten aus einem Dimer und einem Monomer entstehen, genauso wie Tetramere aus Trimer und Monomer oder zwei Dimeren gebildet werden. Sie können sich also erst dann bilden, wenn der nächst kleinere Cluster bereits vorliegt, weswegen die entsprechenden Banden im Schwingungsspektrum verzögert auftreten.

In den Spektren der übersichtlicheren Amid A-Region in Abbildung 4-11 fällt zum einen auf, dass im IR-Spektrum die höherfrequente Dimerbande bei $3395 \mathrm{~cm}^{-1}$ mit zunehmender Konzentration schneller wächst als die niederfrequente. Außerdem taucht im Raman-Spektrum eine verzögerte Bande bei $3386 \mathrm{~cm}^{-1}$ auf, während die beiden Dimerbanden mit abnehmender Düsentemperatur ungefähr gleich schnell wachsen. Die Entwicklung der Banden lässt auf ein Trimer schließen, wobei es sich nicht um ein lineares Trimer handeln kann, da die beiden NHStreckschwingungen der beiden als Wasserstoffbrückendonor fungierenden Monomereinheiten zwei Banden ergeben müssten, die auf Grund des kooperativen Effekts unterschiedlich stark zum Dimer rotverschoben sind. Außerdem müssten wegen fehlender Symmetrie die Banden 
sowohl IR- als auch Raman-aktiv sein und im Spektrum zu gleichen Wellenzahlen erscheinen.

Eine mögliche Erklärung für die geringe Verschiebung ist die Ausbildung von cyclischen Trimeren, wie sie bereits für Pyrrol festgestellt wurde [228]. Im cyclischen Trimer führt die Ringspannung zur Schwächung der Wasserstoffbrückenbindung, so dass die NH-Streckschwingungen des Trimers gegenüber dem Dimer nicht so stark bis gar nicht verschoben werden Zudem würde eine näherungsweise lokale Symmetrie diese nahezu äquivalent erscheinen lassen, so dass das Trimer zwei benachbarte asymmetrische (und damit IR-aktive) und eine symmetrische (und Raman-aktive) NH-Streck-Normalmode hat, was die unterschiedlichen Bandenpositionen in IR und Raman erklärt (Davydov-Splitting [220, 229, 230]).

Diese These lässt sich mittels quantenchemischer Rechnungen überprüfen. Eine Geometrieoptimierung auf dem bereits etablierten B3LYP-D3(BJ)/aVTZ-Niveau liefert neben dem linearen Trimer $\left(t t t_{1}\right)$ auch zwei stabile cyclische Trimerstrukturen. Diese haben eine Fass-artige Struktur, bei der die NMA-Monomereinheiten entweder gleich ausgerichtet (parallel, $t t t_{\mathrm{p}}$ ) oder eines der Monomere entgegengestzt ausgerichtet ist (antiparallel, $t t t_{\mathrm{a}}$ ) (siehe Abb.4-16).
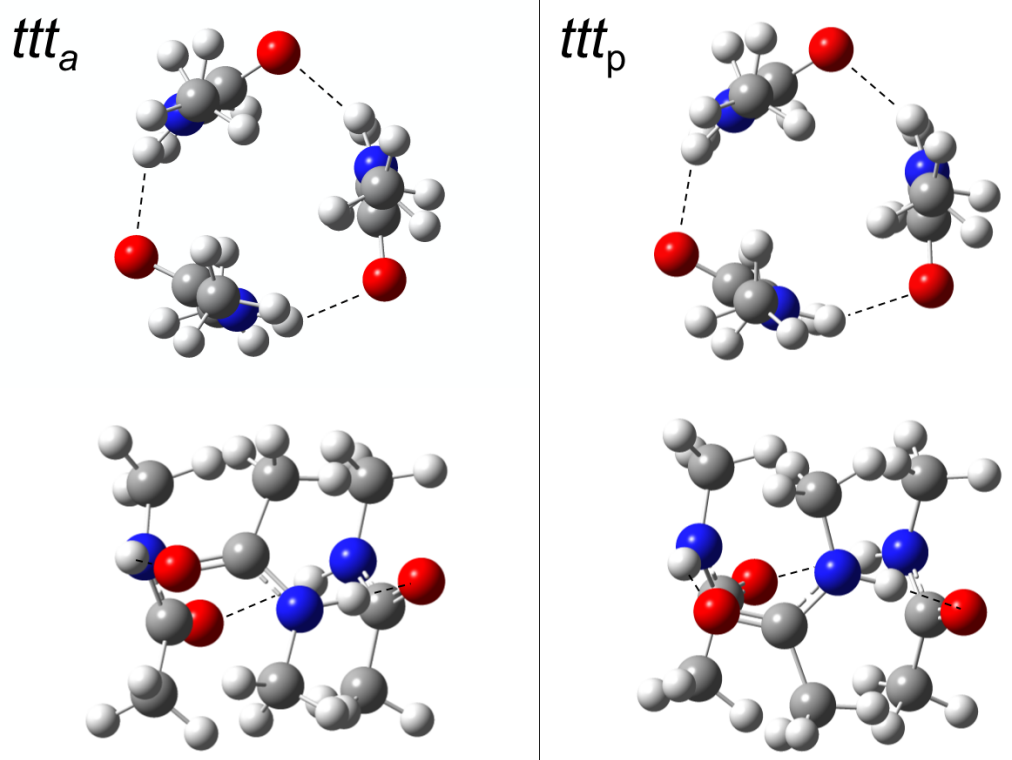

Abb. 4-16. Molekülstrukturen der beiden cyclischen trans-NMA Trimere auf B3LYP-D3(BJ)/aVTZ-Niveau. Die Amidgruppen der Monomereinheiten sind dabei entweder gleich ausgerichtet (parallel, $t t t_{p}$ ) und es liegt $C_{3}$-Symmetrie vor oder eine der drei Monomere hat eine antiparallele Ausrichtung (ttta). (veröffentlicht auf figshare.com als "ttt.png“ (DOI: 10.6084/m9.figshare.4233110.v1), publiziert unter der CC BY 4.0 Lizenz [https://creativecommons.org/licenses/by/4.0/])

Das $t t t_{\mathrm{p}}$ weist dabei $C_{3}$-Symmetrie auf und ist mit einem nullpunktskorrigierten Energieunterschied von $0.5 \mathrm{~kJ} \cdot \mathrm{mol}^{-1}$ vernachlässigbar stabiler als $t t t_{\mathrm{a}}$ (siehe Tab. 4-12). Allerdings ist der Energieunterschied der beiden zyklischen Isomere zum linearen Trimer mit ca. $17 \mathrm{~kJ} \cdot \mathrm{mol}^{-1}$ sehr groß. Die harmonischen Frequenzrechnungen ergeben keine imaginären Frequenzen (siehe Tab. 4-12). Damit gibt es laut Theorie zwei isoenergetische zyklische NMA-Trimere, die energetisch deutlich unterhalb dem allgemein postulierten linearen Trimer liegen und ein echtes Minimum darstellen. Für diese Strukturen ergibt sich ein $\mathrm{N}-\mathrm{H} \cdots \mathrm{C}=\mathrm{O}$-Bindungswinkel von $109^{\circ}$ für $t t t_{\mathrm{p}}$ und $104-110^{\circ}$ für $t t t_{\mathrm{a}}$ und damit ca. $10^{\circ}$ kleiner als in der einfachen Wasserstoffbrücke im $t t_{C}$-Dimer. Mit dieser Ringspannung geht eine Verlängerung der Wasserstoffbrücken- 
Tab. 4-12. Berechnete Eigenschaften der cyclischen NMA-Trimere $t t t_{a}$ und $t t t_{p}$, sowie des linearen Trimers $t t t_{1}$ auf B3LYP/aVTZ-Niveau mit und ohne Dispersionskorrektur (Strukturen, sie Abb. 4-16] [212]. Für eine Erklärung der Bezeichnungen, siehe Tab. 4-10. Thermodynamische Größen für 298.15 K bei 1 atm berechnet.

\begin{tabular}{|c|c|c|c|c|c|c|}
\hline & \multicolumn{2}{|l|}{$t t t_{\mathrm{a}}$} & \multicolumn{2}{|l|}{$t t t_{\mathrm{p}}$} & \multicolumn{2}{|l|}{$t t t_{1}$} \\
\hline & B3LYP-D3(BJ) & B3LYP & B3LYP-D3(BJ) & B3LYP & B3LYP-D3(BJ) & B3LYP \\
\hline$\Delta E_{\mathrm{el}} / \mathrm{kJ} \cdot \mathrm{mol}^{-1}$ & 0.0 & 0.0 & 0.1 & 0.3 & 21 & -10 \\
\hline$\Delta E_{0} / \mathrm{kJ} \cdot \mathrm{mol}^{-1}$ & 0.0 & 0.0 & 0.5 & 0.3 & 17 & -12 \\
\hline$\Delta H^{\ominus} / \mathrm{kJ} \cdot \mathrm{mol}^{-1}$ & 0.0 & - & 0.0 & - & 20 & - \\
\hline$S^{\ominus} / \mathrm{kJ} \cdot \mathrm{mol}^{-1} \cdot \mathrm{K}^{-1}$ & 0.67 & - & 0.66 & - & 0.76 & - \\
\hline$\Delta G^{\ominus} / \mathrm{kJ} \cdot \mathrm{mol}^{-1}$ & 6.2 & - & 8.8 & - & 0.0 & - \\
\hline \multirow[t]{3}{*}{$\alpha(\mathrm{H} \cdots \mathrm{O}=\mathrm{C}) /^{\circ}$} & 104 & 110 & 109 & 114 & - & - \\
\hline & 105 & 115 & 109 & 114 & 141 & 137 \\
\hline & 110 & 110 & 109 & 114 & 118 & 142 \\
\hline \multirow[t]{3}{*}{$d(\mathrm{H} \cdots \mathrm{O}) / \mathrm{pm}$} & 212 & 226 & 207 & 224 & - & - \\
\hline & 210 & 224 & 207 & 224 & 189 & 194 \\
\hline & 207 & 225 & 207 & 224 & 188 & 195 \\
\hline$\tilde{v}_{\min } / \mathrm{cm}^{-1}$ & 39 & 32 & 45 & 30 & 5 & 5 \\
\hline$\mu / \mathrm{D}$ & 0.4 & 0.3 & 1.2 & 0.7 & 11 & 15 \\
\hline
\end{tabular}

bindungen um ca. $20 \mathrm{pm}$ gegenüber dem linearen Trimer und eine untypische Koordination am Sauerstoffatom einher. Eine sehr große Zahl an theoretischen Publikationen zu NMA-Aggregaten [19, 51, 180, 181, 195, 207, 208, 210, 224, 231,-236] bis hin zu aktuellen Arbeiten [182, 194] beinhalten eine offenkettige Struktur als energetisch günstigstes Trimer und es wird stellenweise die Existenz eines zyklischen Clusters ausgeschlossen [237] oder nur für das allcis-Trimer gefunden [222]. Damit ist dies das erste Mal neben der zeitgleich zu und unabhängig von dieser Arbeit entstandenen Studie von Maeyama et al. [21], dass zyklische Strukturen als stabilste Konformationen für das all-trans-Trimer von NMA vorhergesagt wurden. Dies erscheint in Anbetracht des sehr deutlichen Energieunterschieds ungewöhnlich und soll nun noch einmal genauer betrachtet werden.

Der Hauptunterschied zu den bisherigen Arbeiten und dieser Arbeit wie auch der von Maeyama et. al. liegt im Einsatz von Methoden mit Dispersionskorrektur. Um den direkten Vergleich zu haben, wurden die gleichen B3LYP-Rechnungen erneut ohne Dispersionskorrektur durchgeführt. Die Ergebnisse (in Tab. 4-12) zeigen nun deutlich, warum bisher keine zyklischen Trimere in Erwägung gezogen wurden: Auch wenn ohne Dispersionskorrektur beide zyklischen Strukturen echte Minima ohne imaginäre Frequenzen ergeben, so hat sich der energetische Differenz zwischen linearer und zyklischer Struktur von +17 auf $-20 \mathrm{~kJ} \cdot \mathrm{mol}^{-1}$ praktisch invertiert.

Die Einbeziehung der skalierten harmonischen Wellenzahlen in die Betrachtung der Amid ASpektren in Abb.4-11 ergibt eine konsistente Zuordnung für die oben betrachteten möglichen Trimerbanden. Ein Unterschied von $10 \mathrm{~cm}^{-1}$ zwischen der höchsten und niedrigsten IR-aktiven Wellenzahl findet sich nicht in der Form zweier getrennter Banden im Spektrum wieder und alle IR-aktiven Normalmoden beider Isomere werden der Bande bei $3395 \mathrm{~cm}^{-1}$ zugeordnet (siehe Tab. 4-13). Für die insgesamt zwei Raman-aktiven Banden der beiden Trimere ergibt sich ein ähnliches Bild. Mit einer Differenz von $5 \mathrm{~cm}^{-1}$ werden beide der Bande bei $3386 \mathrm{~cm}^{-1}$ zugeordnet. Es ist auch hier wieder eine generelle Überschätzung der Verschiebung durch die 
Rechnung erkennbar, allerdings nicht so sehr wie bei den Dimeren. Dies lässt sich mit der oben beschriebenen Ringspannung und der nicht-klassichen Koordination erklären, die zu einer Reduzierung der diagonalen Anharmonizität der NH-Streckschwingung führen. Allgemein können die Rechnungen jedoch als Bestätigung der getroffenen Bandenzuordnung angesehen werden, so dass die Trimerzuordnung in [51] revidiert werden muss. Die als Dimer und Trimer bezeichneten Banden haben ihre Ursache vielmehr in den beiden isoenergetischen Dimeren (vgl. Abschnitt 4.3) und das Trimer ist unterhalb der höherfrequenten Dimerbande zu finden.

Tab. 4-13. Berechnete Normalmoden der zyklischen ttt-Trimere von NMA für die $\mathrm{N}-\mathrm{H}$ - und $\mathrm{C}=\mathrm{O}-\mathrm{Streckschwingung}$ sowie die Amid III-Mode des Donor- und Akzeptormoleküls auf B3LYP-D3(BJ)/aVTZ-Niveau und Zuordnung zu den experimentellen Raman-Banden ( $\left.\tilde{v}_{\mathrm{Ra}}\right)$ und FTIR-Banden $\tilde{v}_{\mathrm{IR}}$ mitsamt relativer Verschiebung zur trans-Monomerbande in Klammern. Die Moden sind in hauptsächlich IR-aktive asymmetrische (as) und hauptsächlich Raman-aktive symmetrische (s) aufgeteilt und beziehen sich auf lokale Symmetrieeffekte in der zyklischen Struktur. Alle weiteren Angaben sind analog zu Tab. 4-11 gewählt. (Weitere Daten der Frequenzrechnungen befinden sich in den Tab. A-15, $A-16$ und $A-18$ im Tabellenwerk in Anhang A.7.2).

\begin{tabular}{|c|c|c|c|c|c|c|}
\hline Mode & Konformer & $x \cdot \omega / \mathrm{cm}^{-1}$ & $A / \mathrm{km} \cdot \mathrm{mol}^{-1}$ & $10^{35} \sigma / \mathrm{m}^{2} \mathrm{sr}^{-1}$ & $\tilde{v}_{\mathrm{Ra}} / \mathrm{cm}^{-1}$ & $\tilde{v}_{\mathrm{IR}} / \mathrm{cm}^{-1}$ \\
\hline $\begin{array}{l}\mathrm{NH}-\mathrm{St} \\
v_{\mathrm{NH}}^{\mathrm{as}}\end{array}$ & $\begin{array}{l}\text { ckschwingur } \\
t t t_{\mathrm{a}} \\
t t t_{\mathrm{p}}\end{array}$ & $\begin{array}{l}\text { gen: } \\
3383(-124) \\
3378(-129) \\
3373(-134) \\
3373(-134)\end{array}$ & $\begin{array}{l}256.73 \\
245.00 \\
298.73 \\
298.88\end{array}$ & $\begin{array}{l}0.32 \\
0.53 \\
0.29 \\
0.29\end{array}$ & - & \} $3395(-113)$ \\
\hline$v_{\mathrm{NH}}^{\mathrm{S}}$ & $\begin{array}{l}t t t_{\mathrm{a}} \\
t t t_{\mathrm{p}}\end{array}$ & $\begin{array}{l}3366(-141) \\
3361(-146)\end{array}$ & $\begin{array}{r}58.06 \\
2.00\end{array}$ & $\begin{array}{l}1.58 \\
1.80\end{array}$ & $3386(-122)$ & - \\
\hline $\begin{array}{l}\mathrm{CO}-\mathrm{St} \\
v_{\mathrm{CO}}^{\text {as }}\end{array}$ & $\begin{array}{l}\text { ckschwingu } \\
t t t_{\mathrm{a}} \\
t t t_{\mathrm{p}}\end{array}$ & $\begin{array}{l}\text { gen: } \\
1690(-29) \\
1690(-29) \\
1688(-31) \\
1688(-31)\end{array}$ & $\begin{array}{l}412.19 \\
392.20 \\
393.09 \\
393.27\end{array}$ & $\begin{array}{l}0.06 \\
0.07 \\
0.07 \\
0.07\end{array}$ & - & - \\
\hline$v_{\mathrm{CO}}^{\mathrm{s}}$ & $\begin{array}{l}t t t_{\mathrm{a}} \\
t t t_{\mathrm{p}}\end{array}$ & $\begin{array}{l}1660(-59) \\
1658(-61)\end{array}$ & $\begin{array}{l}0.87 \\
3.40\end{array}$ & $\begin{array}{l}0.63 \\
0.59\end{array}$ & \} $1661(-59)$ & - \\
\hline $\begin{array}{l}\text { Amid I } \\
v_{\text {III }}^{\text {as }}\end{array}$ & $\begin{array}{l}\text { Schwingung } \\
t t t_{\mathrm{a}} \\
t t t_{\mathrm{p}}\end{array}$ & $\begin{array}{l}1306(+39) \\
1308(+41)\end{array}$ & $\begin{array}{l}156.73 \\
172.74\end{array}$ & $\begin{array}{l}0.08 \\
0.29\end{array}$ & - & - \\
\hline$v_{\mathrm{III}}^{\mathrm{s}}$ & $t t t_{\mathrm{a}}$ & $\begin{array}{l}1302(+35) \\
1301(+34) \\
1302(+35) \\
1302(+35)\end{array}$ & $\begin{array}{l}3.45 \\
4.90 \\
3.94 \\
3.93\end{array}$ & $\begin{array}{l}0.42 \\
0.26 \\
0.23 \\
0.23\end{array}$ & \} $1285(+27)$ & - \\
\hline
\end{tabular}

Mit den Ergebnissen der Frequenzrechnung in Tabelle 4-13 kann ein Zuordnungsversuch in der komplizierten Bandenstruktur um die Amid I- und die Amid III-Bande in den Spektren in Abbildung 4-10 unternommen werden. Die Theorie ergibt hier, dass beide zyklischen TrimerStrukturen mit einem Unterschied von maximal $2 \mathrm{~cm}^{-1}$ sowohl für die Amid I als auch die Amid III nicht unterscheidbar sein werden. Im FTIR-Spektrum ist kein Trimersignal erkennbar, allerdings entsprechen die skalierten Verschiebungen im Raman-Spektrum sehr gut der Bande bei $1661 \mathrm{~cm}^{-1}$ für die Amid I und relativ gut der Bande bei $1285 \mathrm{~cm}^{-1}$ oberhalb der Amid IIIBande des Monomers. Deren Blauverschiebung durch die zyklische Koordination wird von der Rechnung deutlich überschätzt. Hier sind aber zusätzliche Einflüsse durch höhere Cluster und eventuell sogar das Dimer nicht auszuschließen, weswegen es in diesem Spektralbereich auch 
für das Trimer eine Zuordnung unter Vorbehalt bleibt.

Ein Vergleich mit den Literaturwerten in Tab. 4-14 ist nur bedingt möglich, da es keine Literaturdaten zu Raman-Spektroskopie von NMA-Clustern gibt und auf Grund der unterschiedlich vorhergesagten Wellenzahlen für IR und Raman kein direkter Vergleich mit IR-Bandenpositionen möglich ist. Aber ein Vergleich mit der Theorie zeigt, dass die Amid I-Bandenpositionen in der Matrix [195] von der Theorie um ca. $10 \mathrm{~cm}^{-1}$ unterschätzt werden und die IRMPD + VUVDaten sich für die Amid I mit einem Unterschied von $20 \mathrm{~cm}^{-1}$ deutlich von der theoretischen Vorhersage unterscheiden. Für die Amid III wird die dortige Bandenposition mit einer Verschiebung von $+50 \mathrm{~cm}^{-1}$ in Bezug auf das Monomersignal von der Theorie um ca. $10 \mathrm{~cm}^{-1}$ unterschätzt. Dies ist vergleichbar mit der Raman-Amid III-Bande in dieser Arbeit und daher plausibel, auch wenn dort eine offenkettige Trimerkonformation angenommen wird.

Tab. 4-14. Literaturvergleich experimenteller Werte der Amid A- $\left(\tilde{v}_{A}^{t t t}\right)$, Amid I- $\left(\tilde{v}_{\mid}^{t t t}\right)$ und Amid III-Banden $\left(\tilde{v}_{\mathrm{lII}}^{t t t}\right)$ des NMATrimers mitsamt relativer Verschiebung zur trans-Monomerbande in Klammern. Alle Angaben in $\mathrm{cm}^{-1}$.

\begin{tabular}{|c|c|c|c|}
\hline Methode & $\tilde{v}_{\mathrm{A}}^{t t t}$ & $\tilde{v}_{1}^{t t t}$ & $\tilde{v}_{\mathrm{lll}}^{t t t}$ \\
\hline Jet - Raman ${ }^{a}$ & $3386(-122)$ & $1661(-59)^{d}$ & $1285(+27)$ \\
\hline Jet - FTIR $^{\mathrm{a}}$ & $3395(-113)$ & - & - \\
\hline Jet - FTIR 51$]$ & $3365(-145) ?$ & - & - \\
\hline IRMPD + VUV & - & $1656(-51)$ & $1298(+50)$ \\
\hline $\mathrm{N}_{2}$-Matrix - FTIR 195. & - & $1663(-43)$ & - \\
\hline Ar-Matrix - FTIR & - & $1670(-38), 1664(-44)$ & - \\
\hline Lösung (in $\left.\mathrm{CCl}_{4}\right)-2 \mathrm{C}$-fsVS 210 & - & $1655(-35)$ & \\
\hline Lösung (in $\mathrm{H}_{2} \mathrm{O}+\mathrm{CCl}_{4}$ ) - FTIR 181 & $3470(-5), 3369(-106), 3354(-121)$ & - & \\
\hline
\end{tabular}

Auch wenn das Jet-Spektrum deutliche Belege für die zyklische Struktur liefert und der dispersionskorrigierte Energieunterschied zum linearen Trimer mit $17 \mathrm{~kJ} \cdot \mathrm{mol}^{-1} \mathrm{sehr}$ groß ist, ist die bisher in der Literatur angenommene lineare Trimerkonformation keineswegs abwegig. Da für eine offenkettige Struktur grundsätzlich eine größere Entropie anzunehmen ist, wird die freie Enthalpie von $t t_{1}$ unter die des zyklischen Trimers fallen. Dies wird laut der thermochemischen Analyse der harmonischen Frequenzrechnung mit Dispersionskorrektur bereits bei einer Temperatur von ca. $228 \mathrm{~K}$ geschehen, unter Normalbedingungen liegt der freie Enthalpieunterschied also bereits bei ca. $6 \mathrm{~kJ} \cdot \mathrm{mol}^{-1}$ (siehe Tab. 4-12). Dies ist konsistent mit Messungen des Dipolmoments in Lösung und als Flüssigkeit [180, 238], der ermittelten Kristallstruktur [48], als auch mit den Messungen von Köddermann und Ludwig [181], die für NMA in Lösung für die NH-Streckschwingung im Trimer drei Banden mit Verschiebungen von $-5,-106$ und $-121 \mathrm{~cm}^{-1}$ finden (siehe Tab. 4-14), was den zu erwartenden Signalen eines Clusters mit einer freien NH-Gruppe und zwei Wasserstoffbrückenbindungen mit zunehmender Kooperativität entspricht.

Wenn die Bindungsenergie der dritten Wasserstoffbrücke ausreicht, trotz der Ringspannung stabile zyklische Trimere zu bilden, dann ist aus thermodynamischer Sicht für alle weiteren isolierten Cluster von NMA eine zyklische Geometrie als stabilste Struktur zu erwarten, wenn entropische Effekte vernachlässigt werden. Bei den Expansionsbedingungen im Jet ist zumin- 
dest für den nächsthöheren Cluster ein zyklisches Tetramer zu erwarten. Demnach wäre auch hier durch die Ringspannung die Rotverschiebung reduziert. Während in den Amid A-Spektren in dem dargestellten Ausschnitt in Abb. 4-11 keine Hinweise auf ein Tetramer oder höhere Oligomere zu finden sind, enthält das Raman-Spektrum der Amid I-III-Region (Abb. 4-10) mehrere Signale, die sich auf höhere Cluster zurückführen lassen könnten. Zum einen liegt auf der niederfrequenten Seite der Amid I-Trimerbande bei $1661 \mathrm{~cm}^{-1}$ (markiert als ttt...) ein Peak bei $1640 \mathrm{~cm}^{-1}$, welcher mit absteigender Düsentemperatur deutlich verzögert hervortritt und möglicherweise auf ein Tetramer zurückzuführen ist. Dies wäre zumindest in Einklang mit der Frequenzrechnung auf B3LYP-D3(BJ)/aVTZ-Niveau (siehe Tab. A-16 im Tabellenwerk in Anhang A.7.2), die für die symmetrische $\mathrm{C}=\mathrm{O}$-Streckschwingung einer zyklischen Tetramerstruktur mit antiparalleler Monomeranordnung und $S_{4}$-Symmetrie $\left(t t t t_{\mathrm{a}}\right)$ eine skalierte harmonische Wellenzahl von $1642 \mathrm{~cm}^{-1}$ vorhersagt. In Anbetracht der typischen Überschätzung der Verschiebung durch B3LYP (siehe oben) kann sich diese genauso gut auf die als ttt... bezeichnete Bande beziehen. Die breite Bande bei $1360 \mathrm{~cm}^{-1}$ oberhalb der Amid III wäre ein zweites Signal, dessen möglicher Ursprung das $t t t t_{\mathrm{a}}$-Tetramer ist. Auch hier stützt sich die Argumentation auf das Bandenwachstum und die skalierten harmonischen Frequenzen, so dass diese Zuordnung unter Vorbehalt geschieht.

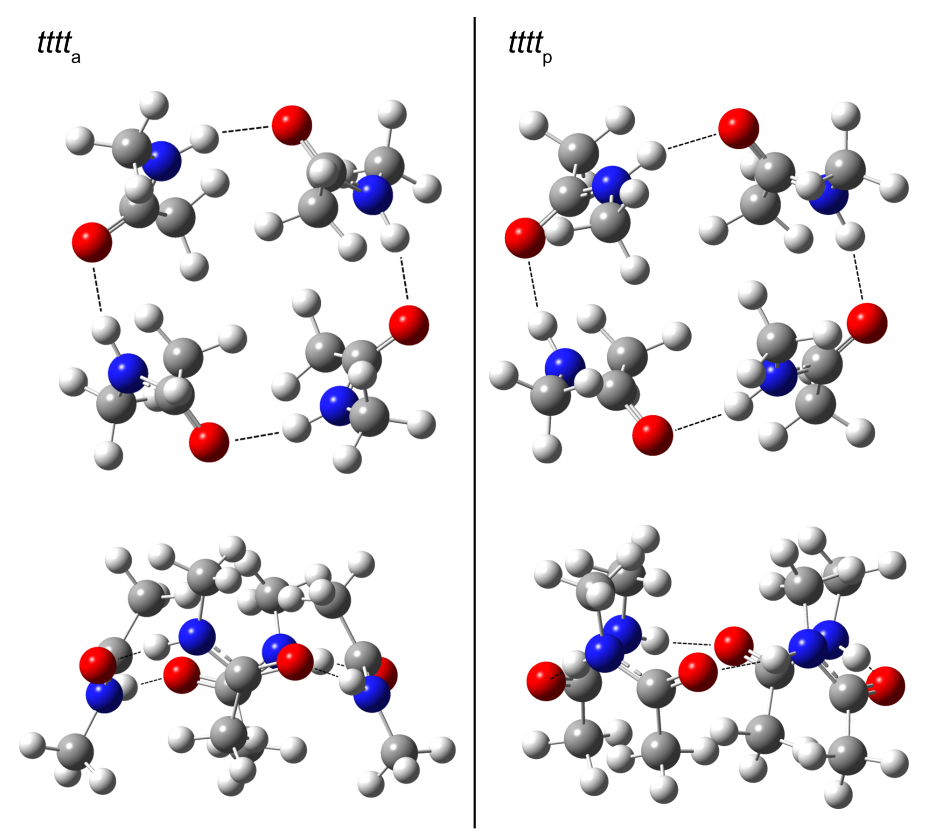

Abb. 4-17. Molekülgeometrien der beiden zyklischen trans-NMA Tetramere auf B3LYP-D3(BJ)/aVTZ-Niveau. Die Amidgruppen der Monomereinheiten sind dabei entweder gleich ausgerichtet (parallel, $t t t t_{p}$ ) und es liegt $C_{2}$-Symmetrie vor, oder die Monomerausrichtung wechselt sich ab und sie haben eine antiparallele Ausrichtung mit $S_{4}$-Symmetrie $\left(t t t t_{a}\right)$.

\subsection{Zusammenfassung und Diskussion}

In diesem Kapitel wird das sekundäre Amid N-Methylacetamid schwingungsspektroskopisch untersucht und die Messergebnisse aus FTIR- und Raman-Jet-Messungen mit quantenchemi- 
schen Rechnungen verglichen. Geometrieoptimierungen und doppelt-harmonische Frequenzrechnungen auf B3LYP-D3(BJ)/aVTZ-Niveau werden, sofern möglich, um anharmonische VPT2Rechnungen ergänzt. Für die untersuchten Spektralbereiche kann erstmals ein Raman-Spektrum im Jet aufgenommen werden, und es können unter Zuhilfenahme skalierter harmonischer Wellenzahlen die gemessenen Banden eindeutig den Amid-Banden von trans- und cisNMA zugeordnet werden.

Für das trans-Monomer ergeben sich aus den Rechnungen $t_{\mathrm{as}}$ und $t_{\mathrm{ss}}$ und für das cis-Monomer das $c_{\mathrm{aa}}$ als energetisch günstigste Konformere und die erstmalig beobachtete Substruktur der Amid A-Bande kann mit Hilfe einer VPT2-Rechnungen für $t_{\mathrm{ss}}$ plausibel auf heiße Banden zurückgeführt werden. Diese Zuordnung sollte aber mit Vorsicht behandelt werden, da die VPT2Methode sehr anfällig für niederfrequente Schwingungen wie die beiden Methyltorsionen im NMA ist [29, 214]. Auch wenn die vorliegende Rechnung keine imaginären anharmonischen Frequenzen aufweist, so geben die berechneten Bandenintensitäten und deren Verschiebung aufgrund von prognostizierten Fermi-Resonanzen Grund zum Zweifel an der Robustheit der Rechnung. Die Übereinstimmung der anharmonischen Kopplungskonstanten mit der experimentellen Verschiebung kann also auch nur durch Zufall so gut sein.

Aus den gut separierten Amid A- und Amid IV-Banden für cis- und trans-NMA kann aus einer Messreihe mit unterschiedlichen Düsentemperaturen der Enthalpieunterschied mittels Van-'tHoff-Plot auf 8(2) bzw. 11(7) kJ.mol ${ }^{-1}$ bestimmt werden. Dies entspricht einem cis-Anteil von ca. $3 \%$. Auch wenn diese Werte im Bereich der vorhandenen Literaturwerte liegen, so ist der Fehler sehr groß. Eine mögliche Ursache für eine fehlerhafte Bestimmung wäre das Vorliegen von Clustern, da deren Bildung das cis-/trans-Verhältnis im Monomer verschiebt. Dies kann hier aber als Fehlerquelle ausgeschlossen werden, da die Raman-Spektren der Temperaturreihen unter Bedingungen aufgenommen werden, bei denen keine signifikanten Signale an den Bandenpositionen der Dimere erkennbar sind und diese sich zuerst bilden würden. Daher ist der große Fehler vor allem auf die nur schwer zu integrierende schwache cis-Bande zurückzuführen. Es ist zudem fraglich, inwieweit die eingestellte Düsentemperatur der tatsächlichen Temperatur im Gasgemisch vor der Expansion entspricht und während der langen Messungen konstant gehalten wurde. Insgesamt kann gesagt werden, dass diese Methode keine exakten Energien liefert, andererseits wird die generelle Annahme des Einfrierens der Konformerenpopulation bestätigt.

Im Hauptteil des Kapitels werden die Aggregate von NMA bis hin zum Tetramer untersucht. Hierbei werden die beiden Clusterbanden im Amid A-Bereich, die bisher als Dimer und Trimer zugeordnet werden [51], erstmalig als Banden zweier isoenergetischer Dimer-Konformationen interpretiert. Dabei wurde eine Fermi-Resonanz durch ergänzende Messungen an deuteriertem NMA ausgeschlossen. Die FTIR-Messungen sind insgesamt in guter Übereinstimmung mit bisherigen Jet-Spektren (ebenda) und offenbaren fehlerhafte Bandenpositionen in der 1800$100 \mathrm{~cm}^{-1}$-Region in einer kurz zuvor veröffentlichten Studie mit „IR multiphoton dissociation“Technik (IRMPD+VUV) [194]. Zudem wurde für das Trimer erstmalig zyklische Aggregate als stabilste Strukturen theoretisch vorhergesagt und spektroskopisch durch die Auswertung komplementärer FTIR- und Raman-Jetspektren in der Amid A-Region bestätigt. Dabei wird gezeigt, 
dass sich die fassförmige Ringgeometrie nur unter Einbeziehung von Dispersionswechselwirkungen als stabilste Struktur ergibt. Dies erklärt das bisherige Übersehen zumindest in der älteren Literatur, als in den quantenchemischen Programmpaketen noch keine Methoden mit Dispersionskorrektur implementiert waren.

Die Amid I-III-Region im Raman erweist sich als zu komplex für eine eindeutige Clusterbandenzuordnung, während im FTIR lediglich die Dimer-Akzeptorbande der CO-Streckschwingung erkennbar ist. Für die im Raman unsichtbare Amid II-Bande treten im IR-Spektrum zwei Banden auf, die unter Vorbehalt einer Fermi-Resonanz der Amid II mit $\mathrm{CH}_{3}$-Gerüstschwingungen zuzuordnen sind. Für das Tetramer ergeben sich theoretisch ebenfalls zyklische Strukturen und spektroskopische Indizen auf der niederfrequenten Seite der Amid I-Monomerbande und blauverschoben zur $v_{\mathrm{III}}^{t}$ der Raman-Spektren.

Abschließend sei angemerkt, dass die Suche nach einem erstmaligen spektroskopischen Nachweis für das theoretisch stabilste $c c$-Dimer erfolglos geblieben ist, was vor allem an der geringen cis-Monomerkonzentration liegt. 


\section{$5 \mathrm{~N}$-Methylformamid}

$N$-Methylformamid (NMF) ist das kleinste sekundäre Amid und besitzt genauso wie NMA eine cis-/trans-Isomerie der C-N-Amidbindung [239]. Eine anfängliche Kontroverse über das stabilste Isomer [34, 73, 239, 241] wurde zugunsten des trans-Konformers entschieden [242] und später durch Messungen mittels Elektronenbeugung in der Gasphase [243] sowie Röntgen[244] und Neutronenbeugung [245] von flüssigem NMF bestätigt.

Der cis-Anteil bei Raumtemperatur wurde aus IR-Spektren in $\mathrm{CCl}_{4}$-Lösung zu $5 \%$ [171] und im Rahmen einer Dipolmessung in Dioxan zu 10\% [246] bestimmt. Außerdem ergaben eine Vielzahl von NMR-Studien in verschiedenen Lösungsmitteln einen cis-NMF-Anteil von 8-11\% [159, 162, 163, 172, 173, 247,-249].

In der Gasphase reichen die Angaben von $5 \%$ bei Raumtemperatur [249] bis hin zu 14\% bei $200{ }^{\circ} \mathrm{C}$ [250]. Es ist also ein deutlich höherer cis-Anteil im Raman-Spektrum zu erwarten als bei NMA. Damit ist NMF ein vielversprechender Kandidat zum Nachweis des cis-cis-Dimers als stabilstes Dimer sekundärer Alkylamide [51].

NMF fehlt gegenüber NMA die carbonylseitige Methylgruppe, was die möglichen stabilen Konformere eines planaren Moleküls auf vier reduziert: $t_{\mathrm{s}}, t_{\mathrm{a}}, c_{\mathrm{s}}$ und $c_{\mathrm{a}}$ 豆 Die Barrierenhöhe für die Rotation der Methylgruppe wurde für trans-NMF mittels Mikrowellenspektroskopie zu $0.65-0.7 \mathrm{~kJ} \cdot \mathrm{mol}^{-1}$ bestimmt [60, 251-253], was einer nahezu freien Drehbarkeit selbst unter Jet-Expansionsbedingungen entspricht. Die Barrierenhöhe liegt für cis-NMF mit $3.6 \mathrm{~kJ} \cdot \mathrm{mol}^{-1}$ [253] allerdings deutlich höher. Dies hat vor allem Auswirkungen auf eine mögliche Tunnelaufspaltung (siehe unten).

Analog zu NMA wurden die stabilsten Strukturen für cis- und trans-NMF mittels B3LYPD3(BJ)/aVTZ-Rechnung bestimmt. Die einzigen beiden Strukturen, die keine imaginären Frequenzen enthalten und somit keinen Sattelpunkt auf der Potentialhyperfläche darstellen, sind $t_{\mathrm{s}}$ und $c_{\mathrm{s}}$, also jeweils mit einem $\mathrm{C}-\mathrm{H}$ der Methylgruppe in syn-Stellung zur C-N-Amidbindung (siehe Abb. 5-1).

Da es auf dem verwendeten Rechenniveau nicht möglich ist die Stellung der Wasserstoffatome einer Methylgruppe mit einer so niedrigen Rotationsbarriere eindeutig zu erfassen [170] und die aus der Methyltorsion resultierenden Konformere höchstens als torsionsangeregte Zustände unterscheidbar sein werden, wird im Folgenden auf eine nähere Bezeichung der Methylgruppenstellung verzichtet. Demnach dienen die in Abbildung 5-1 dargestellten Molekülstrukturen als Grundlage für alle Frequenzrechnungen und Geometrieoptimierungen von möglichen NMF-Aggregaten (siehe Abschnitt5.3).

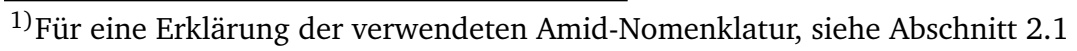




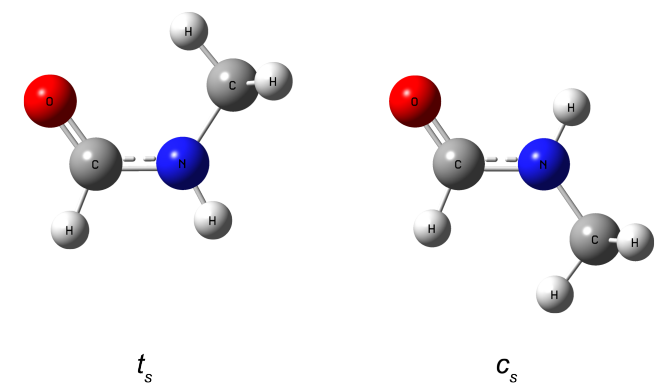

Abb. 5-1. Minimumstrukturen von cis- und trans-NMF nach Geometrieoptimierung auf B3LYP-D3(BJ)/aVTZ-Niveau.

\subsection{Schwingungsspektroskopie des Monomers}

Auch wenn NMF bisher nicht annähernd so umfassend untersucht wurde wie NMA, gibt es doch eine Reihe von Arbeiten zur Schwingungsspektroskopie dieses Moleküls. Diese setzen sich, neben klassischen IR-Studien in der Lösung [171, 178, 240], in der Gasphase [73, 239], in der Stickstoff- [68], Argon- [249, 254, 255] und Neon-Matrix [254] auch aus FTIR- und RamanJetstudien aus der Suhm-Gruppe [51, 112, 175, 256] zusammen. Dabei liegt der Fokus auf unterschiedlichen Aspekten, denn während Albrecht et al. [51] die Clusterbildung von transNMF an der Amid A- und Amid I-Bande mittels FTIR-Spektroskopie untersuchten und dies durch die Arbeit von Lee [256] um ein mögliches cc-NMF-Signal in der Amid A-Region ergänzt wurde, liegt der Schwerpunkt der Arbeit von Otto [112] auf der Bestimmung des cis-transEnergieunterschieds aus dem Raman-Jet-Spektrum der Amid A-Bande und einer Diskussion des $c c$-Dimers im Raman-Spektrum.

Amid A Abbildung 5-2 zeigt im oberen Teil das Raman-Jet-Spektrum der Amid A-Region von NMF. Die bei unterschiedlichen Düsentemperaturen aufgenommenen Spektren zeigen einen dominanten Doppelpeak bei 3501 und $3498 \mathrm{~cm}^{-1}$ und eine schwächere Bande bei $3459 \mathrm{~cm}^{-1}$, an deren niederfrequenter Seite mit zunehmender Temperatur ein Signal bei $3456 \mathrm{~cm}^{-1}$ erkennbar wird.

Der Vergleich mit den Literaturwerten in Tabelle 5-1 ermöglicht direkt eine Zuordnung der Peaks bei 3501 und $3459 \mathrm{~cm}^{-1}$ zu trans-NMF bzw. cis-NMF. Der Wert für trans-NMF stimmt im Rahmen der spektralen Auflösung mit den FTIR-Jetdaten von Albrecht et al. [51] und Lee [256], den Raman-Spektren von Otto [112] sowie den Werten aus der Neon-Matrix [254] überein und ist in guter Übereinstimmung mit den weiteren Matrixwerten, wenn typische MatrixVerschiebungen (siehe Abschnitt 4.1 berücksichtigt werden. Ähnliches gilt für cis-NMF, wo die experimentellen Literaturwerte der Jet- und Matrix-Studien maximal um $6 \mathrm{~cm}^{-1}$ nach unten hin abweichen.

Die angegebenen Literaturwerte für IR-Messungen in der Gasphase weichen dagegen deutlich von den hier gemessenen Werten ab. Dies lässt sich durch thermische Verschiebung gepaart mit einer deutlich schlechteren spektralen Auflösung erklären [51]. Die Werte für flüssiges NMF und NMF in Lösung zeigen die typische Rotverschiebung aufgrund der intermolekularen Wechselwirkungen [226] und die Messung von Suzuki [241] zusätzlich eine Bande bei $3070 \mathrm{~cm}^{-1}$, 

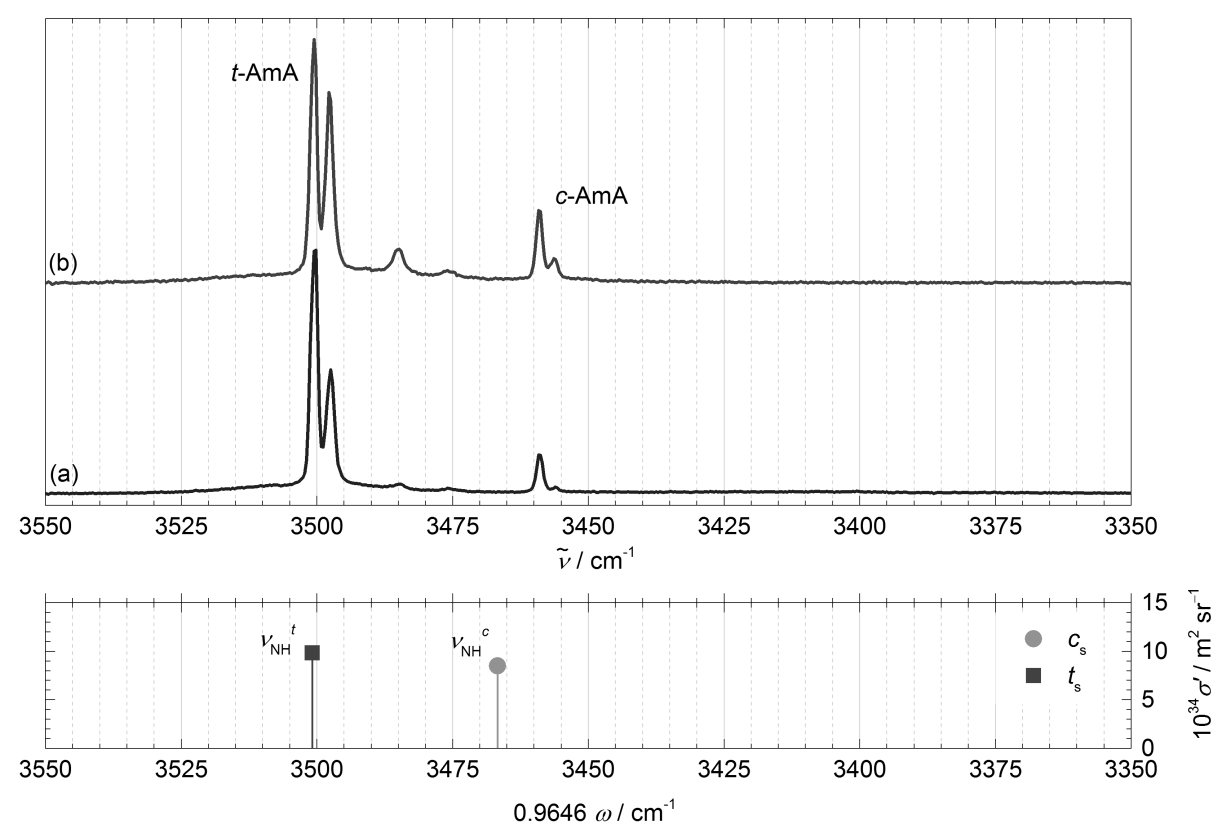

Abb. 5-2. curry-Jet-Spektren vom NMF-Monomer im NH-Streckbereich bei Düsentemperaturen von $100^{\circ} \mathrm{C}$ (a) und $190^{\circ} \mathrm{C}(\mathrm{b})$, normiert auf die Fundamentale der trans-Amid A-Bande $(t-A m A)$ bei $3501 \mathrm{~cm}^{-1}$. Darunter Strichspektren des jeweils stabilsten trans- (Rechteck) und cis-Konformers (rund) auf B3LYP-D3(BJ)/aVTZ-Niveau Die Wellenzahlskalierung mit dem Faktor 0.9646 bezieht die theoretisch vorhergesagte harmonische Wellenzahl der $\mathrm{N}-\mathrm{H}$-Streckschwingung von trans-NMF $\left(v_{\mathrm{NH}}^{t}\right)$ auf den experimentellen Wert von $t$-AmA.

die sich gut als Amid B-Bande deuten lässt (siehe Abschnitt 2.2).

Die Zuordnung wird auch durch quantenchemische Rechnungen unterstützt. Dabei zeigt die doppelt-harmonische Frequenzrechnung auf B3LYP-D3(BJ)/aVTZ-Niveau bei einer Skalierung auf die trans-Monomerbande, dass die Wellenzahl der cis-Bande um $9 \mathrm{~cm}^{-1} \mathrm{zu}$ hoch abgeschätzt wurde. Für diese, wie auch weitere B3LYP-Rechnungen (siehe Tab. 5-2), liegt der unskalierte harmonische Wert für die trans-Amid A-Banden zwischen 124 und $130 \mathrm{~cm}^{-1}$ über dem Messwert von $3501 \mathrm{~cm}^{-1}$, während die harmonische Wellenzahldifferenz zwischen beiden Isomeren mit $-35 \mathrm{~cm}^{-1}$ stabil ist. Im Vergleich dazu liegt der Unterschied zwischen harmonischer Theorie und experimentellem Wert mit - (180-194) $\mathrm{cm}^{-1}$ für die MP2-Rechnungen deutlich höher. Wie schon bei NMA weist die harmonische Rechnung mit dem PW91 ${ }_{\mathrm{XC}}$-Funktional [188] nahezu keine Verschiebung zum Experiment auf. Die einzig vorliegende anharmonische Rechnung zu beiden NMF-Konformeren ist eine VPT2-Rechnung auf der Grundlage einer dispersionskorrigierten B3LYP-Rechnung mit cc-pVTZ-Basissatz. Die Anharmonizität beträgt $-166 \mathrm{~cm}^{-1}$ für trans-NMF und $-167 \mathrm{~cm}^{-1}$ für das cis-Konformer.

Alle Rechnungen verorten die höchstfrequente Normalmode zu $100 \% 22$ in der lokalen N-HStreckschwingung, so dass dieser aus der Theorie stammende Begriff synonym mit der experimentellen Bezeichnung „Amid A“ ist und im Folgenden gleichermaßen verwendet wird.

Die zwei schwächeren Nebenpeaks auf der jeweils niederfrequenten Seite der beiden Amid AFundamentalbanden bei $3498 \mathrm{~cm}^{-1}$ und $3456 \mathrm{~cm}^{-1}$ nehmen mit steigender Düsentemperatur im Vergleich zur jeweiligen Fundamentalen zu. Da aufgrund der Messbedingungen und im Ver-

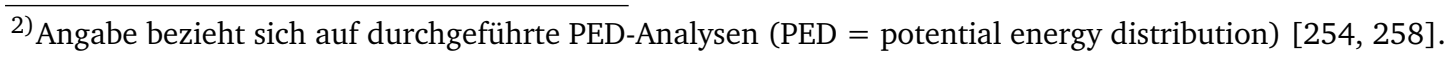


Tab. 5-1. Vergleich experimenteller Werte der Amid A-Bande von trans- $\left(\tilde{v}_{\mathrm{A}}^{t}\right)$ und cis-NMF $\left(\tilde{v}_{\mathrm{A}}^{c}\right)$ und weiterer Banden oberhalb von $3050 \mathrm{~cm}^{-1}$ (Alle Angaben in $\mathrm{cm}^{-1}$ ).

\begin{tabular}{|c|c|c|c|}
\hline Methode & $\tilde{v}_{\mathrm{A}}^{t}$ & $\tilde{v}_{\mathrm{A}}^{c}$ & weitere Banden \\
\hline \multicolumn{4}{|l|}{ Jet-Expansion: } \\
\hline Raman $^{\mathrm{a}}$ & 3501,3498 & 3459,3456 & 3485 \\
\hline Raman 112 & 3501,3498 & 3458 & - \\
\hline FTIR 51256 & 3501 & 3452 & - \\
\hline \multicolumn{4}{|l|}{ Matrix: } \\
\hline $\mathrm{N}_{2}-\mathrm{IR} 68$ & 3490 & 3452 & - \\
\hline $\mathrm{Ar}-\mathrm{IR} 249$ & 3493 & 3456 & - \\
\hline $\mathrm{Ar}-\mathrm{FTIR} 255$ & 3494 & 3456 & - \\
\hline $\mathrm{Ar}-\mathrm{FTIR} 254$ & 3494 & 3456 & - \\
\hline $\mathrm{Ne}-\mathrm{FTIR} 254$ & 3502,3501 & 3452 & - \\
\hline \multicolumn{4}{|l|}{ Gasphase: } \\
\hline IR 239] & 3480 & - & $3052^{c}$ \\
\hline $\operatorname{IR} 73$ & $3504,3494,3482,3471$ & - & - \\
\hline \multicolumn{4}{|l|}{ Lösung: } \\
\hline $\mathrm{CCl}_{4}-\mathrm{IR} 171$ & 3466 & 3429 & - \\
\hline $\mathrm{CCl}_{4}-\mathrm{IR}^{\mathrm{b}} 2 \mathrm{240}$ & 3468 & 3428 & $3389^{d}$ \\
\hline $\mathrm{CCl}_{4}-\mathrm{IR} 178$ & 3460 & 一 & 一 \\
\hline \multicolumn{4}{|l|}{ Flüssigkeit: } \\
\hline IR 257. & 3301 & - & - \\
\hline IR 241 & 3300 & - & 3070 (Amid B) \\
\hline Raman 258. & 3295 & - & - \\
\hline
\end{tabular}

a: aus dieser Arbeit; ${ }^{b}: 0.005 \mathrm{M}^{c}$ : Kombinationsbande, siehe $[71] ;{ }^{\mathrm{d}}:$ zugeordnet als Clusterbande + CO-Streck-Oberton.

Tab. 5-2. Harmonische Wellenzahlen der $\mathrm{N}-\mathrm{H}$-Streckschwingung $v_{\mathrm{NH}}$ von trans- und cis-NMF; berechnet mit unterschiedlichen theoretischen Methoden. Die den Berechnungen zugrundeliegenden Konformationen (Konf.) sind angegeben, soweit sie aus den jeweiligen Veröffentlichungen hervorgehen. Alle Angaben in $\mathrm{cm}^{-1}$.

\begin{tabular}{|c|c|c|c|c|c|}
\hline Methode & $\tilde{v}_{\mathrm{NH}}^{t}$ & $\tilde{v}_{\mathrm{NH}}^{c}$ & $\Delta_{c-t}$ & weitere Banden & Konf. \\
\hline \multicolumn{6}{|l|}{ doppelt-harmonisch: } \\
\hline $\mathrm{HF} / 6-31+\mathrm{G}^{*}[258]$ & 3895 & - & - & - & $t_{\mathrm{a}}$ \\
\hline $\mathrm{B} 3 \mathrm{LYP} / 6-311+\mathrm{G}^{*} 51$ & 3625 & - & - & - & $t_{\mathrm{s}} / c_{\mathrm{s}}$ \\
\hline B3LYP/VTZ 255 & 3631 & 3596 & -35 & - & $t_{\mathrm{s}} / c_{\mathrm{s}}$ \\
\hline B3LYP-D3(BJ)/aVTZa & 3629 & 3594 & -35 & - & $t_{\mathrm{s}} / c_{\mathrm{s}}$ \\
\hline$P W 91 \times C / 6-31+G * b$ & 3505 & - & - & - & $t_{\mathrm{s}} / c_{\mathrm{s}}$ \\
\hline MP2/aVTZ 259] & 3681 & 3631 & -50 & - & $t_{\mathrm{a}} / c_{\mathrm{s}}$ \\
\hline CASPT2(6-5)/VTZ 259]. & 3696 & 3656 & -40 & - & $t_{\mathrm{s}} / c_{\mathrm{s}}$ \\
\hline \multicolumn{6}{|l|}{ anharmonisch: ${ }^{\mathrm{c}}$} \\
\hline B3LYP-D3(BJ)/VTZ + VPT2 ${ }^{\mathrm{a}}$ & 3468 [3634] & 3432 [3599] & $-36[-35]$ & $3486\left(2 v_{\mathrm{CO}}^{t}\right)$ & $t_{\mathrm{s}} / c_{\mathrm{s}}$ \\
\hline MP2/6-311++G(2d,2p) ${ }^{b}+$ VPT2 254 & 3550 [3695] & 3483 [3650] & $-67[-45]$ & - & $t_{\mathrm{a}} /-$ \\
\hline \multicolumn{6}{|l|}{ experimentell: d } \\
\hline Jet - Raman ${ }^{\mathrm{a}}$ & 3501 & 3459 & -43 & 3485 & $-1-$ \\
\hline
\end{tabular}

a: aus dieser Arbeit; ${ }^{\text {b }}$ : Counterpoise-Korrektur (CP) [der harmonischen Rechnung] [150]; ${ }^{\mathrm{c}}$ : harmonische Werte in eckigen Klammern; d: siehe auch Tab. 5-1 
gleich mit den Ergebnissen zu NMF-Aggregaten und entsprechenden Literaturwerten (siehe Abschnitt 5.3) Cluster als Ursache ausgeschlossen werden können, deutet dies auf heiße Banden hin, wie sie schon bei NMA diskutiert wurden (siehe Abschnitt4.1). Diese Schlussfolgerung für die $t$-Amid A-Bande wird ebenfalls von K. Otto [112] gemacht und das um $-4 \mathrm{~cm}^{-1}$ gegenüber der Fundamentalen verschobene Signal wird als heiße Bande der Methyltorsionsschwingung interpretiert.

Tabelle 5-3 stellt der experimentellen Verschiebung die Kopplungskonstanten einer B3LYPD3(BJ)/VTZ + VPT2-Rechnung gegenüber. VPT2 zeigt bekanntermaßen Schwächen bei der Erfassung von Methyltorsionen [29, 214]. Dennoch ergeben sich für beide Isomere des NMFMonomers VPT2-Rechnungen ohne imaginäre anharmonische Frequenzen. Die resultierenden anharmonischen Konstanten der NH-Streckschwingung sind in Tabelle 5-3 zu finden. Das niedrigste Schwingungsniveau entspricht der Methyltorsion und müsste nach Boltzmann das am stärksten besetzte sein. Die berechnete Kopplungskonstante von $+1.4 \mathrm{~cm}^{-1}$ würde aber eine Blauverschiebung bedeuten, was sich nicht mit der Beobachtung im Spektrum deckt. Auch die bisher noch nicht diskutierte schwache Bande bei $3485 \mathrm{~cm}^{-1}$ wäre generell als heiße Bande erklärbar, allerdings fehlt hier ebenfalls eine passende Kopplungskonstante in der VPT2Rechnung. Ähnliches findet sich für cis-NMF, wo der berechneten Verschiebung von $-3.8 \mathrm{~cm}^{-1}$ für eine Kopplung der NH-Streck mit der niedrigsten Mode einer experimentelle Differenz von $-2 \mathrm{~cm}^{-1}$ gegenübersteht. Die anharmonische Rechnung ergibt also für die Struktur der Amid ABande von trans- und cis-NMF keine zufriedenstellende Erklärung, was auf die bereits erwähnten Probleme dieser Methode mit den Methyltorsionsschwingungen zurückzuführen ist.

Dies kann allerdings eher als Beleg dafür gewertet werden, dass die VPT2-Methode trotz fehlender imaginärer Frequenzen an der Methyltorsion scheitert, als dass es die Zuordnung als heiße Bande widerlegt.

Wie bereits für NMA diskutiert, kann auch bei NMF durch die geringe Tiefe des Potentials der Methyltorsion eine Tunnelaufspaltung hervorgerufen werden, die zu den Energieniveaus A und dem zweifach entarteten E führt. Diese wurden in einer Mikrowellenstudie genauer untersucht und deren Energieunterschied wird auf ca. $3 \mathrm{~cm}^{-1}$ abgeschätzt [60].

Tab. 5-3. Hypothetische experimentelle und theoretische anharmonische Konstanten $x_{i, j}^{\exp }$ und $x_{i, j}^{\mathrm{VPT} 2}$ für die Amid ABande von trans- und cis-NMF aus dem Jet-Ramanspektrum in Abb. 5-2 und ausgewählten niederfrequenten Normalmoden $j$ basierend auf dem Vergleich mit VPT2-Rechnungen des $t_{\mathrm{s}}$-Konformers (B3LYP-D3(BJ)/VTZ). Zusätzlich sind die berechneten harmonischen und anharmonischen Wellenzahlen $\tilde{v}_{j}^{\text {harm }}$ bzw. $\tilde{v}_{j}^{\mathrm{VPT} 2}$ der niederfrequenten Normalmode $j$ angegeben.

\begin{tabular}{|c|c|c|c|c|c|}
\hline$i$ & $j$ & $\tilde{v}_{j}^{\text {harm }} / \mathrm{cm}^{-1}$ & $\tilde{v}_{j}^{\mathrm{VPT} 2} / \mathrm{cm}^{-1}$ & $x_{i, j}^{\mathrm{VPT} 2} / \mathrm{cm}^{-1}$ & $x_{i, j}^{\exp } / \mathrm{cm}^{-1}$ \\
\hline \multicolumn{6}{|c|}{ trans-NMF: } \\
\hline 1 & 21 & 67 & 34 & 1.4 & -3 \\
\hline 1 & $2 \times 21$ & 134 & 39 & 2.8 & \\
\hline 1 & 20 & 268 & 290 & -10.9 & -16 \\
\hline 1 & 19 & 537 & 513 & -10.3 & -16 \\
\hline \multicolumn{6}{|c|}{ cis-NMF: } \\
\hline 1 & 21 & 106 & 119 & -3.8 & -3 \\
\hline
\end{tabular}


Amid I-III Die in Abbildung 5-3 dargestellten Raman-Jetspektren in der Region zwischen 1800 und $1100 \mathrm{~cm}^{-1}$ umfassen die Amidbanden I-III und bei $1550-1350 \mathrm{~cm}^{-1}$ die CH-Deformationsschwingungen als breite Bande mit drei prominenten Peaks bei 1515, 1448 und $1386 \mathrm{~cm}^{-1}$.

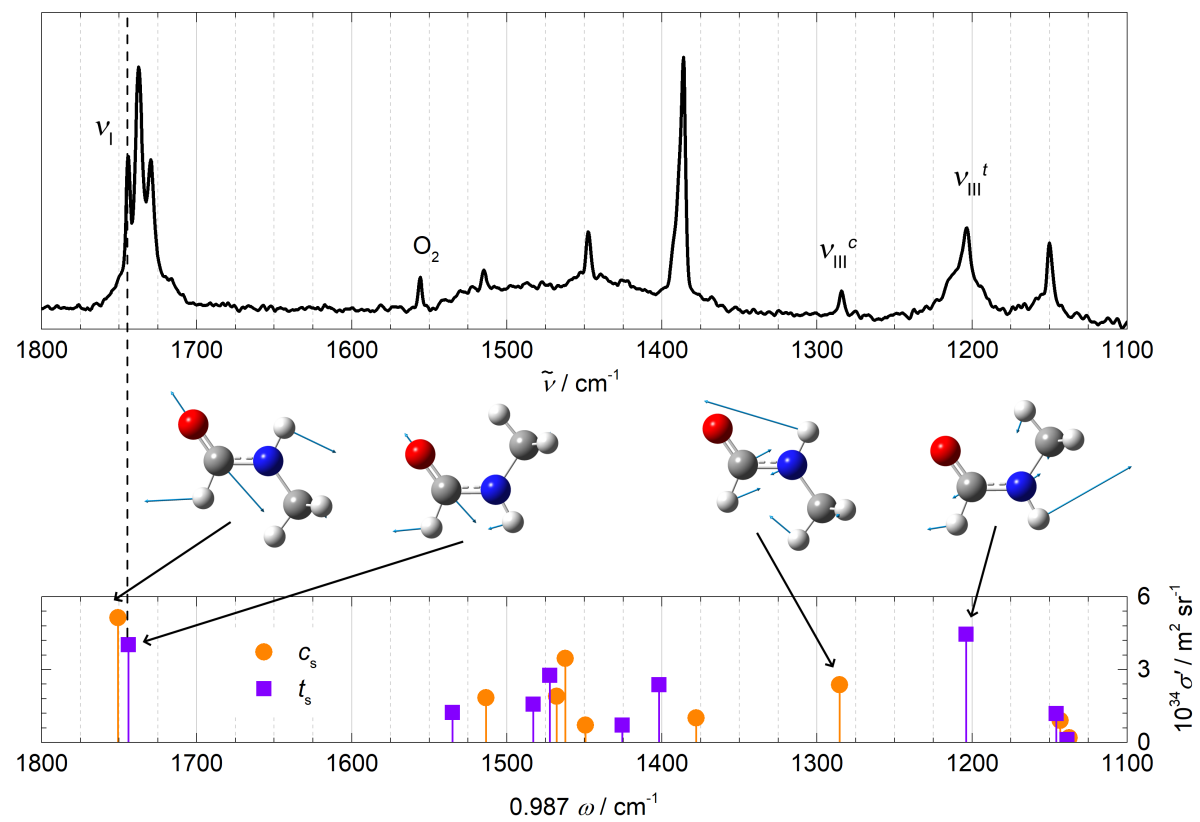

Abb. 5-3. curry-Jet-Spektrum des NMF-Monomers im Amid I-III-Bereich bei einer Düsentemperatur von $100^{\circ} \mathrm{C}$. Darunter Strichspektren des jeweils stabilsten trans- (Rechteck, violett) und cis-Konformers (rund, orange) auf B3LYPD3(BJ)/aVTZ-Niveau und an den jeweiligen Molekülstrukturen illustrierten Normalmoden. Die Wellenzahlskalierung bezieht die theoretische Linienposition der CO-Streckschwingung des trans-Amids auf das Signal bei $1744 \mathrm{~cm}^{-1}$.

Die Amid I-Bande weist eine Substruktur aus drei Peaks bei 1744, 1738 und $1729 \mathrm{~cm}^{-1}$ auf, die ihren Ursprung in heißen Übergängen haben kann, wie sie bereits für die Amid A-Bande diskutiert wurden. In der Literatur stehen für eine Zuordnung nur IR-spektroskopische Daten zur Verfügung. Sowohl im Jet [51] als auch in der Argon- [255] und Neon-Matrix [254] findet sich keine vergleichbare Substruktur, jedoch eine breite Bande, deren Intensitätsmaximum eher auf der hochfrequenten Seite liegt. Die in Tabelle 5-4 aufgeführten Bandenpositionen deuten am ehesten auf den niederfrequentesten der drei Peaks bei $1729 \mathrm{~cm}^{-1}$ als Fundamentale der CO-Streckschwingung hin. Die Abweichung ist mit $-2 \mathrm{~cm}^{-1}$ bis $+4 \mathrm{~cm}^{-1} \mathrm{zu}$ den Matrixwerten allerdings in Anbetracht der typischen Verschiebungen aufgrund auftretender Matrixeffekte erstaunlich gering und bezogen auf die Neon-Matrix auch in die falsche Richtung.

Eine ergänzende filet-Jet-Messung in Abb. 5-4 zeigt deutliche Unterschiede in der Amid IBandenform des Monomers. So steht den drei gut separierten Peaks im Raman-Spektrum eine breite unsymmetrische Bande im IR gegenüber. Die IR-Bande weist aber drei „Dips“ auf, die sich genau den genannten Raman-Peaks zuordnen lassen. Die fehlende Struktur in den IRSpektren ist also nicht nur einer schlechteren spektralen Auflösung geschuldet, sondern es ist im IR-Spektrum der CO-Streckschwingung offenbar kein Q-Zweig vorhanden oder nur schwach ausgeprägt. Um die Zuordnung der drei Raman-Signale zu klären, wurden Raman-Jet-Spektren bei verschiedenen Düsentemperaturen aufgenommen, um mögliche heiße Banden von der Fundamentalen zu unterscheiden. Die Spektren in Abbildung 5-4 (unten) sind auf den Peak 


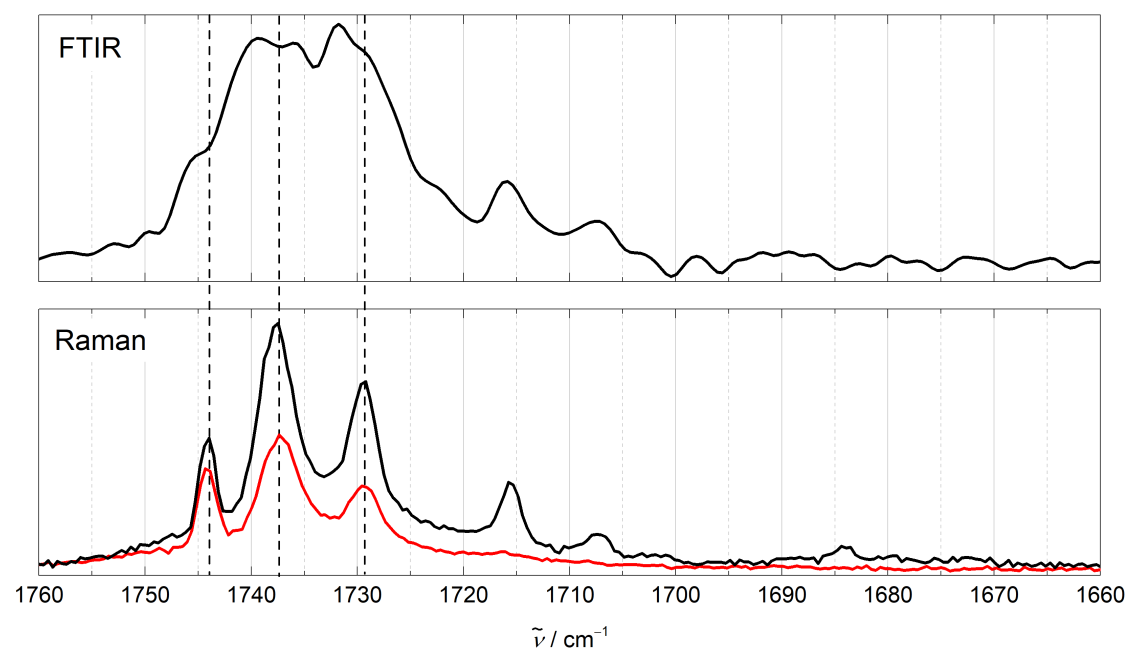

Abb. 5-4. Vergleich von FTIR- und Raman-Spektrum zur Zuordnung der CO-Streckfundamentalen von trans-NMF. Oben: FTIR-Spektrum des filet-Jets. Unten: curry-Jet-Spektren bei einer Düsentemperatur von $100^{\circ} \mathrm{C}$ (rot) und bei Raumtemperatur (schwarz). Die gestrichelten Linien illustrieren die Zuordnung der Raman-Peaks zu den IR-Dips. Details zu den Messungen der Raman-Spektren befinden sich im Raman-Spektrenverzeichnis in Tab. A-6 in Anhang A.6.1 und das FTIR-Spektrum entspricht dem Datensatz $L$ in Tab. A-8 in Anhang A.6.2 (Das Ramanspektrum bei Raumtemperatur wurde aufgenommen von Katharina A. E. Meyer.)

bei $1744 \mathrm{~cm}^{-1}$ skaliert und zeigen eine Zunahme der beiden niederfrequenteren Signale mit abnehmender Düsentemperatur, was der Interpretation als heiße Banden widerspricht. Gleichzeitig werden für das Spektrum bei Raumtemperatur (schwarz) auf der niederfrequenten Seite mögliche Clusterbanden sichtbar, so dass auch mit der Temperaturreihe eine eindeutige Interpretation der Amid I-Bande nicht möglich ist.

Eine weitere Möglichkeit der Klärung ist der Vergleich mit dem entsprechenden Raman-Spektrum von NMA (siehe Abb. 4-10). Doch auch hiermit ist eine klare Zuordnung nicht möglich, da die Amid I-Bande des strukturell sehr ähnlichen NMA anstatt einer vergleichbaren Substruktur ein einzelnes, schmales Signal aufweist, das auch auf der hochfrequenten Seite eines breiteren Sockels liegt. Damit handelt es sich jedoch eindeutig um den kalten Übergang. Weder die Literatur noch ein Vergleich mit NMA-Spektren oder eine Messreihe unter Variation der Düsentemperatur liefern eindeutige Hinweise zur Zuordnung der Amid I-Fundamentalen von transNMF. Aufgrund der Interpretation der Amid A-Bande (siehe oben) und der Beobachtung, dass in den Literaturspektren das Intensitätsmaximum auf der hochfrequenten Seite der Bande liegt, wird im Folgenden unter Vorbehalt die Fundamentale dem Signal bei $1744 \mathrm{~cm}^{-1}$ zugeordnet. Dies wird im Zusammenhang mit der Interpretation der Clusterbanden in Abschnitt 5.3 noch weiter analysiert. ${ }^{3)}$

In der Literatur findet sich neben den genannten Peaks noch ein Signal bei $1731 \mathrm{~cm}^{-1}$ in der Argon- und bei $1741 \mathrm{~cm}^{-1}$ in der Neon-Matrix. Diese werden in beiden Arbeiten als Amid IBande des cis-Konformers interpretiert. Unter Berücksichtigung des vorliegenden cis-/trans-

\footnotetext{
3) Als weiteres Indiz für diese Zuordnung wird in der abschließenden Diskussion dieser Arbeit substanzübergreifend die typische Überschätzung der experimentellen Bandenposition durch harmonische Frequenzrechnungen herangezogen (siehe Abschnitt 9.1).
} 
Konformerenverhältnisses (siehe Abschnitt 5.2) ist keine intensive cis-NMF-Bande in der JetExpansion zu erwarten und kann somit nicht einer der vorliegenden Banden zugeordnet werden.

Tab. 5-4. Vergleich experimenteller Werte $\tilde{v}_{\mid}, \tilde{v}_{\mid l}, \tilde{v}_{|| l}$ der Amid I-III-Banden von trans-NMF (cis-NMF in Klammern) und $\mathrm{CH}-$ Gerüstschwingungen zwischen 1800 und $1100 \mathrm{~cm}^{-1}$. Alle Angaben in $\mathrm{cm}^{-1}$.

\begin{tabular}{|c|c|c|c|c|}
\hline Methode & $\tilde{v}_{1}^{b}$ & $\tilde{v}_{\mid l} \mathrm{~b}$ & $\tilde{v}_{I I I}{ }^{b}$ & weitere Banden \\
\hline \multicolumn{5}{|l|}{ Jet-Expansion: } \\
\hline Raman $^{\mathrm{a}}$ & 1744 & - & $1203(1284)$ & $1738,1729,1515,1448,1386,1150$ \\
\hline FTIR $[51]$ & 1732 & - & - & - \\
\hline \multicolumn{5}{|l|}{ Matrix: } \\
\hline $\mathrm{N}_{2}-\mathrm{IR} 68$ & 1721 & 1528 & $1207(1302)$ & $1467,1458,1410,1392,1148$ \\
\hline $\mathrm{Ar}-\mathrm{FTIR} 249$ & $1725\left(1722^{\mathrm{C}}\right)$ & 1517 & $1205(1293)$ & $1464,1456,1409,1393,1146$ \\
\hline $\mathrm{Ar}-\mathrm{FTIR} 255$ & $1725(1731)$ & 1518 & $1206(1293)$ & $1465,1457,1409,1394,1147$ \\
\hline $\mathrm{Ne}-\mathrm{FTIR} 254$ & $1731(1741)$ & $1515^{\mathrm{c}}$ & $1205(1285)$ & $1473,1423,1412,1150$ \\
\hline \multicolumn{5}{|l|}{ Gasphase: } \\
\hline IR 62 250 & 1727 & 1490 & $1200(1280)$ & $1415,1375,1290,1268$ \\
\hline IR 239 & 1724 & 1498 & 1201 & $1411,1293,1273$ \\
\hline $\operatorname{IR} 73$ & 1733 & 1494 & 1201 & $1460,1409,1376,1291,1270$ \\
\hline \multicolumn{5}{|l|}{ Lösung: } \\
\hline $\mathrm{CCl}_{4}-\mathrm{IR} 178$ & 1698 & - & 1248 & - \\
\hline $\mathrm{H}_{2} \mathrm{O}-\mathrm{IR} 260$ & 1661 & - & - & - \\
\hline DMSO - IR 260] & 1679 & - & - & - \\
\hline \multicolumn{5}{|l|}{ Flüssigkeit: } \\
\hline IR [239] & 1658 & 1539 & 1244 & $1415,1383,1333$ \\
\hline Raman 258] & 1655 & 1546 & 1246 & $1454,1414,1385$ \\
\hline
\end{tabular}

Die Amid II-Bande ist typischerweise kaum Raman-aktiv, dennoch befindet sich die RamanJet-Bande bei $1515 \mathrm{~cm}^{-1}$ in guter Übereinstimmung mit den in Argon- und Neon-Matrixmessungen gefundenen Positionen der Amid II-Bande von trans-NMF (siehe Tab. 5-4).

Auf der niederfrequenten Seite des breiten Bereichs der $\mathrm{CH}$-Deformationsschwingungen befindet sich die Region der Amid III-Bande, in der im Raman-Jetspektrum in Abbildung 5-3 eine schwache Bande bei $1284 \mathrm{~cm}^{-1}$ sowie zwei mittelstarke bei $1203 \mathrm{~cm}^{-1}$ und $1150 \mathrm{~cm}^{-1} \mathrm{zu}$ finden sind. Die beiden Matrixarbeiten von Crespo-Otero et al. [255] und Sałdyka et al. [254], die für alle weiteren Banden bisher sehr gute Übereinstimmung lieferten, ergeben als Zuordnung die Amid III-Bande von trans-NMF bei $1203 \mathrm{~cm}^{-1}$ und die entsprechende cis-Bande bei $1284 \mathrm{~cm}^{-1}$, während der Peak bei $1150 \mathrm{~cm}^{-1}$ einer weiteren CH-Deformationsschwingung zuzuordnen ist.

Auch hier wurden harmonische und anharmonische Frequenzrechnungen durchgeführt, die in Tabelle 5-5 gemeinsam mit gerechneten Frequenzen aus der Literatur aufgeführt sind. Die auf die $v_{\mathrm{I}}^{t}$ skalierten, harmonischen Frequenzen der Standard-B3LYP-Rechnung mit Dispersionskorrektur sind zusätzlich auch noch als Strichspektrum in Abbildung 5-3 den Ergebnissen der Raman-Jet-Messungen gegenübergestellt. Die auf Grund der experimentellen Literaturwerte getroffene Zuordnung deckt sich mit den quantenchemischen Vorhersagen der DFT-Rechnung für die Amid I- und Amid III-Banden. Diese bestätigt die Beobachtung in der Argon- und Neon-Matrix, dass die cis-Amid I- um $6-10 \mathrm{~cm}^{-1}$ oberhalb der trans-Bande liegt. Im vorlie- 
genden Raman-Spektrum ist, trotz eines um den Faktor 1.3 größeren Streuquerschnitts (siehe Tab. A-21) gegenüber der CO-Streckschwingung von trans-NMF, kein Signal oberhalb der transNMF-Fundamentalen bei $1744 \mathrm{~cm}^{-1}$ erkennbar, welches cis-NMF zugeordnet werden könnte.

Anders sieht es bei der Amid III-Bande des cis-Konformers aus, deren berechnete Bandenposition bei $1275 \mathrm{~cm}^{-1}$ (harmonisch, skaliert; siehe Tab. A-23) sich in guter Übereinstimmung mit dem Wert von $1284 \mathrm{~cm}^{-1}$ im Raman-Jet befindet, sodass hier die experimentelle Zuordnung von theoretischer Seite als bestätigt angesehen werden kann.

Tab. 5-5. Harmonische und anharmonische Wellenzahlen $\tilde{v}_{\mid}, \tilde{v}_{\| \mid}, \tilde{v}_{\| \mid}$der Amid I-III Normalmoden $\left(v_{\mid}, v_{||}\right.$und $\left.v_{\mid I I}\right)$ von trans-NMF und cis-NMF (Werte in Klammern), berechnet mit unterschiedlichen Methoden. Die den Berechnungen zugrunde liegenden Konformationen (Konf.) sind angegeben, soweit sie aus den jeweiligen Veröffentlichungen hervorgehen. Alle Angaben in $\mathrm{cm}^{-1}$.

\begin{tabular}{|c|c|c|c|c|}
\hline Methode / Basissatz & $v_{1}{ }^{\mathrm{b}}$ & $v_{\| I}{ }^{b}$ & $v_{\mid I I}{ }^{b}$ & Konf. \\
\hline $\mathrm{HF} / 6-31+\mathrm{G}^{*} 258$ & 1945 & 1693 & 1346 & $t_{\mathrm{a}}$ \\
\hline $\mathrm{B} 3 \mathrm{LYP} / 6-311+\mathrm{G}^{\star}[51$ & 1777 & & & \\
\hline B3LYP/VTZ 255] & $1783(1791)$ & $1557(1535)$ & $1219(1302)$ & $t_{\mathrm{s}} / c_{\mathrm{s}}$ \\
\hline B3LYP-D3(BJ)/VTZ ${ }^{\mathrm{a}}$ & $1783(1793)$ & $1557(1535)$ & $1220(1303)$ & $t_{\mathrm{s}} / c_{\mathrm{s}}$ \\
\hline B3LYP-D3(BJ)/VTZ + VPT2 ${ }^{a}$ & $1752(1759)$ & $1515(1493)$ & $1188(1279)$ & $t_{\mathrm{s}} / c_{\mathrm{s}}$ \\
\hline B3LYP-D3(BJ)/aVTZ ${ }^{\mathrm{a}}$ & $1767(1774)$ & $1555(1533)$ & $1220(1302)$ & $t_{\mathrm{s}} / c_{\mathrm{s}}$ \\
\hline$P W 91 \times C / 6-31+G * C$ & 1742 & 1505 & 1279 & $t_{\mathrm{s}} / c_{\mathrm{s}}$ \\
\hline$M P 2 / 6-311++G(2 d, 2 p)^{c}[254$ & $1748(1763)$ & $1560(1490)$ & $1242(1314)$ & $t_{\mathrm{a}} /-$ \\
\hline MP2/6-311++G(2d,2p) ${ }^{c}+$ VPT2 254 & $1707(1725)$ & $1487(1452)$ & $1217(1286)$ & $t_{\mathrm{a}} /-$ \\
\hline MP2/aVTZ [259] & $1757(1771)$ & $1551(1483)$ & $1243(1312)$ & $t_{\mathrm{a}} / c_{\mathrm{s}}$ \\
\hline CASPT2(6-5)/VTZ 259. & $1780(1791)$ & 1573 (1495) & $1243(1319)$ & $t_{\mathrm{s}} / c_{\mathrm{s}}$ \\
\hline \multicolumn{5}{|l|}{ experimentell: } \\
\hline Jet - Raman ${ }^{a}$ & 1744 & $1515^{d}$ & 1203 (1284) & $-1-$ \\
\hline
\end{tabular}

Die vorliegenden anharmonischen VPT2-Rechnungen versagen für die CO-Streck-Fundamentale, wobei mit B3LYP die experimentelle Bandenposition über- und mit MP2 unterschätzt wird (siehe Tab. 5-5). Werden allerdings die jeweiligen Wellenzahlen von harmonischer und anharmonischer Rechnung betrachtet, so ergibt sich für B3LYP + VPT2 eine Differenz von $-31 \mathrm{~cm}^{-1}$, während für MP2 + VPT2 eine Differenz von $-41 \mathrm{~cm}^{-1}$ vorliegt. Hier besteht also ein Unterschied in der Anharmonizität von lediglich $10 \mathrm{~cm}^{-1}$ zwischen B3LYP und MP2, während sich die absoluten anharmonischen Wellenzahlen beider Methoden um $45 \mathrm{~cm}^{-1}$ unterscheiden.

Unter der Annahme, dass die Anharmonizität tatsächlich zwischen -31 und $-41 \mathrm{~cm}^{-1}$ liegt, wären alle Rechnungen mit harmonischen Frequenzen zwischen 1760 und $1770 \mathrm{~cm}^{-1}$ in sehr guter Übereinstimmung mit den experimentellen Werten der Raman-Jet-Messungen. Dies trifft auf die B3LYP-D3(BJ)/aVTZ-Rechnung zu. Ein Vergleich mit NMA, für das nur anharmonische Rechnungen auf B3LYP-D3(BJ)/VTZ-Niveau vorliegen, ergibt eine Wellenzahldifferenz von $-28 \mathrm{~cm}^{-1}$ und somit eine ähnliche Anharmonizität wie bei NMF.

Die VPT2-Anharmonizitäten für die Amid II-Bande von trans-NMF ergeben sich $\mathrm{zu}-42 \mathrm{~cm}^{-1}$ für B3LYP-D3(BJ)/VTZ und $-73 \mathrm{~cm}^{-1}$ für die MP2-Rechnung. Wenn die harmonischen Frequenzen aller B3LYP-Rechnungen und MP2-Rechnungen mit der VPT2-Anharmonizität der B3LYP-Rechnung korrigiert werden, dann finden sich gute bis sehr gute Übereinstimmungen mit dem experimentellen Wert von $1515 \mathrm{~cm}^{-1}$. Dies ist darauf zurückzuführen, dass der ver- 
wendete Basissatz in der MP2-VPT2-Rechnung zu klein ist. Daher ist in diesem Fall die Korrektur einer guten harmonischen Methode mit den VPT2-Anharmonizitäten der B3LYP-D3(BJ)/VTZRechnung die beste Wahl für anharmonische Korrekturen.

Die Normalmodenanalyse der harmonischen Frequenzrechnung liefert für die im Vergleich zu anderen Amiden (siehe z. B. Abb. 5-3) ungewohnt starke Bande bei $1386 \mathrm{~cm}^{-1}$ eine Erklärung. Sie entspricht der nahezu vollständig am Carbonyl-Wasserstoff lokalisierten CH-Knickschwingung, die daher so nur bei Formamiden vorkommen kann.

Weitere Amidbanden Der Vollständigkeit halber wurden auch Raman-Jet-Messungen im niederfrequenten Teil des Spektrums (unterhalb von $1000 \mathrm{~cm}^{-1}$ ) durchgeführt. In Abbildung 5-5 sind drei Ausschnitte dargestellt, wobei das rechte und mittlere Spektrum aus derselben Messung und gemeinsam mit dem linken Spektrum aus der gleichen Messreihe stammen (siehe Tab. A-6 in Anhang A.6.1 und deren Intensitäten also direkt vergleichbar sind. Der Doppelpeak bei 952 und $946 \mathrm{~cm}^{-1}$ im linken Teilspektrum kann eindeutig der Amid IV-Bande des trans-NMF zugeordnet werden. Dies ist konsistent mit IR-Messungen in diversen Matrizen, in der Gasphase und genauso mit Messungen in flüssigem NMF (siehe Tab. 5-6). Die Zuordnung als Amid-Bande ist aber noch relativ jung [19] und für Formamide nur eingeschränkt gültig (keine Unterscheidung in Amid IVa und IVb). Der niederfrequente Nebenpeak lässt sich analog zur Amid A-Bande als heiße Bande erklären. Die ebenfalls in der Abbildung zu sehende Gegenüberstellung harmonischer B3LYP-D3(BJ)/aVTZ-Frequenzen zeigt eine sehr gute Übereinstimmung mit der Messung. Auch wenn die Passgenauigkeit von harmonischer Theorie und Experiment an dieser Stelle sicherlich dem Zufall geschuldet ist, so lässt sich im niederfrequenten Bereich ein geringer Unterschied aller gerechneten Frequenzen von der jeweiligen experimentellen Bandenposition mit der typischerweise geringeren Anharmonizität niederfrequenter Gerüstmoden erklären.

Die schwache, aber scharfe Bande bei $606 \mathrm{~cm}^{-1}$ lässt sich auf den ersten Blick mithilfe der experimentellen Werte der IR-Messungen in der Argon-Matrix (und mit einiger Abweichung auch denen der $\mathrm{N}_{2}$-Matrix) als cis-Amid IV erklären. Die Theorie zeigt, dass die Zuordnung richtig ist, allerdings aus den falschen Gründen. Die B3LYP-Rechnung liefert, genauso wie die in der Literatur verfügbaren Frequenzrechnungen, zwei Normalmoden mit nahezu gleicher Wellenzahl um $600 \mathrm{~cm}^{-1}$, allerdings unterschiedlicher Symmetrie (siehe Tab. 5-8). Die in der Molekülebene stattfindenden Deformationsschwingungen ( $A^{\prime}$-Symmetrierasse) der Amid IV-Mode von cis-NMF sind nahezu ausschließlich Raman-aktiv, wohingegen die $A^{\prime \prime}$-symmetrische Torsionsschwingung fast ausschließlich IR-aktiv ist (siehe Tab. 5-7). Das Signal in den IR-Spektren der Literatur ist daher nicht die Amid IV-Bande, liegt aber an der ungefähr gleichen Position im Spektrum, weswegen sie in Tabelle 5-6 in Klammern aufgeführt ist. Die echte Amid IV-Bande von cis-NMF ist also nicht in den IR-Spektren der Literatur sichtbar und es liegt keine RamanMessung in Gasphase, Matrix oder Jet-Expansion vor, so dass die Zuordnung letztendlich einzig aufgrund der quantenchemischen Rechnung erfolgt.

Als letzte Bande findet sich im rechten Spektrum in Abbildung 5-5 eine breite und unsymmetrische Bande mit zwei identifizierbaren Peaks bei 289 und $276 \mathrm{~cm}^{-1}$. In der experimentellen 

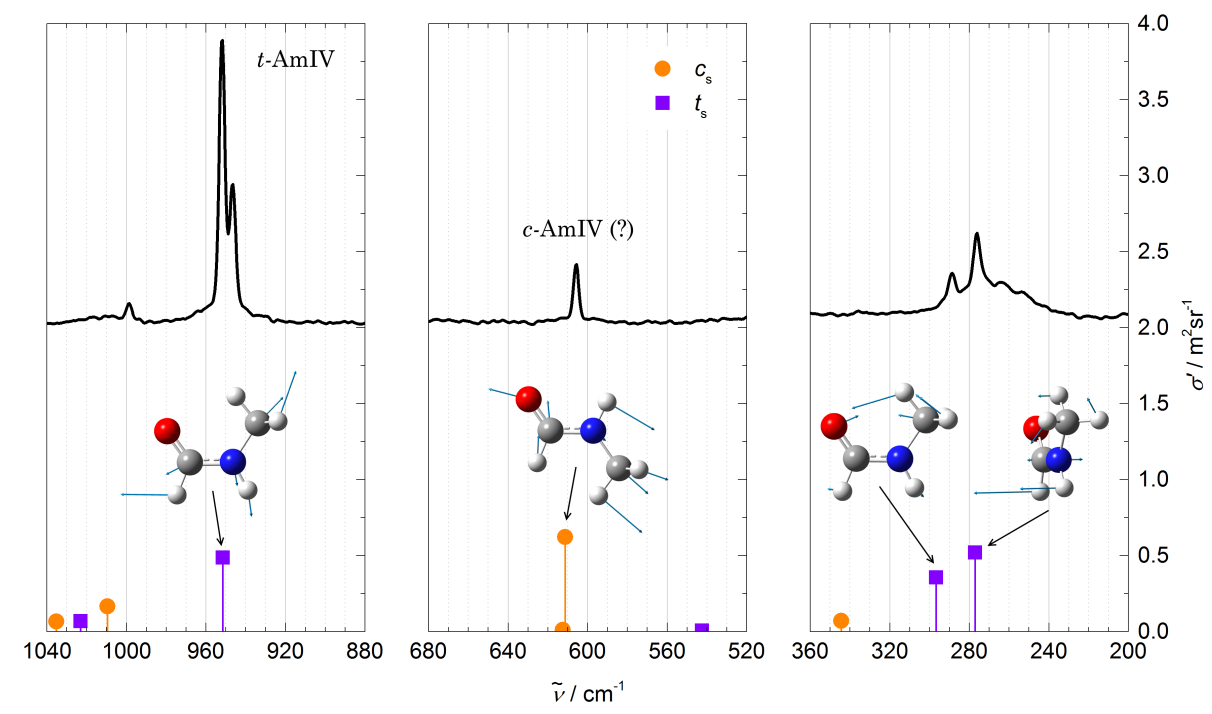

Abb. 5-5. curry-Jet-Spektren des NMF-Monomers im sogenannten Fingerprintbereich bei $100^{\circ} \mathrm{C}$. Darunter Strichspektren des jeweils stabilsten trans- (Rechteck, violett) und cis-Konformers (rund, orange) auf harmonischem B3LYPD3(BJ)/aVTZ-Niveau und an den jeweiligen Molekülstrukturen illustrierten Normalmoden. Die Wellenzahlen sind unskaliert.

Tab. 5-6. Vergleich ausgewählter experimenteller Werte $\tilde{v}_{I V}$ der Amid IV-Banden von trans- und cis-NMF. Werte in Klammern sind die Werte einer IR-aktiven Normalmode, die nicht der Symmetrie der nur wenig IR-aktiven Amid IV entspricht (siehe auch Tab. 5-8, diese aber möglicherweise überlagert. Alle Angaben in $\mathrm{cm}^{-1}$.

\begin{tabular}{|c|c|c|c|}
\hline Methode & $\tilde{v}_{\mathrm{IV}}^{t}$ & $\tilde{v}_{\mathrm{IV}}^{c}$ & weitere trans-Banden \\
\hline \multicolumn{4}{|l|}{ Jet-Expansion: } \\
\hline $\operatorname{Raman}^{\mathrm{a}}$ & 952,946 & 606 & 289,276 \\
\hline \multicolumn{4}{|l|}{ Matrix: } \\
\hline$N_{2}-I R[68$ & 951 & (629) & 237 \\
\hline Ar - FTIR 249 & 947 & $(602)$ & - \\
\hline $\mathrm{Ar}-\mathrm{FTIR} 25$ & 948 & (602) & - \\
\hline $\mathrm{Ar}-\mathrm{FTIR} 254$ & 948 & $(602)$ & - \\
\hline $\mathrm{Ne}-\mathrm{FTIR}$ 254 & 950 & - & - \\
\hline \multicolumn{4}{|l|}{ Gasphase: } \\
\hline IR 239. & $958,946,933$ & - & - \\
\hline IR 73 & $961,949,935$ & - & - \\
\hline FTIR 261]. & - & $(579)$ & - \\
\hline \multicolumn{4}{|l|}{ Flüssigkeit: } \\
\hline IR 62 & 958 & - & - \\
\hline IR 257. & 956 & - & 356,302 \\
\hline IR 73 & 958 & - & 356,302 \\
\hline Raman 258 & 962 & - & 360,297 \\
\hline
\end{tabular}


Tab. 5-7. Raman-Streuquerschnitte und IR-Intensitäten für die niederfrequenten Moden von NMF aus der Frequenzrechnung auf B3LYP-D3(BJ)/aVTZ-Niveau. Angegeben sind die unskalierte harmonische Wellenzahl für cis- $\left(\omega^{c}\right)$ und transNMF $\left(\omega^{t}\right)$, die IR-Intensität $A$ und der differentielle Streuquerschnitt $\sigma^{\prime}$ nach GI. 3-12 bei $T=298 \mathrm{~K}$ und $532.2885 \mathrm{~nm}$ Laserwellenlänge. (Details zur Rechenmethode, siehe Abschnitt 3.4

\begin{tabular}{cclccc}
\hline$\omega^{t} / \mathrm{cm}^{-1}$ & $\omega^{c} / \mathrm{cm}^{-1}$ & Symmetrierasse & $A / \mathrm{km} \cdot \mathrm{mol}^{-1}$ & $10^{35} \sigma^{\prime} / \mathrm{m}^{2} \mathrm{sr}^{-1}$ & Zuordnung \\
\hline \multirow{3}{*}{1023} & 1035 & $A^{\prime \prime}$ & 2 & 0.7 & \\
& & $A^{\prime \prime}$ & 0.03 & 0.7 & \\
952 & 1010 & $A^{\prime}$ & 41 & 1.7 & \\
767 & & $A^{\prime}$ & 19 & 4.9 & $v_{\mathrm{IV}}^{t}$ \\
& \multirow{2}{*}{613} & $A^{\prime}$ & 0.7 & 0.2 & \\
543 & 611 & $A^{\prime \prime}$ & 110 & 0.1 & \\
& & $A^{\prime}$ & 13 & 6.2 & $v_{\mathrm{IV}}^{c}$ \\
297 & \multirow{2}{*}{344} & $A^{\prime \prime}$ & 41 & 0.06 & \\
277 & & $A^{\prime}$ & 8 & 0.7 & \\
\hline
\end{tabular}

Literatur liefert nur die IR-Studie in der $\mathrm{N}_{2}$-Matrix [68] mit $237 \mathrm{~cm}^{-1}$ eine Bandenposition in der Nähe der hier gemessenen. Die Zunahme der anharmonischen Wellenzahlen der „weiteren trans-Banden" gegenüber den entsprechenden harmonischen Werten in den anharmonischen Rechnungen in Tabelle 5-8 ist ein Indiz für das Versagen der VPT2-Rechnung. Daher wird zur Interpretation der Bande ebenfalls in erster Linie auf die Ergebnisse der dispersionskorrigierten B3LYP-Rechnung zurückgegriffen. Hier fallen zwei Moden des trans-NMF auf, die einer Schwingung in $\left(A^{\prime}\right)$ und aus der Ebene heraus $\left(A^{\prime \prime}\right)$ entsprechen. Die Bande bei $276 \mathrm{~cm}^{-1}$ lässt sich versuchsweise letzterer zuordnen und wäre damit nach der Definition für die Amidbanden von NMA [19] die Amid VIII-Bande. Für den höherfrequenten Peak gibt es keine AmidbandenBezeichnung.

Tab. 5-8. Vergleich Raman-aktiver harmonischer Wellenzahlen der Amid IV-Normalmoden von trans-NMF $\left(v_{\mathrm{IV}}^{t}\right)$ und cis-NMF $\left(v_{\text {IV }}^{c}\right)$, berechnet mit unterschiedlichen Methoden. Die den Berechnungen zugrunde liegenden Konformationen (Konf.) sind angegeben, soweit sie aus den jeweiligen Veröffentlichungen hervorgehen.

\begin{tabular}{|c|c|c|c|c|c|}
\hline Methode / Basissatz & $v_{\mathrm{IV}}^{t}\left(A^{\prime}\right)$ & $v_{\mathrm{IV}}^{c}\left(A^{\prime}\right)$ & weitere trans-Banden ${ }^{d}$ & weitere cis-Banden ${ }^{d}$ & Konf. \\
\hline $\begin{array}{l}\text { theoretisch: } \\
\text { HF/6-31+G* 258. } \\
\text { B3LYP/6-31++G*b } 249 . \\
\text { B3LYP/VTZ 255. }\end{array}$ & $\begin{array}{l}1030 \\
955 \\
948\end{array}$ & $\begin{array}{l}- \\
608 \\
612\end{array}$ & $\begin{array}{l}291\left(A^{\prime}\right), 260\left(A^{\prime \prime}\right) \\
294\left(A^{\prime}\right), 276\left(A^{\prime \prime}\right) \\
-\end{array}$ & $\begin{array}{l}- \\
617\left(A^{\prime \prime}\right) \\
622\left(A^{\prime \prime}\right)\end{array}$ & $\begin{array}{l}t_{\mathrm{a}} \\
t_{\mathrm{s}} / c_{\mathrm{s}} \\
t_{\mathrm{s}} / c_{\mathrm{s}}\end{array}$ \\
\hline $\begin{array}{l}\text { B3LYP-D3(BJ)/VTZ }{ }^{\text {a }} \\
\text { B3LYP-D3(BJ)/VTZ + VPT2 }\end{array}$ & $\begin{array}{l}955 \\
934\end{array}$ & $\begin{array}{l}613 \\
606\end{array}$ & $\begin{array}{l}299\left(A^{\prime}\right), 268\left(A^{\prime \prime}\right) \\
292\left(A^{\prime}\right), 290\left(A^{\prime \prime}\right)\end{array}$ & $\begin{array}{l}620\left(A^{\prime \prime}\right) \\
600\left(A^{\prime \prime}\right)\end{array}$ & $t_{\mathrm{s}} / c_{\mathrm{s}}$ \\
\hline $\begin{array}{l}\text { B3LYP-D3(BJ)/aVTZ } \\
\text { PW91 xc/6-31+G*c } 188\end{array}$ & $\begin{array}{l}952 \\
990\end{array}$ & $\begin{array}{l}611 \\
-\end{array}$ & $\begin{array}{l}297\left(A^{\prime}\right), 277\left(A^{\prime \prime}\right) \\
332\left(A^{\prime}\right), 204\left(A^{\prime \prime}\right)\end{array}$ & $\begin{array}{l}613\left(A^{\prime \prime}\right) \\
-\end{array}$ & $\begin{array}{l}t_{\mathrm{s}} / c_{\mathrm{s}} \\
t_{\mathrm{s}} / c_{\mathrm{s}}\end{array}$ \\
\hline $\begin{array}{l}\text { MP2/6-311++G(2d,2p })^{c} 254 \\
\text { MP2/6-311++G(2d,2p })^{c}+\text { VPT2 } 254\end{array}$ & $\begin{array}{l}974 \\
961\end{array}$ & $\begin{array}{l}613 \\
604\end{array}$ & $\begin{array}{l}270\left(A^{\prime}\right), 209\left(A^{\prime \prime}\right) \\
219\left(A^{\prime}\right), 339\left(A^{\prime \prime}\right)\end{array}$ & $\begin{array}{l}596\left(A^{\prime \prime}\right) \\
571\left(A^{\prime \prime}\right)\end{array}$ & $t_{\mathrm{a}} / ?$ \\
\hline $\begin{array}{l}\text { MP2/aVTZ 259 } \\
\text { CASPT2(6-5)/VTZ 259. }\end{array}$ & $\begin{array}{l}981 \\
982\end{array}$ & $\begin{array}{l}611 \\
616\end{array}$ & $\begin{array}{l}263\left(A^{\prime}\right), 219\left(A^{\prime \prime}\right) \\
300\left(A^{\prime}\right), 242\left(A^{\prime \prime}\right)\end{array}$ & $\begin{array}{l}596\left(A^{\prime \prime}\right) \\
603\left(A^{\prime \prime}\right)\end{array}$ & $\begin{array}{l}t_{\mathrm{a}} / c_{\mathrm{s}} \\
t_{\mathrm{s}} / c_{\mathrm{s}}\end{array}$ \\
\hline 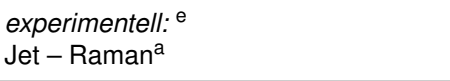 & 952 & 606 & 289,276 & - & - \\
\hline
\end{tabular}

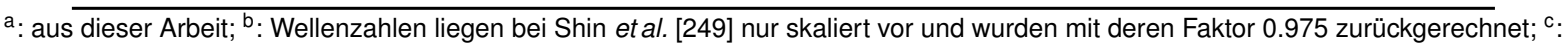

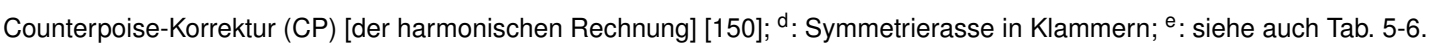




\section{2 cis-/trans-Isomerisierung}

Die Werte für die Barrierenhöhe der cis-/trans-Isomerisierung von NMF liegen in der Literatur experimentell zwischen $88-94 \mathrm{~kJ} \cdot \mathrm{mol}^{-1}$ für NMF in Lösung und $72-89 \mathrm{~kJ} \cdot \mathrm{mol}^{-1}$ aus theoretischen Berechnungen möglicher Übergangszustände (siehe Tab. 5-9) (4) Dabei sind die $88 \mathrm{~kJ} \cdot \mathrm{mol}^{-1}$ aus NMR-Messungen von einer, in einer Kryofalle ausgefrorenen Gasphase bei $540^{\circ} \mathrm{C}$ [262] der Messwert, der am ehesten den Bedingungen in einer Jet-Expansion entspricht. Die experimentellen Daten zeigen, dass die Entropie des trans-NMF höher ist als bei cis-NMF, was sich auch in den theoretischen Werten wiederspiegelt. Bei der Barrierenhöhe ist ein Einfrieren des Konformerenverhältnisses vor der Expansion anzunehmen und es kann daher die Bestimmung des Energieunterschieds von cis- und trans-NMF aus den Raman-Jetspektren ana$\log$ zu der von NMA in Abschnitt 4.2 durchgeführt werden. Die Bestimmung erfolgt aus den gut separierten Amid A-Banden mitsamt den als heiße Banden interpretierten Nebensignalen.

Tab. 5-9. Experimentelle und theoretische Energieunterschiede zwischen cis- und trans-NMF sowie Isomerisierungsbarrieren. (Eine umfassende Übersicht liefert Tab. A-24 im Tabellenwerk A.8 im Anhang.).

\begin{tabular}{|c|c|c|c|c|}
\hline \multicolumn{5}{|l|}{ experimentell } \\
\hline Methode & Bedingungen & $\Delta G / \mathrm{kJ} \cdot \mathrm{mol}^{-1 \mathrm{a}}$ & $\Delta H / \mathrm{kJ} \cdot \mathrm{mol}^{-1}$ & $\Delta E_{\mathrm{A}} / \mathrm{kJ} \cdot \mathrm{mol}^{-1 \mathrm{f}}$ \\
\hline Raman - Jet ${ }^{b}$ & $\mathrm{He} / 373.15-483.15 \mathrm{~K}$ & $7.5(373.15)$ & $6(2)$ & - \\
\hline IR-Matrix 68 & $\mathrm{N}_{2} / 298-770 \mathrm{~K}$ & - & 5.4 & - \\
\hline IR-Matrix 249. & $\operatorname{Ar} / 298-473 \mathrm{~K}$ & - & $7.4 \pm 0.7$ & - \\
\hline IR 250 & gasförmig, $473 \mathrm{~K}$ & $8.0(473)$ & 6.7 & - \\
\hline NMR 161 & in 1,2-Dichlorethan & $6.7 \pm 2.6(333.15)$ & $21 \pm 15$ & 94 \\
\hline NMR 262 & in 1,2-Dichlorethan & $6.0(263)$ & $6.1 \pm 0.2$ & 88 \\
\hline NMR 262 & gasförmig; cryogenic trapping & $9.2(814)$ & - & - \\
\hline NMR 263 & in $\mathrm{H}_{2} \mathrm{O}$ & $6.0 \pm 0.2(298)$ & $5.8 \pm 0.2$ & - \\
\hline NMR 263 & in $\mathrm{CDCl}_{3}$ & $4.7 \pm 0.2(298)$ & $3.7 \pm 0.2$ & - \\
\hline NMR $\overline{249}$ & in $\mathrm{CDCl}_{3}$ & $5.9 \pm 0.2(298)$ & - & - \\
\hline
\end{tabular}

\begin{tabular}{|c|c|c|c|c|c|}
\hline \multicolumn{6}{|l|}{ theoretisch } \\
\hline Methode & $\Delta E / \mathrm{kJ} \cdot \mathrm{mol}^{-1} \mathrm{~g}$ & $\Delta G / \mathrm{kJ} \cdot \mathrm{mol}^{-1 \mathrm{a}}$ & $\Delta H / \mathrm{kJ} \cdot \mathrm{mol}^{-1}$ & $\Delta E_{\mathrm{A}} / \mathrm{kJ} \cdot \mathrm{mol}^{-1 \mathrm{f}}$ & Konf. \\
\hline $\mathrm{HF} / 6-31+\mathrm{G}(\mathrm{d}) 203$ & 5.1 & - & - & $85.7 / 78.1^{e}$ & n.a. \\
\hline $\mathrm{HF} / 6-31 \mathrm{G}(\mathrm{d}, \mathrm{p}) 203$ & 4.5 & - & - & $85.0 / 76.7^{e}$ & n.a. \\
\hline B3LYP/6-31G* 163 264 & 3.9 & $4.3(298.15)$ & 3,5 & - & $t_{\mathrm{s}} / c_{\mathrm{s}}$ \\
\hline B3LYP/VTZ 255 & 4.0 & - & - & 88.7 & $t_{\mathrm{s}} / c_{\mathrm{s}}$ \\
\hline B3LYP/aVTZ 255. & 4.4 & $6.6(?)$ & - & - & $t_{\mathrm{s}} / c_{\mathrm{s}}$ \\
\hline B3LYP-D3(BJ)/aVTZ b & $4.8[4.6]$ & $7.8(373.15)$ & $4.4^{i}$ & - & $t_{\mathrm{s}} / c_{\mathrm{s}}$ \\
\hline BHandHLYP/aVTZ 265 & {$[4.7]$} & - & - & 78.9 & $t_{\mathrm{s}} / c_{\mathrm{s}}$ \\
\hline MP2/6-311+G** 266 & 6.4 & - & - & $79.7 / 84.3^{\mathrm{e}}$ & $t_{\mathrm{a}} / c_{\mathrm{s}}$ \\
\hline MP2/VTZ 265 & {$[5.2]$} & - & - & 74.0 & $t_{\mathrm{s}} / c_{\mathrm{s}}$ \\
\hline MP2/aVTZZ265 & [6.1] & - & - & 74.7 & $t_{\mathrm{s}} / c_{\mathrm{s}}$ \\
\hline $\operatorname{CcsD}\left(T^{*}\right)-F 12 a / a V T Z{ }^{\text {h }} 265$ & {$[5.7]$} & - & - & 72.0 & $t_{\mathrm{s}} / c_{\mathrm{s}}$ \\
\hline
\end{tabular}

n. a.: nicht angegeben; ${ }^{a}$ : (Temperatur in $\left.\mathrm{K}\right){ }^{\mathrm{b}}{ }^{\mathrm{a}}$ : aus dieser Arbeit; ${ }^{\mathrm{c}}$ : freie Enthalpie $\Delta G$ (Temperatur in $\left.\mathrm{K}\right)$ ); ${ }^{\mathrm{d}}$ : Counterpoise-Korrektur (CP) 150]; " : zwei mögliche Übergangszustände; ${ }^{\mathrm{f}}$ : Barriere bezogen auf trans-NMF; ${ }^{\text {: }}$ elektronische [nullpunktskorrigierte] Energieunterschiede; ${ }^{\mathrm{h}}$ : Geometrie MP2/aVTZ optimiert; ' : aus Van 't-Hoff-Plot auf Basis der berechneten $\Delta G$-Werte im Temperaturbereich $373.15-483.15 \mathrm{~K}$.

Eine solche Bestimmung aus den Raman-Jetspektren wurde bereits gemeinsam mit K. Otto im Rahmen ihrer Doktorarbeit [112] durchgeführt. Niedrige Substanzkonzentrationen gepaart mit Einschränkungen bei der Düsentemperatur führten dort allerdings zu einer schwachen cis-

\footnotetext{
4) Eine erste Berechnung der Barriere zu $117-119 \mathrm{~kJ} \cdot \mathrm{mol}^{-1}$ mittels einer einfachen Normalmodenanalyse [241] wird später von LaPlanche und Rogers [159] angezweifelt.
} 
Amid A-Bande, die sich nur mit einer großen Unsicherheit integrieren ließ. Der neu eingebaute heizbare Sättiger erlaubt höhere Substanzkonzentrationen und die damit erhöhte Clusterbildungswahrscheinlichkeit wird durch höhere Düsentemperaturen bis $210^{\circ} \mathrm{C}$ kompensiert, um ein reines Monomerspektrum zu erhalten. Die sechs Spektren bei unterschiedlichen Temperaturen von 100 bis $210^{\circ} \mathrm{C}$ sind in Abbildung 5-6 dargestellt.

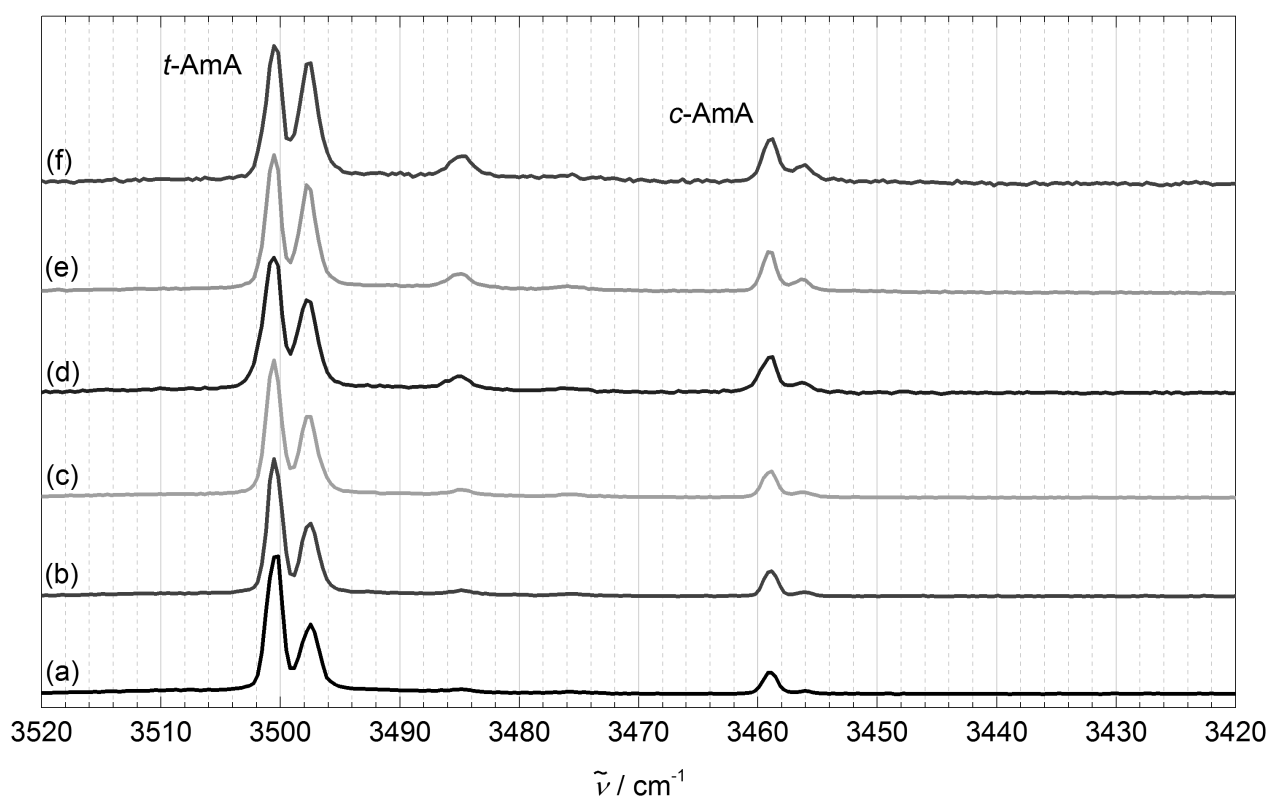

Abb. 5-6. Temperaturmessreihe von NMF im NH-Streckbereich. Die Temperaturen vor der Expansion, $\vartheta_{\mathrm{H}} /{ }^{\circ} \mathrm{C}$, betragen von unten nach oben: 100, 130, 150, 170, 190, 210. (Intensitäten aller gezeigten Spektren normiert auf die $t$-Amid AFundamentale bei $3501 \mathrm{~cm}^{-1}$ )

Eine Berechnung des cis-Monomeranteils erfolgt durch die Berechnung des um die theoretischen Streuquerschnitte korrigierten Verhältnisses der integrierten Banden von cis- und transNMF (siehe Abschnitt 4.2). Daraus ergibt sich ein Anteil von $8 \%$ cis-NMF für die Messung bei $100{ }^{\circ} \mathrm{C}$ bis hin zu $15 \%$ in der $210^{\circ} \mathrm{C}$-Messung.

Der Van-'t-Hoff-Plot ${ }^{5)}$ in Abbildung 5-7 zeigt ebenso wie die Spektrenreihe in Abbildung 5-6 einen Sprung zwischen den experimentellen Datenpunkten bei $150^{\circ} \mathrm{C}$ und $170^{\circ} \mathrm{C}$, der seine Ursache in einer systematisch fehlerhaften Temperaturmessung haben müsste. Die beiden Temperaturblöcke entstammen jedoch zwei zeitlich weiter auseinanderliegenden unterschiedlichen Messserien (siehe Tab. A-6 in Anhang A.6.1 für Details zu den Messungen). Eine getrennte Auswertung liefert zwei lineare Regressionen, aus deren Steigungen sich gemäß Gl. 2-7 eine Enthalpiedifferenz von 5(1) kJ·mol ${ }^{-1}$ für den Fit der hohen Temperaturen und 6(2) kJ.mol ${ }^{-1}$ für die Messungen bei niedrigeren Temperaturen berechnen lässt. Zum Vergleich ergibt der van 't-Hoff-Plot der thermochemischen Daten einer harmonischen B3LYP-D3(BJ)/aVTZ-Frequenzrechnung einen Enthalpieunterschied von 4.392(6) $\mathrm{kJ} \mathrm{mol}^{-1}$, wobei dieser Fehler ausschließ-

${ }^{5)}$ In $K$ enthaltene theoretische Streuquerschnitte haben laut Gl. 2-7 keinen Einfluss auf die über die Steigung im Van 't-Hoff-Plot bestimmten Enthalpien. 


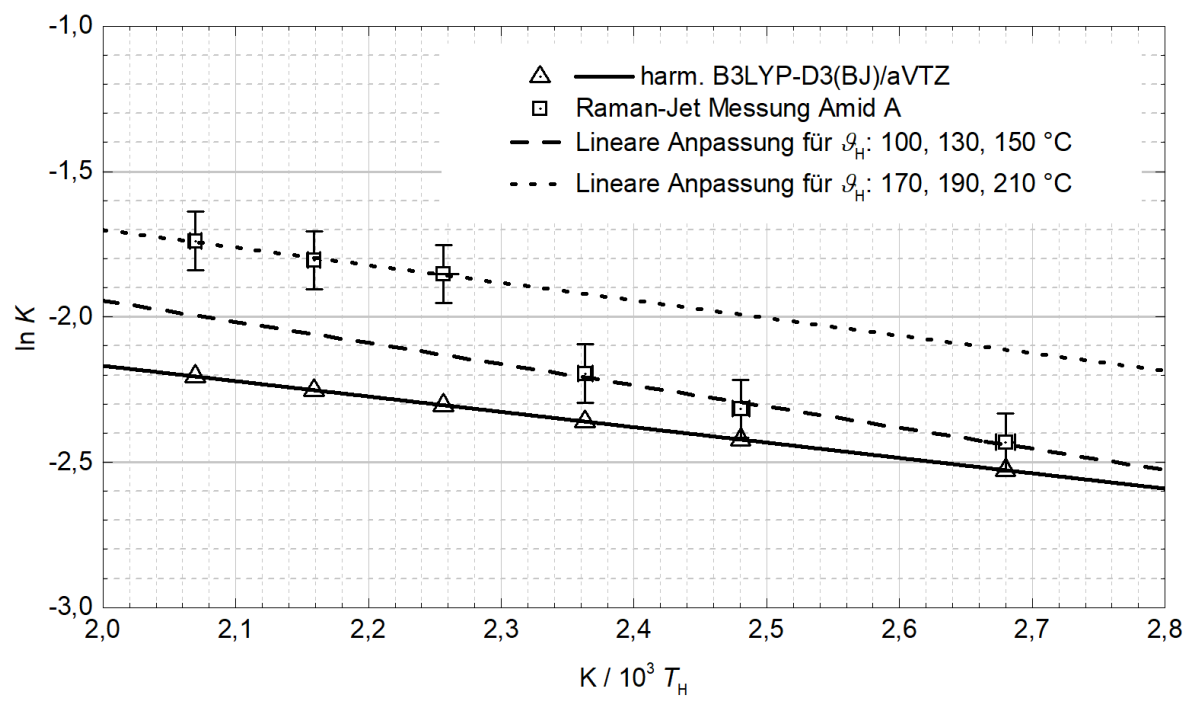

Abb. 5-7. Van-'t Hoff-Plots für die cis-/trans-Isomerisierung von NMF. Oben: Auftragung nach Gleichung 2-7 aus den Intensitätsverhältnissen $F$ der Amid A-Banden für $\vartheta_{\mathrm{H}}=170,190,210^{\circ} \mathrm{C}$ in Abb. 5-6 und den aus B3LYP-D3(BJ)/aVTZ berechneten Streuquerschnitten $\sigma^{\prime}$ (siehe auch Gl. 2-5. Die Fehler-gewichtete lineare Anpassung ergibt für die Steigung $b=-(6.1 \pm 1.4) \cdot 10^{2} \mathrm{~K}$.

Mitte: Analoge Auftragung mit den Intensitätsverhältnissen der nicht-normierten Amid A-Banden für $\vartheta_{\mathrm{H}}=100,130$, $150^{\circ} \mathrm{C}$ in Abb. 5-6. Die Fehler-gewichtete lineare Anpassung ergibt für die Steigung $b=-(7.3 \pm 2.2) \cdot 10^{2} \mathrm{~K}$.

Unten: Auf Grundlage der mit dem Gaussiantool "freqchk“ für die jeweiligen Temperaturen bestimmten freien Enthalpien aus harmonischen Frequenzrechnungen auf B3LYP-D3(BJ)/aVTZ-Niveau. Steigung der linearen Anpassung: $b=-5.282(7) \cdot 10^{2} \mathrm{~K}$. 
lich der linearen Regression entspricht. Zur Einordnung des Fehlers liefert die direkte, harmonisch in der starren Rotatornäherung durchgeführte Berechnung bei $443.15 \mathrm{~K}$ einen Enthalpieunterschied von 4.39(1) kJ.mol ${ }^{-1}$. Dies zeigt, dass der Fehler der linearen Regression nicht signifikant zum Gesamtfehler beiträgt.

Die für die beiden Temperaturbereiche getrennt bestimmten Enthalpiedifferenzen unterscheiden sich um $1 \mathrm{~kJ} \cdot \mathrm{mol}^{-1}$, was für beide innerhalb des abgeschätzten Fehlerbereichs liegt. Sie liegen außerdem im Bereich der Literaturwerte aus IR-Matrix-Messungen [68, 249] (siehe Tab. 5-9]. Auch wenn Otto [112] wegen starker Schwankungen in ihren Messwerten aufgrund apparativer Defizite in der Aufrechterhaltung der Düsentemperatur auf eine Auswertung ihrer NMF-Temperaturreihe in einem Van-'t-Hoff-Plot verzichtet hat, so liegen die Rohdaten für die Peakflächenverhältnisse aus fünf Messreihen im Anhang ihrer Arbeit vor. Daraus ergeben sich mittels Van-'t-Hoff-Plot Enthalpiedifferenzen von $1.2-3.0 \mathrm{~kJ} \cdot \mathrm{mol}^{-1}$, was zwar deutlich unter den Werten aus dieser Arbeit liegt, aber unter Berücksichtigung der dort beschriebenen apparativen Probleme können diese Werte als gute Übereinstimmung gewertet werden. 


\subsection{Dimerisierung}

In diesem Abschnitt wird untersucht, inwieweit die Beobachtungen zu den Aggregaten von NMA in Kapitel 4.3 und 4.4 auch auf NMF zutreffen. Dabei wird ebenfalls erwartet, dass sich aufgrund des hohen Anteils an trans-Monomer in der Expansion hauptsächlich tt-Dimere und ttt-Trimere bilden. Der cis-Monomer-Anteil ist im Vergleich zu NMA mit 8-15\% (siehe Abschnitt 5.2) jedoch deutlich größer, so dass der spektroskopische Nachweis von gemischten ct-Dimeren und des theoretisch stabilsten cc-Dimers möglich erscheint. Desfrançois et al. [45] schätzen die Dimerverteilung im Molekularstrahl von NMF in Helium bei einer Düsentemperatur von $370 \mathrm{~K}$ grob auf 26, 71 und $3 \%$ für $c c$, tt und $c t$ ab. Die Abschätzung kombiniert berechnete Bindungsenergien der Cluster mit den relativen Anteilen der Monomerkonformere vor der Dimerisierung. Im curry-Jet zeigt sich, dass einmal in der Expansion gebildete Cluster sich nicht umgruppieren und da die Isomerisierungsbarriere zu hoch für eine innere Umwandlung ist, dominieren hier statisitische Effekte.

Amid A Im oberen Teil von Abbildung 5-8 ist eine Serie von curry-Jetspektren mit von (a) bis (e) zunehmender NMF-Konzentration bei konstanter Düsentemperatur abgebildet. Die Abbildung zeigt den Amid A-Bereich, wo in den Spektren neben den bereits diskutierten Monomersignalen eine Vielzahl von Banden hinzukommen. Dabei sind die Banden bei 3395 und $3113 \mathrm{~cm}^{-1}$ in allen Spektren deutlich vorhanden, während die anderen erst mit zunehmender Konzentration sichtbar werden. Daher handelt es sich bei beiden Banden voraussichtlich um Dimersignale. Die Rotverschiebung sowie die bereits angesprochene zu erwartende Verteilung der möglichen Dimere legt für erstere eine Zuordnung als Donorbande des tt-Dimers nahe. Dies wird im Vergleich mit den Literaturwerten in Tabelle 5-10 bestätigt. Die Bandenposition stimmt unter Berücksichtigung der Messunsicherheit mit der in FTIR-Jet-Messungen [51, 256] überein und befindet sich im Rahmen üblicher Matrixeffekte in guter Übereinstimmung mit den Ergebnissen in zwei FTIR-Studien in der Argon-Matrix [249, 267]. Dies bedeutet eine Rotverschiebung gegenüber der trans-Monomerbande um $106 \mathrm{~cm}^{-1}$ und ergibt somit eine Bandenposition unterhalb derjenigen der beiden $t t$-Dimere von NMA (siehe Tab. 4-9 in Abschnitt 4.3).

Die zugehörige Akzeptorbande befindet sich in der Argon-Matrix bei $3478 \mathrm{~cm}^{-1}$, was eine Verschiebung von $-16 \mathrm{~cm}^{-1}$ gegenüber dem Monomer bedeutet [267]. In Abbildung 5-9 ist ein Ausschnitt der Raman-Jetspektren der Amid A-Region mit dem Fokus auf die tt-Dimerbanden dargestellt. Hier zeigt sich eine kleine zunehmende Bande bei $3485 \mathrm{~cm}^{-1}$. Diese ist auch um $-16 \mathrm{~cm}^{-1}$ gegenüber dem trans-Monomer verschoben. Allerdings wächst die Bande bei $3498 \mathrm{~cm}^{-1}$ ebenfalls mit zunehmender NMF-Konzentration in der Expansion. Hier liegt die Rotverschiebung wie bei der vermuteten Akzeptorbande des tt-Dimers von NMA bei $3 \mathrm{~cm}^{-1}$.

Beide Banden wurden zusätzlich bereits in Abschnitt 5.1 als heiße Banden des trans-Monomers zugeordnet. Die Messbedingungen der vorliegenden Spektrenserie variieren jedoch lediglich in der Sättigertemperatur bei konstanter Temperatur des Heizwegs und der Düse (siehe Abschnitt 3.1.1 und Tab. A-6 in Anhang A.6.1), weshalb eine Veränderung heißer Banden eigentlich nicht zu erwarten ist, es sei denn die Heizstrecke ist nicht ausreichend, um die einge- 


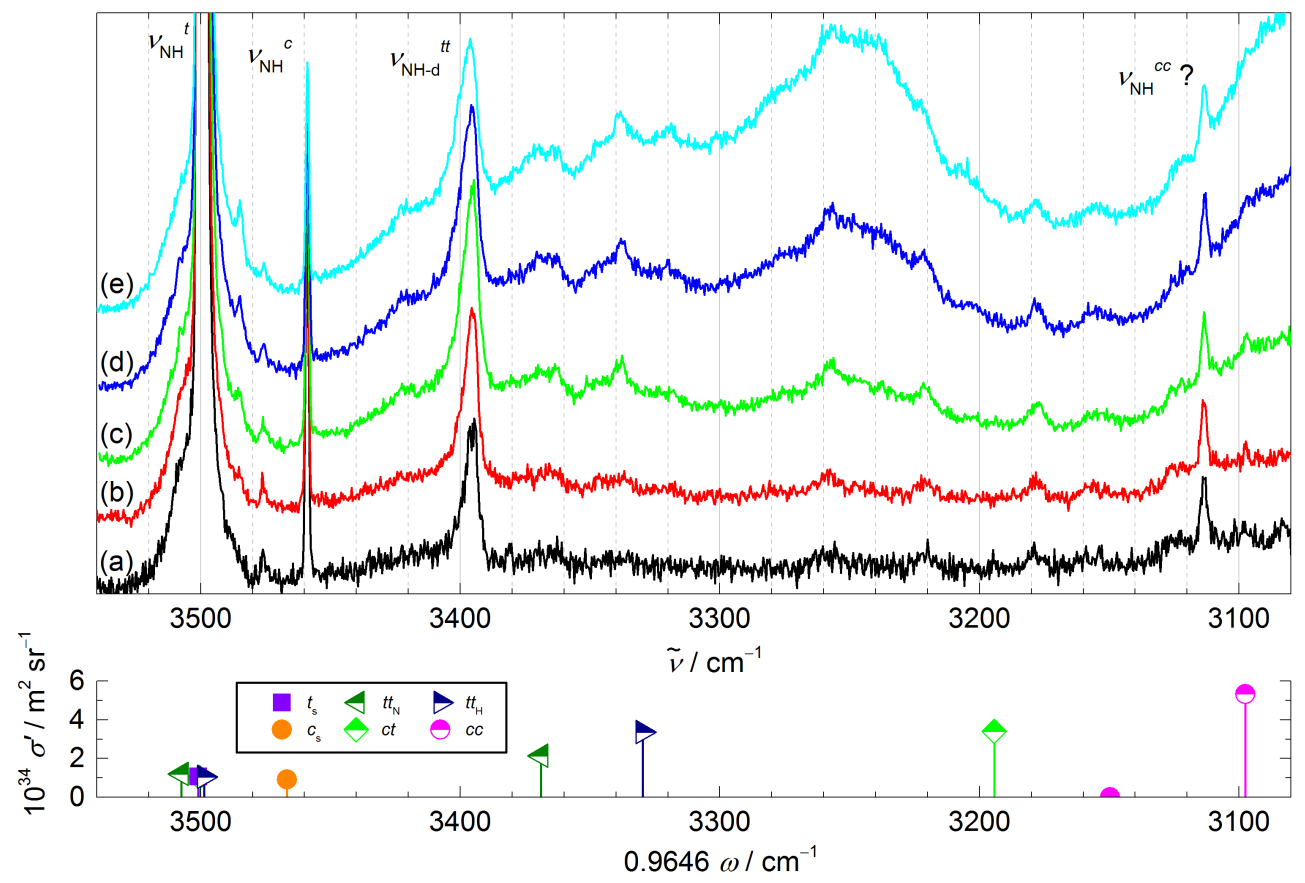

Abb. 5-8. Raman-Jetspektren zur Clusterbildung von NMF in der Amid A-Region. Jet-Expansion mit Helium als Trägergas bei konstanter Düsentemperatur $\vartheta=70^{\circ} \mathrm{C}$ und mit von (a) bis (e) zunehmender NMF-Konzentration. Die Spektren sind in ihrer Intensität auf die $v_{\mathrm{NH}}^{t}$-Bande normiert. Außerdem ist die Zuordnung der $\mathrm{NH}$-Donorbande des stabilsten ttDimers $\left(v_{\mathrm{NH}-\mathrm{d}}^{t t}\right)$ und die vorläufige Zuordnung des cc-Dimers $\left(v_{\mathrm{NH}}^{t t}\right)$ angegeben. Weitere Banden bei 3367, 3338, 3319, $3257,3222,3178,3155$ und $3123 \mathrm{~cm}^{-1}$. Im unteren Teil befinden sich die auf die $v_{\mathrm{NH}}^{t}$-Bandenposition skalierten harmonischen Frequenzen der stabilsten Monomer- und Dimerstrukturen aus B3LYP-D3(BJ)/aVTZ-Rechnungen. (Ausführliche Informationen zu den Messbedingungen befinden sich in Tab. A-6 im Raman-Spektrenverzeichnis in Anhang A.6.1 Details zu den quantenchemischen Rechnung sind in den Tabellen 5-11] und A-20 enthalten.)

Tab. 5-10. Experimentelle und theoretische Bandenpositionen des tt-Dimers von NMF. Angegeben sind die Werte (in $\mathrm{cm}^{-1}$ ) für die freie $\left(v_{\mathrm{NH}-\mathrm{a}}^{t t}\right)$ und die Wasserstoffbrücken-gebundene $\mathrm{N}-\mathrm{H}$-Streckschwingung $\left(v_{\mathrm{NH}-\mathrm{d}}^{t t}\right)$, sowie die freie $\mathrm{C}=\mathrm{O}-$ Streckschwingung des Akzeptors $\left(v_{\mathrm{CO}-\mathrm{a}}^{t t}\right)$ und des Donors $\left(v_{\mathrm{NH}-\mathrm{d}}^{t t}\right)$. In Klammern dahinter die relative Verschiebung zum Monomer. Alle Angaben in $\mathrm{cm}^{-1}$.

\begin{tabular}{|c|c|c|c|c|c|}
\hline Methode & Geometrie & $v_{\mathrm{NH}-\mathrm{d}}^{t t}$ & $v_{\mathrm{NH}-\mathrm{a}}^{t t}$ & $v_{\mathrm{CO}-\mathrm{d}}^{t t}$ & $v_{\mathrm{CO}-\mathrm{a}}^{t t}$ \\
\hline \multicolumn{6}{|l|}{ experimentell: } \\
\hline Jet - Raman ${ }^{\mathrm{a}}$ & & $3395(-106)$ & - & $1715(-29)$ & $1707(-37)$ \\
\hline Jet - FTIR $^{\mathrm{a}, \mathrm{c}}$ & & - & - & $1715(-29)$ & $1707(-37)$ \\
\hline Jet - FTIR $51 \quad 256$ & & $3394(-107)$ & - & - & $1708(-24)$ \\
\hline Ar-Matrix - FTIR 267] & & $3412(-82)$ & $3478(-16)$ & $1712(-14)$ & $1702(-23)$ \\
\hline Ar-Matrix - FTIR 249] & & $3412(-81)$ & - & - & - \\
\hline \multicolumn{6}{|l|}{ theoretisch: } \\
\hline $\mathrm{B} 3 L Y P / 6-311+\mathrm{G}^{*}$ [51] & $t t_{\mathrm{H}}$ & $3515(-110)$ & - & $1766(-11)$ & $1751(-26)$ \\
\hline \multirow{2}{*}{ B3LYP-D3/aVTZb 267} & $t t_{\mathrm{H}}$ & $3457(-167)$ & $3623(-1)$ & $1751(-13)$ & $1737(-27)$ \\
\hline & $t t_{N}$ & $3490(-134)$ & $3634(+10)$ & $1749(-15)$ & $1739(-25)$ \\
\hline \multirow{2}{*}{ B3LYP-D3(BJ)/aVTZa } & $t t_{\mathrm{H}}$ & $3452(-177)$ & $3627(-2)$ & $1755(-12)$ & $1741(-26)$ \\
\hline & $t t_{\mathrm{N}}$ & $3492(-137)$ & $3636(+7)$ & $1756(-11)$ & $1743(-24)$ \\
\hline
\end{tabular}

a: aus dieser Arbeit; ${ }^{b}$ : Wellenzahlen liegen bei Crespo-Otero et al. 267] nur skaliert vor und wurden mit deren Faktoren $0.96384\left(v_{\mathrm{NH}}\right)$ und $0.97812\left(v_{\mathrm{CO}}\right) z_{\text {zurückgerechnet; }}{ }^{\mathrm{c}}$ : Verschiebungen beziehen sich auf die als $t$-Monomer angenommene Schulter bei $1744 \mathrm{~cm}^{-1}$. 


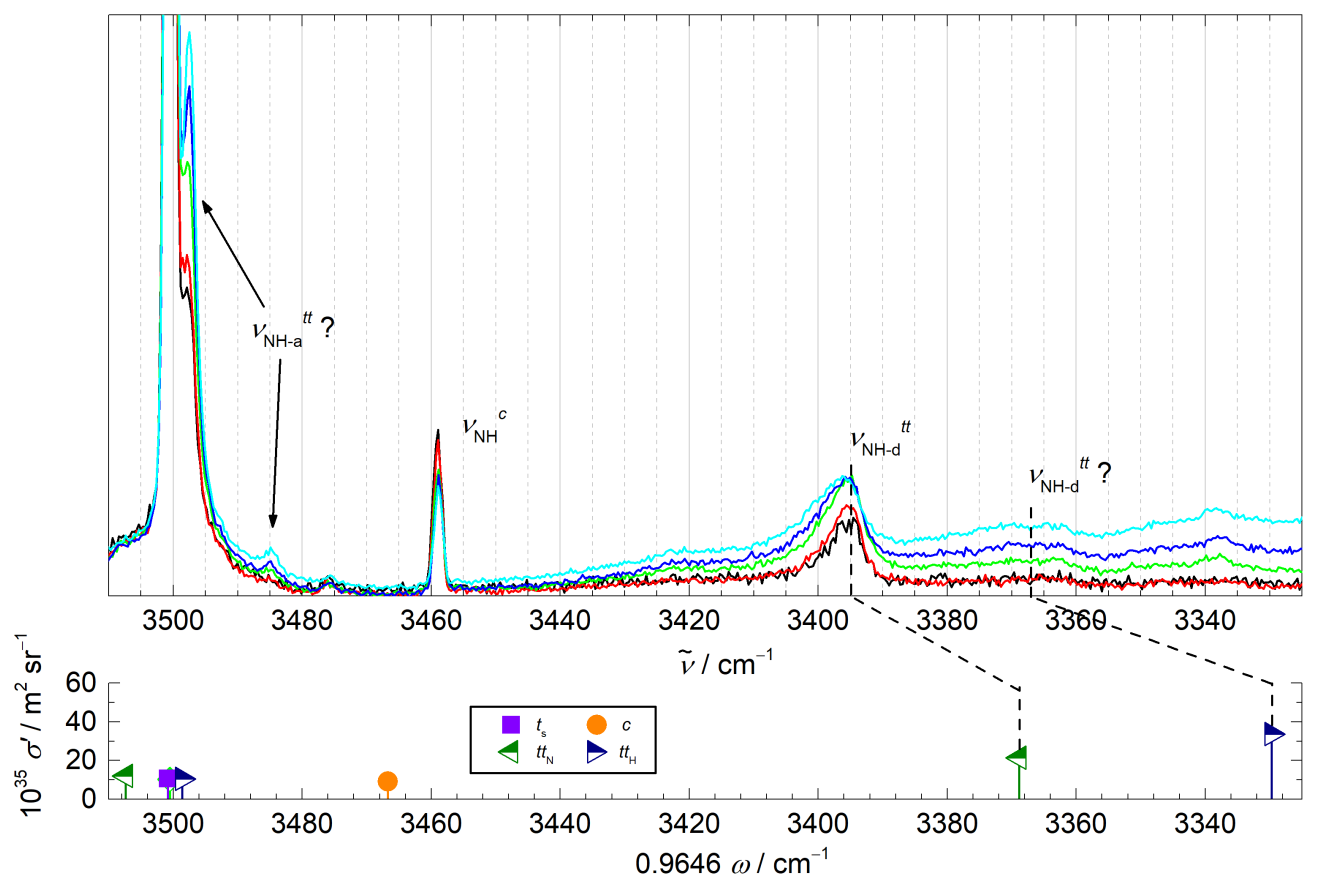

Abb. 5-9. Ausschnitt der Raman-Jetspektren von NMF in der Amid A-Region mit Fokus auf die tt-Dimerbanden. Spektren wie in Abb. 5-8 also normiert auf die Intensität des $t$-Monomers. Die Strickspektren sind die, auf die $v_{\mathrm{NH}}^{t}$-Bandenposition skalierten, harmonischen Frequenzen der stabilsten Monomer- und Dimerstrukturen aus B3LYP-D3(BJ)/aVTZ-Rechnungen. (Ausführliche Informationen zu den Messbedingungen befinden sich in Tab. A-6 im Raman-Spektrenverzeichnis in Anhang A.6.1. Details zu den quantenchemischen Rechnung sind in den Tabellen 5-11 und A-20 enthalten.) 
stellte Temperatur der Düse auch im Gasgemisch zu erreichen, so dass die unterschiedlichen Sättigertemperaturen die Intensitäten der heißen Banden beeinflussen. Auch wenn die Messungen zur cis-/trans-Isomerisierung bei NMA und NMF bei deutlich höheren Düsentemperaturen nahelegen, dass die Heizstrecke ausreichend ist, kann dieser Einfluss nicht gänzlich ausgeschlossen werden, denn die Isomerisierungsrate fällt rasch mit abnehmender Temperatur. Eine andere mögliche Erklärung ist die Veränderung der Bedingungen in der Expansion durch die steigende NMF-Konzentration. Mehr NMF in der Expansion erhöht die Clusterbildung und die dadurch freiwerdende zusätzliche Bindungsenergie kann von dem entsprechend abnehmenden Helium-Anteil schlechter abgeführt werden und somit die Expansionstemperatur leicht erhöhen. Insgesamt ist die Indizienlage nicht hinreichend für eine sichere Zuordnung der DimerAkzeptorbande.

Die Zuordnung des Peaks bei $3113 \mathrm{~cm}^{-1}$ in Abbildung 5-8 ist nicht eindeutig geklärt. Otto [112] diskutiert ausführlich, inwiefern es sich um die Bande der symmetrischen NH-Streckschwingung eines zyklischen $c c$-Dimers handeln kann, kommt aber durch eine statistische Abschätzung des Populationsverhältnisses von cc- zu tt-Dimer aus dem Bandenverhältnis der Monomerkonformere zu dem Schluss, dass das gemessene Signal um eine Größenordnung zu groß für das cc-Dimer ist. Um den Ursprung der Bande genauer zu untersuchen, sind in Abbildung 5-10 erneut die Spektren aus Abbildung 5-8 dargestellt, hier jedoch mit dem Fokus auf die $v_{\mathrm{NH}}^{c}$ und die $v_{\mathrm{NH}-\mathrm{d}}^{t t}$-Bande, sowohl normiert auf die $t$-Monomer- als auch auf die $3113 \mathrm{~cm}^{-1}$ Bande. Der Ausschnitt zeigt eine mit zunehmender NMF-Konzentration abnehmende cis-Monomerbande sowie eine zunehmende $t t$-Bande für beide Normierungen. Diese Beobachtung spricht gegen die These, dass es sich bei dem Signal bei $3113 \mathrm{~cm}^{-1}$ um die cc-Dimerbande handelt, da für diese die cis-Monomerkonzentration zwar abnehmen würde, aber die beobachtete Abnahme vergleichbar stark bei dem auf auf das $t$-Monomer skalierte Spektrum auftritt. Daher handelt es sich bei der $3113 \mathrm{~cm}^{-1}$-Bande eher um ein $t$-Monomersignal. Auch die sehr schmale Bandenform spricht eher für ein Monomer- als für ein Dimersignal. Die zugehörigen Clusterbanden dieser selektiven cis-Aggregation sind allerdings nicht im Spektrum erkennbar.

Amid I-III Im Rahmen dieser Arbeit wurden erstmalig Raman-Jetspektren der Amid I-Region auch unter Clusterbedingungen aufgenommen. Dieser sind in Abbildung 5-11. FTIR-Spektren des filet-Jets gegenüber gestellt. Die Position der Amid I-Bande des Monomers bei $1744 \mathrm{~cm}^{-1}$ wurde bereits diskutiert und wird im Folgenden durch die Einbeziehung von Clusterspektren und der Interpretation der darin auftretenden Banden bestätigt.

Die Clusterspektren der Raman-Jetmessung in Abbildung 5-11 sind auf die vermeintliche Fundamentale bei $1744 \mathrm{~cm}^{-1}$ normiert und zeigen zwei neue Banden bei 1715 und $1707 \mathrm{~cm}^{-1}$, die gegenüber der Fundamentalen um -29 bzw. $-37 \mathrm{~cm}^{-1}$ verschoben sind. Diese Banden finden sich an gleicher Position ebenfalls in den darüber abgebildeten FTIR-Jet-Spektren. Beide Banden lassen sich durch den Vergleich mit der Literatur dem $t t$-Dimer zuordnen, wobei die höherfrequente die Donor- $\left(v_{\text {CO-d }}^{t t}\right)$ und die niederfrequente die Akzeptorbande $\left(v_{\text {CO-a }}^{t t}\right)$ der COStreckschwingung ist. Demnach findet sich auch in vorherigen FTIR-Jetmessungen [51] eine Bande bei $1707 \mathrm{~cm}^{-1}$, die der CO-Streckschwingung in der Wasserstoffbrücke des Dimers zu- 

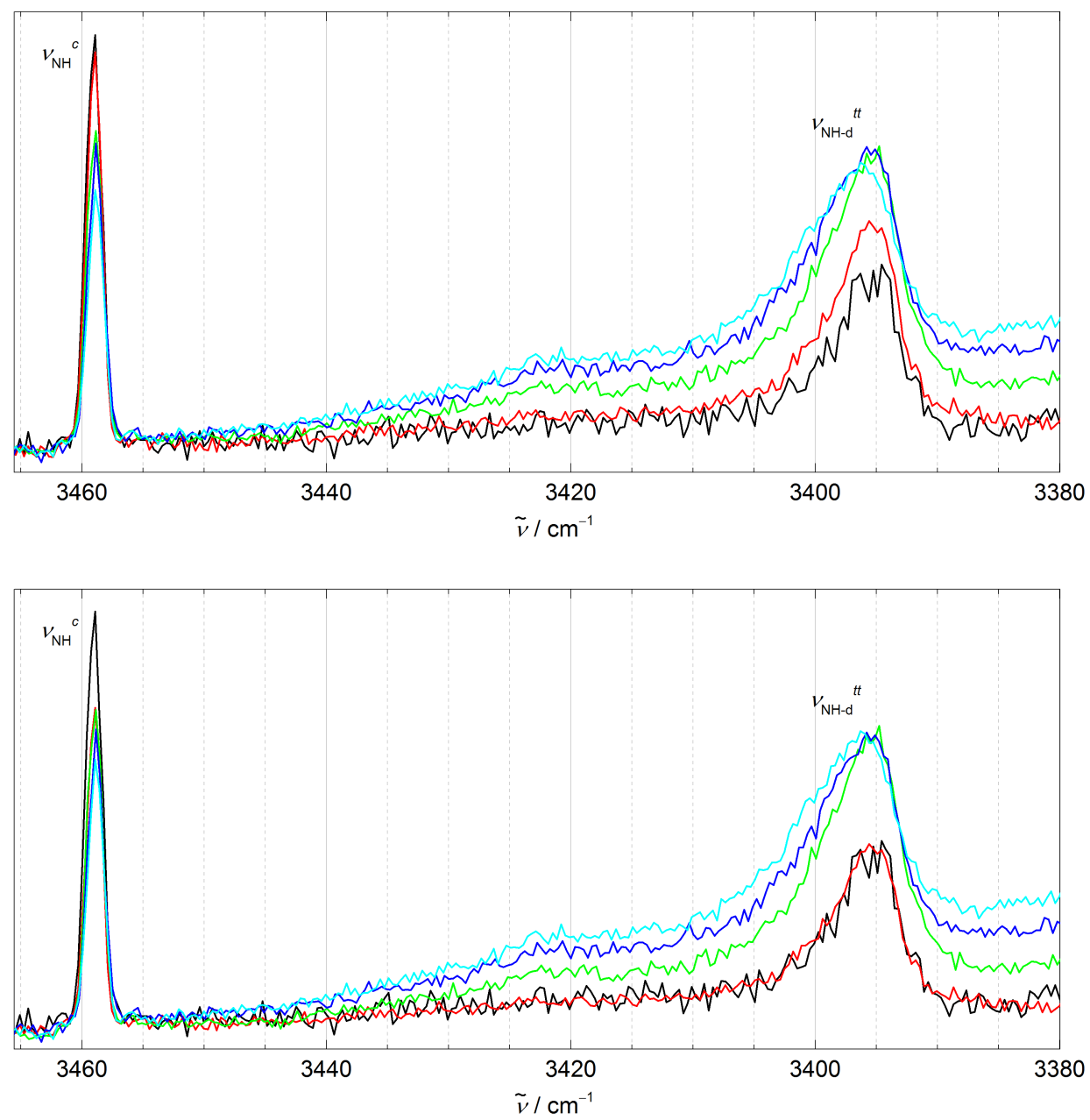

Abb. 5-10. Raman-Jetspektren von NMF in der Amid A-Region zum möglichen Nachweis des cc-Dimers. Zu sehen ist ein Ausschnitt der gleichen Spektren wie in Abb. 5-8 Oben: Normiert auf die $t$-Monomerbande. Unten: Normiert auf die $3113 \mathrm{~cm}^{-1}$-Bande. (Ausführliche Informationen zu den Messbedingungen befinden sich in Tab. A-6 im RamanSpektrenverzeichnis in Anhang A.6.1 Details zu den quantenchemischen Rechnung sind in den Tabellen 5-11 und A-20 enthalten.) 
geordnet wird. In Argon-Matrixexperimenten [267] sind beide Banden vorhanden, weichen jedoch in ihren absoluten Werten aufgrund von Matrix-Effekten leicht ab.

Zusätzlich zu den beiden Dimerbanden nehmen auch die bisher als Monomerbanden zugeordneten Peaks bei 1738 und $1729 \mathrm{~cm}^{-1}$ mit steigender NMF-Konzentration zu. Zusammen mit der bereits diskutierten Beobachtung, dass die Intensität dieser Banden mit zunehmender Düsentemperatur abnimmt, ergibt sich eher eine Zuordnung als weitere Dimerbanden und nicht als heiße Monomerbanden. Dieser Interpretation widerspricht jedoch die Tatsache, dass beide Banden im nahezu clusterfreien Spektrum bei $100^{\circ} \mathrm{C}$ Düsentemperatur in Abbildung [5-4 mit hoher Intensität auftreten. Eine mögliche Erklärung wäre eine Überlagerung von heißen Monomerbanden mit weiteren Dimerbanden, wodurch sich die typischen Veränderungen der Peakintensität bei Änderung der Konzentration und Düsentemperatur teilweise kompensieren würden.

An der niederfrequenten Flanke der Amid I-Bande im FTIR-Spektrum in Abbildung 5-11 befindet sich bei $1724 \mathrm{~cm}^{-1}$ eine Schulter, die ebenfalls mit zunehmender Substanzkonzentration zunimmt, was auf eine Clusterbande schließen lässt. Die Interpretation dieser möglichen Bande ist aber reine Spekulation und Bedarf weiterer Messungen, die nicht Bestandteil dieser Arbeit sind.

Quantenchemische Rechnungen Auch für die Aggregate von NMF wurden harmonische Frequenzrechnungen auf B3LYP-D3(BJ)/aVTZ-Niveau durchgeführt. Die zugrunde liegenden Minimumgeometrien der stabilsten Cluster sind auf dem gleichen Niveau berechnet worden und in Abbildung 5-12 dargestellt sowie die zugehörigen Eigenschaften in Tabelle 5-11 zusammengefasst. Es ergeben sich analog zu NMA zwei tt-Dimere deren struktureller Unterschied in der Akzeptorstelle der Wasserstoffbrückenbindung am Carbonyl-Sauerstoff liegt. Auch die Nomenklatur wurde analog gewählt, wobei hier aufgrund der nicht vorhandenen Methylgruppe statt $t t_{\mathrm{C}}$ die Abkürzung $t t_{\mathrm{H}}$ gewählt wird. Die NMF-Dimere $t t_{\mathrm{N}}$ und $t t_{\mathrm{H}}$ haben einen nullpunktskorrigierten Energieunterschied von $1.4 \mathrm{~kJ} \cdot \mathrm{mol}^{-1}$ mit $t t_{\mathrm{N}}$ als das stabilere Isomer. Mit einer nullpunktskorrigierten Dissoziationsenergie von $52.8 \mathrm{~kJ} \cdot \mathrm{mol}^{-1}$ als mit Abstand am stabilsten erweist sich auch für NMF das zyklische $c c$-Dimer mit zwei Wasserstoffbrückenbindungen und $C_{2 \mathrm{~h}}$-Symmetrie. Es ist damit um $23.6 \mathrm{~kJ} \cdot \mathrm{mol}^{-1}$ stabiler als das $t t_{\mathrm{N}}$-Isomer. Aufgrund des höheren cis-Monomeranteils von NMF (siehe Abschnitt 5.2) sind gemischte Dimere möglicherweise im Spektrum erkennbar, weswegen gezielt nach gemischten Dimerstrukturen gesucht und als

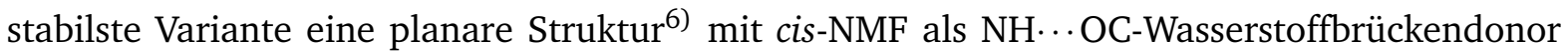
gefunden wurde. Sie besitzt zusätzlich zur NH ... OC-Wasserstoffbrückenbindung auch noch eine $\mathrm{CH}$ - OC-Wechselwirkung, in der das Wasserstoffatom am Carbonyl-Kohlenstoff als Donor fungiert (siehe Abb. 5-12). Dies führt zu einer nullpunktskorrigierten Dissoziationsenergie von $34.3 \mathrm{~kJ} \cdot \mathrm{mol}^{-1}$ und somit ist die als $c t$-Dimer bezeichnete Struktur $5.1 \mathrm{~kJ} \cdot \mathrm{mol}^{-1}$ stabiler als das $t t_{\mathrm{N}}$-Dimer. Beim Vergleich mit den Dimerstrukturen von NMA, $t t_{\mathrm{N}}$ und $t t_{\mathrm{C}}$ (siehe Tab. 4-10), zeigt sich, dass die fehlende Methylgruppe im NMF keinen Einfluss auf den $\mathrm{H} \cdots \mathrm{O}=\mathrm{C}$-Bindungswinkel bei Koordination an das N-seitige freie Elektronenpaar des Akzeptor-Sauerstoffa-

6) „Planar“ meint hier, dass alle Atome bis auf je zwei Wasserstoffatome der Methylgruppen in einer Ebene liegen. 


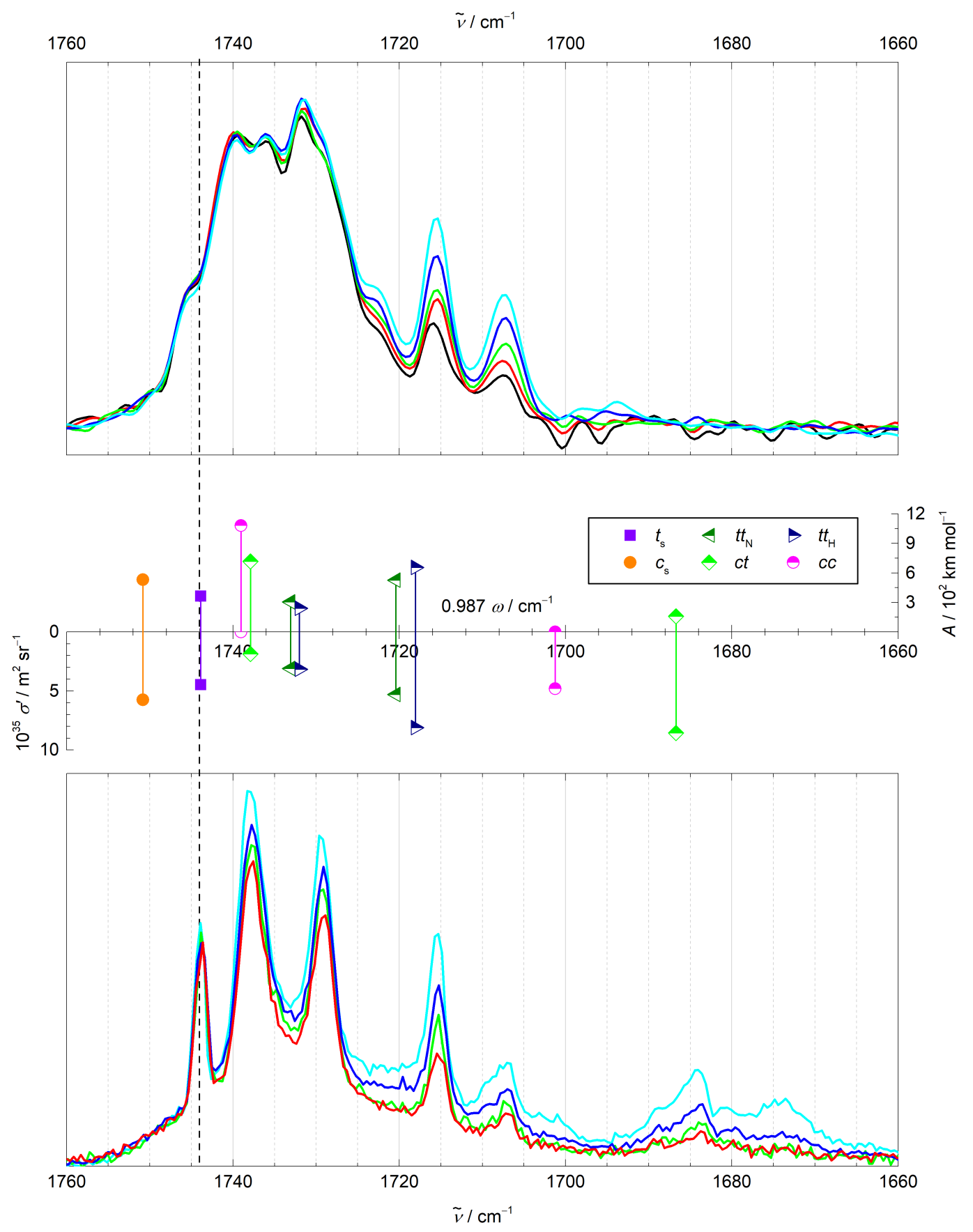

Abb. 5-11. Raman- und FTIR-Jetspektren zur Clusterbildung von NMF in der Amid I-Region.

Oben: FTIR-Jetspektren mit Helium als Trägergas bei zunehmender NMF-Konzentration (schwarz, rot, grün, blau, cyan). Unten: Raman-Jetspektren mit Helium als Trägergas mit von rot über grün, blau bis zu cyan hin zunehmender Konzentration bei konstanter Düsentemperatur.

Mitte: Strichspektren harmonischer Frequenzen $\omega$ aus B3LYP-D3(BJ)/aVTZ-Rechnungen mit einer Skalierung auf die $v_{\perp}^{t}$ (Faktor: 0.987, gestrichelte Linie); nach oben sind die IR-Intensitäten $A$ und nach unten die spezifischen RamanStreuquerschnitte $\sigma^{\prime}$ aufgetragen.

(Details zu den Messungen der Raman-Spektren befinden sich im Raman-Spektrenverzeichnis in Tab. A-6 in Anhang A.6.1 und die FTIR-Spektren schwarz, rot, grün, blau und cyan entsprechen den Datensätzen I-M in Tab. A-8 in Anhang A.6.2 (Daten aufgenommen von Katharina A. E. Meyer); Details zu den Frequenzrechnungen befinden sich in Tab. A-20 in Anhang A.8) 
toms hat, wohingegen das $t t_{\mathrm{H}}$-Isomer von NMF mit $112^{\circ}$ einen geringfügig kleineren Winkel $\alpha$ aufweist, als das NMA-Isomer $t t_{C}$. Für NMF wurde die gleiche Rechnung auch ohne Dispersionskorrektur durchgeführt (siehe Tab. 4-10). Dies führt einerseits zu einer Umkehrung der energetischen Reihenfolge bei den $t t$-Isomeren, wo nun $t t_{\mathrm{H}} \mathrm{um} 0.9 \mathrm{~kJ} \cdot \mathrm{mol}^{-1}$ (nullpunktskorrigiert) stabiler ist, andererseits zu einer Vergrößerung von $\alpha$ um 15-17 für beide $t t$-Dimere, während die Länge der Wasserstoffbrückenbindung um 5-6 pm abnimmt. Dies entspricht, bis auf die energetische Situation, den Beobachtungen für Rechnungen ohne Dispersionskorrektur bei NMA. Die Unterschiede zwischen $t t_{\mathrm{N}}$ und $t t_{\mathrm{H}}$ in der Geometrie der Wasserstoffbrückenbindung mit und ohne Dispersionskorrektur sind jedoch für NMF aufgrund der fehlenden Methylgruppe am Carbonyl-Kohlenstoff größer. Ursächlich sind hier also, wie schon bei NMA, sterische Gründe.
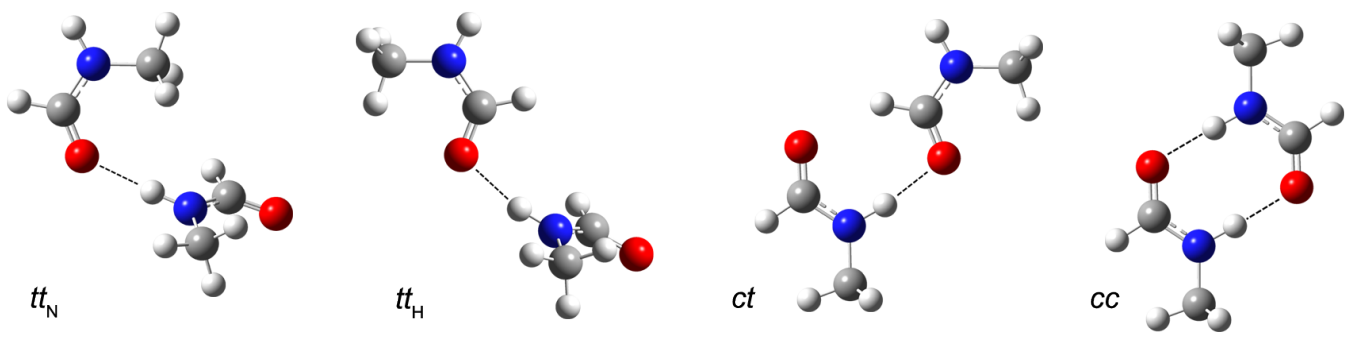

Abb. 5-12. Molekülgeometrien der trans-trans-, cis-trans- und cc-NMF Minimumstrukturen auf B3LYP-D3(BJ)/aVTZNiveau. Die Indizes beziehen sich auf das Amin-seitige $\left(t t_{N}\right)$ oder das Formyl-seitige $\left(t t_{H}\right)$ freie Elektronenpaar des Carbonyl-Sauerstoffs, das als Akzeptor der Wasserstoffbrückenbindung fungiert. Gestrichelte Linien zeigen intermolekulare Wasserstoffbrückenbindungen an. Relative Energien, Dissoziationsenergien und geometrische Parameter sind in Tabelle 5-11 aufgeführt.

Tab. 5-11. Berechnete Eigenschaften der NMF-Dimere $t t_{\mathrm{N}}, t t_{\mathrm{H}}$ und $c c$, sowie des gemischten Dimers $c t$ auf B3LYP/aVTZ-Niveau mit und ohne Dispersionskorrektur. Angegeben sind die relativen elektronischen und nullpunktskorrigierten Energien $\Delta E_{\text {el }}$ bzw. $\Delta E_{0}$, sowie Dissoziationsenergien $D_{\text {el }}$ und $D_{0}$. Länge der Wasserstoffbrückenbindung $d(\mathrm{H} \cdots \mathrm{O})$, der Bindungswinkel der Wasserstoffbrückenbindung mit der $\mathrm{C}=\mathrm{O}$-Bindung $\alpha(\mathrm{H} \cdots \mathrm{O}=\mathrm{C})$, die niedrigste Frequenz in doppelt-harmonischer Näherung $\tilde{v}_{\min }$ und die Dipolmomente $\mu / \mathrm{D}$.

\begin{tabular}{|c|c|c|c|c|c|c|c|c|}
\hline & \multicolumn{2}{|c|}{$t t_{N}$} & \multicolumn{2}{|c|}{$t t_{\mathrm{H}}$} & \multicolumn{2}{|c|}{$c c$} & \multicolumn{2}{|c|}{$c t$} \\
\hline & D3(BJ) & ohne & D3(BJ) & ohne & D3(BJ) & ohne & D3(BJ) & ohne \\
\hline$\Delta E_{\mathrm{el}} / \mathrm{kJ} \cdot \mathrm{mol}^{-1}$ & 26.4 & 24.4 & 27.9 & 22.9 & 0.0 & 0.0 & 19.8 & 17.5 \\
\hline$\Delta E_{0} / \mathrm{kJ} \cdot \mathrm{mol}^{-1}$ & 23.6 & 20.7 & 25.0 & 19.8 & 0.0 & 0.0 & 18.5 & 16.1 \\
\hline$D_{\mathrm{el}} / \mathrm{kJ} \cdot \mathrm{mol}^{-1}$ & 33.5 & 23.9 & 32.0 & 25.3 & 69.5 & 57.1 & 44.9 & 35.2 \\
\hline$D_{0} / \mathrm{kJ} \cdot \mathrm{mol}^{-1}$ & 29.2 & 20.7 & 27.8 & 21.7 & 52.8 & 49.8 & 34.3 & 29.5 \\
\hline$\alpha(\mathrm{H} \cdots \mathrm{O}=\mathrm{C}) /^{\circ}$ & 139 & 154 & 112 & 129 & 120 & 121 & 107 & 108 \\
\hline$d(\mathrm{H} \cdots \mathrm{O}) / \mathrm{pm}$ & 193 & 199 & 192 & 197 & 182 & 186 & 186 & 190 \\
\hline$\tilde{v}_{\min } / \mathrm{cm}^{-1}$ & 21 & 9 & 18 & 13 & 34 & 36 & 34 & 36 \\
\hline$\mu / \mathrm{D}$ & 7.3 & 8.0 & 8.0 & 8.9 & 0.0 & 0.0 & 2.3 & 2.4 \\
\hline
\end{tabular}

Vergleich von Theorie und Experiment Für die Amid A-Region befinden sich die harmonischen Frequenzen als skaliertes Strichspektrum (Faktor: 0.9646) im unteren Teil von Abbildung 5-8, Um die beiden berechneten, energetisch sehr ähnlichen Dimere $t t_{\mathrm{N}}$ und $t t_{\mathrm{H}}$ der Bande bei $3395 \mathrm{~cm}^{-1}$ im Ramanspektrum der Amid A-Region (siehe Abb. 5-9) zuzuordnen, bietet sich der Vergleich mit den Dimeren des strukturell sehr ähnlichen NMA an. Im Amid A- 
Spektrum von NMA finden sich zwei Dimerbanden, die der Donor-NH-Streckschwingung der beiden NMA-Dimere $t t_{\mathrm{N}}$ und $t t_{\mathrm{C}}$ zugeordnet sind. Diese beiden Signale sind um $-112 \mathrm{~cm}^{-1}$ und $-141 \mathrm{~cm}^{-1}$ gegenüber der $t$-Monomerbande verschoben und liegen damit $29 \mathrm{~cm}^{-1}$ auseinander (siehe Tab. 4-9). Der energetische Unterschied wird auf B3LYP-D3(BJ)/aVTZ-Niveau zu $0.1 \mathrm{~kJ} \cdot \mathrm{mol}^{-1}$ berechnet und ist damit um eine Größenordnung geringer als bei den NMF-Dimeren mit $1.4 \mathrm{~kJ} \cdot \mathrm{mol}^{-1}$ (vgl. Tab. 4-10 und Tab. 5-11), weshalb für das $t_{\mathrm{H}}-\mathrm{NMF}$, wenn überhaupt, nur ein sehr schwaches Signal erwartet werden kann. Zur Abschätzung der $t t_{\mathrm{H}}$-Bandenposition werden die Ergebnisse der harmonischen B3LYP-D3(BJ)/aVTZ-Frequenzrechnungen von NMA und NMF verglichen. Dies geschieht unter der Annahme, dass aufgrund der Ähnlichkeit der Dimerstrukturen die gleiche theoretische Methode eine vergleichbare Differenz der Bandenpositionen bei ähnlicher Überschätzung voraussagt.

Für NMA überschätzt die harmonische Rechnung mit einer Differenz von $35 \mathrm{~cm}^{-1}$ den experimentellen Abstand um $21 \%$ (siehe Tab. 4-11). Die Rechnungen für NMF ergeben mit einer Differenz der harmonischen Wellenzahlen von $t t_{\mathrm{N}^{-}}$und $t t_{\mathrm{H}^{-N M F}}$ von $40 \mathrm{~cm}^{-1}$ (siehe Tab. 5-12) und $21 \%$ Überschätzung eine hypothetische Bandenposition von $34 \mathrm{~cm}^{-1}$ unterhalb der Bande bei $3395 \mathrm{~cm}^{-1}$, also bei ca. $3361 \mathrm{~cm}^{-1}$. An dieser Stelle ist im Raman-Spektrum in Abbildung 5-9 tatsächlich eine sehr breite Bande mit geringer Intensität zu beobachten, deren Position im Amid A-Übersichtsspektrum in Abbildung 5-8 zu $3367 \mathrm{~cm}^{-1}$ bestimmt wird. Die energetischen Verhältnisse der beiden NMF-Dimere und die Analogie zu NMA legen eine Zuordnung von $t t_{\mathrm{N}}-\mathrm{NMF}$ zur $3395 \mathrm{~cm}^{-1}$ - und $t t_{\mathrm{H}}$ zur $3367 \mathrm{~cm}^{-1}$-Bande nahe. Damit lässt sich grob das maximale $t t$-Dimerverhältnis abschätzen. Hierzu wird ein Peakflächenverhältnis von $t t_{\mathrm{H}}$ : $t t_{\mathrm{N}}=2.3: 6.1$ für die höchstkonzentrierte Messung abgelesen (cyanfarbenes Spektrum (e) in Abb. 5-8 und 5-9), was nach einer Korrektur um die theoretischen differentiellen Streuquerschnitte (siehe Tab. 5-12) mit Gleichung 2-5 einem maximalen Stoffmengenanteil von $19 \% t t_{\mathrm{H}}$ entspricht. Das ergibt zusammen mit dem berechneten Energieunterschied von $1.4 \mathrm{~kJ} \cdot \mathrm{mol}^{-1}$ nach Boltzmann eine maximale Schwingungstemperatur von $117 \mathrm{~K}$.

Für die Akzeptorbande ergibt sich für $t t_{\mathrm{H}}$ mit einer Verschiebung von $-2 \mathrm{~cm}^{-1}$ eine sehr gute Übereinstimmung mit der experimentellen Bande bei $3498 \mathrm{~cm}^{-1}$, die bereits als mögliche Akzeptorbande diskutiert wurde, während für $t t_{\mathrm{N}}$ eine Blauverschiebung $\mathrm{um} 7 \mathrm{~cm}^{-1}$ berechnet wird. Sowohl der relative Energieunterschied als auch die näher am Experiment liegende, berechnete Donor-Bandenposition spricht für $t t_{\mathrm{N}}$ als das vorherrschende Dimer in der NMFGasphase. Allerdings ist die Vorhersage einer Blauverschiebung für die freie NH-Streckschwingung unrealistisch, so dass hier die Rechnung $\mathrm{zu} t_{\mathrm{H}}$ die bessere Übereinstimmung mit dem Experiment liefert (siehe auch Abb. 5-9).

In der Literatur wurde von Desfrançois et al. [45] in einer empirischen Rechnung $t t_{\mathrm{N}}$ als stabilstes trans-trans-Dimer bestimmt. Zu der Argon-Matrix-Studie von Crespo-Otero et al. [267] gehört auch eine gründliche Suche nach möglichen Dimerstrukturen auf B3LYP-D3/aVTZ-Niveau, die nicht nur $t t_{\mathrm{N}}$ um $1.3 \mathrm{~kJ} \cdot \mathrm{mol}^{-1}$ stabiler als $t t_{\mathrm{H}}$ vorhersagt, sondern auch die in Abbildung 5-12 dargestellten gleichen Geometrien für das gemischte $c t$-Dimer und das zyklische $c c$ Dimer findet. Die oben bereits diskutierte Präferenz im $t t$-Dimer für die H-seitige Koordination am Akzeptor-Sauerstoffatom in Rechnungen ohne Dispersionskorrektur stimmt mit den B3LYP- 
Rechnungen von Tan et al. [268] und Albrecht et al. [51] überein. Die wenigen in der Literatur durchgeführten Frequenzrechnungen zu NMF-Dimeren sind in Tabelle 5-10 zusammengefasst.

Unter der Voraussetzung, dass die von Lee [256] bei $3177 \mathrm{~cm}^{-1}$ im FTIR-Spektrum gefundene Bande sich tatsächlich der antisymmetrischen NH-Streckschwingung im cc-Dimer zuordnen lässt und sich die Überschätzung der Rotverschiebung durch die B3LYP-D3(BJ)-Rechnung von somit $47 \mathrm{~cm}^{-1}$ (vgl. Tab. 5-12) auch auf die entsprechend Raman-aktive, symmetrische NHStreckschwingung übertragen lässt, wäre die gesuchte $c c$-Dimerbande um $3123 \mathrm{~cm}^{-1} \mathrm{zu}$ finden. Dies liegt sehr nahe an der bereits dem $t$-Monomer zugeordneten Bande bei $3113 \mathrm{~cm}^{-1}$. Tatsächlich findet sich im Spektrum in Abb. 5-8 eine sehr kleines, breites Signal bei $3123 \mathrm{~cm}^{-1}$. Eine Überprüfung dieser Zuordnung durch eine Normierung der Spektren auf diese Bande ist aufgrund der geringen Intensität nicht möglich. Daher kann aufgrund der stark vereinfachten Annahmen keine eindeutige Zuordnung zum $c c$-Dimer getroffen werden. Weiterhin steht der möglichen $c c$-Dimerbande im FTIR bei $3177 \mathrm{~cm}^{-1}$ ein schwaches Signal bei $3178 \mathrm{~cm}^{-1}$ im Raman-Jet gegenüber, wobei es sich aber aufgrund des für $C_{2 \mathrm{~h}}$-symmetrische Moleküle geltenden Alternativverbots nicht um die antisymmetrische NH-Streckschwingung handeln kann.

Von den vielen weiteren Banden in Abbildung [5-8 lässt sich keine eindeutig dem gemischten Dimer zuordnen.

Tab. 5-12. Berechnete Normalmoden der $t t-$, $c t$ - und $c c$-Dimere von NMF für die $\mathrm{N}-\mathrm{H}$ - und $\mathrm{C}=\mathrm{O}-\mathrm{Streckschwingung}$ des Donor- und Akzeptormoleküls auf B3LYP-D3(BJ)/aVTZ-Niveau und Zuordnung zu den experimentellen RamanBanden $\left(\tilde{v}_{\mathrm{Ra}}\right)$ und FTIR-Banden $\tilde{v}_{\mathrm{IR}}$. Relative Verschiebungen zur entsprechenden Monomerbande stehen in runden Klammern. In eckigen Klammern stehen experimentelle Werte, deren Zuordnung nicht eindeutig geklärt ist. Angegeben sind zudem die harmonischen Wellenzahlen $\omega$, die IR-Intensität $A$ und der differentielle Raman-Streuquerschnitt $\sigma^{\prime}$ bei $532.274 \mathrm{~nm}$ Anregungswellenlänge und $90^{\circ}$ Streugeometrie nach GI. 3-13. In Klammern ist die relative Verschiebung zur harmonischen Wellenzahl des entsprechenden Monomerkonformers angegeben. Die Buchstaben „a“ und „d“ im Index stehen für Akzeptor und Donor und wechseln für das zyklische cc-Dimer zu „as“ und „" für die antisymmetrische und symmetrische Streckschwingung (Weitere Daten der Frequenzrechnungen befinden sich in den Tab. A-20 und A-21 im Tabellenwerk in Anhang A.8).

\begin{tabular}{|c|c|c|c|c|c|c|}
\hline \multicolumn{2}{|c|}{ Konformer } & $\omega / \mathrm{cm}^{-1}$ & $A / \mathrm{km} \cdot \mathrm{mol}^{-1}$ & $10^{34} \sigma^{\prime} / \mathrm{m}^{2} \mathrm{sr}^{-1}$ & $\tilde{v}_{\mathrm{Ra}} / \mathrm{cm}^{-1}$ & $\tilde{v}_{\mathrm{IR}} / \mathrm{cm}^{-1}$ \\
\hline \multicolumn{7}{|c|}{ NH-Streckschwingungen: } \\
\hline \multirow[t]{3}{*}{$v_{\mathrm{NH}-\mathrm{a}}$} & $t t_{\mathrm{H}}$ & $3627(-2)$ & 35 & 1.06 & \multirow{2}{*}[3498(-3)]{} & - \\
\hline & $t t_{N}$ & $3636(+7)$ & 37 & 1.20 & & - \\
\hline & $c t$ & $3629( \pm 0)^{\mathrm{a}}$ & 33 & 1.02 & - & - \\
\hline \multirow[t]{3}{*}{$v_{\mathrm{NH}-\mathrm{d}}$} & $t t_{\mathrm{H}}$ & $3452(-177)$ & 662 & 3.37 & \multirow{2}{*}{$3395(-106)$} & \multirow{2}{*}{$3394(-107)^{\mathrm{c}}$} \\
\hline & $t t_{N}$ & $3492(-137)$ & 511 & 2.13 & & \\
\hline & $c t$ & $3312(-282)^{b}$ & 766 & 3.40 & - & - \\
\hline \multirow{2}{*}{$\begin{array}{l}v_{\mathrm{NH}-\mathrm{as}} \\
v_{\mathrm{NH}-\mathrm{s}}\end{array}$} & \multirow{2}{*}{$c c$} & $3265(-329)$ & 1595 & 0 & - & \multirow{2}{*}{$\begin{array}{l}{[3177(-282)]^{\mathrm{C}}} \\
-\end{array}$} \\
\hline & & $3211(-383)$ & 0 & 5.33 & - & \\
\hline \multicolumn{7}{|c|}{ CO-Streckschwingungen: } \\
\hline \multirow[t]{3}{*}{$v_{\mathrm{CO}-\mathrm{d}}$} & $t t_{\mathrm{H}}$ & $1755(-12)$ & 240 & 0.32 & \multirow{2}{*}[1729(-15)]{$^{d}$} & \multirow{2}{*}[1729(-15)]{$^{d}$} \\
\hline & $t t_{N}$ & $1756(-11)$ & 305 & 0.31 & & \\
\hline & $c t$ & $1761(-13)^{b}$ & 717 & 0.19 & - & - \\
\hline \multirow[t]{3}{*}{$v_{\mathrm{CO}-\mathrm{a}}$} & $t t_{\mathrm{H}}$ & $1741(-26)$ & 656 & 0.81 & \multirow{2}{*}[1715(-29)]{$^{d}$} & \multirow{2}{*}[1715(-29)]{$^{d}$} \\
\hline & $t t_{N}$ & $1743(-24)$ & 527 & 0.53 & & \\
\hline & $c t$ & $1709(-58)^{\mathrm{a}}$ & 160 & 0.86 & [1684] & - \\
\hline$v_{\text {CO-as }}$ & \multirow{2}{*}{$c c$} & $1762(-12)$ & 1082 & 0 & - & - \\
\hline$v_{\mathrm{CO}-\mathrm{s}}$ & & $1724(-50)$ & 0 & 0.48 & - & - \\
\hline
\end{tabular}


Die Frequenzrechnung ergibt für die beiden $t t$-Dimere jeweils für Akzeptor- und Donor-COStreckschwingung fast die gleichen Bandenpositionen mit ähnlich großen IR-Intensitäten bzw. Raman-Aktivitäten, sodass auch der Vergleich der skalierten harmonischen Frequenzen (Faktor 0.987) mit den FTIR- und Raman-Jetspektren der Amid I-Region in Abbildung 5-11 keine Präferenz für eines der beiden Isomere zeigt. Deren skalierte Schwingungswellenzahlen berechnen sich zu $(1733 \pm 1) \mathrm{cm}^{-1}$ für die CO-Streckschwingung des Wasserstoffbrückendonors (freie Carbonylgruppe) und $(1719 \pm 1) \mathrm{cm}^{-1}$ für diejenige des -akzeptors. In Anbetracht der üblichen Überschätzung der Rotverschiebung von Clustern durch die B3LYP-Rechnung, wird die oben getroffene Zuordnung der beiden Dimerbanden bei 1715 und $1707 \mathrm{~cm}^{-1}$ als Donor- und Akzeptorbande des $t t$-Isomers nicht bestätigt. Vielmehr deuten Verschiebungen von $-11 \mathrm{~cm}^{-1}$ und $-12 \mathrm{~cm}^{-1}$ für die Donor- sowie $-24 \mathrm{~cm}^{-1}$ und $-26 \mathrm{~cm}^{-1}$ für die Akzeptorbande aus den harmonischen Frequenzrechnungen auf eine Zuordnung zu dem, als Überlappung von heißer Bande und Dimerbande interpretierten, Signal bei $1729 \mathrm{~cm}^{-1}\left(-15 \mathrm{~cm}^{-1}\right)$ und $\mathrm{zu}$ der Bande bei $1715 \mathrm{~cm}^{-1}\left(-29 \mathrm{~cm}^{-1}\right)$.

Für das eigentlich gesuchte $c c$-Dimer sagt die Frequenzrechnung mit $-12 \mathrm{~cm}^{-1}$ eine nur schwach verschobene Bande für die IR-aktive Mode der CO-Streckschwingung voraus, die, wenn überhaupt vorhanden, dann vollständig von der breiten und intensiven Amid I-Bande des IR-Spektrums überdeckt wird. Für die komplementäre Schwingung im Raman-Spektrum wird eine harmonische Schwingungswellenzahl von $1724 \mathrm{~cm}^{-1}$ berechnet und wird somit um $-50 \mathrm{~cm}^{-1}$ gegenüber dem cis-Monomer verschoben vorhergesagt. Dies entspricht einer skalierten Wellenzahl von $1702 \mathrm{~cm}^{-1}$, was unter Berücksichtigung der bereits angesprochenen Überschätzung der Rotverschiebung in guter Übereinstimmung mit der $1707 \mathrm{~cm}^{-1}$-Bande im Raman-Spektrum ist. Dieser Bande wurde weiter oben bereits als Akzeptorbande der CO-Streckschwingung des $t t$-NMF zugeordnet. Die voraussichtliche Bandenposition des cc-Dimers fällt also mit einer Bande des in der Expansion statistisch häufiger gebildeten tt-NMF-Clusters zusammen und wird daher ebenfalls nicht nachzuweisen sein.

Der skalierte Wert der Normalschwingung des trans-Akzeptors von ct-NMF liegt bei $1687 \mathrm{~cm}^{-1}$. Dieses Dimer hat ähnlich zum cc-Dimer eine zyklische Struktur, die neben der NH ‥ OC-Wasserstoffbrücke eine zweite lokale intermolekulare Wechselwirkung zwischen der CH-Gruppe des trans-Konformers und der Carbonylgruppe des cis-Konformers besitzt (siehe Abb. 5-12). Dies führt, trotz der $C_{1}$-Symmetrie des Dimers, zu lokalen Symmetrien der Normalmoden von NHund CO-Streckschwingung und damit zu einer Unterscheidung in dominant IR- und Ramanaktive Moden. Daher ist die zweite CO-Streckbande hauptsächlich Raman-aktiv, was in den Strichspektren in Abbildung 5-11 dargestellt ist. Hier findet sich mit einer Bande bei $1684 \mathrm{~cm}^{-1}$ eine mögliche Entsprechung im Raman-Spektrum. Dies ist zwar in sehr guter Übereinstimmung mit der Theorie, aber alleine zu wenig für eine sichere Zuordnung.

\subsection{Trimere und höhere Aggregate}

Sowohl die Spektren der Amid I-Bande als auch die im N-H-Streckbereich zeigen einige weitere Signale, wie z. B. die große, breite Bande bei ca. $3250 \mathrm{~cm}^{-1}$ im Raman-Jetspektrum in Abbil- 
dung 5-8, die ihren Ursprung in größeren Clustern haben können. In diesem Abschnitt soll der Schwerpunkt auf die nächstgrößeren Aggregate, nämlich die NMF-Trimere gelegt werden. Dazu werden die entsprechenden Spektren in den Abbildungen des vorherigen Abschnitts auf die identifizierten Dimerbanden normiert, um Banden größerer Aggregate aufzudecken.

Amid A Diese Darstellung erfolgt für die Raman-Spektren im NH-Streckbereich in Abbildung 5-13 durch eine Normierung auf die $t t$-Dimerbande bei $3395 \mathrm{~cm}^{-1}$. Auf deren niederfrequenter Seite sind zwei Banden bei 3367 und $3338 \mathrm{~cm}^{-1}$ erkennbar, die mit zunehmender Konzentration (von Spektrum (a) bis (e)) an Intensität zunehmen, wobei Wachstum und Form der Bande bei $3338 \mathrm{~cm}^{-1}$ darunterliegende Banden größerer Cluster nahelegen. Die Bande bei $3367 \mathrm{~cm}^{-1}$ wurde im vorherigen Abschnitt aufgrund analoger Verschiebungen im NMA als $\mathrm{NH}$-Streck-Donorbande des $t t_{\mathrm{H}}$-Dimers zugeordnet. Die vorliegende Darstellung belegt, dass hier Cluster größer als Dimere vorliegen, weshalb eine Interpretation als $t t_{\mathrm{H}}$-Dimer zumindest unvollständig ist. Dies entspricht einer Rotverschiebung von 134 und $163 \mathrm{~cm}^{-1}$ gegenüber dem trans-Monomer und lediglich 28 und $57 \mathrm{~cm}^{-1}$ gegenüber dem $t t$-Dimer und ist deutlich geringer, als aufgrund des kooperativen Effekts in Wasserstoffbrücken-gebundenden Amidketten [195, 225] für Trimere zu erwarten ist. Dies spricht, analog zu den Ergebnissen zu NMA, ebenfalls für ein zyklisches Trimer. Bei den drei NMF-Molekülen sollten sich gegenüber NMA aufgrund der fehlenden Methylgruppe aus sterischen Gründen die intermolekularen Abstände verringern und damit die Ringspannung eines zyklischen NMF-Trimers erhöhen. Auch für NMF wären durch eine parallele und eine antiparallele Anordnung von drei trans-Monomermolekülen zwei Trimere denkbar, die durch einen geringen Energieunterschied beide in der Jet-Expansion populiert sein können und ursächlich für die beiden gefundenen Banden sind. Daher wird zunächst einmal von einem zyklischen $t t t$-Trimer bei NMF ausgegangen.

Amid I-III Das FTIR-Spektrum im CO-Streckbereich (Abb. 5-14, oben) ist auf das Dimersignal bei $1715 \mathrm{~cm}^{-1}$ normiert. Dabei ist ein Wachstum der Bande bei $1707 \mathrm{~cm}^{-1}$ mit zunehmender NMF-Konzentration erkennbar. Dies deutet auf ein Trimersignal hin, das intensitätsmäßig unterhalb der bereits den Dimeren zugeordneten Bande liegt und unterstützt die Hypothese eines zyklischen Trimers mit Ringspannung, da in diesem Fall keinerlei Verschiebung zum Dimer vorliegt.

In der gleichen Abbildung unten befindet sich das auf die Dimersignale normierte RamanSpektrum. Es ist erkennbar, dass die Bande bei $1674 \mathrm{~cm}^{-1}$ mit steigender Sättigertemperatur und damit auch zunehmender NMF-Konzentration insbesondere für die höchste Konzentration (cyanfarbenes Spektrum) an Intensität gewinnt. Auch die Bande bei $1684 \mathrm{~cm}^{-1}$ zeigt eine geringe Zunahme, was bedeuten würde, dass die Schwingungswellenzahl der CO-Streckschwingung eine Rotverschiebung von 55 und $45 \mathrm{~cm}^{-1}$ gegenüber dem trans-Monomer erfährt.

Der Vergleich mit dem zyklischen NMA-Trimer, dessen Verschiebung bei $-59 \mathrm{~cm}^{-1}$ für die asymmetrische CO-Streckschwingung liegt (siehe Tab. 4-13), ist ein Indiz für eine Zuordnung vor allem der Bande bei $1674 \mathrm{~cm}^{-1}$ als Signal eines zyklischen NMF-Trimers.

Der große Unterschied in der Trimer-Bandenposition zwischen dem FTIR- und dem Raman- 


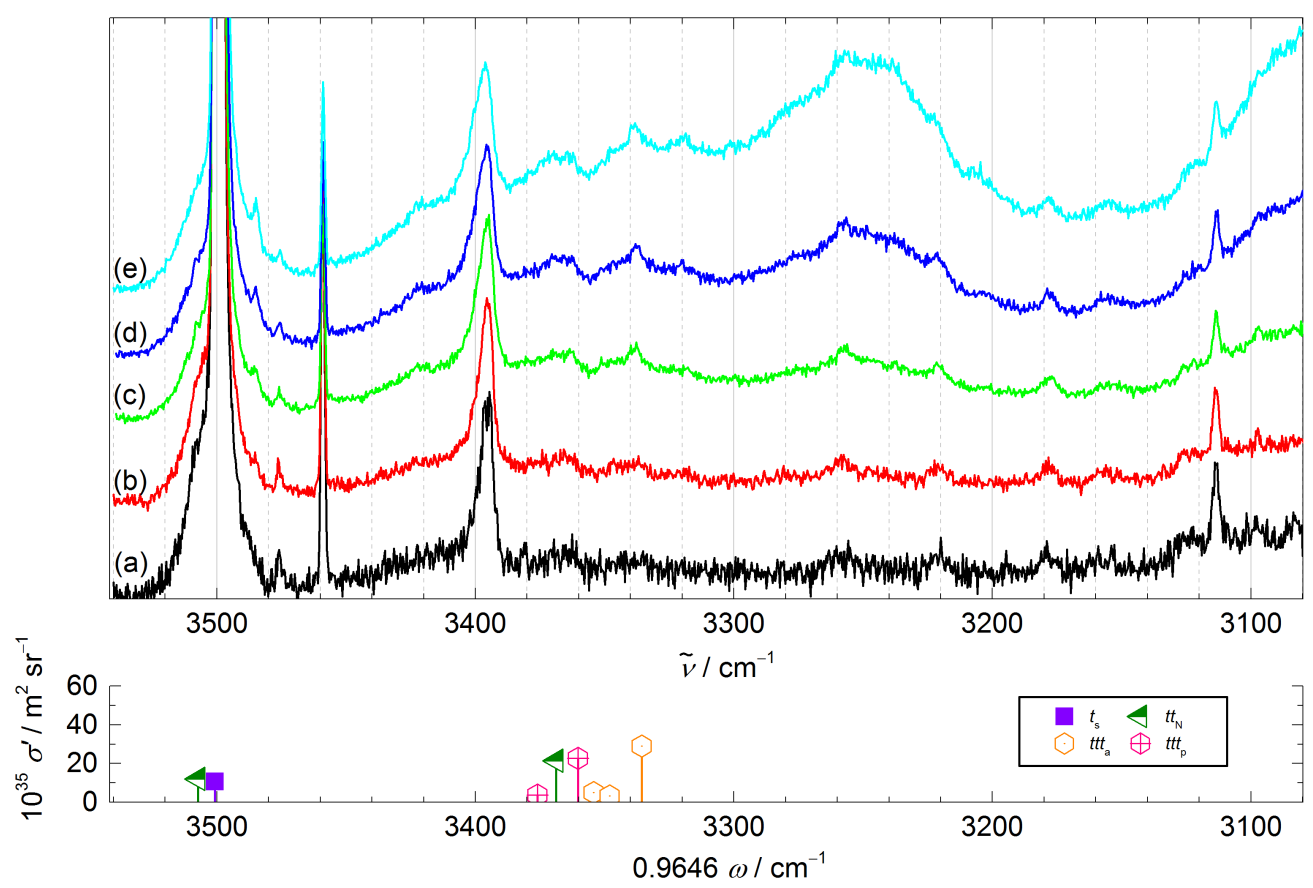

Abb. 5-13. Auf das $t t$-Dimer von NMF normierte Raman-Jetspektren in der Amid A-Region zum Nachweis möglicher Trimerbanden. Gleiche Spektren und Rechnungen wie in Abb. 5-8 jedoch auf die $v_{A}^{t t}$-Bande bei $3395 \mathrm{~cm}^{-1}$ normiert. (Ausführliche Informationen zu den Messbedingungen befinden sich in Tab. A-6 im Raman-Spektrenverzeichnis in Anhang A.6.1 Details zu den quantenchemischen Rechnung sind in den Tabellen 5-11 und A-20 enthalten.)

Spektrum von $33 \mathrm{~cm}^{-1}$ spricht ebenfalls für ein zyklisches Trimer, dessen lokale Symmetrien der CO-Streckschwingung für eine Art Alternativverbot sorgen. Es sollte sich also im Spektrum, genauso wie beim NMA-Trimer, zwischen einer Raman-aktiven, symmetrischen Normalmode und zwei IR-aktiven, asymmetrischen Normalmoden unterscheiden lassen.

Dabei sei angemerkt, dass eine direkte Vergleichbarkeit von NMF und NMA nur bedingt gegeben ist, da insbesondere in kleinen zyklischen Aggregaten mit hoher Ringspannung der sterische Einfluss des Einzelmoleküls eine signifikante Rolle spielt. Inwieweit die fehlende Methylgruppe im NMF Einfluss auf die Wasserstoffbrückenbindungslänge $d$ und den intermolekularen Bindungswinkel $\alpha$ und damit auch indirekt auf die Verschiebung der an der Wasserstoffbrücke beteiligten Amidbanden hat, wird nun unter Einbeziehung der Ergebnisse quantenchemischer Rechnungen analysiert.

Quantenschemische Rechnungen Da es bisher keine experimentellen Daten zu Trimeren von NMF gibt, wird die weitere Interpretation der Spektren auf den Vergleich mit NMA und die Ergebnisse harmonischer Frequenzrechnungen aufgebaut. Die Frequenzrechnungen auf B3LYPD3(BJ)/aVTZ-Niveau wurden auch für das NMF-Trimer mit und ohne Dispersionskorrektur durchgeführt. Dabei ergeben sich die beiden vermuteten zyklischen Trimere, die hier analog zu NMA als $t t t_{\mathrm{p}}$ und $t t t_{\mathrm{a}}$ entsprechend einer parallelen (p) oder antiparallelen (a) Ausrichtung der Amidgruppen zueinander bezeichnet werden. Zusammen mit einer möglichen Konforma- 

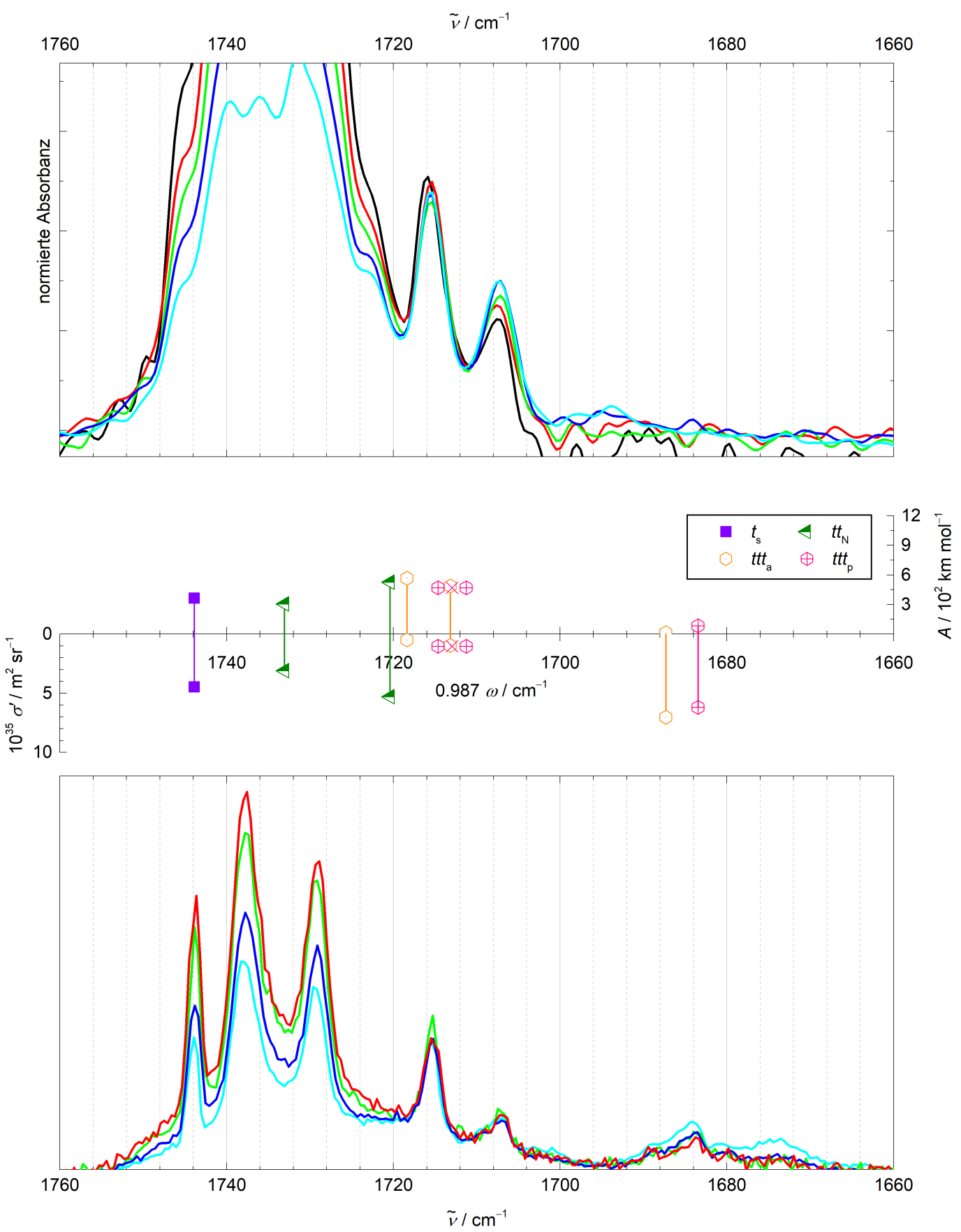

Abb. 5-14. Auf das $t t$-Dimer von NMF normierte FTIR- (oben) und Raman-Jetspektren (unten) in der Amid I-Region zum Nachweis möglicher Trimerbanden. Gleiche Spektren wie in Abb. 5-11] jedoch auf die $v_{1}^{t t}$-Bande normiert (Details zu den Messungen den Frequenzrechnungen, siehe ebenda.). Die Strichspektren in der Mitte entsprechen denen in Abb. 5-11 ergänzt um die skalierten Frequenzen der CO-Streckschwingungsmoden in den zyklischen Trimeren $t t t_{a}$ und $t t t_{p}$ auf B3LYP-D3(BJ)/aVTZ-Niveau. Die Darstellung von $t t t_{p}$ im Strichspektrum visualisiert die zweifache Entartung der zugrundeliegenden Schwingungsmode. Deren tatsächliche Position wird durch ein kleines $\mathrm{x}$ zwischen den beiden Symbolen gekennzeichnet. 


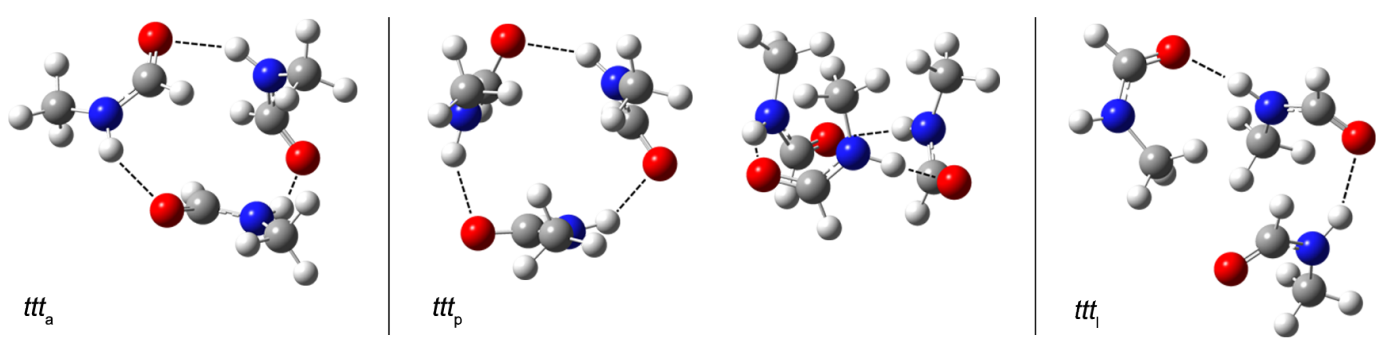

Abb. 5-15. Molekülstrukturen der beiden zyklischen trans-NMF Trimere $t t t_{a}$ und $t t t_{p}$ und des linearen Trimers $t t t_{\text {| }}$ auf B3LYP-D3(BJ)/aVTZ-Niveau. Die Amidgruppen der Monomereinheiten sind dabei entweder gleich ausgerichtet (parallel, $\left.t t t_{p}\right)$ und es liegt $C_{3}$-Symmetrie vor oder eines der drei Monomere hat eine antiparallele Ausrichtung $\left(t t t_{a}\right)$. Relative Energien, Dissoziationsenergien und geometrische Parameter sind in Tabelle 5-13 aufgeführt.

tion für ein lineares Trimer sind die Strukturen in Abbildung 5-15 dargestellt. Die optimierte Geometrie des $t t t_{\mathrm{p}}$-NMF besitzt, wie das entsprechende NMA-Trimer, $C_{3}$-Symmetrie. Durch die nicht vorhandene Methylgruppe am Carbonyl-Kohlenstoffatom und die daraus resultierende verminderte sterische Hinderung bei der Clusterbildung führt dies bei $t t t_{\mathrm{a}}$-NMF zu einer asymmetrischen zyklischen Struktur in der ein NMF-Molekül nach außen ragt und die für $t t t_{\mathrm{a}}$-NMA berechnete, fassartige Struktur aufgibt. Wie stark die Verzerrung der Fassstruktur im $t t t_{\mathrm{a}}-\mathrm{NMF}$ ist, wird auch durch die Bindungswinkel $\alpha$ verdeutlicht, die sich für das NMF-Trimer um $13^{\circ}$ unterscheiden (siehe Tab.5-13), während der Unterschied bei NMA nur bei $6^{\circ}$ liegt.

Die Struktur von $t t_{1}$-NMF wird nur aufgrund einer einheitlichen Nomenklatur als „linear“ bezeichnet, auch wenn diese hier stark gekrümmt ist, was durch Dispersionswechselwirkungen der Methylgruppen mit dem freien Sauerstoffatom stabilisiert wird. Dies wird aus der gleichen Rechnung ohne Dispersionskorrektur ersichtlich, die für das stabilste offenkettige Trimer eine deutlich „linearere“ Struktur ergibt, die ohne Dispersionskorrektur ein Dipolmoment von 13.6 D aufweist, während die in Abbildung 5-15 abgebildete Struktur ein Dipolmoment von 4.2 D besitzt (siehe Tab.5-13).

Tab. 5-13. Berechnete Eigenschaften der zyklischen NMF-Trimere $t t t_{a}$ und $t t t_{p}$, sowie des linearen Trimers $t t t_{1}$ auf B3LYP/aVTZ-Niveau mit und ohne Dispersionskorrektur (zugehörige Strukturen befinden sich in Abb. 5-15. Für eine Erklärung der Bezeichnungen, siehe Tab. 5-11

\begin{tabular}{|c|c|c|c|c|c|c|}
\hline & \multicolumn{2}{|l|}{$t t t_{\mathrm{a}}$} & \multicolumn{2}{|l|}{$t t t_{p}$} & \multicolumn{2}{|l|}{$t t t_{\mid}$} \\
\hline & B3LYP-D3(BJ) & B3LYP & B3LYP-D3(BJ) & B3LYP & B3LYP-D3(BJ) & B3LYP \\
\hline$\Delta E_{\mathrm{el}} / \mathrm{kJ} \cdot \mathrm{mol}^{-1}$ & 0.0 & 0.0 & 3.3 & 4.2 & 9.7 & 4.0 \\
\hline$\Delta E_{0} / \mathrm{kJ} \cdot \mathrm{mol}^{-1}$ & 0.0 & 0.0 & 2.0 & 3.0 & 8.6 & 1.4 \\
\hline$D_{\mathrm{el}} / \mathrm{kJ} \cdot \mathrm{mol}^{-1}$ & 92.2 & 59.8 & 89.0 & 55.5 & 82.6 & 55.8 \\
\hline$D_{0} / \mathrm{kJ} \cdot \mathrm{mol}^{-1}$ & 80.4 & 49.7 & 78.3 & 46.7 & 71.8 & 48.3 \\
\hline \multirow[t]{3}{*}{$\alpha(\mathrm{H} \cdots \mathrm{O}=\mathrm{C}) /{ }^{\circ}$} & 112 & 112 & 106 & 111 & 112 & 151 \\
\hline & 105 & 104 & & & 139 & 133 \\
\hline & 99 & 106 & & & - & - \\
\hline \multirow[t]{3}{*}{$d(\mathrm{H} \cdots \mathrm{O}) / \mathrm{pm}$} & 200 & 218 & 202 & 216 & 198 & 194 \\
\hline & 204 & 212 & & & 188 & 193 \\
\hline & 204 & 211 & & & - & - \\
\hline$\tilde{v}_{\min } / \mathrm{cm}^{-1}$ & 20 & 21 & 10 & 3.8 & 27 & 6.8 \\
\hline$\mu / D$ & 1.0 & 0.9 & 3.1 & 2.3 & 4.2 & 13.6 \\
\hline
\end{tabular}


Die Gegenüberstellung der zwei zyklischen Trimere und des stabilsten „linearen“ Trimers von NMF in der Tabelle ergibt mit den dispersionskorrigierten B3LYP-Rechnungen $t t t_{\mathrm{a}}$ als stabilste Trimerstruktur. Es ist bezogen auf die Nullpunktsenergie um $2.0 \mathrm{~kJ} \cdot \mathrm{mol}^{-1}$ stabiler als das $t t t_{\mathrm{p}}$-Trimer und um ca. $9 \mathrm{~kJ} \cdot \mathrm{mol}^{-1}$ stabiler als die offenkettige Struktur. Anders als für NMA wird $t t t_{1}$ unter Vernachlässigung der Dispersionskorrektur nicht zum stabilsten Trimer, allerdings reduziert sich der Unterschied zu $t t t_{\mathrm{a}}$ auf $1.4 \mathrm{~kJ} \cdot \mathrm{mol}^{-1}$ und ist somit stabiler als das $C_{3^{-}}$symmetrische zyklische Isomer. Während sich die beiden zyklischen Isomere bezüglich des Bindungswinkels $\alpha$ und der Wasserstoffbrückenlänge $d$ kaum unterscheiden, wenn für die asymmetrische Struktur der jeweilige Mittelwert betrachtet wird, führt die Dispersionskorrektur generell zu kleineren Werten für $\alpha$ und größeren Bindungsabständen. Die Dispersionskorrektur sorgt also erwartungsgemäß für eine stärkere intermolekulare Wechselwirkung, reduziert also Molekülabstände und erhöht die Ringspannung.

Die Struktur von NMF-Trimeren findet in der Literatur nur geringe Beachtung. Es gibt eine Reihe von Arbeiten, die die supramolekulare Struktur von flüssigem NMF sowohl experimentell als auch theoretisch untersucht haben [49, 244, 245, 269, 270], wobei jedoch nur vereinzelt auf lokale Strukturen eingegangen wird. So werden in der Arbeit von Hammami et al. [270] in einer Kombination von Röntgen-Streuexperimenten und DFT-Rechnungen für das NMF-Trimer drei stabile Strukturen gefunden. Dabei bilden drei cis-NMF-Moleküle ein zyklisches Trimer als stabilste Struktur, während für ttt-NMF nur eine lineare Struktur postuliert wird. Dies deckt sich mit einfachen $a b$ initio-Rechnungen (Restricted Hartree-Fock mit 3-21G-Basissatz), die für trans-NMF erst ab einer Clustergröße von fünf Molekülen eine zyklische Struktur vorschlagen [49].

Ein potentiell zyklischer ttt-NMF-Cluster wird erstmals von Desfrançois et al. [45] am Rande einer Studie an Amid-Clustern im Molekularstrahl mit der Rydberg-Elektronen-Transfermethode erwähnt. Hier ist von einem ttt-Trimer mit einem permanenten Dipolmoment $\mu=0.36 \mathrm{D}$ die Rede, was auf eine zyklische Form hindeutet. Tan et al. [268] haben eine systematische Untersuchung des kooperativen Effekts in NMF-Oligomeren auf B3LYP/VTZ-Niveau durchgeführt, ohne jedoch auf zyklische Strukturen näher einzugehen.

DFT-Rechnungen auf B3LYP/6-31G*-Niveau mit Counterpoise-Korrektur ergeben zyklische Strukturen als stabilste Cluster für alle NMF-Oligomere mit Clustergrößen $n=2-6$ [264]. Hier wird erstmalig das ttt-NMF als sogenanntes head-to-tail-verknüpftes Trimer beschrieben, in der die zwischenmolekularen Wechselwirkungen hauptsächlich auf NH... OC-Wasserstoffbrückenbindungen basieren.

Vergleich von Theorie und Experiment Um die postulierten zyklischen Strukturen weiter zu bestätigen, werden in den Abbildungen 5-13 und 5-14 die Ergebnisse der doppelt-harmonischen Frequenzrechnungen der beiden stabilsten zyklischen Trimerstrukturen $t t t_{\mathrm{a}}$ und $t t t_{\mathrm{p}}$ als skalierte Strichspektren den gemessenen Spektren gegenüber gestellt. In der Amid A-Region liegen lediglich Raman-Jetmessungen vor, so dass nur die Betrachtung der symmetrischen NH-Streckschwingung sinnvoll ist. Die skalierten Werte der Rechnungen liegen bei einer Verschiebung von $-171 \mathrm{~cm}^{-1}$ für $t t t_{\mathrm{a}}$ und $-145 \mathrm{~cm}^{-1}$ für $t t t_{\mathrm{p}}$ und entsprechen damit sehr gut 
den Signalen bei $3338 \mathrm{~cm}^{-1}$ und $3367 \mathrm{~cm}^{-1}$ im Raman-Spektrum, deren Rotverschiebung sie erwartungsgemäß überschätzen (siehe Tab. 5-14). Dies bestätigt die oben getroffene erste Zuordnung der beiden Banden als Trimerbanden von NMF und ist zudem konsistent mit den Ergebnissen aus der Geometrieoptimierung in Tabelle 5-13, aus denen für das $t t t_{\mathrm{a}}$-Trimer aufgrund des stabileren Clusters die intensivere und aufgrund der geringeren Ringspannung eine stärker rotverschobene Bande zu erwarten wäre. Auffällig ist, dass die berechneten und skalierten Frequenzen für die Trimere wesentlich besser zum Spektrum passen, als die für das $t t_{N^{-}}$ Dimer (siehe Abschnitt „Dimere“), wo die Rotverschiebung deutlich überschätzt wird.

Tab. 5-14. Berechnete Normalmoden der zyklischen $t t$-Trimere von NMF für die $\mathrm{N}-\mathrm{H}$ - und $\mathrm{C}=\mathrm{O}-\mathrm{Streckschwingung}$ des Donor- und Akzeptormoleküls auf B3LYP-D3(BJ)/aVTZ-Niveau und Zuordnung zu den experimentellen Raman-Banden $\left(\tilde{v}_{R a}\right)$ und FTIR-Banden $\tilde{v}_{I R}$ mitsamt relativer Verschiebung zur trans-Monomerbande in Klammern. Die Moden sind in hauptsächlich IR-aktive asymmetrische (as) und hauptsächlich Raman-aktive symmetrische (s) aufgeteilt und beziehen sich auf lokale Symmetrieeffekte in der zyklischen Struktur. Alle weiteren Angaben sind analog zu Tab. 5-12 gewählt. (Weitere Daten der Frequenzrechnungen befinden sich in den Tab. A-20 und A-21 im Tabellenwerk in Anhang A.8).

\begin{tabular}{|c|c|c|c|c|c|c|}
\hline Mode & Konformer & $\omega / \mathrm{cm}^{-1}$ & $A / \mathrm{km} \cdot \mathrm{mol}^{-1}$ & $10^{35} \sigma^{\prime} / \mathrm{m}^{2} \mathrm{sr}^{-1}$ & $\tilde{v}_{\mathrm{Ra}} / \mathrm{cm}^{-1}$ & $\tilde{v}_{\mathrm{IR}} / \mathrm{cm}^{-1}$ \\
\hline \multicolumn{7}{|c|}{ NH-Streckschwingungen: } \\
\hline \multirow[t]{4}{*}{$v_{\mathrm{NH}}^{\mathrm{as}}$} & $t t t_{\mathrm{a}}$ & $3477(-152)$ & 362 & 0.50 & \multirow{4}{*}{-} & \multirow{4}{*}{-} \\
\hline & & $3471(-158)$ & 463 & 0.33 & & \\
\hline & $t t t_{\mathrm{p}}$ & $3500(-129)$ & 377 & 0.38 & & \\
\hline & & $3500(-129)$ & 377 & 0.38 & & \\
\hline \multirow{2}{*}{$v_{\mathrm{NH}}^{\mathrm{S}}$} & $t t t_{\mathrm{a}}$ & $3458(-171)$ & 14 & 2.91 & \multirow{2}{*}{$\begin{array}{l}3338(-163) \\
3367(-134)\end{array}$} & \multirow{2}{*}{-} \\
\hline & $t t t_{\mathrm{p}}$ & $3484(-145)$ & 5 & 2.27 & & \\
\hline \multicolumn{7}{|c|}{ CO-Streckschwingungen: } \\
\hline$v_{\mathrm{CO}}^{\text {as }}$ & $t t t_{\mathrm{a}}$ & $1741(-26)$ & 566 & 0.05 & \multirow{4}{*}{-} & \multirow{4}{*}{$1707(-22)$} \\
\hline & & $1736(-31)$ & 488 & 0.10 & & \\
\hline & $t t t_{\mathrm{p}}$ & $1736(-31)$ & 469 & 0.10 & & \\
\hline & & $1736(-31)$ & 469 & 0.10 & & \\
\hline \multirow{2}{*}{$v_{\mathrm{CO}}^{\mathrm{s}}$} & $t t t_{\mathrm{a}}$ & $1710(-57)$ & 13 & 0.70 & \multirow{2}{*}{\} $1674(-55)$} & \multirow{2}{*}{-} \\
\hline & $t t t_{\mathrm{p}}$ & $1706(-61)$ & 83 & 0.62 & & \\
\hline
\end{tabular}

In der Amid I-Region ergeben sich zum einen zwei asymmetrische CO-Streckschwingungen $v_{\mathrm{CO}}^{\text {as }}$, die nahezu ausschließlich IR-aktiv sind und für das $t t t_{\mathrm{a}}$-Trimer bei $1741 \mathrm{~cm}^{-1}$ und $1736 \mathrm{~cm}^{-1}$ liegen, während für das $t t t_{\mathrm{p}}$ eine zweifach entartete Normalschwingung bei $1736 \mathrm{~cm}^{-1}$ berechnet wird. Zum anderen liegt eine symmetrische Normalmode $v_{\mathrm{CO}}^{\mathrm{s}}$ vor, die hauptsächlich Raman-aktiv ist und mit $1710 \mathrm{~cm}^{-1}$ für $t t t_{\mathrm{a}}$ und $1706 \mathrm{~cm}^{-1}$ für $t t t_{\mathrm{p}}$ mit ca. $59 \mathrm{~cm}^{-1}$ deutlich weiter rotverschoben ist als die $v_{\mathrm{CO}}^{\text {as }}$-Moden (Skalierungsfaktor der Wellenzahlen $x=0.987$; siehe Tab. 5-14).

Die Rechnungen ergeben also die erwartete IR/Raman-Komplementarität der CO-Streckschwingungen und unterstützen die getroffene Zuordnung der Banden bei $1707 \mathrm{~cm}^{-1}$ im FTIRSpektrum und bei $1674 \mathrm{~cm}^{-1}$ im Raman-Spektrum als Trimerbanden durch vergleichbare Rotverschiebungen gegenüber dem $t$-Monomer. Wenn anstatt der relativen Verschiebungen die normierten Wellenzahlen der Rechnungen mit den Bandenpositionen des Experiments verglichen werden, wäre auch eine Zuordnung der Bande bei $1684 \mathrm{~cm}^{-1}$ als Trimerbande legitim.

Die beiden berechneten Konformere $t t t_{\mathrm{a}}$ und $t t t_{\mathrm{p}}$ sind im Spektrum nicht unterscheidbar, wobei aufgrund der Daten in Tabelle 5-13 davon ausgegangen werden kann, dass das $t t t_{\mathrm{a}}{ }^{-}$ 
Trimer das stabilere Konformer ist und somit den Hauptanteil des Trimersignals in den Spektren verursacht.

\subsection{Zusammenfassung und Diskussion}

In diesem Kapitel wird $N$-Methylformamid als das kleinste sekundäre Amid untersucht und damit die Arbeit zu NMA im vorangegangenen Kapitel ergänzt. DFT-Rechnungen auf B3LYPD3(BJ)/aVTZ-Niveau ergeben sowohl für cis- als auch trans-NMF-Monomere die Methylgruppe in syn-Stellung als stabilste Konformation, wobei $t_{\mathrm{s}}$ das globale Minimum darstellt.

Die Raman-spektroskopische Untersuchung des Monomers erfolgte in drei Spektralbereichen: in der Amid A-Region oberhalb von $3000 \mathrm{~cm}^{-1}$ sowie erstmalig in der Region der Amidbanden I-III zwischen 1800 und $1100 \mathrm{~cm}^{-1}$ und im niederfrequenten Bereich zwischen 1000 und $200 \mathrm{~cm}^{-1}$. Dabei lassen sich die Banden in den Spektren durch den Vergleich mit Literaturwerten und den Ergebnissen skaliert doppelt-harmonischer Frequenzrechnungen eindeutig den Amidmoden A, I, III und IV zuordnen. Für alle genannten Banden bis auf Amid I ist das weniger stabile cis-Konformer im Spektrum neben dem dominanten trans-Konformer erkennbar.

Durch die gute Auflösung und das sehr gute Signal-zu-Rauschen-Verhältnis ist in der Amid ABande und der Amid-I-Bande eine Substruktur erkennbar, die mithilfe von Temperaturreihen auf heiße Banden zurückgeführt werden können. Dabei erweisen sich anharmonische Frequenzvorhersagen auf Basis der VPT2-Methode trotz ausschließlich reeller Frequenzen durch deutliche Abweichungen der berechneten Verschiebung von der experimentellen Bandenposition als wenig hilfreich. Die Probleme von VPT2-Rechnungen bei Molekülen mit sehr flachen Torsionspotentialen, wie es z. B. bei Methylgruppen der Fall ist, wurden bereits in der Diskussion für NMA ausgeführt (siehe Abschnitt 4.5) und bekommen hier aufgrund der im Vergleich zu NMA niedrigeren Barriere der $N$-Methylgruppe eine noch größere Bedeutung.

Die Raman-Messungen im niederfrequenten Bereich zeigen erstmals eine echte Amid IV-Bande des cis-NMF. Da diese Bande als Normalschwingung in der Molekülebene laut der durchgeführten Frequenzrechnungen nahezu keine IR-Aktivität besitzt, kann sie nicht der Bande in den IR-Spektren aus der Literatur entsprechen. Hierbei handelt es sich vielmehr um die Bande einer IR-aktiven Normalmode, die einer Torsionsschwingung entspricht und somit laut Definition keine Amidbande darstellt, allerdings im Spektrum an einer ähnlichen Position zu finden ist.

Das cis-/trans-Isomerisierungsgleichgewicht ist im Vergleich zu NMA bei NMF mit 8-15\% (siehe Abschnitt 5.2) deutlich weiter Richtung cis-NMF verschoben, weshalb die cis-Signale auch bei niedrigen Temperaturen noch gut erkennbar sind. Eine zu NMA analoge Ermittlung des Enthalpieunterschieds aus dem Van-'t-Hoff-Plot wird hier nur für die Amid A-Bande durchgeführt und ergibt für zwei Messungen unterschiedlicher Temperaturbereiche eine Differenz von 5(1) bzw. 6(2) kJ.mol ${ }^{-1}$. Die sehr hohe relative Messungenauigkeit ist auf die konservative Fehlerabschätzung bei der Integration der Peakflächen zurückzuführen, die sich im fehlergewichteten Van-'t-Hoff-Plot niederschlagen. Dennoch liegen die beiden Ergebnisse der getrennt ausgewerteten Messreihen innerhalb der Messungenauigkeit des jeweils anderen. Sie übertreffen allerdings leicht den Wert aus der harmonischen DFT-Rechnung von $4.4 \mathrm{~kJ} \cdot \mathrm{mol}^{-1}$. Die 
Schwächen dieser Methode zur Enthalpiebestimmung wurden bereits in der Diskussion für NMA ausgeführt.

Für das Dimer als kleinstmögliches Molekülaggregat ergeben die DFT-Rechnungen die zwei fast isoenergetischen trans-trans-Dimere, $t t_{\mathrm{N}}$ und $t t_{\mathrm{H}}$, das mit Abstand stabilste $c c$-Dimer und das gemischte $c t$-Dimer.

Es wurden FTIR- und erstmalig auch Ramanspektren der Amid A- und Amid I-Bande von NMF unter clusterbildenden Expansionsbedingungen aufgenommen. Die Variation der NMFKonzentration bei konstanter Düsentemperatur liefert Spektrenserien, aus denen durch den Vergleich mit FTIR-Literaturwerten und den Frequenzrechnungen eine eindeutige Zuordnung einzelner Peaks als tt-Dimerbanden hervorgeht. Aufgrund eines nullpunktskorrigierten energetischen Unterschieds von $1.4 \mathrm{~kJ} \cdot \mathrm{mol}^{-1}$ dominiert in der Amid A-Region des Ramanspektrums die $t t_{\mathrm{N}}$-Donorbande. Der Vergleich mit den Clusterspektren und harmonischen Frequenzrechnungen auf B3LYP-D3(BJ)/aVTZ-Niveau von NMA ermöglicht eine vorläufige Zuordnung der Donorbande des $t t_{\mathrm{H}}-\mathrm{NMF}$ und eine grobe Abschätzung des $t t_{\mathrm{H}}$-Dimeranteils. Die in Abschnitt 5.3 gemachte Abschätzung liegt zu hoch, da Banden höherer Cluster mit einbezogen wurden, die sich mit der $t t_{\mathrm{H}}$-Bande überlagern (siehe Abschnitt 5.4). Eine erneute Abschätzung, diesmal mit einem Spektrum, das kein intensives Signal bei $3338 \mathrm{~cm}^{-1}$ hat und somit kaum Trimersignale aufweist (schwarzes Spektrum (a) in Abb. 5-8 und 5-9), ergibt einen Anteil von maximal $14 \% t t_{\mathrm{H}}-\mathrm{NMF}$ und somit eine maximale Schwingungstemperatur von $92 \mathrm{~K}$. Dieses plausible Ergebnis unterstützt den Ansatz, aus experimentell und theoretisch ermittelten Daten von NMA aufgrund struktureller Ähnlichkeiten eine grobe Abschätzung von Werten für NMF zu machen.

Die entsprechenden tt-Akzeptorbanden können im Raman-Spektrum aufgrund einer Überlagerung mit den heißen Banden des $t$-Monomers nicht eindeutig zugeordnet werden.

Genauso wie bei NMA ist im Raman-Spektrum für NMF die Zuordnung der Banden im Amid I-Bereich aufgrund einer Vielzahl möglicher Dimerbanden nicht eindeutig. Der Vergleich mit Literaturwerten aus FTIR-Messungen legt eine Zuordnung der beiden Banden bei $1715 \mathrm{~cm}^{-1}$ und $1707 \mathrm{~cm}^{-1}$ als Streckschwingung der tt- Akzeptor- bzw. tt-Donor-Carbonylgruppe nahe. Entsprechend normierte Spektren zeigen aber, dass sich in den Banden bei $1738 \mathrm{~cm}^{-1}$ und $1729 \mathrm{~cm}^{-1}$ heiße Banden des $t$-Monomers mit signifikanten Anteilen von $t t$-Dimerbanden überlagern. Es liegen also sehr wahrscheinlich Resonanzen vor und eine eindeutige Zuordnung der vier Dimerbanden zur Akzeptor- oder Donorschwingung sowie zu den Dimerstrukturen $t t_{\mathrm{N}}$ oder $t t_{\mathrm{H}}$ ist nicht möglich.

Auf der Suche nach der Bande des cc-Dimers im Ramanspektrum kann eine bereits in der Doktorarbeit von K. Otto [112] wegen der ungewöhnlich hohen Peakintensität angezweifelte Zuordnung der Bande bei $3113 \mathrm{~cm}^{-1}$ ausgeschlossen werden. Es handelt sich hierbei vielmehr mit hoher Wahrscheinlichkeit um ein Signal des $t$-Monomers, das auf Oberton- oder Kombinationsbanden zurückzuführen ist und durch Resonanzen eine Intensitätsverstärkung erfährt. Aufgrund einer vereinfachten Abschätzung der Überschätzung der Rotverschiebung des $c c$-Dimersignals durch die B3LYP-D3(BJ)/aVTZ-Rechnungen gegenüber des zu erwartenden experimentellen Wertes, könnte die kleine Bande bei $3123 \mathrm{~cm}^{-1}$ der $c c$-Dimerbande entsprechen. 
Wahrscheinlicher ist jedoch eine, durch komplizierte Resonanzen hervorgerufene, breite Verteilung der gesuchten Bande, ähnlich dem strukturell ähnlichen Ameisensäure-Dimer [29, 271]. Aufgrund der Überlagerung mit dem C-H-Streckbereich, wäre ein Raman-spektroskopischer Nachweis nur mit Hilfe von Experimenten mit $N$-deuteriertem NMF möglich.

Durch eine Normierung auf das Dimersignal lassen sich im Amid A-Raman-Spektrum zwei Trimer-Banden finden, die durch den Vergleich mit DFT-Frequenzrechnungen den beiden zyklischen Strukturen $t t t_{\mathrm{a}}$ und $t t t_{\mathrm{p}}$ zugeordnet werden können. Zudem gibt eine Gegenüberstellung von FTIR- und Ramanspektren im Amid I-Bereich Aufschluss über die Komplementarität der CO-Streckmoden aufgrund lokaler Symmetrien in Trimerclustern. Zusammen mit den durchgeführten DFT-Rechnungen belegen diese Spektren, dass auch bei NMF-Trimeren mit $t t t_{\mathrm{a}}$ eine zyklische Geometrie - ähnlich derjenigen bei NMA - die stabilste Konformation darstellt. Anders als bei NMA ist hierfür eine Berücksichtigung der Dispersionswechselwirkungen mit einer D3(BJ)-Korrektur nicht zwingend notwendig. Ein Vergleich der berechneten Trimerstrukturen von NMF und NMA wird in der abschließenden Diskussion in Kapitel 9 angestellt.

Zwischen 3350 und $3140 \mathrm{~cm}^{-1}$ entstehen mit zunehmender NMF-Konzentration eine Reihe von kleineren Banden, die jedoch von einer großen, breiten Bande überlagert werden, welche auf die Bildung größerer Aggregate zurückzuführen ist. Eine Untersuchung von Tetrameren und größeren Clustern ist mit dem curry-Jet nur eingeschränkt möglich und ist nicht Gegenstand dieser Arbeit.

Es kann abschließend festgestellt werden, dass es trotz des gegenüber NMA erhöhten cis-Monomeranteils bei NMF von 8-15\% in den hier vorliegenden Messungen keinen klaren Hinweis auf die cc-Dimerbande gibt, auch wenn aus allen quantenchemischen Methoden dies eindeutig als das stabilste Dimer hervorgeht. Ein experimenteller Nachweis des $c c$-Dimers erscheint beim Betrachten der Ergebnisse der quantenchemischen Rechnungen insgesamt eher unwahrscheinlich, da in einer Messung mit statistischer Dimerbildung immer die $t t$-Dimere dominieren werden, solange der cis-Monomeranteil vor der Expansion nicht signifikant erhöht wird. 


\section{$6 \mathrm{~N}$-Phenylformamid}

Inwieweit ein geringer cis-Monomeranteil in der Expansion ursächlich für den fehlenden eindeutigen Nachweis des cis-cis-Dimers in FTIR- und Raman-Schwingungsspektren ist, konnte auch mit NMF nicht geklärt werden (siehe Abschnitt 5). Daher wird nun mit $N$-Phenylformamid $(\mathrm{NPhF})$ ein sekundäres Amid untersucht, dessen cis-Anteil in Lösung mittels NMR-Spektroskopie konzentrationsabhängig zu 27-55\% ermittelt wurde [272]. Dies wird durch weitere Studien mit NMR- [248, 273, 274] , IR- [171, 275] und Mikrowellenspektroskopie [276, 277] bestätigt. Für festes NPhF konnte gezeigt werden, dass cis- und trans-Form im Verhältnis 1:1 als Co-Kristall vorliegen [278]. Der niedrigste cis-Anteil wurde mit $6.5 \%$ in 2-Photonen-REMPI-Spektren einer Jet-Expansion in Helium und Argon gefunden [47], was jedoch immer noch über dem cis-Anteil der meisten bisher diskutierten Alkylamide liegt. Wenn also der cis-cisDimernachweis bisher an der Konzentration des cis-Monomers gescheitert ist, ist NPhF ein aussichtsreicher Kandidat für den spektroskopischen Nachweis eines cis-cis-Clusters als stabilstes Dimer.

$\mathrm{NPhF}$ ist bei Raumtemperatur ein weißer Feststoff, wenig wasserlöslich, schmilzt bei ca. $46^{\circ} \mathrm{C}$ und besitzt eine Siedetemperatur von $271^{\circ} \mathrm{C}$ [152]. Industriell wird es z. B. in der Synthese des Fungizids Mepanipyrim eingesetzt [279]. Anders als NMF und NMA zählt es nicht zu den Modellpeptiden, weswegen diese Untersuchungen nur eine eingeschränkte biochemische Relevanz haben. Dennoch ist NPhF als aromatisch-substituiertes, sekundäres Amid ein interessantes System zur ergänzenden Untersuchung der cis-/trans-Isomerisierung und Clusterbildung sekundärer Amide. Hierbei sind vor allem die Fragen nach dem Einfluss der aromatischen Substitution auf die Molekülgeometrie und die Isomerisierungsbarriere sowie nach den intermolekularen Wechselwirkungen mit der Phenylgruppe in Dimer- und Trimeraggregaten von Interesse.

\subsection{Struktur und Schwingungsspektroskopie des Monomers}

Aufgrund der eingeschränkten Rotation um die C-N-Bindung der Amidgruppe, liegen auch bei $\mathrm{NPhF}$ cis- und trans-Konformere vor. In diesem Fall ist das cis-Konformer jedoch nicht planar, sondern hat einen Diederwinkel $\varphi^{1)}$, der in Mikrowellenexperimenten zu 34.7(5) ${ }^{\circ}$ [276] und in einer Kombination aus Mikrowellen- und Gasphasen-Elektronenbeugungsdaten zu $36.7^{\circ}$ [280]

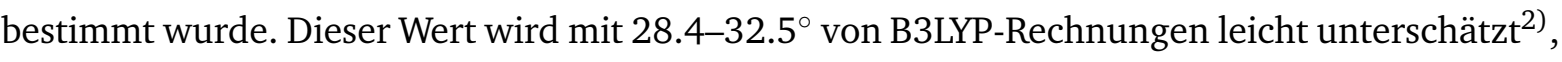
während MP2-Rechnungen mit 36.2-42.9 den Winkel treffen bzw. leicht überschätzen [276,

\footnotetext{
${ }^{1)}$ Definition: siehe Abschnitt2.1. In der entsprechenden Literatur der Mikrowellenspektroskopie wird dieser Winkel üblicherweise mit $\tau$ bezeichnet.

${ }^{2)}$ Diese Arbeit, Blanco et al. [276] und Marochkin und Dorofeeva [280
} 
280, 281]. Aufgrund dieser nicht-planaren Struktur gibt es zwei isoenergetische, enantiomere Konformationen von cis-NPhF [281]. Die Molekülstrukturen der cis- und trans-Konformere in Abbildung 6-1 dargestellt.
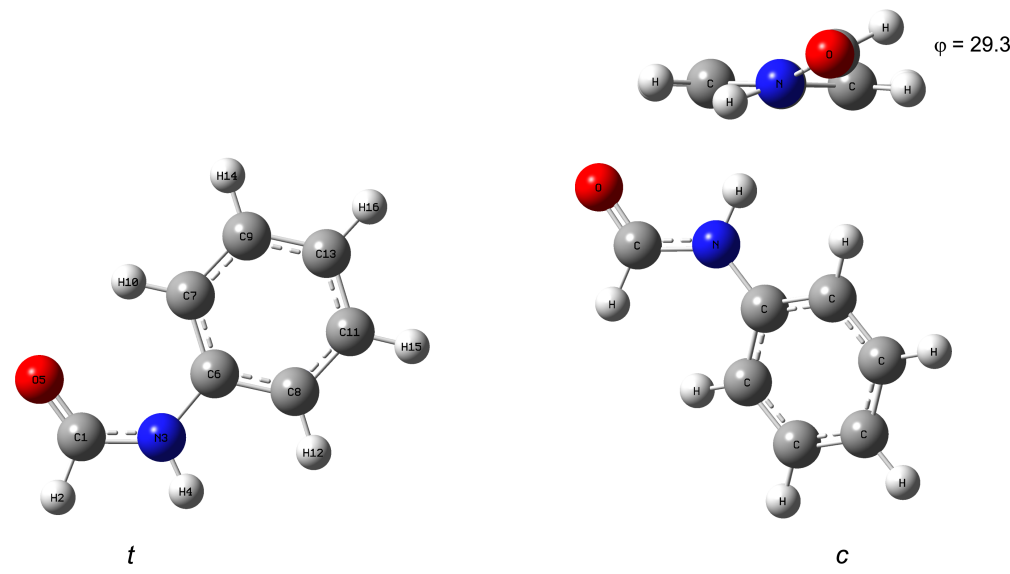

Abb. 6-1. Minimumstrukturen von trans- und cis-NPhF nach Geometrieoptimierung auf B3LYP-D3(BJ)/aVTZ-Niveau $\left(E_{0}(t)=E_{0}(c)-2.3 \mathrm{~kJ} \cdot \mathrm{mol}^{-1}\right)$. Während $t-\mathrm{NPhF}$ planar und damit $C_{s}$-symmetrisch ist, ist die Phenylgruppe bei $c$-NPhF ca. $29.3^{\circ}$ aus der Amidebene gedreht (rechts oben).

Die Höhe der Barriere zwischen den beiden cis-Konformationen wurde aus Mikrowellenspektren zu 1.82(2) $\mathrm{kJ} \cdot \mathrm{mol}^{-1}$ bestimmt [276]. Die Geometrie am Sattelpunkt entspricht einer planaren Struktur mit $C_{\mathrm{S}}$-Symmetrie. Diese Barriere führt zu einer Tunnelaufspaltung von $0.1245 \mathrm{~cm}^{-1}$ [276], die aber in den hier vorgelegten Spektren aufgrund der spektralen Auflösung des curry-Jets (siehe Abschnitt 3.1.4) nicht sichtbar ist. Für die Zwecke dieser Arbeit kann cis-NPhF in Anbetracht der niedrigen Umwandlungsbarriere als quasiplanar mit näherungsweise doppelter Zustandssumme betrachtet werden.

trans-NPhF hat eine planare Molekülstruktur, die durch eine Resonanzerweiterung des konjugierten Systems der Amidgruppe auf das gesamte Molekül [278] und durch schwache, elektrostatische $\mathrm{C}-\mathrm{H} \cdots \mathrm{O}$-Wechselwirkungen stabilisiert wird [47]. In beiden Konformeren liegen repulsive Wechselwirkungen zwischen der Amidgruppe und dem ortho-Wasserstoffatom im Phenylring vor. Zusätzlich wird im cis-NPhF die $\mathrm{C}-\mathrm{H} \cdots \mathrm{O}$-Wechselwirkung des trans-Konformers durch eine weitere repulsive Wechselwirkung ersetzt. Dies verhindert für $c-\mathrm{NPhF}$ eine planare, resonanzstabilisierte Struktur, was zur geringeren Stabilität gegenüber $t$-NPhF beiträgt.

Die Barrierenhöhe der Isomerisierung der C-N-Bindung in Amiden wird neben sterischen Effekten auch zu einem wesentlichen Anteil durch Resonanzstabilisierung (also dem partiellen Doppelbindungscharakter) dieser Bindung beeinflusst [282].

Der Vollständigkeit halber sei erwähnt, dass eine $180^{\circ}$-Rotation der Phenylgruppe im $t$-NPhF erwartungsgemäß nur ein Maximum auf der Potentialkurve bei einem Diederwinkel von ca. $90^{\circ}$ durchläuft, das auf B3LYP/6-311 + + G(2df,p)-Niveau zu $16.4 \mathrm{~kJ} \cdot \mathrm{mol}^{-1}$ bestimmt wurde [283]. Bei $c-N P h F$ liegt neben einer etwas niedrigeren Rotationsbarriere von ca. $11 \mathrm{~kJ} \cdot \mathrm{mol}^{-1}$ 3) auch eine sehr viel niedrigere Racemisierungsbarriere von ca. $0.9 \mathrm{~kJ} \cdot \mathrm{mol}^{-1}$ [3] und einem planaren

${ }^{3)}$ Aus einem PES-Scan entlang der Drehung um den $\mathrm{C}=\mathrm{N}-\mathrm{C}=\mathrm{C}$-Diederwinkel auf B3LYP-D3(BJ)/aVTZ-Niveau aus dieser Arbeit. 
Übergangszustand vor. Die DFT-Rechnung ergibt also für die Racemisierung einen Wert, der nur ungefähr halb so groß ist wie der Wert aus den Mikrowellenexperimenten.

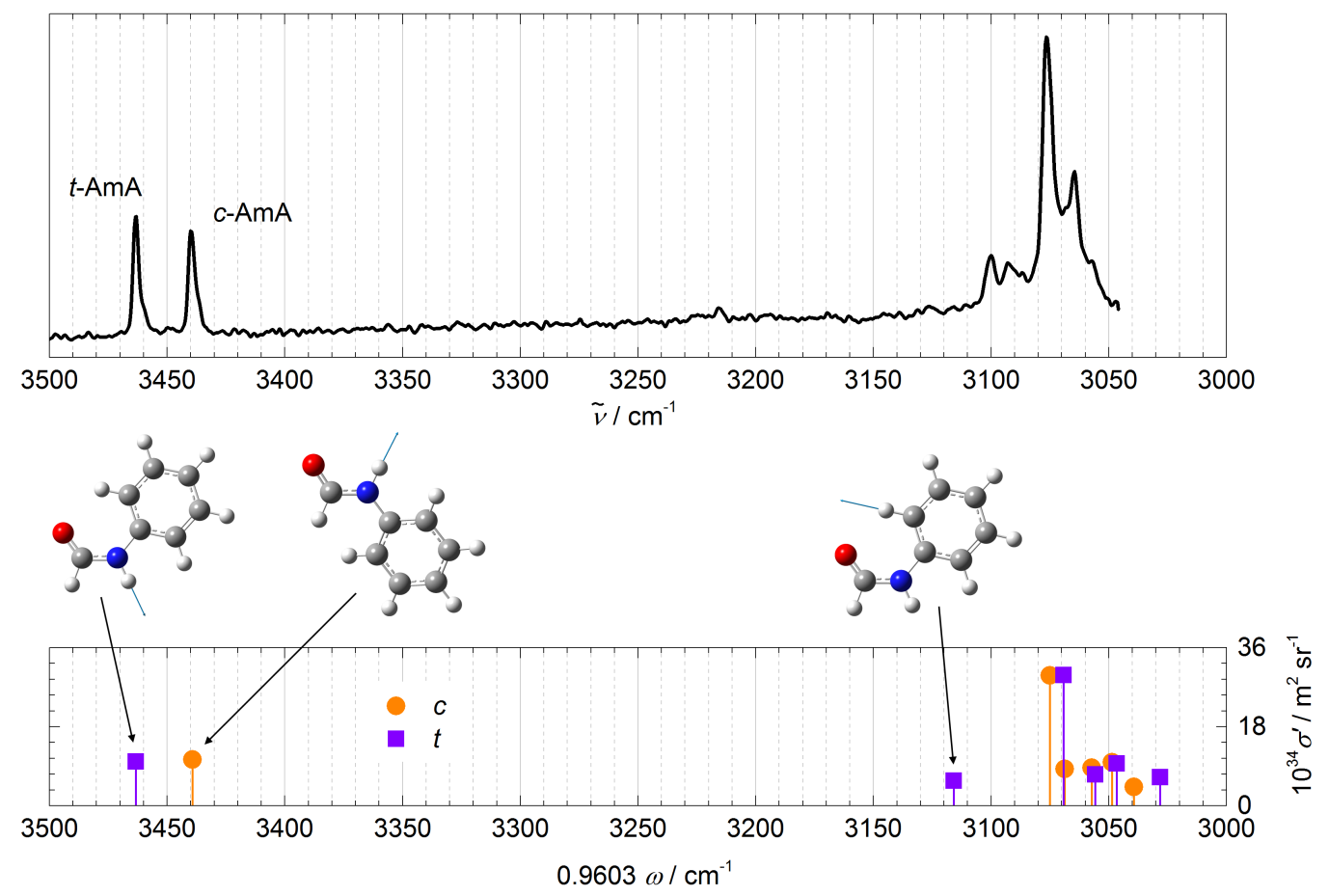

Abb. 6-2. curry-Jet-Spektrum der NPhF-Monomere im NH- und CH-Streckbereich bei $\vartheta_{\mathrm{H}}=130^{\circ} \mathrm{C}$. Darunter Strichspektren des jeweils stabilsten trans- (Rechteck, violett) und cis-Konformers (rund, orange) auf B3LYP-D3(BJ)/aVTZ-Niveau und deren, an den jeweiligen Molekülstrukturen illustrierten, Normalmoden. Die Wellenzahlskalierung bezieht die theoretische NH-Streckfrequenz von $t$-NPhF auf $t$-AmA. (Ausführliche Informationen zu den Messbedingungen befinden sich in Tab. A-6 im Raman-Spektrenverzeichnis in Anhang A.6.1 Details zu den quantenchemischen Rechnungen sind in Tabelle A-25 enthalten.)

Die erstmalige Aufnahme von Raman-Jetspektren von $N$-Phenylformamid stellt eine Ergänzung zu den FTIR-Jet-Spektren von Albrecht [175] dar. Das Raman-Spektrum in Abbildung 6-2 zeigt den Bereich oberhalb von $3000 \mathrm{~cm}^{-1}$, wo zwei Banden bei $3463 \mathrm{~cm}^{-1}$ und $3440 \mathrm{~cm}^{-1} \mathrm{zu}$ erkennen sind. Der Vergleich mit spektroskopischen Daten in der Literatur (siehe Tab. 6-1) lässt eine eindeutige Zuordnung als Amid A-Bande des trans- bzw. cis-Konformers zu. Dabei liegt eine sehr gute Übereinstimmung mit den Daten anderer Jet-Experimente vor. Die IR-Daten in Lösung weisen hierzu eine Rotverschiebung von $29-44 \mathrm{~cm}^{-1}$ auf, die typisch für Schwingungsspektren von Lösungen ist. Der Abstand zwischen trans- und cis-Bande von $23 \mathrm{~cm}^{-1}$ im RamanSpektrum ist in guter Übereinstimmung mit den Flüssigkeitsspektren.

Die sehr intensive cis-Bande bestätigt die eingangs formulierte Vermutung eines erhöhten cisAnteils im NPhF. Daraus resultierende energetische Betrachtungen finden sich im Abschnitt 6.2 . Eine klar erkennbare Substruktur aufgrund heißer Banden, wie sie sowohl bei NMA als auch bei NMF zu finden ist, liegt hier aufgrund der fehlenden Methylgruppen nicht vor.

In Abbildung 6-2 (oben) befindet sich im Raman-Spektrum neben den Amid A-Banden auch eine breite Bandenansammlung zwischen $3050 \mathrm{~cm}^{-1}$ und $3110 \mathrm{~cm}^{-1}$ mit zwei intensiven Maxima bei $3064 \mathrm{~cm}^{-1}$ und $3075 \mathrm{~cm}^{-1}$. Hierbei handelt es sich um die Banden der CH-Streckschwingungen des aromatischen Ringes, welche typischerweise oberhalb von $3000 \mathrm{~cm}^{-1} \mathrm{zu}$ 
Tab. 6-1. Vergleich experimenteller Werte der Amid A-Bande von trans- ( $t$-AmA) und cis-NPhF $(c-A m A)$ mit harmonischen Frequenzrechnungen zur Normalmode der $\mathrm{NH}$-Streckschwingung $\left(v_{\mathrm{NH}}\right)$. Zusätzlich ist die jeweilige Wellenzahldifferenz $\Delta_{c-t}$ angegeben. (Alle Angaben in $\mathrm{cm}^{-1}$ )

\begin{tabular}{|c|c|c|c|}
\hline experimentelle Methode & $t$-AmA & $c-A m A$ & $\Delta_{c-t}$ \\
\hline \multicolumn{4}{|l|}{ Jet-Expansion: } \\
\hline Raman $^{\mathrm{a}}$ & 3463 & 3440 & -23 \\
\hline FTIR [175] & 3464 & 3441 & -23 \\
\hline IR-UV 284 & 3463.2 & 3439.5 & -23.7 \\
\hline IR-UV 285 & 3463 & 3441 & -22 \\
\hline \multicolumn{4}{|l|}{ Lösung: } \\
\hline $\mathrm{CCl}_{4}-\mathrm{IR}[171$ & 3434 & 3408 & -26 \\
\hline $\mathrm{CCl}_{4}-\mathrm{IR} 275$ & 3434 & 3407 & -27 \\
\hline $\mathrm{CCl}_{4}-\mathrm{IR} 178$ & 3420 & 3400 & -20 \\
\hline theoretische Methode / Basisatz & $v_{\mathrm{NH}}^{t}$ & $\nu_{\mathrm{NH}}^{c}$ & $\Delta_{c-t}$ \\
\hline \multicolumn{4}{|l|}{ doppelt-harmonisch: } \\
\hline$\omega \mathrm{B} 97 \mathrm{X}-\mathrm{D} / \mathrm{aVTZ}[286$ & 3654 & 3631 & -22 \\
\hline$B 3 L Y P / 6-31+G * b, c$ [285] & 3593 & 3574 & -19 \\
\hline $\mathrm{B} 3 \mathrm{LYP} / 6-311+\mathrm{G}^{*} 175$ & 3603 & 3585 & -18 \\
\hline B3LYP/VTZ C 284 & 3606 & 3581 & -25 \\
\hline B3LYP-D3(BJ)/aVTZa & 3607 & 3581 & -26 \\
\hline B3LYP/def2TZVPa & 3595 & 3570 & -25 \\
\hline B3LYP-D3(BJ)/def2TZVPa & 3598 & 3574 & -24 \\
\hline
\end{tabular}

a: aus dieser Arbeit; ${ }^{\text {b }: ~ B S S E-k o r r i g i e r t ; ~}{ }^{\text {c}}$ : Wellenzahlen liegen nur skaliert vor $\left(\tilde{v}_{\text {sc }}\right)$ und wurden entsprechend zurückgerechnet: 285]: $\tilde{v}_{\mathrm{sc}}=0.977 \cdot \tilde{v}_{\text {calc }},[284]: \tilde{v}_{\mathrm{sc}}=\tilde{v}_{\text {calc }} \cdot \exp \left(-1.25 \cdot 10^{-5} \mathrm{~cm} \cdot \tilde{v}_{\text {calc }}\right)$.

finden sind. Weitere Peaks dieser Gruppe liegen bei 3057, 3065, 3087, 3093 und $3100 \mathrm{~cm}^{-1}$, lassen sich aber nicht eindeutig einem Konformer zuordnen.

Die Zuordnung der Amid A-Banden wird durch harmonische Frequenzrechnungen auf B3LYPD3(BJ)/aVTZ-Niveau unterstützt. Die unskalierten Werte liegen aufgrund der nicht berücksichtigten Anharmonizität ca. $140 \mathrm{~cm}^{-1}$ oberhalb der experimentellen Positionen, allerdings befindet sich die Differenz zwischen cis- und trans-Bande mit $\Delta_{c-t}=-26 \mathrm{~cm}^{-1}$ in sehr guter Übereinstimmung mit dem Experiment. Neben ergänzend durchgeführten B3LYP-Rechnungen mit def2-TZVP-Basissatz sowohl mit als auch ohne Dispersionskorrektur liegen in der Literatur noch weitere Frequenzrechnungen mit dem $\omega$ B97X-D- und dem B3LYP-Funktional vor (siehe Tab. 6-1). Diese befinden sich bezüglich $\Delta_{c-t}$ in guter Übereinstimmung mit dem Experiment. Die auf die trans-Amid A-Bande skalierten Werte der $\mathrm{N}-\mathrm{H}$ - und $\mathrm{C}-\mathrm{H}$-Streckschwingungen beider Konformere sind in Abbildung 6-2 als Strichspektren dargestellt.

Die Frequenzrechnung ergibt für $t-\mathrm{NPhF}$ eine Normalmode bei $3245 \mathrm{~cm}^{-1}$ ]), die fast vollständig als $\mathrm{C}-\mathrm{H}$-Streckschwingung an der carbonylseitigen ortho-C-H-Gruppe im Phenylring $\left(\mathrm{C}_{7}-\mathrm{H}_{10}\right.$, siehe Abb. 6-1) lokalisiert ist. Diese liegt, im Vergleich zu den anderen C-H-Streckschwingungsmoden, isoliert und zu höheren Wellenzahlen verschoben. Da keine äquivalente Mode im $c$-NPhF vorliegt, könnte die entsprechende Bande als Referenz für die Veränderung des Anteils an $t$-NPhF-Monomer in der Expansion für Untersuchungen der Clusterbildung oder der cis-/trans-Isomerisierung dienen. Es ist jedoch im Raman-Spektrum im vorhergesagten Wellenzahlbereich keine signifikante Bande erkennbar, die sich dieser $\mathrm{C}-\mathrm{H}$-Streckschwingungsmode

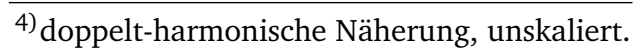


eindeutig zuordnen ließe (siehe Abb. 6-2). 


\section{2 cis-/trans-Isomerisierung}

Alle experimentellen und bis auf eine MP2/6-31G*-Rechnung [281] auch alle theoretischen Studien zur cis-/trans-Isomerie finden $t$-NPhF als stabilstes Konformer. Über die Höhe der Isomerisierungsbarriere $\Delta E_{\mathrm{A}}$ finden sich kaum Angaben in der Literatur. Die einzig verfügbaren Daten liefern B3LYP-Rechnungen, die je nach gefundenem Übergangszustand Barrierenhöhen von 68.2-88.2 $\mathrm{kJ}^{-\mathrm{mol}^{-1}}$ angeben [276, 287] und eine NMR-Analyse bei $349 \mathrm{~K}$, die eine freie Aktivierungsenthalpie von 74.1(8) $\mathrm{kJ} \cdot \mathrm{mol}^{-1}$ angibt [273]. Diese Barrierenhöhen lassen die Annahme zu, dass während der Expansion keine Relaxation stattfindet, die Besetzungsverhältnisse im thermodynamischen Gleichgewicht vor der Expansion also „eingefroren“ werden [116]. Für $\mathrm{NPhF}$ wurde dies bereits ausführlich von Manea et al. [47] diskutiert.

Tab. 6-2. Theoretische und experimentelle cis-trans-Energieunterschiede und Enthalpien für die Isomerisierungsreaktion trans-NPhF $\rightleftharpoons$ cis-NPhF sowie Isomerisierungsbarrieren $\Delta E_{\mathrm{A}}$.

\begin{tabular}{|c|c|c|c|c|}
\hline \multicolumn{5}{|l|}{ experimentell } \\
\hline Methode & Bedingungen & $\Delta G_{\exp } / \mathrm{kJ} \cdot \mathrm{mol}^{-1 \mathrm{a}}$ & $\Delta H / \mathrm{kJ} \cdot \mathrm{mol}^{-1}$ & $\Delta E_{\mathrm{A}} / \mathrm{kJ} \cdot \mathrm{mol}^{-1 \mathrm{f}}$ \\
\hline Raman - Jet ${ }^{b}$ & $\theta_{\mathrm{H}}=353.15-403.15 \mathrm{~K}$ & $0.35 \pm 0.28(363.15)^{i}$ & $5.7 \pm 4.9$ & - \\
\hline IR 275] & in $\mathrm{CCl}_{4} T=297-341 \mathrm{~K}$ & $1.5(297)$ & $2.6 \pm 0.3$ & - \\
\hline FT-MW - Jet 276 & in $\mathrm{He}$ & $2.1 \pm 1.8(373)^{j}$ & - & - \\
\hline FT-MW - Jet 277 & in $\mathrm{Ne}$ & $3.3 \pm 1.0(385)$ & - & - \\
\hline REMPI - Jet 47 & in $\mathrm{He}$ & $10.5(373)$ & - & - \\
\hline NMR 273. & in $\mathrm{CDCl}_{3} / 349 \mathrm{~K}$ & - & - & $74.1 \pm 0.8(349)^{\mathrm{c}}$ \\
\hline NMR 248. & in $\mathrm{CH}_{2} \mathrm{Cl}_{2} / \mathrm{CD}_{2} \mathrm{Cl}_{2}$ & $0.59(298)$ & - & - \\
\hline NMR 274 & in $\mathrm{CDCl}_{3}$ & $0.2 \pm 0.1(298)$ & - & - \\
\hline
\end{tabular}

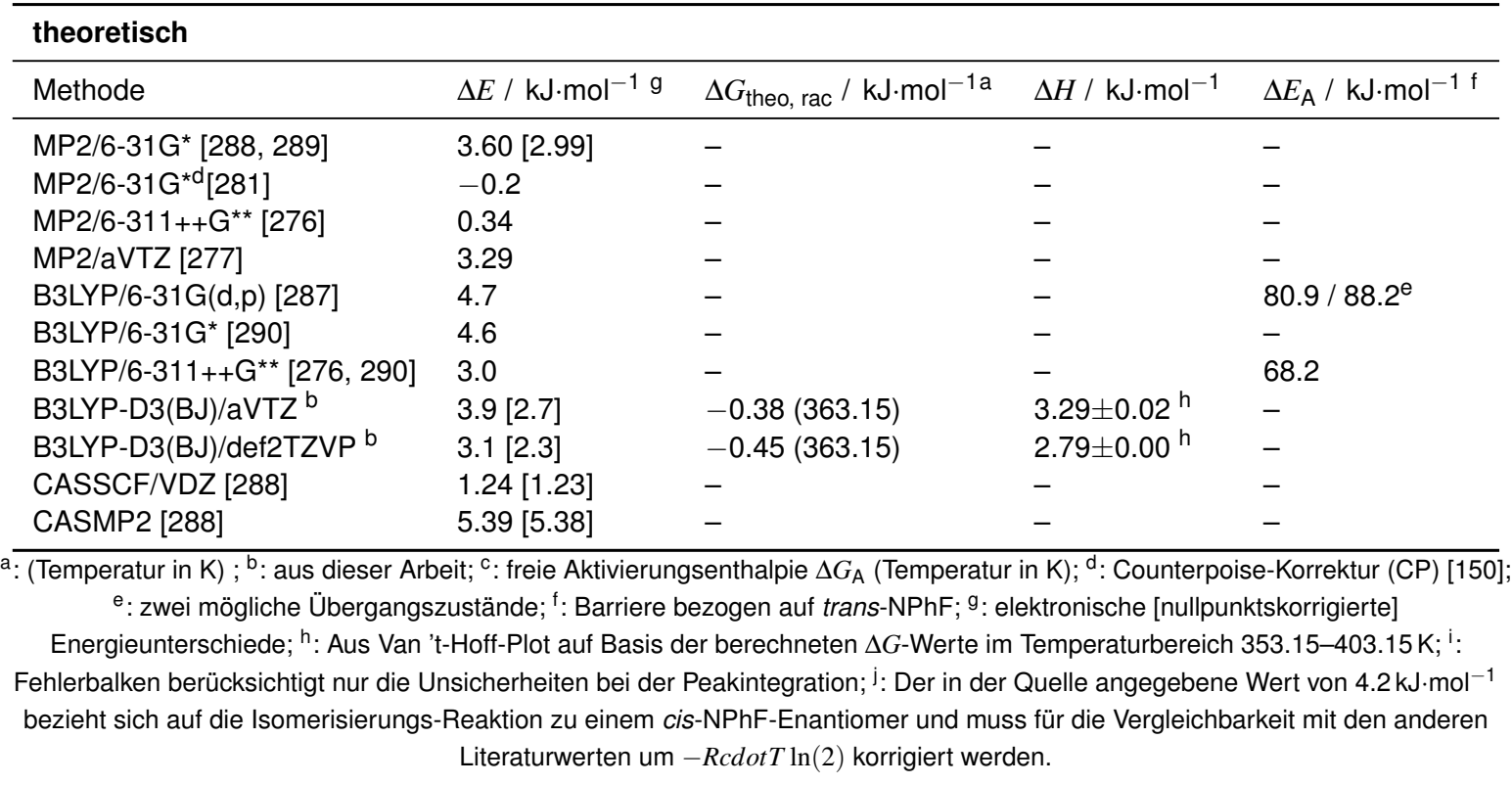

Analog zu NMA und NMF (siehe Abschnitte 4.2 und 5.2) wird der Enthalpieunterschied zwischen beiden Konformeren mittels Van-'t-Hoff-Analyse einer Temperaturreihe von RamanJetspektren bestimmt (siehe Abb. 6-3). Betrachtet wird das cis-/trans-Peakflächenverhältnis $F$ der beiden gut separierten Amid A-Banden. Daraus lässt sich mit dem Verhältnis der theoretisch bestimmten Streuquerschnitte (Tab. A-25) durch Gleichung 2-5 das Besetzungsverhältnis $K$ 


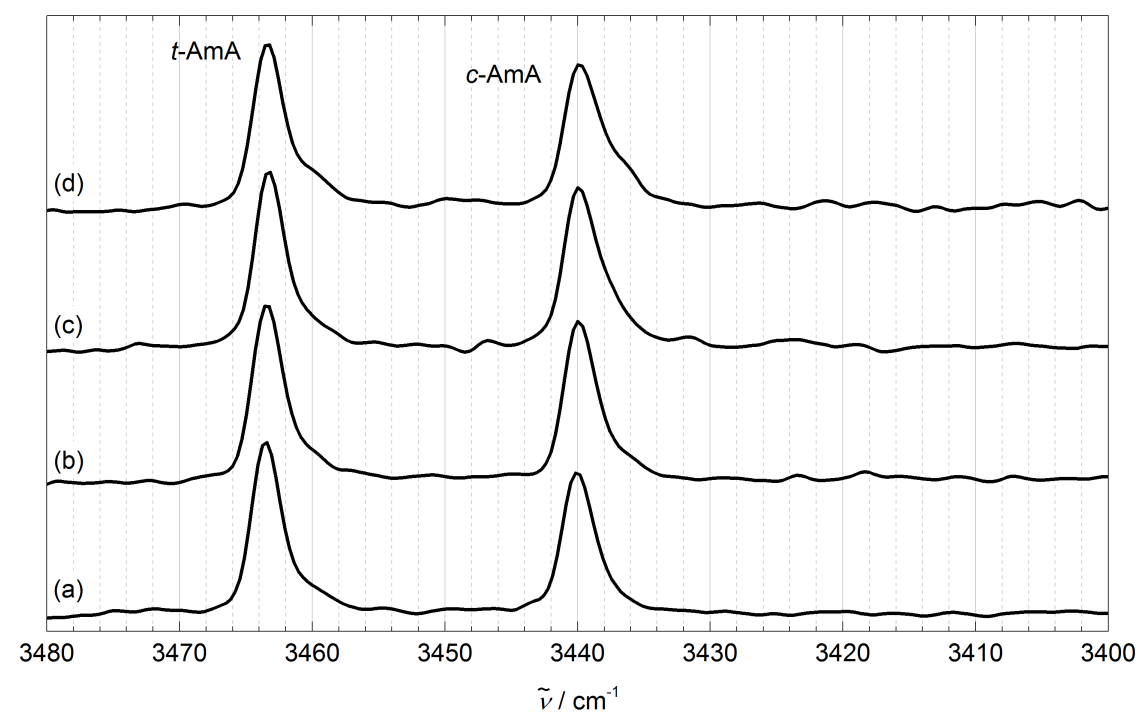

Abb. 6-3. Temperaturmessreihe von NPhF im NH-Streckbereich. Die Temperaturen vor der Expansion, $\vartheta_{\mathrm{H}} /{ }^{\circ} \mathrm{C}$, betragen von (a) bis (d): 80, 90, 110, 130. Die Spektren sind normiert auf die $t$-AmA-Bande bei $3463 \mathrm{~cm}^{-1}$. (Ausführliche Informationen zu den Messbedingungen befinden sich in Tab. A-6 im Raman-Spektrenverzeichnis in Anhang A.6.1.)

bestimmen, welches für die Expansionstemperaturen $\vartheta_{\mathrm{H}}=80-130^{\circ} \mathrm{C}$ einen cis-Anteil von $42.7-$ $50.5 \%$ ergibt.

Die unterschiedlichen Streuquerschnitte beeinflussen im Van-'t-Hoff-Plot nur den Y-Achsenabschnitt und können daher bei der Enthalpiebestimmung mittels Gleichung 2-7 vernachlässigt werden. Im Van-'t-Hoff-Plot in Abbildung 6-4 wird daher $\ln F$ anstatt $\ln K$ aufgetragen. Es ergeben sich Enthalpieunterschiede von $6(5) \mathrm{kJ} \cdot \mathrm{mol}^{-1}$ für die Raman-Messdaten und $3.29(2) \mathrm{kJ} \cdot \mathrm{mol}^{-1}$ aus dem Plot der harmonischen B3LYP-D3(BJ)/aVTZ-Daten.

Auffällig ist hierbei das große Fehlerintervall (siehe Fehlerbalken im Plot), welches auf die große Unsicherheit bei der Integration der Banden zurückzuführen ist. Zur Ermittlung dieses Fehlers wurden die Banden dreimal unabhängig voneinander integriert und deren arithmetisches Mittel ist die Grundlage für die Berechnung von $\ln F$. Die konservativ abgeschätzten Fehlergrenzen der Peakflächeninhalte, $\Delta F_{c i s}$ und $\Delta F_{\text {trans }}$, haben gemäß der Gleichungen 2-9 und 2-8 Einfluss auf den für den Fit relevanten Fehler von $\ln F$ (siehe Abschnitt 2.4). Der im Vergleich dazu kleine Fehlerbalken der reziproken Temperatur basiert auf beobachteten Temperaturschwankungen am PID-Controller der Düse von \pm 2 K. Für die Standardabweichung des Fits der Daten aus der harmonischen Rechnung gilt wie für die Van-'t-Hoff-Plots in den vorherigen Kapiteln, dass dies nicht signifikant zum Gesamtfehler beiträgt (siehe Abschnitt5.2).

Für die freie Enthalpiedifferenz $\Delta G$ der Reaktion trans-NPhF $\rightleftharpoons$ cis- $\mathrm{NPhF}$ finden sich in der Literatur für ähnliche Temperaturen $(\Delta T=10 \mathrm{~K})$ deutliche Unterschiede zwischen den Mikrowellen-Jet-, REMPI-Jet-Daten und dem Wert aus dieser Arbeit (siehe Tab. 6-2). Ein experimenteller Wert für den Enthalpieunterschied $\Delta H$ findet sich nur bei Suzuki et al. [275], der allerdings in Lösung gemessen wurde. Diese Werte stimmen aber mit denen der RamanJet-Messungen dieser Arbeit im Rahmen der großen Messunsicherheit des Raman-Experiments 


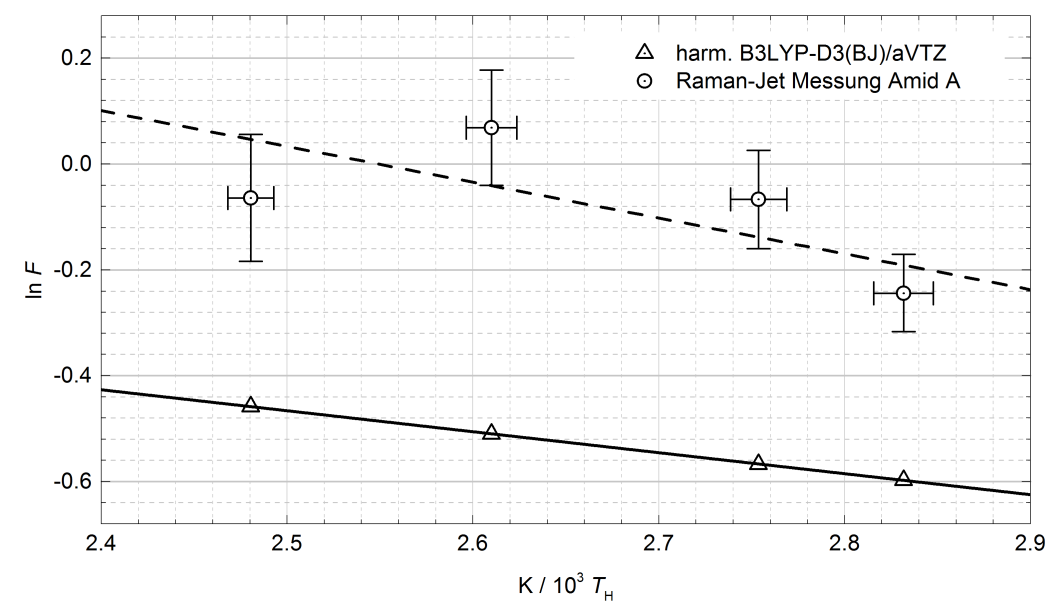

Abb. 6-4. Van-'t-Hoff-Plot für die cis-/trans-Isomerisierung von NPhF. Oben: Aus den Intensitäten der nicht-normierten Amid A-Banden der Spektren in Abb. 6-3 Die instrumentell gewichtete lineare Anpassung ergibt für die Steigung $b=-(6.8 \pm 5.9) \cdot 10^{2} \mathrm{~K}$.

Unten: Auf Grundlage der, mit dem Gaussiantool „freqchk“ für die jeweiligen Temperaturen bestimmten, freien Enthalpien aus harmonischen Frequenzrechnungen auf B3LYP-D3(BJ)/aVTZ-Niveau. Steigung der linearen Anpassung: $b=-3.96(2) \cdot 10^{2} \mathrm{~K}$.

miteinander überein.

Auf Seiten der quantenchemischen Rechnungen liegen in der Literatur keine Werte für den Enthalpieunterschied vor. Beim Vergleich der elektronischen Energiedifferenzen stimmen die durchgeführten B3LYP-Rechnungen mit $3.1 \mathrm{~kJ} \cdot \mathrm{mol}^{-1}$ und $3.9 \mathrm{~kJ} \cdot \mathrm{mol}^{-1}$ mit anderen B3LYPRechnungen [276, 287, 290] und den MP2-Rechnungen von Ullrich et al. [288] und Aviles Moreno et al. [277] gut überein. Die einzige nullpunktskorrigierte Energie in der Literatur liegt für die MP2-Rechnung mit double- $\zeta$-Basissatz vor [288, 289] und liegt mit $2.99 \mathrm{~kJ} \cdot \mathrm{mol}^{-1}$ nur geringfügig über denen der B3LYP-Rechnungen mit 2.3 und $2.7 \mathrm{~kJ} \cdot \mathrm{mol}^{-1}$.

Mithilfe der durchgeführten Frequenzrechnungen lassen sich für die im Experiment verwendeten Düsentemperaturen theoretische $\Delta G_{\text {theo }}$-Werte bestimmen, aus denen mit Gleichung 2-3 Gleichgewichtskonstanten $K_{\text {theo, u }}$ berechnet werden können. Die zugrunde liegende Reaktion berücksichtigt jedoch, anders als die experimentellen Werte, nicht das Vorliegen von zwei isoenergetischen Enantiomeren des cis-NPhF. Da die Rechnung sich nur auf ein Enantiomer bezieht, ist der experimentelle cis-Anteil doppelt so groß und das unkorrigierte $K_{\text {theo, u }}$ aus den GAUSSIAN-Rechnungen muss daher in ein $K_{\text {theo, rac }}=2 \cdot K_{\text {theo, u }}$ umgerechnet werden, damit die über Gleichung 2-3 zugehörigen $\Delta G_{\text {theo, rac }}$-Werte mit den experimentell bestimmten $\Delta G_{\text {exp }^{-}}$ Werten vergleichbar sind.

Ein Vergleich dieser theoretischen freien Enthalpien mit den aus gemessenen Bandenintensitäten und berechneten Streuquerschnitten ermittelten semi-experimentellen Werten findet sich in Tabelle 6-3. Hier zeigt sich eine große Diskrepanz zwischen den experimentellen Werten. Im Experiment liegt mit Ausnahme der Messung bei 383.15 K der Wert für die Gleichgewichtskonstante $K_{\exp }$ unter 1 . Der überwiegende Teil der NPhF-Moleküle liegt also in der trans-Kon- 
formation vor. Dagegen liegen die $K_{\text {theo, rac }}$-Werte der B3LYP/aVTZ-Rechnung allesamt über 1. In der Theorie überwiegt also bei den untersuchten Temperaturen bereits das cis-NPhF. Unter Berücksichtigung des Fehlerintervalls der Peakintegration (siehe Tab. 6-3) liegen die $K_{\text {theo, rac }}{ }^{-}$ Werte nur noch geringfügig über den experimentellen Werten. Diese Diskrepanz lässt sich auf die berechneten Entropien der doppelt-harmonischen Frequenzrechnungen zurückführen, insbesondere auf den Entropiegewinn durch Racemisierung.

Tab. 6-3. Vergleich theoretisch und semi-experimentell bestimmter Gleichgewichtskonstanten $K$ der Isomerisierungsreaktion trans-NPhF $\rightleftharpoons$ cis-NPhF. Die experimentellen Gleichgewichtskonstanten $K_{\text {exp }}$ sind aus den um die Streuquerschnitte der B3LYP/aVTZ-Frequenzrechnung korrigierten Peakflächenverhältnissen der Raman-Spektren berechnet worden (siehe Abb. 6-3) und beziehen sich auf das racemische Gemisch beider cis-Enantiomere. Auf B3LYP-D3(BJ)/aVTZNiveau berechnete freie Enthalpien für die Reaktion eines cis-Enantiomers $\left(\Delta G_{\text {theo, u }}\right)$ und des racemischen Gemisches $\left(\Delta G_{\text {theo, rac }}\right)$ liefern über Gleichung 2-3 die entsprechenden Gleichgewichtskonstanten $K_{\text {theo, u }}$ und $K_{\text {theo, rac }}$.

\begin{tabular}{llllll}
\hline Temperatur $T / \mathrm{K}$ & $\Delta G_{\text {theo, }}^{\ominus} / \mathrm{kJ} \cdot \mathrm{mol}^{-1}$ & $K_{\text {theo, u }}$ & $\Delta G_{\text {theo, rac }}^{\ominus} / \mathrm{kJ} \cdot \mathrm{mol}^{-1}$ & $K_{\text {theo, rac }}$ & $K_{\text {exp }}$ \\
\hline 353.15 & 1.75 & 0.55 & -0.28 & 1.10 & $0.75 \pm 0.06$ \\
363.15 & 1.71 & 0.57 & -0.38 & 1.14 & $0.89 \pm 0.09$ \\
383.15 & 1.63 & 0.60 & -0.58 & 1.20 & $1.02 \pm 0.12$ \\
403.15 & 1.54 & 0.63 & -0.79 & 1.26 & $0.89 \pm 0.11$ \\
\hline
\end{tabular}




\subsection{Dimerisierung}

Für das gesuchte $c c-\mathrm{NPhF}$ als vermeintlich stabilstes Dimer gab es die ersten experimentellen Indizien in der H-NMR-Studie von Bourn et al. [272], worin nachgewiesen wurde, dass das Gleichgewichts-cis-trans-Verhältnis in $\mathrm{CDCl}_{3}$-Lösung mit abnehmender NPhF-Konzentration zunimmt. Dies wird auf die Stabilisierung des cis-Konformers durch eine ringförmige $c c$ Dimerbildung zurückgeführt, was in einer späteren NMR-Studie wieder aufgegriffen wurde [291].

Die erstmalige Zuordnung von Amid A-Banden zu NPhF-Clustern erfolgte in IR-Spektren einer $\mathrm{CCl}_{4}$-Lösung [275], wobei hier nur grob zwischen Clusterbanden des trans-Typs bei ca. $3280 \mathrm{~cm}^{-1}$ und des cis-Typs bei $3220 \mathrm{~cm}^{-1}$ unterschieden wird. Dies entspricht einer Rotverschiebung von $154 \mathrm{~cm}^{-1}$ bzw. $187 \mathrm{~cm}^{-1}$ bezüglich der trans- bzw. cis-Amid A des Monomers.

Insgesamt beschränken sich die Untersuchungen zur Clusterbildung von $N$-Phenylformamid auf die Mikrosolvation in Wasser [281, 292, 293] sowie die Umordnung dieser Aggregate [294]. Außerdem gibt es noch eine Untersuchung zur Clusterbildung mit Stickstoff und Argon [286].

Zur Selbstaggregation von $\mathrm{NPhF}$ in der Gasphase gibt es neben den FTIR-Jet-Experimenten von Albrecht [175] und den dazu ergänzend durchgeführten B3LYP-Rechungen keine Literaturdaten.

Eine zweite Raman-Messreihe in der Amid A-Region einer NPhF/Helium-Jetexpansion ist in Abbildung 6-5 (unten) dargestellt. Die drei Spektren mit von (b) nach (d) zunehmender NPhF-Konzentration bei konstanter Düsentemperatur $\vartheta_{\mathrm{H}}=130^{\circ} \mathrm{C}$ sind um das nominell clusterärmste Spektrum mit der höchsten Düsentemperatur $\left(\vartheta_{\mathrm{H}}=120^{\circ} \mathrm{C}\right)$ der Messreihe zur cis/trans-Isomerisierung (Abb.6-3(d)) ergänzt (Abb.6-5 (a)). Die Spektren sind auf die Amid ABande des cis-Monomers normiert. In der gegenüber Abbildung 6-2 stark vergrößerten Darstellung sind eine Reihe schwacher Banden zu erkennen, deren Intensität mit steigender Konzentration zunimmt. So findet sich ein Bandenpaar bei $3381 \mathrm{~cm}^{-1}$ und $3367 \mathrm{~cm}^{-1}$ und eine sehr breite, unsymmetrische Bande im Bereich bei ca. $3166-3300 \mathrm{~cm}^{-1}$ mit drei prominenten Peaks bei 3221, 3215 und $3196 \mathrm{~cm}^{-1}$. Weiterhin befinden sich noch zwei schwache Signale bei $3411 \mathrm{~cm}^{-1}$ und $3321 \mathrm{~cm}^{-1}$. Inwieweit die Intensität der Bande bei $3141 \mathrm{~cm}^{-1}$ und der Doppelpeak bei $3129 / 3126 \mathrm{~cm}^{-1}$ mit steigender Konzentration zunimmt, ist nicht klar zu erkennen.

Die Verschiebung des Bandenpaars bei $3381 / 3367 \mathrm{~cm}^{-1}$ liegt mit -82 bis $-96 \mathrm{~cm}^{-1}$ bezogen auf das trans-Monomer in der gleichen Größenordnung wie die der $t t$-Dimer-Donorbanden von NMF mit $-106 \mathrm{~cm}^{-1}$ (siehe Tab. 5-12) und NMA mit $-112 \mathrm{~cm}^{-1}$ und $-141 \mathrm{~cm}^{-1}$ (siehe $\mathrm{Tab}$ 4-11).

Im oberen Teil der Abbildung 6-5] sind die FTIR-Jetspektren von Albrecht [175] bei $130^{\circ} \mathrm{C}$ (e) und $150^{\circ} \mathrm{C}$ (f) Substanztemperatur dargestellt. Hier findet sich ein analoges Bandenpaar bei $3382 / 3368 \mathrm{~cm}^{-1}$, welches als die Donorbanden eines gemischten trans-cis-Dimers5) bzw. eines trans-trans-Homodimers interpretiert wird.

\footnotetext{
${ }^{5)}$ In der zitierten Arbeit heißt das Dimer cis-trans. Dies wird hier angepasst, um mit der bisher verwendeten Konvention die Cluster in der Reihenfolge H-Brückendonor, -akzeptor zu benennen, konsistent zu sein.
} 


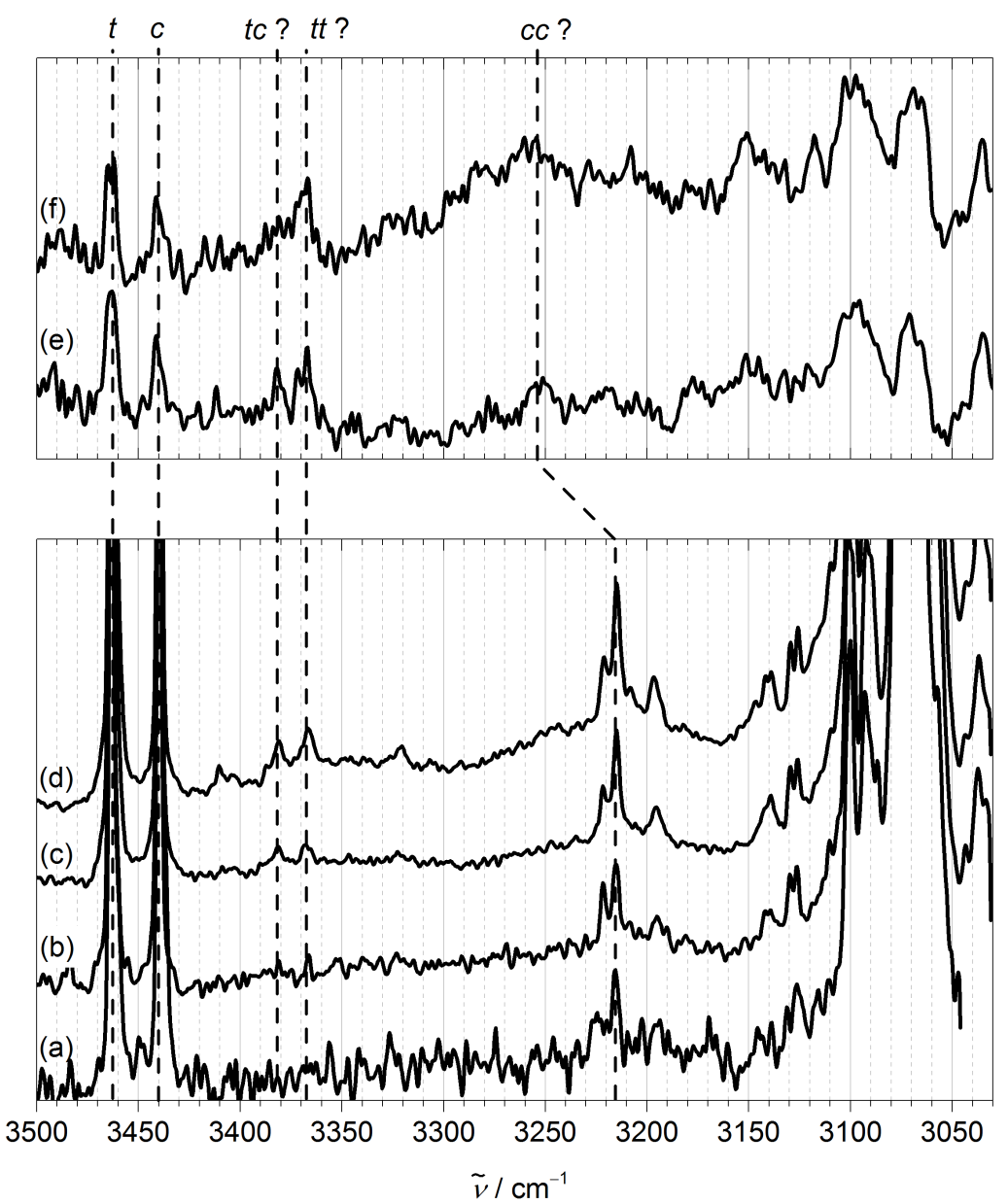

Abb. 6-5. FTIR- und Raman-Jetspektren zur Clusterbildung von NPhF in der Amid A-Region. Spektren aus Jet-Expansionen mit Helium als Trägergas und jeweils auf die cis-Monomerbande normiert.

Oben: FTIR-Jetspektren mit von (e) bis (f) zunehmender NPhF-Konzentration inklusive (? = vorläufiger) Bandenzuordnung (aus [175]).

Unten: Raman-Jetspektrum bei Düsentemperaturen $\vartheta=120-130^{\circ} \mathrm{C}$ und mit von (a) bis (d) zunehmender NPhF-Konzentration. (Ausführliche Informationen zu den FTIR-Messbedingungen sind in [175] dokumentiert. Einzelheiten zu den Raman-Messungen befinden sich in Tab. A-6 im Raman-Spektrenverzeichnis in Anhang A.6.1 Details zu den quantenchemischen Rechnungen sind in den Tabellen 6-5 und A-26 enthalten.) 
Zu der breiten Bande bei 3166-3300 $\mathrm{cm}^{-1}$ findet sich im Jet-FTIR eine ähnliche Bande zwischen 3165 und $3305 \mathrm{~cm}^{-1}$, jedoch ohne vergleichbar intensive Einzelpeaks, aber mit einem Maximum bei $3255 \mathrm{~cm}^{-1}$. Diese Bande wird dem $c c$-Dimer zugeordnet. Die Existenz eines unstrukturierten Multipletts anstatt des zu erwartenden Einzelpeaks wird bei Albrecht [175] mit einer Fermi-Resonanz mit der Kombinationsbande aus Carbonyl-Streckschwingung und $\mathrm{N}-\mathrm{H}$ Knickschwingung erklärt. Es ist dabei anzunehmen, dass die resultierende Verschiebung bis in die, auch im FTIR verhältnismäßig intensiven, C-H-Streckschwingungsbanden unterhalb von $3165 \mathrm{~cm}^{-1}$ hineinreicht [175]. Auffällig ist hierbei, dass im Raman-Spektrum die beiden Peaks bei 3221 und $3215 \mathrm{~cm}^{-1}$ als intensivste Signale dieser breiten Bande bereits im clusterärmsten Spektrum (Abb. 6-5 (a)) klar erkennbar sind, was auf das Dimerbandenpaar bei $3381 / 3367 \mathrm{~cm}^{-1}$ im gleichen Spektrum jedoch nicht zutrifft.

Die gefundenen Banden in den Raman- und FTIR-Jetspektren sind mitsamt vorläufiger Zuordnung in Tabelle 6-4 zusammengefasst.

Tab. 6-4. Experimentelle Amid A-Bandenpositionen von NPhF aus Raman- und FTIR-Jetspektren mitsamt Zuordnung. Alle Angaben in $\mathrm{cm}^{-1}$.

\begin{tabular}{|c|c|c|c|c|}
\hline \multicolumn{2}{|c|}{ Raman Jet $^{b}$} & \multicolumn{3}{|c|}{ FTIR Jeta,b } \\
\hline 3463 & $t$ & 3464 & & $t$ \\
\hline 3440 & $c$ & 3441 & & $c$ \\
\hline 3411 & $?$ & - & & - \\
\hline 3381 & {$\left[t c_{\mathrm{N}-\mathrm{a}}, t t_{\mathrm{N}-\mathrm{d}}, c t_{\mathrm{d}}\right]$} & 3382 & & {$\left[t c_{\mathrm{H}-\mathrm{d}}\right]$} \\
\hline 3367 & $t c_{\mathrm{N}-\mathrm{d}}$ & 3368 & & {$\left[t t_{\mathrm{H}-\mathrm{d}}\right]$} \\
\hline 3321 & $?$ & - & & - \\
\hline 3221 & ) & & & ) \\
\hline 3215 & & 3255 & $3305-3165$ & c \\
\hline 3196 & & - & & $>\mathrm{cc}$ \\
\hline- & $c c$ & 3152 & & $\int$ \\
\hline 3141 & & - & & - \\
\hline 3129 & & - & & - \\
\hline 3126 & & - & & - \\
\hline
\end{tabular}

Quantenchemische Rechnungen Mit dem hohen cis-NPhF-Anteil in der Jet-Expansion von ca. $50 \%$ (siehe oben) ergibt sich für die cc-Dimerbildung bei einer rein statistischen Betrachtung eine Bildungswahrscheinlichkeit von $25 \%$. Mit ebenfalls $25 \%$ Wahrscheinlichkeit wird ein $t t$-Dimer gebildet. Auf gemischte Dimere entfallen somit $50 \%$ Bildungswahrscheinlichkeit. Damit werden in der Expansion neben den trans-trans-Konformeren und dem gesuchten ciscis-Dimer auch gemischte Dimere in signifikanter Menge erwartet. Dieser Umstand macht eine Bandenzuordnung auf Basis der experimentellen Ergebnisse, nicht zuletzt wegen weniger Vergleichsmöglichkeiten in der Literatur, unmöglich. Daher wird an dieser Stelle in B3LYP-Rechnungen sowohl mit als auch ohne Dispersionskorrektur die relative Stabilität verschiedener Dimerstrukturen ermittelt und die harmonischen Frequenzen der N-H-Streckschwingung dem gemessenen Spektrum gegenübergestellt. Anders als in den bisherigen Molekülen wird hier statt „aug-cc-pVTZ“ auf den kleineren triple- $\zeta$-Basissatz „def2-TZVP“ zurückgegriffen, um die maximal verfügbare Rechenzeit auch mit den deutlich größeren NPhF-Clustern einzuhalten. 
Die Verwendung von def2-TZVP ist ein guter Kompromiss aus Performance und Genauigkeit und in diesem Zusammenhang gut erprobt [57, 295].

Durch Geometrieoptimierungen auf B3LYP-D3(BJ)/def2-TZVP-Niveau können die stabilsten Strukturen der Homodimervarianten $t t$ und $c c$ sowie gemischter Dimere einmal mit cis-NPhF als Wasserstoffbrückendonormolekül (ct) und einmal mit $t-\mathrm{NPhF}$ als Donormolekül $(t c)$ berechnet werden. Für $t t$ - und $t c-\mathrm{NPhF}$ werden im Folgenden jeweils zwei Strukturen unterschieden, die entweder mit dem $\mathrm{H}$-seitigen (Index $\mathrm{H}$ ) oder dem $\mathrm{N}$-seitigen (Index $\mathrm{N}$ ) freien Elektronenpaar des Akzeptor-Sauerstoffatoms eine Wasserstoffbrücke ausbilden (siehe auch die Clusternomenklatur von NMA in Abschnitt 4.3). Die sechs stabilsten Dimerstrukturen sind in Abbildung 6-6 dargestellt und die zugehörigen Eigenschaften in Tabelle 6-5 zusammengefasst.
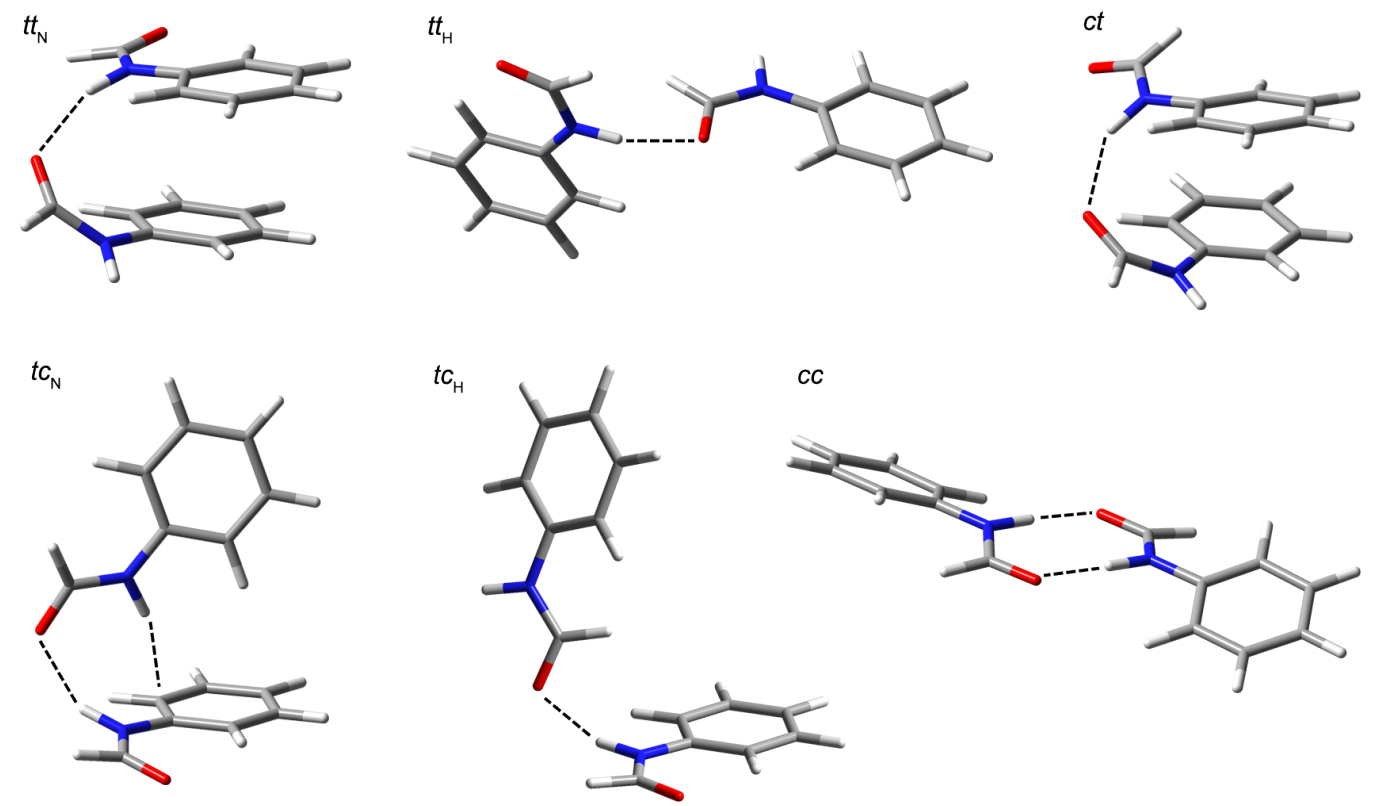

Abb. 6-6. NPhF-Dimerstrukturen auf B3LYP-D3(BJ)/def2-TZVP-Niveau. Gestrichelte Linien zeigen intermolekulare Wasserstoffbrückenbindungen an. Relative Energien, Dissoziationsenergien und geometrische Parameter sind in Tabelle 6-5 aufgeführt.

Tab. 6-5. Berechnete Eigenschaften der NPhF-Dimerstrukturen auf B3LYP/def2-TZVP-Niveau mit und ohne Dispersionskorrektur. Angegeben sind die relativen elektronischen und nullpunktskorrigierten Energien $\Delta E_{\mathrm{el}}$ bzw. $\Delta E_{0}$, sowie Dissoziationsenergien $D_{\mathrm{el}}$ bzw. $D_{0}$ (alle in $\mathrm{kJ} \cdot \mathrm{mol}^{-1}$ ). Die Länge der $\mathrm{N}-\mathrm{H} \cdots \mathrm{O}=\mathrm{C}$-Wasserstoffbrückenbindung $d$ (in pm), der $\mathrm{H} \cdots \mathrm{O}=\mathrm{C}$-Bindungswinkel der Wasserstoffbrückenbindung $\alpha$ (in ${ }^{\circ}$ ), die niedrigste Frequenz in doppelt-harmonischer Näherung $\tilde{v}_{\min }\left(\right.$ in $\mathrm{cm}^{-1}$ ) und die Dipolmomente $\mu$ (in D).

\begin{tabular}{|c|c|c|c|c|c|c|c|c|c|c|c|}
\hline & \multirow{2}{*}{$\frac{t t_{\mathrm{N}}}{\mathrm{D} 3(\mathrm{BJ})^{\mathrm{a}}}$} & \multicolumn{2}{|c|}{$t t_{\mathrm{H}}$} & \multicolumn{2}{|c|}{$c c$} & \multicolumn{2}{|c|}{$c t$} & \multicolumn{2}{|c|}{$t c_{N}$} & \multicolumn{2}{|c|}{$t c_{\mathrm{H}}$} \\
\hline & & D3(BJ) & ohne & D3(BJ) & ohne & D3(BJ) & ohne & D3(BJ) & ohne & D3(BJ) & ohne \\
\hline$\Delta E_{\mathrm{el}}$ & 19.6 & 29.3 & 25.7 & 0.0 & 0.0 & 26.1 & 28.7 & 20.8 & 27.4 & 31.5 & 27.5 \\
\hline$\Delta E_{0}$ & 19.5 & 29.1 & 24.9 & 0.0 & 0.0 & 25.1 & 26.8 & 20.3 & 26.2 & 30.5 & 25.8 \\
\hline$D_{\mathrm{el}}$ & 47.7 & 38.0 & 27.1 & 73.6 & 57.9 & 44.3 & 26.6 & 49.7 & 27.9 & 39.0 & 27.8 \\
\hline$D_{0}$ & 43.1 & 33.5 & 23.4 & 67.3 & 51.9 & 39.8 & 23.3 & 44.6 & 23.9 & 34.5 & 24.3 \\
\hline$\alpha$ & 128 & 106 & 124 & 121 & 124 & 127 & 171 & 116 & 129 & 105 & 125 \\
\hline$d$ & 202 & 195 & 198 & 182 & 187 & 208 & 199 & 204 & 201 & 196 & 198 \\
\hline$\tilde{v}_{\text {min }}$ & 19 & 9 & 8 & 13 & 14 & 7 & 6 & 15 & 12 & 11 & 7 \\
\hline$\mu$ & 6.0 & 6.9 & 7.9 & 0.034 & 0.021 & 6.6 & 4.1 & 4.7 & 6.5 & 7.0 & 8.4 \\
\hline
\end{tabular}


Das ringförmige cis-cis-Dimer erweist sich, wie bei NMF und NMA, mit zwei $\mathrm{N}-\mathrm{H} \cdots \mathrm{O}=\mathrm{C}$ Wasserstoffbrücken als das deutlich stabilste mit $19.5 \mathrm{~kJ} \cdot \mathrm{mol}^{-1}$ Unterschied ${ }^{6)} \mathrm{zum} \mathrm{tt}_{\mathrm{N}}$-Dimer, aber im Vergleich zu NMF und NMA mit etwas weniger Energievorsprung, da jetzt die $t t$-Dimersierung Aromatenstacking erlaubt. Ein direkter Vergleich der Rechnungen ist wegen der unterschiedlichen Basissätze jedoch nicht möglich. Das cis-cis-Dimer ist aufgrund der nicht-planaren cis-Monomerstruktur nur $C_{2}$-symmetrisch, hat aber mit $121^{\circ}$ nahezu perfekte H-Brücken-Bindungswinkel und damit eine zyklische Struktur ohne nennenswerte Ringspannung sowie eine Wasserstoffbrückenbindungslänge von lediglich $182 \mathrm{pm}$. Beides zusammen lässt eine starke Rotverschiebung für die N-H-Streckschwingung erwarten.

Als zweitstabilste Struktur ergibt sich das $t t_{\mathrm{N}}$-Konformer, das zusätzlich zur $202 \mathrm{pm}$ langen $\mathrm{N}-\mathrm{H} \cdots \mathrm{O}=\mathrm{C}$-Wasserstoffbrücke eine dispersive $\pi-\pi$-Wechselwirkung der beiden Phenylgruppen aufweist. Der damit verbundene Energiegewinn ist groß genug, um die planare Geometrie beider trans-Monomermoleküle aufzugeben. Deren Diederwinkel betragen $\varphi=15.5^{\circ}$ am Donor und $\varphi=41.6^{\circ}$ am Akzeptor. Ohne die Berücksichtigung der Dispersionskorrektur in der Rechnung gibt es keine stabile Konformation mit dem N-seitigen freien Elektronenpaar des Sauerstoffatoms als H-Brückenakzeptor.

Die nominell drittstabilste Struktur ist das gemischte $t c_{\mathrm{N}}$-Dimer, das mit einem Energieunterschied von lediglich $0.8 \mathrm{~kJ} \cdot \mathrm{mol}^{-1}$ als de facto isoenergetisch $\mathrm{zu}_{\mathrm{N}}$ angesehen werden kann. Seine Stabilität erhält es durch eine zusätzlich zur $\mathrm{N}-\mathrm{H} \cdots \mathrm{O}=\mathrm{C}$-Wasserstoffbrücke auftretende $\mathrm{N}-\mathrm{H} \cdots \pi$-Wechselwirkung der $\mathrm{N}-\mathrm{H}$-Gruppe des Akzeptormoleküls mit dem Phenylring des Donors. Das trans-Monomermolekül verliert hierbei seine planare Struktur und hat einen Diederwinkel von $\varphi=12.3^{\circ}$. Durch diese zweite Wechselwirkung ist auch für die N-H-Schwingung eine deutliche Rotverschiebung gegenüber dem cis-Monomer zu erwarten.

Die $t c_{\mathrm{H}}$-Dimerstruktur entspricht dem von Albrecht [175] gefundenen stabilsten gemischten Dimer. In der Struktur liegt keine zusätzliche $\mathrm{N}-\mathrm{H} \cdots \pi$-Wasserstoffbrücke vor (siehe Abb. A-11 in Anhang A.9.1) und sie ist gegenüber $t c_{N}$ um $10.2 \mathrm{~kJ} \cdot \mathrm{mol}^{-1}$ benachteiligt. Der Vergleich der nullpunktskorrigierten Energien ohne Dispersionskorrektur ergibt allerdings einen Energieunterschied von $0.4 \mathrm{~kJ} \cdot \mathrm{mol}^{-1}$ zugunsten von $t c_{\mathrm{H}}$. Zur Zeit der oben genannten Arbeit war die empirische Dispersionskorrektur noch nicht in GAUSSIAN implementiert, weshalb dort $t c_{\mathrm{H}}-\mathrm{NPhF}$ als das präferierte gemischte Dimer angegeben ist.

Energetisch folgt noch das ct-Dimer mit dem cis-Konformer als Donormolekül, das $4.8 \mathrm{~kJ} \cdot \mathrm{mol}^{-1}$ über dem $t c_{N}$-Dimer liegt und strukturell eine hohe Ähnlichkeit zur $t t_{\mathrm{N}}$-Struktur aufweist. Der $\varphi$-Diederwinkel im cis-Donormolekül des ct-Dimers ist mit $34.8^{\circ} \mathrm{im}$ Vergleich zu den $15.5^{\circ} \mathrm{im}$ trans-Donor des $t t_{\mathrm{N}}$-Dimers mehr als doppelt so groß, was zu einem größeren Abstand der Phenylgruppen und damit zu geringeren $\pi-\pi$-Wechselwirkungen führt.

Das $t t_{\mathrm{H}}$-Konformer ist mit $29.1 \mathrm{~kJ} \cdot \mathrm{mol}^{-1}$ gegenüber dem $c c$-Konformer als globale Minimumstruktur die energetisch zweit-ungünstigste unter den vorgestellten Dimerstrukturen und wird nur noch vom $t c_{\mathrm{H}}$-Konformer übertroffen. Aufgrund des Energieunterschieds sind beide für die Clusterbildung in der Jet-Expansion als nicht relevant anzunehmen. Es handelt sich zudem

${ }^{6)}$ Falls nicht explizit erwähnt, sind angegebene Energien die relativen, nullpunktskorrigierten Energien der Rechnung mit Dispersionskorrektur ( $\Delta E_{0}$ in Tab. 6-5). 
um die $t t$-Dimerstruktur, auf deren Grundlage die Bandenzuordnungen im FTIR-Jetspektrum erfolgte [175]. Dies wird wieder durch die dort durchgeführte Geometrieoptimierung ohne Dispersionskorrektur plausibel, worin $t t_{\mathrm{H}}$ das einzig stabile $t t$-Konformer ist und eine dem $t t_{\mathrm{N}}$ entsprechende Startgeometrie in der Optimierung zum $t t_{\mathrm{H}}$ konvergiert. Ein ähnliches Verhalten zeigt die ct-Dimerstruktur, deren Geometrie ebenfalls durch sekundäre Dispersionswechselwirkungen stabilisiert wird. Die zugehörigen Strukturen ohne Dispersionskorrektur finden sich in Abbildung A-11 in Anhang A.9.1.

Wenn nicht die Gesamtenergie der Cluster verglichen wird, sondern die nullpunktskorrigierte Wechselwirkungsenergie (oder Dissoziationsenergie) $\Delta D_{0}$, also der gesamte Energiegewinn der Clusterbildung gegenüber den isolierten Monomeren in der jeweils im Dimer vorliegenden qualitativen Konformation, dann ergibt sich im Wesentlichen die gleiche energetische Abfolge der beschriebenen Strukturen. Einzig $t t_{\mathrm{N}}$ hat eine um $1.5 \mathrm{~kJ} \cdot \mathrm{mol}^{-1}$ geringere Wechselwirkungsenergie als $t c_{\mathrm{N}}$ und $t c_{\mathrm{H}}$ ist gegenüber $t t_{\mathrm{H}} \mathrm{um} 1 \mathrm{~kJ} \cdot \mathrm{mol}^{-1}$ begünstigt.

Vergleich von Theorie und Experiment Die harmonischen N-H-Streckschwingungsfrequenzen der drei stabilsten Dimere, $c c, t t_{\mathrm{N}}$ und $t c_{\mathrm{N}}$, sind als Strichspektren in Abbildung 6-7 dargestellt und auf das trans-Monomer skaliert (Faktor: 0.962).

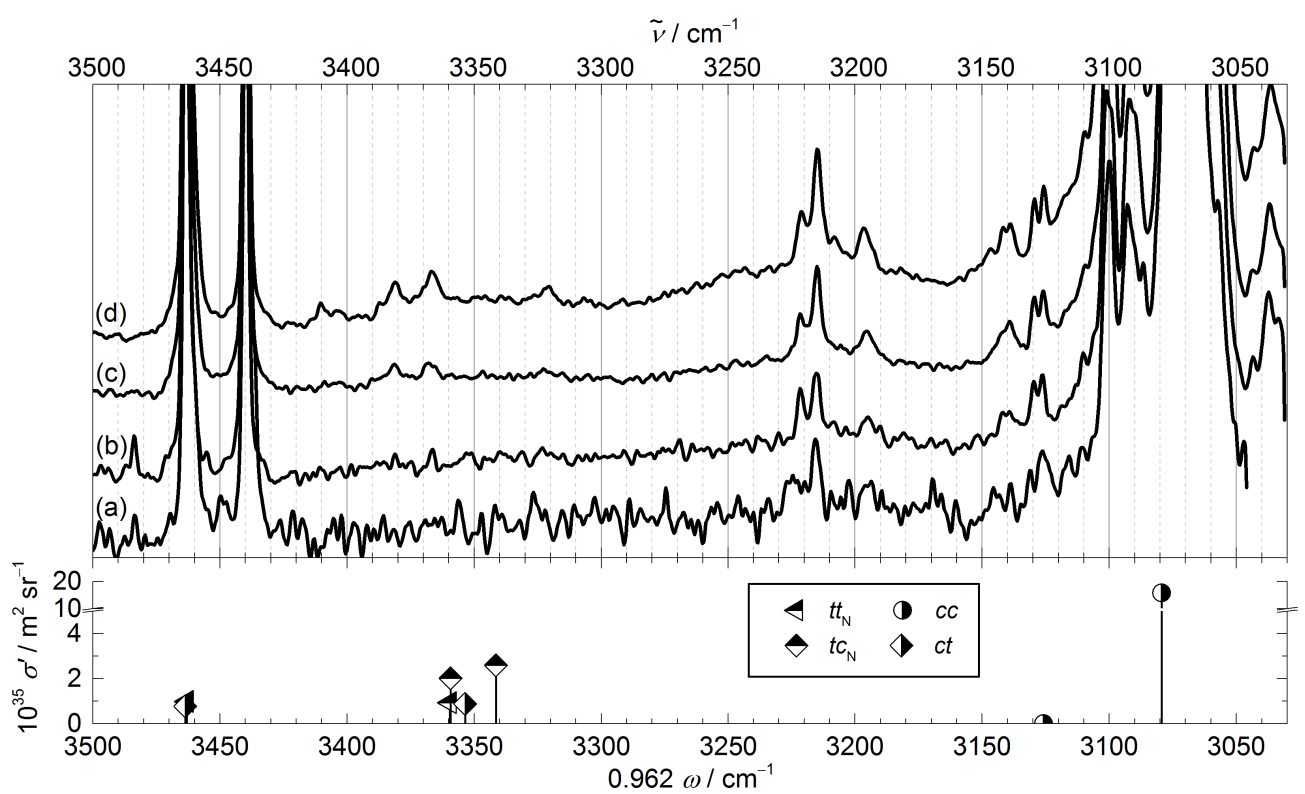

Abb. 6-7. Raman-Jetspektren zur Clusterbildung von NPhF und theoretische Bandenpositionen in der Amid A-Region. Spektren wie in Abb. 6-5 Im unteren Teil befinden sich die auf die $t$-AmA-Bandenposition skalierten harmonischen Frequenzen der stabilsten Monomer- und Dimerstrukturen aus B3LYP-D3(BJ)/def2-TZVP-Rechnungen. (Ausführliche Informationen zu den Messbedingungen befinden sich in Tab. A-6 im Raman-Spektrenverzeichnis in Anhang A.6.1 Details zu den quantenchemischen Rechnungen sind in den Tabellen 6-5 und A-26 enthalten.)

Die skalierte Position der beiden N-H-Strecknormalmoden von $t c_{\mathrm{N}}$ befinden sich in Anbetracht der typischen Überschätzung der Rotverschiebung durch die B3LYP-Rechnung in guter Übereinstimmung mit dem Bandenpaar bei 3381 und $3367 \mathrm{~cm}^{-1}\left(3382\right.$ und $3368 \mathrm{~cm}^{-1}$ im FTIR). Zur genaueren Überprüfung dieser Zuordnung werden wieder die Differenzen der unskalierten, harmonischen Werte verwendet (siehe Tab. 6-4). Demnach liegen die berechneten 
Positionen für Donor- und Akzeptor-NH-Streckschwingung $19 \mathrm{~cm}^{-1}$ auseinander. Mit einer bereits für NMF und NMA angenommenen Überschätzung der Verschiebung von $21 \%$ (siehe Abschnitt 5.3) liegt der zu erwartende Unterschied zwischen Donor- und Akzeptorbande bei ca. $16 \mathrm{~cm}^{-1}$, was in Anbetracht der groben Abschätzung in guter Übereinstimmung mit der experimentellen Differenz der beiden Banden von $14 \mathrm{~cm}^{-1}$ ist und die Zuordnung bekräftigt.

Für die Position der $t t_{\mathrm{N}}$-Donorbande des nominell stabilsten Dimers ergibt die Rechnung mit $3492 \mathrm{~cm}^{-1}$ die gleiche unskalierte Position wie für die Akzeptorbande des $t c_{\mathrm{N}}$-Dimers (siehe Tab. 6-4) und trägt entsprechend zur Bandenintensität bei. Aus statistischer Sicht ist die Bildung beider Dimere gleich wahrscheinlich, solange keine Relaxation in der Expansion stattfindet. Während eine cis/trans-Isomerisierung der Monomere in den Clustern aufgrund der hohen Barriere ausgeschlossen werden kann, wären Umlagerungen wie z. B. von $c t$ in $t c_{N}$ eher denkbar, obwohl auch hier die Wasserstoffbrückenbindung erst gelöst werden muss. Durch eine solche Relaxation wäre der Anteil des $t c_{N}$-Dimers höher und dessen Banden würden im Spektrum überwiegen. Im Raman-Spektrum sind jedoch beide Banden in etwa gleich intensiv, was unter Einbeziehung der berechneten Streuquerschnitte (siehe Tab. 6-6) bedeutet, dass beide Dimere ähnlich häufig gebildet werden und der höhere Streuquerschnitt von $t c_{N}$ bei $3367 \mathrm{~cm}^{-1}$ durch den $t t_{\mathrm{N}}$-Anteil an der $3381 \mathrm{~cm}^{-1}$-Bande kompensiert wird.

Im FTIR-Spektrum in Abbildung 6-5 (f) ist die $3368 \mathrm{~cm}^{-1}$-Bande intensiver als diejenige bei $3382 \mathrm{~cm}^{-1}$. Dabei ergibt sich aus den Rechnungen, dass die IR-Intensität der höherfrequenten $t c_{N}$-Akzeptorbande um den Faktor 5.6 größer ist als die niederfrequente $t c_{N}$-Donorbande (siehe Tab. 6-6). Zusätzlich dürfte auch hier die $t t_{\mathrm{N}}$-Donorschwingung aufgrund ihrer hohen IR-Intensität einen signifikanten Einfluss auf die $3382 \mathrm{~cm}^{-1}$-Bande haben. Die FTIR-Ergebnisse stehen somit im Widerspruch zu der oben vorgestellten Zuordnung im Ramanspektrum.

Tab. 6-6. Berechnete N-H-Streck-Normalmoden der drei stabilsten NPhF-Dimere auf B3LYP-D3(BJ)/def2-TZVP-Niveau und Zuordnung zu den experimentellen Raman-Banden ( $\left.\tilde{v}_{\mathrm{Ra}}\right)$ und FTIR-Banden $\tilde{v}_{\mathrm{IR}}$. Relative Verschiebungen zum entsprechenden Monomerwert stehen in runden Klammern; experimentelle Werte, deren Zuordnung nicht eindeutig geklärt ist, in eckigen Klammern. Angegeben sind die harmonischen Wellenzahlen $\omega$, die IR-Intensität $A$ und der differentielle Raman-Streuquerschnitt $\sigma^{\prime}$ bei $532.269 \mathrm{~nm}$ Anregungswellenlänge und $90^{\circ}$ Streugeometrie nach Gl. 3-13 Die Buchstaben „a“ und „d“ im Index stehen für Akzeptor und Donor und wechseln für das zyklische cc-Dimer zu "as“ und ",“ für die antisymmetrische und symmetrische Streckschwingung (Weitere Daten der Frequenzrechnungen zu diesen und weiteren Dimerstrukturen befinden sich in den Tab. A-26 im Tabellenwerk in Anhang A.9.2).

\begin{tabular}{|c|c|c|c|c|c|c|}
\hline \multicolumn{2}{|c|}{ Konformer } & \multirow{2}{*}{$\frac{\omega / \mathrm{cm}^{-1}}{3600(+2)}$} & \multirow{2}{*}{$\frac{A / \mathrm{km} \cdot \mathrm{mol}^{-1}}{31}$} & \multirow{2}{*}{$\frac{10^{34} \sigma^{\prime} / \mathrm{m}^{2} \mathrm{sr}^{-1}}{0.98}$} & \multirow{2}{*}{$\frac{\tilde{v}_{\mathrm{Ra}} / \mathrm{cm}^{-1}}{-}$} & \multirow{2}{*}{$\frac{\tilde{v}_{\mathrm{IR}} / \mathrm{cm}^{-1}}{-}$} \\
\hline$t t_{N}$ & $v_{\mathrm{NH}-\mathrm{a}}$ & & & & & \\
\hline & $v_{\mathrm{NH}-\mathrm{d}}$ & $3492(-106)$ & 195 & 0.92 & {$[3381(-82)]$} & {$[3368(-96)]^{c}$} \\
\hline \multirow[t]{2}{*}{$t c_{N}$} & $v_{\mathrm{NH}-\mathrm{a}}$ & $3492(-82)^{\mathrm{a}}(-106)^{\mathrm{b}}$ & 431 & 2.01 & $3381(-59)^{\mathrm{a}}(-82)^{\mathrm{b}}$ & \multirow{2}{*}[3382(-59)]{$^{a, c}$} \\
\hline & $v_{\mathrm{NH}-\mathrm{d}}$ & $3473(-125)^{b}$ & 77 & 2.60 & $3367(-96)^{b}$ & \\
\hline \multirow[t]{2}{*}{$c t$} & $v_{\mathrm{NH}-\mathrm{a}}$ & $3600(+2)$ & 27 & 0.76 & $(-)$ & \\
\hline & $v_{\mathrm{NH}-\mathrm{d}}$ & $3486(-134)^{\mathrm{a}}(-112)^{\mathrm{b}}$ & 149 & 0.87 & $(-)$ & \\
\hline \multirow[t]{2}{*}{$c c$} & $v_{\mathrm{NH}-\mathrm{as}}$ & $3249(-325)$ & 1929 & 0.00 & - & {$[3255(-186)]^{c, d}$} \\
\hline & $v_{\mathrm{NH}-\mathrm{s}}$ & $3201(-373)$ & 0.0 & 15.8 & {$[3215(-225)]^{d}$} & - \\
\hline
\end{tabular}

Die harmonische B3LYP-D3(BJ)-Rechnung verortet die Raman-aktive, symmetrische N-HStreckschwingung des cc-Dimers mit $3201 \mathrm{~cm}^{-1}$ mitten im C-H-Streckbereich. Damit ist sie 
gegenüber der Frequenz der entsprechenden Normalmode im c-Monomer um $-373 \mathrm{~cm}^{-1}$ verschoben. Dem steht im Raman-Spektrum eine Verschiebung von $-225 \mathrm{~cm}^{-1}$ gegenüber, wenn der intensivste Einzelpeak bei $3215 \mathrm{~cm}^{-1}$ als Referenz für die breite Bande bei $3166-3300 \mathrm{~cm}^{-1}$ genommen wird. Dies entspricht einer Überschätzung der Rotverschiebung um $66 \%$. Wenn die bisher angenommenen $21 \%$ angesetzt werden, wäre die cc-Dimerbande im Spektrum bei $3132 \mathrm{~cm}^{-1}$ zu erwarten. Dies bekräftigt die schon aufgrund der Breite und Struktur der Bande getroffene Vermutung, dass hier eine Fermi-Resonanz vorliegt und die cc-Dimerbande bis in den Bereich der aromatischen $\mathrm{C}-\mathrm{H}-\mathrm{Streckschwingungen} \mathrm{hineinreicht.} \mathrm{Damit} \mathrm{kann} \mathrm{auch} \mathrm{die} \mathrm{langge-}$ zogene hochfrequente Flanke der intensivsten $\mathrm{CH}$-Streckschwingungsbande mitsamt der leicht zunehmenden Peaks bei 3141, 3129 und $3126 \mathrm{~cm}^{-1}$ versuchsweise dem $c c$-Dimer zugeordnet werden.

Es existieren aufgrund der lokalen Symmetrie des zyklischen Dimers mit $v_{\mathrm{NH}}^{a s}$ und $v_{\mathrm{NH}}^{s}$ eine anti- und eine symmetrische $\mathrm{N}-\mathrm{H}$-Strecknormalmode, die entsprechend nahezu vollständig IR- bzw. Raman-aktiv sind. Die B3LYP-D3(BJ)-Rechnung ergibt für diese beiden Moden mit $3249 \mathrm{~cm}^{-1}$ und $3201 \mathrm{~cm}^{-1}$ um $48 \mathrm{~cm}^{-1}$ unterschiedliche Frequenzen (siehe Tab. 6-6). Eine solche Verschiebung ist in der Gegenüberstellung von FTIR- und Raman-Spektren in Abbildung 6-5 zwischen den Maxima im FTIR- $\left(3255 \mathrm{~cm}^{-1}\right)$ und im Raman-Spektrum $\left(3215 \mathrm{~cm}^{-1}\right)$ der potentiellen $c c$-Dimerbande zu erkennen und beträgt folglich $40 \mathrm{~cm}^{-1}$. Wenn nun wieder die Überschätzung von $21 \%$ für den theoretischen Wert angenommen wird, dann ergäbe dies einen Unterschied von $39.7 \mathrm{~cm}^{-1}$, was ein weiteres Indiz für die $c c$-Dimerzuordnung ist.

Weiterhin wäre neben den zur Resonanz beitragenden Moden auch interessant, wie groß der Anteil der N-H-Streckschwingungsnormalmode an der Intensität der Peakgruppe um $3215 \mathrm{~cm}^{-1}$ im Raman-Spektrum ist. Wenn das Verhältnis zwischen den Bandenintegralen der Peakgruppe und einer anderen reinen NH-Streckschwingungsbande (z B. bei $3367 \mathrm{~cm}^{-1}$ ) ähnlich den zugeordneten Streuquerschnitten ist, dann ist der Anteil der N-H-Streckschwingung an der Gesamtintensität der resonanten Bande hoch. In diesem Fall steht ein experimentelles Verhältnis von 1:10 einem theoretischen von 1:6 gegenüber (Donor-N-H-Streckschwingung des $t c_{\mathrm{N}}$-Dimers : symmetrischer $\mathrm{N}-\mathrm{H}$-Streckschwingung des cc-Dimers). In Anbetracht einer hohen Unsicherheit in der theoretischen Berechnung der Raman-Aktivität von Moden mit starken intermolekularen Wechselwirkungen, kann dieses Verhältnis als ähnlich groß angesehen werden.

Die beiden Banden bei 3411 und $3321 \mathrm{~cm}^{-1}$ skalieren wie die anderen Dimerbanden mit zunehmender Konzentration. Diese Banden sind aber weder im FTIR-Spektrum klar erkennbar, noch liefern die gerechneten Dimerstrukturen passende Bandenpositionen, weshalb eine Zuordnung an dieser Stelle ausbleiben muss. 


\subsection{Trimerbildung}

Weder der experimentelle Nachweis noch quantenchemische Rechungen von NPhF-Trimeren sind in der Literatur bekannt. Die Clusterspektren in Abbildung 6-7 weisen keine Banden auf, die auf Trimere hindeuten. Dies entspricht den Erwartungen für diese Messreihe, da diese im Vergleich zu den FTIR-Jet-Messungen von Albrecht [175] eine deutlich geringere Substanzkonzentration aufweisen und dort schon keine Trimerbanden erkennbar sind. Dennoch wurden DFT-Rechnungen zur Struktur der Trimere gemacht, um die Möglichkeit der Bildung von zyklischen Aggregaten zu überprüfen, wie sie für NMA und NMF gefunden wurden (siehe Abschnitte 4.4 und 5.4).

Die B3LYP-D3(BJ)-Geometrieoptimierungen ergeben, anders als bei den kleineren Amiden, nur eine stabile zyklische $t t t$-Trimerstruktur, die hier analog als $t t t_{\mathrm{a}}$ bezeichnet wird (siehe Abb. 6-8). Ein $t t t_{\mathrm{p}}$-Trimer, bei dem die Phenylreste auf der gleichen Seite stehen, ergibt aufgrund der viel größeren sterischen Hinderung keine Minimumstruktur. Dieses Trimer ist gemeinsam mit $t t-c$ - und $c-c c-\mathrm{NPhF}$ eines der drei stabilsten Trimere von den hier untersuchten Strukturen bezogen auf die nullpunktskorrigierte, absolute Energie der Rechnung mit Dispersionskorrektur. Der maximale energetische Unterschied der drei Strukturen liegt bei $0.8 \mathrm{~kJ} \cdot \mathrm{mol}^{-1}$, was in DFT-Rechnungen nicht signifikant ist. Als weitere Strukturen sind das zyklische ${ }^{\prime} c_{\mathrm{cyc}^{-}}$ Trimer mit einem Energieunterschied von $11.4 \mathrm{~kJ} \cdot \mathrm{mol}^{-1}$ und $t$-tc-NPhF mit $10.1 \mathrm{~kJ} \cdot \mathrm{mol}^{-1}$ bezüglich $t t t_{\mathrm{a}}$ deutlich abgeschlagen (siehe Tab.6-7).7)
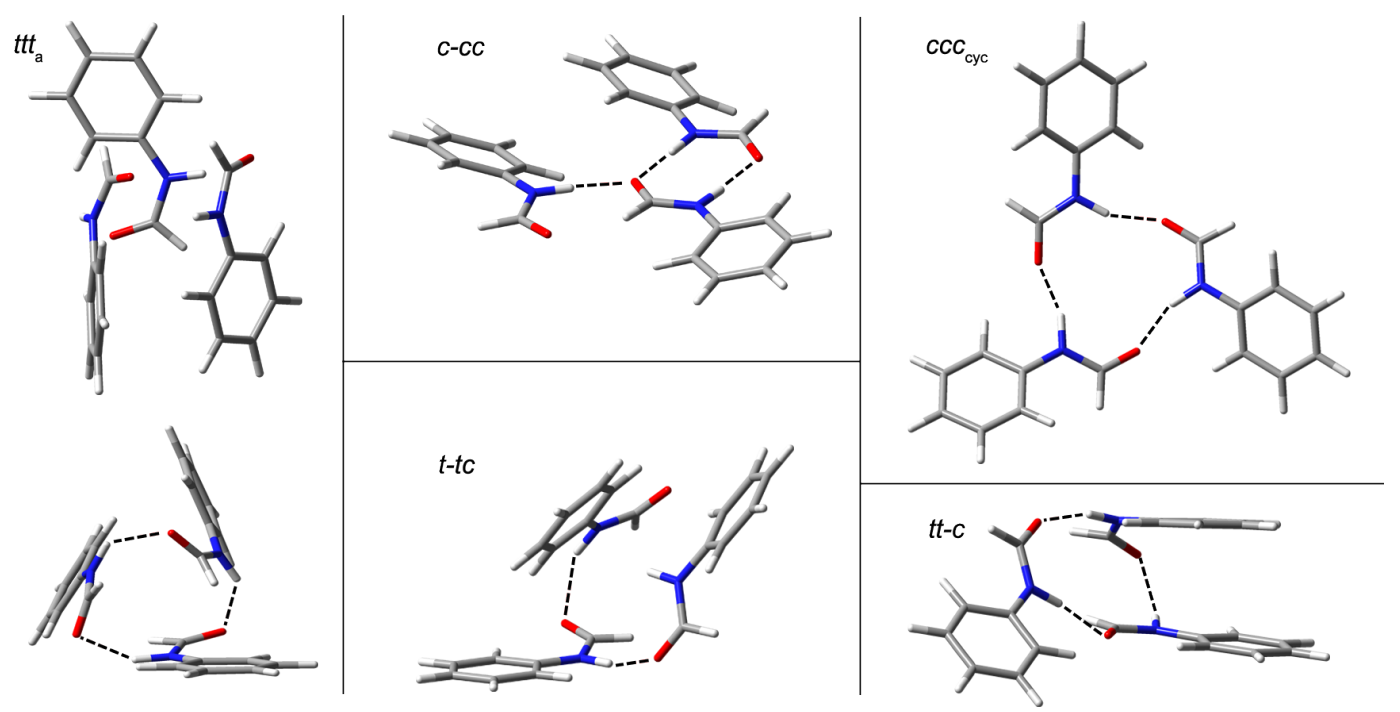

Abb. 6-8. Ausgewählte NPhF-Trimerstrukturen auf B3LYP-D3(BJ)/def2-TZVP-Niveau. Gestrichelte Linien zeigen intermolekulare Wasserstoffbrückenbindungen an. Die Amidgruppe eines der drei Monomere im $t t t_{a}$ hat eine antiparallele Ausrichtung. Relative Energien, Dissoziationsenergien und geometrische Parameter sind in Tabelle 6-7]aufgeführt.

Dieses Bild verändert sich grundlegend, wenn die gleichen Rechnungen ohne Dispersionskorrektur durchgeführt werden. Dann sind die beiden reinen cis-NPhF Cluster die stabilsten, während tt-c- mit $\Delta E_{0}=11.1 \mathrm{~kJ} \cdot \mathrm{mol}^{-1}$ und $t t t_{\mathrm{a}}-\mathrm{NPhF}$ mit $\Delta E_{0}=20.3 \mathrm{~kJ} \cdot \mathrm{mol}^{-1}$ deutlich dar-

\footnotetext{
${ }^{7)}$ Die diskutierten Strukturen stellen lediglich eine Auswahl denkbarer Trimergeometrien dar. Eine vollständige Analyse der Potentialhyperfläche liegt nicht vor und andere stabile Trimerstrukturen sind nicht auszuschließen.
} 
über liegen. Dies hängt mit der stabilisierenden Wirkung dispersiver Wechselwirkungen der Phenylgruppen in den beiden letztgenannten Clustern zusammen. Auch die anderen beiden Trimerstrukturen büßen Stabilität ein, was an der Verlängerung der Wasserstoffbrückenbindungen $\left(d(\mathrm{H} \cdots \mathrm{O})\right.$ in Tab. 6-7) und der deutlich reduzierten Wechselwirkungsenergie $D_{0}$ erkennbar ist. Dieser Effekt ist aber gering gegenüber $t t-c$ - und $t t t_{\mathrm{a}}-\mathrm{NPhF}$. Die zugehörigen Strukturen finden sich in Abbildung A-11 in Anhang A.9.1. Für t-tc-NPhF ist die Geometrioptimierung ohne Dispersionskorrektur gescheitert.

Tab. 6-7. Berechnete Eigenschaften ausgewählter NPhF-Trimerstrukturen auf B3LYP/def2-TZVP-Niveau mit und ohne Dispersionskorrektur „D3“ (zugehörige Strukturen mit D3(BJ) befinden sich in Abb. 6-8 und ohne in Abb. A-11 in Anhang A.9.1. Für eine Erklärung der Bezeichnungen, siehe Tab. 6-5

\begin{tabular}{|c|c|c|c|c|c|c|c|c|c|c|}
\hline & \multicolumn{2}{|c|}{$t t t_{a}$} & \multirow{2}{*}{$\begin{array}{c}\text { ttt } \\
\text { ohne }\end{array}$} & \multicolumn{2}{|c|}{$t t-c$} & \multirow{2}{*}{$\begin{array}{c}t-t c \\
\text { D3 }\end{array}$} & \multicolumn{2}{|c|}{$c-c c$} & \multicolumn{2}{|c|}{$c c C_{\text {cyc }}$} \\
\hline & D3 & ohne & & D3 & ohne & & D3 & ohne & D3 & ohne \\
\hline$\Delta E_{\mathrm{el}} / \mathrm{kJ} \cdot \mathrm{mol}^{-1}$ & 0.0 & 18.7 & 22.6 & 3.4 & 10.0 & 13.2 & 5.1 & 0.0 & 16.7 & 0.7 \\
\hline$\Delta E_{0} / \mathrm{kJ} \cdot \mathrm{mol}^{-1}$ & 0.0 & 20.3 & 22.2 & 0.3 & 11.1 & 10.1 & 0.8 & 0.0 & 11.4 & 0.1 \\
\hline$D_{\mathrm{el}} / \mathrm{kJ} \cdot \mathrm{mol}^{-1}$ & 124.0 & 64.4 & 60.4 & 123.8 & 75.6 & 114.0 & 128.3 & 90.8 & 116.8 & 90.1 \\
\hline$D_{0} / \mathrm{kJ} \cdot \mathrm{mol}^{-1}$ & 110.8 & 54.8 & 53.0 & 112.8 & 65.8 & 103.0 & 117.0 & 80.5 & 106.4 & 80.3 \\
\hline \multirow[t]{3}{*}{$\alpha(\mathrm{H} \cdots \mathrm{O}=\mathrm{C}) /^{\circ}$} & 102 & 114 & - & 103 & 161 & - & 108 & 107 & 150 & 156 \\
\hline & 103 & 105 & 130 & 147 & 102 & 100 & 122 & 123 & & \\
\hline & 101 & 106 & 121 & 114 & 108 & 136 & 121 & 107 & & \\
\hline \multirow[t]{3}{*}{$d(\mathrm{H} \cdots \mathrm{O}) / \mathrm{pm}$} & 228 & 219 & - & 218 & 217 & - & 183 & 194 & 186 & 191 \\
\hline & 223 & 212 & 193 & 190 & 207 & 213 & 181 & 191 & & \\
\hline & 213 & 208 & 194 & 183 & 192 & 185 & 184 & 187 & & \\
\hline$\tilde{v}_{\min } / \mathrm{cm}^{-1}$ & 15 & 9 & 4 & 6 & 7 & 9 & 9 & 10 & 5 & 8 \\
\hline$\mu / \mathrm{D}$ & 1.2 & 0.59 & 10.7 & 2.5 & 2.3 & 3.6 & 2.9 & 3.3 & 1.4 & 0.88 \\
\hline
\end{tabular}

Da in einer Jet-Expansion der simultane Stoß von drei Monomermolekülen zwar generell möglich, aber sehr unwahrscheinlich ist, kann vereinfacht davon ausgegangen werden, dass die Edukte jedes in der Expansion gebildeten Trimers ein Dimer und ein Monomer sind. Damit erfolgt die Trimer-Bildung im Wesentlichen aus den drei häufigsten Dimeren $t t_{N^{-}}, t c_{N}$ und $c c$ NPhF. Es bilden sich bevorzugt Strukturen, für die im Dimer nur eine minimale Änderung nötig ist, also für deren Bildung z. B. keine Wasserstoffbrücke im Dimer gelöst werden muss, wie bei der Bildung des $c c c_{c y c}-\mathrm{NPhF}$. Dies gilt ebenfalls für die $\mathrm{N}-\mathrm{H} \cdots \pi$-Wechselwirkung im $t c_{\mathrm{N}}$-Dimer, welche bei der Bildung des $t$-tc-Trimers gelöst werden muss. Als besonders wahrscheinlich kann die Bindung eines weiteren Moleküls an $c c-\mathrm{NPhF}$ angesehen werden. Das Dimer bleibt als Substruktur erhalten und hat zwei gleichwertige Akzeptorstellen. $t t t_{\mathrm{a}}-\mathrm{NPhF}$ gibt die $\pi-\pi$ Wechselwirkung im $t t_{\mathrm{N}}$-Dimer für zwei zusätzliche Wasserstoffbrückenbindungen auf. Im $t t-c$ Trimer kommen durch das $c$-Monomer ebenfalls zwei H-Brücken hinzu, die $t t_{\mathrm{N}}$-Dimerstruktur mit den besagten dispersiven Wechselwirkungen bleibt hier jedoch erhalten.

Eine offenkettige Trimerstruktur $t t_{1}$, ähnlich den bei NMF und NMA diskutierten, ist für NPhF selbst ohne Dispersionskorrektur das instabilste der gefundenen Trimere (siehe Tab. 6-7 und Abb. A-11 in Anhang A.9.1). Mit Berücksichtigung der Dispersion konvergiert $t t t_{1}$ zum zyklischen $t t t_{\mathrm{a}}$. 


\subsection{Zusammenfassung und Diskussion}

Mit diesem Kapitel werden die bisherigen Untersuchungen zu aliphatisch substituierten sekundären Amiden um Raman-spektroskopische Daten und quantenchemische Rechnungen für das aromatisch substituierte $\mathrm{N}$-Phenylformamid ergänzt. Es liegen mit cis-NPhF und trans-NPhF zwei stabile Konformationsisomere der Amidbindung vor, wobei auch hier das planare transIsomer als die globale Minimumstruktur durch Experimente und quantenchemische Rechnungen in der Literatur festgestellt wird. $c$-NPhF ist mit einem Diederwinkel $\varphi \approx 30^{\circ}$ nicht-planar und somit existieren zwei isoenergetische Enantiomere, die durch eine Drehung um $\varphi$ ineinander überführt werden können. Die durch die Isomerisierungsbarriere hervorgerufene Tunnelaufspaltung ist mit $0.1245 \mathrm{~cm}^{-1}$ [276] im curry-Jet jedoch nicht auflösbar.

Eine zu NMA und NMF analog durchgeführte Ermittlung des Enthalpieunterschieds aus dem Van-'t-Hoff-Plot ergibt eine Differenz von 6(5) $\mathrm{kJ} \mathrm{mol}^{-1}$. Dies liegt knapp über den Werten aus Jet-FT-Mikrowellenmessungen [276, 277] und denjenigen eines Fits der B3LYP-D3(BJ)/aVTZRechungen mit 3.29(2) kJ.mol ${ }^{-1}$. Ein zusätzlich durchgeführter Vergleich der freien Enthalpien der Isomersierungsreaktion trans- $\mathrm{NPhF} \rightleftharpoons$ cis-NPhF offenbart für den untersuchten Temperaturbereich, dass in den Rechnungen das Gleichgewicht auf Seiten des cis-NPhF liegt, während die Experimente das Gleichgewicht auf der Eduktseite verorten. Die Unterschiede zwischen experimentellen und theoretischen Werten fallen aber gering aus. Auffällig ist die Nähe aller Werte zu $\Delta G=0$, was allerdings aufgrund der gemachten Näherungen auch eine Folge günstiger Fehlerkompensationen sein kann.

Unter Berücksichtigung des sehr großen Fehlerbalkens im Van-'t-Hoff-Plot, welcher bereits ausführlich für NMA diskutiert wurde und aus der ungenauen Bandenintegration resultiert, ist dieses Ergebnis konsistent zu Theorie und Literatur.

Die erstmalige Untersuchung von $N$-Phenylformamid mittels Raman-Jet-Spektroskopie erfolgt in der $\mathrm{N}-\mathrm{H}$ - und $\mathrm{C}-\mathrm{H}$-Streckschwingungsregion oberhalb von $3000 \mathrm{~cm}^{-1}$. Die Amid ABanden von trans- und cis-NPhF sind deutlich getrennt und besitzen, anders als NMF und NMA, keine erkennbare Substruktur. Das cis-/trans-Intensitätsverhältnis liegt bei ungefähr 1:1. Dieser ungewöhnlich hohe cis-Anteil von ca. $50 \%$, bei einem mit NMF vergleichbaren Enthalpieunterschied beider Konformere (siehe Abschnitt 5.2), hat seine Ursache hauptsächlich in der Entartung durch die cis-NPhF-Enantiomere.

Im Raman-Spektrum sind die Bandenpositionen der N-H-Streckschwingung des cis- und trans-Monomers mit $3462 \mathrm{~cm}^{-1}$ bzw. $3440 \mathrm{~cm}^{-1}$ in sehr guter Übereinstimmung mit den Ergebnissen einer früheren FTIR-Jet-Studie von Albrecht [175] und verschiedenen IR-UV-Experimenten [284, 285].

Eine Zuordnung der C-H-Streckschwingungsbanden ist nicht eindeutig möglich, da laut B3LYP-D3(BJ)/aVTZ-Rechnung die Normalmoden von cis- und trans-NPhF kaum unterschiedliche Frequenzen aufweisen und somit die Banden im Spektrum überlagert sind. Darüber hinaus ist eine solche Zuordnung für aromatische Systeme wenig zielführend, da hier Schwingungsnormalmoden in der Regel kaum lokalisiert sind.

Darüber hinaus werden Ramanspektren der Amid A-Bande von NPhF unter clusterbildenden 
Expansionsbedingungen gemessen. Durch den 50\%-Anteil an cis-Monomer in der Expansion sind gleichermaßen die Homodimere $t t$ - und $c c-\mathrm{NPhF}$ sowie die gemischten $c t$ - und $t c$-Dimere zu erwarten. Diese Abschätzung gilt nur unter der Annahme, dass die Clusterbildung statistisch erfolgt und keine Relaxation in eine energetisch günstigere Clusterkonformation stattfindet, die Barrieren zur Umlagerung also hinreichend hoch sind.

Die Variation der Substanzkonzentration in der Expansion bei konstanter Düsentemperatur liefert Spektrenserien, die durch eine Normierung auf die cis-Amid A-Bande des Monomers Clusterbanden offenbaren. Die Normierung erfolgt hier auf das cis-Monomer, da die B3LYPD3(BJ)-Frequenzrechnungen für die verschiedenen Dimere die N-H-Streck-Akzeptorbande an der Position des $t$-Monomers vorhersagen. Damit wird die Intensität dieser Bande zu einem wahrscheinlich geringen aber unbekannten Anteil durch Dimere hervorgerufen und ist somit für eine Monomer-Normierung nicht geeignet.

Von den möglichen Dimerbanden legt das Bandenpaar bei 3381/3367 $\mathrm{cm}^{-1}$, aufgrund der guten Übereinstimmung der experimentellen Bandendifferenz mit den berechneten Verschiebungen der B3LYP-D3(BJ)/aVTZ-Frequenzrechnung, eine Zuordnung als Akzeptor- und Donorbande des gemischten $t c_{N}$-Dimers nahe. Zusätzlich lassen die berechneten Frequenzen der NH-Streckschwingung vom Donormolekül im $c t$ - und $t t_{\mathrm{N}}-\mathrm{NPhF}$ sowie vom Akzeptor des $t c_{\mathrm{N}^{-}}$ Dimers auf einen Beitrag dieser Cluster zur $3381 \mathrm{~cm}^{-1}$-Bande schließen. Damit sollte bei statistischer Dimerbildung diese Bande gegenüber der bei $3367 \mathrm{~cm}^{-1}$ auch unter Berücksichtigung der jeweiligen Raman-Streuquerschnitte deutlich intensiver sein. Tatsächlich besitzt jedoch die niederfrequente der beiden Banden im Raman-Spektrum mindestens gleich viel und im FTIRSpektrum deutlich mehr Intensität, was gegen diese Interpretation für die Dimerbandenzuordnung spricht. Eine mögliche Erklärung wäre eine Relaxation in der Expansion, so dass die Bildung der angesprochenen Cluster zusätzlich zur Stoßwahrscheinlichkeit thermodynamischen Aspekten unterliegt. Es könnte zum Beispiel das ct-Dimer in das um ca. $5 \mathrm{~kJ} \cdot \mathrm{mol}^{-1}$ stabilere $t c_{N}$-Konformer relaxieren. Zur Überprüfung der These wäre eine Untersuchung des Potentialverlaufs des Reaktionspfades einer solchen Isomerisierung notwendig, auf dem in jedem Fall der Bruch der Wasserstoffbrücke im ct-Dimer stattfindet. Im Rahmen der vorliegenden Messungen ist aber aufgrund des schlechten Signal-zu-Rauschen-Verhältnis die Aussagekraft von Analysen der Bandenintensität eingeschränkt.

Die Zuordnung steht außerdem im Widerspruch zur Interpretation von Albrecht [175]. Dort werden auf Grundlage nicht-dispersionskorrigierter B3LYP-Rechnungen die Peaks des Bandenpaares im FTIR-Spektrum (dort: $3382 / 3368 \mathrm{~cm}^{-1}$ ) dem $t c_{\mathrm{H}^{-}}$bzw. $t t_{\mathrm{H}^{-}}$-Dimer ${ }^{8)}$ unter Vorbehalt zugeordnet. Es wird außerdem die Möglichkeit in Betracht gezogen, dass beide Banden von zwei unterschiedlichen, aber energetisch ähnlichen $t t$-Dimeren stammen können. Da zu der Zeit eine Dispersionskorrektur noch nicht allgemein zur Verfügung stand, war es nicht möglich, Konformere wie $t t_{\mathrm{N}}$ und $t c_{\mathrm{N}}$ als stabilste Dimerstrukturen auf Basis des $t$-Monomers zu finden. Die berechneten Bandenpositionen der stattdessen gefundenen $t t_{\mathrm{H}}$ und $t c_{\mathrm{H}}$ haben lediglich eine Differenz von $4 \mathrm{~cm}^{-1}$ (B3LYP/6-311+G*), was nicht konsistent mit dem Abstand der experimentellen Banden von $19 \mathrm{~cm}^{-1}$ ist. Dies ist ein weiteres Beispiel dafür, dass bei Struk-

\footnotetext{
$\overline{{ }^{8)} \text { Die Bezeichnung ist aus Konsistenzgründen }}$ an die Nomenklatur in dieser Arbeit angepasst.
} 
turoptimierungen von Clustern ohne Dispersionskorrektur Geometrien übersehen werden, die in guter Übereinstimmung mit den experimentellen Ergebnissen sind.

Ergänzend sei angemerkt, dass eine $\mathrm{X}-\mathrm{H} \cdots \mathrm{X}$-Wasserstoffbrücke typischerweise eine größere Rotverschiebung als eine $\mathrm{X}-\mathrm{H} \cdots \pi$-Wasserstoffbrücke hervorruft, was mit der getroffenen Zuordnung übereinstimmt. Allerdings konnte gezeigt werden, dass dies beispielsweise im Fall von Methanol und Benzofuran mit einer Hydroxygruppe als Wasserstoffbrückendonor nicht der Fall ist und somit die Rechnung eine genau umgekehrte Zuordnung vorhersagt [296]. Bekannte Tendenzen zur Bandenverschiebung durch $\mathrm{X}-\mathrm{H} \cdots \pi$-Wechselwirkungen im Vergleich zu $\mathrm{X}-\mathrm{H} \cdots \mathrm{X}$-Wasserstoffbrücken sind also nicht ohne Weiteres auf andere Systeme übertragbar.

Auch für NPhF wird das zyklische $c c$-Konformer als stabilste Dimerstruktur vorhergesagt und ist aufgrund des hohen $c$-Monomeranteils als Bande im Spektrum erkennbar. Diese ist durch Fermi-Resonanzen sehr breit und reicht von ca. $3221 \mathrm{~cm}^{-1}$ bis in den Bereich der C-H-Streckschwingungen, wo eine genauere Lokalisierung aufgrund von Überlagerungen nicht möglich ist. Zum cc-Dimer gehört eine Gruppe von Peaks um $3215 \mathrm{~cm}^{-1}$ im Raman-Spektrum. Deren Unterschied in der Bandenposition zum FTIR-Spektrum ist in guter Übereinstimmung mit der berechneten Differenz zwischen Raman-aktiver, symmetrischer und IR-aktiver, antisymmetrischer N-H-Streckschwingung, was die getroffene Zuordnung bekräftigt und vermuten lässt, dass diese Peakgruppe als Teil der Fermi-Resonanz einen großen Anteil der N-H-Streckschwingung auf sich vereinigt. Ein Intensitätsvergleich mit einer reinen $\mathrm{N}-\mathrm{H}$-Streckschwingungsbande eines anderen Dimers und deren Streuquerschnittsverhältnis unterstützt diese Vermutung.

Als schwächerer Resonanzpartner wird die Kombinationsbande aus $\mathrm{C}=\mathrm{O}$-Streckschwingung und der N-H-Knickschwingung angenommen [175]. Eine erste Überprüfung dieser Vermutung könnte durch anharmonische Frequenzrechnungen des $c c$-Dimers erfolgen, was ein notwendiger nächster Schritt in der Aufklärung des Schwingungsspektrums von NPhF ist.

In den Raman-Spektren ist die Bande bei $3215 \mathrm{~cm}^{-1}$ beim ansonsten clusterfreien Spektrum mit niedrigster NPhF-Konzentration (Abb. 6-5 (a)) noch klar erkennbar. Eine mögliche Erklärung ist die Bildung von $c c$-Dimeren schon vor der Expansion. Hierfür muss ausreichend Monomer zur Verfügung stehen, was bei einem cis-Anteil von ca. 50 \% für NPhF gegeben ist und die freie Standardbildungsenthalpie des Dimers muss kleiner sein als die zweier c-Monomere. Eine Abschätzung aus den B3LYP-D3(BJ)/def2-TZVP-Frequenzrechnungen ergibt eine freie Enthalpiedifferenz von $\Delta G \approx-7 \mathrm{~kJ} \cdot \mathrm{mol}^{-1}$ bei $373.15 \mathrm{~K}$, also der maximalen Düsentemperatur der curry-Jet-Experimente. Bei der gleichen Temperatur ergibt sich für $t c_{N}$ ein $\Delta G$-Wert von ca. $10 \mathrm{~kJ} \cdot \mathrm{mol}^{-1}$. Auch wenn Standardbildungsenthalpien aus harmonischen Frequenzrechnungen nur eine grobe Abschätzung darstellen, muss die Möglichkeit der cc-Dimerbildung unter Präexpansionsbedingungen in Betracht gezogen werden. Dies ließe sich durch ein Gasphasenspektrum überprüfen. Wenngleich ein solches Spektrum für NPhF nicht vorliegt, finden sich bei Carbonsäuredimeren mit einem ähnlichen zwischenmolekularem Bindungsmotiv, wie z. B. Ameisensäure, bereits Dimere in der Gasphase [297].

Die Bildung von Trimeren in den Raman-Messungen ist aufgrund der im Vergleich zur JetFTIR-Messung niedrigen NPhF-Konzentration nicht zu erwarten, da schon im FTIR-Spektrum keine Trimerbanden zu erkennen sind. Eine dennoch durchgeführte quantenchemische Analy- 
se möglicher NPhF-Trimerstrukturen findet neben einem zyklischen $t t t_{\mathrm{a}}$-Trimer, wie es analog auch für NMF und NMA gefunden wurde, vor allem Addukte von $c$ - oder $t$-Monomereinheiten an die stabilsten Dimere als energetisch günstigste Trimerstrukturen. Diese haben auch aus stoßtheoretischer Sicht die höchste Bildungswahrscheinlichkeit. Die Notwendigkeit der Dispersionskorrektur bei der Geometrieoptimierung hat sich bei NMA, NMF und Imidazol [57] vor allem bei den Trimeren in der energetischen Konkurrenz zwischen offenkettiger und zyklischer Struktur gezeigt. Bei NPhF ist jedoch ein offenkettiges Trimer auch ohne Dispersionskorrektur die instabilste der gefundenen Strukturen.

Eine Erhöhung der NPhF-Konzentration, wie es für den Nachweis von Trimeren notwendig wäre, ist in der vorliegenden Konfiguration des curry-Jets nicht möglich. Es werden aufgrund des geringen Dampfdrucks (siehe Tab. 3-1) für die vorliegenden Clusterspektren sehr hohe Sättigertemperaturen von bis zu $95^{\circ} \mathrm{C}$ benötigt. Dabei muss durch eine mindestens $10^{\circ} \mathrm{C}$ wärmere Düse eine Kondensation in der Düsenzuleitung ausgeschlossen werden. Hier kompensiert die clusterreduzierende Wirkung einer geheizten Düse die Erhöhung der Clusterbildung mit höheren Substanzkonzentrationen durch eine höhere Sättigertemperatur. Zudem ist NPhF bei Raumtemperatur fest und tendiert zur Kondensation an den Komponenten innerhalb der nichtheizbaren Vakuumkammer, was insbesondere die Funktionalität der Optiken einschränkt und nur kurze Messperioden ermöglicht sowie häufige und umfangreiche Reinigungsarbeiten erfordert. Diese experimentellen Schwierigkeiten sind der Grund, warum sich in diesem Kapitel mit den Spektren oberhalb von $3000 \mathrm{~cm}^{-1}$ auf nur einen Messbereich beschränkt wird. Die Ramanspektroskopische Untersuchung der Amid I-Bande steht also noch aus und könnte weiteren Aufschluss über die Bildung des cc-Dimers liefern. 



\section{Längerkettige Alkylamide}

Die Messungen an NPhF lassen vermuten, dass sterisch anspruchsvolle Substituenten auf mindestens einer Seite der Amidgruppe das Isomerisierungsgleichgewicht zum cis-Konformer verschieben können. Als Gruppen kommen neben Ringsystemen wie im NPhF unter anderem auch verzweigte Alkylreste (z. B. eine tert-Butylgruppe) oder längerkettige $n$-Alkylreste in Frage. Ein Beispiel für verzweigte Alkylreste ist $N$-tert-Butylformamid (NtBF) das bei NMR-Messungen [159] und IR-Messungen [240] in Lösung einen cis-Anteil von 18-20\% zeigt. In einer JetFTIR-Studie von Albrecht [175] konnte das cis-Konformer gar nicht nachwiesen werden.

LaPlanche und Rogers [159] nennen auch $N$-Ethylformamid (NEF) als Beispiel für ein unverzweigtes Alkylamid, in dem das cis-Konformer spektroskopisch nachgewiesen werden kann, finden aber gleichzeitig keine cis-Konformere von Acetamiden (inklusive NMA) in ihren Spektren, deren Nachweis wiederum im IR-Spektrum als $\mathrm{CCl}_{4}$-Lösung [171] und im Raman-Jet-Spektrum gelungen ist (siehe Abschnitt 4). Daher soll in diesem Kapitel überprüft werden, inwieweit eine Untersuchung weiterer sekundärer Alkylamide auf das cis-trans-Isomerenverhältnis im RamanJet-Spektrum sinnvoll wäre.

Im Allgemeinen lassen sich unverzweigte sekundäre $N$-Alkylamide mit der Konstitutionsformel $\mathrm{H}_{2 n+1} \mathrm{C}_{n} \mathrm{CONHC}_{m} \mathrm{H}_{2 m+1}$ beschreiben und ergeben für $m=n$, also einer gleichmäßigen Verlängerung der beiden Alkylreste, eine homologe Reihe. Diese soll hier für $m=n=1,2,3$ genauer betrachtet werden. Der Fall $m=n=1$ ergibt das bereits in Abschnitt 4 beschriebene NMA. Die Fortsetzung der Reihe ergibt $N$-Ethylpropionamid (NEP, $m=n=2$ ) und $N$-Propylbutanamid (NPB, $m=n=3$ ) (siehe Abb. 7-1).

Darüber hinaus wurden die unsymmetrisch substituierten Alkylamide $(m \neq n)$ bis $m=3 ; n=2$ ebenfalls untersucht. Während $n=0$ die Alkylformamide $N$-Methylformamid (NMF, $m=1$, siehe Abschnitt 5), NEF ( $m=2$ ) und $N$-Propylformamid (NPF, $m=3$ ) beinhaltet, sind mit $m=0$ die primären Alkylamide erfasst, für die keine cis-trans-Isomerisierung beobachtet werden kann. Weitere unsymmetrisch substituierte $N$-Alkylamide, die in diesem Kapitel betrachtet werden, sind: $N$-Ethylacetamid (NEA, $m=2 ; n=1$ ), $N$-Propylacetamid (NPA, $m=3 ; n=1$ ), $N$-Methylpropionamid (NMP, $m=1, n=2$ ) und $N$-Propylpropionamid (NPP, $m=3, n=2$ ).

Bei der Betrachtung von sekundären Amiden mit längeren $n$-Alkylketten könnte ein anderer Effekt zusätzlich Einfluss auf das cis-trans-Isomerenverhältnis haben: Die unverzweigten Alkylketten auf beiden Seiten der Amidgruppe sollten ab einer bestimmten Kettenlänge aufgrund von Dispersionswechselwirkungen neben der energetisch günstigsten all-trans-Konformation ${ }^{1)}$ eine haarnadelförmige Molekülstruktur bewirken. Diese Haarnadelstruktur konnte für Alkane

\footnotetext{
${ }^{1)}$ Der Begriff all-trans wird in den genannten Referenzen für einen Wert von $180^{\circ}$ für alle Diederwinkel entlang der Molekülkette verwendet und entspräche einer all-anti-Konformation nach der in dieser Arbeit verwendeten Dieder-Nomenklatur.
} 
ab einer Kettenlänge von 17-18 C-Atomen [117, 204] und für Alkylbenzole ab einer Alkylkettenlänge von 8 C-Atomen [298] nachgewiesen werden. Bei unverzweigten $N$-Alkylamiden wäre es grundsätzlich denkbar, dass solche Dispersionswechselwirkungen zwischen den Alkylsubstituenten zu einer Verschiebung des cis-trans-Isomerenverhältnis führen können. Dazu werden in diesem Kapitel eine Reihe von $N$-Alkylamiden theoretisch hinsichtlich des Energieunterschieds zwischen dem energetisch niedrigsten cis- und trans-Konformer untersucht.

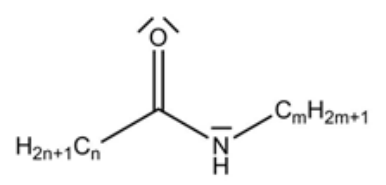

\begin{tabular}{|c|cccc|}
\hline$n=$ & $n=$ & 1 & 2 & 3 \\
\hline 0 & \multicolumn{4}{|c|}{ (primäre Amide) } \\
1 & NMF & NMA & NMP & $\ldots$ \\
2 & NEF & NEA & NEP & $\ldots$ \\
3 & NPF & NPA & NPP & NPB \\
\hline
\end{tabular}

Abb. 7-1. Allgemeine Strukturformel für sekundäre Alkylamide mit den tabellarisch sortierten Abkürzungen der Molekülnamen für unterschiedliche Seitenkettenlängen $m$ und $n$ (fett: Alkylamide der homologen Reihe; weitere Abkürzungen: NMP: N-Methylpropionamid, NEF: N-Ethylformamid, NEA: $N$-Ethylacetamid, NPF: N-Propylformamid, NPA: $N$-Propylacetamid, NPP: N-Propylpropionamid).

Für alle genannten und in Abb. 7-1 tabellierten Amide wurde zuerst die energetisch günstigste Molekülstruktur für das cis- und trans-Konformer durch einen relaxierten Scan bei Rotation um die Diederwinkel $\varphi, \varphi^{\prime}, \varphi^{\prime \prime}, \varphi^{\prime \prime \prime}$ und $\psi, \psi^{\prime}, \psi^{\prime \prime}, \psi^{\prime \prime \prime}$ auf B3LYP-D3(BJ)/def2TZVP-Niveau gesucht. Diese können neben den bekannten syn- und anti-Stellungen (vgl. Abschnitt 2.1) mit Ausnahme der terminalen Methylgruppen zusätzlich noch eine synclinale $( \pm s c)$ Stellung für Diederwinkel von $\pm(30-90)^{\circ}$ und eine anticlinale $( \pm a c)$ Stellung für Diederwinkel von $\pm(90-$ $150)^{\circ}$ annehmen.

Dieses vieldimensionale Optimierungsproblem wurde durch sukkzessive Optimierungen von jeweils einem $\varphi, \psi$-Diederwinkelpaar auf drei Dimensionen ${ }^{2)}$ reduziert. Hierbei wurde angenommen, dass der Einfluss der Alkylreste auf die Gesamtenergie hauptsächlich durch sterische Abstoßung und Dispersionswechselwirkungen zustande kommt, so dass der Einfluss der Diederwinkel mit zunehmendem Abstand von der Amidbindung sinkt. Zum Beispiel wurde als Ausgangspunkt für die gleichzeitige Optimierung von $\varphi^{\prime}$ und $\psi^{\prime}$ die Struktur mit optimierten $\varphi$ und $\psi$ verwendet. Der jeweils letzte Diederwinkel entspricht der Torsion der terminalen Methylgruppen. Diese nimmt in allen Rechnungen für längerkettige Amide immer die anti-Stellung ein und wird daher und aufgrund des geringen Einflusses auf die Gesamtenergie nicht genauer analysiert. ${ }^{3)}$

Die Geometrien aller lokalen Minima des letzten Scans wurden auf B3LYP-D3(BJ)/aVTZNiveau nachoptimiert, um eine Vergleichbarkeit zu den Ergebnissen für NMF und NMA zu haben. Zur Bestimmung des nullpunktskorrigierten Energieunterschieds und um sicher zu stellen, dass es sich um eine echte Minimumstruktur handelt, wurde eine harmonische Frequenzrechnung auf dem gleichen Niveau durchgeführt. Die Ergebnisse dieser Rechnungen für die energetisch günstigsten Strukturen sowie die all-trans-Strukturen des cis- und trans-Konformers für

\footnotetext{
${ }^{2)}$ Gemeint sind die beiden Diederwinkel und die Energie des Moleküls.

${ }^{3)}$ In diesem Fall sind anti- und synclinale Konformation aufgrund der $120^{\circ}$-Periodizität der Methyltorsion äquivalent.
} 
jedes Amid sind im Anhang in Tabelle A-33 zusammengefasst. In Abbildung 7-2 werden die Energieunterschiede der jeweils günstigsten cis- und trans-Konformere samt Strukturformeln veranschaulicht. Dabei sind die Amide mit gleicher $N$-seitiger Alkylkette ( $m=$ const) zusammengefasst.

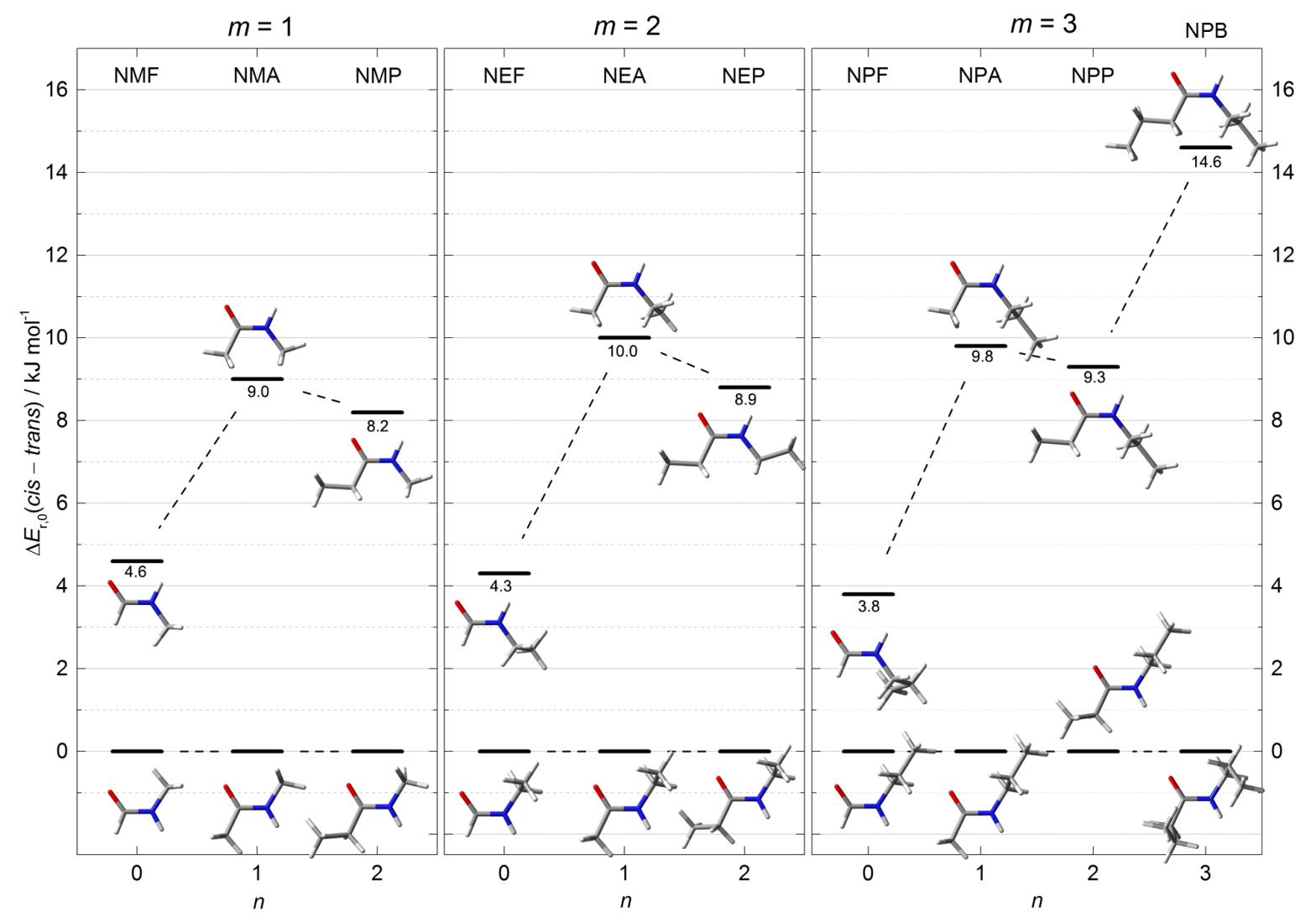

Abb. 7-2. cis/trans-Energieunterschied aller untersuchten sekundären Alkylamide. Dargestellt sind die nullpunktskorrigierten Energien auf B3LYP-D3(BJ)/aVTZ-Niveau mit den entsprechenden Molekülstrukturen. Weitere Details finden sich in Tab. A-33.

Beim Blick auf die Molekülstrukturen fallen einige Tendenzen und Besonderheiten auf: Der Vergleich der carbonylseitigen Alkylreste ergibt ab $n=2$ einen grundsätzlichen Unterschied zwischen cis- und trans-Konformer. Während bei allen cis-Strukturen die all-trans-Variante stets die bevorzugte ist, zeigt sich für alle trans-Strukturen ein gauche-Diederwinkel für $\psi$ und bei NPB zusätzlich für $\psi^{\prime}$.

Die $N$-seitigen Alkylreste verhalten sich auf den ersten Blick weit weniger systematisch. Die trans-Konformere der $N$-Ethylamide und der $N$-Propylamide (mit Ausnahme von NPB) haben zwar alle einen synclinalen Diederwinkel $\varphi \approx 85^{\circ}$ und darüber hinaus alle weiteren Diederwinkel in anti-Stellung, aber bei Betrachtung der cis-Konformere scheint eine Systematik zu fehlen. Bei den $N$-Methylamiden ist NMF das einzige Molekül, bei dem die $N$-Methylgruppe in syn-Stellung steht, ${ }^{4)}$ Bei den cis- $N$-Ethylamiden zeigen NEF und NEA unterschiedlich starke gauche-Orientierungen für $\varphi$, während bei NEP die all-trans-Konformation für die $N$-seitige Alkylkette bevorzugt wird. Bei den $N$-Propylamiden fällt NPF mit einem gauche-Dieder für $\varphi^{\prime}$ aus

\footnotetext{
4) Die Stellung der terminalen Methylgruppen ist bereits ausführlich in den Abschnitten 4 und 5 diskutiert worden.
} 
der Reihe.

Diese „Unregelmäßigkeiten“ lassen sich relativieren, wenn man die anderen Strukturen der jeweiligen Moleküle betrachtet (siehe Tab. A-33). Da zeigt sich für NEP mit dem $c_{+}$sca, aa-Konformer eine Struktur mit einem Energieunterschied von $\Delta E_{\mathrm{r}, 0}=0.6 \mathrm{~kJ} / \mathrm{mol}$ und dem nahezu gleichen $\varphi$-Diederwinkel wie im cis-NEA. Ähnliches findet sich für NPF, das mit $c_{+ \text {acaa }}$ im Vergleich zum cis-Minimum ein um $0.6 \mathrm{~kJ} / \mathrm{mol}$ höheres Konformer besitzt, das im Vergleich zu NPA, NPP und NPB zwar ein um ca. $20^{\circ}$ anderes $\varphi$ hat, jedoch für $\varphi^{\prime}$ und $\varphi^{\prime \prime}$ ebenfalls in anti-Stellung ist. Diese Energieunterschiede liegen innerhalb des Unsicherheitsbereichs von B3LYP-Rechnungen, so dass hier nicht von signifikanten Unregelmäßigkeiten gesprochen werden kann.

Die Betrachtung der Energieunterschiede zwischen den Konformeren zeigt eine generelle Bevorzugung der trans-Konformation um ca. $4 \mathrm{~kJ} / \mathrm{mol}$ bei den drei $N$-Alkylformamiden bis hin zu $14.6 \mathrm{~kJ} / \mathrm{mol}$ für NPB. Der Energieunterschied nimmt dabei nicht konstant zu, sondern zeigt in jeder Gruppe von $N$-Alkylamiden mit gleichem $m$ ein ähnliches Muster. Die Energiedifferenz wird von den Formamiden zu den Acetamiden ungefähr verdoppelt und dann zu den Propionamiden hin wieder etwas geringer (zwischen $0.5 \mathrm{~kJ} / \mathrm{mol}$ von NPA zu NPP und $1.1 \mathrm{~kJ} / \mathrm{mol}$ von NEA zu NEP).

Entlang der homologen Reihe NMA-NEP-NPB ist NPB das einzige Molekül mit einer signifikanten Veränderung des Energieunterschieds. NPB ist insgesamt das interessanteste Molekül in dieser Untersuchung, denn es weist nicht nur den größten Energieunterschied aller betrachteten Amide auf, sondern ist das erste Amid, bei dem beide Alkylketten lang genug sind, dass Dispersionswechselwirkungen zwischen den Ketten erwartet werden können. Dies zeigt die Molekülstruktur des trans-Konformers (hier aus einer B3LYP-D3(BJ)/aVTZ-Rechnung), das eine hohe strukturelle Ähnlichkeit mit der Haarnadelstruktur von $n$-Oktan und Butansäureproylester (BSPE) besitzt (siehe Abb. 7-3).

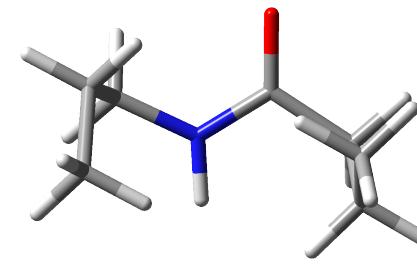

trans-N-Propylbutanamid

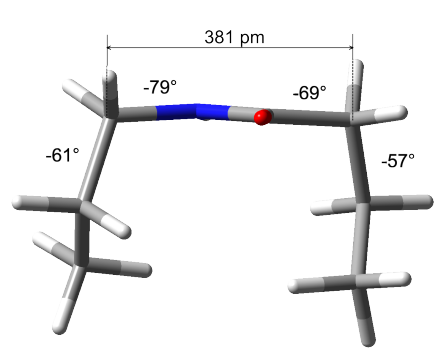

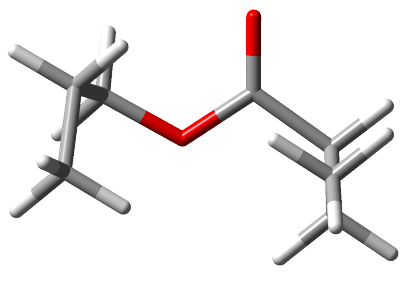

Butansäurepropylester

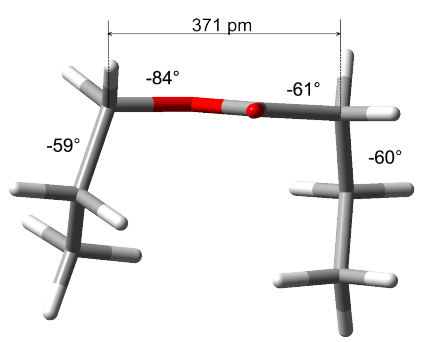

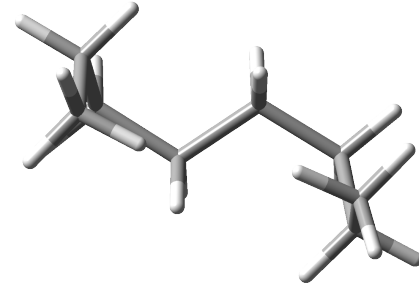

n-Oktan

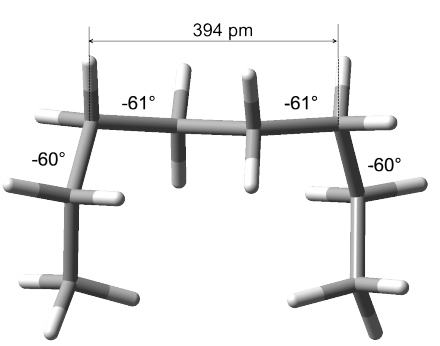

Abb. 7-3. Haarnadel-Molekülstrukturen von trans- $N$-Propylformamid, Butansäurepropylester und $n$-Oktan aus B3LYPD3(BJ)/def2TZVP-Rechnungen mit Angabe der gauche-Diederwinkel und der Haarnadelbreite.

Die Haarnadelkonformere von n-Oktan und BSPE sind rein hypothetischer Natur. Die dis- 
kutierten Strukturen sind dennoch lokale Minima auf der Potentialhyperfläche und erst eine Kettenverlängerung auf $n$-Heptadekan bzw. Pentansäurebutylester liefert spektroskopisch nachweisbare Haarnadelstrukturen [117, 299]. Alle drei zeigen in der Haarnadel das bekannte gauche-gauche-trans-gauche-gauche-Motiv [204], wobei alle gauche-Diederwinkel nahezu $60^{\circ}$ betragen, bis auf die an Heteroatomen, die aufgrund der freien Elektronenpaare im Ester bzw. dem delokalisierten $\pi$-Elektronensystem im Amid mit 79-84 etwas größer ausfallen.

Die Breite der Haarnadelstruktur nimmt von $n$-Oktan über $t$-NPB hin zu BSPE ab, was der allgemeinen Tendenz $\measuredangle(\mathrm{C}-\mathrm{O}-\mathrm{C})<\measuredangle(\mathrm{C}-\mathrm{N}-\mathrm{C})<\measuredangle(\mathrm{C}-\mathrm{C}-\mathrm{C})$ entspricht, und liegt zwischen 3.73.9 ̊. Bei cis-NPB liegt die Breite der Amidgruppe hingegen mit 3.0 A deutlich niedriger als im trans-Konformer und keines der niederenergetischen Molekülstrukturen zeigt eine Haarnadelstruktur. Die cis-Konformation führt möglicherweise zu einer zu großen räumlichen Nähe für die Alkylketten, so dass sterische Effekte die der Dispersion überwiegen.

Der hohe Energieunterschied zwischen cis- und trans-NPB ist auf die Dispersionswechselwirkungen zwischen den Alkylketten zurückzuführen. Jede weitere Verlängerung der Alkylketten würde diese Wechselwirkungen noch vergrößern, während parallel ausgerichtete Alkylketten für das cis-Konformer aus sterischen Gründen keine Option darstellen, so dass für alle größeren unverzweigten $\mathrm{N}$-Alkylamide das trans-Konformer das energetisch günstigste sein wird. 



\section{$8 \gamma$-Butyrolactam}

Im Rahmen der Untersuchung der Isomerisierung von sekundären Amiden ist die Untersuchung von $\gamma$-Butyrolactam $(\gamma \mathrm{BL})$ interessant, da es aufgrund struktureller Einschränkungen (siehe Abschnitt 8.1) ausschließlich in der cis-Konformation vorliegt.

$\gamma$ BL ist ein cyclisches sekundäres Amid der Summenformel $\mathrm{C}_{4} \mathrm{H}_{7} \mathrm{NO}$ und gehört daher zur Stoffklasse der Lactame. Es ist ein gesättigter, $N$-heterocyclischer Fünfring, der bei $25^{\circ} \mathrm{C}$ schmilzt und einen Siedepunkt von $251^{\circ} \mathrm{C}$ hat [152]. Weitere, verbreitete Bezeichnungen sind Pyrrolidin-2-on, 2-Pyrrolidon oder Butyrolactam. Es ist ein wichtiges Zwischenprodukt in der industriellen Herstellung des Polymers Povidon (PVP), das eine breite Anwendung vor allem in der Medizin und der Kosmetikindustrie hat [300, 301]. $\gamma$ BL ist die Lactamform des Neurotransmitters $\gamma$-Aminobuttersäure (GABA) und wurde als endogener Bestandteil des Blutplasmas von Säugetieren (u.a auch Menschen) nachgewiesen [302, 303].

\subsection{Struktur und Isomerisierung}

Monomer Das $\gamma$ BL-Monomer ist nicht planar, sondern hat eine dem Cyclopentan ähnliche Briefumschlag-Konformation (siehe Abb. 8-1). Es liegt also eine planare Chiralität vor, die zwei enantiomere Konformationen ermöglicht. Deren elektronische Isomerisierungsbarriere liegt bei $3.6 \mathrm{~kJ} \cdot \mathrm{mol}^{-1}$ (B3LYP-D3(BJ)/aVTZ) mit einer planaren Struktur als Übergangszustand.
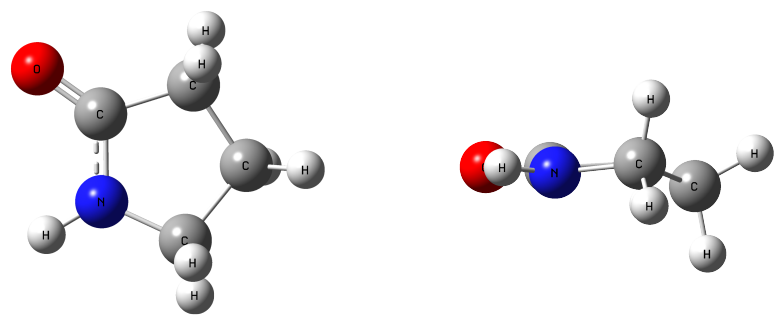

Abb. 8-1. Minimumstruktur des $\gamma$-Butyrolactam-Monomers nach Geometrieoptimierung auf B3LYP-D3(BJ)/aVTZ-Niveau. Exemplarische Darstellung des $S$-Enantiomers als eines von zwei isoenergetischen Konformationsisomeren. In der Seitenansicht (rechts) ist die Briefumschlag-Konformation zu erkennen.

Wie alle Lactame mit einer Ringgröße $n<9$ wird auch $\gamma$ BL durch die Ringspannung in die für sekundäre Amide allgemein energetisch ungünstigere cis-Konformation gezwungen [304, 305]. Dies macht eine Betrachtung der cis-/trans-Isomerisierung, wie sie für die anderen Amide durchgeführt wurde, unnötig und das in Abschnitt 2.1 vorgestellte Nomenklatursystem vereinfacht sich damit für $\gamma \mathrm{BL}$ wie folgt: Es werden Monomere, Dimere und Trimere entsprechend mit M, D oder T bezeichnet. Weitere strukturelle Besonderheiten, wie z. B. die enantiomeren 
Formen des Monomers, werden durch entsprechende Indizes (,$S$ ) gekennzeichnet und im Folgenden bei erstmaliger Erwähnung eingeführt.

$\gamma$-Butyrolactam unterliegt einer Konstitutionsisomerie in Form einer Lactam-Lactim-Tautomerie (siehe Abb. 8-2] [306, 307]. Der elektronische Energieunterschied zwischen den beiden tautomeren Formen berechnet sich zu $56.5 \mathrm{~kJ} \cdot \mathrm{mol}^{-1}$ auf B3LYP-D3(BJ)/aVTZ-Niveau zugunsten des Lactams. Die Tautomerisierung erfolgt durch einen konzertierten Protonentransfer im cyclischen Dimer und hat eine Aktivierungsbarriere von ca. $209 \mathrm{~kJ} \cdot \mathrm{mol}^{-1}$ [307]. Aufgrund des Energieunterschieds und der Barrierenhöhe ist die Bildung der Lactim-Form thermodynamisch ungünstig und kinetisch gehindert, sodass deren Vorliegen für die Gasphase vernachlässigt werden kann [306].

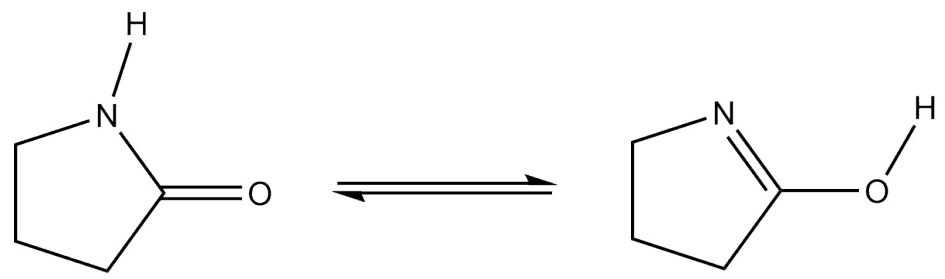

Abb. 8-2. Lactam-Lactim-Tautomerie in $\gamma$-Butyrolactam. Elektronischer Energieunterschied auf B3LYP-D3(BJ)/aVTZNiveau: $E_{\mathrm{el}}($ Lactam $)=E_{\mathrm{el}}($ Lactim $)-56.5 \mathrm{~kJ} \cdot \mathrm{mol}^{-1}$.

Dimer Quantenchemische Rechnungen auf B3LYP-D3(BJ)/aVTZ-Niveau ergeben zwei stabile Dimerstrukturen $\mathrm{D}_{2 \mathrm{H}}$ und $\mathrm{D}_{1 \mathrm{H}}$ (siehe Abb. 8-3). 1) Das cyclische $\mathrm{D}_{2 \mathrm{H}}$ bildet zwei $\mathrm{N}-\mathrm{H} \cdots \mathrm{O}=\mathrm{C}$ Wasserstoffbrückenbindungen aus. Dagegen hat $\mathrm{D}_{1 \mathrm{H}}$ nur eine Wasserstoffbrücke, die allerdings durch eine $\mathrm{C}-\mathrm{H} \cdots \mathrm{O}=\mathrm{C}$-Wechselwirkung stabilisiert wird. In der Literatur werden diese beiden Strukturen als DHBD und SHBD (doubly / singly hydrogen bonded dimer) diskutiert [306, 308]. Dielektrische Polarisationsmessungen von $\gamma$ BL in Lösung ergeben ein Gleichgewicht zwischen beiden Dimeren, das weit auf der Seite des $\mathrm{D}_{2 \mathrm{H}}$-Konformers liegt (90\% in $0.3 \mathrm{M}$ Benzollösung) [309]. Dieses Gleichgewicht wird in polarem Lösemittel in Richtung des $\mathrm{D}_{1 \mathrm{H}}$-Dimers verschoben, da polare Lösemittel die Öffnung einer Wasserstoffbrücke stabilisieren [310]. Im Kristall liegt $\gamma$ BL nur in der $\mathrm{D}_{2 \mathrm{H}}$-Form vor [311].

Die Ergebnisse der B3LYP-Rechnungen in Tabelle 8-1 weisen erwartungsgemäß das Dimer mit den zwei Wasserstoffbrücken $\left(\mathrm{D}_{2 \mathrm{H}}\right)$ mit einem nullpunktskorrigierten Energieunterschied von $23.2 \mathrm{~kJ} \cdot \mathrm{mol}^{-1}$ als das stabilere der beiden aus. Dabei ist die Geometrie der Wasserstoffbrücken in beiden Konformeren sehr ähnlich. Beide haben $(\mathrm{H} \cdots \mathrm{O}=\mathrm{C})$-Bindungswinkel von nahezu $120^{\circ}$ und die Bindungslänge $d$ ist mit $186 \mathrm{pm}$ bei $\mathrm{D}_{1 \mathrm{H}}$ nur geringfügig länger als bei $\mathrm{D}_{2 \mathrm{H}}$ mit $181 \mathrm{pm}$.

Jedes der beiden Dimere kann entweder aus zwei Monomeren gleicher Chiralität zusammengesetzt sein (z. B. $M_{S} M_{S}$ ) oder als Racemat vorliegen $\left(M_{S} M_{R}\right)$. B3LYP-D3(BJ)-Rechnungen ergeben für $M_{R} M_{R}$ und $M_{R} M_{S}$ in $D_{2 H}$-Geometrie einen nicht signifikanten nullpunktskorrigierten Energieunterschied von $0.03 \mathrm{~kJ} \cdot \mathrm{mol}^{-1}$. Die jeweilige $\mathrm{N}-\mathrm{H}$-Streckschwingungsmode liegt zudem

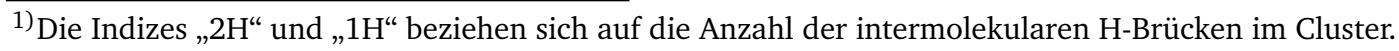


$\mathrm{D}_{2 \mathrm{H}}$
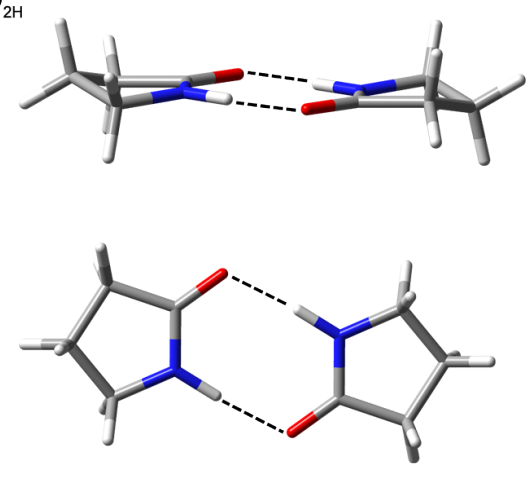

$D_{1 H}$
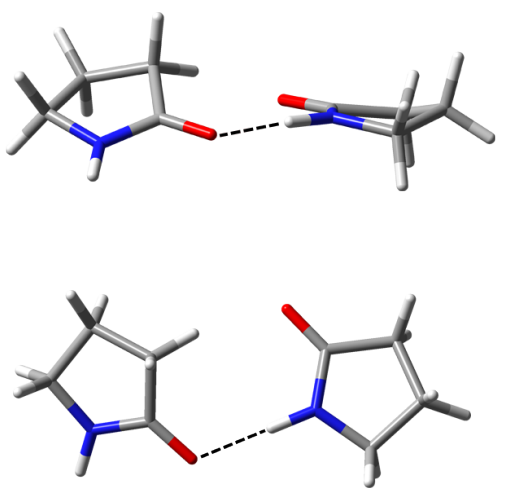

Abb. 8-3. Die beiden stabilsten Strukturen des $\gamma$-Butyrolactam-Dimers nach Geometrieoptimierung auf B3LYPD3(BJ)/aVTZ-Niveau. Exemplarische Darstellung des $C_{i}$-symmetrischen $D_{2 H}$ aus einem $M_{S}$ - und einem $M_{R}$-Monomer.

bei der gleichen harmonischen Wellenzahl. Daher sind alle Berechnungen des $\mathrm{D}_{2 \mathrm{H}}$-Dimers in der höhersymmetrischen $\left(C_{\mathrm{i}}\right) \mathrm{M}_{\mathrm{R}} \mathrm{M}_{\mathrm{S}}$-Form durchgeführt worden.

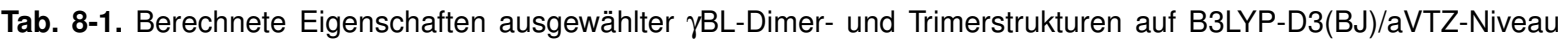
(zugehörige Strukturen befinden sich in Abb. 8-3 und A-12 in Anhang A.10.1. Angegeben sind die relativen elektronischen und nullpunktskorrigierten Energien $\Delta E_{\text {el }}$ bzw. $\Delta E_{0}$, sowie Dissoziationsenergien $D_{\text {el }}$ bzw. $D_{0}$ (alle in $\mathrm{kJ} \cdot \mathrm{mol}^{-1}$ ). Die Länge der $\mathrm{N}-\mathrm{H} \cdots \mathrm{O}=\mathrm{C}$-Wasserstoffbrückenbindung $d$ (in pm), der $\mathrm{H} \cdots \mathrm{O}=\mathrm{C}$-Bindungswinkel der Wasserstoffbrückenbindung $\alpha$ (in ${ }^{\circ}$ ), die niedrigste Frequenz in doppelt-harmonischer Näherung $\tilde{v}_{\min }\left(\right.$ in $\mathrm{cm}^{-1}$ ) und die Dipolmomente $\mu$ (in D).

\begin{tabular}{|c|c|c|c|c|}
\hline & $\mathrm{D}_{2 \mathrm{H}}$ & $D_{1 H}$ & $\mathrm{~T}_{\text {cyc }}$ & $T_{M+D}$ \\
\hline$\Delta E_{\mathrm{el}} / \mathrm{kJ} \cdot \mathrm{mol}^{-1}$ & 0.0 & 24.7 & 4.4 & 0.0 \\
\hline$\Delta E_{0} / \mathrm{kJ} \cdot \mathrm{mol}^{-1}$ & 0.0 & 23.2 & 3.1 & 0.0 \\
\hline$D_{\mathrm{el}} / \mathrm{kJ} \cdot \mathrm{mol}^{-1}$ & 70.6 & 45.9 & 112.4 & 116.8 \\
\hline$D_{0} / \mathrm{kJ} \cdot \mathrm{mol}^{-1}$ & 64.7 & 41.5 & 103.4 & 106.5 \\
\hline \multirow[t]{3}{*}{$\alpha(\mathrm{H} \cdots \mathrm{O}=\mathrm{C}) /^{\circ}$} & 118 & 117 & 145 & 116 \\
\hline & & & & 113 \\
\hline & - & - & & 118 \\
\hline \multirow[t]{3}{*}{$d(\mathrm{H} \cdots \mathrm{O}) / \mathrm{pm}$} & 181 & 186 & 183 & 211 \\
\hline & & & & 192 \\
\hline & - & - & & 177 \\
\hline$\tilde{v}_{\min } / \mathrm{cm}^{-1}$ & 24 & 20 & 11 & 17 \\
\hline$\mu / D$ & 0 & 2.4 & 0.057 & 4.3 \\
\hline
\end{tabular}

Größere Cluster Größere Aggregate werden in der Literatur in erster Linie für $\gamma B L$ in Lösung diskutiert [309, 312, 315]. Dabei zeigen 2D-IR-Messungen, dass $\mathrm{D}_{1 \mathrm{H}}$ der Grundbaustein für die Bildung offenkettiger Oligomere ist [316]. Die Entstehung von zyklischen Trimeren, wie bei NMA und NMF, wird für $\gamma$ BL zumindest in unpolarem Lösungsmittel ausgeschlossen [309]. Allgemein nimmt die Bildungswahrscheinlichkeit zyklischer Cluster mit zunehmender Clustergröße aus entropischen Gründen ab [306]. Über die Trimerbildung in der Gasphase oder der Matrix gibt es keine Angaben in der Literatur. Von theoretischer Seite ist, neben dem bereits erwähnten cyclischen Trimer $\left(\mathrm{T}_{\mathrm{cyc}}\right)$ mit $C_{3}$-Symmetrie, auch ein Addukt eines Monomers und eines $\mathrm{D}_{2 \mathrm{H}}$-Dimers $\left(\mathrm{T}_{\mathrm{M}+\mathrm{D}}\right)$ denkbar. Die berechneten Strukturen der jeweils stabilsten Varianten 
sind in Abbildung A-12 in Anhang A.10.1 dargestellt. Deren Eigenschaften sind in Tabelle 8-1 aufgeführt und die Ergebnisse der Frequenzrechnungen finden sich in den Tabellen A-27 und A-28 in Anhang A.10.2.

Die nullpunktskorrigierte Energie $E_{0}$ des zyklischen Trimers liegt laut B3LYP-Rechnung $3.1 \mathrm{~kJ} \cdot \mathrm{mol}^{-1}$ über der des $\mathrm{T}_{\mathrm{M}+\mathrm{D}}$. Beide Konformere haben drei $\mathrm{N}-\mathrm{H} \cdots \mathrm{O}=\mathrm{C}$-Wasserstoffbrücken. Bei $\mathrm{T}_{\mathrm{M}+\mathrm{D}}$ stammen zwei dieser Wasserstoffbrücken aus dem $\mathrm{D}_{2 \mathrm{H}}$-Dimer. Die Anordnung des dritten $\gamma$ BL-Moleküls legt zusätzlich zur dritten $\mathrm{N}-\mathrm{H} \cdots \mathrm{O}=\mathrm{C}$-Wasserstoffbrücke eine stabilisierende $\mathrm{C}-\mathrm{H} \cdots \mathrm{O}=\mathrm{C}$-Wechselwirkung nahe.

Ein Vergleich mit den größeren Clustern von Ameisensäure [317], das ebenfalls Dimere mit zwei Wasserstoffbrückenbindungen bildet, lässt vermuten, dass bei $\gamma \mathrm{BL}$ in analoger Weise Tetramere in Form von zwei gestapelten $D_{2 \mathrm{H}}$-Dimeren gebildet werden. Diese sind möglicherweise überproportional stabiler als die hier postulierten Trimere. Tetramere und noch größere Cluster von $\gamma$ BL sind allerdings nicht Gegenstand dieser Arbeit.

\subsection{Schwingungsspektroskopie}

Bedingt durch die cis-Konformation des $\gamma$ BL-Monomers sind als Dimere nur cis-cis-Aggregate zu erwarten. Die schwingungsspektroskopische Untersuchung der Clusterbildung von $\gamma$ BL ermöglicht damit den Nachweis des cis-cis-Dimers eines sekundären Amids. Dieser ist in dieser Arbeit bisher entweder aufgrund zu geringer cis-Monomer-Konzentrationen (bei NMA und NMF, siehe Abschnitte 4.3 und 5.3 oder wegen einer Überlagerung mit den $\mathrm{CH}$-Streckschwingungsmoden eines aromatischen Restes (bei NPhF, siehe Abschnitt 6.3) gescheitert und soll nun für $\gamma \mathrm{BL}$ erfolgen.

Amid I Das Raman-Jetspektrum von $\gamma$ BL zeigt in der Amid I-III-Region zwischen 1800 und $1200 \mathrm{~cm}^{-1}$ (Abb. 8-4 (unten)) eine intensive und schmale Bande bei $1765 \mathrm{~cm}^{-1}$. Dem steht im FTIR-Jetspektrum von Albrecht [175] (Abb. 8-4 (oben)) eine Bande bei $1767 \mathrm{~cm}^{-1}$ gegenüber, die dem $\gamma$ BL-Monomer zugeordnet wird. Zusätzlich befinden sich auf der niederfrequenten Seite der Ramanbande bei $1756 \mathrm{~cm}^{-1}$ und $1750 \mathrm{~cm}^{-1}$ zwei Peaks, die ebenfalls dem Monomer zugeordnet werden und vermutlich auf Fermi-Resonanzen zurückzuführen sind. Das Pendant im FTIR-Spektrum ist möglicherweise eine kleine Bande bei $1748 \mathrm{~cm}^{-1}$.

Die Normierung der Ramanspektren einer $\gamma$ BL-Konzentrationsreihe bei konstanter Düsentemperatur auf die Monomerbande zeigt eine breite Clusterbande bei $1691 \mathrm{~cm}^{-1}$ mit einer deutlichen Bandenschulter bei ca. $1711 \mathrm{~cm}^{-1}$. Zusätzlich ist ein sehr schwaches Signal bei $1735 \mathrm{~cm}^{-1}$ erkennbar. Die Banden skalieren ungefähr gleich und werden Dimeren zugeordnet, wobei aus den oben genannten Gründen hierfür am ehesten das Dimer $\mathrm{D}_{2 \mathrm{H}}$ infrage kommt. Für eine solche Struktur wäre durch die vorliegende Symmetrie eine Davidov-Aufspaltung anzunehmen. Demnach müsste die symmetrische $\mathrm{C}=\mathrm{O}$-Streckschwingung im Raman-Spektrum gegenüber der antisymmetrischen $\mathrm{C}=\mathrm{O}$-Streckschwingung im FTIR einen Unterschied in der Bandenposition aufweisen. Der Vergleich mit dem Monomer-normierten FTIR-Jetspektrum (Abb. 8-4 (oben)) zeigt eine zum Raman-Spektrum um $38 \mathrm{~cm}^{-1}$ unterschiedliche Bande bei 


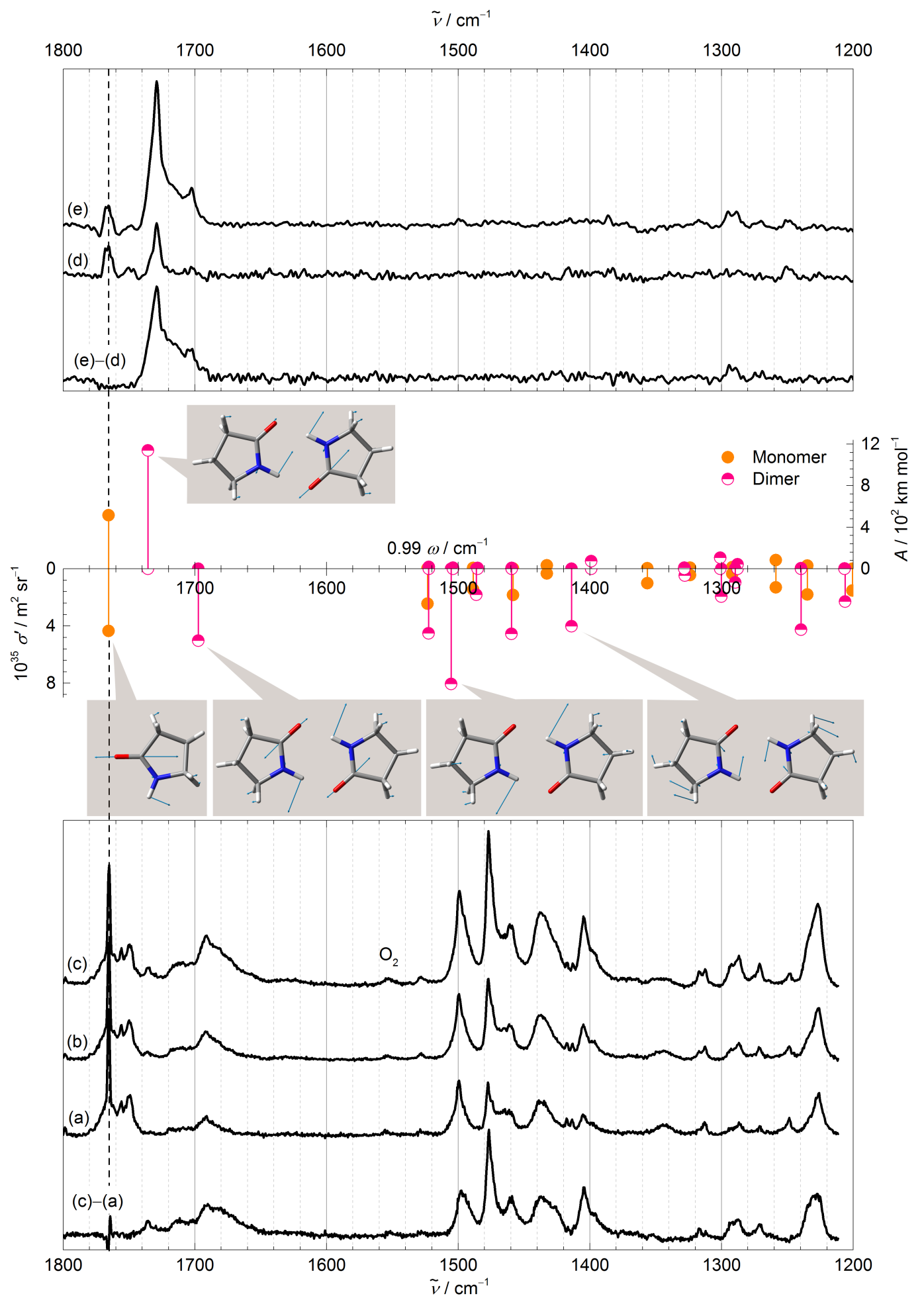

Abb. 8-4. FTIR- und Raman-Jet-Spektren von $\gamma$-Butyrolactam in der Amid I-III-Region.

Oben: Auf die Intensität der Monomerbande normierte FTIR-Jetspektren mit von (d) zu (e) zunehmender $\gamma$ BL-Konzentration (aus [175]) und Differenzspektrum (e)-(d).

Mitte: Auf die Monomerbandenposition skalierte Strichspektren (Faktor 0.99, gestrichelte Linie) des Monomers und des cyclischen Dimers auf B3LYP-D3(BJ)/aVTZ-Niveau mit IR-Aktivitäten (nach oben) und Raman-Streuquerschnitten (nach unten) sowie Illustrationen ausgewählter Normalmoden.

Unten: Auf die Intensität der Monomerbande normierte Raman-Jetspektren mit von (a) zu (c) zunehmender $\gamma B \mathrm{BL}-K o n-$ zentration und Differenzspektrum (c)-(a).

(Details zu den Messungen der Raman-Spektren befinden sich im Raman-Spektrenverzeichnis in Tab. A-6 in Anhang A.6.1. Details zu den Frequenzrechnungen befinden sich in Tab. A-28 in Anhang A.10.2) 
$1729 \mathrm{~cm}^{-1}$. Diese wird dem Dimer zugeordnet [175]. Im FTIR-Spektrum dominiert das Dimersignal auch bei der Messung mit geringerer Konzentration das des Monomers (siehe Abb. 8-4 (d)). Ein zusätzlicher Clusterpeak im FTIR bei $1705 \mathrm{~cm}^{-1}$ lässt sich nicht sicher zuordnen. Trotz dessen Nähe zur Raman-Dimerbande bei $1691 \mathrm{~cm}^{-1}$ ist ein Zusammenhang aus Symmetriegründen sehr unwahrscheinlich.

Die Raman-Spektren bei unterschiedlichen $\gamma$ BL-Konzentrationen weisen im Vergleich zueinander keine Indizien für das Vorliegen von Trimeren oder größeren Clustern auf.

Darüber hinaus liefert das Raman-Spektrum zwischen $1500 \mathrm{~cm}^{-1}$ und $1227 \mathrm{~cm}^{-1}$ eine Reihe sehr intensiver C-H-Knickschwingungsbanden. Das Differenzspektrum (c)-(a) in Abbildung 8-4 zeigt den hohen Dimeranteil dieser Banden. Hier liegt so gut wie keine Verschiebung der Bandenposition vom Monomer zum Dimer vor, was eine Unabhängigkeit der $\mathrm{CH}$ Knickschwingungsfrequenz von der Clusterbildung belegt.

Diese Banden sind typischerweise wenig IR-aktiv und daher in FTIR-Spektren kaum erkennbar. Dabei zeigt das FTIR-Differenzspektrum (e)-(d) in Abbildung 8-4, dass die von Albrecht [175] bei $1498 \mathrm{~cm}^{-1}$ gefundene sehr schwache Bande, die als N-H-Knickschwingung des Dimers zugewiesen wird, im Rauschen untergeht und nicht als signifikantes Signal betrachtet werden kann.

Tab. 8-2. Vergleich von Amid I-Bandenpositionen des Monomers und der Dimere von $\gamma \mathrm{BL}$ im Raman-Spektrum mit Literaturdaten anderer experimenteller Techniken. Alle Werte sind in $\mathrm{cm}^{-1}$ angegeben. Literaturwerte von IR-Messungen in Lösung finden sich in Tab. A-32 in Anhang A.10.2

\begin{tabular}{lllll}
\hline & $\mathrm{M}$ & $\mathrm{D}_{2 \mathrm{H}}$ & $\mathrm{D}_{1 \mathrm{H}}{ }^{\mathrm{d}}$ & weitere Banden \\
\hline Jet-Expansion: & & & & \\
Ramana,b & 1765 & 1691 & - & $1756^{\mathrm{c}}, 1750^{\mathrm{c}}, 1735^{\mathrm{c}},(1711)$ \\
$\mathrm{FTIR}$ 175] & 1767 & 1729 & - & $1748,1703^{\mathrm{e}}$ \\
Matrix: & & & & \\
$\mathrm{N}_{2}-\mathrm{IR}[308$ & 1734 & 1699 & $1712 / 1680$ & $1724^{\mathrm{c}}$ \\
$\begin{array}{l}\text { Gasphase: } \\
\text { IR 318 }\end{array}$ & 1735 & - & - & -
\end{tabular}

a: aus dieser Arbeit; ${ }^{\mathrm{b}}$ : Bandenposition am Intensitätsmaximum; ${ }^{\mathrm{c}}$ : vermutlich Fermi-Resonanzen; ${ }^{\mathrm{d}}$ : antisymmetrisch / "symmetrisch“ (siehe Text und Abb. A-13 in Anhang A.10.1; ; : vermutlich symmetrische Streckschwingung des Tetramers, das durch die Stapelung zweier Dimere aktiviert wird.

In $\mathrm{N}_{2}$-Matrix-Experimenten liegt die $\mathrm{D}_{2 \mathrm{H}}$-Dimerbande bei $1699 \mathrm{~cm}^{-1}$ und damit $-35 \mathrm{~cm}^{-1}$ gegenüber dem Monomer verschoben (siehe Tab. 8-2). Es liegt somit eine zu den FTIR-Jetmessungen vergleichbare Rotverschiebung vom Monomer zum Dimer vor. Die $\mathrm{N}_{2}$-Matrixdaten unterliegen jedoch den für diese Methode typischen Verschiebungen [319, 320]. Die Fixierung in der Matrix verhindert zudem die Einstellung des $\mathrm{D}_{2 \mathrm{H}} \leftrightarrow \mathrm{D}_{1 \mathrm{H}}$-Gleichgewichts, so dass auch das energetisch ungünstigere offene $\mathrm{D}_{1 \mathrm{H}}$-Dimer bei $1712 \mathrm{~cm}^{-1}$ im Spektrum sichtbar ist.

Amid A Das Raman-Spektrum von $\gamma$ BL in Abbildung 8-5(a) zeigt in der Region oberhalb von $3000 \mathrm{~cm}^{-1}$ eine starke Einzelbande bei $3484 \mathrm{~cm}^{-1}$. Der Vergleich mit den Jet-FTIR-Ergebnissen aus der Literatur ergibt hierfür eindeutig eine Zuordnung als Amid A-Bande des Monomers (siehe Tab. 8-3). In der Literatur finden sich zudem Werte aus $\mathrm{N}_{2}$-Matrix-Experimenten und IR- 
Gasphasen-Messungen die für das $\gamma$ BL-Monomer mit 3473 bzw. $3474 \mathrm{~cm}^{-1}$ ca. $10 \mathrm{~cm}^{-1}$ unterhalb der im Jet gemessenen Bandenpositionen liegen (siehe Tab. 8-3).

Das Spektrum zeigt für zunehmende Substanzkonzentrationen in einer auf das Monomer normierten Darstellung (Abb. 8-5(b,c)), dass eine Reihe von Peaks unterhalb von $3400 \mathrm{~cm}^{-1}$ mit zunehmender $\gamma$ BL-Konzentration an Intensität gewinnen und von einer breiten Anhebung der Basislinie begleitet werden. Während der Basislinienverlauf auf große Cluster hinweist, legen die übrigen Banden aufgrund ihres Wachstums gegenüber dem Monomer nahe, dass es sich um Banden von Dimeren und größeren Clustern in Form von gestapelten Dimeren handelt. Die Bandenstruktur deutet auf starke Resonanzen hin, wie sie für das NPhF bereits diskutiert wurden (siehe Abschnitt 6.3).

Im Gegensatz zu den Jet-FTIR-Spektren in Abbildung 8-5(d,e) sind die Clusterbanden im Raman-Spektrum allesamt weniger intensiv als das Monomersignal. Im FTIR-Spektrum sind die dem Dimer zugeordneten Banden zwischen $3275-3047 \mathrm{~cm}^{-1}$ alle intensiver als das Monomer bei $3485 \mathrm{~cm}^{-1}$. Besonders intensiv ist ein einzelner Peak bei $3121 \mathrm{~cm}^{-1}$. An der Stelle der $3121 \mathrm{~cm}^{-1}$-Bande im FTIR stehen im Raman-Spektrum drei ähnlich intensive Banden bei 3117, 3101 und $3074 \mathrm{~cm}^{-1}$. Eine breite Bande bei ca. $3245-3140 \mathrm{~cm}^{-1}$ liegt in FTIR- und Raman-Spektrum vor und wird von Albrecht [175] dem Dimer zugeordnet. Die auffälligen Peaks dieser Bande unterschieden sich und liegen im FTIR bei $3195 \mathrm{~cm}^{-1} / 3181 \mathrm{~cm}^{-1}$ und im RamanSpektrum bei 3192 und $3174 \mathrm{~cm}^{-1}$. In den Spektren beider Techniken findet sich auch ein Dublett bei $3377 \mathrm{~cm}^{-1} / 3369 \mathrm{~cm}^{-1}$ und eine kleinere Bande bei $3259 \mathrm{~cm}^{-1}$. Während Albrecht [175] beide Banden nicht explizit erwähnt, führt sie eine FTIR-Bande bei $3413 \mathrm{~cm}^{-1}$ an. Hierfür findet sich keine Entsprechung im Raman-Spektrum. Bei der $3377 \mathrm{~cm}^{-1} / 3369 \mathrm{~cm}^{-1}$-Bande im Raman-Spektrum und der $3413 \mathrm{~cm}^{-1}$-Bande im FTIR-Spektrum handelt es sich vermutlich jeweils um den Oberton der symmetrischen bzw. antisymmetrischen Carbonylstreckschwingung im $D_{2 \mathrm{H}}$-Dimer.

Tab. 8-3. Vergleich von Amid A-Bandenpositionen des Monomers und der Dimere von $\gamma \mathrm{BL}$ im Raman-Spektrum mit Literaturdaten anderer experimenteller Techniken. „ausgewählte Bandenmaxima“ beinhalten alle signifikanten Peaks im Spektrum, die nicht bereits andernorts erwähnt sind. Alle Werte sind in $\mathrm{cm}^{-1}$ angegeben. Literaturwerte von IRMessungen in Lösung finden sich in Tab. A-32 in Anhang A.10.2

\begin{tabular}{lllll}
\hline & $\mathrm{M}$ & $\mathrm{D}_{2 \mathrm{H}}$ & $\mathrm{D}_{1 \mathrm{H}}{ }^{\mathrm{c}}$ & ausgewählte Bandenmaxima \\
\hline $\begin{array}{l}\text { Jet-Expansion: } \\
\text { Ramana,b }\end{array}$ & 3484 & $<3400$ & - & $3372,3260,3206,3192,3174,3117,3101,3074$ \\
$\mathrm{FTIR}[175]$ & 3485 & $3275-3047$ & - & $3195,3181,3413$ \\
Matrix: & & & $3473 / \approx 3231$ \\
$\mathrm{~N}_{2}-\mathrm{IR}[308]$ & 3473 & $\approx 3194$ & $-\quad$ Anhang A.10.1].
\end{tabular}

Für die Dimere ergeben sich in der Matrix Bandenpositionen bei ca. $3194 \mathrm{~cm}^{-1}$ für $\mathrm{D}_{2 \mathrm{H}}$ und $3231 \mathrm{~cm}^{-1}$ für $\mathrm{D}_{1 \mathrm{H}}$, die mit dem angenommenen Dimer-Bereich der Jet-Spektren übereinstimmen. 
Abgesehen von der starken Anhebung der Basislinie für höhere $\gamma$ BL-Konzentrationen zeigt das Raman-Spektrum keine einzelnen Banden, die auf Trimere oder höhere Cluster zurückzuführen sind.

$\mathrm{Zu}$ niedrigen Wellenzahlen hin ist das Spektrum aufgrund der Bildbreite der CCD-Kamera des curry-Jets abgeschnitten. Der Aufnahmebereich ist ein Kompromiss aus einer möglichst vollständigen Darstellung der Amid A-Clusterbanden bei gleichzeitiger Abbildung des Monomersignals als Referenz für die Normierung.

Quantenchemische Rechnungen Analog zu den Untersuchungen der bereits vorgestellten Amide wurden auch für $\gamma$ BL harmonische DFT-Frequenzrechnungen durchgeführt. Mit den Rechnungen auf B3LYP-D3(BJ)/aVTZ-Niveau liegen harmonische Frequenzen für Monomer und beide Dimer-Konformationen erstmals mit Dispersionskorrektur vor ${ }^{2)}$

Die harmonischen Rechnungen werden für das Monomer durch eine anharmonische VPT2Rechnung erweitert und den experimentellen Ergebnissen sowie einer weiteren VPT2-Rechnung aus der Literatur in Tabelle A-31 gegenüber gestellt. Die durchgeführte VPT2-Rechnung ergibt mit $3467 \mathrm{~cm}^{-1}$ eine $\mathrm{NH}$-Streckschwingungsfrequenz, die $18 \mathrm{~cm}^{-1}$ niedriger als die Amid ABande im FTIR liegt. Für die Amid I-Bande liegt der berechnete Wert hingegen $3 \mathrm{~cm}^{-1}$ höher als die Bande im FTIR-Jet. Neben den in dieser Arbeit durchgeführten VPT2-Rechnungen liegt in der Literatur nur eine weitere Arbeit mit VPT2-Frequenzen auf Basis einer MP2-Rechnung mit

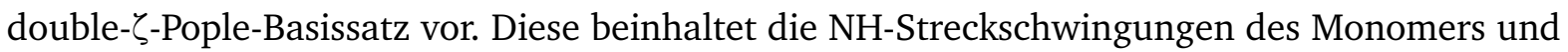
beider Dimere sowie der CO-Streckschwingung des Monomers (siehe Tab. A-31). Für die NHStreckschwingungsfrequenz des $\mathrm{D}_{2 \mathrm{H}}$-Clusters lässt sich lediglich feststellen, dass die Rechnung den Bereich trifft, in dem experimentell die Bande vermutet wird.

$\mathrm{D}_{2 \mathrm{H}}$ ist $C_{\mathrm{i}}$-symmetrisch (strenggenommen nur für die Aggregation von einem $\mathrm{M}_{\mathrm{S}}$ - und einem $\mathrm{M}_{\mathrm{R}}$-Monomer ${ }^{3)}$ ) und die Normalschwingungen unterliegen dem Alternativverbot. Daher gibt es zu jeder antisymmetrischen IR-aktiven Schwingung ein symmetrisches Raman-aktives Pendant. Ein Vergleich der experimentellen Ergebnisse mit einer doppelt-harmonischen Frequenzrechnung zu $\mathrm{D}_{2 \mathrm{H}}$ ergibt für die Raman-aktive Mode der $\mathrm{C}=\mathrm{O}$-Streckschwingung eine Verschiebung von $-69 \mathrm{~cm}^{-1}$ gegenüber $-74 \mathrm{~cm}^{-1}$ im Experiment (siehe Tab. 8-4). Die entsprechende FTIRVerschiebung beträgt theoretisch $-30 \mathrm{~cm}^{-1}$ und liegt in den Spektren bei $-38 \mathrm{~cm}^{-1}$.

Für die CO-Streckschwingung von Ameisensäuredimeren wurde allerdings gezeigt, dass zum Vergleich von harmonischen B3LYP-Rechnungen und experimentellen Ergebnissen aufgrund von günstiger Kompensation der anharmonischen Effekte die Betrachtung der Davidov-Aufspaltung besser geeignet ist als die relative Verschiebung zum jeweiligen Monomersignal [321]. Die Davidov-Aufspaltung zwischen symmetrischer und antisymmetrischer CO-Streckschwingung des $D_{2 \mathrm{H}^{-}}$Dimers beträgt $38 \mathrm{~cm}^{-1}$ im Experiment und $39 \mathrm{~cm}^{-1}$ für die B3LYP-D3(BJ)/aVTZ-

${ }^{2)}$ Die Ergebnisse werden zusätzlich anderen DFT-Rechnungen aus der Literatur und den experimentellen Ergebnissen in Tab. A-31 in Anhang A.10.2 gegenüber gestellt.

${ }^{3)}$ Auch wenn die $C_{i}$-Symmetrie streng genommen nur für das diastereomere Dimer aus $M_{S}$ und $M_{R}$ gilt, zeigen die harmonischen Frequenzrechnungen für die beiden enantiomeren Formen $\left(M_{R} M_{R}\right.$ und $\left.M_{S} M_{S}\right)$ IR-Aktivitäten und Raman-Streuqerschnitte, die einem Alternativverbot gleichkommen. Aufgrund der planaren Chiralität des $\gamma$ BLMonomers (siehe oben) werden die $S$ - und $R$-Konfigurationen mit den entsprechenden Indizes gekennzeichnet, falls eine Unterscheidung nötig ist. 


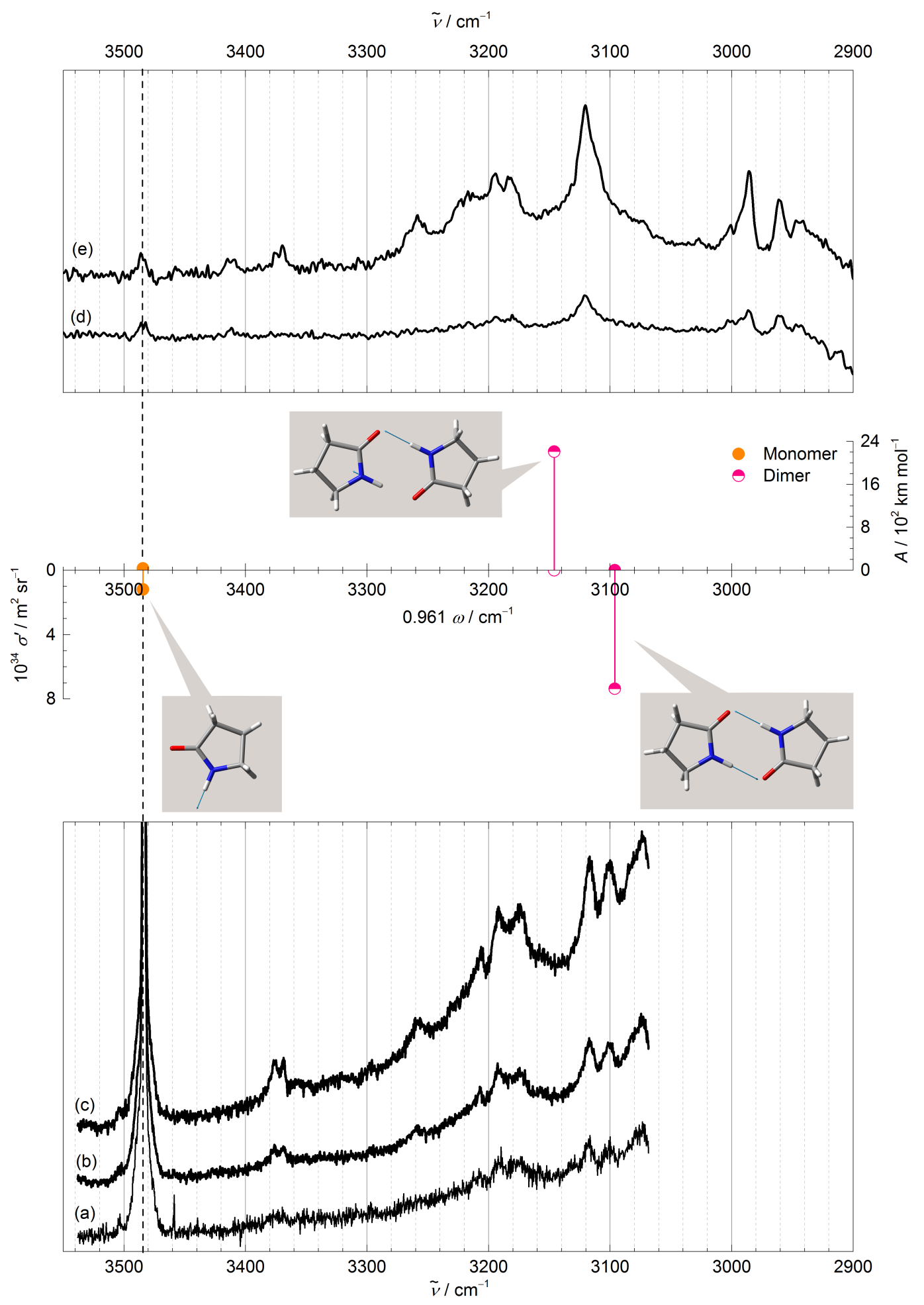

Abb. 8-5. FTIR- und Raman-Jet-Spektren von $\gamma$-Butyrolactam in der Amid A-Region.

Oben: Auf die Intensität der Monomerbande normierte FTIR-Jetspektren mit von (d) zu (e) zunehmender $\gamma$ BL-Konzentration (aus [175]).

Mitte: Auf die Monomerbandenposition skalierte Strichspektren (Faktor 0.961, gestrichelte Linie) des Monomers und des cyclischen Dimers auf B3LYP-D3(BJ)/aVTZ-Niveau mit IR-Aktivitäten (nach oben) und Raman-Streuquerschnitten (nach unten) sowie Illustrationen ausgewählter Normalmoden.

Unten: Auf die Intensität der Monomerbande normierte Raman-Jetspektren mit von (a) zu (c) zunehmender $\gamma B$ BL-Konzentration.

(Details zu den Messungen der Raman-Spektren befinden sich im Raman-Spektrenverzeichnis in Tab. A-6 in Anhang A.6.1. Details zu den Frequenzrechnungen befinden sich in Tab. A-27 in Anhang A.10.2) 
Rechnungen, was je nach Betrachtungsweise die getroffene Bandenzuordnung oder die gewählte quantenchemische Methode bestätigt, ${ }^{4)}$

Diese Gegenüberstellung ist für die harmonischen NH-Streckschwingungsfrequenzen des Dimers aufgrund des nicht scharf lokalisierbaren Bandenursprungs der Amid-A-Bande nicht möglich.

Tab. 8-4. Berechnete $\mathrm{N}-\mathrm{H}$-Streck- und $\mathrm{C}=\mathrm{O}-\mathrm{Streck}-$ Normalmoden der zwei stabilsten $\gamma \mathrm{BL}$-Dimere auf B3LYPD3(BJ)/aVTZ-Niveau und Zuordnung zu den experimentellen Raman-Banden ( $\left.\tilde{v}_{\text {Ra }}\right)$ und FTIR-Banden $\left(\tilde{v}_{\mid R}\right)$. Relative Verschiebungen zum entsprechenden Monomerwert stehen in runden Klammern. Angegeben sind die harmonischen Wellenzahlen $\omega$, die IR-Intensität $A$ und der differentielle Raman-Streuquerschnitt $\sigma^{\prime}$ bei $532.2847 \mathrm{~nm}$ Anregungswellenlänge und $90^{\circ}$ Streugeometrie nach Gl. 3-13. Die Buchstaben „a“ und „d“ im Index stehen für Akzeptor und Donor und wechseln für cyclische Cluster zu „as" und „s“ für die antisymmetrische und symmetrische Streckschwingung (Weitere Daten der Frequenzrechnungen zu diesen Dimerstrukturen befinden sich in den Tab. A-27 A-28 und A-29 im Tabellenwerk in Anhang A.10.2).

\begin{tabular}{|c|c|c|c|c|c|c|}
\hline \multicolumn{2}{|c|}{ Konformer } & $\omega / \mathrm{cm}^{-1}$ & $A / \mathrm{km} \cdot \mathrm{mol}^{-1}$ & $10^{34} \sigma^{\prime} / \mathrm{m}^{2} \mathrm{sr}^{-1}$ & $\tilde{v}_{\mathrm{Ra}} / \mathrm{cm}^{-1}$ & $\tilde{v}_{\mathrm{IR}} / \mathrm{cm}^{-1}$ \\
\hline \multirow[t]{4}{*}{$\mathrm{D}_{2 \mathrm{H}}$} & $v_{\mathrm{NH} \text {-as }}$ & $3274(-352)$ & 2208 & 0 & - & 一 \\
\hline & $v_{\mathrm{NH}-\mathrm{s}}$ & $3222(-404)$ & 0 & 7.37 & - & - \\
\hline & $v_{\text {CO-as }}$ & $1753(-30)$ & 1139 & 0 & - & $1729(-38) a, b$ \\
\hline & $v_{\mathrm{CO}-\mathrm{s}}$ & $1714(-69)$ & 0 & 0.50 & $1691(-74)^{b}$ & - \\
\hline \multirow[t]{4}{*}{$\mathrm{D}_{1 \mathrm{H}}$} & $v_{\mathrm{NH}-\mathrm{a}}$ & $3625(-1)$ & 35 & 1.20 & - & 一 \\
\hline & $v_{\mathrm{NH}-\mathrm{d}}$ & $3362(-264)$ & 899 & 3.95 & - & - \\
\hline & $v_{\mathrm{CO}-\mathrm{as}}$ & $1762(-21)$ & 1056 & 0.11 & - & - \\
\hline & $v_{\mathrm{CO}-\mathrm{s}}$ & $1734(-49)$ & 106 & 0.87 & - & - \\
\hline
\end{tabular}

\subsection{Zusammenfassung und Diskussion}

In diesem Kapitel werden erstmalig Raman-Jet-Spektren von $\gamma$-Butyrolactam im Bereich von $3060-3540 \mathrm{~cm}^{-1}$ (Amid A- und C-H-Streckschwingungsbanden) und 1200-1800 $\mathrm{cm}^{-1}$ (Amid IIII- und C-H-Knickschwingungsbanden) vorgestellt. Diese Spektren werden mit den FTIR-JetSpektren von Albrecht [175] verglichen. Die Bandenpositionen des Monomers sind sowohl für die Amid A- als auch für die Amid I-Bande mit Abweichungen von $-1 \mathrm{~cm}^{-1}$ bzw. $-2 \mathrm{~cm}^{-1}$ in guter Übereinstimmung mit den FTIR-Messungen.

Die Amid I-Bande des cis-Monomers ist bei NMF und NMA neben der dominanten transMonomerbande nicht zu erkennen, sollte dort aber laut skalierter Frequenzrechnung unter der höherfrequenten Flanke des trans-Peaks liegen (siehe Abschnitte 5.1 und 4.1). Durch die cyclische Molekülkonstitution liegt $\gamma$ BL nur als cis-Konformer vor, weswegen das Raman-Spektrum von $\gamma$ BL die isolierte Bandenposition für ein sekundäres Amid in cis-Konformation zeigt. Diese Bandenposition weicht allerdings mit $1765 \mathrm{~cm}^{-1}$ deutlich von den anzunehmenden Positionen für NMA $\left(\approx 1720 \mathrm{~cm}^{-1}\right)$ und NMF $\left(\approx 1744 \mathrm{~cm}^{-1}\right)$ ab.

Weiterhin werden in diesem Kapitel die Untersuchungen zu Clustern sekundärer Amide mit $\gamma$ BL abgeschlossen. Durch das ausschließliche Vorliegen der cis-Konformation im Monomer,

\footnotetext{
4) Dieser Konflikt der Perspektiven zwischen Theoretikern und Experimentalwissenschaftlern lässt sich nicht ohne weiteres lösen, ist jedoch die Triebkraft für aktuelle Benchmarking-Forschungsprojekte [52].
} 
ist in den Spektren als Dimer-Cluster nur die cis-cis-Form zu erwarten. Die Dimersignale im FTIR- und Raman-Spektrum sollten sich aufgrund des $C_{\mathrm{i}}$-symmetrischen $\mathrm{D}_{2 \mathrm{H}}$-Aggregats und dem daraus resultierenden Alternativverbot komplementär verhalten. Dieser Effekt ist für die Amid I-Bande deutlich zu erkennen, wo die Raman-aktive Dimerbande um $-74 \mathrm{~cm}^{-1}$ und die IR-aktive um $-38 \mathrm{~cm}^{-1}$ gegenüber der jeweiligen Monomerbande (Raman: $1765 \mathrm{~cm}^{-1}$; FTIR: $1767 \mathrm{~cm}^{-1}$ ) verschoben ist. Im Amid A-Bereich sorgen jedoch starke Resonanzen dafür, dass die Banden sehr breit sind und in beiden Spektren kein Signal eindeutig als Bandenzentrum des Dimers zugeordnet werden kann. Es kann lediglich der Bereich unterhalb von $3400 \mathrm{~cm}^{-1}$ als Dimerbandenbereich angegeben werden. Allerdings deckt dieser Bereich die berechneten Verschiebungen des Signals gegenüber der Monomerbande von $-352 \mathrm{~cm}^{-1}$ (FTIR) und $-404 \mathrm{~cm}^{-1}$ (Raman) ab.

Für das $\gamma$ BL-Dimer finden sich in der Literatur zwei Konformationen, die eine oder zwei N$\mathrm{H} \cdot \mathrm{O}=\mathrm{C}$-Wasserstoffbrücken bilden. Die in den Jet-Spektren gefundenen Dimersignale können aus verschiedenen Gründen dem cyclischen Dimer mit zwei Wasserstoffbrücken $\left(\mathrm{D}_{2 \mathrm{H}}\right)$ zugeordnet werden. Mehrere Studien zum $\gamma$ BL-Dimer verorten das Gleichgewicht zwischen offenem $\left(D_{1 H}\right)$ und geschlossenem Dimer in Lösungen weit auf der Seite des cyclischen Dimers [309, 314] und führen die Bildung des um $23.2 \mathrm{~kJ} \cdot \mathrm{mol}^{-1}$ (B3LYP-D3(BJ)/aVTZ) instabileren offenen Dimers auf eine Stabilisierung durch Lösemittel zurück. Damit kann sowohl für die Gasphase als auch für die Jet-Expansion das offene Dimer vernachlässigt werden. Außerdem weisen die Bandenstruktur und die starke Rotverschiebung eine Ähnlichkeit zum cc-Dimer des $N$-Phenylformamids auf (siehe Abschnitt 6.3). Wie bei NPhF lässt sich auch hier die Vielzahl an weit verstreuten Peaks am ehesten mit Fermi-Resonanzen erklären. 



\section{Zusammenfassung und Ausblick}

In dieser Arbeit werden schwerpunktmäßig kleine Amide sowohl hinsichtlich der Energieunterschiede zwischen cis- und trans-Konformeren als auch deren Aggregation zu Clustern bis hin zum Trimer untersucht. Hierzu wird ein multispektroskopischer Ansatz mit einem Schwerpunkt auf Raman-Jet-Spektroskopie gewählt. Für die untersuchten Substanzen bedarf es einer apparativen Anpassung des Heizsystems des curry-Jets. Die experimentellen Ergebnisse werden quantenchemischen DFT-Frequenzrechnungen gegenübergestellt und daraus Banden zugeordnet, wobei die Spektrenauswertung durch ein neues Auswertprogramm vereinfacht und um verschiedene Optionen ergänzt wird.

\subsection{Schwingungsspektroskopie und Isomerisierung}

Die cis-/trans-Enthalpiedifferenz wird für NMA, NMF und NPhF in der Jet-Expansion aus Temperaturmessreihen mittels linearer Raman-Spektroskopie über Van-'t-Hoff-Plots bestimmt. Für alle drei Amide werden Raman-Jet-Spektren der Amid-A- und Amid-I-Bande aufgenommen, wobei der Fokus auf die Amid-A-Bande gelegt wird, da in diesem Bereich jeweils eindeutig getrennte trans- und cis-Banden vorliegen, die laut DFT-Rechnung ihren Ursprung nahezu vollständig in der N-H-Streckschwingung haben. Die trans-Amid-A-Bande ist bei allen drei untersuchten Amiden die höherfrequente Bande. Gegenüber den ähnlich positionierten Amid-ABanden von $t$-NMF $\left(3501 \mathrm{~cm}^{-1}\right)$ und $t$-NMA $\left(3508 \mathrm{~cm}^{-1}\right)$ liegt die von $t$-NPhF mit $3463 \mathrm{~cm}^{-1}$ bei deutlich niedrigeren Wellenzahlen. Dies ist ein spektroskopisches Indiz für die Schwächung der N-H-Bindung, welche z.B. durch $\mathrm{N}-\mathrm{H} \cdots \mathrm{X}$-Wasserstoffbrückenbindungen hervorgerufen werden kann. In diesem Fall liegt die Ursache dieser Rotverschiebung wahrscheinlich in der Resonanzerweiterung durch den Phenylring in einer planaren trans-Konformation und in der $\mathrm{C}-\mathrm{H} \cdots \mathrm{O}=\mathrm{C}-$ Wechselwirkung zwischen der ortho-C $-\mathrm{H}$-Gruppe des Phenylrings und der Carbonylgruppe.

Bei NMA und NMF ist eine Substruktur der Banden erkennbar, die mithilfe anharmonischer VPT2-Rechnungen auf durch die Torsionsschwingung(en) der Methylgruppe(n) hervorgerufene, heiße Banden zurückgeführt werden kann. Diese Interpretation der Rechnungen steht allerdings in Anbetracht der Unzuverlässigkeit von VPT2 in Bezug auf die Berechnung von Torsionsschwingungen mit niedrigen Barrieren, wie z. B. Methyltorsionen [29, 214], unter Vorbehalt. Das Fehlen einer solchen Substruktur im Amid-A-Spektrum von NPhF ist jedoch konsistent mit einer Interpretation der Peaks als heiße Banden der Methyltorsion.

In einem ergänzenden Projekt zu dieser Arbeit werden die Raman- und FTIR-Jet-Spektren des heterozyklischen Amins Imidazol (Im) aufgenommen (siehe Abb. A-1 und A-2 in An- 
hang A.3). Die dort ebenfalls auftretenden heißen Banden lassen sich durch den Vergleich mit VPT2-Rechnungen den zugrunde liegenden niederfrequenten Normalmoden $v_{k}$ zuordnen. Da die Wellenzahldifferenz zur Fundamentalen $v_{i}$ genau der zugehörigen Anharmonizitätskonstanten $x_{i, k}$ entspricht, können aus dem Raman-Spektrum experimentelle Werte für $x_{i, k}$ sowohl für die NH-Streckschwingung $\left(v_{1}\right)$ als auch die erste $\mathrm{CH}$-Streckschwingungsmode $v_{2}$ bestimmt und der Theorie entgegengestellt werden. Die guten Übereinstimmungen für die aufgeführten Anharmonizitätskonstanten mit Unterschieden zwischen Theorie und Experiment von höchstens $2 \mathrm{~cm}^{-1}$ (siehe Tab. A-2) sind jedoch kein Beleg für eine besondere Eignung des VPT2Ansatzes für die Beschreibung der Anharmonizität der N-H- und C-H-Streckschwingungen im Imidazol. Dies zeigt der Vergleich der mit VPT2 berechneten anharmonischen Verschiebungen gegenüber dem harmonischen Wert einer B3LYP-D3(BJ)-Rechnung. Oberflächlich scheinen die VPT2-Vorhersagen in guter Übereinstimmung mit dem Experiment und eine einfache Skalierung der harmonischen Werte ein legitimer Ansatz, allerdings zeigen die berechneten diagonalen Anharmonizitätskonstanten der VPT2-Rechnung, dass diese Übereinstimmung in erster Linie auf eine günstige Fehlerkompensation zurückzuführen ist [57]. Die Raman-Spektren von Imidazol sind damit ein interessanter Datensatz für das Benchmarking von Methoden zur Berechnung anharmonischer Frequenzen.

Für die in der Literatur häufig als charakteristischer Marker verwendete Amid-I-Bande können cis- und trans-Konformere der hier untersuchten Amide nicht im Spektrum unterschieden werden, was mit den B3LYP-D3(BJ)/aVTZ-Rechnungen, die den Abstand zwischen trans- und cis-Amid-I-Bande auf ca. $7 \mathrm{~cm}^{-1}$ (NMF und NMA) berechnen, konsistent ist. Allerdings liegt die berechnete Frequenz einer harmonischen Normalschwingung typischerweise oberhalb des experimentellen anharmonischen Wertes. So liegen die harmonischen Frequenzen der CO-Streckschwingung von trans-NMA, trans-NMF und $\gamma \mathrm{BL}$ um 19(4) $\mathrm{cm}^{-1}$ oberhalb der entsprechenden Amid I-Banden. Eine ähnlich große Differenz ist für die Amid I-Bande von cis-NMA und cisNMF zu erwarten, sofern keine Resonanzen vorliegen (siehe Tab.9-1).

Tab. 9-1. Vergleich der harmonischen (harm.) CO-Streckschwingungsfrequenzen mit den experimentellen (exp.) Amid IBandenpositionen im Raman-Jet-Spektrum von NMA, NMF und $\gamma B \mathrm{BL}$. Doppelt-harmonische Rechnungen auf B3LYPD3(BJ)/aVTZ-Niveau. Alle Angaben in $\mathrm{cm}^{-1}$.

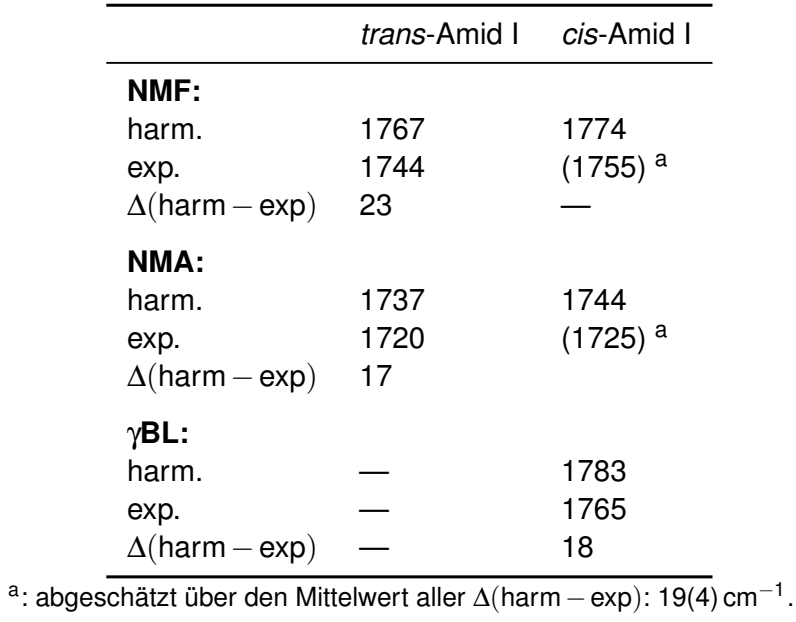


Der Vergleich mit den Raman-Spektren verortet die Amid I-Bande von cis-NMA und cis-NMF in der höherfrequenten Flanke der jeweiligen trans-Amid I-Bande (Abb. 4-4 und 5-3). In beiden Fällen ist an der berechneten Position im Spektrum kein Signal mit signifikanter Intensität erkennbar.

Diese Abschätzung ist zudem ein weiteres Indiz dafür, dass die Zuordnung der Bande bei $1744 \mathrm{~cm}^{-1}$ als Amid I-Bande von trans-NMF richtig ist, denn für die Banden bei $1738 \mathrm{~cm}^{-1}$ und $1729 \mathrm{~cm}^{-1}$ wäre die Differenz zum harmonischen Wert mit $29 \mathrm{~cm}^{-1}$ und $38 \mathrm{~cm}^{-1}$ deutlich größer als die $17 \mathrm{~cm}^{-1}$ bei trans-NMA und $18 \mathrm{~cm}^{-1}$ bei $\gamma \mathrm{BL}$ (vgl. Abschnitt „Amid I-III“ in Kapitel 5.1).

Aufgrund des Fehlens von cis-Amid I-Banden in den Raman-Spektren der untersuchten Amide (mit Ausnahme von $\gamma B \mathrm{BL}$ ) wird die Van-'t-Hoff-Analyse der cis-/trans-Isomerisierung mit den Amid-A-Spektren durchgeführt und für NMA exemplarisch auch auf die ebenfalls gut separierte Amid-IV-Bande angewendet. Es ergeben sich aus den Van-'t-Hoff-Plots für die drei untersuchten Amide cis-/trans-Enthalpieunterschiede von $5-8 \mathrm{~kJ} \cdot \mathrm{mol}^{-1}$, wobei jeweils eine trans-Konformation die stabilste ist (siehe Tabelle 9-2).

Tab. 9-2. cis-/trans-Isomerisierungsenthalpien für die Amide NMF, NMA und NPhF aus den Van-'t-Hoff-Plots experimenteller Raman-Jet-Temperaturmessreihen $\left(\Delta_{\mathrm{r}} H_{\mathrm{exp}}^{\ominus}\right)$ und den thermodynamischen Daten harmonischer Frequenzrechnungen auf B3LYP-D3(BJ)/aVTZ-Niveau $\left(\Delta_{\mathrm{r}} H_{\text {theo }}^{\ominus}\right)$.

\begin{tabular}{llll}
\hline Amid & $\Delta_{\mathrm{r}} H_{\text {exp }}^{\ominus} / \mathrm{kJ} \cdot \mathrm{mol}^{-1}$ & $\Delta_{\mathrm{r}} H_{\text {theo }}^{\ominus} / \mathrm{kJ} \cdot \mathrm{mol}^{-1}$ \\
\hline NMF & $5(1), \quad 6(2)^{\mathrm{a}}$ & $4.392(6)$ \\
NMA & $8(2)$ & $8.506(7)$ \\
NPhF & $6(5)$ & $3.929(2)$ \\
\cline { 2 - 2 } & & 3 a zwei getrennte Auswertungen des Van-'t-Hoff-Plots (siehe Abschnitt5.2.
\end{tabular}

Auch wenn diese Ergebnisse aufgrund der hohen Messunsicherheiten keine signifikante Tendenz erkennen lassen, so ist die Zunahme des cis-Anteils von NMA über NMF bis NPhF bei qualitativer Betrachtung der Spektren erkennbar, was eine Abnahme des Energieunterschieds bedeuten würde. Der große Fehlerbalken in den Van-'t-Hoff-Plots aller drei Substanzen hat seine Ursache in Schwierigkeiten bei der Durchführung und Auswertung der Messungen. So lässt sich nicht feststellen, ob vor der Expansion das thermische Gleichgewicht bei Düsentemperatur eingestellt wird. Auf Seiten der Auswertung liegt die Hauptfehlerquelle in der Integration der Spektren, insbesondere der wenig intensiven cis-Banden bei NMF und NMA, die aufgrund des geringen Signal-zu-Rauschen-Verhältnisses nicht eindeutig integriert werden können. Dies ließe sich durch längere oder häufigere Messungen verbessern. Hier wird es allerdings schwierig, mit dem vorliegenden curry-Setup Expansions- und Messbedingungen (so z. B. Substanzkonzentration und Temperatur) über einen langen Zeitraum konstant zu halten. Daher finden sich weiter unten Vorschläge zur apparativen Weiterentwicklung des curry-Jets.

Auf theoretischer Seite ergeben die B3LYP-Rechnungen die ebenfalls in Tabelle 9-2 zusammengefassten Enthalpieunterschiede. Die gemessenen Werte sind also im Rahmen der Messungenauigkeit in Übereinstimmung mit den jeweiligen Ergebnissen der quantenchemischen Rechnungen.

Ergänzend zu den diskutierten experimentellen und theoretischen Untersuchungen werden 
die cis-/trans-Energieunterschiede von $N$-Alkylamiden mit Kettenlängen $m, n \leq 3{ }^{1}$ ) rein quantenchemisch bestimmt. Dabei kann die Hypothese, wonach das Gleichgewicht mit steigender Alkylkettenlänge zum cis-Konformer verschoben werden müsste, da dieses durch zunehmende intramolekulare Dispersionswechselwirkungen der Alkylreste stabilisiert wird, nicht bestätigt werden. Die quantenchemische Untersuchung symmetrisch substituierter $N$-Alkylamide ergibt für NPB mit $14.6 \mathrm{~kJ} \cdot \mathrm{mol}^{-1}$ einen höheren Energieunterschied $\Delta E_{\mathrm{r}, 0}$ (cis-trans) als NMA mit $9.0 \mathrm{~kJ} \cdot \mathrm{mol}^{-1}$. Dies wird plausibel, wenn die Struktur der trans- $N$-Alkylamide mit derjenigen zur Haarnadel gefalteten $n$-Alkane gleicher Länge verglichen wird. Die Breite der Haarnadel in den gebogenen Alkanen entspricht in etwa der Breite einer trans-Amidgruppe, weswegen die Bildung des cis-Konformers nicht durch intramolekulare Wechselwirkungen zwischen Alkylresten eines haarnadelförmig angeordneten $N$-Alkylamids stabilisiert wird. Es sei erwähnt, dass vermeintliche Trends im cis-/trans-Energieunterschied von $N$-Alkylamiden verschiedener Alkylkettenlängen mit Vorsicht zu interpretieren sind, da hier die Differenzen oftmals in der Größenordnung der anzunehmenden Unsicherheiten der zugrundeliegenden DFT-Rechnungen liegen. Aufgrund der bedingten Zuverlässigkeit der theoretischen Vorhersagen dürfte es auch in Zukunft interessant sein, diese Moleküle experimentell zu untersuchen.

Abschließend lässt sich zur Bestimmung von Isomerisierungsenthalpien festhalten, dass der curry-Jet in dem hier vorgestellten Aufbau nur für deren grobe Abschätzung geeignet ist. Neben der häufig verwendeten Amid-A-Bande wird erstmals die niederfrequente Amid-IV-Bande des Raman-Spektrums für beide NMA-Konformere eindeutig zugeordnet. Sie ist aufgrund ihrer guten cis-/trans-Separation vergleichbar gut geeignet, um das Konformerenverhältnis zu bestimmen. Die Amid-IV-Bande stellt für Amide somit eine gute Alternative für solche quantitativen Untersuchungen dar, wenn es gelingt diese eindeutig zuzuordnen.

Eine Erhöhung des cis-cis-Dimeranteils in der Expansion lässt sich nicht auf thermodynamischem Wege über die Substanztemperatur vor der Expansion realisieren, da eine höhere Düsentemperatur zwar grundsätzlich den cis-Monomeranteil in der Expansion erhöht, gleichzeitig aber die Dimerkonzentration abnimmt, da eine Temperaturerhöhung einer Clusterbildung entgegen wirkt. Hier wären eine Isomerisierung durch einen Katalysator vor der Düse (siehe Cox et al. [322, 323] und Cox und Lectka [324]) oder die Photoisomerisierung mittels Laser [35, 81, 325] mögliche Ansätze für die Zukunft.

\subsection{Molekülaggregate}

Eine zentrale Motivation dieser Arbeit ist der spektroskopische Nachweis von cis-cis-Dimeren sekundärer Amide. Deren höhere Stabilität relativ zu trans-trans- oder gemischten Dimeren wird aufgrund der zweiten $\mathrm{N}-\mathrm{H} \cdots \mathrm{O}=\mathrm{C}$-Wasserstoffbrücke allgemein angenommen und hier durch dispersionskorrigierte DFT-Rechnungen für die drei Amide NMA, NMF und NPhF bestätigt (siehe Tab. 9-3).

Für einen Nachweis von Clusterbanden im Raman-Spektrum werden in erster Linie AmidA- und Amid-I-Banden untersucht, da diese in der Regel als scharfe, intensive und gut isolierte

\footnotetext{
${ }^{1)}$ Die Systematik der Alkylkettenbezeichung findet sich in Abb. 7-1.
} 
Tab. 9-3. Nullpunktskorrigierte Dissoziationsenergien $D_{0}$ für Dimere von NMA, NMF und NPhF aus B3LYP-D3(BJ)Rechnungen. Alle Werte in $\mathrm{kJ} \cdot \mathrm{mol}^{-1}$. Sofern nicht anders angegeben, hat das stabilste $t t$-Dimer $t t_{\mathrm{N}}$-Geometrie.

\begin{tabular}{cccc}
\hline & $t t$ & $c c$ & Basissatz \\
\hline NMA & $32^{a}$ & 63 & $a V T Z$ \\
NMF & $28^{\text {b }} ; 29$ & 53 & aVTZ \\
NPhF & 43 & 52 & def2TZVP \\
\cline { 2 - 2 } a: $t t_{N}$ und $t t_{C}$ isoenergetisch; ${ }^{b}: t t_{H}$-Geometrie.
\end{tabular}

Peaks auftreten. Hier lässt sich feststellen, dass sich für die drei Amide die Amid-I-Banden der Cluster nicht eindeutig zuordnen lassen, so dass sich die folgenden Erläuterungen nur auf die Amid-A-Bande beziehen.

Trotz der hohen Stabilität bleibt der eindeutige Nachweis eines $c c$-Konformers für die genannten Amide bisher aus. Dies wird für NMA auf die geringe cis-Monomerkonzentration in der Expansion zurückgeführt, denn ein cis-Monomeranteil von ca. $3 \%$ unter den im curry-Jet optimalen Expansionsbedingungen ist zu gering, um im Spektrum das Dimer als signifikante Bande zuzuordnen.

Ein interessanter Befund dieser Arbeit ist, dass trotz eines leicht höherem (8-15\% bei NMF) und sehr hohem (ca. $50 \%$ bei NPhF) cis-Monomeranteil im Raman-Spektrum keine $c c$-Dimerbanden eindeutig nachweisbar sind. Daher wird mit $\gamma$ BL ein sekundäres Amid, das in der Gasphase ausschließlich in cis-Konformation vorliegt, auf Dimerbildung untersucht. Hier liegt ein sehr breites Dimersignal vor, das starke Resonanzen mit anderen Schwingungsmoden vermuten lässt und Ähnlichkeiten in Verschiebung und Breite zu NPhF aufweist. Für NMF und NMA wird ebenfalls angenommen, dass die $\mathrm{N}-\mathrm{H}$-Streckschwingungsbanden der cc-Dimere eine starke Verbreiterung durch Resonanzen erfährt, da die O-H-Streckschwingungsbande des strukturell ähnlichen Ameisensäuredimers ebenfalls stark resonanzverbreitert ist [29, 271, 326]. Dass also keine N-H-Streckschwingungsbande des cc-Dimers im Raman-Spektrum für hohe cisMonomerkonzentrationen wie bei NPhF erkennbar ist, liegt wahrscheinlich an dem durch Resonanzen stark verbreiterten Signal.

Für NMA finden sich im Raman-Jet-Spektrum Evidenzen für zwei tt-Dimere, deren Banden $29 \mathrm{~cm}^{-1}$ auseinander liegen. Auf theoretischer Seite ergeben sich hierfür zwei isoenergetische Dimergeometrien, die sich im freien Elektronenpaar des Sauerstoffatoms unterscheiden, das als Wasserstoffbrücken-Akzeptorstelle dient. Deren Donorbandenpositionen liegen in doppeltharmonischer Näherung $32 \mathrm{~cm}^{-1}$ auseinander.

Die DFT-Rechnungen ergeben auch für NMF zwei nahezu isoenergetische Dimerstrukturen $\left(\Delta E_{0} \approx 1 \mathrm{~kJ} \cdot \mathrm{mol}^{-1}\right.$, siehe Tab. 9-3), die, mit zu den NMA-Dimeren analoger Geometrie, ebenfalls zwei getrennte Amid-A-Donor-Banden erwarten lassen $\left(\Delta \tilde{v}=40 \mathrm{~cm}^{-1}\right.$ nach B3LYPD3(BJ)/aVTZ). Anders als bei NMA lässt sich aber nur eine Bande sicher als tt-Dimerbande zuordnen, wenngleich eine um $28 \mathrm{~cm}^{-1}$ niedrigere Bande geringer Intensität durch den Vergleich mit NMA und den DFT-Rechungen sowie einer Plausibilitätsprüfung der aus dem Peakverhältnis ermittelten Schwingungstemperatur die zweite $t t$-Dimerbande sein kann. Bei NPhF, wo aufgrund des cis-/trans-Konformerenverhältnisses im Monomer von nahezu 1:1 nun auch die Bildung gemischter Dimere in Betracht gezogen werden muss, lässt sich keine der Banden 
im Amid-A-Raman-Spektrum einer der in den Rechnungen gefundenen Dimerkonformationen eindeutig zuordnen.

Eines der zentralen Ergebnisse dieser Arbeit ist der spektroskopische Nachweis der Bildung von ringförmigen Trimerclustern in der Expansion für NMA. Sowohl eine verhältnismäßig geringe Rotverschiebung der Donorbanden der N-H-Streckschwingung des Trimers gegenüber der Fundamentalen des Monomers als auch die unterschiedlichen Bandenpositionen im FTIRSpektrum sind klare Hinweise auf gespannte Wasserstoffbrückenbindungen und Symmetrieeffekte, wie sie eine zyklische Trimerstruktur aufweist. Die auf B3LYP-D3(BJ)/aVTZ-Niveau stabilsten Trimerkonformationen sind beide zyklisch mit einem nullpunktskorrigierten Energieunterschied zur offenen Anordnung von $\approx 17 \mathrm{~kJ} \cdot \mathrm{mol}^{-1}$. Dass dieses eindeutige Ergebnis noch nicht Einzug in die Literatur gefunden hat, liegt in der Dispersionskorrektur der DFTRechnung begründet. Dieses Werkzeug ist erst seit vergleichsweise kurzer Zeit in den gängigen quantenchemischen Rechenprogrammen verfügbar. Die Vergleichsrechnung ohne Dispersionskorrektur ergibt für die offenkettige Variante von NMA, dass diese um $12 \mathrm{~kJ} \cdot \mathrm{mol}^{-1}$ stabiler ist als die zyklische, was die bisherige Literaturlage erklärt.

Es zeigt sich also am Beispiel NMA, dass der Verzicht auf Dispersionskorrektur in DFT-Rechnungen für die Suche nach der stabilsten Geometrie von Wasserstoffbrücken-gebundenen Clustern grob falsche Ergebnisse liefern kann.

Auch wenn im NMF-Spektrum keine Trimersignale erkennbar sind und eine nennenswerte Trimerbildung für NPhF aufgrund von apparativen Limitierungen ausgeschlossen werden kann, postulieren analoge DFT-Rechnungen ebenfalls zyklische Geometrien als stabilste Clusterstrukturen. Für NMF und NPhF wird dieses Resultat jedoch auch ohne Dispersionskorrektur erhalten.

Die Länge der Wasserstoffbrücke $d$ und der Bindungswinkel der Wasserstoffbrückenbindung mit der $\mathrm{C}=\mathrm{O}$-Bindung $\alpha$ der berechneten zyklischen Trimerstrukturen nehmen von NMA hin zu NMF ab. Die Abnahme von $d$ bedeutet einen geringeren Abstand der Moleküle im Cluster, was vor allem mit der im NMF nicht vorhandenen Methylgruppe und der damit verbundenen verminderten sterischen Hinderung zusammenhängt. Die Abnahme des Bindungswinkels ist ebenfalls eine Folge der geringeren sterischen Hinderung und sollte für NMF zu einer erhöhten Ringspannung gegenüber NMA führen.

Im Imidazol-Projekt steht die Bildung von Imidazol-Trimeren im Mittelpunkt. Durch den Vergleich der experimentellen Ergebnisse mit harmonischen Frequenzrechnungen auf B3LYPD3(BJ)/def2TZVP-Niveau kann auch hier für das stabilste Trimer eine zyklische Geometrie nachgewiesen werden. Die sehr asymmetrische Anordnung der Imidazolmoleküle im Cluster mit drei $\mathrm{N}-\mathrm{H} \cdots \mathrm{N}$-Wasserstoffbrückenbindungen liefert drei Trimerbanden (siehe Raman-Spektren in Abb. A-2 in Anhang A.3. . Auch hier ist auf theoretischer Seite die Ringanordnung der Monomere im Aggregat nur unter Berücksichtigung der Dispersionskorrektur stabiler als die offenkettige Anordnung.

Es zeigt sich also, dass auch jenseits der kleinen sekundären Amide zyklische Clustergeometrien zumindest für die Gasphase in Betracht gezogen werden müssen. Deren relative Stabilität in DFT-Rechnungen kann dabei gegenüber der offenkettigen Geometrie erheblich von der Ver- 
wendung einer Dispersionskorrektur abhängen. Die durchgängige Konsistenz mit allen hier vorgestellten Ergebnissen ohne nennenswerte Einbußen in der Rechendauer zeigt eindeutig, dass eine Dispersionskorrektur bei DFT-Methoden standardmäßig verwendet werden sollte.

\subsection{Apparative Entwicklungen des curry-Jets}

Im Rahmen dieser Arbeit ist ein verbessertes Sättigermodell entwickelt worden, welches bis zu einer Temperatur von $\vartheta_{\text {sat }} \approx 100^{\circ} \mathrm{C}$ erfolgreich betrieben werden kann. Dies ermöglicht nun Messungen mit Substanzen mit einem besonders niedrigen Dampfdruck, wie z. B. das in dieser Arbeit untersuchte $N$-Phenylformamid oder Imidazol (siehe Tab. 3-1). Die heizbaren Leitungen wurden erneuert und durch den Ersatz des x-Hahns durch zwei Kugelhähne die zulässige Betriebstemperatur von $80^{\circ} \mathrm{C}$ auf $210^{\circ} \mathrm{C}$ angehoben (begrenzt durch die Eigenschaften der verwendeten O-Ringe). Problematisch ist der sehr hohe Substanzverbrauch in diesem Sättiger, was aufgrund der benötigten Messdauer für ein gutes Signal-zu-Rauschen-Verhältnis eine Vermessung von geringen Substanzmengen erschwert.

Für die Zukunft könnten folgende Verbesserungen eingebracht werden:

- Einbau eines heizbaren Bodens analog dem Deckel im heizbaren Sättiger, so dass der Sättiger mit Boden-, Deckel und Manteltemperatur drei unabhängig regelbare Temperaturen hat. Dies sollte sowohl die langen Aufheizzeiten als auch die Diskrepanz zwischen gemessener und tatsächlicher Sättigertemperatur reduzieren.

- Ersatz der Magnetventile am Reservoir zur Einstellung des Stagnationsdrucks durch einen (digitalen) Druckminderer oder Mass-Flow-Controller, um eine wirklich kontinuierliche Expansion zu ermöglichen. Dies würde das eruptive Aufwirbeln der Substanz insbesondere in den vorgeschalteten kühlbaren Sättigern beenden. Es gäbe zudem einen konstanten Hintergrunddruck während der Expansion und ein definiert einstellbaren Stagnationsdruck.

- Der Druckmesskopf zur Bestimmung des Stagnationsdrucks sollte in der Nähe der VakuumDurchführung der Substanzleitung, also hinter dem heizbaren Sättiger anstatt im Reservoir positioniert werden, da durch die enge Rohrleitung und insbesondere im Fall des Betriebes des heizbaren Sättigers mit zwei Fritten und einem Rückschlagventil der wahre Stagnationsdruck an der Düse unterhalb dem gemessenen im Reservoir liegen wird.

- Zur vollständigen Untersuchung des Expansionsprofils bietet sich eine Ausstattung der Düsenhalterung mit absolut positionierbaren Aktuatoren für die $x$ - und $z$-Richtung an. Mit einem Skript an den Software-Schnittstellen der Kamera und der Aktuatorensteuerung ließe sich so die Expansion automatisch vermessen.

- Um cis-cis-Dimere von Amiden spektral sichtbar zu machen oder die Isomerisierungskinetik zu untersuchen, wäre die Entwicklung eines Düsenkörpers mit einer Durchflusskammer für einen immobilisierten Katalysator zur Isomerisierung direkt vor der Düse denkbar. 
- Der verbliebene Wellschlauch zwischen Vakuumdurchführung und Düsenkörper sollte durch eine heizbare, flexible Alternative mit geringerer Innenoberfläche ersetzt werden, um Substanzablagerungen bei geheizten Experimenten zu vermeiden.

Im Rahmen dieser Arbeit wurde die komplette Auswertungsroutine für alle curry-Jet-Spektren von MatLAB auf die nicht-proprietäre und quelloffene Programmiersprache Python 3 portiert. Das dabei geschaffene Modul madras ermöglicht eine modulare Zusammenstellung des Skripts je nach gewünschter Auswertung. Zudem ist durch die Programmstruktur eine unkomplizierte Erweiterung um neue Funktionen möglich. Hier sind z. B. eine automatisierte Linienzuordnung in den Kalibrierspektren (siehe Arbeit von M. Gawrilow) oder eine Option zur Glättung der Spektren sinnvolle Weiterentwicklungen. 


\section{A Anhang}

\section{A.1 Publikationen}

Teile dieser Arbeit wurden im Vorfeld veröffentlicht:

- Forsting, Thomas; Gottschalk, Hannes C.; Hartwig, Beppo; Mons, Michel; Suhm, Martin A. (2017): Correcting the record: the dimers and trimers of trans-N-methylacetamide. In: Phys. Chem. Chem. Phys. 19 (17), 10727-10737.

(DOI: 10.1039/C6CP07989J)

- Forsting, Thomas; Zischang, Julia; Suhm, Martin A.; Eckhoff, Marco; Schröder, Benjamin; Mata, Ricardo A. (2019): Strained hydrogen bonding in imidazole trimer. A combined infrared, Raman, and theory study. In: Phys. Chem. Chem. Phys. 21 (11), 5989-5998. (DOI: $10.1039 /$ c9cp00399a)

- Forsting, Thomas; Suhm, Martin (2019): Curry-Jet SETUP. figshare. Dataset. (DOI: 10.6084/m9.figshare.6395840.v1)

- Suhm, Martin; Forsting, Thomas; Mons, Michel; Hartwig, Beppo; Gottschalk, Hannes Christian (2016): ttt.png. figshare. Dataset.

(DOI: 10.6084/m9.figshare.4233110.v1)

\section{A.2 Verwendete Chemikalien}

In Tab. A-1 findet sich eine Liste der verwendeten Gase und vermessenen Chemikalien. Alle Daten beziehen sich auf die Angaben im Datenblatt des Herstellers.

Tab. A-1. Verwendete Chemikalien

\begin{tabular}{lllllll}
\hline Kürzel & Langname & CAS-Nr. & Hersteller & Produktnummer & Charge & Reinheit \\
\hline NMF & N-Methylformamid & $123-39-7$ & Sigma Aldrich & 473936-100ML & MKBV5064V & $99 \%$ \\
NMA & $N$-Methylacetamid & $79-16-3$ & Sigma-Aldrich & M26305-500G & BCBR7263V & $\geq 99 \%$ \\
NMP & $N$-Methylpropionamid & $1187-58-2$ & TCl & M0488-25ML & - & $>99.0 \%$ \\
gBL & $\gamma$-Butyrolactam & $616-45-5$ & Sigma Aldrich & P7,437-0 & S56167; S38821-118 & $99 \%$ \\
NPhF & N-Phenylformamid & $103-70-8$ & Sigma Aldrich & $13,332-9$ & S46925 & $99 \%$ \\
Im & Imidazol & $288-32-4$ & abcr & AB109053 & 1115039 & $99 \%$ \\
He & Helium & $7440-59-7$ & Linde & & - & $99.996 \%(4.6)$ \\
$N_{2}$ & Stickstoff & $7727-37-9$ & Air Liquide & AlphaGaz N & - & $99.999 \%(5.0)$ \\
\hline
\end{tabular}




\section{A.3 Imidazol}

Dieser Abschnitt ist eine Zusammenstellung einiger Ergebnisse zur Clusterbildung von Imidazol aus Forsting et al. [57], die in der Diskussion in Kapitel 9 eingebracht werden.

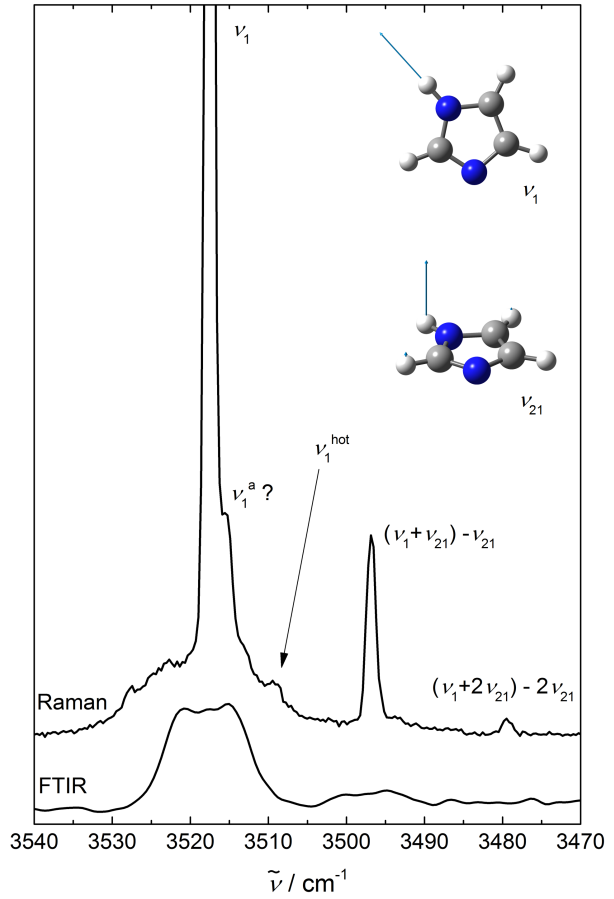

Abb. A-1. Raman-Jet-Spektren (Düsentemperatur $\left.\vartheta_{D}=120^{\circ} \mathrm{C}\right)$ und FTIR-Jet-Spektren $\left(\vartheta_{D}=150 / 130^{\circ} \mathrm{C}\right)$ der $\mathrm{NH}$-Streckschwingung des Imidazolmonomers. Bandenzuordnung für die Fundamentale $(\mathrm{M})$ und verschiedene heiBe Banden. Vorläufige Zuordnungen sind mit ? markiert. Dargestellte Strukturformeln und Normalmoden basieren auf B3LYP-D3(BJ)/def2-TZVP-Rechungen. (aus: Forsting etal. [57], publiziert unter der CC BY 3.0 Lizenz [https://creativecommons.org/licenses/by/3.0/]) 


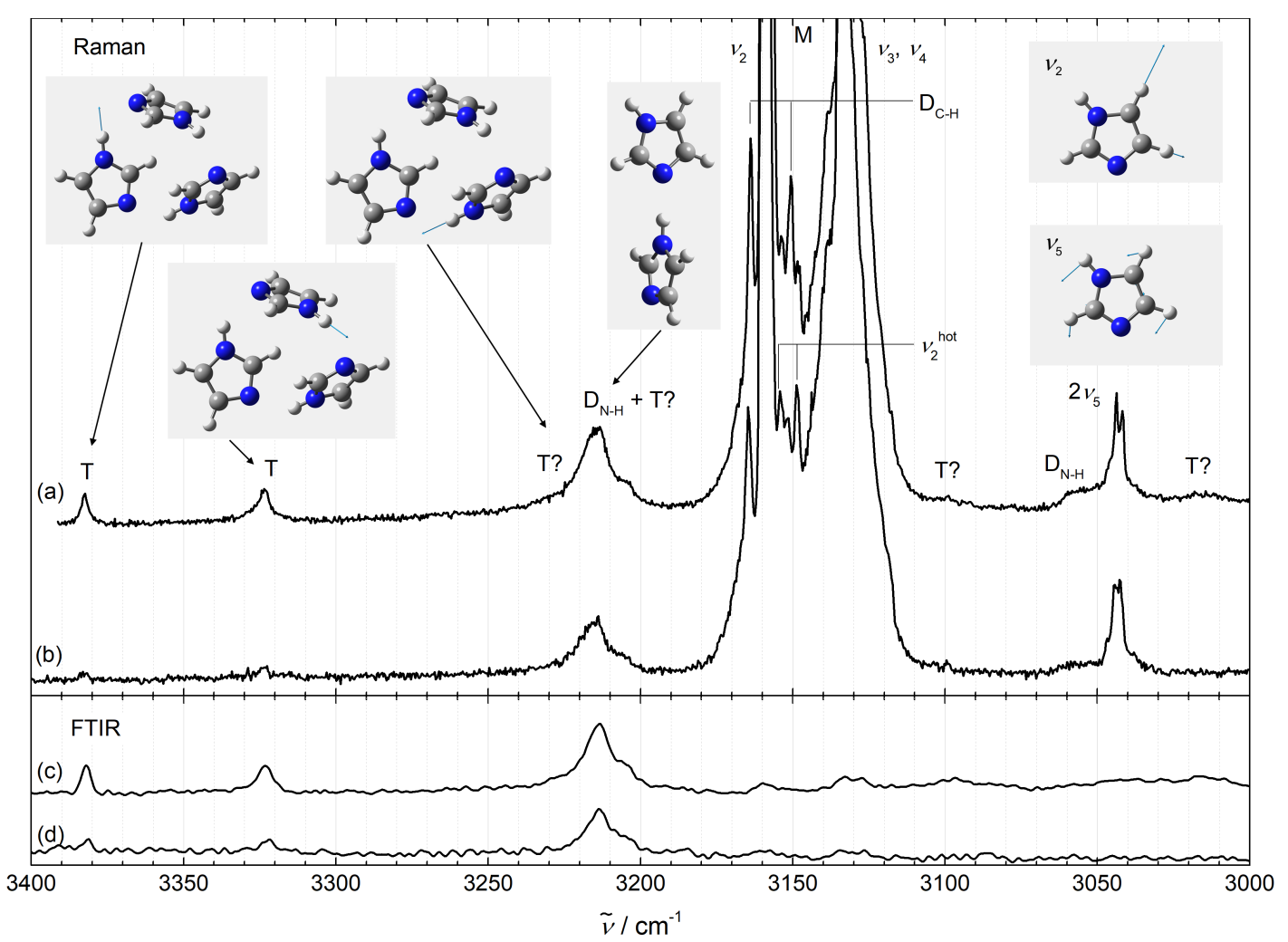

Abb. A-2. Raman-Jet-Spektren (a,b; Düsentemperatur $\vartheta_{\mathrm{D}}=120^{\circ} \mathrm{C}$ ) und FTIR-Jet-Spektren $\left(\mathrm{c}, \mathrm{d} ; \vartheta_{\mathrm{D}}=150 / 130^{\circ} \mathrm{C}\right.$ ) von Imidazol mit Bandenzuordnungen für das Monomer (M), Dimer (D) und Trimer ( $T$ ). Vorläufige Zuordnungen sind mit ? markiert. Die jeweils oberen Spektren $(\mathrm{a}, \mathrm{c})$ sind höher konzentriert. Skaliert auf ähnliche Intensitäten der $\mathrm{CH}$ Bande bei $3159 \mathrm{~cm}^{-1}$ innerhalb der Methode und auf die NH-Streckschwingungsbande bei $3214 \mathrm{~cm}^{-1}$ zwischen den Methoden. Dargestellte Geometrien und Normalmoden entsprechen den jeweils stabilsten Strukturen basierend auf B3LYP-D3(BJ)/def2-TZVP-Rechnungen. (adaptiert aus: Forsting et al. [57], publiziert unter der CC BY 3.0 Lizenz [https://creativecommons.org/licenses/by/3.0/]) 
Tab. A-2. Experimentelle Anharmonizitätskonstanten $x_{1, k}$ und $x_{2, k}$ sowie relative Bandintensitäten $I_{k} / I_{i}$ für das Imidazol Monomer aus Raman-Jet-Spektren (Abb. A-2) basierend auf dem Vergleich mit VPT2-Rechnungen (B3LYP-D3(BJ)/def2TZVP - VPT2-Ergebnisse sind mit VTZ-Basis gerechnet). (aus: Forsting et al. [57], publiziert unter der CC BY 3.0 Lizenz [https://creativecommons.org/licenses/by/3.0/])

\begin{tabular}{cccccc}
\hline$i$ & $k$ & $\tilde{v}_{k}^{\mathrm{VPT} 2} / \mathrm{cm}^{-1}$ & $x_{i, k}^{\mathrm{VPT} 2} / \mathrm{cm}^{-1}$ & $x_{i, k}^{\mathrm{exp}} / \mathrm{cm}^{-1}$ & $I_{k} / I_{i}{ }^{\mathrm{a}}$ \\
\hline 1 & 12 & 1071 & -8 & -9 & $0.01^{\mathrm{b}}$ \\
1 & 21 & $531^{*}$ & -20 & -21 & $0.14^{\mathrm{b}}$ \\
1 & $2 \times 21$ & $1087^{*}$ & -40 & -39 & $0.01^{\mathrm{b}}$ \\
\hline 2 & 13 & 1056 & -8 & -6 & $\geq 0.005^{\mathrm{c}}$ \\
2 & 16 & 869 & -5 & -6 & $\geq 0.01^{\mathrm{c}}$ \\
2 & 18 & 731 & -13 & -11 &
\end{tabular}

a : höher als der entsprechende Wert der harmonischen Rechnung. ${ }^{\mathrm{b}}$ : aus dem Spektrum in Abb. A-1, ${ }^{\mathrm{c}}$ : Angegebene Untergrenzen basieren auf Spektrum (a) in Abb. A-2 unter Berücksichtigung von Bandenüberlappungen. 


\section{A.4 Komponenten des curry-Jets}

Die folgende Tabelle (A-3) listet alle relevanten Bauteile und Komponenten des curry-Jets mit Hersteller, genauer Typenbezeichnung und den wichtigsten Eigenschaften auf. 
Tab. A-3. curry-Jet: Bauteile und Komponenten. Die fettgedruckten Abkürzungen beziehen sich auf die Abb. 3-1 und 3-3. Die genannten Spezifikationen entsprechen den Herstellerangaben.

\begin{tabular}{|c|c|c|c|c|}
\hline Bauteil / Komponente & Hersteller & Bezeichner / Artikelnummer & Spezifikationen & Referenzen \\
\hline Laser & Spectra-Physics & Millennia eV & $\begin{array}{l}\text { Dauerstrich } 532 \mathrm{~nm} ; \max .20 / 25 \mathrm{~W} \text {; Strahldurchmesser @ 1/ } \mathrm{e}^{2}: 2.3 \mathrm{~mm} \text {; Divergenz: } \\
2 \theta<(0.5 \pm 10 \%) \mathrm{mrad}\end{array}$ & Handbuch \\
\hline Laserkühler & Solid State & Thermorack 401 & $\begin{array}{l}\text { Kühltemperatur: } 5-45^{\circ} \mathrm{C} \text {; Reproduzierbarkeit: } \pm 0.05^{\circ} \mathrm{CUmgebungstemperatur:} 5- \\
40^{\circ} \mathrm{C} \text {; Kühlmittel: demin. } \mathrm{H}_{2} \mathrm{O}\end{array}$ & Handbuch \\
\hline CCD-Kamera & Princeton Instruments & PyLoN 400B & $\begin{array}{l}1340 \times 400 \text { Pixel }\left(26.8 \times 8.0 \mathrm{~mm}^{2}\right) \text {; Pixelgröße: } 20 \times 20 \mu \mathrm{m}^{2} ; \text { Dunkelrauschen: } 0.3 e^{-} / \mathrm{px} / \mathrm{h} \\
@-120^{\circ} \mathrm{C} ; T_{\min }=-120^{\circ} \mathrm{C} \text {; Ausleserauschen: } 3 e^{-} @ 100 \mathrm{kHz} ; 16 \text { bit; } 40 \mathrm{~mm} \text { Shutter }\end{array}$ & SpecSheet \\
\hline Vakuumkammer & Tech. Werkst. des Inst. & - & $80 \times 40 \times 60 \mathrm{~cm}^{3}(\mathrm{BHT}) ;$ Leckrate: $0.015 \mathrm{mbar} \mathrm{h}^{-1} ;$ schwarz lackiert & [112] \\
\hline $\begin{array}{l}\text { Reservoir } \\
\text { Kalibrierlampen }\end{array}$ & $\begin{array}{l}\text { Tech. Werkst. des Inst. } \\
\text { L.O.T. Oriel GmbH }\end{array}$ & - & Volumen: $4.7 \mathrm{~L}$; max. 3.0 bar; $10-25^{\circ} \mathrm{C}$ & 31,327 \\
\hline Mikroaktuatoren & $\begin{array}{l}\text { Newport } \\
\text { Newport }\end{array}$ & $\begin{array}{l}\text { CMA-25CC } \\
\text { LTA-HS }\end{array}$ & $\begin{array}{l}\text { Gleichstrommotor; Mindestschrittweite: }<0.5 \mu \mathrm{m} \text {; Verstellweg: } 25 \mathrm{~mm} \\
\text { Gleichstrommotor, Mindestschrittweite: } 0.1 \mu \mathrm{m} \text {; Auflösung: } 0.035 \mu \mathrm{m}\end{array}$ & $31]$ \\
\hline \multicolumn{5}{|l|}{ Vakuumpumpen } \\
\hline Drehschieberpumpe & Dr.-Ing. K. Busch GmbH & UNO $101 \mathrm{~S}$ & $100 \mathrm{~m}^{3} / \mathrm{h}$ & [31] [ [ \\
\hline & Pfeiffer Vacuum & Okta 500 AM - PP W32 100 B & $560 \mathrm{~m}^{3} / \mathrm{h} @ 50 \mathrm{~Hz}$ & (Datenblatt) \\
\hline Walzkolbenpumpen & Pfeiffer Vacuum & WKP 250 AM - PP W22 100 A & $270 \mathrm{~m}^{3} / \mathrm{h} @ 50 \mathrm{~Hz}$ & (Handbuch) \\
\hline \multicolumn{5}{|l|}{ heizbares System } \\
\hline Magnetventil MV3 & Norgren & 8572100.9402 & stromlos gesperrt; max. $200^{\circ} \mathrm{C}$ Fluidtemperatur ; max. 16 bar & (Katalog) \\
\hline Rückschlagventil RV & Swagelok & SS-6C-MM-1/3 & Edelstahl + FKM; Öffnungsdruck: 0.03 bar; max. $190^{\circ} \mathrm{C}$ bei $150 \mathrm{bar}$ & (Katalog) \\
\hline Kugelhähne & Swagelok & SS-42GXS6MM & 3/2; max. $148^{\circ} \mathrm{C} ; \max .70$ bar & (Katalog) \\
\hline PID-Regler & Schneider Electric & Eurotherm 3216 & Kalibriergenauigkeit: $< \pm 0.25 \%$ der Anzeige; CJC-Genauigkeit: ${ }^{1)}< \pm 1^{\circ} \mathrm{C}$ & (Manual) \\
\hline & RS Components & $\mathrm{E} 6 \mathrm{C}$ & Genauigkeit: $0.1 \%$ des Messbereichs, CJC-Genauigkeit: $\pm 1{ }^{\circ} \mathrm{C}$ & (Datenblatt) \\
\hline \multicolumn{5}{|l|}{ optische Komponenten } \\
\hline Monochromator & GCA/McPherson & Model 2051 & $\begin{array}{l}f=1000 \mathrm{~mm} ; f / 8.7 \text {; Gitter: } 1200 / \mathrm{mm}, 110 \mathrm{~mm}^{2} ; \text { Auflösung: } 0.01 \mathrm{~nm}(10 \mu \mathrm{m} \text { Eintrittss- } \\
\text { palt, } 1200 / \mathrm{mm} \text { Gitter, } 1 \text {. Ordnung) }\end{array}$ & [31], (Manual) \\
\hline Kantenfilter KF & LOT-Quantum Design GmbH & REFUS532-25 USLR & OD $6.0 @ 532.0 \mathrm{~nm}, T>90 \% @ \lambda>533.7 \mathrm{~nm}$ & 112] \\
\hline Achromat L3 & Edmund Optics & L45-354 & $\varnothing=50 \mathrm{~mm} ; f=350 \mathrm{~mm} ; f / 7$ & [31] \\
\hline Kameraobjektiv 0 & Nikon & Nikkor Al-S 50/1.2 & $f=50 \mathrm{~mm} ; f / 1.2 ; 46^{\circ}$ Bildwinkel & (Handbuch) \\
\hline Fokuslinse F1 & Qioptiq & G312300322 & $\begin{array}{l}\text { plankonvex, } f=50 \mathrm{~mm}, \varnothing=22.4 \mathrm{~mm} \text {; ARB2-Beschichtung: } R<0.5 \%, \text { AOI }=0^{\circ}, \lambda= \\
450-700 \mathrm{~nm} \text {, Zerstörschwelle: } 30 \mathrm{~J} / \mathrm{cm}^{2}(11 \mathrm{~ns}, 1064 \mathrm{~nm}, 10 \mathrm{~Hz})\end{array}$ & (Katalog) \\
\hline Eingangsfenster F1 & Qioptiq & G390112322 & Quarz; hohe Parallelität und Planität $(\lambda / 20) ; \varnothing=25.4 \mathrm{~mm}$; ARB2-Beschichtung (s. o.) & (Katalog) \\
\hline Ausgangsfenster F2 & Qioptiq & G390406322 & Quarz; hohe Planität $(\lambda / 10) ; \varnothing=63 \mathrm{~mm}$; ARB2-Beschichtung (s. o.) & (Katalog) \\
\hline Laserspiegel M & Vision Lasertechnik & OP04LM-R25-532-45 & 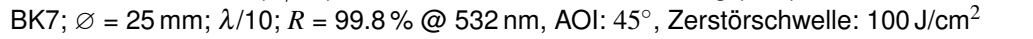 & (Katalog) \\
\hline
\end{tabular}




\section{A.5 Input-Zeilen der GAUSSIAN-Rechnungen}

In Tab. A-4 finden sich die verwendeten Input-Zeilen der Gaussian-Rechnungen für die durchgeführten DFT-Rechnungen.

Tab. A-4. Gaussian-Input-Zeilen der durchgeführten DFT-Rechnungen (Erklärung der verwendeten Methoden, siehe Abschnitt 3.3.

\begin{tabular}{ll}
\hline Methode / Basissatz & GAUSSIAN Input-Zeile \\
\hline B3LYP-D3(BJ)/aVTZ & \# b3lyp aug-cc-pvtz empiricaldispersion=gd3bj opt=tight int=SuperFine \\
& freq=Raman \\
B3LYP-D3(BJ)/def2TZVP & \# b3lyp def2TZVP empiricaldispersion=gd3bj opt=tight int=SuperFine \\
Ereq=Raman & $\begin{array}{l}\text { \# b3lyp cC-pvtz empiricaldispersion=gd3bj opt=tight int=SuperFine } \\
\text { freq=Anharm }\end{array}$ \\
\hline
\end{tabular}

\section{A.6 Spektrenverzeichnis}

\section{A.6.1 Raman-Spektren}

Das in Tab. A-6 gezeigte Verzeichnis liefert Details zu den Messbedingungen der in den jeweiligen Abbildungen gezeigten Raman-Spektren. Alle Raman-Spekten wurden am curry-Jet aufgenommen (siehe Abschnitt 3.1). Die Bezeichnung der Messungen erfolgt nach dem Datum des Messtages im JJMMTT-Format und am gleichen Messtag mit Kleinbuchstaben in alphabetischer Reihenfolge.

Falls nicht alle Einzelscans einer Messung in der weiteren Auswertung berücksichtigt wurden (weil z. B. die Expansionsbedingungen noch nicht konstant waren oder die Substanz im Sättiger vorzeitig zur Neige ging), sind die verwendeten Scans in eckigen Klammern hinter dem Namen der Messung vermerkt.

Während der Entstehung der Messdaten unterlag der curry-Jet einer ganzen Reihe von apparativen Änderungen. Die jeweils aktuelle Konfiguration der Apparatur ist in Spalte „Version“ angegeben und die entsprechenden Details der Version finden sich in Tab. A-5, Insbesondere wurden auch zwei unterschiedlich leistungsstarke Laser der gleichen Baureihe verwendet (Spectra-Physics Millennia eV 20 W (Mi20) und 25 W (Mi25)). Dabei lag während einiger Messungen die maximal mögliche Leistung $P_{\mathrm{L}}$, ist unterhalb der nominellen Laserleistung $P_{\mathrm{L}, \text { nom }}$. In diesem Fall findet sich in der Spalte ${ }_{2} P_{\mathrm{L}} / \mathrm{W}$ “ die Darstellung $\left[P_{\mathrm{L}, \text { ist }}\right] /\left[P_{\mathrm{L}}\right.$, soll $]$. Die Angabe der Sättigerversionen „V3“ oder „V4“ bezieht sich auf die Beschreibung der Sättigerhistorie in Abschnitt 3.1.2. Details zu den genannten Komponenten finden sich in Tab. A-3. Alle Messungen wurden mit Helium als Trägergas durchgeführt. Die Spaltöffnung $d_{\text {Spalt }}$ am Monochromator beträgt $75 \mu \mathrm{m}$, wenn nicht anders erwähnt.

Die Auswertung fand mit den Curry.finning()- und Curry.smooth()-Methoden der aktuellsten Version der madras-Klasse statt (siehe hierzu die Abschnitte 3.1.5 und A.12). Anderweitige Auswertungen sind gesondert vermerkt. 
Tab. A-5. Unterschiedliche Konfigurationen des curry-Jet-Aufbaus mit Versionsnummern.

\begin{tabular}{llllll}
\hline Version & Laser & Sättiger & CCD-Kamera & Heizleitung & Sonstiges \\
\hline 1a & Mi25 & V3 + Messingkern & Spec-10 & X-Hahn + Analogregler & $\lambda / 2$-Plättchen \\
1b & Mi25 & V3 + Messingkern & Spec-10 & X-Hahn + Analogregler & - \\
2 & Mi25 & V3 + Edelstahlkern & Pylon & X-Hahn + Analogregler & - \\
3 & Mi25 & V3 + Edelstahlkern + Eurotherm & Pylon & X-Hahn + Analogregler & - \\
4 & Mi25 & V3 + Edelstahlkern + Eurotherm + & Pylon & X-Hahn + Analogregler & - \\
& & Füllkörper & & & - \\
5 & Mi25 & V4 & Pylon & X-Hahn + Analogregler & - \\
$6 a$ & Mi20 & V4 & Pylon & X-Hahn + Analogregler & - \\
$6 b$ & Mi20 & V4 & Pylon & X-Hahn + Eurotherm & - \\
$6 \mathrm{c}$ & Mi20 & V4 + Kugelhähne & Pylon & X-Hahn + Eurotherm & - \\
7 & Mi25 & V4 + Kugelhähne & Pylon & X-Hahn + Eurotherm & Pylon \\
8 & Mi25 & V4 + Kugelhähne & 3/2-Kugelhähne + Festverrohrung + & - \\
& & & Eurotherm & \\
9 & Mi25 & V4 + Kugelhähne + Deckelheizung & Pylon & 3/2-Kugelhähne + Festverrohrung + & - \\
& & & Eurotherm & \\
\end{tabular}


Tab. A-6. Raman-Spektrenverzeichnis: Messbedingungen der den dargestellten Spektren zugrundeliegenden Messungen am curry-Jet.

\begin{tabular}{|c|c|c|c|c|c|c|c|c|c|c|}
\hline Abbildung & Messung [Scans] & Messzeit & $d_{\mathbf{D}} / \mathbf{m m}$ & $\vartheta_{\mathrm{H}} /{ }^{\circ} \mathbf{C}$ & $\vartheta_{\mathbf{S}} 1^{\circ} \mathbf{C}$ & $p_{\mathbf{S}} /$ bar & $\lambda_{\mathrm{MC}} / \mathrm{nm}$ & $P_{\mathbf{L}} / \mathrm{W}$ & Version & Bemerkungen \\
\hline 3-4: $\bigcirc$ & 180611_a & $20 \times 0.005 \mathrm{~s}$ & - & - & - & - & 532.0 & 25 & 9 & $d_{\text {Spalt }}=10 \mu \mathrm{m}$; ohne Kantenfilter \\
\hline 3-4: $\triangle$ & 180611_b & $20 \times 0.005 \mathrm{~s}$ & - & - & - & - & 532.0 & 25 & 9 & ohne Kantenfilter \\
\hline $3-5$ & 180418_a1-180418_a28 & $60 \times 0.005 \mathrm{~s}$ & - & - & - & - & 532.0 & $0.1-25$ & 9 & ohne Kantenfilter \\
\hline 4-2: links & 160504_c & $10 \times 180 s$ & 1 & 130 & 45 & 0.35 & 646.0 & 25 & 3 & - \\
\hline \multirow{2}{*}{ 4-2. Mitte } & 160503_b & $10 \times 180 \mathrm{~s}$ & 1 & 130 & 42.5 & 0.35 & 571.0 & 25 & 3 & - \\
\hline & 160503_c & $10 \times 180 s$ & 1 & 130 & 42.5 & 0.35 & 586.0 & 25 & 3 & - \\
\hline 4-2: rechts & 160503_a & $10 \times 180 s$ & 1 & 130 & 42.5 & 0.35 & 556.0 & 25 & 3 & - \\
\hline 4-3: oben & 160526_g & $10 \times 360 s$ & 1 & 190 & 50 & 0.35 & 646.0 & 25 & 3 & - \\
\hline 4-3: unten & 160504_c & $10 \times 180 s$ & 1 & 130 & 45 & 0.35 & 646.0 & 25 & 3 & - \\
\hline \multirow[t]{2}{*}{ 4-4 } & 160503_b & $10 \times 180 s$ & 1 & 130 & 42.5 & 0.35 & 571.0 & 25 & 3 & - \\
\hline & 160503_c & $10 \times 180 s$ & 1 & 130 & 42.5 & 0.35 & 586.0 & 25 & 3 & - \\
\hline 4-5: oben & 160526_a & $10 \times 360 s$ & 1 & 190 & 50 & 0.35 & 556.0 & 25 & 3 & \\
\hline 4-5; unten & 160503_a & $10 \times 180 s$ & 1 & 130 & 42.5 & 0.35 & 556.0 & 25 & 3 & - \\
\hline A-8: schwarz & 160422_b[2-16] & $15 \times 420 s$ & 1 & 115 & 40 & 0.35 & 649.0 & 25 & 2 & - \\
\hline A-8: blau & 160510_a[7-16] & $10 \times 420 s$ & 1 & 170 & 45 & 0.35 & 649.0 & 25 & 3 & - \\
\hline A-8: orange & 160511_b[6,8-12] & $6 \times 420 s$ & 1 & 190 & 45 & 0.35 & 649.0 & 25 & 3 & - \\
\hline 4-8: schwarz & $160530 \_b[7-16]$ & $10 \times 420 s$ & 1 & 130 & 50 & 0.35 & 556.0 & 25 & 4 & - \\
\hline 4-8: rot & $160530 \_c$ & $16 \times 420 s$ & 1 & 150 & 50 & 0.35 & 556.0 & 25 & 4 & - \\
\hline \multirow[t]{2}{*}{ 4-8: grün } & 160531_a[10-16] & $7 \times 420 s$ & 1 & 170 & 50 & 0.35 & 556.0 & 25 & 4 & - \\
\hline & 160531_b[2] & $1 \times 420 s$ & 1 & 170 & 50 & 0.35 & 556.0 & 25 & 4 & - \\
\hline 4-8: blau & 160531_c & $16 \times 420 s$ & 1 & 190 & 50 & 0.35 & 556.0 & 25 & 4 & - \\
\hline 4-10: (a) & 161103_d[3-9] & $7 \times 420 s$ & 3 & 180 & 60 & 0.45 & 578.0 & 25 & 5 & - \\
\hline 4-10; (b) & 161103 & $16 \times 420 s$ & 3 & 100 & 60 & 0.45 & 578.0 & 25 & 5 & - \\
\hline 4-10: (c) & 161103_b & $16 \times 420 s$ & 3 & 90 & 60 & 0.45 & 578.0 & 25 & 5 & - \\
\hline 4-10: (d) & $161102 \_b[1-15]$ & $15 \times 420 s$ & 3 & 80 & 60 & 0.45 & 578.0 & 25 & 5 & - \\
\hline 4-10; (e) & 161101_b[2-16] & $15 \times 420 s$ & 3 & 70 & 60 & 0.45 & 578.0 & 25 & 5 & - \\
\hline 4-11: (a) & 160617_a & $16 \times 504 \mathrm{~s}$ & 3 & 170 & 60 & 0.45 & 647.0 & 20 & 4 & - \\
\hline
\end{tabular}


Tab. A-6. Raman-Spektrenverzeichnis: Messbedingungen der den dargestellten Spektren zugrundeliegenden Messungen am curry-Jet (Fortsetzung).

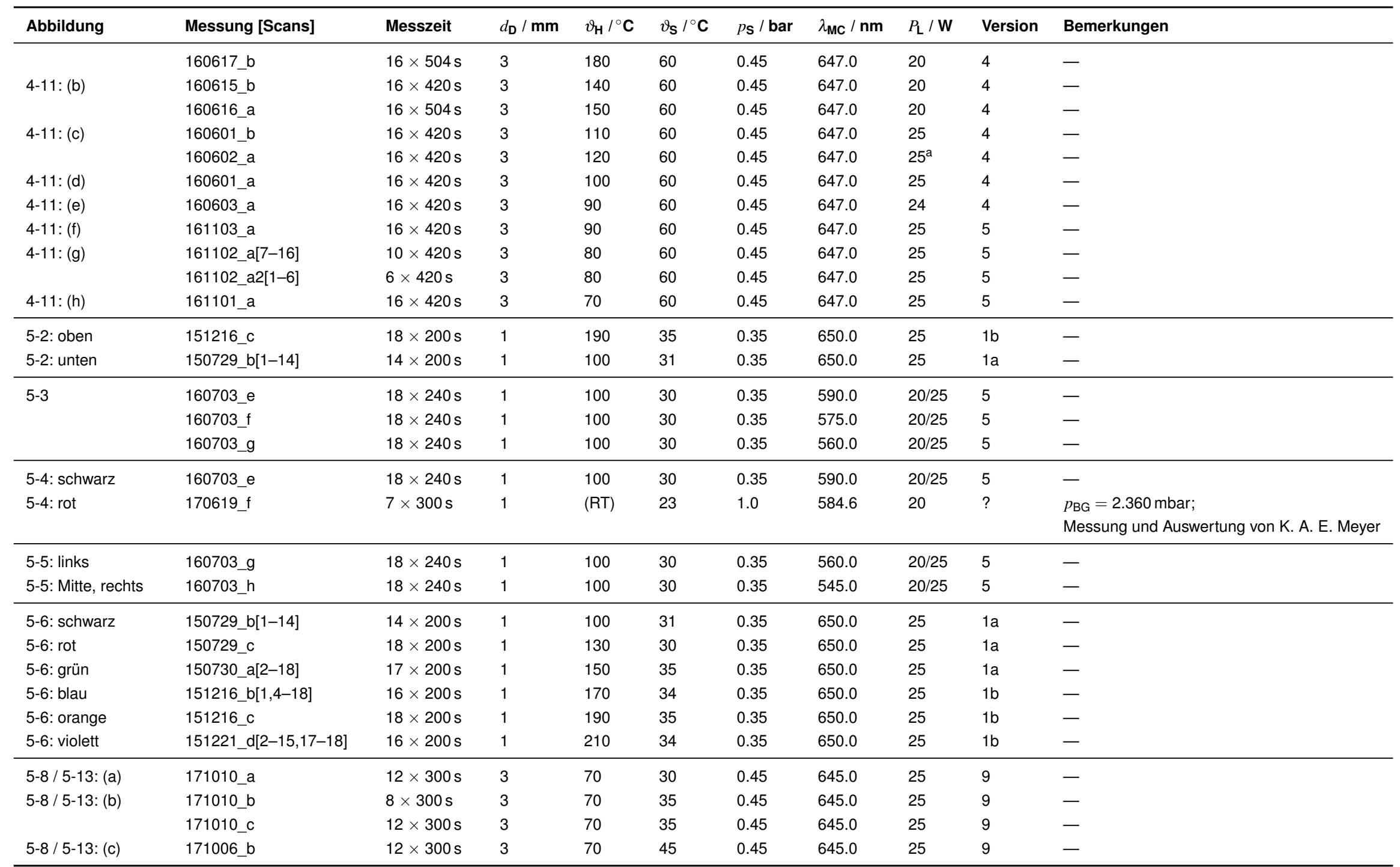

Fortsetzung auf der folgenden Seite ... 
Tab. A-6. Raman-Spektrenverzeichnis: Messbedingungen der den dargestellten Spektren zugrundeliegenden Messungen am curry-Jet (Fortsetzung).

\begin{tabular}{|c|c|c|c|c|c|c|c|c|c|c|}
\hline Abbildung & Messung [Scans] & Messzeit & $d_{\mathbf{D}} / \mathbf{m m}$ & $\vartheta_{\mathrm{H}} /{ }^{\circ} \mathbf{C}$ & $\vartheta_{\mathrm{S}} /{ }^{\circ} \mathbf{C}$ & $p_{\mathbf{S}} /$ bar & $\lambda_{\mathrm{MC}} / \mathrm{nm}$ & $P_{\mathrm{L}} / \mathrm{W}$ & Version & Bemerkungen \\
\hline & 171006_c & $12 \times 300 s$ & 3 & 70 & 45 & 0.45 & 645.0 & 25 & 9 & - \\
\hline \multirow[t]{2}{*}{$5-8$ 5-13: (d) } & 171006_d[2-6] & $5 \times 300 s$ & 3 & 70 & 50 & 0.45 & 645.0 & 25 & 9 & - \\
\hline & 171006_e & $12 \times 300 s$ & 3 & 70 & 50 & 0.45 & 645.0 & 25 & 9 & - \\
\hline \multirow{2}{*}{$5-8$ 5-13: (e) } & $171010 \_d[4-9]$ & $6 \times 300 s$ & 3 & 70 & 55 & 0.45 & 645.0 & 25 & 9 & - \\
\hline & 171010_e & $12 \times 300 s$ & 3 & 70 & 55 & 0.45 & 645.0 & 25 & 9 & - \\
\hline \begin{tabular}{|l|l:}
$5-9$ & $5-10$ \\
schwarz
\end{tabular} & 171010_a & $12 \times 300 s$ & 3 & 70 & 30 & 0.45 & 645.0 & 25 & 9 & - \\
\hline 5-9 $5-10$ rot & 171010_b & $8 \times 300 s$ & 3 & 70 & 35 & 0.45 & 645.0 & 25 & 9 & - \\
\hline \multirow[t]{2}{*}{ 5-9 5-10; grün } & 171006_b & $12 \times 300 s$ & 3 & 70 & 45 & 0.45 & 645.0 & 25 & 9 & - \\
\hline & 171006_c & $12 \times 300 s$ & 3 & 70 & 45 & 0.45 & 645.0 & 25 & 9 & - \\
\hline \multirow[t]{2}{*}{$5-9$ 5-10 blau } & $171006 \_d[2-6]$ & $5 \times 300 s$ & 3 & 70 & 50 & 0.45 & 645.0 & 25 & 9 & - \\
\hline & 171006_e & $12 \times 300 s$ & 3 & 70 & 50 & 0.45 & 645.0 & 25 & 9 & - \\
\hline \multirow{2}{*}{ 5-9 5-10; cyan } & 171010_d[4-9] & $6 \times 300 s$ & 3 & 70 & 55 & 0.45 & 645.0 & 25 & 9 & - \\
\hline & 171010_e & $12 \times 300 s$ & 3 & 70 & 55 & 0.45 & 645.0 & 25 & 9 & - \\
\hline 5-11, 5-14 rot & 170127_q & $16 \times 504 s$ & 3 & 70 & 30 & 0.45 & 578.0 & 20 & $6 a$ & - \\
\hline 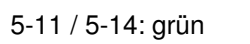 & 170203_b & $16 \times 504 s$ & 3 & 70 & 35 & 0.45 & 578.0 & 20 & $6 a$ & - \\
\hline $6-2$ & 170305_d[3-12] & $10 \times 600 \mathrm{~s}$ & 1 & 130 & 40 & 0.35 & 644.0 & 20 & $6 b$ & - \\
\hline 6-3: (a) & 170305_a & $12 \times 600 s$ & 1 & 80 & 40 & 0.35 & 644.0 & 20 & $6 b$ & - \\
\hline 6-3: (b) & 170305_b & $12 \times 600 s$ & 1 & 90 & 40 & 0.35 & 644.0 & 20 & $6 b$ & - \\
\hline 6-3: (c) & $170305 \_c$ & $12 \times 600 s$ & 1 & 110 & 40 & 0.35 & 644.0 & 20 & $6 b$ & - \\
\hline 6-3: (d) & 170305_d[3-12] & $10 \times 600 s$ & 1 & 130 & 40 & 0.35 & 644.0 & 20 & $6 b$ & - \\
\hline 6-5: $6-7:$ (a) & 170305_d[3-12] & $10 \times 600 s$ & 1 & 130 & 40 & 0.35 & 644.0 & 20 & $6 b$ & - \\
\hline 6-5 (b-7: (b) & $171124 \_a$ & $14 \times 300 s$ & 3 & 120 & 65 & 0.65 & 643.5 & 25 & 9 & - \\
\hline 6-5 6-7: (c) & 171124_e & $12 \times 300 s$ & 3 & 120 & 80 & 0.65 & 643.5 & 25 & 9 & - \\
\hline $\begin{array}{ll}6-5 & \text { 6-7: (d) } \\
\end{array}$ & 171124_h & $12 \times 300 s$ & 3 & 120 & 95 & 0.65 & 643.5 & 25 & 9 & - \\
\hline $8-4:$ (a) & 171027_a[4-12] & $9 \times 300 s$ & 3 & 100 & 60 & 0.45 & 578.0 & 25 & 9 & - \\
\hline \multirow[t]{2}{*}{ 8-4: (b) } & $171027 \_b[2-8]$ & $7 \times 300 s$ & 3 & 100 & $60-70$ & 0.45 & 578.0 & 25 & 9 & - \\
\hline & 171027 _c & $12 \times 300 s$ & 3 & 100 & 70 & 0.45 & 578.0 & 25 & 9 & - \\
\hline 8-4:(c) & $171027 \_\mathrm{d}[3-11]$ & $9 \times 300 s$ & 3 & 100 & $70-80$ & 0.45 & 578.0 & 25 & 9 & - \\
\hline
\end{tabular}


Tab. A-6. Raman-Spektrenverzeichnis: Messbedingungen der den dargestellten Spektren zugrundeliegenden Messungen am curry-Jet (Fortsetzung).

\begin{tabular}{|c|c|c|c|c|c|c|c|c|c|c|}
\hline Abbildung & Messung [Scans] & Messzeit & $d_{\mathbf{D}} / \mathbf{m m}$ & $\vartheta_{\mathbf{H}} /{ }^{\circ} \mathbf{C}$ & $\vartheta_{\mathbf{S}} /{ }^{\circ} \mathbf{C}$ & $p_{\mathbf{S}} /$ bar & $\lambda_{\mathrm{MC}} / \mathrm{nm}$ & $P_{\mathrm{L}} / \mathbf{W}$ & Version & Bemerkungen \\
\hline & 171027_e[1-11] & $11 \times 300 s$ & 3 & 100 & 80 & 0.45 & 578.0 & 25 & 9 & - \\
\hline \multirow[t]{3}{*}{ A-2: (a) } & 170928_d[7-12] & $6 \times 300 \mathrm{~s}$ & 3 & 120 & $80-95$ & 1.0 & 638.7 & 25 & 9 & - \\
\hline & 170928_e & $12 \times 300 \mathrm{~s}$ & 3 & 120 & 95 & 1.0 & 638.7 & 25 & 9 & - \\
\hline & 170928_f[1-7] & $7 \times 300 s$ & 3 & 120 & 95-100 & 1.0 & 638.7 & 25 & 9 & - \\
\hline \multirow[t]{3}{*}{ A-2: (b) } & 170907_c & $7 \times 300 \mathrm{~s}$ & 3 & 120 & 80 & 1.0 & 640.2 & 25 & 9 & - \\
\hline & 170907_d & $4 \times 300 \mathrm{~s}$ & 3 & 120 & $80-85$ & 1.0 & 640.2 & 25 & 9 & - \\
\hline & 170907_e[1-2] & $2 \times 300 s$ & 3 & 120 & 85 & 1.0 & 640.2 & 25 & 9 & - \\
\hline
\end{tabular}

a: Laserleistung nicht über die gesamte Messung stabil. 


\section{A.6.2 FTIR-Spektren}

Tab. A-7. Unterschiedliche Konfigurationen des FTIR-Spektrometers im filet-Jet. Die Scan-Geschwindigkeit liegt für alle Varianten bei $80 \mathrm{kHz}$.

\begin{tabular}{lllll}
\hline & I & II & III & IV \\
\hline Lichtquelle: $^{\mathrm{a}}$ & $\mathrm{W} 150$ & Globar & Globar & Globar \\
Blende: & $3.5 \mathrm{~mm}$ & $7.5 \mathrm{~mm}$ & $4.0 \mathrm{~mm}$ & $4.0 \mathrm{~mm}$ \\
Optiken: & $\mathrm{CaF}_{2}$ & $\mathrm{KBr}$ & $\mathrm{KBr}$ & $\mathrm{KBr}$ \\
Filter:b $_{\text {Detektor: }}^{\mathrm{F} 13 \mathrm{a}}$ & $\mathrm{F} 2$ & $\mathrm{~F} 4$ & $\mathrm{~F} 18 \mathrm{a}$ \\
Vorwiderstand: & $2.2 \mathrm{k} \Omega$ & $3.1 \mathrm{k} \Omega$ & $1.0 \mathrm{k} \Omega, 2.0 \mathrm{~V}$ & $1.0 \mathrm{k} \Omega, 2.0 \mathrm{~V}$ \\
\hline
\end{tabular}

a: W150 - Wolfram-Glühlampe (150 W); ${ }^{\text {b }: ~ g r u p p e n i n t e r n e ~ B e z e i c h n u n g ; ~}{ }^{\mathrm{c}}$ : neuer MCT-Sandwich-Detektor. 
Tab. A-8. FTIR-Spektrenverzeichnis: Messbedingungen der den dargestellten Spektren zugrundeliegenden Messungen am filet-Jet. Die Einzelscans der unterschiedlichen Messungen wurden untereinander verglichen und in „Datensätzen“ zusammengefasst. Alle Messungen wurden mit Helium als Trägergas und in einer der drei Konfigurationen I, II oder

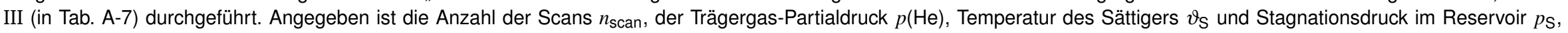
sowie die Pumpzeit zwischen zwei Expansionen $\left(t_{\mathrm{vac}}\right)$. Zudem ist für Gasphasenmessungen ein Hintergrunddruck $p_{\mathrm{BG}}$ der Messkammer angegeben (fehlt für Jet-Messungen). Die Datensätze I-M wurden von K. Meyer aufgenommen und sind in ihrem Laborjournal „September 2016 - Oktober 2017“ dokumentiert.

\begin{tabular}{|c|c|c|c|c|c|c|c|c|}
\hline Messung [Scans] & $n_{\text {scan }}$ & $p(\mathrm{He}) / \mathrm{bar}$ & $\vartheta_{\mathrm{S}} /{ }^{\circ} \mathbf{C}$ & $p_{\mathbf{S}} /$ bar & $p_{\mathrm{BG}} / \mathrm{bar}$ & $t_{\mathrm{vac}} / \mathbf{s}$ & Konfiguration & Bemerkungen \\
\hline \multicolumn{9}{|c|}{ Datensatz A (285 Scans): } \\
\hline 161026_a-d & 285 & 1.6 & 22 & - & 0.23 & - & III & Gasphase \\
\hline \multicolumn{9}{|c|}{ Datensatz $B_{1}(1000$ Scans): } \\
\hline 161020_a-i & 1000 & 1.6 & 22 & 0.75 & - & 30 & III & - \\
\hline \multicolumn{9}{|c|}{ Datensatz $B_{2}$ (800 Scans): } \\
\hline 161021_a-h & 800 & 1.6 & 24 & 0.75 & - & 30 & III & - \\
\hline \multicolumn{9}{|c|}{ Datensatz C (2600 Scans): } \\
\hline 160720_a & 250 & 1.6 & 15 & 0.75 & - & 20 & I & - \\
\hline 160721_c & 400 & 1.6 & 15 & 0.75 & - & 20 & I & - \\
\hline 160722_a & 350 & 1.6 & 15 & 0.75 & - & 20 & I & - \\
\hline 160825_d & 1000 & 1.6 & 15 & 0.75 & - & 20 & I & - \\
\hline 160826_a[000-599] & 600 & 1.6 & 20 & 0.75 & - & 20 & I & - \\
\hline \multicolumn{9}{|c|}{ Datensatz D (800 Scans): } \\
\hline 160719_a & 25 & 1.6 & 20 & 0.75 & - & 20 & I & - \\
\hline 160719_b & 275 & 1.6 & 20 & 0.75 & - & 20 & I & - \\
\hline 160721_a & 100 & 1.6 & 20 & 0.75 & - & 20 & I & - \\
\hline 160721_b & 400 & 1.6 & 20 & 0.75 & - & 20 & I & - \\
\hline \multicolumn{9}{|c|}{ Datensatz E (750 Scans): } \\
\hline 160719_c & 250 & 1.6 & 25 & 0.75 & - & 20 & I & - \\
\hline 160720_b & 300 & 1.6 & 25 & 0.75 & - & 20 & I & - \\
\hline 160720_c & 200 & 1.6 & 25 & 0.75 & - & 20 & I & - \\
\hline \multicolumn{9}{|c|}{ Datensatz F (2000 Scans): } \\
\hline 160929_f, h, i, k & 400 & 1.25 & 20 & 0.75 & - & 25 & II & - \\
\hline 160929_m + n & 400 & 1.25 & 22 & 0.75 & - & 25 & II & - \\
\hline 160929_t[000-299] & 300 & 1.25 & 24 & 0.75 & - & 25 & II & - \\
\hline 160930_a, c, d & 300 & 1.25 & 24 & 0.75 & - & 25 & II & - \\
\hline 161005_f, g, i, j, l, m & 600 & 1.25 & 22 & 0.75 & - & 25 & II & - \\
\hline
\end{tabular}

Fortsetzung auf der folgenden Seite ... 
Tab. A-8. FTIR-Spektrenverzeichnis: Messbedingungen der den dargestellten Spektren zugrundeliegenden Messungen am filet-Jet (Fortsetzung).

\begin{tabular}{|c|c|c|c|c|c|c|c|c|}
\hline Messung [Scans] & $n_{\text {scan }}$ & $p(\mathrm{He}) / \mathrm{bar}$ & $\vartheta_{\mathbf{S}} /{ }^{\circ} \mathbf{C}$ & $p_{\mathbf{S}} /$ bar & $p_{\mathrm{BG}} /$ bar & $t_{\mathrm{vac}} / \mathrm{s}$ & Konfiguration & Bemerkungen \\
\hline \multicolumn{9}{|c|}{ Datensatz G (285 Scans): } \\
\hline 161026_f, g, i & 285 & 1.6 & 22 & - & 0.23 & - & I & Gasphase \\
\hline \multicolumn{9}{|c|}{ Datensatz H (570 Scans): } \\
\hline 160929_o, q & 285 & 1.25 & 22 & - & 0.23 & - & II & Gasphase \\
\hline 160929_s, u & 285 & 1.25 & 24 & - & 0.23 & - & II & Gasphase \\
\hline \multicolumn{9}{|c|}{ Datensatz I (100 Scans): } \\
\hline $170330 \_f$ & 100 & 1.6 & 25 & 0.75 & - & 20 & IV & - \\
\hline \multicolumn{9}{|c|}{ Datensatz J (50 Scans): } \\
\hline 170330_p & 50 & 1.6 & 25 & 0.75 & - & 20 & IV & - \\
\hline \multicolumn{9}{|c|}{ Datensatz K (100 Scans): } \\
\hline 170330_u & 100 & 1.6 & 25 & 0.75 & - & 20 & IV & - \\
\hline \multicolumn{9}{|c|}{ Datensatz L (50 Scans): } \\
\hline 170331_c & 50 & 1.6 & 15 & 0.75 & - & 20 & IV & - \\
\hline \multicolumn{9}{|c|}{ Datensatz M (100 Scans): } \\
\hline 170331_l+m & 100 & 1.6 & 15 & 0.75 & - & 20 & IV & - \\
\hline
\end{tabular}




\section{A.6.3 NMR-Spektren}

Von allen verwendeten Substanzen (bis auf NMP) wurden ${ }^{1} \mathrm{H}$-NMR- und ${ }^{13} \mathrm{C}-\mathrm{NMR}$-Spektren aufgenommen. Als Lösungsmittel (LSM) wurde durchgehend Chloroform- $d\left(\mathrm{CDCl}_{3}\right)$ eingesetzt und gegen Tetramethylsilan (TMS) als Standard gemessen.

\section{${ }^{1} \mathrm{H}-\mathrm{NMR}$}

Die ${ }^{1}$-NMR-Spektren (Abb. A-3, A-4, A-5, A-6, A-7) zeigen für alle Substanzen eine hohe Reinheit. Es finden sich lediglich Spuren von Wasser $\left(\mathrm{H}_{2} \mathrm{O}\right)$, auf Grund der hygroskopischen Eigenschaften von Amiden und auch Imidazol.

Bei NMF und NPhF lassen sich cis (c)- und trans ( $t$ )-Form unterscheiden. Das cis-Konformer des NMA ist zwar erkennbar (mit * gekennzeichnet), aber das Signal zu niedrig für eine Auswertung.

Die Zuordnung und Positionen der Banden aller Amide ist in sehr guter Übereinstimmung mit Abraham et. al.[328]. Die Zuordnung des Imidazolspektrums erfolgte mit Hilfe der „Spectral Database for Organic Compounds (SDBS)“[329].

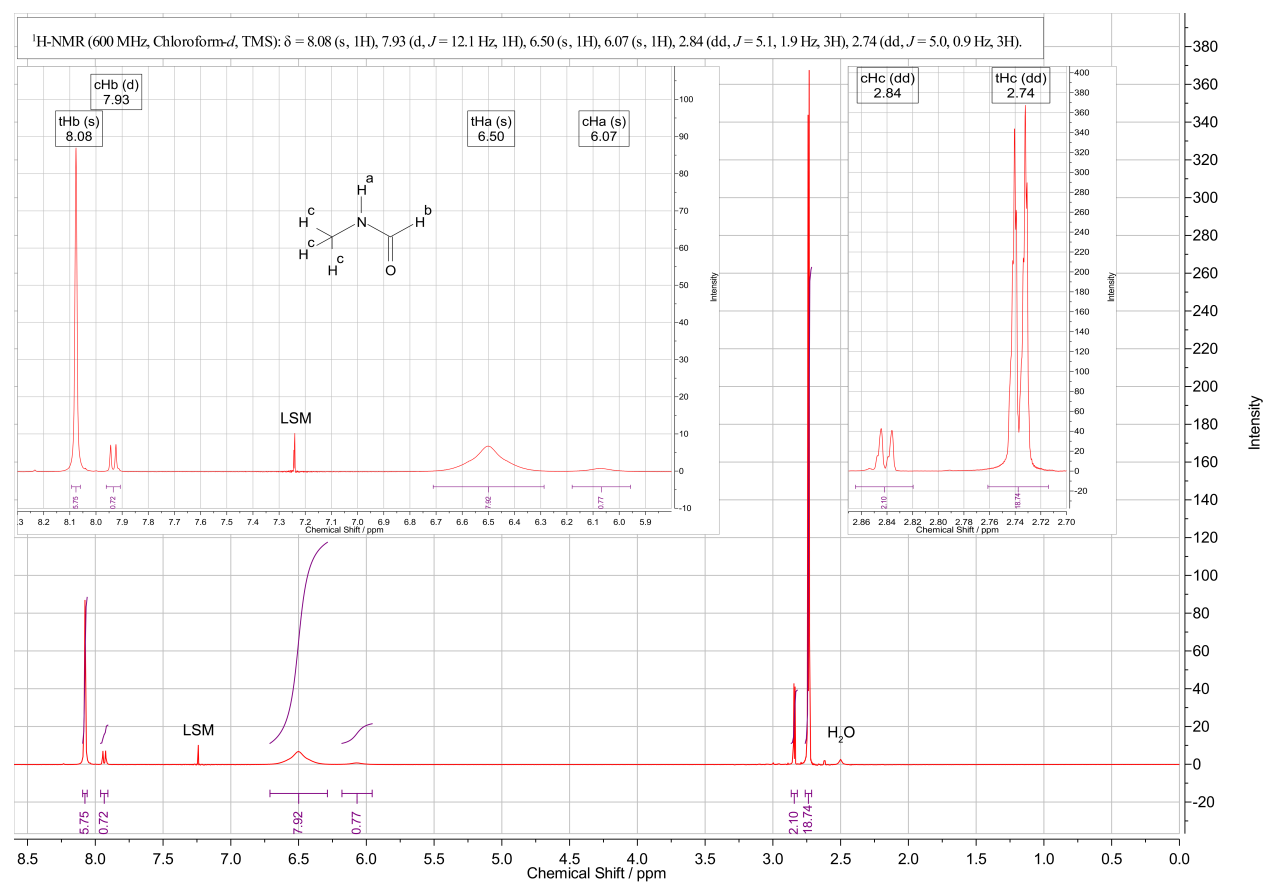

Abb. A-3. ${ }^{1} \mathrm{H}-\mathrm{NMR}$-Spektrum von N-Methylformamid. cis $(c)$ - und trans $(t)$-Konformere unterscheidbar (siehe Präfix der Zuordnung). 


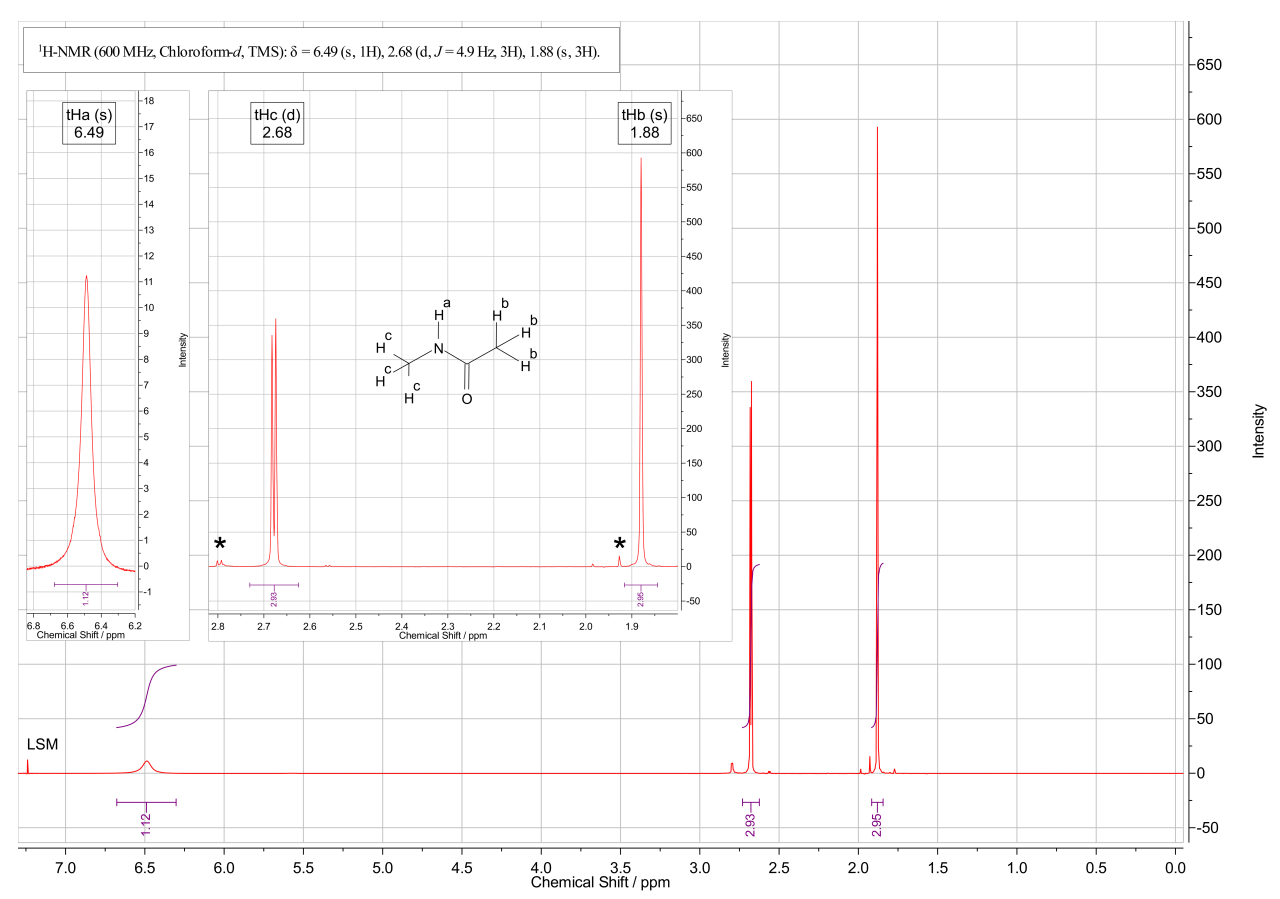

Abb. A-4. ${ }^{1} \mathrm{H}-\mathrm{NMR}-S p e k t r u m$ von $N$-Methylacetamid. cis $(c)$ - und trans $(t)$-Konformere erkennbar $\left({ }^{*}\right)$.

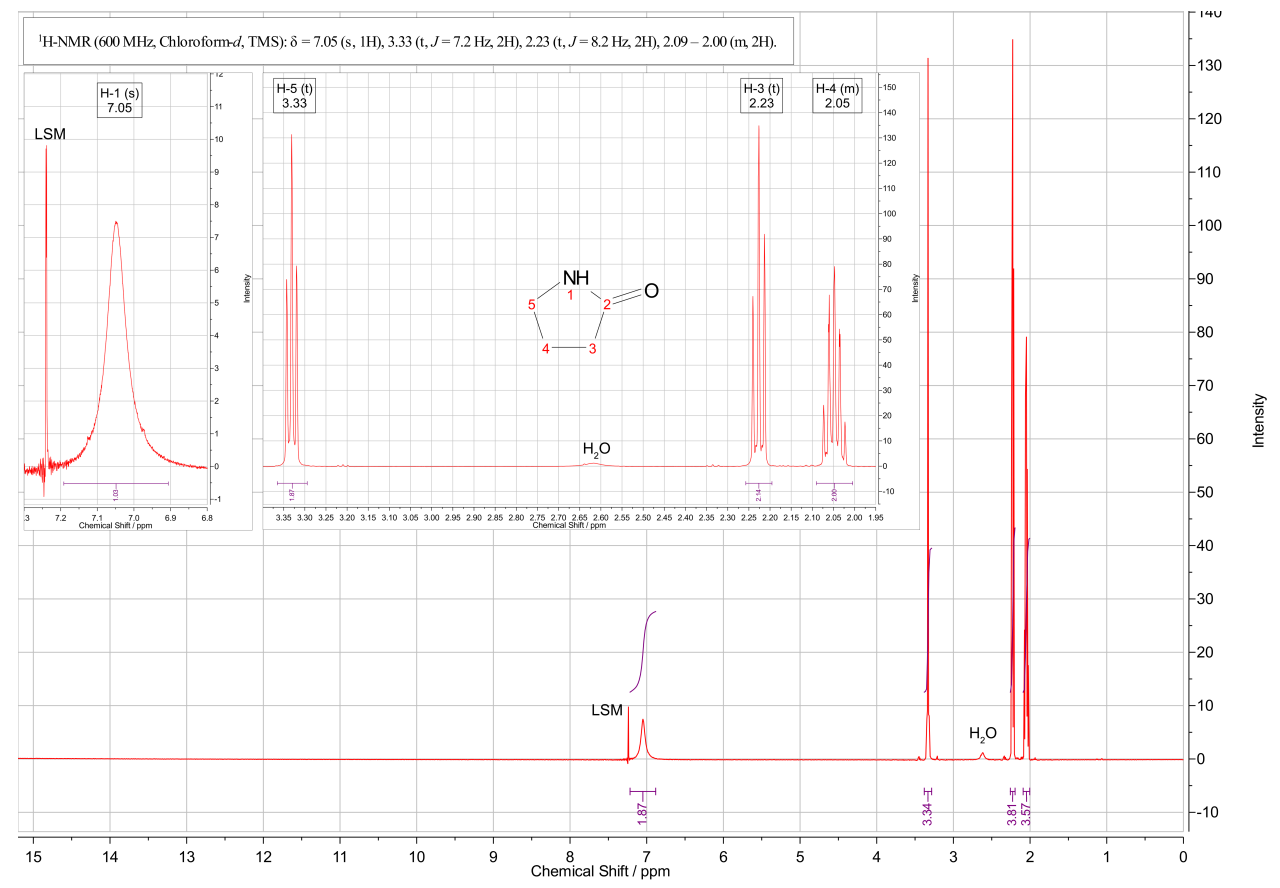

Abb. A-5. ${ }^{1} \mathrm{H}$-NMR-Spektrum von $\gamma$-Butyrolactam. 


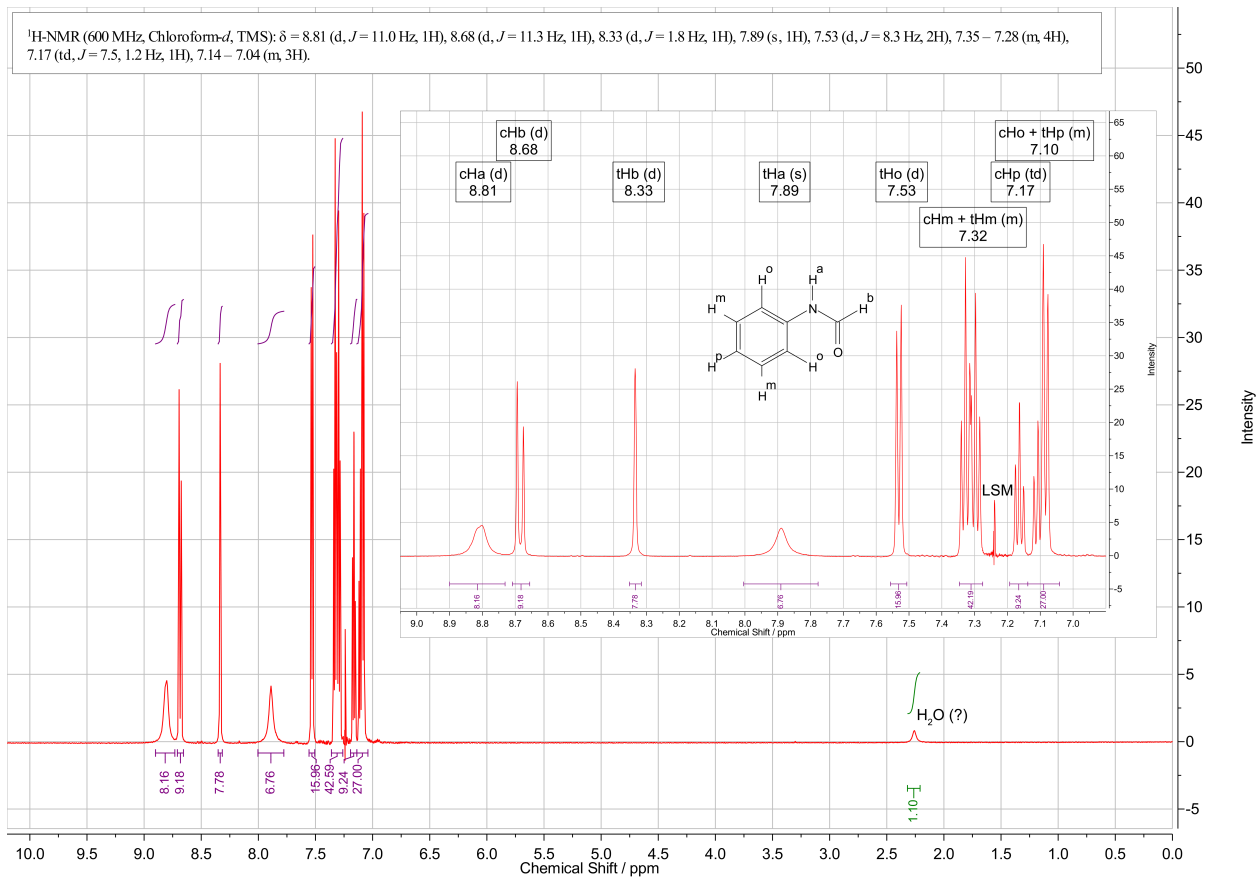

Abb. A-6. ${ }^{1} \mathrm{H}$-NMR-Spektrum von $N$-Phenylformamid.cis $(c)$ - und trans $(t)$-Konformere unterscheidbar (siehe Präfix der Zuordnung). Die Zuordnung des Peaks bei 2.25 ppm ist vorläufig, denn er entspricht nicht der typischen Verschiebung von Wasser.

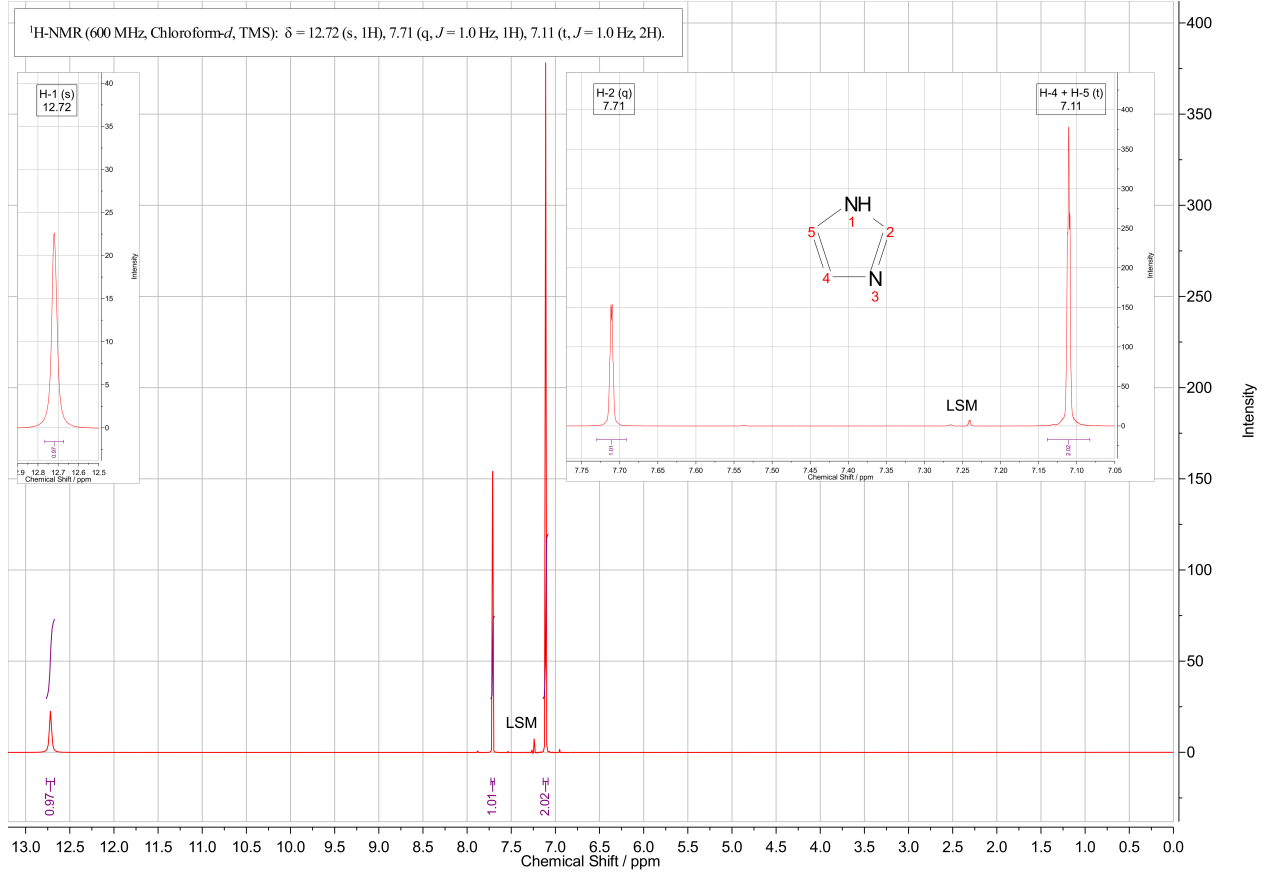

Abb. A-7. ${ }^{1} \mathrm{H}$-NMR-Spektrum von Imidazol. 


\section{${ }^{13} \mathrm{C}-\mathrm{NMR}$}

Ergänzend aufgenommene ${ }^{13} \mathrm{C}$-NMR-Spektren ergeben folgende Verschiebungen samt Zuordnung (Indizes: q - quartär, o - ortho, $\mathrm{m}$ - meta, $\mathrm{p}$ - para):

NMF: ${ }^{13} \mathrm{C}-\mathrm{NMR}(101 \mathrm{MHz}$, Chloroform-d, TMS): $\delta / \mathrm{ppm}=165.28$ (cis-CH, 1C), 162.14 (trans$\mathrm{CH}, 1 \mathrm{C}), 27.82\left(\right.$ cis- $\left.\mathrm{CH}_{3}, 1 \mathrm{C}\right), 24.49$ (trans- $\mathrm{CH}_{3}, 1 \mathrm{C}$ ).

NMA: ${ }^{13} \mathrm{C}-\mathrm{NMR}\left(101 \mathrm{MHz}\right.$, Chloroform-d, TMS): $\delta / \mathrm{ppm}=171.06$ (trans- $\mathrm{C}_{\mathrm{q}}, 1 \mathrm{C}$ ), 25.98 (trans- $\mathrm{CH}_{3}, 1 \mathrm{C}$ ), 22.59 (trans $-\mathrm{CH}_{3}, 1 \mathrm{C}$ ).

gBL: ${ }^{13} \mathrm{C}-\mathrm{NMR}\left(101 \mathrm{MHz}\right.$, Chloroform-d, TMS): $\delta / \mathrm{ppm}=179.35\left(\mathrm{C}_{\mathrm{q}}, 1 \mathrm{C}\right), 42.16(\mathrm{CH}, 1 \mathrm{C})$, $30.00(\mathrm{CH}, 1 \mathrm{C}), 20.51(\mathrm{CH}, 1 \mathrm{C})$.

NPhF: ${ }^{13} \mathrm{C}-\mathrm{NMR}(101 \mathrm{MHz}$, Chloroform-d, TMS): $\delta / \mathrm{ppm}=162.97(\mathrm{CH}, 1 \mathrm{C}), 159.43(\mathrm{CH}$, 1C), $136.91\left(\mathrm{C}_{\mathrm{q}}, 1 \mathrm{C}\right), 136.72\left(\mathrm{C}_{\mathrm{q}}, 1 \mathrm{C}\right), 129.60(\mathrm{CH}, 2 \mathrm{C}), 128.94(\mathrm{CH}, 2 \mathrm{C}), 125.13(\mathrm{CH}, 1 \mathrm{C})$, 124.66 (CH, 1C), $120.02(\mathrm{CH}, 2 \mathrm{C}), 118.66$ (CH, 2C).

Im: ${ }^{13} \mathrm{C}-\mathrm{NMR}(101 \mathrm{MHz}$, Chloroform-d, TMS): $\delta / \mathrm{ppm}=135.09(\mathrm{CH}, 1 \mathrm{C}), 121.67(\mathrm{CH}, 2 \mathrm{C})$. 


\section{A.7 N-Methylacetamid}

\section{A.7.1 Ergänzende Abbildungen}

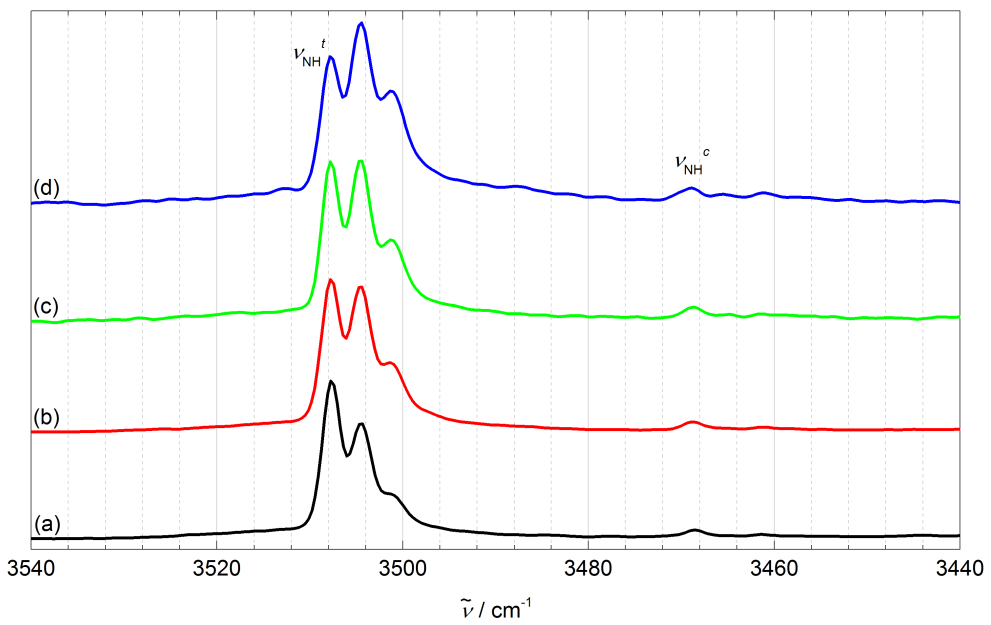

Abb. A-8. Temperaturmessreihe von NMA im NH-Streckbereich. Die Temperaturen vor der Expansion, $\vartheta_{\mathrm{H}} /{ }^{\circ} \mathrm{C}$, betragen von (a) nach (e): 115, 150, 170, 210. Die Spektren sind normiert auf $v_{\mathrm{NH}}^{t}$ bei $3508 \mathrm{~cm}^{-1}$. Dadurch ist zwar keine Temperaturabhängigkeit des cis-/trans-Verhältnisses zu erkennen, allerdings wird hier durch das relative Bandenwachstum deutlich, dass die Banden bei 3504 und $3501 \mathrm{~cm}^{-1}$ heiße Banden sind (Details zu den Messungen finden sich im Raman-Spektrenverzeichnis in Tab. A-6. 


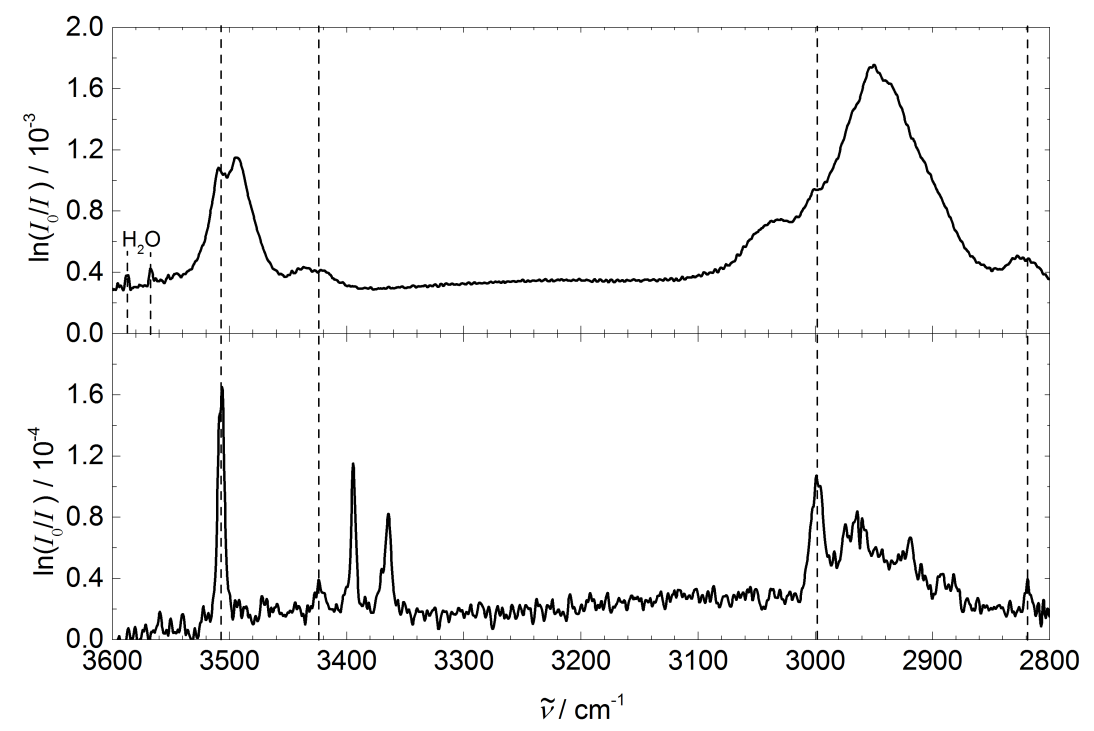

Abb. A-9. NMA: FTIR-Jet-(unten) und Gasphasenspektrum (oben) zwischen 3600 und $2800 \mathrm{~cm}^{-1}$. Die gestrichelten Linien beziehen sich auf einige Jet-Banden, die sich nur in mittelmäßiger Übereinstimmung mit den Banden in der Gasphase befinden, was möglicherweise auf die in Abschnitt 4.1 diskutierten Kombinationen mit heißen Methyltorsionsbanden zurückzuführen ist. Der in Abschnitt 4.3 besprochene Oberton der $\mathrm{C}=\mathrm{O}$-Streckschwingung bei $3425 \mathrm{~cm}^{-1}$ ist in der Gasphase klar erkennbar. Zusätzliche kleine Linien oberhalb der Amid A in der Gasphase lassen sich Wasser zuordnen und bestätigen die Kalibrierung des FTIR-Jets auf $0.2 \mathrm{~cm}^{-1}$ genau. (adaptiert aus: Forsting et al. 212. publiziert unter der CC BY 3.0 Lizenz [https://creativecommons.org/licenses/by/3.0/]) 


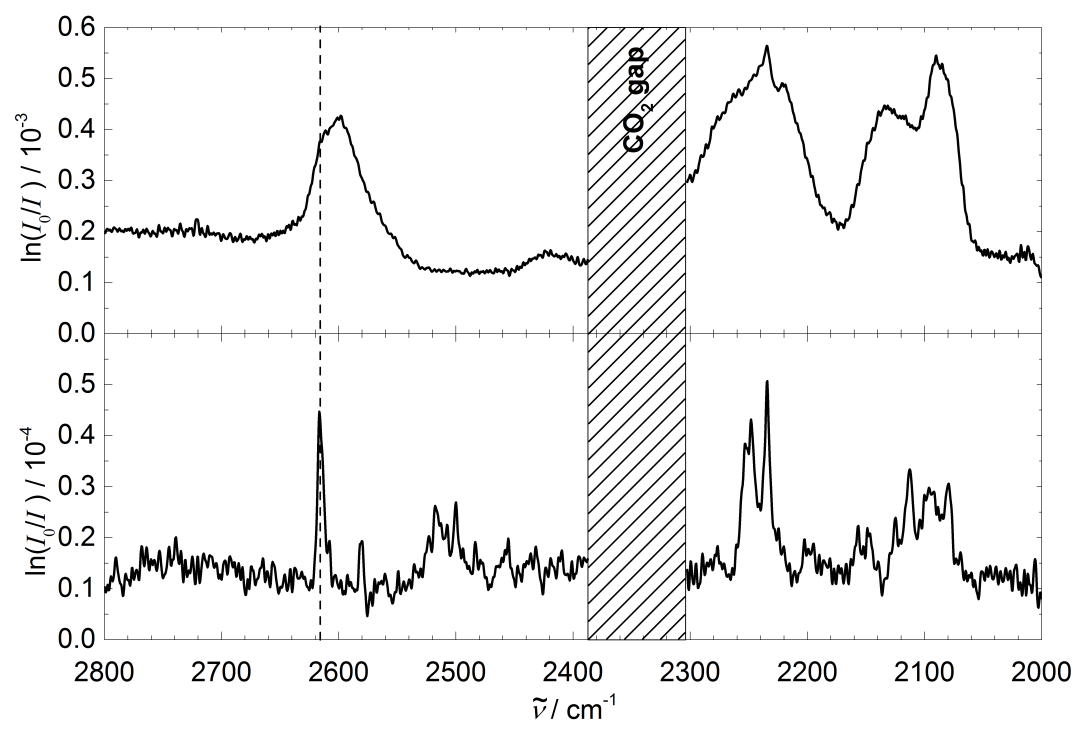

Abb. A-10. NMA-d $d_{7}$ : FTIR-Jet-(unten) und Gasphasenspektrum (oben) zwischen 2800 und $2000 \mathrm{~cm}^{-1}$. Die trans- und cis-Amid A-Banden bei 2616 bzw. $2580 \mathrm{~cm}^{-1}$ im Jet bildet in der Gasphase eine breite Bande bei ca. $2598 \mathrm{~cm}^{-1}$, woran die effektive Kühlung im Jet gut erkennbar ist. Der $t t$-Dimer Doppelpeak liegt bei 2518 und $2500 \mathrm{~cm}^{-1}$. Der CD-Streckbereich scheint beim Vergleich von Jet und Gasphase temperatursensitiv zu sein. Der als " $\mathrm{CO}_{2}$ gap“ bezeichnete Bereich ist der Absorptionsbereich der asymmetrischen Streckschwingung von $\mathrm{CO}_{2}$, die einen hohen IR Absorptionsquerschnitt besitzt. Durch Konzentrationsschwankungen von $\mathrm{CO}_{2}$ zwischen Hintergrund- und Substanzmessung enthält der Bereich eine Reihe zufälliger Signale positiver und negativer Intensität, die ausgeblendet wurden. (aus: Forsting et al. [212. publiziert unter der CC BY 3.0 Lizenz [https://creativecommons.org/licenses/by/3.0/]) 


\section{A.7.2 Tabellenwerk}


Tab. A-9. Experimentelle Banden von trans-NMA (cis-NMA) im Bereich der Amid I-III-Banden und CH-Gerüstschwingungen zwischen 1800 und $1100 \mathrm{~cm}^{-1}$.

\begin{tabular}{|c|c|c|c|c|}
\hline Methode & $\tilde{v}_{l}^{b}$ & $\tilde{v}_{\|} \mathrm{b}$ & $\tilde{v}_{\mid I I} b$ & weitere Banden \\
\hline \multicolumn{5}{|l|}{ Jet-Expansion: } \\
\hline $\operatorname{Raman}^{\mathrm{a}}$ & 1720 & - & 1258 & 1440vb, 1369, 1178, 1168 \\
\hline $\mathrm{FTIR}^{\mathrm{a}}$ & 1720 & 1534 & 1258 & $1490,1475,1451,1423,1370$ \\
\hline FTIR $[51]$ & 1722 & - & - & - \\
\hline IRMPD + VUVe 194 & 1707 & 1519 & 1248 & $1478,1415,1364$ \\
\hline \multicolumn{5}{|l|}{ Matrix: } \\
\hline$N_{2}-\operatorname{IR} 68$ & 1707 & $1511(1485)$ & $1266(1325)$ & $1445,1432,1419,1370,1168$ \\
\hline $\mathrm{N}_{2}-\mathrm{IR} 74$ & - & 1524,1511 & 1265 & - \\
\hline $\mathrm{N}_{2}-\mathrm{IR} 195$ & 1706 & - & - & - \\
\hline $\mathrm{Ar}-\mathrm{IR}[74$ & 1708 & 1523 & 1264 & - \\
\hline $\mathrm{Ar}-\mathrm{IR}[195$ & 1708 & - & - & - \\
\hline para- $\mathrm{H}_{2}-\mathrm{IR} 176$ & 1710 & 1513 & 1261 & $1473,1449,1444,1430,1420,1369$ \\
\hline \multicolumn{5}{|l|}{ Gasphase: } \\
\hline $\mathrm{FTIR}^{\mathrm{a}}$ & 1730,1715 & - & 1254 & $1534,1493,1372$ \\
\hline FTIR $^{d}$ 62] & $1718^{\mathrm{d}}$ & 1487 & 1247 & $1435,1415,1375,1160$ \\
\hline FTIR 73 & 1731,1713 & 1497 & 1257 & 1426,1377 \\
\hline FTIR 72 & 1731,1713 & 1535,1500 & 1256,1247 & - \\
\hline FTIR & 1731,1714 & 1499 & 1255 & - \\
\hline UVRR [177] & 1728 & 1500 & 1259 & - \\
\hline Präresonanz-Raman [75] & 1736 & - & - & - \\
\hline \multicolumn{5}{|l|}{ Lösung: } \\
\hline $\mathrm{CCl}_{4}-\mathrm{IR} 62$ & 1700 & 1500 & 1260 & - \\
\hline $\mathrm{CCl}_{4}-\mathrm{IR} 178$ & 1691 & - & 1300 & - \\
\hline $\mathrm{CCl}_{4}-2 \mathrm{C}$-fsVS 210 & 1690 & - & - & - \\
\hline $\mathrm{H}_{2} \mathrm{O}$ - Präresonanz-Raman 75 & 1640 & - & - & - \\
\hline $\mathrm{H}_{2} \mathrm{O}-\mathrm{IR} 81$ & 1620 & 1580 & 1315 & 1418,1378 \\
\hline $\mathrm{H}_{2} \mathrm{O}-\mathrm{IR} 260$ & 1628 & - & - & - \\
\hline $\mathrm{H}_{2} \mathrm{O}-$ UVRR 81 & - & $1581(1496)$ & 1316 & 1382 \\
\hline $\mathrm{H}_{2} \mathrm{O}-$ UVRR 82 & $1627(1623)$ & $1581(1495)$ & 1318 & - \\
\hline $\mathrm{H}_{2} \mathrm{O}-$ UVRR 35 & - & $1580(1496)$ & 1316 & - \\
\hline $\mathrm{H}_{2} \mathrm{O}-$ Raman 88 & 1646,1626 & 1584,1566 & 1313 & - \\
\hline \multicolumn{5}{|l|}{ Flüssigkeit: } \\
\hline Präresonanz-Raman 80 & 1650 & 1560 & 1305 & $1450,1415,1375,1160$ \\
\hline Präresonanz-Raman [75] & 1673 & - & - & - \\
\hline IR 69 & 1640 & 1550 & 1290 & \\
\hline IR 6263 & 1653 & 1567 & 1299 & \\
\hline FTIR |183] & 1658 & 1559 & 1296 & - \\
\hline Raman [69] & 1655 & - & 1302 & - \\
\hline Raman 88 & 1630 & 1579,1566 & 1302 & - \\
\hline
\end{tabular}

a: aus dieser Arbeit; ${ }^{\mathrm{b}}$ : () = cis-Bande; ${ }^{\mathrm{c}}: T=200^{\circ} \mathrm{C}$, diese frühen Werte weichen um ca. $-10 \mathrm{~cm}^{-1}$ gegenüber den späteren Werten in der Gasphase ab und sind nur der historischen Vollständigkeit halber gelistet; ${ }^{d}$ : kein Dublett vorhanden; ${ }^{\mathrm{e}}$ : IR multiphoton 
Tab. A-10. Experimentelle und theoretische Amid IV-Banden von NMA. Die den Berechnungen zugrunde liegenden Konformationen (Konf.) sind angegeben, soweit sie aus den jeweiligen Veröffentlichungen hervorgehen.

\begin{tabular}{|c|c|c|c|c|}
\hline Methode & trans-Amid IVa & cis-Amid IV & trans-Amid IVb & Konf. \\
\hline \multicolumn{5}{|l|}{ experimentell: } \\
\hline Jet - Raman ${ }^{a}$ & 870 & 799 & 622 & \\
\hline $\mathrm{N}_{2}$-Matrix $-\mathrm{IR}^{\mathrm{b}} 68$ & 857 & - & 619 & \\
\hline Gasphase - FTIR 62 $\left(T=200^{\circ} \mathrm{C}\right)$ & 805 & - & - & \\
\hline Gasphase - FTIR|73| & 812 & - & 626 & \\
\hline Gasphase - Resonanz-Raman 177] & - & - & 626 & \\
\hline Lösung (in $\mathrm{CCl}_{4}$ ) $-\mathrm{IR} 62$ & 881 & - & 627 & \\
\hline Lösung (in $\mathrm{H}_{2} \mathrm{O}$ ) - UVRR 81 & 881 & - & 628 & \\
\hline Lösung (in $\mathrm{H}_{2} \mathrm{O}$ ) - UVRR 82 & 886 & 822 & - & \\
\hline Lösung (in $\mathrm{H}_{2} \mathrm{O}$ ) - UVRR 35 & - & - & 632 & \\
\hline Lösung (in $\mathrm{H}_{2} \mathrm{O}$ ) - IR-Raman 88 & 883 & - & 632 & \\
\hline Flüssigkeit - Präresonanz-Raman[80] & 885 & - & 627 & \\
\hline Flüssigkeit - IR 69 & 880 & - & - & \\
\hline Flüssigkeit - IR 62 & 881 & - & 627 & \\
\hline Flüssigkeit - FTIR $1 \overline{83}$ & 874 & - & 628 & \\
\hline Flüssigkeit - Raman[69] & - & - & 627 & \\
\hline \multicolumn{5}{|l|}{ theoretisch - doppelt-harmonisch: } \\
\hline $\mathrm{HF} / 6-31 \mathrm{G} * 66,174$ & $861^{\mathrm{c}}$ & $798^{\mathrm{C}}$ & $649,639^{c, d}$ & $t_{\mathrm{as}} / c_{\mathrm{aa}}$ \\
\hline $\mathrm{MP} 2 / 6-311++\mathrm{G}^{* \star}[330]$ & 886 & - & 616 & $t_{\mathrm{as}}$ \\
\hline MP2/VTZ[186] & 878 & 823 & 630 & $t_{\mathrm{ss}} / c_{\mathrm{aa}}$ \\
\hline MP2/aVTZ[186] & 881 & 823 & 629 & $t_{\mathrm{ss}} / c_{\mathrm{aa}}$ \\
\hline MP2/VQZ 186 & 886 & - & 632 & $t_{\mathrm{ss}} / c_{\mathrm{aa}}$ \\
\hline CASPT2/VTZ[186 & 887 & - & 621 & $t_{\mathrm{ss}} / c_{\mathrm{aa}}$ \\
\hline B3LYP/6-31+G* 189 & 879 & - & 623 & $t_{\text {as }}$ \\
\hline B3LYP/6-311++G* 183 & 867 & - & 622 & $t_{\mathrm{ss}} / c_{\mathrm{aa}}$ \\
\hline B3LYP-D3(BJ)/def2TZVPa & 869 & 806 & 624 & $t_{\mathrm{ss}} / c_{\mathrm{aa}}$ \\
\hline B3LYP-D3(BJ)/aVTZa & 874 & 805 & 625 & $t_{\mathrm{as}} / c_{\mathrm{aa}}$ \\
\hline$P W 91 X C / 6-31+G^{*} 188$ & 849 & - & 607 & $t_{\mathrm{ss}} / c_{\mathrm{aa}}$ \\
\hline \multicolumn{5}{|l|}{ theoretisch - anharmonisch: ${ }^{\mathrm{e}}$} \\
\hline B3LYP/6-31+G*(3MR-PES) + VCI[189 & 869 & - & 619 & $t_{\mathrm{as}} /-$ \\
\hline B3LYP/6-31+G*(3MR-PES) + VPT2[189] & 861 & - & 614 & $t_{\text {as }} /-$ \\
\hline B3LYP-D3(BJ)/VTZ + VPT2 ${ }^{\mathrm{a}}$ & 847 [869] & - & 618 [635] & $t_{\mathrm{sS}} /-$ \\
\hline MP2/DZP(2MR-PES) + cc-VSCF $[185]^{d}$ & 891 & - & 636 & $t_{\text {as }} /-$ \\
\hline MP2/DZP(DZP pair potential) + cC-VSCF 190] & 883 & - & 715 & $-1-$ \\
\hline MP2/aVTZ(part. 3MR-PES) + VCI|191 & 886 & - & 633 & $t_{\mathrm{as}} /-$ \\
\hline
\end{tabular}

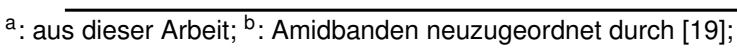

c: Werte skaliert; ${ }^{\mathrm{d}}$ : nicht eindeutig aus PED; ${ }^{\mathrm{e}}$ : später korrigiert durch [190]; ${ }^{\mathrm{e}}$ : harmonische Werte in eckigen Klammern. 
Tab. A-11. Vergleich der B3LYP-D3(BJ)-Rechnungen mit den Basissätzen def2TZVP, def2QZVP, cc-pVTZ, aug-cc-pVTZ, aug-cc-pVQZ für alle acht NMA-Konformere. Zusätzlich zu den relativen Energien ist für die Minimumskonformation des jeweiligen Basissatzes die absolute Energie $E_{\mathrm{min}}$ el in Hartree angegeben.

\begin{tabular}{|c|c|c|c|c|c|c|c|c|}
\hline & $t_{\mathrm{aa}}$ & $t_{\mathrm{as}}$ & $t_{\mathrm{sa}}$ & $t_{\mathrm{ss}}$ & $c_{\mathrm{aa}}$ & $c_{\text {as }}$ & $c_{\mathrm{sa}}$ & $c_{\mathrm{ss}}$ \\
\hline \multicolumn{9}{|c|}{ def2TZVP: $\left(E_{\mathrm{min}, \mathrm{el}}=-248.6442691 E_{\mathrm{h}}\right)$} \\
\hline$\Delta E_{\mathrm{el}} / \mathrm{kJ} \cdot \mathrm{mol}^{-1}$ & 1.83 & 0.35 & 1.43 & 0.00 & 8.68 & 11.81 & 10.10 & 14.89 \\
\hline$\Delta E_{0} / \mathrm{kJ} \cdot \mathrm{mol}^{-1}$ & - & - & - & 0.46 & 9.22 & - & - & - \\
\hline$v_{x} \min / \mathrm{cm}^{-1}$ & -91.30 & -34.09 & -90.46 & 49.78 & 59.90 & -107.37 & -96.51 & -124.92 \\
\hline \multicolumn{9}{|c|}{ def2QZVP: $\left(E_{\min , \text { el }}=-248.6633896 E_{\mathrm{h}}\right)$} \\
\hline$\Delta E_{\mathrm{el}} / \mathrm{kJ} \cdot \mathrm{mol}^{-1}$ & 1.41 & 0.00 & 1.49 & 0.06 & 8.87 & 11.99 & 10.49 & 15.17 \\
\hline$\Delta E_{0} / \mathrm{kJ} \cdot \mathrm{mol}^{-1}$ & - & 0.00 & - & 0.51 & - & - & - & - \\
\hline$v_{x} \min / \mathrm{cm}^{-1}$ & -91.30 & 28.46 & -90.92 & 31.10 & -14.55 & -106.35 & -98.54 & -124.92 \\
\hline \multicolumn{9}{|c|}{$c c-p V T Z:\left(E_{\min , \text { el }}=-248.6375021 E_{\mathrm{h}}\right)$} \\
\hline$\Delta E_{\mathrm{el}} / \mathrm{kJ} \cdot \mathrm{mol}^{-1}$ & & 0.82 & & 0.00 & 8.94 & & & \\
\hline$\Delta E_{0} / \mathrm{kJ} \cdot \mathrm{mol}^{-1}$ & & - & & 0.00 & 8.89 & & & \\
\hline$v_{x} \min / \mathrm{cm}^{-1}$ & & -58.53 & & 55.53 & 49.52 & & & \\
\hline \multicolumn{9}{|c|}{ aug-cc-pVTZ: $\left(E_{\min , \text { el }}=-248.6420289 E_{\mathrm{h}}\right)$} \\
\hline$\Delta E_{\mathrm{el}} / \mathrm{kJ} \cdot \mathrm{mol}^{-1}$ & 1.35 & 0.00 & 1.44 & 0.05 & 8.80 & 11.93 & 10.42 & 15.10 \\
\hline$\Delta E_{0} / \mathrm{kJ} \cdot \mathrm{mol}^{-1}$ & - & 0.00 & - & 0.49 & 9.02 & - & - & - \\
\hline$v_{x} \min / \mathrm{cm}^{-1}$ & -87.02 & 32.08 & -88.73 & 33.29 & 10.32 & -105.96 & -97.00 & -129.81 \\
\hline \multicolumn{9}{|c|}{ aug-cc-pVQZ: $\left(E_{\min , \text { el }}=-248.6602389 E_{\mathrm{h}}\right)$} \\
\hline$\Delta E_{\mathrm{el}} / \mathrm{kJ} \cdot \mathrm{mol}^{-1}$ & 1.36 & 0.00 & 1.46 & 0.06 & 8.90 & 12.01 & 10.48 & 15.17 \\
\hline$\Delta E_{0} / \mathrm{kJ} \cdot \mathrm{mol}^{-1}$ & - & 0.00 & - & 0.51 & - & - & - & - \\
\hline$v_{x} \min / \mathrm{cm}^{-1}$ & -88.55 & 30.45 & -90.15 & 31.49 & -12.87 & -107.30 & -96.65 & -132.88 \\
\hline
\end{tabular}

Tab. A-12. Berechnete Amid A/B-Bandenpositionen von trans-NMA (cis-NMA) mit unterschiedlichen Methoden. Die den Berechnungen zugrunde liegenden Konformationen (Konf.) sind angegeben, soweit sie aus den jeweiligen Veröffentlichungen hervorgehen.

\begin{tabular}{|c|c|c|c|c|}
\hline Methode & $\tilde{v}_{\mathrm{NH}}^{t}$ & $\tilde{v}_{\mathrm{NH}}^{c}$ & weitere Banden & Konf. \\
\hline \multicolumn{5}{|l|}{ doppelt-harmonisch: } \\
\hline $\mathrm{HF} / 6-31 \mathrm{G}^{\star \mathrm{b}} \quad 66 \quad 174$ & 3510 & 3471 & - & $t_{\mathrm{as}} / c_{\mathrm{aa}}$ \\
\hline $\mathrm{MP} 2 / 6-311++\mathrm{G}^{\star \star}[330]$ & 3689 & - & - & $t_{\mathrm{as}} /-$ \\
\hline MP2/VTZ 186 & 3697 & 3649 & - & $t_{\mathrm{ss}} / c_{\mathrm{aa}}$ \\
\hline MP2/aVTZ 186 & 3690 & 3638 & - & $t_{\mathrm{ss}} / c_{\mathrm{aa}}$ \\
\hline MP2/VQZ 186] & 3704 & - & - & $t_{\mathrm{ss}} / c_{\mathrm{aa}}$ \\
\hline CASPT2/VTZ [186] & 3706 & - & - & $t_{\mathrm{ss}} / c_{\mathrm{aa}}$ \\
\hline $\mathrm{B} 3 \mathrm{LYP} / 6-31+\mathrm{G}^{*}[181]$ & 3643 & 3603 & - & $t_{\mathrm{sa}} / c_{\mathrm{ss}}$ \\
\hline$B 3 L Y P / 6-31+G^{*}[189]$ & 3643 & - & - & $t_{\mathrm{as}} /-$ \\
\hline B3LYP/6-311++G & 3644 & - & - & $t_{\mathrm{ss}} / c_{\mathrm{aa}}$ \\
\hline B3LYP-D3(BJ)/def2TZVPa & 3638 & 3605 & - & $t_{\mathrm{ss}} / c_{\mathrm{aa}}$ \\
\hline B3LYP-D3(BJ)/aVTZ ${ }^{\mathrm{a}}$ & 3657 & 3616 & - & $t_{\mathrm{as}} / c_{\mathrm{aa}}$ \\
\hline BPW91/6-31G**331 & 3583 & - & - & $t_{\mathrm{as}} /-$ \\
\hline BPW91/6-31G(d,p) [187] & 3576 & 3544 & - & $t_{\mathrm{ss}} / c_{\mathrm{aa}}$ \\
\hline$P W 91 \times C / 6-31+G^{*} 188$ & 3543 & 3519 & - & $t_{\mathrm{ss}} / c_{\mathrm{aa}}$ \\
\hline \multicolumn{5}{|l|}{ anharmonisch: ${ }^{\mathrm{d}}$} \\
\hline BPW91/6-31G(d,p) + electrostatic DFT map 331$]$ & 3428 & - & - & $t_{\mathrm{as}} /-$ \\
\hline B3LYP/6-31+G*(3MR-PES) + VCl 189 & 3479 & - & - & $t_{\mathrm{as}} /-$ \\
\hline B3LYP/6-31+G*(3MR-PES) + VPT2 [189] & 3466 & - & - & $t_{\mathrm{as}} /-$ \\
\hline B3LYP-D3(BJ)/VTZ + VPT2 ${ }^{\mathrm{a}}$ & 3478 [3650] & - & $3436\left(2 v_{\mathrm{CO}}\right)$ & $t_{\mathrm{ss}} /-$ \\
\hline MP2/DZP(2MR-PES) + cc-VSCF $185^{c}$ & 3523 & - & - & $t_{\mathrm{as}} /-$ \\
\hline MP2/DZP(DZP pair potential) + cC-VSCF 190 & 3542 & - & - & $-1-$ \\
\hline MP2/aVTZ(part. 3MR-PES) + VCI [191] & 3544 & - & - & $t_{\mathrm{as}} /-$ \\
\hline \multicolumn{5}{|l|}{ Molekulardynamik (MD) - Simulation: } \\
\hline
\end{tabular}

\footnotetext{
${ }^{a}$ : aus dieser Arbeit; ${ }^{b}$ : Werte skaliert; ${ }^{c}$ : später korrigiert durch [190]; ${ }^{\text {d: }}$ harmonische Werte in eckigen Klammern.
} 
Tab. A-13. Berechnete Amid I-III-Bandenpositionen von trans-NMA (cis-NMA) mit unterschiedlichen Methoden. Die den Berechnungen zugrunde liegenden Konformationen (Konf.) sind angegeben, soweit sie aus den jeweiligen Veröffentlichungen hervorgehen.

\begin{tabular}{|c|c|c|c|c|}
\hline Methode / Basissatz & $\tilde{v}_{1}^{t}\left(\tilde{v}_{1}^{c}\right)$ & $\tilde{v}_{\| \mid}^{t}\left(\tilde{v}_{\|\|}^{c}\right)$ & $\tilde{v}_{\text {III }}^{t}\left(\tilde{v}_{\text {III }}^{c}\right)$ & Konf. \\
\hline \multicolumn{5}{|l|}{ doppelt-harmonisch: } \\
\hline $\mathrm{HF} / 6-31 \mathrm{G}^{* \mathrm{e}} 66,174$ & $1700(1717)$ & $1512(1481)$ & 1266 & $t_{\mathrm{as}} / c_{\mathrm{aa}}$ \\
\hline $\mathrm{HF} / 6-311++\mathrm{G}^{\star \star}$ & 1912 & - & - & $t_{\mathrm{as}}$ \\
\hline$M P 2 / 6-311++G * * 202$ & 1756 & - & - & $t_{\text {as }}$ \\
\hline$M P 2 / 6-311++G^{* *} 330$ & 1759 & 1562 & 1291 & $t_{\mathrm{as}}$ \\
\hline MP2/VTZ 186 & $1770(1777)$ & $1578(1545)$ & 1284 & $t_{\mathrm{ss}} / c_{\mathrm{aa}}$ \\
\hline MP2/aVTZ 186] & $1740(1750)$ & $1559(1544)$ & 1289 & $t_{\mathrm{ss}} / c_{\mathrm{aa}}$ \\
\hline MP2/VQZ 186 & 1754 & 1563 & 1292 & $t_{\mathrm{ss}} / c_{\mathrm{aa}}$ \\
\hline CASPT2/VTZ 186 & 1759 & 1571 & 1296 & $t_{\mathrm{ss}} / c_{\mathrm{aa}}$ \\
\hline B3LYP/D95++(d,p)e 332 & 1692 & - & - & - \\
\hline $\mathrm{B} 3 \mathrm{LYP} / 6-31+\mathrm{G}^{*} 189$ & 1751 & 1560 & 1292 & $t_{\text {as }}$ \\
\hline $\mathrm{B} 3 \mathrm{LYP} / 6-311++\mathrm{G}^{* *} 202$ & 1741 & - & - & $t_{\mathrm{as}}$ \\
\hline B3LYP/6-311++G** 183. & 1744 & 1560 & 1266 & $t_{\mathrm{ss}} / c_{\mathrm{aa}}$ \\
\hline B3LYP-D3(BJ)/def2TZVPa & $1749(1755)$ & $1563(1523)$ & $1273(1349)$ & $t_{\mathrm{ss}} / c_{\mathrm{aa}}$ \\
\hline B3LYP-D3(BJ)/aVTZ ${ }^{\mathrm{a}}$ & $1737(1744)$ & $1543(1523)$ & $1280(1347)$ & $t_{\mathrm{as}} / c_{\mathrm{aa}}$ \\
\hline BPW91/6-311+G(3df,2p) 78 & 1696 & 1508 & 1225 & $t_{\text {as }}$ \\
\hline BPW $91 / 6-31 \mathrm{G}\left(\mathrm{d}^{0.25}\right)^{\mathrm{c}} \mid 78$ & 1653 & 1521 & 1243 & $t_{\text {as }}$ \\
\hline BPW91/6-31G** 331$]$ & 1733 & 1507 & 1241 & $t_{\text {as }}$ \\
\hline BPW91/6-31G(d,p) [187] & $1736(1741)$ & $1522(1493)$ & $1231(1308 ?)$ & $t_{\mathrm{ss}} / c_{\mathrm{aa}}$ \\
\hline BPW91/aVDZ [78] & 1684 & 1510 & 1223 & $t_{\text {as }}$ \\
\hline $\mathrm{PW} 91 \mathrm{XC} / 6-31+\mathrm{G}^{*}$ & $1706(1709)$ & $1528(1496)$ & $1244(1322)$ & $t_{\mathrm{ss}} / c_{\mathrm{aa}}$ \\
\hline \multicolumn{5}{|l|}{ anharmonisch: ${ }^{f}$} \\
\hline BPW91/6-31G(d,p) + electrostatic DFT map 331] & 1724 & 1495 & 1229 & $t_{\text {as }}$ \\
\hline $\mathrm{B} 3 \mathrm{LYP} / 6-31+\mathrm{G}^{*}(3 \mathrm{MR}-\mathrm{PES})+\mathrm{VCl}$ 189 & 1725 & 1537 & 1272 & $t_{\text {as }}$ \\
\hline B3LYP/6-31+G*(3MR-PES) + VPT2 189 & 1725 & 1505 & 1256 & $t_{\text {as }}$ \\
\hline B3LYP-D3(BJ)/VTZ + VPT2 ${ }^{\mathrm{a}}$ & $1727[1755]$ & $1511[1563]$ & $1245[1272]$ & $t_{\mathrm{SS}}$ \\
\hline MP2/DZP(2MR-PES) + cc-VSCFd 185 & 1751 & 1547 & 1283 & $t_{\text {as }}$ \\
\hline MP2/DZP(DZP pair potential) + cc-VSCF [190] & 1749 & 1536 & 1284 & - \\
\hline MP2/aVTZ(part. 3MR-PES) + VCI 191] & 1727 & 1519 & 1253 & $t_{\text {as }}$ \\
\hline \multicolumn{5}{|l|}{ Molekulardynamik (MD) - Simulation: } \\
\hline Car-Parrinello (BLYP basiert) 209] & 1609 & 1458 & 1189 & $t_{\mathrm{aa}} / c_{\mathrm{aa}}$ \\
\hline
\end{tabular}

a: aus dieser Arbeit; ${ }^{\mathrm{b}}$ : ()$=$ cis-Bande; $^{\mathrm{c}}$ : diffusere d-Polarisationsfunktionen im Basissatz (0.8 ist Standard);

d: später korrigiert durch [190]; ${ }^{\text {e: }}$ skalierte Wellenzahlen; ${ }^{\text {f }}$ : harmonische Werte in eckigen Klammern. 
Tab. A-14. Experimentelle und theoretische Energieunterschiede zwischen cis- und trans-NMA, sowie Isomerisierungsbarrieren.

\begin{tabular}{|c|c|c|c|c|}
\hline \multicolumn{5}{|l|}{ experimentell } \\
\hline Methode & Bedingungen & $\Delta G / \mathrm{kJ} \cdot \mathrm{mol}^{-1 \mathrm{a}}$ & $\Delta H / \mathrm{kJ} \cdot \mathrm{mol}^{-1}$ & $\Delta E_{\mathrm{A}} / \mathrm{kJ} \cdot \mathrm{mol}^{-1 \mathrm{f}}$ \\
\hline Raman - Jet ${ }^{b}$ & $\theta_{\mathrm{H}}=388.15-483.15 \mathrm{~K}$ & $12.7(388.15)$ & $8 \pm 2$ & - \\
\hline Raman 34 ] & flüssig & - & - & 58.6 \\
\hline NMR 161 & in 1,2-Dichlorethan & $11.7 \pm 1.5(333.15)$ & $12 \pm 15$ & 95 \\
\hline NMR 161 & in $\mathrm{H}_{2} \mathrm{O}$ & $10.4 \pm 2.5(333.15)$ & $14 \pm 15$ & 97 \\
\hline 2D-NMR 196 & in $\mathrm{H}_{2} \mathrm{O} / 293-313 \mathrm{~K}$ & $10.2(293)$ & 15.3 & - \\
\hline 2D-NMR 196 & in DMSO / 293-313K & $11.4(293)$ & 1.8 & $98 \pm 11$ \\
\hline 2D-NMR 196 & in Aceton / 293-313 K & $7.1(293)$ & 7.1 & - \\
\hline IR-Matrix 68 & $\mathrm{~N}_{2} / T_{\text {Düse }}=298-770 \mathrm{~K}$ & - & 9.6 & - \\
\hline UVRR 35 & in $\mathrm{H}_{2} \mathrm{O}$ & $10.9 \pm 1.7$ (n. a.) & - & $57.7 \pm 3.3$ \\
\hline UVRR 82 & in $\mathrm{H}_{2} \mathrm{O}$ & - & $7.1 \pm 0.8$ & - \\
\hline
\end{tabular}

\begin{tabular}{|c|c|c|c|c|c|}
\hline \multicolumn{6}{|l|}{ theoretisch } \\
\hline Methode & $\Delta E / \mathrm{kJ} \cdot \mathrm{mol}^{-1} \mathrm{~g}$ & $\Delta G / \mathrm{kJ} \cdot \mathrm{mol}^{-1 \mathrm{a}}$ & $\Delta H / \mathrm{kJ} \cdot \mathrm{mol}^{-1}$ & $\Delta E_{\mathrm{A}} / \mathrm{kJ} \cdot \mathrm{mol}^{-1 \mathrm{f}}$ & Konf. \\
\hline $\mathrm{HF} / 4-31 \mathrm{G}^{*} 174$ & 10.3 & - & - & - & $t_{\mathrm{as}}^{\mathrm{j}} / \mathrm{c}_{\mathrm{aa}}$ \\
\hline $\mathrm{HF} / 6-31 \mathrm{G}(\mathrm{d}, \mathrm{p}) 202$ 203. & 10.6 & $9.4(298.15)$ & 12.9 & $68.4 / 87.2^{\mathrm{e}}$ & n. a. \\
\hline $\mathrm{HF} / 6-31+\mathrm{G}(\mathrm{d}) 202203$ & 11.0 & $17.2(298.15)$ & 8.2 & $70.0 / 88.3^{\mathrm{e}}$ & n. a. \\
\hline $\mathrm{HF} / 6-311++\mathrm{G}^{\star \star} 202$ & 11.8 & $18.8(298.15)$ & 9.0 & - & $t_{\mathrm{as}}$ \\
\hline MP2/6-31G* 198 & 9.3 & $10.6(298)$ & 8.9 & $70.4 / 88.7^{e}$ & $t_{\mathrm{sa}} / c_{\mathrm{aa}}$ \\
\hline MP2/6-31G* 199 & $9.4[10.4]$ & $15.4(298)$ & 9.3 & $69.5 / 87.8^{\mathrm{e}}$ & $t_{\mathrm{as}} / c_{\mathrm{aa}}$ \\
\hline$M P 2 / 6-311++G(d, p) d$ & {$[11.2]$} & - & - & - & $t_{\mathrm{as}}$ \\
\hline MP2/6-311++G* 202 & 9.5 & $16.0(298.15)$ & 12.6 & - & $t_{\mathrm{as}}$ \\
\hline MP2/aVTZ d,i 44 & 9.5 & - & - & - & $t_{\mathrm{ss}} / c_{\mathrm{aa}}$ \\
\hline MP2/aVTZ 186 & 10.3 & - & - & - & $t_{\mathrm{ss}} / c_{\mathrm{aa}}$ \\
\hline MP2/DZP 185 & 10.4 & - & - & - & $t_{\mathrm{as}}$ \\
\hline MP4\#/6-31G* 198 & 9.3 & $10.6(298)$ & 9.0 & $69.7 / 87.4^{\mathrm{e}}$ & $t_{\mathrm{sa}} / c_{\mathrm{aa}}$ \\
\hline BPW91/6-31G $\times 187$ & 11.0 & - & - & - & $t_{\mathrm{ss}} / c_{\mathrm{aa}}$ \\
\hline$B 3 L Y P / 6-31 G^{*} 333$ & 10.3 & - & - & - & $t_{\mathrm{ss}} / c_{\mathrm{aa}}$ \\
\hline$B 3 L Y P / 6-31+G^{*} \mathrm{U}$ & $9.6[10.1]$ & - & - & - & $t_{\mathrm{sa}} / c_{\mathrm{ss}}$ \\
\hline B3LYP/6-311++G** 202 & 10.4 & $9.2(298.15)$ & 12.6 & - & $t_{\mathrm{as}}$ \\
\hline B3LYP/6-311++G(3df,3pd) 20 & 9.6 & - & - & $78.2 / 91.6^{\mathrm{e}}$ & $t_{\mathrm{as}}$ \\
\hline B3LYP-D3(BJ)/def2TZVP b & 8.7 [9.2] & $10.9(388.15)$ & $8.4^{\mathrm{h}}$ & - & $t_{\mathrm{ss}} / c_{\mathrm{aa}}$ \\
\hline B3LYP-D3(BJ)/aVTZ b & $8.8[9.0]$ & $6.4(388.15)$ & $8.5^{\mathrm{k}}$ & - & $t_{\mathrm{as}} / \mathrm{c}_{\mathrm{aa}}$ \\
\hline LC-BLYP/6-311++G(d,p) d 21 & {$[10.7]$} & - & - & - & $t_{\mathrm{as}}$ \\
\hline
\end{tabular}

n. a.: nicht angegeben; UVRR: UV-Resonanz-Raman-Spektroskopie; ${ }^{\mathrm{a}}$ : (Temperatur in $\mathrm{K}$ ) $;^{\mathrm{b}}$ : aus dieser Arbeit; ${ }^{\mathrm{c}}$ : freie Enthalpie $\Delta G$ (Temperatur in K)); ${ }^{d}$ : Counterpoise-Korrektur (CP) 150$]$; ${ }^{e}$ : zwei mögliche Übergangszustände; ${ }^{f}$ : Barriere bezogen auf trans-NMA; 9 : elektronische [nullpunktskorrigierte] Energieunterschiede; ${ }^{\mathrm{h}}$ : Mittelwert der $\Delta H$-Werte der Frequenzrechnungen im Temperaturbereich 388.15-483.15K; ' : Geometrie MP2/aVDZ optimiert; ' : trans-Konformer in [66]; ${ }^{\mathrm{k}}$ : Aus Van 't-Hoff-Plot auf Basis der berechneten $\Delta G$ -

Werte im Temperaturbereich 388.15-483.15K. 
Tab. A-15. NH-Streckschwingungsmoden aus der Frequenzrechnung auf B3LYP-D3(BJ)/aVTZ-Niveau (doppelt-harmonische Näherung) für die stabilsten Monomere und Cluster von NMA. Angegeben sind die harmonische Wellenzahl $\omega$ (unskaliert und skaliert), die IR-Intensität $A$, die Raman-Aktivität $R$, das Depolarisationsverhältnis für polarisiertes Licht $\delta_{k}$ und der, aus der korrigierten Raman-Aktivität (Gl. 3-16 berechneten, differentielle Raman-Streuquerschnitt $\sigma^{\prime}$ bei $532 \mathrm{~nm}$ Anregungswellenlänge und $90^{\circ}$ Streugeometrie (Gl. 3-13). Details zur Rechenmethode, siehe Abschnitt 3.4

\begin{tabular}{|c|c|c|c|c|c|c|}
\hline Konf. & $\omega / \mathrm{cm}^{-1}$ & $0.959 \omega / \mathrm{cm}^{-1}$ & $A / \mathrm{km} \cdot \mathrm{mol}^{-1}$ & $R / \mathrm{A}^{4} \cdot \mathrm{amu}^{-1}$ & $\delta_{k}$ & $10^{35} \sigma^{\prime} / \mathrm{m}^{2} \mathrm{sr}^{-1}$ \\
\hline$c_{\mathrm{aa}}$ & 3616 & 3467 & 29.48 & 93.21 & 0.16 & 0.99 \\
\hline$t_{\mathrm{as}}$ & 3657 & 3507 & 25.57 & 76.42 & 0.16 & 0.79 \\
\hline$t t_{\mathrm{C}}$ & $\begin{array}{l}3653 \\
3469\end{array}$ & $\begin{array}{l}3504 \\
3326\end{array}$ & $\begin{array}{r}34.20 \\
578.49\end{array}$ & $\begin{array}{r}85.89 \\
223.21\end{array}$ & $\begin{array}{l}0.16 \\
0.25\end{array}$ & $\begin{array}{l}0.89 \\
2.54\end{array}$ \\
\hline$t t_{N}$ & $\begin{array}{l}3652 \\
3504\end{array}$ & $\begin{array}{l}3503 \\
3360\end{array}$ & $\begin{array}{r}33.09 \\
507.80\end{array}$ & $\begin{array}{r}81.75 \\
175.17\end{array}$ & $\begin{array}{l}0.18 \\
0.23\end{array}$ & $\begin{array}{l}0.85 \\
1.96\end{array}$ \\
\hline$c c$ & $\begin{array}{l}3277 \\
3225\end{array}$ & $\begin{array}{l}3143 \\
3093\end{array}$ & $\begin{array}{r}2033.42 \\
0.00\end{array}$ & $\begin{array}{r}0.00 \\
528.70\end{array}$ & $\begin{array}{l}0.00 \\
0.28\end{array}$ & $\begin{array}{l}0.00 \\
6.78\end{array}$ \\
\hline$t t t_{a}$ & $\begin{array}{l}3528 \\
3522 \\
3510\end{array}$ & $\begin{array}{l}3383 \\
3378 \\
3366\end{array}$ & $\begin{array}{r}256.73 \\
245.00 \\
58.06\end{array}$ & $\begin{array}{r}29.00 \\
47.96 \\
141.57\end{array}$ & $\begin{array}{l}0.51 \\
0.31 \\
0.05\end{array}$ & $\begin{array}{l}0.32 \\
0.53 \\
1.58\end{array}$ \\
\hline$t t t_{p}$ & $\begin{array}{l}3517 \\
3517 \\
3505\end{array}$ & $\begin{array}{l}3373 \\
3373 \\
3361\end{array}$ & $\begin{array}{r}298.73 \\
298.88 \\
2.00\end{array}$ & $\begin{array}{r}26.38 \\
26.36 \\
161.13\end{array}$ & $\begin{array}{l}0.75 \\
0.75 \\
0.03\end{array}$ & $\begin{array}{l}0.29 \\
0.29 \\
1.80\end{array}$ \\
\hline$t t t t_{a}$ & $\begin{array}{l}3387 \\
3375 \\
3375 \\
3352\end{array}$ & $\begin{array}{l}3248 \\
3236 \\
3236 \\
3214\end{array}$ & $\begin{array}{r}19.67 \\
1089.07 \\
1089.07 \\
0.00\end{array}$ & $\begin{array}{r}166.62 \\
13.32 \\
13.32 \\
374.80\end{array}$ & $\begin{array}{l}0.75 \\
0.75 \\
0.75 \\
0.06\end{array}$ & $\begin{array}{l}1.97 \\
0.16 \\
0.16 \\
4.51\end{array}$ \\
\hline$t_{t t t}$ & $\begin{array}{l}3406 \\
3396 \\
3393 \\
3373\end{array}$ & $\begin{array}{l}3266 \\
3257 \\
3254 \\
3234\end{array}$ & $\begin{array}{r}52.72 \\
965.18 \\
1021.08 \\
29.30\end{array}$ & $\begin{array}{r}152.79 \\
14.31 \\
8.86 \\
349.61\end{array}$ & $\begin{array}{l}0.75 \\
0.71 \\
0.23 \\
0.07\end{array}$ & $\begin{array}{l}1.79 \\
0.17 \\
0.10 \\
4.17\end{array}$ \\
\hline
\end{tabular}


Tab. A-16. CO-Streckschwingungsmoden aus der Frequenzrechnung auf B3LYP-D3(BJ)/aVTZ-Niveau (doppelt-harmonische Näherung) für die stabilsten Monomere und Cluster von NMA. (Erklärungen zu den angegeben Größen finden sich in Tab. A-15

\begin{tabular}{ccccccc}
\hline Konf. & $\omega / \mathrm{cm}^{-1}$ & $0.99 \omega / \mathrm{cm}^{-1}$ & $A / \mathrm{km} \cdot \mathrm{mol}^{-1}$ & $R / \mathrm{A}^{4} \cdot \mathrm{amu}^{-1}$ & $\delta_{k}$ & $10^{35} \sigma^{\prime} / \mathrm{m}^{2} \mathrm{sr}^{-1}$ \\
\hline$c_{\mathrm{aa}}$ & 1745 & 1727 & 431.74 & 16.57 & 0.31 & 0.52 \\
$t_{\mathrm{as}}$ & 1737 & 1719 & 283.01 & 11.51 & 0.28 & 0.36 \\
$t t_{\mathrm{C}}$ & 1727 & 1709 & 213.37 & 8.10 & 0.50 & 0.26 \\
& 1708 & 1691 & 505.47 & 25.83 & 0.22 & 0.83 \\
$t t_{\mathrm{N}}$ & 1728 & 1710 & 191.04 & 7.99 & 0.62 & 0.25 \\
& 1713 & 1696 & 526.35 & 17.47 & 0.13 & 0.56 \\
$\mathrm{cc}$ & 1729 & 1711 & 921.44 & 0.00 & 0.00 & 0.00 \\
& 1692 & 1676 & 0.00 & 12.36 & 0.16 & 0.40 \\
$t t t_{\mathrm{a}}$ & 1707 & 1690 & 412.19 & 1.79 & 0.75 & 0.06 \\
& 1707 & 1690 & 392.20 & 2.33 & 0.75 & 0.07 \\
& 1677 & 1660 & 0.87 & 19.10 & 0.08 & 0.63 \\
$t t t_{\mathrm{p}}$ & 1705 & 1688 & 393.09 & 2.24 & 0.75 & 0.07 \\
& 1705 & 1688 & 393.27 & 2.24 & 0.75 & 0.07 \\
& 1675 & 1658 & 3.40 & 17.94 & 0.06 & 0.59 \\
$t t t t_{\mathrm{a}}$ & 1699 & 1682 & 12.66 & 5.42 & 0.75 & 0.17 \\
& 1686 & 1669 & 681.76 & 0.90 & 0.75 & 0.03 \\
& 1686 & 1669 & 681.76 & 0.90 & 0.75 & 0.03 \\
& 1659 & 1642 & 0.00 & 31.65 & 0.08 & 1.05 \\
$t t t t_{\mathrm{p}}$ & 1699 & 1682 & 20.92 & 4.33 & 0.75 & 0.14 \\
& 1694 & 1677 & 651.08 & 0.87 & 0.74 & 0.03 \\
& 1684 & 1667 & 547.54 & 0.19 & 0.59 & 0.01 \\
& 1670 & 1653 & 3.43 & 28.45 & 0.10 & 0.94 \\
\hline
\end{tabular}


Tab. A-17. Amid II-Schwingungsmoden aus der Frequenzrechnung auf B3LYP-D3(BJ)/aVTZ-Niveau (doppelt-harmonische Näherung) für die stabilsten Monomere und Cluster von NMA. (Erklärungen zu den angegeben Größen finden sich in Tab. A-15

\begin{tabular}{|c|c|c|c|c|c|c|}
\hline Konf. & $\omega / \mathrm{cm}^{-1}$ & $0.99 \omega / \mathrm{cm}^{-1}$ & $A / \mathrm{km} \cdot \mathrm{mol}^{-1}$ & $R / \mathrm{A}^{4} \cdot \mathrm{amu}^{-1}$ & $\delta_{k}$ & $10^{35} \sigma^{\prime} / \mathrm{m}^{2} \mathrm{sr}^{-1}$ \\
\hline$c_{\mathrm{aa}}$ & 1523 & 1508 & 32.43 & 6.11 & 0.58 & 0.23 \\
\hline$t_{\mathrm{as}}$ & 1543 & 1528 & 188.44 & 1.82 & 0.57 & 0.07 \\
\hline$t t_{\mathrm{C}}$ & $\begin{array}{l}1594 \\
1556\end{array}$ & $\begin{array}{l}1578 \\
1541\end{array}$ & $\begin{array}{l}170.56 \\
242.09\end{array}$ & $\begin{array}{l}0.60 \\
1.10\end{array}$ & $\begin{array}{l}0.72 \\
0.35\end{array}$ & $\begin{array}{l}0.02 \\
0.04\end{array}$ \\
\hline$t t_{N}$ & $\begin{array}{l}1592 \\
1556\end{array}$ & $\begin{array}{l}1576 \\
1540\end{array}$ & $\begin{array}{l}166.68 \\
178.38\end{array}$ & $\begin{array}{l}0.58 \\
1.22\end{array}$ & $\begin{array}{l}0.69 \\
0.57\end{array}$ & $\begin{array}{l}0.02 \\
0.04\end{array}$ \\
\hline$c c$ & $\begin{array}{l}1565 \\
1556\end{array}$ & $\begin{array}{l}1550 \\
1540\end{array}$ & $\begin{array}{r}0.00 \\
42.53\end{array}$ & $\begin{array}{r}32.61 \\
0.00\end{array}$ & $\begin{array}{l}0.20 \\
0.00\end{array}$ & $\begin{array}{l}1.17 \\
0.00\end{array}$ \\
\hline$t t t_{a}$ & $\begin{array}{l}1595 \\
1589 \\
1587\end{array}$ & $\begin{array}{l}1579 \\
1574 \\
1572\end{array}$ & $\begin{array}{r}358.64 \\
49.82 \\
17.72\end{array}$ & $\begin{array}{l}0.14 \\
0.61 \\
0.79\end{array}$ & $\begin{array}{l}0.61 \\
0.68 \\
0.63\end{array}$ & $\begin{array}{l}0.00 \\
0.02 \\
0.03\end{array}$ \\
\hline$t t t_{p}$ & $\begin{array}{l}1592 \\
1587 \\
1587\end{array}$ & $\begin{array}{l}1577 \\
1571 \\
1571\end{array}$ & $\begin{array}{r}328.59 \\
48.33 \\
48.08\end{array}$ & $\begin{array}{l}0.21 \\
0.59 \\
0.59\end{array}$ & $\begin{array}{l}0.00 \\
0.75 \\
0.75\end{array}$ & $\begin{array}{l}0.01 \\
0.02 \\
0.02\end{array}$ \\
\hline$t t t t_{a}$ & $\begin{array}{l}1618 \\
1616 \\
1616 \\
1615\end{array}$ & $\begin{array}{l}1602 \\
1600 \\
1600 \\
1599\end{array}$ & $\begin{array}{r}321.43 \\
169.19 \\
169.19 \\
0.00\end{array}$ & $\begin{array}{l}0.38 \\
0.22 \\
0.22 \\
0.23\end{array}$ & $\begin{array}{l}0.75 \\
0.75 \\
0.75 \\
0.02\end{array}$ & $\begin{array}{l}0.01 \\
0.01 \\
0.01 \\
0.01\end{array}$ \\
\hline$t t t t_{p}$ & $\begin{array}{l}1619 \\
1616 \\
1609 \\
1606\end{array}$ & $\begin{array}{l}1602 \\
1600 \\
1593 \\
1590\end{array}$ & $\begin{array}{r}69.09 \\
85.74 \\
331.41 \\
228.51\end{array}$ & $\begin{array}{l}0.91 \\
0.28 \\
0.17 \\
1.92\end{array}$ & $\begin{array}{l}0.13 \\
0.17 \\
0.56 \\
0.50\end{array}$ & $\begin{array}{l}0.03 \\
0.01 \\
0.01 \\
0.07\end{array}$ \\
\hline
\end{tabular}


Tab. A-18. Amid III-Schwingungsmoden aus der Frequenzrechnung auf B3LYP-D3(BJ)/aVTZ-Niveau (doppelt-harmonische Näherung) für die stabilsten Monomere und Cluster von NMA. (Erklärungen zu den angegeben Größen finden sich in Tab. A-15,

\begin{tabular}{lllrclc}
\hline Konf. & $\omega / \mathrm{cm}^{-1}$ & $0.99 \omega / \mathrm{cm}^{-1}$ & $A / \mathrm{km} \cdot \mathrm{mol}^{-1}$ & $R / \mathrm{A}^{4} \cdot \mathrm{amu}^{-1}$ & $\delta_{k}$ & $10^{35} \sigma^{\prime} / \mathrm{m}^{2} \mathrm{sr}^{-1}$ \\
\hline$c_{\mathrm{aa}}$ & 1347 & 1333 & 127.74 & 4.49 & 0.74 & 0.19 \\
$t_{\mathrm{as}}$ & 1280 & 1267 & 82.49 & 7.05 & 0.50 & 0.32 \\
$t t_{\mathrm{C}}$ & 1316 & 1303 & 94.93 & 6.44 & 0.39 & 0.29 \\
& 1295 & 1282 & 76.79 & 13.73 & 0.43 & 0.62 \\
$t t_{\mathrm{N}}$ & 1315 & 1302 & 93.09 & 6.51 & 0.39 & 0.29 \\
& 1294 & 1281 & 61.85 & 6.62 & 0.53 & 0.30 \\
$c c$ & 1378 & 1365 & 0.00 & 5.30 & 0.73 & 0.22 \\
& 1377 & 1363 & 61.73 & 0.00 & 0.00 & 0.00 \\
$t t t_{\mathrm{a}}$ & 1319 & 1306 & 156.73 & 1.84 & 0.10 & 0.08 \\
& 1315 & 1302 & 3.45 & 9.53 & 0.28 & 0.42 \\
& 1314 & 1301 & 4.90 & 5.93 & 0.67 & 0.26 \\
$t t t_{\mathrm{p}}$ & 1321 & 1308 & 172.74 & 6.48 & 0.01 & 0.29 \\
& 1315 & 1302 & 3.94 & 5.24 & 0.75 & 0.23 \\
& 1315 & 1302 & 3.93 & 5.24 & 0.75 & 0.23 \\
$t t t t_{\mathrm{a}}$ & 1344 & 1330 & 134.56 & 8.54 & 0.75 & 0.37 \\
& 1339 & 1326 & 25.57 & 6.26 & 0.75 & 0.27 \\
& 1339 & 1326 & 25.57 & 6.26 & 0.75 & 0.27 \\
& 1335 & 1322 & 0.00 & 12.01 & 0.00 & 0.52 \\
$t t t t_{\mathrm{p}}$ & 1341 & 1327 & 119.89 & 17.33 & 0.22 & 0.75 \\
& 1340 & 1327 & 42.50 & 5.83 & 0.41 & 0.25 \\
& 1335 & 1322 & 20.82 & 3.17 & 0.37 & 0.14 \\
& 1334 & 1321 & 29.75 & 6.77 & 0.67 & 0.30 \\
\hline & & & & & &
\end{tabular}

Tab. A-19. Amid IV-Schwingungsmoden aus der Frequenzrechnung auf B3LYP-D3(BJ)/aVTZ-Niveau (doppelt-harmonische Näherung) für die stabilsten Monomere und Cluster von NMA. (Erklärungen zu den angegeben Größen finden sich in Tab. A-15

\begin{tabular}{cccccccc}
\hline \multicolumn{2}{l}{ Konformer } & $\omega / \mathrm{cm}^{-1}$ & $0.99 \omega / \mathrm{cm}^{-1}$ & $A / \mathrm{km} \cdot \mathrm{mol}^{-1}$ & $R / \mathrm{A}^{4} \cdot \mathrm{amu}^{-1}$ & $\delta_{k}$ & $10^{35} \sigma^{\prime} / \mathrm{m}^{2} \mathrm{sr}^{-1}$ \\
\hline$t_{\mathrm{as}}$ & $(\mathrm{IVa})$ & 874 & 865.45 & 5.41 & 8.04 & 0.06 & 0.58 \\
& $(\mathrm{IVb})$ & 625 & 618 & 5.37 & 6.31 & 0.19 & 0.66 \\
\multicolumn{2}{c}{$c_{\mathrm{aa}}$} & 805 & 797.36 & 0.66 & 14.22 & 0.06 & 1.13 \\
\hline
\end{tabular}




\section{A.8 N-Methylformamid}

Ergänzende Tabellen zum Kapitel 5 .

Tab. A-20. NH-Streckschwingungsmoden aus der Frequenzrechnung auf B3LYP-D3(BJ)/aVTZ-Niveau (doppelt-harmonische Näherung) für die stabilsten Monomere und Cluster von NMF. Angegeben sind die harmonische Wellenzahl $\omega$ (unskaliert und skaliert), die IR-Intensität $A$, die Raman-Aktivität $R$, das Depolarisationsverhältnis für polarisiertes Licht $\delta_{k}$ und der, aus der korrigierten Raman-Aktivität (Gl. 3-16 berechneten, differentielle Raman-Streuquerschnitt $\sigma^{\prime}$ bei $532 \mathrm{~nm}$ Anregungswellenlänge und $90^{\circ}$ Streugeometrie (Gl. 3-13). Details zur Rechenmethode, siehe Abschnitt 3.4

\begin{tabular}{|c|c|c|c|c|c|c|}
\hline Konf. & $\omega / \mathrm{cm}^{-1}$ & $0.9646 \omega / \mathrm{cm}^{-1}$ & $A / \mathrm{km} \cdot \mathrm{mol}^{-1}$ & $R / \mathrm{A}^{4} \cdot \mathrm{amu}^{-1}$ & $\delta_{k}$ & $10^{34} \sigma^{\prime} / \mathrm{m}^{2} \mathrm{sr}^{-1}$ \\
\hline$c_{\mathrm{s}}$ & 3594 & 3467 & 26.84 & 85.98 & 0.1587 & 0.92 \\
\hline$t_{\mathrm{S}}$ & 3629 & 3501 & 24.78 & 102.05 & 0.1827 & 1.07 \\
\hline$t t_{N}$ & $\begin{array}{l}3636 \\
3492\end{array}$ & $\begin{array}{l}3507 \\
3369\end{array}$ & $\begin{array}{r}37.13 \\
510.58\end{array}$ & $\begin{array}{l}114.52 \\
189.64\end{array}$ & $\begin{array}{l}0.199 \\
0.235\end{array}$ & $\begin{array}{l}1.20 \\
2.13\end{array}$ \\
\hline$t t_{\mathrm{H}}$ & $\begin{array}{l}3627 \\
3452\end{array}$ & $\begin{array}{l}3499 \\
3330\end{array}$ & $\begin{array}{r}35.48 \\
661.93\end{array}$ & $\begin{array}{l}100.33 \\
293.82\end{array}$ & $\begin{array}{l}0.1774 \\
0.2596\end{array}$ & $\begin{array}{l}1.06 \\
3.37\end{array}$ \\
\hline$c c$ & $\begin{array}{l}3265 \\
3211\end{array}$ & $\begin{array}{l}3150 \\
3098\end{array}$ & $\begin{array}{r}1594.59 \\
0.00\end{array}$ & $\begin{array}{r}0.00 \\
413.10\end{array}$ & $\begin{array}{l}0 \\
0.2664\end{array}$ & $\begin{array}{l}0.00 \\
5.33\end{array}$ \\
\hline$c t$ & $\begin{array}{l}3629 \\
3312\end{array}$ & $\begin{array}{l}3501 \\
3194\end{array}$ & $\begin{array}{r}33.29 \\
766.46\end{array}$ & $\begin{array}{r}96.53 \\
276.95\end{array}$ & $\begin{array}{l}0.1684 \\
0.2726\end{array}$ & $\begin{array}{l}1.02 \\
3.40\end{array}$ \\
\hline$t t t_{\mathrm{a}}$ & $\begin{array}{l}3477 \\
3471 \\
3458\end{array}$ & $\begin{array}{l}3354 \\
3348 \\
3336\end{array}$ & $\begin{array}{r}361.78 \\
463.03 \\
14.13\end{array}$ & $\begin{array}{r}44.48 \\
28.67 \\
254.82\end{array}$ & $\begin{array}{l}0.5929 \\
0.7384 \\
0.0826\end{array}$ & $\begin{array}{l}0.50 \\
0.33 \\
2.91\end{array}$ \\
\hline$t t t_{p}$ & $\begin{array}{l}3500 \\
3500 \\
3484\end{array}$ & $\begin{array}{l}3376 \\
3376 \\
3360\end{array}$ & $\begin{array}{r}376.58 \\
376.57 \\
4.62\end{array}$ & $\begin{array}{r}33.58 \\
33.52 \\
201.11\end{array}$ & $\begin{array}{l}0.75 \\
0.75 \\
0.0444\end{array}$ & $\begin{array}{l}0.38 \\
0.38 \\
2.27\end{array}$ \\
\hline$t t t_{1}$ & $\begin{array}{l}3635 \\
3475 \\
3434\end{array}$ & $\begin{array}{l}3506 \\
3352 \\
3312\end{array}$ & $\begin{array}{r}38.26 \\
276.54 \\
592.55\end{array}$ & $\begin{array}{r}116.82 \\
78.93 \\
179.52\end{array}$ & $\begin{array}{l}0.1985 \\
0.2484 \\
0.1944\end{array}$ & $\begin{array}{l}1.23 \\
0.89 \\
2.08\end{array}$ \\
\hline$t t t t_{a}$ & $\begin{array}{l}3308 \\
3293 \\
3293 \\
3262\end{array}$ & $\begin{array}{l}3191 \\
3177 \\
3177 \\
3147\end{array}$ & $\begin{array}{r}176.63 \\
1694.36 \\
1694.36 \\
0.00\end{array}$ & $\begin{array}{r}222.92 \\
42.08 \\
42.08 \\
567.44\end{array}$ & $\begin{array}{l}0.75 \\
0.75 \\
0.75 \\
0.0698\end{array}$ & $\begin{array}{l}2.74 \\
0.52 \\
0.52 \\
7.14\end{array}$ \\
\hline$t t t t_{p}$ & $\begin{array}{l}3393 \\
3392 \\
3283 \\
3270\end{array}$ & $\begin{array}{l}3273 \\
3271 \\
3167 \\
3154\end{array}$ & $\begin{array}{r}974.87 \\
11.58 \\
1874.63 \\
0.85\end{array}$ & $\begin{array}{r}3.88 \\
227.98 \\
1.59 \\
487.86\end{array}$ & $\begin{array}{l}0.75 \\
0.2884 \\
0.75 \\
0.2133\end{array}$ & $\begin{array}{l}0.05 \\
2.69 \\
0.02 \\
6.11\end{array}$ \\
\hline
\end{tabular}


Tab. A-21. CO-Streckschwingungsmoden aus der Frequenzrechnung auf B3LYP-D3(BJ)/aVTZ-Niveau (doppelt-harmonische Näherung) für die stabilsten Monomere und Cluster von NMF. (Erklärungen zu den angegeben Größen finden sich in Tab. A-20

\begin{tabular}{|c|c|c|c|c|c|c|}
\hline Konf. & $\omega / \mathrm{cm}^{-1}$ & $0.979 \omega / \mathrm{cm}^{-1}$ & $A / \mathrm{km} \cdot \mathrm{mol}^{-1}$ & $R / \mathrm{A}^{4} \cdot \mathrm{amu}^{-1}$ & $\delta_{k}$ & $10^{34} \sigma^{\prime} / \mathrm{m}^{2} \mathrm{sr}^{-1}$ \\
\hline$c_{\mathrm{s}}$ & 1774 & 1737 & 531.47 & 18.90 & 0.2985 & 0.58 \\
\hline$t_{\mathrm{s}}$ & 1767 & 1730 & 363.74 & 14.59 & 0.2759 & 0.45 \\
\hline$t t_{\mathrm{N}}$ & $\begin{array}{l}1756 \\
1743\end{array}$ & $\begin{array}{l}1719 \\
1706\end{array}$ & $\begin{array}{l}305.21 \\
526.96\end{array}$ & $\begin{array}{l}10.06 \\
17.00\end{array}$ & $\begin{array}{l}0.6924 \\
0.0887\end{array}$ & $\begin{array}{l}0.31 \\
0.53\end{array}$ \\
\hline$t t_{\mathrm{H}}$ & $\begin{array}{l}1755 \\
1741\end{array}$ & $\begin{array}{l}1718 \\
1704\end{array}$ & $\begin{array}{l}240.49 \\
655.98\end{array}$ & $\begin{array}{l}10.27 \\
26.01\end{array}$ & $\begin{array}{l}0.6898 \\
0.175\end{array}$ & $\begin{array}{l}0.32 \\
0.81\end{array}$ \\
\hline$c c$ & $\begin{array}{l}1762 \\
1724\end{array}$ & $\begin{array}{l}1725 \\
1687\end{array}$ & $\begin{array}{r}1081.96 \\
0.00\end{array}$ & $\begin{array}{r}0.00 \\
15.21\end{array}$ & $\begin{array}{l}0 \\
0.2218\end{array}$ & $\begin{array}{l}0.00 \\
0.48\end{array}$ \\
\hline$c t$ & $\begin{array}{l}1761 \\
1709\end{array}$ & $\begin{array}{l}1724 \\
1673\end{array}$ & $\begin{array}{l}717.18 \\
160.20\end{array}$ & $\begin{array}{r}6.09 \\
26.75\end{array}$ & $\begin{array}{l}0.585 \\
0.1445\end{array}$ & $\begin{array}{l}0.19 \\
0.86\end{array}$ \\
\hline$t t t_{\mathrm{a}}$ & $\begin{array}{l}1741 \\
1736 \\
1710\end{array}$ & $\begin{array}{l}1704 \\
1699 \\
1674\end{array}$ & $\begin{array}{r}566.08 \\
487.57 \\
12.61\end{array}$ & $\begin{array}{r}1.56 \\
3.06 \\
21.99\end{array}$ & $\begin{array}{l}0.7342 \\
0.6572 \\
0.1229\end{array}$ & $\begin{array}{l}0.05 \\
0.10 \\
0.70\end{array}$ \\
\hline$t t t_{p}$ & $\begin{array}{l}1736 \\
1736 \\
1706\end{array}$ & $\begin{array}{l}1699 \\
1699 \\
1670\end{array}$ & $\begin{array}{r}469.36 \\
469.37 \\
83.47\end{array}$ & $\begin{array}{r}3.32 \\
3.31 \\
19.34\end{array}$ & $\begin{array}{l}0.75 \\
0.75 \\
0.0587\end{array}$ & $\begin{array}{l}0.10 \\
0.10 \\
0.62\end{array}$ \\
\hline$t t t_{1}$ & $\begin{array}{l}1750 \\
1739 \\
1717\end{array}$ & $\begin{array}{l}1714 \\
1703 \\
1681\end{array}$ & $\begin{array}{l}679.53 \\
202.09 \\
200.83\end{array}$ & $\begin{array}{r}2.31 \\
6.11 \\
20.67\end{array}$ & $\begin{array}{l}0.5241 \\
0.3467 \\
0.1215\end{array}$ & $\begin{array}{l}0.07 \\
0.19 \\
0.66\end{array}$ \\
\hline$t t t t_{a}$ & $\begin{array}{l}1737 \\
1725 \\
1725 \\
1697\end{array}$ & $\begin{array}{l}1701 \\
1688 \\
1688 \\
1661\end{array}$ & $\begin{array}{r}13.72 \\
936.01 \\
936.01 \\
0.00\end{array}$ & $\begin{array}{r}10.34 \\
0.98 \\
0.98 \\
29.31\end{array}$ & $\begin{array}{l}0.75 \\
0.75 \\
0.75 \\
0.1238\end{array}$ & $\begin{array}{l}0.32 \\
0.03 \\
0.03 \\
0.95\end{array}$ \\
\hline$t t t t_{p}$ & $\begin{array}{l}1735 \\
1730 \\
1717 \\
1703\end{array}$ & $\begin{array}{l}1699 \\
1694 \\
1681 \\
1667\end{array}$ & $\begin{array}{r}100.81 \\
1144.17 \\
389.94 \\
31.75\end{array}$ & $\begin{array}{r}4.43 \\
0.30 \\
4.50 \\
33.38\end{array}$ & $\begin{array}{l}0.6835 \\
0.75 \\
0.75 \\
0.1347\end{array}$ & $\begin{array}{l}0.14 \\
0.01 \\
0.14 \\
1.07\end{array}$ \\
\hline
\end{tabular}


Tab. A-22. Amid II-Schwingungsmoden aus der Frequenzrechnung auf B3LYP-D3(BJ)/aVTZ-Niveau (doppelt-harmonische Näherung) für die stabilsten Monomere und Cluster von NMF. (Erklärungen zu den angegeben Größen finden sich in Tab. A-20

\begin{tabular}{|c|c|c|c|c|c|c|}
\hline Konf. & $\omega / \mathrm{cm}^{-1}$ & $0.979 \omega / \mathrm{cm}^{-1}$ & $A / \mathrm{km} \cdot \mathrm{mol}^{-1}$ & $R / \mathrm{A}^{4} \cdot \mathrm{amu}^{-1}$ & $\delta_{k}$ & $10^{34} \sigma^{\prime} / \mathrm{m}^{2} \mathrm{sr}^{-1}$ \\
\hline$c_{\mathrm{s}}$ & 1533 & 1501 & 16.73 & 6.16 & 0.7436 & 0.23 \\
\hline$t_{\mathrm{s}}$ & 1555 & 1523 & 99.76 & 4.15 & 0.6625 & 0.15 \\
\hline$t t_{N}$ & $\begin{array}{l}1582 \\
1547\end{array}$ & $\begin{array}{l}1549 \\
1514\end{array}$ & $\begin{array}{l}107.32 \\
118.78\end{array}$ & $\begin{array}{l}3.57 \\
6.99\end{array}$ & $\begin{array}{l}0.2702 \\
0.3335\end{array}$ & $\begin{array}{l}0.13 \\
0.25\end{array}$ \\
\hline$t t_{\mathrm{H}}$ & $\begin{array}{l}1585 \\
1566\end{array}$ & $\begin{array}{l}1551 \\
1534\end{array}$ & $\begin{array}{l}110.28 \\
111.02\end{array}$ & $\begin{array}{l}4.41 \\
2.81\end{array}$ & $\begin{array}{l}0.2375 \\
0.564\end{array}$ & $\begin{array}{l}0.16 \\
0.10\end{array}$ \\
\hline$c c$ & $\begin{array}{l}1559 \\
1557\end{array}$ & $\begin{array}{l}1527 \\
1524\end{array}$ & $\begin{array}{r}31.94 \\
0.00\end{array}$ & $\begin{array}{r}0.00 \\
24.50\end{array}$ & $\begin{array}{l}0 \\
0.2261\end{array}$ & $\begin{array}{l}0.00 \\
0.88\end{array}$ \\
\hline$c t$ & $\begin{array}{l}1566 \\
1557\end{array}$ & $\begin{array}{l}1534 \\
1525\end{array}$ & $\begin{array}{r}114.90 \\
3.60\end{array}$ & $\begin{array}{r}2.93 \\
11.80\end{array}$ & $\begin{array}{l}0.1135 \\
0.3949\end{array}$ & $\begin{array}{l}0.10 \\
0.43\end{array}$ \\
\hline$t t t_{\mathrm{a}}$ & $\begin{array}{l}1602 \\
1596 \\
1592\end{array}$ & $\begin{array}{l}1569 \\
1562 \\
1558\end{array}$ & $\begin{array}{r}81.85 \\
146.35 \\
87.49\end{array}$ & $\begin{array}{l}4.59 \\
4.29 \\
3.23\end{array}$ & $\begin{array}{l}0.1433 \\
0.3129 \\
0.2399\end{array}$ & $\begin{array}{l}0.16 \\
0.15 \\
0.11\end{array}$ \\
\hline$t t t_{p}$ & $\begin{array}{l}1586 \\
1582 \\
1582\end{array}$ & $\begin{array}{l}1553 \\
1549 \\
1549\end{array}$ & $\begin{array}{r}162.82 \\
42.42 \\
42.42\end{array}$ & $\begin{array}{l}6.02 \\
1.11 \\
1.11\end{array}$ & $\begin{array}{l}0.001 \\
0.75 \\
0.75\end{array}$ & $\begin{array}{l}0.21 \\
0.04 \\
0.04\end{array}$ \\
\hline$t t t_{1}$ & $\begin{array}{l}1606 \\
1592 \\
1545\end{array}$ & $\begin{array}{l}1572 \\
1559 \\
1513\end{array}$ & $\begin{array}{l}107.30 \\
109.65 \\
111.79\end{array}$ & $\begin{array}{l}1.21 \\
3.34 \\
5.67\end{array}$ & $\begin{array}{l}0.3706 \\
0.2603 \\
0.2484\end{array}$ & $\begin{array}{l}0.04 \\
0.12 \\
0.21\end{array}$ \\
\hline$t t t t_{a}$ & $\begin{array}{l}1624 \\
1617 \\
1617 \\
1614\end{array}$ & $\begin{array}{l}1590 \\
1583 \\
1583 \\
1580\end{array}$ & $\begin{array}{r}0.00 \\
229.45 \\
229.45 \\
90.34\end{array}$ & $\begin{array}{r}24.95 \\
2.02 \\
2.02 \\
6.84\end{array}$ & $\begin{array}{l}0.031 \\
0.75 \\
0.75 \\
0.75\end{array}$ & $\begin{array}{l}0.85 \\
0.07 \\
0.07 \\
0.24\end{array}$ \\
\hline$t t t t_{p}$ & $\begin{array}{l}1621 \\
1620 \\
1615 \\
1615\end{array}$ & $\begin{array}{l}1587 \\
1586 \\
1582 \\
1581\end{array}$ & $\begin{array}{r}11.73 \\
101.61 \\
304.11 \\
97.39\end{array}$ & $\begin{array}{r}16.00 \\
0.04 \\
0.51 \\
0.64\end{array}$ & $\begin{array}{l}0.1757 \\
0.75 \\
0.75 \\
0.2783\end{array}$ & $\begin{array}{l}0.55 \\
0.00 \\
0.02 \\
0.02\end{array}$ \\
\hline
\end{tabular}


Tab. A-23. Amid III-Schwingungsmoden aus der Frequenzrechnung auf B3LYP-D3(BJ)/aVTZ-Niveau (doppelt-harmonische Näherung) für die stabilsten Monomere und Cluster von NMF. (Erklärungen zu den angegeben Größen finden sich in Tab. A-20

\begin{tabular}{|c|c|c|c|c|c|c|}
\hline Konf. & $\omega / \mathrm{cm}^{-1}$ & $0.979 \omega / \mathrm{cm}^{-1}$ & $A / \mathrm{km} \cdot \mathrm{mol}^{-1}$ & $R / \mathrm{A}^{4} \cdot \mathrm{amu}^{-1}$ & $\delta_{k}$ & $10^{34} \sigma^{\prime} / \mathrm{m}^{2} \mathrm{sr}^{-1}$ \\
\hline$c_{\mathrm{s}}$ & 1302 & 1275 & 117.90 & 5.94 & 0.3558 & 0.27 \\
\hline$t_{\mathrm{s}}$ & 1220 & 1194 & 78.02 & 10.79 & 0.5677 & 0.53 \\
\hline$t t_{\mathrm{N}}$ & $\begin{array}{l}1264 \\
1242\end{array}$ & $\begin{array}{l}1238 \\
1216\end{array}$ & $\begin{array}{l}62.36 \\
40.18\end{array}$ & $\begin{array}{l}6.80 \\
7.20\end{array}$ & $\begin{array}{l}0.5107 \\
0.5965\end{array}$ & $\begin{array}{l}0.32 \\
0.34\end{array}$ \\
\hline$t t_{\mathrm{H}}$ & $\begin{array}{l}1264 \\
1237\end{array}$ & $\begin{array}{l}1238 \\
1211\end{array}$ & $\begin{array}{l}67.17 \\
70.88\end{array}$ & $\begin{array}{r}8.11 \\
16.78\end{array}$ & $\begin{array}{l}0.6101 \\
0.4293\end{array}$ & $\begin{array}{l}0.38 \\
0.80\end{array}$ \\
\hline$c c$ & $\begin{array}{l}1375 \\
1367\end{array}$ & $\begin{array}{l}1346 \\
1338\end{array}$ & $\begin{array}{r}0.00 \\
206.60\end{array}$ & $\begin{array}{r}17.32 \\
0.00\end{array}$ & $\begin{array}{l}0.2973 \\
0\end{array}$ & $\begin{array}{l}0.73 \\
0.00\end{array}$ \\
\hline$c t$ & $\begin{array}{l}1353 \\
1235\end{array}$ & $\begin{array}{l}1324 \\
1209\end{array}$ & $\begin{array}{r}119.96 \\
95.40\end{array}$ & $\begin{array}{r}8.74 \\
16.51\end{array}$ & $\begin{array}{l}0.3034 \\
0.406\end{array}$ & $\begin{array}{l}0.38 \\
0.79\end{array}$ \\
\hline$t t t_{a}$ & $\begin{array}{l}1275 \\
1272 \\
1265\end{array}$ & $\begin{array}{l}1248 \\
1245 \\
1238\end{array}$ & $\begin{array}{r}30.55 \\
63.24 \\
5.92\end{array}$ & $\begin{array}{r}12.93 \\
4.88 \\
17.55\end{array}$ & $\begin{array}{l}0.746 \\
0.5098 \\
0.2498\end{array}$ & $\begin{array}{l}0.60 \\
0.23 \\
0.82\end{array}$ \\
\hline$t t t_{p}$ & $\begin{array}{l}1270 \\
1270 \\
1266\end{array}$ & $\begin{array}{l}1243 \\
1243 \\
1239\end{array}$ & $\begin{array}{r}5.50 \\
5.50 \\
113.20\end{array}$ & $\begin{array}{l}6.57 \\
6.56 \\
6.86\end{array}$ & $\begin{array}{l}0.75 \\
0.75 \\
0.0235\end{array}$ & $\begin{array}{l}0.30 \\
0.30 \\
0.32\end{array}$ \\
\hline$t t t_{1}$ & $\begin{array}{l}1281 \\
1263 \\
1244\end{array}$ & $\begin{array}{l}1254 \\
1236 \\
1218\end{array}$ & $\begin{array}{l}43.60 \\
46.10 \\
45.23\end{array}$ & $\begin{array}{r}9.66 \\
10.61 \\
6.98\end{array}$ & $\begin{array}{l}0.3434 \\
0.456 \\
0.5441\end{array}$ & $\begin{array}{l}0.44 \\
0.50 \\
0.33\end{array}$ \\
\hline$t t t t_{\mathrm{a}}$ & $\begin{array}{l}1307 \\
1300 \\
1300 \\
1290\end{array}$ & $\begin{array}{l}1280 \\
1273 \\
1273 \\
1263\end{array}$ & $\begin{array}{r}43.96 \\
42.94 \\
42.94 \\
0.00\end{array}$ & $\begin{array}{r}34.20 \\
4.57 \\
4.57 \\
14.33\end{array}$ & $\begin{array}{l}0.75 \\
0.75 \\
0.75 \\
0.0121\end{array}$ & $\begin{array}{l}1.53 \\
0.21 \\
0.21 \\
0.65\end{array}$ \\
\hline$t t t t_{p}$ & $\begin{array}{l}1294 \\
1293 \\
1288 \\
1288\end{array}$ & $\begin{array}{l}1267 \\
1266 \\
1261 \\
1261\end{array}$ & $\begin{array}{l}51.14 \\
15.17 \\
41.60 \\
20.56\end{array}$ & $\begin{array}{r}7.06 \\
6.13 \\
43.61 \\
2.14\end{array}$ & $\begin{array}{l}0.5715 \\
0.75 \\
0.3202 \\
0.75\end{array}$ & $\begin{array}{l}0.32 \\
0.28 \\
1.99 \\
0.10\end{array}$ \\
\hline
\end{tabular}


Tab. A-24. Experimentelle und theoretische Energieunterschiede zwischen cis- und trans-NMF, sowie Isomerisierungsbarrieren (erweiterte Liste von Tab. 5-9 in Abschnitt5.2.

\begin{tabular}{|c|c|c|c|c|}
\hline \multicolumn{5}{|l|}{ experimentell } \\
\hline Methode & Bedingungen & $\Delta G / \mathrm{kJ} \cdot \mathrm{mol}^{-1 \mathrm{a}}$ & $\Delta H / \mathrm{kJ} \cdot \mathrm{mol}^{-1}$ & $\Delta E_{\mathrm{A}} / \mathrm{kJ} \cdot \mathrm{mol}^{-1 \mathrm{f}}$ \\
\hline Raman - Jet ${ }^{b}$ & $\mathrm{He} / 373.15-483.15 \mathrm{~K}$ & $7.5(373.15)$ & $6(2)$ & - \\
\hline IR-Matrix 68 & $\mathrm{N}_{2} / 298-770 \mathrm{~K}$ & - & 5.4 & - \\
\hline IR-Matrix 249. & $\operatorname{Ar} / 298-473 \mathrm{~K}$ & - & $7.4 \pm 0.7$ & - \\
\hline IR 250 & gasförmig, $473 \mathrm{~K}$ & $8.0(473)$ & 6.7 & - \\
\hline Raman 34 & flüssig & - & - & 58.6 \\
\hline NMR 161 & in 1,2-Dichlorethan & $6.7 \pm 2.6(333.15)$ & $21 \pm 15$ & 94 \\
\hline NMR 262 & in 1,2-Dichlorethan & $6.0(263)$ & $6.1 \pm 0.2$ & 88 \\
\hline NMR 262 & gasförmig; cryogenic trapping & $9.2(814)$ & - & - \\
\hline NMR 263 & in $\mathrm{H}_{2} \mathrm{O}$ & $6.0 \pm 0.2(298)$ & $5.8 \pm 0.2$ & - \\
\hline NMR 263 & in $\mathrm{CDCl}_{3}$ & $4.7 \pm 0.2(298)$ & $3.7 \pm 0.2$ & - \\
\hline NMR 249 & in $\mathrm{CDCl}_{3}$ & $5.9 \pm 0.2(298)$ & - & - \\
\hline
\end{tabular}

\begin{tabular}{|c|c|c|c|c|c|}
\hline \multicolumn{6}{|l|}{ theoretisch } \\
\hline Methode & $\Delta E / \mathrm{kJ} \cdot \mathrm{mol}^{-1} \mathrm{~g}$ & $\Delta G / \mathrm{kJ} \cdot \mathrm{mol}^{-1 \mathrm{a}}$ & $\Delta H / \mathrm{kJ} \cdot \mathrm{mol}^{-1}$ & $\Delta E_{\mathrm{A}} / \mathrm{kJ} \cdot \mathrm{mol}^{-1 \mathrm{f}}$ & Konf. \\
\hline $\mathrm{HF} / 4-31 \mathrm{G} 334$ & 5.6 & - & - & - & $t_{\mathrm{s}} / c_{\mathrm{s}}$ \\
\hline $\mathrm{HF} / 6-31+\mathrm{G}^{\times} \mid 258$ & 5.5 & - & - & - & $t_{\mathrm{a}} / c_{\mathrm{s}}$ \\
\hline$H F / 6-31+G(d) 203$ & 5.1 & - & - & $85.7 / 78.1^{e}$ & n.a. \\
\hline $\mathrm{HF} / 6-31 \mathrm{G}(\mathrm{d}, \mathrm{p})$ 203 & 4.5 & - & - & $85.0 / 76.7^{e}$ & n. a. \\
\hline $\begin{array}{llll} & B L Y P / 6-31 G^{*} & 163 & 264 \\
\end{array}$ & 3.9 & $4.3(298.15)$ & 3,5 & - & $t_{\mathrm{s}} / c_{\mathrm{s}}$ \\
\hline B3LYP/6-31++G** 249 & [3.6] & - & - & - & $t_{\mathrm{s}} / c_{\mathrm{s}}$ \\
\hline B3LYP/6-311++G(d,p) 263] & [4.5] & - & - & - & n.a. \\
\hline B3LYP/TZV c 270 & 6.8 & - & - & - & $t_{\mathrm{a}} / c_{\mathrm{a}}$ \\
\hline B3LYP/VTZ 255 & 4.0 & - & - & 88.7 & $t_{\mathrm{s}} / c_{\mathrm{s}}$ \\
\hline B3LYP/aVTZZ 255. & 4.4 & $6.6(?)$ & - & - & $t_{\mathrm{s}} / c_{\mathrm{s}}$ \\
\hline B3LYP-D3(BJ)/aVTZ ${ }^{b}$ & $4.8[4.6]$ & $7.8(373.15)$ & $4.4^{i}$ & - & $t_{\mathrm{s}} / c_{\mathrm{s}}$ \\
\hline BHandHLYP/aVTZ 265 & [4.7] & - & - & 78.9 & $t_{\mathrm{s}} / c_{\mathrm{s}}$ \\
\hline LC-BLYP/6-311++G(d,p) 21 & {$[6.0]$} & - & - & - & $t_{\mathrm{a}} / ?$ \\
\hline$P W 91 \times c / 6-31+G^{* d} 188$ & 3.8 & - & - & - & $t_{\mathrm{s}} / c_{\mathrm{s}}$ \\
\hline$M P 2 / 6-31 G^{* *} 253$ & 5.6 & - & - & - & $t_{\mathrm{s}} / c_{\mathrm{s}}$ \\
\hline$M P 2 / 6-311+G^{* *} 266$ & 6.4 & - & - & $79.7 / 84.3^{\mathrm{e}}$ & $t_{\mathrm{a}} / c_{\mathrm{s}}$ \\
\hline MP2/6-311++G(d,p) 335 & 6.3 & - & - & - & $t_{\mathrm{s}} / c_{\mathrm{s}}$ \\
\hline MP2/6-311++G(d,p) 21 & [5.8] & - & - & - & $t_{\mathrm{a}} / ?$ \\
\hline MP2/VTZ 265 & [5.2] & - & - & 74.0 & $t_{\mathrm{s}} / c_{\mathrm{s}}$ \\
\hline MP2/aVTZZ 265 & [6.1] & - & - & 74.7 & $t_{\mathrm{s}} / c_{\mathrm{s}}$ \\
\hline MP2/aVTZ 259 & 6.5 & - & - & - & $t_{\mathrm{a}} / c_{\mathrm{s}}$ \\
\hline $\operatorname{CcSD}\left(T^{\star}\right)-F 12 a / a V T Z^{h}$ & [5.7] & - & - & 72.0 & $t_{\mathrm{s}} / c_{\mathrm{s}}$ \\
\hline $\operatorname{CCSD}\left(T^{*}\right)-F 12 a / a V T Z^{j}$ & [5.6] & - & - & 72.6 & $t_{\mathrm{s}} / c_{\mathrm{s}}$ \\
\hline $\operatorname{CcsD}(T) / 6-311++G^{* * k} 263$ & [6.2] & - & - & - & n.a. \\
\hline CASSCF $^{\prime} 255$ & 3.9 & - & - & - & $t_{\mathrm{s}} / c_{\mathrm{s}}$ \\
\hline MS-CASPT2' 255 & 4.8 & - & - & - & $t_{\mathrm{s}} / c_{\mathrm{s}}$ \\
\hline CASPT2(6-5)/VIL 259. & 4.7 & - & - & - & $t_{\mathrm{s}} / c_{\mathrm{s}}$ \\
\hline
\end{tabular}

n. a.: nicht angegeben; ${ }^{\text {a: }}$ (Temperatur in $\left.\mathrm{K}\right) ;^{\mathrm{b}}$ : aus dieser Arbeit; ${ }^{\mathrm{c}}$ : freie Enthalpie $\Delta G$ (Temperatur in $\left.\mathrm{K}\right)$ ); ${ }^{\mathrm{d}}$ : Counterpoise-Korrektur (CP) 150]; e : zwei mögliche Übergangszustände; ' : Barriere bezogen auf trans-NMF; g: elektronische [nullpunktskorrigierte]

Energieunterschiede; ${ }^{\mathrm{h}}$ : Geometrie MP2/aVTZ optimiert; ${ }^{\mathrm{i}}$ : aus Van 't-Hoff-Plot auf Basis der berechneten $\Delta G$-Werte im Temperaturbereich 373.15-483.15K; ${ }^{j}$ : Geometrie BHandHLYP/aVTZ optimiert; ${ }^{\mathrm{k}}$ : Geometrie B3LYP/6-311++G** optimiert; ': Geometrie SA-3-CASSCF(10,8)/6-31G(d) optimiert. 


\section{A.9 N-Phenylformamid}

\section{A.9.1 Ergänzende Abbildungen}

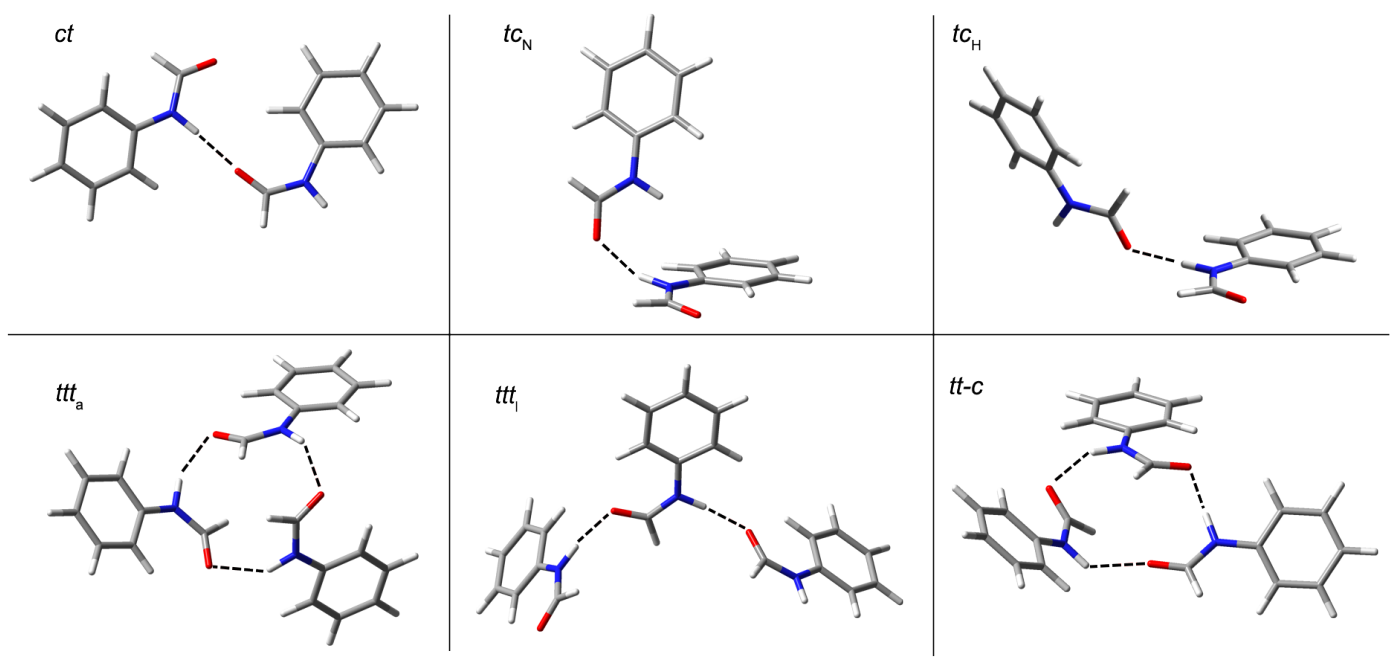

Abb. A-11. Minimumstrukturen ohne Dispersionskorrektur ausgewählter NPhF-Cluster. Dargestellt sind auf B3LYP/def2TZVP-Niveau optimierte Geometrien von $c t-, t c_{\mathrm{N}^{-}}, t c_{\mathrm{H}^{-}}, t t t_{\mathrm{a}^{-}}, t t t_{\left.\right|^{-}}$und $t t-c-N P h F$. Gestrichelte Linien zeigen intermolekulare Wasserstoffbrückenbindungen an. Relative Energien, Dissoziationsenergien und geometrische Parameter sind in den Tabellen 6-5 und 6-7 aufgeführt.

\section{A.9.2 Tabellenwerk}

Tab. A-25. NH- und CH-Streckschwingungsmoden für cis- und trans-NPhF auf B3LYP-D3(BJ)/aVTZ-Niveau. Angegeben sind die harmonische Wellenzahl $\omega$ (unskaliert und skaliert), die IR-Intensität $A$, die Raman-Aktivität $R$, das Depolarisationsverhältnis für linear polarisiertes Licht $\delta_{k}$ und der, aus der korrigierten Raman-Aktivität (GI. 3-16) berechneten, differentielle Raman-Streuquerschnitt $\sigma^{\prime}$ bei $532.269 \mathrm{~nm}$ Anregungswellenlänge und $90^{\circ}$ Streugeometrie (GI. 3-13). Details zur Rechenmethode, siehe Abschnitt 3.4

\begin{tabular}{ccccccc}
\hline Konf. & $\omega / \mathrm{cm}^{-1}$ & $0.9595 \omega / \mathrm{cm}^{-1}$ & $A / \mathrm{km} \cdot \mathrm{mol}^{-1}$ & $R / \mathrm{A}^{4} \cdot \mathrm{amu}^{-1}$ & \multicolumn{1}{c}{$\delta_{k}$} & $10^{34} \sigma^{\prime} / \mathrm{m}^{2} \mathrm{sr}^{-1}$ \\
\hline \multirow{6}{*}{ cis } & 3581 & 3436 & 28.72 & 103.88 & 0.1196 & 1.05 \\
& 3202 & 3072 & 2.97 & 240.21 & 0.0977 & 2.97 \\
& 3196 & 3066 & 16.13 & 71.33 & 0.2357 & 0.83 \\
& 3184 & 3055 & 13.24 & 82.49 & 0.6062 & 0.86 \\
& 3175 & 3046 & 0.12 & 92.58 & 0.5605 & 0.98 \\
& 3165 & 3037 & 7.01 & 39.88 & 0.5605 & 0.43 \\
& 2990 & 2869 & 39.64 & 51.20 & 0.3747 & 0.63 \\
\hline \multirow{6}{*}{ trans } & 3182 & 3460 & 21.55 & 100.61 & 0.1280 & 1.00 \\
& 3607 & 3113 & 4.94 & 48.39 & 0.1655 & 0.57 \\
& 3173 & 3067 & 12.67 & 241.60 & 0.1122 & 2.97 \\
& 3153 & 3044 & 18.00 & 69.80 & 0.7263 & 0.71 \\
& 3026 & 0.30 & 89.83 & 0.5336 & 0.96 \\
\hline
\end{tabular}


Tab. A-26. NH-Streckschwingungsmoden aus der Frequenzrechnung auf B3LYP-D3(BJ)/def2TZVP-Niveau (doppeltharmonische Näherung) für die stabilsten Monomere und Cluster von NPhF. Angegeben sind die harmonische Wellenzahl $\omega$ (unskaliert und skaliert), die IR-Intensität $A$, die Raman-Aktivität $R$, das Depolarisationsverhältnis für linear polarisiertes Licht $\delta_{k}$ und der, aus der korrigierten Raman-Aktivität (GI. 3-16) berechneten, differentielle Raman-Streuquerschnitt $\sigma^{\prime}$ bei $532.269 \mathrm{~nm}$ Anregungswellenlänge und $90^{\circ}$ Streugeometrie (Gl. 3-13. Details zur Rechenmethode, siehe Abschnitt 3.4

\begin{tabular}{|c|c|c|c|c|c|c|}
\hline Konf. & $\omega / \mathrm{cm}^{-1}$ & $0.9595 \omega / \mathrm{cm}^{-1}$ & $A / \mathrm{km} \cdot \mathrm{mol}^{-1}$ & $R / \mathrm{A}^{4} \cdot \mathrm{amu}^{-1}$ & $\delta_{k}$ & $10^{34} \sigma^{\prime} / \mathrm{m}^{2} \mathrm{sr}^{-1}$ \\
\hline$c$ & 3574 & 3430 & 28.92 & 99.71 & 0.1411 & 1.00 \\
\hline$t$ & 3598 & 3452 & 21.27 & 96.38 & 0.153 & 0.95 \\
\hline$t t_{N}$ & $\begin{array}{l}3600 \\
3492\end{array}$ & $\begin{array}{l}3454 \\
3351\end{array}$ & $\begin{array}{r}31.35 \\
194.68\end{array}$ & $\begin{array}{l}99.90 \\
86.34\end{array}$ & $\begin{array}{l}0.1817 \\
0.0942\end{array}$ & $\begin{array}{l}0.98 \\
0.92\end{array}$ \\
\hline$t t_{\mathrm{H}}$ & $\begin{array}{l}3597 \\
3427\end{array}$ & $\begin{array}{l}3451 \\
3289\end{array}$ & $\begin{array}{r}28.80 \\
598.81\end{array}$ & $\begin{array}{r}85.34 \\
375.00\end{array}$ & $\begin{array}{l}0.1236 \\
0.2113\end{array}$ & $\begin{array}{l}0.86 \\
3.94\end{array}$ \\
\hline$c c$ & $\begin{array}{l}3249 \\
3201\end{array}$ & $\begin{array}{l}3118 \\
3071\end{array}$ & $\begin{array}{r}1928.68 \\
0.0094\end{array}$ & $\begin{array}{c}0.018 \\
1331.46\end{array}$ & $\begin{array}{l}0.75 \\
0.1873\end{array}$ & $\begin{array}{r}0.00 \\
15.82\end{array}$ \\
\hline$c t$ & $\begin{array}{l}3600 \\
3486\end{array}$ & $\begin{array}{l}3455 \\
3345\end{array}$ & $\begin{array}{r}26.84 \\
149.25\end{array}$ & $\begin{array}{l}77.75 \\
80.95\end{array}$ & $\begin{array}{l}0.1662 \\
0.0872\end{array}$ & $\begin{array}{l}0.76 \\
0.87\end{array}$ \\
\hline$t c_{N}$ & $\begin{array}{l}3492 \\
3473\end{array}$ & $\begin{array}{l}3351 \\
3333\end{array}$ & $\begin{array}{r}431.42 \\
77.15\end{array}$ & $\begin{array}{l}206.60 \\
241.84\end{array}$ & $\begin{array}{l}0.3283 \\
0.1097\end{array}$ & $\begin{array}{l}2.01 \\
2.60\end{array}$ \\
\hline$t c_{\mathrm{H}}$ & $\begin{array}{l}3571 \\
3430\end{array}$ & $\begin{array}{l}3426 \\
3291\end{array}$ & $\begin{array}{c}37.721 \\
452.62\end{array}$ & $\begin{array}{l}105.79 \\
240.15\end{array}$ & $\begin{array}{l}0.1251 \\
0.1786\end{array}$ & $\begin{array}{l}1.08 \\
2.55\end{array}$ \\
\hline$t t t_{a}$ & $\begin{array}{l}3536 \\
3527 \\
3501\end{array}$ & $\begin{array}{l}3393 \\
3384 \\
3360\end{array}$ & $\begin{array}{l}117.34 \\
112.12 \\
126.35\end{array}$ & $\begin{array}{r}46.67 \\
113.65 \\
134.72\end{array}$ & $\begin{array}{l}0.1794 \\
0.1684 \\
0.1206\end{array}$ & $\begin{array}{l}0.47 \\
1.16 \\
1.42\end{array}$ \\
\hline$t t-c$ & $\begin{array}{l}3508 \\
3430 \\
3267\end{array}$ & $\begin{array}{l}3366 \\
3291 \\
3135\end{array}$ & $\begin{array}{l}101.74 \\
664.10 \\
913.85\end{array}$ & $\begin{array}{r}50.67 \\
169.61 \\
559.89\end{array}$ & $\begin{array}{l}0.0641 \\
0.1629 \\
0.2296\end{array}$ & $\begin{array}{l}0.55 \\
1.82 \\
6.32\end{array}$ \\
\hline$t-t c$ & $\begin{array}{l}3523 \\
3502 \\
3377\end{array}$ & $\begin{array}{l}3380 \\
3360 \\
3240\end{array}$ & $\begin{array}{l}131.40 \\
198.41 \\
584.58\end{array}$ & $\begin{array}{l}151.63 \\
105.08 \\
219.90\end{array}$ & $\begin{array}{l}0.2091 \\
0.1145 \\
0.1744\end{array}$ & $\begin{array}{l}1.52 \\
1.11 \\
2.40\end{array}$ \\
\hline$c-c c$ & $\begin{array}{l}3292 \\
3253 \\
3219\end{array}$ & $\begin{array}{l}3158 \\
3121 \\
3088\end{array}$ & $\begin{array}{r}866.82 \\
1716.62 \\
17.27\end{array}$ & $\begin{array}{l}336.07 \\
143.24 \\
544.87\end{array}$ & $\begin{array}{l}0.2025 \\
0.2439 \\
0.2722\end{array}$ & $\begin{array}{l}3.79 \\
1.62 \\
6.21\end{array}$ \\
\hline$c c c_{\mathrm{cyc}}$ & $\begin{array}{l}3362 \\
3362 \\
3337\end{array}$ & $\begin{array}{l}3226 \\
3226 \\
3202\end{array}$ & $\begin{array}{r}1287.10 \\
1287.08 \\
3.55\end{array}$ & $\begin{array}{l}108.55 \\
108.53 \\
923.72\end{array}$ & $\begin{array}{l}0.75 \\
0.75 \\
0.1153\end{array}$ & $\begin{array}{r}1.00 \\
1.00 \\
10.57\end{array}$ \\
\hline
\end{tabular}




\section{A.10 $\gamma$-Butyrolactam}

\section{A.10.1 Ergänzende Abbildungen}
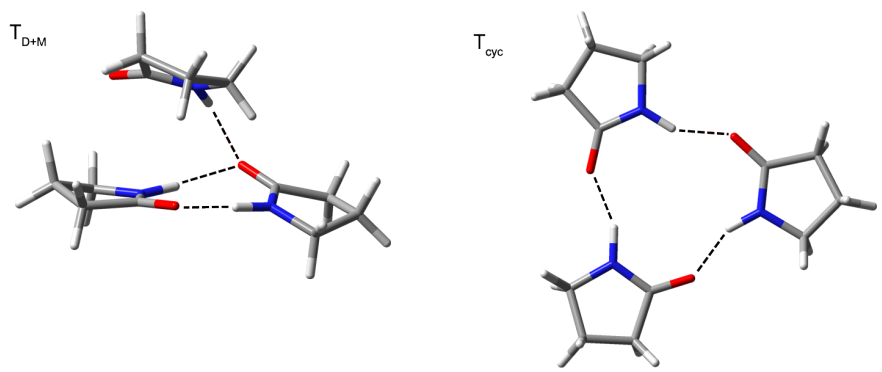

Abb. A-12. Zwei stabile Strukturen des $\gamma$-Butyrolactam-Trimers nach Geometrieoptimierung auf B3LYP-D3(BJ)/aVTZNiveau.

\section{A.10.2 Tabellenwerk}

Tab. A-27. NH-Streckschwingungsmoden aus der Frequenzrechnung auf B3LYP-D3(BJ)/aVTZ-Niveau (doppelt-harmonische Näherung) für das Monomer und die stabilsten Cluster von $\gamma \mathrm{BL}$. Angegeben sind die harmonische Wellenzahl $\omega$ (unskaliert und skaliert), die IR-Intensität $A$, die Raman-Aktivität $R$, das Depolarisationsverhältnis für linear polarisiertes Licht $\delta_{k}$ und der, aus der korrigierten Raman-Aktivität (Gl. 3-16) berechneten, differentielle Raman-Streuquerschnitt $\sigma^{\prime}$ bei $532.2847 \mathrm{~nm}$ Anregungswellenlänge und $90^{\circ}$ Streugeometrie (Gl. 3-13. (Details zur Rechenmethode, siehe Abschnitt 3.4

\begin{tabular}{llccccc}
\hline Konf. & $\omega / \mathrm{cm}^{-1}$ & $0.961 \omega / \mathrm{cm}^{-1}$ & $A / \mathrm{km} \cdot \mathrm{mol}^{-1}$ & $R / \mathrm{A}^{4} \cdot \mathrm{amu}^{-1}$ & \multicolumn{1}{c}{$\delta_{k}$} & $10^{34} \sigma^{\prime} / \mathrm{m}^{2} \mathrm{sr}^{-1}$ \\
\hline $\mathrm{M}_{S}$ & 3626 & 3484 & 32.13 & 124.40 & 0.21 & 1.19 \\
$\mathrm{D}_{2 \mathrm{H}}$ & 3274 & 3146 & 2208.13 & 0.00 & 0 & 0.00 \\
& 3222 & 3096 & 0.00 & 654.37 & 0.2977 & 7.37 \\
$\mathrm{D}_{1 \mathrm{H}}$ & 3625 & 3484 & 35.13 & 125.64 & 0.2071 & 1.20 \\
& 3362 & 3231 & 898.91 & 376.13 & 0.2923 & 3.95 \\
$\mathrm{~T}_{\mathrm{cyc}}$ & 3371 & 3240 & 1588.12 & 188.69 & 0.75 & 1.73 \\
& 3371 & 3240 & 1588.12 & 188.69 & 0.75 & 1.73 \\
& 3340 & 3210 & 0.13 & 719.19 & 0.116 & 8.22 \\
$\mathrm{~T}_{\mathrm{D}+\mathrm{M}}$ & 3548 & 3409 & 287.60 & 145.07 & 0.2005 & 1.44 \\
& 3398 & 3265 & 630.29 & 143.94 & 0.3087 & 1.48 \\
& 3163 & 3040 & 959.35 & 294.62 & 0.3015 & 3.42 \\
\hline
\end{tabular}




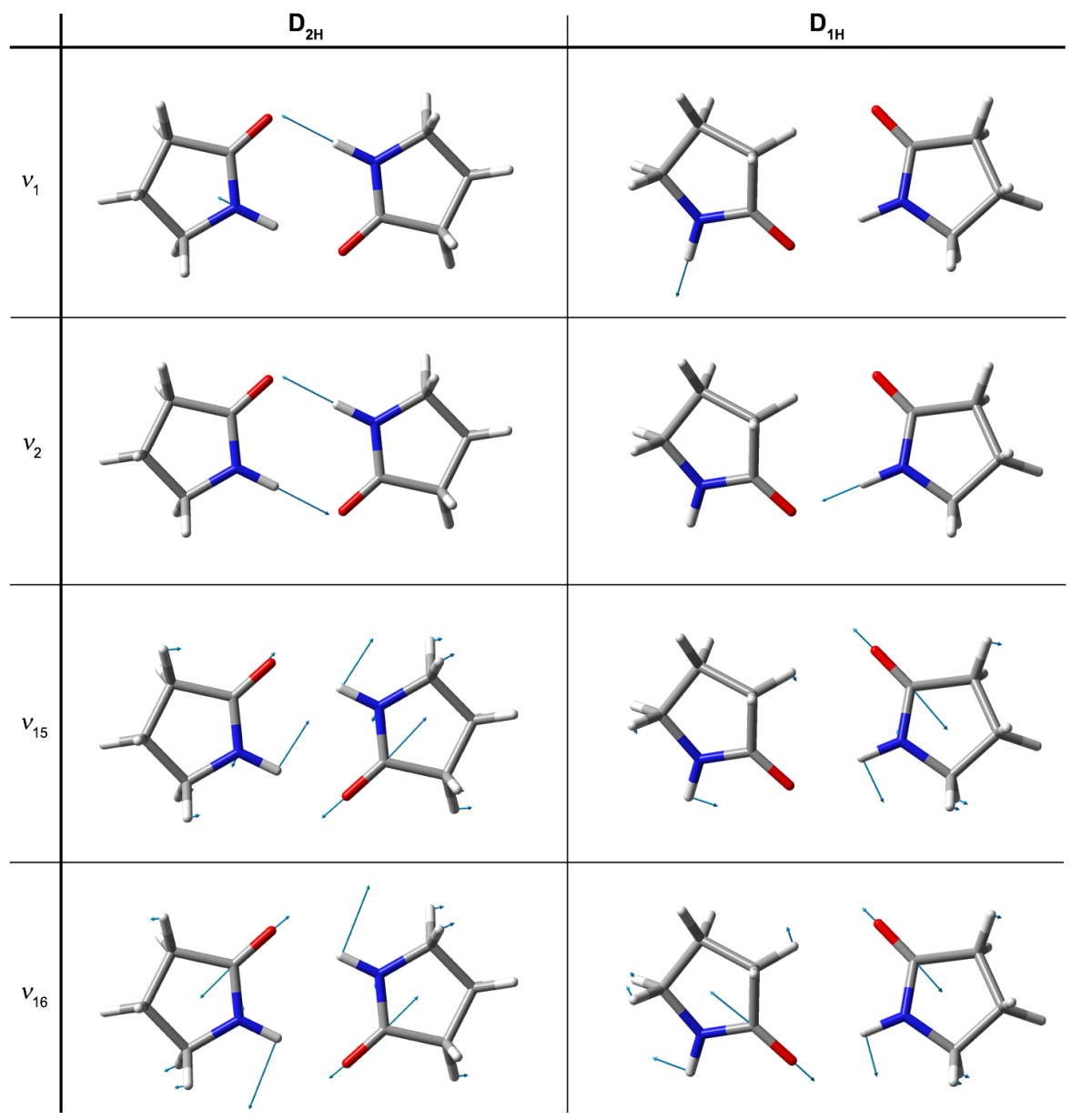

Abb. A-13. Illustrierte Normalkoordinaten der $\mathrm{N}-\mathrm{H}-\left(v_{1,2}\right)$ und $\mathrm{C}=\mathrm{O}-\mathrm{Streckschwingungsmode}\left(v_{15}, 16\right)$ von $\mathrm{D}_{2 \mathrm{H}^{-}}$und

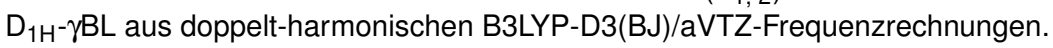

Tab. A-28. CO-Streckschwingungsmoden aus der Frequenzrechnung auf B3LYP-D3(BJ)/aVTZ-Niveau (doppelt-harmonische Näherung) für das Monomer und die stabilsten Cluster von $\gamma B$ L. Angaben wie in Tab. A-27. Für Details zur Rechenmethode siehe Abschnitt 3.4

\begin{tabular}{llccccc}
\hline Konf. & $\omega / \mathrm{cm}^{-1}$ & $0.99 \omega / \mathrm{cm}^{-1}$ & $A / \mathrm{km} \cdot \mathrm{mol}^{-1}$ & $R / \mathrm{A}^{4} \cdot \mathrm{amu}^{-1}$ & \multicolumn{1}{c}{$\delta_{k}$} & $10^{34} \sigma^{\prime} / \mathrm{m}^{2} \mathrm{sr}^{-1}$ \\
\hline $\mathrm{M}_{S}$ & 1783 & 1765 & 516.23 & 16.65 & 0.4164 & 0.44 \\
$\mathrm{D}_{2 \mathrm{H}}$ & 1753 & 1736 & 1139.30 & 0.00 & 0 & 0.00 \\
& 1714 & 1697 & 0.00 & 17.23 & 0.2232 & 0.50 \\
$\mathrm{D}_{1 \mathrm{H}}$ & 1762 & 1745 & 1055.90 & 4.28 & 0.7407 & 0.11 \\
& 1734 & 1717 & 105.93 & 30.01 & 0.2093 & 0.87 \\
$\mathrm{~T}_{\mathrm{cyc}}$ & 1753 & 1735 & 1108.85 & 12.54 & 0.75 & 0.02 \\
& 1753 & 1735 & 1108.86 & 12.54 & 0.75 & 0.02 \\
& 1702 & 1685 & 0.35 & 48.94 & 0.1311 & 0.004 \\
$\mathrm{~T}_{\mathrm{D}+\mathrm{M}}$ & 1762 & 1744 & 700.72 & 7.07 & 0.3441 & 0.19 \\
& 1732 & 1720 & 740.46 & 0.26 & 0.6416 & 0.007 \\
& 1700 & 1683 & 42.92 & 16.62 & 0.1488 & 0.50 \\
\hline
\end{tabular}


Tab. A-29. Weitere Schwingungsmoden in der Amid I-III-Region aus der Frequenzrechnung auf B3LYP-D3(BJ)/aVTZNiveau (doppelt-harmonische Näherung) für das Monomer und das cyclische Dimer von $\gamma B \mathrm{BL}$. Angaben wie in Tab. A-27. Für Details zur Rechenmethode siehe Abschnitt 3.4

\begin{tabular}{|c|c|c|c|c|c|c|}
\hline Konf. & $\omega / \mathrm{cm}^{-1}$ & $0.99 \omega / \mathrm{cm}^{-1}$ & $A / \mathrm{km} \cdot \mathrm{mol}^{-1}$ & $R / \mathrm{A}^{4} \cdot \mathrm{amu}^{-1}$ & $\delta_{k}$ & $10^{35} \sigma^{\prime} / \mathrm{m}^{2} \mathrm{sr}^{-1}$ \\
\hline \multirow[t]{14}{*}{$\mathrm{M}_{S}$} & 1002 & 992 & 11.36 & 1.87 & 0.701 & 0.96 \\
\hline & 1074 & 1063 & 16.65 & 2.30 & 0.7373 & 1.08 \\
\hline & 1097 & 1086 & 0.43 & 0.54 & 0.721 & 0.25 \\
\hline & 1191 & 1179 & 4.20 & 0.45 & 0.7215 & 0.19 \\
\hline & 1213 & 1201 & 0.81 & 3.64 & 0.5211 & 1.53 \\
\hline & 1247 & 1235 & 30.43 & 4.56 & 0.6552 & 1.80 \\
\hline & 1271 & 1259 & 84.38 & 3.34 & 0.6233 & 1.29 \\
\hline & 1305 & 1292 & 16.03 & 0.95 & 0.7156 & 0.35 \\
\hline & 1337 & 1324 & 10.10 & 1.19 & 0.4464 & 0.45 \\
\hline & 1370 & 1356 & 6.17 & 2.90 & 0.7403 & 1.00 \\
\hline & 1447 & 1433 & 31.99 & 0.93 & 0.3456 & 0.33 \\
\hline & 1473 & 1458 & 3.71 & 5.82 & 0.7408 & 1.83 \\
\hline & 1504 & 1489 & 6.81 & 4.75 & 0.729 & 1.45 \\
\hline & 1539 & 1523 & 3.22 & 7.89 & 0.5195 & 2.45 \\
\hline \multirow[t]{28}{*}{$\mathrm{D}_{2 \mathrm{H}}$} & 1011 & 1001 & 0.00 & 6.11 & 0.7498 & 3.07 \\
\hline & 1012 & 1002 & 8.68 & 0.00 & 0 & 0.00 \\
\hline & 1090 & 1079 & 0.00 & 3.30 & 0.7071 & 1.52 \\
\hline & 1093 & 1082 & 9.55 & 0.00 & 0 & 0.00 \\
\hline & 1103 & 1092 & 0.00 & 1.79 & 0.5938 & 8.29 \\
\hline & 1105 & 1094 & 8.73 & 0.00 & 0 & 0.00 \\
\hline & 1190 & 1178 & 0.00 & 1.05 & 0.5946 & 4.46 \\
\hline & 1190 & 1179 & 7.18 & 0.00 & 0 & 0.00 \\
\hline & 1218 & 1206 & 0.00 & 5.51 & 0.5507 & 2.28 \\
\hline & 1219 & 1207 & 2.27 & 0.00 & 0 & 0.00 \\
\hline & 1252 & 1240 & 5.60 & 0.00 & 0 & 0.00 \\
\hline & 1252 & 1240 & 0.00 & 11.00 & 0.7333 & 4.25 \\
\hline & 1301 & 1288 & 41.75 & 0.00 & 0 & 0.00 \\
\hline & 1303 & 1290 & 0.00 & 2.40 & 0.352 & 0.96 \\
\hline & 1313 & 1300 & 0.00 & 4.97 & 0.4009 & 1.94 \\
\hline & 1314 & 1301 & 105.80 & 0.00 & 0 & 0.00 \\
\hline & 1341 & 1328 & 0.00 & 1.23 & 0.2766 & 0.48 \\
\hline & 1342 & 1328 & 10.43 & 0.00 & 0 & 0.00 \\
\hline & 1413 & 1399 & 73.28 & 0.00 & 0 & 0.00 \\
\hline & 1428 & 1414 & 0.00 & 12.22 & 0.7197 & 4.01 \\
\hline & 1474 & 1459 & 8.76 & 0.00 & 0 & 0.00 \\
\hline & 1474 & 1460 & 0.00 & 14.47 & 0.7192 & 4.55 \\
\hline & 1500 & 1485 & 7.21 & 0.00 & 0 & 0.00 \\
\hline & 1501 & 1486 & 0.00 & 5.84 & 0.684 & 1.81 \\
\hline & 1519 & 1504 & 9.56 & 0.00 & 0 & 0.00 \\
\hline & 1521 & 1505 & 0.00 & 24.15 & 0.2985 & 8.05 \\
\hline & 1538 & 1522 & 17.41 & 0.00 & 0 & 0.00 \\
\hline & 1538 & 1522 & 0.00 & 14.66 & 0.5533 & 4.51 \\
\hline
\end{tabular}

Tab. A-30. NH- und CO-Streckschwingungsmoden aus der Frequenzrechnung auf B3LYP-D3(BJ)/def2TZVP-Niveau (doppelt-harmonische Näherung) für das $M_{S}$-Monomer und das $D_{2 H}$-Dimer von $\gamma B L$. Angaben wie in Tab. $A-27$ Für Details zur Rechenmethode siehe Abschnitt 3.4

\begin{tabular}{llrrll}
\hline Konf. & $\omega / \mathrm{cm}^{-1}$ & $A / \mathrm{km} \cdot \mathrm{mol}^{-1}$ & $R / \mathrm{A}^{4} \cdot \mathrm{amu}^{-1}$ & \multicolumn{1}{l}{$\delta_{k}$} & $10^{34} \sigma^{\prime} / \mathrm{m}^{2} \mathrm{sr}^{-1}$ \\
\hline $\mathrm{M}_{S}$ & 3620 & 32.18 & 123.08 & 0.2405 & 1.16 \\
& 1793 & 488.46 & 10.99 & 0.5537 & 0.28 \\
\hline $\mathrm{D}_{2 \mathrm{H}}$ & 3279 & 2140.85 & 0.00 & 0 & 0 \\
& 3228 & 0.00 & 660.79 & 0.2952 & 7.4 \\
& 1761 & 1116.84 & 0.00 & 0 & 0 \\
& 1724 & 0.00 & 12.60 & 0.2970 & 0.36 \\
\hline
\end{tabular}


Tab. A-31. Harmonische sowie anharmonische $\mathrm{N}-\mathrm{H}$ - und $\mathrm{C}=\mathrm{O}-\mathrm{Streckschwingungsfrequenzen} \mathrm{des} \mathrm{Monomers} \mathrm{und} \mathrm{der}$ Dimere von $\gamma \mathrm{BL}$. Experimentelle Raman- und FTIR-Daten aus Jet-Experimenten sind zum Vergleich aufgeführt. Alle Werte sind in $\mathrm{cm}^{-1}$ angegeben.

\begin{tabular}{|c|c|c|c|c|c|c|}
\hline & \multicolumn{3}{|c|}{$v_{\mathrm{NH}}$} & \multicolumn{3}{|c|}{$v_{\mathrm{CO}}$} \\
\hline & M & $\mathrm{D}_{2 \mathrm{H}}{ }^{\mathrm{g}}$ & $D_{1 H}{ }^{g}$ & M & $\mathrm{D}_{2 \mathrm{H}}{ }^{\mathrm{g}}$ & $\mathrm{D}_{1 \mathrm{H}} \mathrm{g}$ \\
\hline \multicolumn{7}{|l|}{ doppelt-harmonisch: } \\
\hline B3PW91/6-311++G** 308 & 3628 & 3317 & $3628 / 3424$ & 1809 & 1775 & $1786 / 1780$ \\
\hline B3PW91/aVDZ 307 & 3644 & 3254 & - & 1800 & 1770 & - \\
\hline B3LYP/6-311+G* 175 & 3628 & 3369 & - & 1789 & 1760 & - \\
\hline B3LYP/6-311++G(d,p) 307. & 3627 & 3328 & - & 1786 & 1755 & - \\
\hline B3LYP/aVDZ 307] & 3621 & 3280 & - & 1777 & 1749 & - \\
\hline B3LYP-D3(BJ)/aVIZa & 3626 & 3274 / 3222 & 3625 / 3362 & 1783 & $1753 / 1714$ & $1762 / 1734$ \\
\hline \multicolumn{7}{|l|}{ anharmonisch: ${ }^{d}$} \\
\hline B3LYP-D3(BJ)/VTZ + VPT2 ${ }^{\mathrm{a}}$ & 3467 [3631] & - & - & 1770 [1801] & - & - \\
\hline $\mathrm{MP} 2 / 6-31 \mathrm{G} \dagger^{\mathrm{e}}+\mathrm{VPT} 2$ 306. & $3559[-]$ & $3151 / 3118[-]$ & $3559 / 3331[-]$ & $1755[-]$ & & - \\
\hline \multicolumn{7}{|l|}{ experimentell: } \\
\hline Raman-Jet ${ }^{\mathrm{a}, \mathrm{f}}$ & 3484 & $<3400$ & - & 1765 & 1691 & - \\
\hline FTIR-Jet 175 & 3485 & $3275-3047$ & - & 1767 & 1729 & - \\
\hline
\end{tabular}

a: aus dieser Arbeit; ${ }^{\mathrm{b}}$ : BSSE-korrigiert; ${ }^{\mathrm{c}}$ : Wellenzahlen liegen nur skaliert vor $\left(\tilde{v}_{\mathrm{sc}}\right)$ und wurden entsprechend zurückgerechnet 308] $\tilde{v}_{\mathrm{sc}}=0.958 \cdot \tilde{v}_{\text {calc }} ;{ }^{d}$ : zugehörige harmonische Daten in eckigen Klammern; ${ }^{e}:$ Kombination aus 6-31G und 6-31G**; : Bandenposition am Intensitätsmaximum; 9: antisymmetrisch / "symmetrisch“" (siehe Text und Abb. A-13 in Anhang A.10.1.

Tab. A-32. Literaturwerte der Amid A und Amid I-Bandenpositionen des Monomers und der Dimere von $\gamma \mathrm{BL}$ in Lösung. Alle Werte sind in $\mathrm{cm}^{-1}$ angegeben.

\begin{tabular}{|c|c|c|c|c|c|c|c|c|}
\hline & \multicolumn{4}{|c|}{ Amid A } & \multicolumn{4}{|c|}{ Amid I } \\
\hline & $M$ & $\mathrm{D}_{2 \mathrm{H}}$ & $D_{1 H} e^{e}$ & weitere Banden & M & $\mathrm{D}_{2 \mathrm{H}}$ & $D_{1 \mathrm{H}}{ }^{e}$ & weitere Banden \\
\hline $\mathrm{CCl}_{4}$ a 308 & 3455 & $\approx 3200$ & - & - & 1714 & 1699 & - & $1729^{c}$ \\
\hline $\mathrm{CCl}_{4} 3 \overline{306}$ & 3452 & 3106 & 3452 / 3360 & - & 1699 & - & - & - \\
\hline $\mathrm{CCl}_{4}[179$ & 3453 & - & - & $3350,3204,3105^{f}$ & - & - & - & - \\
\hline $\mathrm{CCl}_{4} \mathrm{~b} 312313$ & 3460 & 3210 & - & - & 1716 & 1701 & - & - \\
\hline $\mathrm{C}_{6} \mathrm{H}_{6}$ b 313 & 3434 & 3209 & - & - & 1715 & 1701 & - & - \\
\hline $\begin{array}{llll}\mathrm{C}_{6} \mathrm{H}_{12} & \text { b } & 312 & 313 \\
\end{array}$ & 3462 & 3205 & - & - & 1732 & 1712 & - & - \\
\hline $\mathrm{CS}_{2} \mathrm{~b} 313$ & 3453 & 3201 & - & - & 1715 & 1701 & - & - \\
\hline $\mathrm{CH}_{2} \mathrm{Cl}_{2} \overline{336}$ & 3437 & 3224 & - & - & - & $1696^{d}$ & - & - \\
\hline
\end{tabular}

${ }^{\mathrm{a}}: c=0.017 \mathrm{M} ;{ }^{\mathrm{b}}: c=0.01 \mathrm{M} ;^{\mathrm{c}}:$ vermutlich Fermi-Resonanzen; ${ }^{\mathrm{d}}:$ nur eine Bande angegeben und $\mathrm{D}_{2 \mathrm{H}}$ als vorherrschende Spezies

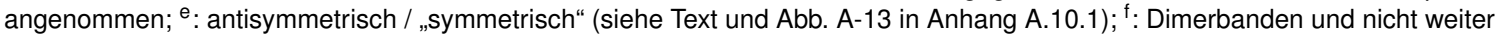
zugeordnet. 


\section{A.11 Längerkettige Amide}

Die cis- und trans-Konformere längerkettiger Alkylamide wurden in Kapitel 7 mit einer eindeutigen Konformationsnomenklatur belegt und deren Energieunterschiede beschrieben und diskutiert. Die in Tabelle A-33 aufgeführten Konformere sind neben den fettgedruckten globalen Minima noch die voll anti-ständigen (all-trans) Konformere und einige nahezu isoenergetische Strukturalternativen zu allen besprochenen Alkylamiden (abgesehen von NMF und NMA, denen ein eigenes Kapitel gewidmet ist.). Angegeben sind die Diederwinkel gemäß der Nomenklatur in Abb. 7-1] und der elektronische und nullpunktskorrigierte Energieunterschied zur globalen Minimumsstruktur des jeweiligen Moleküls. 
Tab. A-33. Diederwinkel (in $\left.{ }^{\circ}\right)$ und relative elektronische $\left(\Delta E_{\mathrm{r}, \text { el }}\right)$ sowie nullpunktskorrigierte $\left(\Delta E_{\mathrm{r}, 0}\right)$ Energien der stabilsten Konformere und der all-trans-Konformere von Amiden mit längeren, unverzweigten Alkylketten. Das jeweils stabilste cis- und trans-Konformer ist fett gedruckt. (Für Details zur Konformationsnomenklatur und Molekülbezeichungen siehe Abschnitt 7)

\begin{tabular}{|c|c|c|c|c|c|c|c|c|c|c|}
\hline Amid & Konformer & $\omega$ & $\varphi$ & $\varphi^{\prime}$ & $\varphi^{\prime \prime}$ & $\psi$ & $\psi^{\prime}$ & $\psi^{\prime \prime}$ & $\Delta E_{\mathrm{r}, \mathrm{el}} / \mathrm{kJ} \cdot \mathrm{mol}^{-1}$ & $\Delta E_{\mathrm{r}, 0} / \mathrm{kJ} \cdot \mathrm{mol}^{-1}$ \\
\hline \multirow[t]{4}{*}{ NMP } & $t_{\mathrm{a}, \mathrm{aa}}$ & 180 & 180 & & & 180 & 180 & & 0.8 & $\mathrm{~N} / \mathrm{A}$ \\
\hline & $t_{\mathrm{s},+\mathrm{aca}}$ & 177 & 9 & & & 139 & 175 & & 0.1 & 0.4 \\
\hline & $t_{\mathrm{a},+\mathbf{a c a}}$ & 177 & 176 & & & 140 & 175 & & 0.0 & 0.0 \\
\hline & $c_{\mathrm{a}, \mathrm{aa}}$ & 0 & 180 & & & 180 & 180 & & 8.1 & 8.2 \\
\hline \multirow[t]{4}{*}{ NEF } & $t_{\mathrm{aa}}$ & 180 & 180 & 180 & & & & & 1.9 & $\mathrm{~N} / \mathrm{A}$ \\
\hline & $t_{+ \text {sca }}$ & -178 & 86 & -170 & & & & & 0.0 & 0.0 \\
\hline & $c_{\mathrm{aa}}$ & 0 & 180 & 180 & & & & & 10.4 & $\mathrm{~N} / \mathrm{A}$ \\
\hline & $\boldsymbol{c}_{+ \text {aca }}$ & 4 & 109 & 179 & & & & & 4.9 & 4.3 \\
\hline \multirow[t]{4}{*}{ NEA } & $t_{\mathrm{aa}, \mathrm{a}}$ & 180 & 180 & 180 & & 180 & & & 3.5 & $\mathrm{~N} / \mathrm{A}$ \\
\hline & $\boldsymbol{t}_{+\mathbf{s c a}, \mathbf{s}}$ & -178 & 85 & 180 & & -2 & & & 0.0 & 0.0 \\
\hline & $c_{\mathrm{aa}, \mathrm{a}}$ & 6 & -170 & 180 & & -179 & & & 10.2 & 10.1 \\
\hline & $\boldsymbol{c}_{+\mathrm{sca}, \mathrm{a}}$ & 9 & 89 & 179 & & -178 & & & 9.4 & 10.0 \\
\hline \multirow[t]{5}{*}{ NEP } & $t_{\mathrm{aa}, \mathrm{aa}}$ & 180 & 180 & 180 & & 180 & 180 & & 3.0 & $\mathrm{~N} / \mathrm{A}$ \\
\hline & $t_{+\mathrm{sca}, \mathrm{sa}}$ & -178 & 85 & 180 & & 2 & 180 & & 2.9 & 3.1 \\
\hline & $\boldsymbol{t}_{+\mathbf{s c a},-\mathbf{a c a}}$ & -175 & 84 & 180 & & -140 & -175 & & 0.0 & 0.0 \\
\hline & $c_{\mathrm{aa}, \mathrm{aa}}$ & -2 & 177 & 180 & & 180 & 180 & & 9.7 & 8.9 \\
\hline & $C_{+ \text {sca, aa }}$ & 10 & 88 & 179 & & -179 & 180 & & 9.3 & 9.5 \\
\hline \multirow[t]{7}{*}{ NPF } & $t_{\text {aaa }}$ & 180 & 180 & 180 & 180 & & & & 2.8 & $\mathrm{~N} / \mathrm{A}$ \\
\hline & $t_{+\mathrm{sc}+\mathrm{sca}}$ & -179 & 84 & 63 & 179 & & & & 0.3 & 0.6 \\
\hline & $t_{+\mathrm{ac}-\mathrm{sca}}$ & -179 & 105 & -63 & 180 & & & & 0.2 & 0.5 \\
\hline & $\boldsymbol{t}_{+ \text {scaa }}$ & -178 & 86 & -179 & 180 & & & & 0.0 & 0.0 \\
\hline & $c_{\text {aaa }}$ & 0 & 180 & 180 & 180 & & & & 11.1 & $\mathrm{~N} / \mathrm{A}$ \\
\hline & $\boldsymbol{c}_{+\mathbf{a c}-\mathbf{s c a}}$ & 5 & 106 & -64 & 180 & & & & 4.1 & 3.9 \\
\hline & $c_{\text {+acaa }}$ & 4 & 107 & 179 & 180 & & & & 5.1 & 4.4 \\
\hline \multirow[t]{8}{*}{ NPA } & $t_{\mathrm{aaa}, \mathrm{a}}$ & 180 & 180 & 180 & 180 & 180 & & & 4.4 & $\mathrm{~N} / \mathrm{A}$ \\
\hline & $t_{+\mathrm{sc}+\mathrm{sca}, \mathrm{s}}$ & -178 & 84 & 63 & 179 & -1 & & & 0.2 & 0.5 \\
\hline & $t_{+\mathrm{ac}-\mathrm{sca}, \mathrm{s}}$ & -179 & 105 & -63 & 180 & -1 & & & 0.2 & 0.6 \\
\hline & $\boldsymbol{t}_{+\mathrm{scaa}, \mathrm{s}}$ & -178 & 85 & -179 & 180 & -1 & & & 0.0 & 0.0 \\
\hline & $C_{\text {aaa, a }}$ & 0 & 180 & 180 & 180 & 180 & & & 11.0 & $\mathrm{~N} / \mathrm{A}$ \\
\hline & $C_{+\mathrm{sc}+\mathrm{sca}, \mathrm{a}}$ & 9 & 88 & 61 & 179 & -178 & & & 9.3 & 10.2 \\
\hline & $c_{+}$ac-sca, a & 7 & 106 & -64 & 178 & 178 & & & 9.0 & 10.2 \\
\hline & $c_{+ \text {scaa, }}$ a & 9 & 89 & 180 & 180 & -178 & & & 9.2 & 9.8 \\
\hline \multirow[t]{7}{*}{ NPP } & $t_{\mathrm{aaa}, \mathrm{aa}}$ & 180 & 180 & 180 & 180 & 180 & 180 & & 4.0 & $\mathrm{~N} / \mathrm{A}$ \\
\hline & $t_{+\mathrm{ac}-\mathrm{sca},+\mathrm{aca}}$ & 180 & 104 & -63 & 180 & 136 & 175 & & 0.0 & 0.5 \\
\hline & $t_{+\mathrm{sc}+\mathrm{sca},+\mathrm{aca}}$ & 180 & 83 & 63 & 179 & 137 & 175 & & 0.1 & 0.4 \\
\hline & $\boldsymbol{t}_{+ \text {scaa, }+ \text { aca }}$ & 180 & 85 & -179 & 180 & 138 & 175 & & 0.0 & 0.0 \\
\hline & $c_{\text {aaa, }}$ aa & 0 & 180 & 180 & 180 & 180 & 180 & & 10.4 & $\mathrm{~N} / \mathrm{A}$ \\
\hline & $\boldsymbol{c}_{+ \text {scaa, }}$ aa & 10 & 88 & 180 & 180 & -179 & 180 & & 9.1 & 9.3 \\
\hline & $C_{+ \text {scaa },+ \text { sca }}$ & 9 & 87 & 180 & 180 & 87 & 176 & & 11.4 & 12.5 \\
\hline \multirow[t]{9}{*}{ NPB } & $t_{\text {aaa, aaa }}$ & 180 & 180 & 180 & 180 & 180 & 180 & 180 & 11.2 & $\mathrm{~N} / \mathrm{A}$ \\
\hline & $t_{+ \text {scaa },+ \text { acaa }}$ & -179 & 85 & -179 & 180 & 126 & 176 & 180 & 6.5 & 5.1 \\
\hline & $t_{+\mathrm{ac}-\mathrm{sca},+\mathrm{ac}-\mathrm{sca}}$ & 180 & 106 & -62 & -179 & 137 & -66 & -179 & 3.9 & 3.3 \\
\hline & $t_{+\mathrm{sc}+\mathrm{sca},+\mathrm{ac}-\mathrm{sca}}$ & -176 & 80 & 61 & 179 & 123 & -65 & -179 & 3.9 & 3.4 \\
\hline & $\boldsymbol{t}_{+\mathbf{s c}+\mathbf{s c a},+\mathbf{s c}+\mathbf{s c a}}$ & -173 & 80 & 61 & 179 & 69 & 57 & 179 & 0.0 & 0.0 \\
\hline & $t_{+\mathrm{scaa},+\mathrm{ac}-\mathrm{sca}}$ & -178 & 84 & -179 & 180 & 129 & -66 & -179 & 4.4 & 3.4 \\
\hline & $C_{\text {aaa, aaa }}$ & 4 & -175 & 180 & 180 & -179 & 180 & 180 & 17.6 & 15.0 \\
\hline & $C_{+ \text {scaa },+ \text { scaa }}$ & 9 & 86 & 180 & 180 & 87 & 177 & 180 & 16.9 & 16.5 \\
\hline & $\boldsymbol{c}_{+ \text {scaa, aaa }}$ & 9 & 88 & 180 & 180 & -178 & 180 & 180 & 16.1 & 14.6 \\
\hline
\end{tabular}




\section{A.12 Auswertskripte}

Spektrenauswertung mit dem Pythonmodul madras.py Vers. 18.07.01 Alle verwendeten Python-Skripte zur Auswertung der Rohdaten des curry-Jets verwenden Python 3.6.0 und Methoden der Curry-Klasse die Teil des Moduls madras.py (Module for Advanced Despiking of RAman Spectra) ist. ${ }^{2)}$ Mit der Curry-Klasse wird ein Objekt <cobject> erzeugt für das u.a. Methoden zum Import und Export von Datensätzen, zum Kalibrieren der Daten, zum Entfernen einzelner Scans aus einem Datensatz, zum „Despiken“ und zur einfachen Basislinienkorrektur zur Verfügung stehen.

Zur visuellen Scananalyse und einem Vergleich des gemittelten Spektrums mit den Einzelscans können die Plot-Methoden Curry.disc_plot () und Curry.despike_plot () verwendet werden. Die Curry-Klasse erlaubt den Spektrenimport als WinSpec/32*SPE-Datei, als LIGHTFIELD *.SPE-Date ${ }^{3)}$ oder als <*.dat>-ASCII-Datei (im TAB-getrennten 2-Spaltenformat aus dem WinSpec/32-Tool "Convert to ASCII").

Die Exportmethode $<$ cobject $>$. export ( $* *$ kwargs) erzeugt eine mehrspaltige, TAB-getrennte ASCII-Datei mit Header. Die erste Datenspalte enthält Pixelwerte (xtype $={ }^{\prime} p x^{\prime}$ ) oder Wellenzahlen (xtype $={ }^{\prime} \mathrm{wn}^{\prime}$, Standardwert, Kalibrierung über convert-Methode notwendig). Weiterhin wird entweder eine Spalte mit gemittelten (und despikten) Daten erzeugt (ytype='fin': Standard, Despiking und Mittelung aus <cobject>.finning() oder ytype=' $\mathrm{avg}^{\prime}$ : Despiking über eine andere Methode (s.o.) und Mittelung mit <cobject $>$. avg ( ) ) oder alle Scans in <cobject $>$ ungemittelt in entsprechend vielen Spalten exportiert (ytype $={ }^{\prime}$ de $s^{\prime}$ : Export der despikten Einzelscans (nicht für finning-Methode) oder ytype $={ }^{\prime}$ raw' : Export der Einzelscans in Rohform). Ansonsten ist die exakte Verwendung der einzelnen Methoden den entsprechenden Kommentaren im Quelltext zu entnehmen.

Die Funktionsweise der unterschiedlichen Despike-Algorithmen ist in Abschnitt 3.1.5 erläutert. Die verwendete Laserwellenlänge, und weitere dynamische Parameter wie der zuletzt verwendete Pfad werden in der Datei < curry_constants. json> abgelegt und bei jeder Objekterzeugung geladen. Zur einfacheren Anwendung wurde eine Reihe von Batch-Programmen geschrieben, die übliche Abfolgen einzelner Curry-Methoden automatisieren:

- cfin.py (Standardauswertung): Import + Kalibrierung + visuelle Scananalyse + Despiking via Curry.finning() + Ergebnisplot + Export(Wellenzahl, gemitteltes Spektrum)

- cdes.py (klassische Auswertung): Import + Kalibrierung + visuelle Scananalyse + Despiking via Curry.despike() + Mittelung via Curry.avg() + Ergebnisplot + Export(Wellenzahl, gemitteltes Spektrum)

- ccal.py (Vorauswertung der Kalibrierspektren): Import + Despiking via Curry . finning () + Ergebnisplot + Export(Pixel, gemitteltes Spektrum)

\footnotetext{
${ }^{2)}$ Modul-Abhängigkeiten des madras-Moduls sind im Quelltext dokumentiert.

${ }^{3)}$ Beide *.SPE-Formate sind Binärdateien, allerdings nicht untereinander kompatibel. Die LIGHTFIELD-Spektren sind, anders als bei WinSpec/32, 0-indiziert.
} 
- craw.py (Kalibrierung und Export der Einzelscans): Import + Kalibrierung + Export(Wellenzahl, Einzelscans)

- cpxraw.py (Export der Einzelscans): Import + Export(Pixel, Einzelscans)

import numpy as np

import json

import os

6 import sys

from tkinter import $\mathrm{Tk}$

from tkinter.filedialog import askopenfilename

from tkinter.filedialog import asksaveasfile

from scipy import signal

11 import matplotlib.pyplot as plt

import matplotlib.widgets as mwid

'"'MADRAS -- (Module for Advanced Despiking of RAman Spectra)

This module contains several classes for data post-processing of the curry-Jet Raman spectrometer of the Suhm Group, Goettingen.

Requirements:

Python 3.6.0 or later with Anaconda 4.3.1 (for Windows users)

Following packages are required (not checked for backwards compatibility):

- SYS

$-O S$

- numpy 1.14.5

- tkinter

- matplotlib 2.1.2

- json

- scipy 1.1.0

, '

class Curry (object) :

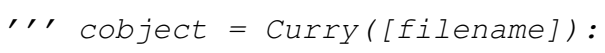


\# Version history:

\# 18.01.1 - first basic running class

\# 18.02.1 - FIRST STABLE RELEASE with all basic functions running

\# 18.04.1 - "finning"-method changed, so that first and last scan are not considered neighbours anymore; MAJOR BUG IN AVERAGING METHOD RESOLVED!

\# 18.05.1 - "finning" algorithm fundamentally changed and older version kept as alternative methods 'fin_median' and 'fin_adjac'

\# 18.07.1 - method Curry.smooth() added.

def _init_(self, fullfilename=None) : self._loadGlobalsettings() self._discscans = np.array([]) \#list of scans discarded via Curry.discard () method

self._levdata $=$ np.array $([])$ self._COFname $=\prime \prime$ \# Inovocation for later COF handling self._.smoothdict $=\{\}$ \# Pre-allocation for dictionary. See self.smooth() for details and definition. self.imp (fullfilename)

"'attributes introduced in various methods:

self._fulldata (from: Curry.imp()) - np.array([])- matrix of imported data set (<self._nscans> columns of y-values of respective scan.) self._fullfilename (from: Curry.imp()) - path and filename of the currently loaded file

self._caldata - (from: Curry.convert()) - raw data matrix for nobel gas calibration

self._reddata - (from: Curry.data matrix emended from certain scans by the Curry.discard() method self._desdata - (from Curry.despike() or Curry.finning()) - despiked data (may be reduced in dimension due to self._discscans)

self._avgdata - (from Curry.avg()) - averaged data

self._pxaxis - x-values (detector scale) in pixel

self._nscans - number of scans

self._wnaxis - x-values (detector scale) in wavenumbers (needs calibration with self._caldata)

self._dindex- list of indices of kept scans after discarding <dindex $>=$ not (<discscans $>-1) " \prime \prime$ 
86

def imp(self, fullfilename=None):

"'This method runs after every initialisation of a Curry object.

The <fullfilename> is opened. If not explicitly given, the dataset will be chosen via tkinter open-File-Dialog.

A sub routine is called according to possible file types "*.dat" (ASCII) or $"$ *.SPE" (bin).

This method returns a *detector-size* by *number of scans* matrix of te dataset stripped off all x-values (detector position)','

def _ getDatatype(fullfilename):

\#-- Method to find out which of the three possible data types (*.SPE, *(3.0).SPE or ASCII) is "fullfilename":

\#-- 0: ASCII; 1: Winspec SPE-file (1-indexed); 2: Lightfield SPE-file (0-indexed)

if fullfilename.split ('.')[1] == "SPE":

with open(fullfilename, 'rb') as fid:

$$
\text { temp_data }=\text { fid.read }()
$$

if temp_data.find $\left(\mathrm{b}^{\prime}<\right.$ ? $\left.\mathrm{xml} \mathrm{l}^{\prime}\right)=-1$ :

$$
\text { datatype }=1
$$

else :

datatype $=2$

elif fullfilename.split('.')[1] == "DAT" or "ASC":

datatype $=0$

else:

print("Curry.imp(): Input file has wrong format! Only *.ASC, *.DAT or *.SPE are allowed! \n------")

datatype $=-1$

return datatype

def _importSPE(fullfilename, version):

"'-- (Modified function from ftp://ftp.princetoninstruments.com/Public /Software/User\%20Writteno20utilities/Python/spe2bin.py)

-- A function to read a SPE file, decode the header and return a single column vertical array (only counts).'"'

with open (fullfilename, ' $r b$ ') as fid:

hdr $=$ np.fromfile(fid, np.uint $16,-1)$

self._globalsettings['det_size'] = int (hdr[21]) \# = detector size (x-

dim) \#\# detector dimension $(y, z)=$ (hdr[328], hdr[723])

dataType $=\operatorname{hdr}[54]$

with open(fullfilename, 'rb') as fid:

if dataType $==0$ :

rawdata $=$ np.fromfile(fid, np.float32) [1025:]

if datatype $==1$ :

rawdata $=$ np.fromfile(fid, np.int 32) [1025: ] 
126

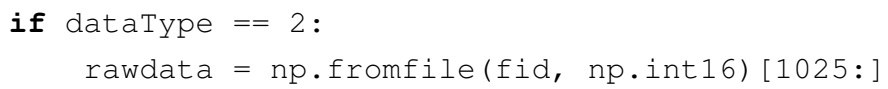

if datatype $==3$ : rawdata $=$ np.fromfile(fid, np.uint16) [1025: ]

rawdata $=$ rawdata [1025: ]

return np.array (rawdata)

def

\#This method only yields reasonable results for the Winspec-"Convert to ASCII" routine with following settings:

\# - FIXME

\#no determination of the length of the data (det_size) possible.

with open(fullfilename, ' $r$ ') as fid:

rawdata $=$ np.loadtxt $($ fid, dtype $=($ int, int $)$

rawdata $=$ rawdata $[:, 1]$

return rawdata

def _reshapeCD (rawdata, datatype):

\# takes raw data fron either ASCII or converted binary SPE files

\# and reshapes them so that every scan is represented by a column.

fulldata = rawdata.reshape( (self._globalsettings['det_size'], -1), order $=^{\prime} \mathrm{F}^{\prime}$ )

[dummy, self._nscans] = fulldata.shape

return fulldata

fullfilename $=$ str(fullfilename)

\#self._loadGlobalsettings()

specImportopt $=\{\}$

_specImportopt ['defaultextension' ] = '*.spe'

_specImportopt ['filetypes'] = [('all files', '*.*'), ('ASCII', ('*.dat', ' *.aSc')), ('SPEC files', '*.SPE')]

_specImportopt ['initialdir'] = self._globalsettings['path']

_specImportopt ['title'] = 'Import Spectrum...'

if not os.path.isfile (_fullfilename):

print('Curry.imp(): Not a valid filename or file is not existent! \ nPlease choose file from input dialog! \n------') self.root $=\mathrm{Tk}()$

self.root.withdraw()

fullfilename $=$ askopenfilename ( $* \star$ _specImportopt)

self.root.destroy()

if not _fullfilename:

print("Curry.imp(): Did not choose file; curry object terminated!") sys.exit()

\#get new path from _fullfilename and put it in the <globalsettings()> dictionary:

self._globalsettings ['path'] = os.path.dirname (_fullfilename) 
171

191

def export (self, defname=None, ytype='fin', xtype=' wn' $^{\prime}$ ) : "'Exports self._findata, self._avgdata, self._desdata or self. fulldata as <ydata> selected by <ytype>=['fin' (default), 'avg', 'des ', 'raw', 'smooth'] with <xtype> = ['wn' (default), 'px'] (preferably wavenumbers if available, otherwise pixels) to file <dfn> (short for default filename).

If valid default filename <defname> is not given, tkinter's **asksavefile **-method is used to choose a filename (However, basename of self. fullfilename is suggested with an appended "_fin", "_avg" or "_des", respectively.)'"'

ytype_string_dict $=\left\{\right.$ 'avg' $^{\prime}$ 'averaged', 'des' : 'despiked', 'raw' : 'full raw ', 'fin' : 'finned'\}

ytype_dict $=\{\}$

xtype_dict $=\left\{{ }^{\prime} x^{\prime}\right.$ : self._pxaxis.reshape $\left.((-1,1))\right\}$

201

specExportopt $=\{\}$

_ specExportopt ['defaultextension'] = '*.dat'

_specExportopt ['filetypes'] = [('all files', '*.*'), ('ASCII', ('*.dat', ' *.asc')), ('SPEC files', '*.SPE')]

_specExportopt ['initialdir'] = self._globalsettings ['path']

try:

_specExportopt ['title'] = 'Export \{\} data...'.format(ytype_string_dict [ytype]) 
211

except (NameError, KeyError, IndexError):

print ('Curry.export(): Wrong value for parameter <ytype>. Changed to " avg" $\backslash n------^{\prime}$

_specExportopt ['title'] ='Export \{\} data...'.format (ytype_string_dict ['avg'])

\section{if type (defname) is not str:}

print('Curry.export(): Not a valid filename or file is not existent!\ nPlease set file and directory via input dialog! \n------')

if $x$ type $==$ ' $p x^{\prime}$ :

defname = os.path.basename (self._fullfilename).split('.')[0]+ ${ }^{\prime}$ _px_\{\}.dat'. format (ytype)

else:

defname = os.path.basename (self._fullfilename).split('.')[0] + ' \{\} .dat' . format (ytype)

_specExportopt ['initialfile'] = defname

root $=\mathrm{Tk}()$

root.withdraw ()

filewrapper $=$ asksaveasfile ( $*$ __specExportopt)

if not filewrapper:

print("Curry.imp(): Did not enter filename for export; curry object terminated!")

sys.exit ()

else:

finalfilename $=\boldsymbol{s t r}($ filewrapper.name)

root.destroy ()

else:

finalfilename $=$ defname

\#constructing export array <expdata>

if $x$ type $=={ }^{\prime} \mathrm{wn}^{\prime}$ :

try:

xtype_dict ['wn'] = self._wnaxis.reshape $((-1,1))$

except AttributeError:

print('Curry.export(): wavenumbers not available for current data, please run Curry.convert(). Pixel values exported instead! \n $\left.-----^{\prime}\right)$

xtype $={ }^{\prime} \mathrm{px}^{\prime}$

elif xtype not in xtype_dict:

print('Curry.export(): Invalid value for <xtype> parameter: Pixel

values exported instead. \n------')

xtype $={ }^{\prime} \mathrm{px}^{\prime}$

$x S=x t y p e \_d i c t[x t y p e]$

if ytype $==$ ' $^{\prime} \mathrm{avg}$ ' :

ytype_dict ['avg'] = self._avgdata.reshape $((-1,1))$

elif ytype $==$ 'des' $^{\prime}$ :

ytype_dict ['des'] = self._desdata

elif ytype $==$ 'fin':

ytype_dict ['fin'] = self._findata 
elif ytype $==$ ' smooth' :

ytype_dict ['smooth'] = self._smoothdata

elif ytype $==$ 'raw' :

ytype_dict ['raw'] = self._fulldata

else:

print('Curry.export(): Invalid value for <ytype> parameter. PROGRAM TERMINATED \n------' )

sys.exit ()

ys = ytype_dict [ytype]

expdata $=\mathrm{np}$.append(xs, ys, axis=1) \# generating export array from chosen $x$-values and $y$-values

\#provide head_string with neccessary header and log information: header_dict $=\{$ 'vers': self._version, 'aut':self._author, 'inpdata': self -_fullfilename, ' lwl': ' -', ' calname': ' -', 'calfit': '-', 'niter': '', 'des': 'Despiked' ,' 'avg': ' ', 'disc': ' -', ', smooth':' ', 'smoothentry' :'

xtype_string_dict $=\left\{{ }^{\prime} \mathrm{px}^{\prime}\right.$ : 'detector position (in px)', ' wn' $^{\prime}$ ' Stokes shift (in $\left.\mathrm{cm} \star \star-1)^{\prime}\right\}$

header_dict ['xdim'] = xtype_string_dict [xtype]

if 'fin' in ytype_dict: header_dict ['des'] = 'Finned'

if ' avg' in ytype_dict: header_dict ['avg'] = ' and averaged'

if 'smooth' in ytype_dict: header_dict ['smooth'] = ' and smoothed' header_dict ['smooth-entry'] = 'Smoothing: Gaussian filter with window size: \{winsize\} px and standard deviation: \{stdev\} px $\backslash \mathrm{n}^{\prime}$. format $(* *$ self. smoothdict)

if ' $w n^{\prime}$ in xtype_dict:

header_dict ['lwl'] = self._olobalsettings ['laserwL' ]

header_dict ['calname'] = self._calname

header_dict ['calfit'] = self._r_squared

if any (self._discscans):

header_dict ['disc'] = self.discscans

try:

header_dict ['niter'] = self._niter

except AttributeError:

pass

head_string $={ }^{\prime}\{$ des $\}\{$ avg $\}\{$ smooth $\}$ data from $\{$ inpdata $\}: \backslash n(b y$ madras. Curry ()

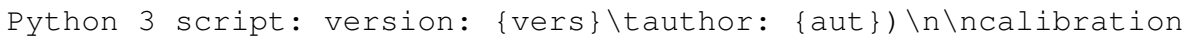
parameters: \nlaser wavelength $=\{l \mathrm{wl}\} \mathrm{nm} \backslash$ ndiscarded $\mathrm{scans}=\{\mathrm{disc}\} \backslash$ ncalibration data $=\{$ calname $\backslash$ ncalibration quality $(R \star \star 2)=\{\mathrm{calfit}\} \backslash \mathrm{n} \backslash$ nx-axis dimension (first data column) : $\{x d i m\} \backslash n\left\{\right.$ smooth-entry ${ }^{\prime}$. format $(* \star$ header_dict)

\#FIXME: cope for despike/finning procedure and niter in head_string 
with open(finalfilename, 'wb') as fid:

np.savetxt (fid, expdata, delimiter $={ }^{\prime} \backslash t^{\prime}$, header=head_string)

print ('Curry.export (): Dataset exported to \{\}$^{\prime}$.format (finalfilename))

\# END OF export()

\section{\#\#\#\#\#\#\#\#\#\#\#\#\#\#\#\#\#\#\#\#\#\#\# \#}

\#\# DATA MANIPULATION: \#\#

\#\#\#\#\#\#\#\#\#\#\#\#\#\#\#\#\#\#\#\#\#

def convert (self, *calname):

"'conversion of self. _pxaxis (x-values in pixel) to wavenumbers (self. —wnaxis) via import and 2nd order polynomial regression of nobel gas calibrarion data"'

Calimportopt $=\{\}$

_calimportopt ['defaultextension'] = '*.dat'

_calimportopt ['filetypes'] = [('ASCII', ('*.dat', '*.asc')), ('all files', $\left.\left.{ }_{\star} . \star^{\prime}\right)\right]$

_calimportopt ['initialdir'] = self._olobalsettings ['path']

_calimportopt ['title'] = 'Import calibration data...'

try:

- calname $=\boldsymbol{s t r}(\mathrm{calname}[0])$

except IndexError:

calname $=$,

if not os.path.isfile (_calname):

print('Curry.convert(): Not a valid calibration filename or file is not existent! \nPlease choose file from input dialog! \n------')

self.root $=\mathrm{Tk}()$

self.root.withdraw ()

_calname = askopenfilename (**_calimportopt)

self.root.destroy ()

if not _calname:

print("Did not choose file; curry object terminated!") sys.exit ()

with open (_calname, ' $r$ ') as fid:

_caldata $=$ np.loadtxt (fid, dtype=float)

self._calname = _calname

self._caldata $=$ _caldata

_calfit = np.polyfit (_caldata [:, 0], _caldata [:, 1], 2, full=True)

r_squared $=1$-__calfit [1] [0]

self._r_squared $=r$ _squared

print('Curry.convert(): polynomial regression with $R \star \star 2=\{: f\} \backslash n------^{\prime}$.

format (r_squared))

self._wnaxis $=1.0 e+7 /$ self._olobalsettings ['laserWL']-1.0e+7/ (_calfit

[0][0]*self._pxaxis**2 + _calfit[0][1]*self._pxaxis + _calfit

[0] [2])

\#END OF convert() 
def despike(self, niter=6, high=1000, low=100, step=50, perm=False): "'classic despiking algorithm.

Be careful: This algorithm is slow and could fail if an unfortunate combination of spikes occur. Make sure to have enough scans (> 6) and your single scans are not too long $(<600 \mathrm{~s})$ to prevent excessive spike occurence. This method is only implemented for the sake of backwards compatibility to former despiking scripts!

data: optional input argument. numpy array of data with each column representing a scan, no $x$-values, no head

niter: optional number of iterations

high: initial upper threshold

low: initial lower theshold

step: step size <high> is decreased with each iteration

FIXME: perm: permutation of adjacent scans'"'

_ data $=$ self._fulldata

print ('Curry.despike() running ...')

$\mathrm{n}=$ niter

self._niter $=$ niter

while $\mathrm{n}>0$ and low $>0$ :

print ('Iteration $\{0\} /\{1\}$ : with bounds $=\{2\}$ and step-size $=\{3\}$ '. format (niter-n+1, niter, (high, low), step))

while high $>=$ low:

for rx, _row_array in enumerate (_data): \# loop through the rows of <data_corr $\rangle$ with row index $\langle r x\rangle$ $\mathrm{mx}=\mathrm{np} \cdot \operatorname{argmax}$ (_row_array) \# $\mathrm{mx}$ is index of maximum in row_array

med = np.median (_row_array) \# find median of row_array

if _row_array[mx] - med >= high:

$I x=m x-1 \quad \# I x$ and $h x$ are the

lower and higher neighbouring row indizes of $\mathrm{mx}$, respectively

$h \mathrm{x}=(\mathrm{mx}+1) \div \operatorname{len}$ (_row_array)

_data $[\mathrm{rx}, \mathrm{mx}]=\mathrm{np} \cdot \operatorname{mean}([$ _row_array $[\mathrm{lx}]$, _row_array $[\mathrm{hx}$ ]]) \# new value for spike from is median of adjacent values

\#loop end--

high $=$ high - step

\#loop end

$\mathrm{n}-=1$

if $\mathrm{n}==0$ :

print ('... Curry.despike() done. \n------') 


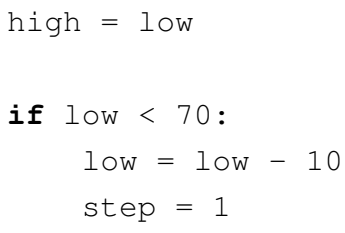

'finning' refers to the brutal yet common practice of cutting of the dorsal fins of living sharks and sell them as delicatesse while the animals die in vain and agony and tries to rise a little awerness to this obnoxious practice

This algorithm loops once through the data set row by row (row dimension is time axis or number of scans and will be refered to as 'track (Tr)') removing every maximum greater than median of Tr with a threshold of the <stddev>-fold of the standard deviation.

Returns averaged numpy array (alternative to Curry.avg()) as self._findata with finned data. -- for alternative versions, see below'"'

\# test for optional input variable <data>:

if data is not None:

- data $=$ data.astype (float)

\section{else:}

\#apply self._reduce upon self._fulldata to eliminate discarded scans: _data $=\operatorname{self}$. reduce $(\mathrm{np} \cdot \operatorname{array}(\operatorname{self}$. _discscans $)-1)$

\#get the dimensions of input data <_data>:

[__detsize, _nscans] = _data. shape

_result $=$ np.zeros ( (_detsize, 1)) \# preallocation of output array

for $m$ in range (__detsize):

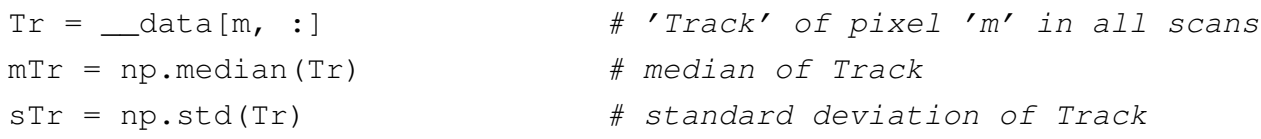
i $+=1$
\#cycle counter 
406

$\operatorname{Tr}[\mathrm{n}]=\mathrm{np} \cdot \operatorname{median}(\operatorname{Tr})$

$\operatorname{chkTr}[\mathrm{n}]=0$

$\mathrm{mTr}=\mathrm{np} . \operatorname{median}(\mathrm{Tr}) \quad$ \# update median of Track

$\operatorname{sTr}=\mathrm{np} . \mathrm{std}(\mathrm{Tr}) \quad$ \# update standard deviation of Track

$\mathrm{n}=$ int (np.argmax $(\mathrm{Tr})$ ) \# update index of max(Track)

$\operatorname{Trx}=\mathrm{np} \cdot \operatorname{multiply}(\operatorname{Tr}, \operatorname{chkTr})$

$\operatorname{mTrx}=\mathrm{np} . \mathbf{s u m}(\operatorname{Trx}) / \mathrm{np} . \mathbf{s u m}(\mathrm{chkTr}) \quad$ \#mean of Tr with all former spikes neglected

_result $[\mathrm{m}]=\operatorname{mTrx}$

print ('Curry.finning(): row: \{\} took \{\} cycles'.format (m, i)) print $\left({ }^{\prime}-----^{\prime}\right)$

if 0 in fresult: \#checks for full substitution of all initial zeros of result

print('Curry.finning(): Rare exception! This couldn''t have happened at all. You crashed the universe! Thanks for nothing... \n------') \# DELME

sys.exit ()

else:

self._findata $=$ _result

return _result

\# END OF finning()

def fin_median(self, data=None, stddev=1.0):

" Alternative version of the finning-algorithm where spiked data points are replaced by the rows median.

This yields a self._desdata array which can be processed with further methods. -- for alternative versions, see below'",

\# test for optional input variable <data>:

if data is not None:

\section{else:}

\#apply self._reduce upon self._fulldata to eliminate discarded scans: _data $=\operatorname{self}$. _reduce (np.array (self._discscans) -1$)$

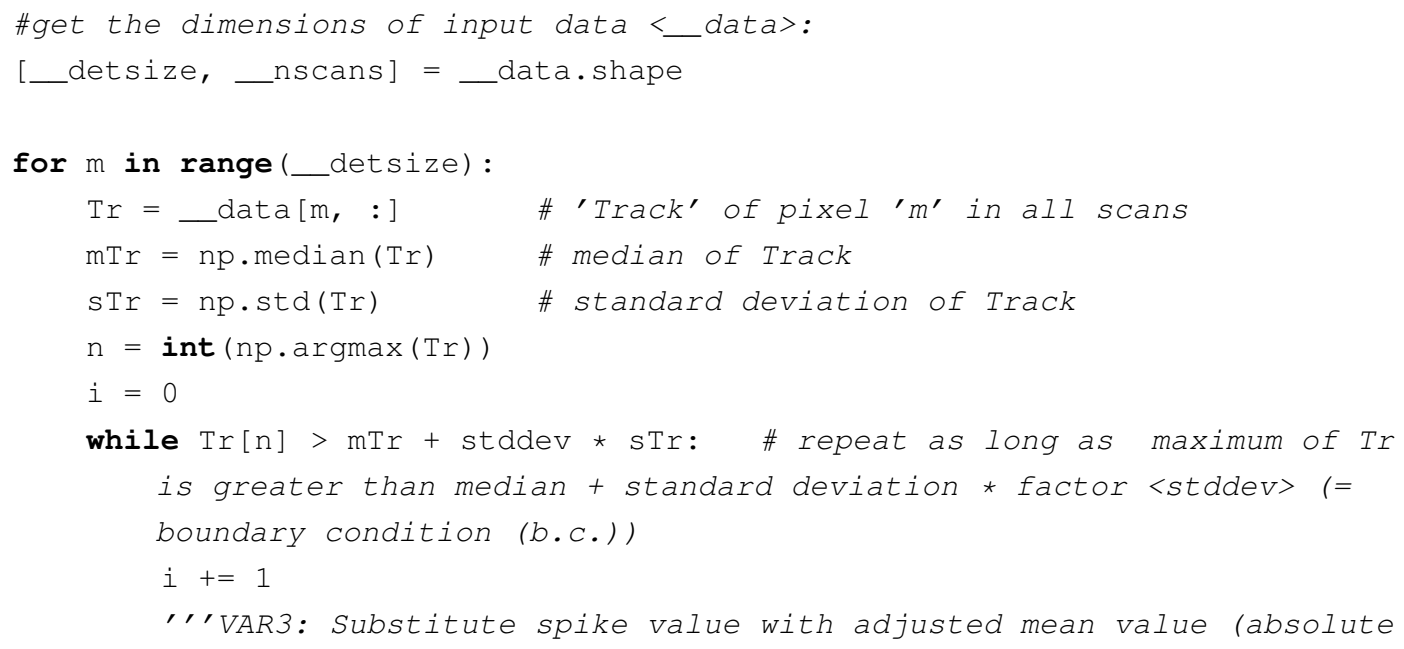


476

mean omitting current position) instead of just of adjacent neighbours. Prevents incomplete despiking!','

$\# \operatorname{Tr}[n]=(n p . \operatorname{sum}(\operatorname{Tr})-\operatorname{Tr}[n]) /(\operatorname{len}(\operatorname{Tr})-1)$

$\operatorname{Tr}[\mathrm{n}]=\mathrm{np} \cdot \operatorname{median}(\operatorname{Tr})$

$\operatorname{mTr}=$ np.median $(\mathrm{Tr}) \quad$ \# update median of Track

$\mathrm{sTr}=\mathrm{np}$.std $(\mathrm{Tr}) \quad$ \# update standard deviation of Track

$\mathrm{n}=$ int (np.argmax (Tr)) \# update index of max (Track)

_data $[\mathrm{m},:]=\operatorname{Tr}$

print ('Curry.finning(): row: \{\} took \{\} cycles'.format (m, i))

print ('------')

self._desdata $=$ _data

return _data

\# END OF fin_median()

def fin_adjac(self, data=None, stddev=1.0):

"'Alternative version of the finning-algorithm where spikes are replaced by the mean of adjacent values.

Needs specila treatment of the first and last value in the row and can trap itself in an endless loop.

Might be useful, if there's a significant change in signal over time. The self._lev method might be a better option!','

\# test for optional input variable <data>:

if data is not None:

else:

_data $=$ data.astype $(\mathbf{f l o a t})$

\#apply self._reduce upon self._fulldata to eliminate discarded scans: _data $=\operatorname{self} . \ldots r e d u c e\left(n p \cdot \operatorname{array}\left(\operatorname{self} . \_\right.\right.$discscans $\left.)-1\right)$

\#get the dimensions of input data <_data>:

[_detsize, _nscans] = _data.shape

for $m$ in range (__detsize):

$\operatorname{Tr}=\ldots \operatorname{data}[\mathrm{m},:]$

$\operatorname{mTr}=\mathrm{np} \cdot \operatorname{mean}(\operatorname{Tr})$

$\operatorname{sTr}=n p . s t d(\operatorname{Tr})$

$\mathrm{n}=\operatorname{int}(\mathrm{np} \cdot \operatorname{argmax}(\operatorname{Tr}))$

n_old = None

$i=0$

while $\operatorname{Tr}[\mathrm{n}]>\mathrm{mTr}+$ stddev * sTr: \# repeat as long as maximum of $\operatorname{Tr}$ is greater than mean + standard deviation * factor <stddev> (= boundary condition (b.c.))

$i+=1$

"'Testing for endless loop trap:" "

if $\mathrm{n}==\mathrm{n}$-old: \#Being stuck in an endless loop ( $\mathrm{n}$ of last cycle ( n_old) is the same as $n$ of this cycle) can only happen if maximum peak and adjacent peaks are of the same value, thus this is only possible for $n==0$, since np.argmax always takes the first value if more than one maximum exists: 
$\operatorname{Tr}[\mathrm{n}+1]=\operatorname{mTr}+$ stddev * sTr \#Next neighbour of trapped value is forced to the current threshold

"'getting lown and highn (lower and higher adjacent row indizes of $n$, respectively): ' ',

if $\mathrm{n}=0$ : \# if spike is the first element of Track, their is no neighbour below, thus the second and third values are used for averaging

lown $=1$

highn $=2$

elif $\mathrm{n}==$ len(Tr) - 1: \# if spike is the last element of Track, their is no neighbour above, thus the second and third to last values are used for averaging:

lown $=\mathrm{n}-2$

highn $=n-1$

else:

lown $=\mathrm{n}-1$

highn $=n+1$

"'Spike is replaced by mean of adjacent values (data replacement considers signal change over time):'"'

$\operatorname{Tr}[\mathrm{n}]=\mathrm{np} \cdot \operatorname{mean}([\operatorname{Tr}[$ lown], $\operatorname{Tr}[$ highn] $])$ \# Replace value at $n$ with mean of adjacent values (see special conditions for boundary values)

$\operatorname{mTr}=\mathrm{np} \cdot \operatorname{mean}(\mathrm{Tr}) \quad$ \# update mean of Track

$\mathrm{sTr}=\mathrm{np} . \mathrm{std}(\mathrm{Tr}) \quad$ \# update standard deviation of Track

$\mathrm{n} \_$old $=\mathrm{n}$

$\mathrm{n}=\operatorname{int}(\mathrm{np} \cdot \operatorname{argmax}(\mathrm{Tr}))$ \# update index of max(Track)

506 _data $[\mathrm{m},:]=\operatorname{Tr}$

print ('Curry.finning(): row: \{\} took \{\} cycles'.format (m, i)) print $\left({ }^{\prime}-----{ }^{\prime}\right)$

self._desdata $=$ _data

return _data

def discard(self, scans):

"'generates numpy array <self._discscans> of scans to discard in further processing from input list <scans>

\# END OF discard() 
def undiscard(self):

"'deletes all discard action done before with Curry.discard()"'

self._discscans = np.array ([])

self._dindex $=\mathrm{np}$. arange $($ self.__nscans $)$

\# END OF undiscard()

def _reduce (self, index, dataset='full') :

"'internal funtion to return a _reddata matrix stripped of all

columns of _data wich are indexed by _ndiscscans-1 (due to 1-indexing in scan numbers).

<return> is a numpy array with all elements greater 0 but not greater than self._nscans.

option <dataset> enables to change between reducing the self. Ifulldata ( default setting 'full') and the already despiked dataset in self.

_ desdata ('des')','

dataset_dict $=\left\{\right.$ ffull'$^{\prime}$ : self._fulldata.astype (float) $\}$

\#Test for self._desdata:

try:

dataset_dict ['des'] = self._desdata.astype (float)

except AttributeError:

print('Curry._reduce(): Hint: No despiking values found so far...')

try:

_data $=$ dataset_dict [dataset]

except :

print('Curry._reduce(): Despiking neccessary for option ' 'des' '. Please run Curry.despike() method on your data first! \n------') sys.exit ()

return np.delete (_data, index, axis=1)

\# END OF reduce()

def lev(self):

" corrects level (or baseline) by shifting the scans by their total mean difference and results in self. levdata'"'

try:

_desdata $=$ self._. desdata

except AttributeError:

print('Despiking neccessary. Please run Curry.despike() on your data first! \n------')

return

\#check for possible discarded data:

_data $=$ self._reduce (np.array (self._discscans)-1)

_ scanmean $=$ np.mean (_data, axis=0)

_ scanmin $=$ np. $\min (\ldots$ scanmean)

_meandiff $=$ _scanmean - _scanmin

self._levdata $=$ _data $-\ldots$ meandiff

\# END OF lev() 
def $\operatorname{avg}(\operatorname{self})$ :

'"'averages input dataset <_data> to a single column averaged data array< self._avgdata>

This method needs a despiked dataset as input for meaningful results, e.g. <self._desdata>:' ',

try:

_desdata $=$ self._desdata

except AttributeError:

print('Despiking neccessary. Please run Curry.despike() method on your data first! \n------')

return

_data $=\operatorname{self} . \ldots r e d u c e(n p \cdot \operatorname{array}(\operatorname{self}$. _discscans $)-1$, dataset='des' $)$

_avgdata $=$ np.mean (_data, axis=1)

self._avgdata $=$ _avgdata

\# END OF avg()

def smooth(self, datatype='fin', winsize=11, stdev=2):

"' smoothing of <data> with gaussian filter window of size <winsize> with a standard deviation <stdev>'"'

data_dict $=\{\}$

if datatype $=={ }^{\prime}$ 'avg':

data_dict $\left[{ }^{\prime}\right.$ avg'] = self._avgdata.reshape $((-1,1))$

elif datatype $==$ 'fin':

data_dict['fin'] = self._findata

else:

print('Curry.smooth(): Wrong input. Only Despiked and averaged or finned data can be smoothed! PROGRAM TERMINATED \n------')

sys.exit ()

data = data_dict [datatype]

win = signal.gaussian (winsize, std=stdev). reshape $((-1,1))$

self._.smoothdata = signal. convolve (data, win, mode='same')/sum(win)

self._smoothdict $=\{$ 'winsize' : winsize, 'stdev': stdev, 'datatype' $:$ datatype

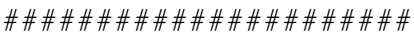

\#\#_globalsettings \#\#

\#\#\#\#\#\#\#\#\#\#\#\#\#\#\#\#\#\#\#\#\# \#

"' this dictionary is load from <curry_constants.json> or newly created upon object creation.

it contains general keys such as <laserWL> or <path>." "'

def _loadglobalsettings (self) :

"'global settings are stored as a dictionary in the json file < curry_constants.json> in the current directory:" "'

try:

ccid = open (' curry_constants.json', 'r')

except IOError:

print ('No <curry_constants.json> found in the current directory!

Standard values are taken! \n------') 
611

616

621

636

641 self._flobalsettings $=\{$ 'laserWL': float(532), 'path': os.getcwd(), ' det_size' : 1340\}

self._storeGlobalsettings()

else :

self._globalsettings = json.load(ccid)

ccid.close ()

\# END OF _loadglobalsettings()

def _storeGlobalsettings (self):

"'store _globalsettings as <curry_constants.json> in current directory:

, ,

with open('curry_constants.json', 'w') as ccid:

json.dump (self._olobalsettings, ccid)

\# END OF _storeGlobalsettings()

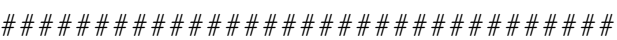

\#\# "SET/GET ARGUMENT" METHODS: \#\#

\#\#\#\#\#\#\#\#\#\#\#\#\#\#\#\#\#\#\#\#\#\#\#\#\#\#\#\#\#\# \#

def setLaserWL(self, laserwavelength):

self._globalsettings ['laserWL'] = float (laserwavelength)

def getLaserWL (self):

return self._globalsettings [' laserWL']

def getDetSize(self):

return self._globalsettings['det_size']

def getPXaxis (self):

return self._pxaxis

def getWNaxis (self):

return self._wnaxis

def getDIndex(self) :

return self.__index

def getcalData(self) :

return self._caldata

def getcalfit (self) :

return self._calfit 
def getFullData(self):

return self._fulldata

661

def getDesData(self) :

return self._desdata

def getFinData(self):

return self._findata

def getLevData(self):

return self._levdata

671

def getAvgData(self) :

return self._avgdata

dstaticmethod

def getVersion():

return Curry._version

estaticmethod

681

def getAuthor():

return Curry._author

\#\#\#\#\#\#\#\#\#\#\#\#\#\#\#\#\#\#\#\#\#\#\#\#\#\#\#\#\#\#\#\#\#\#\#

\#\# curry object file handling (COF): \#\#

686

\#\#\#\#\#\#\#\#\#\#\#\#\#\#\#\#\#\#\#\#\#\#\#\#\#\#\#\#\#\#\#\#\#

"'to save the current curry object as it is, you can use the save method. retrieving an curry object is done by the load method.'"'

def load(self, *filename): \#FIXME: To be implemented in a later version

pass

696

def save(self, *filename):

"'Save current object to "curry object file" (*.cof)

If no filename is given, the one stored in _COFname is used (and overwritten!).

If _COFname is empty, an askopenfile dialog is used!','

701 \#FIXME: To be implemented in a later version

pass 
def disc_plot (self) :

def _checkButtons_action(label):

index = labels.index $(1 \mathrm{abel})$

lines [index]. set_visible (not lines [index].get_visible())

plt.draw ( )

def _radioButtons_action(label):

try :

xdata $=$ x_dict $[$ label]

except (NameError, TypeError, KeyError):

radio.set_active (0)

print ('Curry.disc_plot(): Using wavenumber scale needs prior calibration via Curry.convert() method. Instead px scale is used! \n------')

else:

[line.set_xdata(xdata) for line in lines]

ax.set_xlim(xlim_dict[label])

plt.draw ()

def_discButton_action (event) :

checked = check.get_status ()

disc_index $=[i$ for $j, i$ in enumerate (_scans) if not checked[j]]

if disc_index:

discscans $=$ np.array (disc_index $)+1$

self.discard (discscans)

else:

print ('Curry.disc_plot(): "DISCARD button": No scans were unchecked , nothing to discard!')

def contButton_action(event) :

plt.close ()

def cancelButton_action(event) :

plt.close ()

sys.exit ( )

\#_ init__

_ scans $=$ np.arange (self._nscans)

ys $=$ self._fulldata

fig, $a x=p l t . s u b p l o t s() \# f i g s i z e=(8,4))$ \#DELME

fig.canvas.set_window_title('All scans of \{\}$^{\prime}$.format (self._ffullfilename))

\#positioning: $\quad$ pos $=$ [left, bottom, width, height]

ax_pos $=[0.3,0.2,0.65,0.75]$

ax_check_pos $=[0.05,0.4,0.15,0.55]$

ax_radio_pos $=[0.05,0.2,0.15,0.15]$ 
ax_discButton_pos $=[0.05,0.05,0.15,0.075]$

ax_contButton_pos $=[0.6,0.05,0.15,0.075]$

ax_cancelButton_pos $=[0.8,0.05,0.15,0.075]$

ax.set_position (ax_pos)

\#prepare $x$-axis:

$\mathrm{pxx}=$ self._pxaxis

pxxlim $=(n p \cdot \min (p x x), n p \cdot \max (p x x))$

$\mathrm{x} \_$dict $=\left\{{ }^{\prime} \mathrm{px}^{\prime}: \mathrm{pxx}\right\}$

xlim_dict $=\left\{{ }^{\prime} x^{\prime}: p x x l i m\right\}$

try:

wnx $=$ self. _wnaxis

except AttributeError:

print('Using wavenumber scale needs prior calibration via Curry.convert

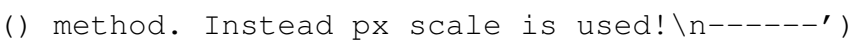

else:

wnxlim $=(n p \cdot \min (w n x), n p \cdot \max (w n x))$

$\mathrm{x} \_$dict $\left[{ }^{\prime} \mathrm{Cm} * *-1^{\prime}\right]=$ wnx

xlim_dict $\left[{ }^{\prime} \mathrm{cm} * *-1^{\prime}\right]=$ wnxlim

\#Invoke line plots:

lines $=\left[a x . p l o t\left(p x x, y s[:, i], l a b e l={ }^{\prime}\{\}^{\prime}\right.\right.$.format $\left.(i+1)\right)[0]$ for $i$ in _.scans] ax.set_xlim(xlim_dict $\left.\left[{ }^{\prime} p x^{\prime}\right]\right)$

776

\#Invoke Checkboxes Axes:

ax_check = plt.axes (ax_check_pos)

labels = [str(line.get_label()) for line in lines]

visibility $=$ [line.get_visible() for line in lines]

781

check = mwid.CheckButtons (ax_check, labels, visibility)

check.on_clicked (_checkButtons_action)

\#Invoke Radiobutton Axes:

ax_radio = plt.axes (ax_radio_pos)

radio = mwid.RadioButtons (ax_radio, ('px', ' $\left.\mathrm{cm} \star \star-1{ }^{\prime}\right)$ )

radio.on_clicked (_radioButtons_action)

\#Invoke discButton:

ax_discButton = plt.axes (ax_discButton_pos)

discButton = mwid. Button (ax_discButton, 'Discard')

discButton.on_clicked(_discButton_action)

\#Invoke contButton:

ax_contButton = plt.axes (ax_contButton_pos)

796

contButton = mwid. Button (ax_contButton, 'Continue')

contButton.on_clicked (_contButton_action)

\#Invoke cancelButton:

ax_cancelButton = plt.axes (ax_cancelButton_pos)

cancelButton = mwid. Button (ax_cancelButton, 'Cancel') 
806

811

821

831

\#Invoke line plots:

\# 1. plot fulldata with respect to discscans to ax bottom

\# 2. check for average data

\# 2.1 if yes: plot avg data to ax_top

\# 2.2 if no: Exit with error message

\# 3. setup button to export results and a cancel button

if self._levdata.any ():

reddata $=$ self. levdata

else:

reddata $=\operatorname{self}$. _reduce $\left(n p \cdot \operatorname{array}\left(\operatorname{self} . \_d i s c s c a n s\right)-1\right)$

ax_bottom.plot (xs, reddata)

ax_bottom.set_xlim(xslim)

if method =='fin':

try :

ax_top.plot (xs, self._findata) 
except AttributeError:

print ('Curry.despike_plot(): No finned data available. Use an appropriate value for "method" argument! \n------') sys.exit()

else:

try:

ax_top.plot (xs, self.__avgdata)

plt.subplots_adjust (bottom=0.2)

861

\#Invoke buttons:

ax_contButton_pos $=[0.6,0.05,0.15,0.075]$

ax_cancelButton_pos $=[0.8,0.05,0.15,0.075]$

\#Invoke contButton:

ax_contButton = plt.axes (ax_contButton_pos)

contButton = mwid. Button (ax_contButton, 'Continue')

contButton.on_clicked(_contButton_action)

\#Invoke cancelButton:

ax_cancelButton = plt.axes (ax_cancelButton_pos)

cancelButton = mwid. Button (ax_cancelButton, 'Cancel')

cancelButton.on_clicked (_cancelButton_action)

plt.show ()

\# END OF despike_plot()

\# END OF CLASS "Curry" \#\#\#\#\#\#\#\#\#\#\#\#\#\#\#\#\#\#\#\#\#\#\#\#\#\#\#\#\#\#\#\#\#\#\#\#\#\#\#\#\#\#\#\#\#

881

if __name__ = = "_main_ " :

cobject $=$ Curry ()

cobject. convert ()

cobject.finning()

886 cobject.export () 



\section{Literaturverzeichnis}

[1] H. Bipp und H. Kieczka, in Ullmann's Encyclopedia of Industrial Chemistry, Hrsg. B. Elvers und F. Ullmann, Wiley-VCH, Weinheim, Germany, 2011, Bd. 16, S. 1-12.

[2] Verband der Chemischen Industrie e.V., Hrsg., Chemiewirtschaft in Zahlen 2019, https: //www.vci.de/vci/downloads-vci/publikation/ chemiewirtschaftin-zahlen-print.pdf (besucht am 04.01.2020).

[3] G. N. Ramachandran und V. Sasisekharan, in Advances in Protein Chemistry, Hrsg. C. B. Anfinsen, Academic Press, New York und London, 1968, Bd. 23, S. 283-437.

[4] T. Miyazawa, in Poly-[alpha]-amino acids, Hrsg. Gerald D. Fasman, Dekker, New York, 1967, Bd. 1, S. 69-103.

[5] K. B. Wiberg, Accounts of Chemical Research, 1999, 32, 922-929.

[6] A. Jabs, M. S. Weiss und R. Hilgenfeld, Journal of Molecular Biology, 1999, 286, 291304.

[7] S. Wawra und G. Fischer, in cis-trans Isomerization in Biochemistry, Hrsg. C. Dugave, Wiley-VCH Verlag GmbH \& Co. KGaA, Weinheim, Germany, 2006, S. 167-193.

[8] G. Fischer, Chemical Society Reviews, 2000, 29, 119-127.

[9] W. J. Wedemeyer, E. Welker und H. A. Scheraga, Biochemistry, 2002, 41, 14637-14644.

[10] F. X. Schmid, R. Grafl, A. Wrba und J. J. Beintema, Proceedings of the National Academy of Sciences of the United States of America, 1986, 83, 872-876.

[11] C. Schiene-Fischer, J. Habazettl, F. X. Schmid und G. Fischer, Nature Structural Biology, 2002, 9, 419-424.

[12] J. Gao, D. A. Bosco, E. T. Powers und J. W. Kelly, Nature Structural \& Molecular Biology, 2009, 16, 684-690.

[13] S. W. Pedersen, G. Hultqvist, K. Strømgaard und P. Jemth, PLOS ONE, 2014, 9, e95619.

[14] V. Sereikaite, T. M. T. Jensen, C. R. O. Bartling, P. Jemth, S. A. Pless und K. Strømgaard, ChemBioChem : A European Journal of Chemical Biology, 2018, 19, 2136-2145.

[15] E. Arunan, G. R. Desiraju, R. A. Klein, J. Sadlej, S. Scheiner, I. Alkorta, D. C. Clary, R. H. Crabtree, J. J. Dannenberg, P. Hobza, H. G. Kjaergaard, A. C. Legon, B. Mennucci und D. J. Nesbitt, Pure and Applied Chemistry, 2011, 83, 1619-1636.

[16] E. Arunan, G. R. Desiraju, R. A. Klein, J. Sadlej, S. Scheiner, I. Alkorta, D. C. Clary, R. H. Crabtree, J. J. Dannenberg, P. Hobza, H. G. Kjaergaard, A. C. Legon, B. Mennucci und D. J. Nesbitt, Pure and Applied Chemistry, 2011, 83, 1637-1641. 
[17] C. Emmeluth, V. Dyczmons, T. Kinzel, P. Botschwina, M. A. Suhm und M. Yáñez, Physical Chemistry Chemical Physics, 2005, 7, 991-997.

[18] P. Zielke, Dissertation, Georg-August Universität, Göttingen, 2007.

[19] W. A. Herrebout, K. Clou und H. O. Desseyn, The Journal of Physical Chemistry A, 2001, 105, 4865-4881.

[20] B. S. Thakkar, J.-S. M. Svendsen und R. A. Engh, The Journal of Physical Chemistry A, 2017, 121, 6830-6837.

[21] T. Maeyama, T. Shimamori und A. Fujii, The Journal of Physical Chemistry A, 2017, 121, 4397-4403.

[22] B. W. Matthews, Protein Science, 2016, 25, 776-777.

[23] V. E. Bondybey, A. M. Smith und J. Agreiter, Chemical Reviews, 1996, 96, 2113-2134.

[24] M. Barranco, R. Guardiola, S. Hernández, R. Mayol, J. Navarro und M. Pi, Journal of Low Temperature Physics, 2006, 142, 1-81.

[25] S. B. Ryali und J. B. Fenn, Berichte der Bunsengesellschaft für physikalische Chemie, 1984, 88, 245-253.

[26] J. P. Toennies und A. F. Vilesov, Angewandte Chemie International Edition, 2004, 43, 2622-2648.

[27] M. Y. Choi, G. E. Douberly, T. M. Falconer, W. K. Lewis, C. M. Lindsay, J. M. Merritt, P. L. Stiles und R. E. Miller, International Reviews in Physical Chemistry, 2006, 25, 1575.

[28] H. E. Hallam, Vibrational spectroscopy of trapped species: [infrared and Raman studies of matrix-isolated molecules, radicals and ions], Wiley and Sons, [London], 1973.

[29] S. Oswald, E. Meyer und M. A. Suhm, The Journal of Physical Chemistry A, 2018, 122, 2933-2946.

[30] K. A. E. Meyer, J. A. Davies und A. M. Ellis, Physical Chemistry Chemical Physics, 2020, 22, 9637-9646.

[31] T. Wassermann, Diss., Georg-August Universität, Göttingen, 2010.

[32] N. O. B. Lüttschwager, Raman spectroscopy of conformational rearrangements at low temperatures: Folding and stretching of alkanes in supersonic jets, Springer, Cham, 2014.

[33] T. N. Wassermann und M. A. Suhm, The Journal of Physical Chemistry A, 2010, 114, 8223-8233.

[34] T. Miyazawa, Bulletin of the Chemical Society of Japan, 1961, 34, 691-696.

[35] P. Li, X. G. Chen, E. Shulin und S. A. Asher, Journal of the American Chemical Society, 1997, 119, 1116-1120.

[36] R. E. Miller, Science, 1988, 240, 447-453. 
[37] M. A. Suhm und F. Kollipost, Physical Chemistry Chemical Physics, 2013, 15, 1070210721.

[38] P. Zielke und M. A. Suhm, Physical Chemistry Chemical Physics, 2006, 8, 2826-2830.

[39] G. Sutherland, in Advances in Protein Chemistry, Hrsg. M. L. Anson, K. Bailey und J. T. Edsall, Academic Press, New York, 1952, Bd. 7, S. 291-318.

[40] S. Krimm und J. Bandekar, in Advances in Protein Chemistry, Hrsg. C. B. Anfinsen, J. T. Edsall und F. M. Richards, Academic Press, New York, London und Sydney, 1986, Bd. 38 , S. 181-364.

[41] W. K. Surewicz und H. H. Mantsch, Biochimica et Biophysica Acta: Protein Structure and Molecular Enzymology, 1988, 952, 115-130.

[42] R. Schweitzer-Stenner, Vibrational Spectroscopy, 2006, 42, 98-117.

[43] R. C. Lord, Applied Spectroscopy, 1977, 31, 187-194.

[44] R. Vargas, J. Garza, R. A. Friesner, H. Stern, B. P. Hay und D. A. Dixon, The Journal of Physical Chemistry A, 2001, 105, 4963-4968.

[45] C. Desfrançois, V. Périquet, S. Carles, J. P. Schermann und L. Adamowicz, Chemical Physics, 1998, 239, 475-483.

[46] C. Dugave, in cis-trans Isomerization in Biochemistry, Hrsg. C. Dugave, Wiley-VCH Verlag GmbH \& Co. KGaA, Weinheim, Germany, 2006, S. 143-166.

[47] V. P. Manea, K. J. Wilson und J. R. Cable, Journal of the American Chemical Society, 1997, 119, 2033-2039.

[48] J. Eckert, M. Barthes, W. T. Klooster, A. Albinati, R. Aznar und T. F. Koetzle, The Journal of Physical Chemistry B, 2001, 105, 19-24.

[49] R. Ludwig, F. Weinhold und T. C. Farrar, The Journal of Chemical Physics, 1997, 107, 499.

[50] R. Ludwig, Journal of Molecular Liquids, 2000, 84, 65-75.

[51] M. Albrecht, C. A. Rice und M. A. Suhm, The Journal of Physical Chemistry A, 2008, 112, 7530-7542.

[52] R. A. Mata und M. A. Suhm, Angewandte Chemie, 2017, 129, 11155-11163.

[53] W. H. James, E. G. Buchanan, C. W. Müller, J. C. Dean, D. Kosenkov, L. V. Slipchenko, L. Guo, A. G. Reidenbach, S. H. Gellman und T. S. Zwier, The Journal of Physical Chemistry A, 2011, 115, 13783-13798.

[54] V. Poterya, V. Profant, M. Fárník, L. Sistík, P. Slavícek und U. Buck, The Journal of Physical Chemistry A, 2009, 113, 14583-14590.

[55] S.-K. Lee, S.-J. Lee, A.-R. Ahn, Y.-S. Kim, A.-R. Min, M.-Y. Choi und R. E. Miller, Bulletin of the Korean Chemical Society, 2011, 32, 885-888.

[56] J. Zischang, Diplomarbeit, Georg-August-Universität, Göttingen, 2010. 
[57] T. Forsting, J. Zischang, M. A. Suhm, M. Eckhoff, B. Schröder und R. A. Mata, Physical Chemistry Chemical Physics, 2019, 21, 5989-5998.

[58] IUPAC-IUB Commission on Biochemical Nomenclature, Pure and Applied Chemistry, 1974, 40, 291-308.

[59] C. Dugave, in cis-trans Isomerization in Biochemistry, Hrsg. C. Dugave, Wiley-VCH Verlag GmbH \& Co. KGaA, Weinheim, Germany, 2006, S. 1-5.

[60] A. C. Fantoni, W. Caminati, H. Hartwig und W. Stahl, Journal of Molecular Structure, 2002, 612, 305-307.

[61] H. M. Randall, R. G. Fowler, N. Fuson und J. R. Dangl, Infrared Determination of Organic Structures, van Nostrand, New York, NY, USA, 1. Aufl., 1949.

[62] T. Miyazawa, T. Shimanouchi und S.-i. Mizushima, The Journal of Chemical Physics, 1956, 24, 408.

[63] T. Miyazawa, T. Shimanouchi und S.-i. Mizushima, The Journal of Chemical Physics, 1958, 29, 611.

[64] A. Warshel, M. Levitt und S. Lifson, Journal of Molecular Spectroscopy, 1970, 33, 84-99.

[65] K. Itoh und T. Shimanouchi, Biopolymers, 1967, 5, 921-930.

[66] N. G. Mirkin und S. Krimm, Journal of Molecular Structure, 1991, 242, 143-160.

[67] S. Krimm, in Infrared analysis of peptides and proteins, Hrsg. B. R. Singh, American Chemical Society, Washington, 2000, Bd. 750, S. 38-53.

[68] S. Ataka, H. Takeuchi und M. Tasumi, Journal of Molecular Structure, 1984, 113, 147160.

[69] S.-i. Mizushima, T. Simanouti, S. Nagakura, K. Kuratani, M. Tsuboi, H. Baba und O. Fujioka, Journal of the American Chemical Society, 1950, 72, 3490-3494.

[70] R. M. Badger und A. D. E. Pullin, The Journal of Chemical Physics, 1954, 22, 1142.

[71] T. Miyazawa, Journal of Molecular Spectroscopy, 1960, 4, 168-172.

[72] Y. V. Venkatachalapathi, D. F. Mierke, J. P. Taulane und M. Goodman, Biopolymers, 1987, 26, 763-773.

[73] R. L. Jones, Journal of Molecular Spectroscopy, 1963, 11, 411-421.

[74] F. Fillaux und C. de Lozé, Chemical Physics Letters, 1976, 39, 547-551.

[75] N. E. Triggs und J. J. Valentini, The Journal of Physical Chemistry, 1992, 96, 6922-6931.

[76] W. K. Surewicz, H. H. Mantsch und D. Chapman, Biochemistry, 1993, 32, 389-394.

[77] T. Measey, A. Hagarman, F. Eker, K. Griebenow und R. Schweitzer-Stenner, The Journal of Physical Chemistry B, 2005, 109, 8195-8205.

[78] J. Kubelka und T. A. Keiderling, The Journal of Physical Chemistry A, 2001, 105, 1092210928. 
[79] L. J. Bellamy, in The Infra-red Spectra of Complex Molecules, Hrsg. L. J. Bellamy, Springer Netherlands, Dordrecht, 1975, S. 231-262.

[80] I. Harada, Y. Sugawara, H. Matsuura und T. Shimanouch, Journal of Raman Spectroscopy, 1975, 4, 91-98.

[81] S. Song, S. A. Asher, S. Krimm und K. D. Shaw, Journal of the American Chemical Society, 1991, 113, 1155-1163.

[82] T. Jordan und T. G. Spiro, Journal of Raman Spectroscopy, 1995, 26, 867-876.

[83] S. A. Asher, A. Ianoul, G. Mix, M. N. Boyden, A. Karnoup, M. Diem und R. SchweitzerStenner, Journal of the American Chemical Society, 2001, 123, 11775-11781.

[84] J. Bandekar, Biochimica et Biophysica Acta: Protein Structure and Molecular Enzymology, 1992, 1120, 123-143.

[85] Y. Wang, R. Purrello und T. G. Spiro, Journal of the American Chemical Society, 1989, 111, 8274-8276.

[86] T. Jordan und T. G. Spiro, Journal of Raman Spectroscopy, 1994, 25, 537-543.

[87] S. Song, S. A. Asher, S. Krimm und J. Bandekar, Journal of the American Chemical Society, 1988, 110, 8547-8548.

[88] X. G. Chen, R. Schweitzer-Stenner, S. A. Asher, N. G. Mirkin und S. Krimm, The Journal of Physical Chemistry, 1995, 99, 3074-3083.

[89] T. Jordan und T. G. Spiro, The Journal of Physical Chemistry A, 1997, 101, 3990-3991.

[90] S. A. Asher, P. Li, Z. Chi, X. G. Chen, R. Schweitzer-Stenner, N. G. Mirkin und S. Krimm, The Journal of Physical Chemistry A, 1997, 101, 3992-3994.

[91] P. Hohenberg und W. Kohn, Physical Review, 1964, 136, B864-B871.

[92] W. Kohn und L. J. Sham, Physical Review, 1965, 140, A1133-A1138.

[93] A. D. Becke, The Journal of Chemical Physics, 1993, 98, 5648.

[94] C. Lee, W. Yang und R. G. Parr, Physical Review B, 1988, 37, 785-789.

[95] A. D. Becke, Physical Review A, 1988, 38, 3098-3100.

[96] F. Jensen, Introduction to Computational Chemistry, Wiley, Chichester, UK und Hoboken, NJ, 3. Aufl., 2017.

[97] P. J. Stephens, F. J. Devlin, C. F. Chabalowski und M. J. Frisch, The Journal of Physical Chemistry, 1994, 98, 11623-11627.

[98] J. P. Perdew und Y. Wang, Physical Review B, 1992, 45, 13244-13249.

[99] S. H. Vosko, L. Wilk und M. Nusair, Canadian Journal of Physics, 1980, 58, 1200-1211.

[100] S. Kristyán und P. Pulay, Chemical Physics Letters, 1994, 229, 175-180.

[101] J. M. Pérez-Jordá, E. San-Fabián und A. J. Pérez-Jiménez, The Journal of Chemical Physics, 1999, 110, 1916-1920. 
[102] Q. Wu und W. Yang, The Journal of Chemical Physics, 2002, 116, 515-524.

[103] U. Zimmerli, M. Parrinello und P. Koumoutsakos, The Journal of Chemical Physics, 2004, 120, 2693-2699.

[104] S. Grimme, Journal of Computational Chemistry, 2004, 25, 1463-1473.

[105] S. Grimme, J. Antony, S. Ehrlich und H. Krieg, The Journal of Chemical Physics, 2010, 132, 154104.

[106] S. Grimme, S. Ehrlich und L. Goerigk, Journal of Computational Chemistry, 2011, 32, $1456-1465$.

[107] L. Goerigk und S. Grimme, Physical Chemistry Chemical Physics, 2011, 13, 6670-6688.

[108] T. N. Wassermann, J. Thelemann, P. Zielke und M. A. Suhm, The Journal of Chemical Physics, 2009, 131, 161108.

[109] H. C. Gottschalk, J. Altnöder, M. Heger und M. A. Suhm, Angewandte Chemie International Edition, 2016, 55, 1921-1924.

[110] H. C. Gottschalk, A. Poblotzki, M. A. Suhm, M. M. Al-Mogren, J. Antony, A. A. Auer, L. Baptista, D. M. Benoit, G. Bistoni, F. Bohle, R. Dahmani, D. Firaha, S. Grimme, A. Hansen, M. E. Harding, M. Hochlaf, C. Holzer, G. Jansen, W. Klopper, W. A. Kopp, L. C. Kröger, K. Leonhard, H. Mouhib, F. Neese, M. N. Pereira, I. S. Ulusoy, A. Wuttke und R. A. Mata, The Journal of Chemical Physics, 2018, 148, 014301.

[111] G. Wedler, Lehrbuch der physikalischen Chemie, Wiley-VCH, Weinheim, 5. Aufl., 2004.

[112] K. E. Otto, Dissertation, Georg-August Universität, Göttingen, 2015.

[113] S. Bocklitz, Dissertation, Georg-August Universität, Göttingen, 2018.

[114] D. H. Levy, Annual Review of Physical Chemistry, 1980, 31, 197-225.

[115] B. Maté, G. Tejeda und S. Montero, The Journal of Chemical Physics, 1998, 108, 26762685.

[116] R. S. Ruoff, T. D. Klots, T. Emilsson und H. S. Gutowsky, The Journal of Chemical Physics, 1990, 93, 3142-3150.

[117] Lüttschwager, Nils O. B., T. N. Wassermann, R. A. Mata und M. A. Suhm, Angewandte Chemie, 2013, 125, 482-485.

[118] Saleh, Bahaa E. A und M. C. Teich, Grundlagen der Photonik, Wiley-VCH, Weinheim, 2. Aufl., 2008.

[119] W. Demtröder, Laserspektroskopie 1: Grundlagen, Springer, Berlin/Heidelberg, 6. Aufl., 2011.

[120] D. J. Schroeder, Astronomical Optics, Academic Press, San Diego, CA, USA, 2. Aufl., 2000.

[121] D. Groom, Experimental Astronomy, 2002, 14, 45-55. 
[122] C. A. Murray und S. B. Dierker, Journal of the Optical Society of America A, 1986, 3, 2151.

[123] G. R. Phillips und J. M. Harris, Analytical Chemistry, 1990, 62, 2351-2357.

[124] W. Hill und D. Rogalla, Analytical Chemistry, 1992, 64, 2575-2579.

[125] Y. Katsumoto und Y. Ozaki, Applied Spectroscopy, 2003, 57, 317-322.

[126] H. Takeuchi, S. Hashimoto und I. Harada, Applied Spectroscopy, 1993, 47, 129-131.

[127] D. Zhang, K. N. Jallad und D. Ben-Amotz, Applied Spectroscopy, 2001, 55, 1523-1531.

[128] D. Zhang und D. Ben-Amotz, Applied Spectroscopy, 2002, 56, 91-98.

[129] D. Zhang, J. D. Hanna und D. Ben-Amotz, Applied Spectroscopy, 2003, 57, 1303-1305.

[130] J. Zhao, Applied Spectroscopy, 2003, 57, 1368-1375.

[131] W. Hill, Applied Spectroscopy, 1993, 47, 2171-2172.

[132] U. B. Cappel, I. M. Bell und L. K. Pickard, Applied Spectroscopy, 2010, 64, 195-200.

[133] S. Li und L. Dai, Applied Spectroscopy, 2011, 65, 1300-1306.

[134] A. Kramida, Yu. Ralchenko, J. Reader und and NIST ASD Team, NIST Atomic Spectra Database (ver. 5.5.6), Gaithersburg, MD, 2018, https://physics.nist.gov/asd (besucht am 02.05.2018).

[135] E. R. Peck und K. Reeder, Journal of the Optical Society of America, 1972, 62, 958.

[136] F. Kollipost, Dissertation, Georg-August Universität, Göttingen, 2015.

[137] M. Heger, Dissertation, Georg-August Universität, Göttingen, 2016.

[138] C. Møller und M. S. Plesset, Physical Review, 1934, 46, 618-622.

[139] M. Head-Gordon, J. A. Pople und M. J. Frisch, Chemical Physics Letters, 1988, 153, 503506.

[140] V. Barone, The Journal of Chemical Physics, 2004, 120, 3059-3065.

[141] V. Barone, The Journal of Chemical Physics, 2005, 122, 14108.

[142] J. Bloino und V. Barone, The Journal of Chemical Physics, 2012, 136, 124108.

[143] F. Weigend und R. Ahlrichs, Physical Chemistry Chemical Physics, 2005, 7, 3297-3305.

[144] T. H. Dunning, The Journal of Chemical Physics, 1989, 90, 1007-1023.

[145] E. R. Davidson, Chemical Physics Letters, 1996, 260, 514-518.

[146] R. A. Kendall, T. H. Dunning und R. J. Harrison, The Journal of Chemical Physics, 1992, 96, 6796-6806. 
[147] M. J. Frisch, G. W. Trucks, H. B. Schlegel, G. E. Scuseria, M. A. Robb, J. R. Cheeseman, G. Scalmani, V. Barone, B. Mennucci, G. A. Petersson, H. Nakatsuji, M. Caricato, X. Li, H. P. Hratchian, A. F. Izmaylov, J. Bloino, G. Zheng, J. L. Sonnenberg, M. Hada, M. Ehara, K. Toyota, R. Fukuda, J. Hasegawa, M. Ishida, T. Nakajima, Y. Honda, O. Kitao, H. Nakai, T. Vreven, J. J. A. Montgomery, J. E. Peralta, F. Ogliaro, M. Bearpark, J. J. Heyd, E. Brothers, K. N. Kudin, V. N. Staroverov, R. Kobayashi, J. Normand, K. Raghavachari, A. Rendell, J. C. Burant, S. S. Iyengar, J. Tomasi, M. Cossi, N. Rega, J. M. Millam, M. Klene, J. E. Knox, J. B. Cross, V. Bakken, C. Adamo, J. Jaramillo, R. Gomperts, R. E. Stratmann, O. Yazyev, A. J. Austin, R. Cammi, C. Pomelli, J. W. Ochterski, R. L. Martin, K. Morokuma, V. G. Zakrzewski, G. A. Voth, P. Salvador, J. J. Dannenberg, S. Dapprich, A. D. Daniels, Ö. Farkas, J. B. Foresman, J. V. Ortiz, J. Cioslowski und D. J. Fox, Gaussian 09, Wallingford CT, 2009.

[148] B. Paizs und S. Suhai, Journal of Computational Chemistry, 1998, 19, 575-584.

[149] P. R. Rablen, J. W. Lockman und W. L. Jorgensen, The Journal of Physical Chemistry A, 1998, 102, 3782-3797.

[150] S. F. Boys und F. Bernardi, Molecular Physics, 1970, 19, 553-566.

[151] T. M. Watson und J. D. Hirst, The Journal of Physical Chemistry A, 2002, 106, 78587867.

[152] D. R. Lide, CRC Handbook of Chemistry and Physics: A Ready-Reference Book of Chemical and Physical Data, CRC Press, Boca Raton, FL, USA, 82. Aufl., 2001.

[153] T. E. Daubert und R. P. Danner, Physical and Thermodynamic Properties of Pure Chemicals: Data Compilation, Taylor \& Francis, Washington, DC, USA, 1989.

[154] C. L. Yaws, Handbook of Vapor Pressure: Volume 1, C1-C4 Compounds, Gulf Pub. Co, Houston, TX, USA, 1994.

[155] J. A. Riddick, W. B. Bunger und T. K. Sakano, Organic Solvents: Physical Properties and Methods of Purification, Wiley, New York, NY, USA, 4. Aufl., 1986, Bd. 2.

[156] F. Ullmann, W. Gerhartz, S. Y. Yamamoto, T. F. Campbell, R. Pfefferkorn und J. F. Rounsaville, Ullmann's Encyclopedia of Industrial Chemistry, VCH Publishing, Deerfield Beach, FL, USA, 5. Aufl., 1985.

[157] S. Kim, P. A. Thiessen, E. E. Bolton, J. Chen, G. Fu, A. Gindulyte, L. Han, J. He, S. He, B. A. Shoemaker, J. Wang, B. Yu, J. Zhang und S. H. Bryant, Nucleic Acids Research, 2016, 44, D1202-13.

[158] G. N. Ramachandran und A. K. Mitra, Journal of Molecular Biology, 1976, 107, 85-92.

[159] L. A. LaPlanche und M. T. Rogers, Journal of the American Chemical Society, 1964, 86, 337-341.

[160] R. H. Barker und G. J. Boudreaux, Spectrochimica Acta Part A: Molecular Spectroscopy, 1967, 23, 727-728.

[161] T. Drakenberg und S. Forsén, J. Chem. Soc. D, 1971, 3, 1404-1405. 
[162] A. Radzicka, L. Pedersen und R. Wolfenden, Biochemistry, 1988, 27, 4538-4541.

[163] A. G. Garcia Martínez, E. T. Vilar, A. G. Fraile und P. Martínez-Ruiz, The Journal of Physical Chemistry A, 2002, 106, 4942-4950.

[164] M. Kimura und M. Aoki, Bulletin of the Chemical Society of Japan, 1953, 26, 429-433.

[165] M. Kitano, T. Fukuyama und K. Kuchitsu, Bulletin of the Chemical Society of Japan, 1973, 46, 384-387.

[166] J. L. Katz und B. Post, Acta Crystallographica, 1960, 13, 624-628.

[167] A. T. Hagler, L. Leiserowitz und M. Tuval, Journal of the American Chemical Society, 1976, 98, 4600-4612.

[168] F. Fillaux und J. Tomkinson, Chemical Physics, 1977, 26, 295-300.

[169] N. Ohashi, J. T. Hougen, R. D. Suenram, F. J. Lovas, Y. Kawashima, M. Fujitake und J. Pyka, Journal of Molecular Spectroscopy, 2004, 227, 28-42.

[170] T. L. Teuteberg, Bachelorarbeit, Georg-August-Universität, Göttingen, 2011.

[171] R. A. Russell und H. W. Thompson, Spectrochimica Acta, 1956, 8, 138-141.

[172] H. Nakanishi und J. D. Roberts, Organic Magnetic Resonance, 1981, 15, 7-12.

[173] M. Liler, J. Chem. Soc., Perkin Trans. 2, 1972, 1, 720-725.

[174] N. G. Mirkin und S. Krimm, Journal of Molecular Structure: THEOCHEM, 1991, 236, 97-111.

[175] M. Albrecht, Biologisch relevante Wasserstoffbrückensysteme im Überschallstrahl: Steuerung der Aggregation durch Substitution, Sierke, Göttingen, 1. Aufl., 2009.

[176] L. O. Paulson und D. T. Anderson, The Journal of Physical Chemistry B, 2011, 115, 13659-13667.

[177] L. C. Mayne und B. Hudson, The Journal of Physical Chemistry, 1991, 95, 2962-2967.

[178] P. U. Bai und K. V. Ramiah, Bulletin of the Chemical Society of Japan, 1971, 44, 313315.

[179] C. Y.-S. Chen und C. A. Swenson, The Journal of Physical Chemistry, 1969, 73, 29993008.

[180] K. Pralat, J. Jadzyn und S. Balanicka, The Journal of Physical Chemistry, 1983, 87, 1385-1390.

[181] T. Köddermann und R. Ludwig, Physical Chemistry Chemical Physics, 2004, 6, 18671873.

[182] Y. Zhou, G. Deng, Y.-Z. Zheng, J. Xu, H. Ashraf und Z.-W. Yu, Scientific Reports, 2016, 6, 36932 .

[183] I. B. Davydova, V. M. Senyavin, O. N. Zefirova, G. M. Kuramshina und Y. A. Pentin, Russian Journal of Physical Chemistry A, 2014, 88, 671-680. 
[184] I. M. Klotz und J. S. Franzen, Journal of the American Chemical Society, 1962, 84, 34613466.

[185] S. K. Gregurick, G. M. Chaban und R. B. Gerber, The Journal of Physical Chemistry A, 2002, 106, 8696-8707.

[186] N. V. Tukachev, V. A. Bataev und I. A. Godunov, Computational and Theoretical Chemistry, 2017, 1113, 82-93.

[187] G. Cuevas, V. Renugopalakrishnan, G. Madrid und A. T. Hagler, Physical Chemistry Chemical Physics, 2002, 4, 1490-1499.

[188] G. V. Papamokos und I. N. Demetropoulos, The Journal of Physical Chemistry A, 2004, 108, 7291-7300.

[189] H. Fujisaki, K. Yagi, K. Hirao und J. E. Straub, Chemical Physics Letters, 2007, 443, 611.

[190] M. Bounouar und C. Scheurer, Chemical Physics, 2006, 323, 87-101.

[191] A. L. Kaledin und J. M. Bowman, The Journal of Physical Chemistry A, 2007, 111, 55935598.

[192] G. Herzberg, Molecular spectra and molecular structure: Volume II: Infrared and Raman Spectra of Polyatomic Molecules, R.E. Krieger Pub. Co, Malabar, Florida, 3. Aufl., 1991.

[193] K.-P. Huber und G. Herzberg, Molecular spectra and molecular structure: 4: Constants of diatomic molecules, van Nostrand Reinhold Comp., New York, 1979.

[194] V. Yatsyna, D. J. Bakker, P. Salen, R. Feifel, A. M. Rijs und V. Zhaunerchyk, Physical Review Letters, 2016, 117, 118101.

[195] H. Torii, T. Tatsumi, T. Kanazawa und M. Tasumi, The Journal of Physical Chemistry B, 1998, 102, 309-314.

[196] J. Zhang und M. W. Germann, Biopolymers, 2011, 95, 755-762.

[197] H. E. Hallam und C. M. Jones, Journal of Molecular Structure, 1970, 5, 1-19.

[198] F. J. Luque und M. Orozco, The Journal of Organic Chemistry, 1993, 58, 6397-6405.

[199] V. Villani, G. Alagona und C. Ghio, Molecular Engineering, 1999, 8, 135-153.

[200] Y. A. Mantz, H. Gerard, R. Iftimie und G. J. Martyna, The Journal of Physical Chemistry $B, 2006,110,13523-13538$.

[201] Y. A. Mantz, D. Branduardi, G. Bussi und M. Parrinello, The Journal of Physical Chemistry $B, 2009,113,12521-12529$.

[202] Y. K. Kang, Journal of Molecular Structure: THEOCHEM, 2001, 546, 183-193.

[203] Y. K. Kang und H. S. Park, Journal of Molecular Structure: THEOCHEM, 2004, 676, 171176.

[204] Lüttschwager, Nils O. B. und M. A. Suhm, Soft Matter, 2014, 10, 4885-4901. 
[205] N. S. Myshakina, Z. Ahmed und S. A. Asher, The Journal of Physical Chemistry B, 2008, 112, 11873-11877.

[206] R. Schweitzer-Stenner, G. Sieler, N. G. Mirkin und S. Krimm, The Journal of Physical Chemistry A, 1998, 102, 118-127.

[207] R. Ludwig, O. Reis, R. Winter, F. Weinhold und T. C. Farrar, The Journal of Physical Chemistry B, 1998, 102, 9312-9318.

[208] M. Akiyama und H. Torii, Spectrochimica Acta, Part A: Molecular and Biomolecular Spectroscopy, 1999, 56, 137-144.

[209] M. P. Gaigeot, R. Vuilleumier, M. Sprik und D. Borgis, Journal of Chemical Theory and Computation, 2005, 1, 772-789.

[210] L. Piatkowski und H. J. Bakker, The Journal of Physical Chemistry A, 2010, 114, 1146211470.

[211] D. J. Nesbitt, Annual Review of Physical Chemistry, 1994, 45, 367-399.

[212] T. Forsting, H. C. Gottschalk, B. Hartwig, M. Mons und M. A. Suhm, Physical Chemistry Chemical Physics, 2017, 19, 10727-10737.

[213] G. Dellepiane, S. Abbate, P. Bosi und G. Zerbi, The Journal of Chemical Physics, 1980, 73, 1040 .

[214] V. Barone, M. Biczysko und J. Bloino, Physical Chemistry Chemical Physics, 2014, 16, 1759-1787.

[215] F. Kollipost, A. V. Domanskaya und M. A. Suhm, The Journal of Physical Chemistry A, 2015, 119, 2225-2232.

[216] B. Mannfors, N. G. Mirkin, K. Palmo und S. Krimm, Journal of Computational Chemistry, 2001, 22, 1933-1943.

[217] S. Ham und M. Cho, The Journal of Chemical Physics, 2003, 118, 6915.

[218] D. A. Dixon, K. D. Dobbs und J. J. Valentini, The Journal of Physical Chemistry, 1994, 98, 13435-13439.

[219] K. Sakota, Y. Shimazaki und H. Sekiya, The Journal of Chemical Physics, 2009, 130, 231105.

[220] R. W. Larsen, P. Zielke und M. A. Suhm, The Journal of Chemical Physics, 2007, 126, 194307.

[221] M. Davies und D. K. Thomas, The Journal of Physical Chemistry, 1956, 60, 767-770.

[222] S. Trabelsi, M. Bahri und S. Nasr, The Journal of Chemical Physics, 2005, 122, 024502.

[223] Z.-Y. Zeng, Y.-S. Wang und S. D. Chao, Computational and Theoretical Chemistry, 2017, 1113, 1-7.

[224] H. Guo und M. Karplus, The Journal of Physical Chemistry, 1992, 96, 7273-7287.

[225] H. Guo und M. Karplus, The Journal of Physical Chemistry, 1994, 98, 7104-7105. 
[226] A. S. Mahadevi und G. N. Sastry, Chemical Reviews, 2016, 116, 2775-2825.

[227] E. Gloaguen und M. Mons, Topics in Current Chemistry, 2015, 364, 225-270.

[228] I. Dauster, C. A. Rice, P. Zielke und M. A. Suhm, Physical Chemistry Chemical Physics, 2008, 10, 2827-2835.

[229] A. S. Davydov, Soviet Physics Uspekhi, 1964, 7, 145-178.

[230] D. Chamma und O. Henri-Rousseau, Chemical Physics, 1999, 248, 53-70.

[231] T. W. Whitfield, G. J. Martyna, S. Allison, S. P. Bates, H. Vass und J. Crain, The Journal of Physical Chemistry B, 2006, 110, 3624-3637.

[232] T. C. Cheam, Journal of Molecular Structure: THEOCHEM, 1992, 257, 57-73.

[233] L. M. Kuznetsova, V. L. Furer und L. I. Maklakov, Journal of Molecular Structure, 1996, 380, 23-29.

[234] M. Huelsekopf und R. Ludwig, Magnetic Resonance in Chemistry, 2001, 39, S127-S134.

[235] X. Jiang und C. Wang, Science China Chemistry, 2010, 53, 1754-1761.

[236] S. Trabelsi, S. Nasr, M. Bahri und M.-C. Bellissent-Funel, The Journal of Physical Chemistry $B, 2006,110,25021-25025$.

[237] R. Ludwig, F. Weinhold und T. C. Farrar, The Journal of Physical Chemistry A, 1997, 101, 8861-8870.

[238] M. M. Omar, Journal of the Chemical Society, Faraday Transactions 1: Physical Chemistry in Condensed Phases, 1980, 76, 711.

[239] R. L. Jones, Journal of Molecular Spectroscopy, 1958, 2, 581-586.

[240] R. Jones, Spectrochimica Acta Part A: Molecular Spectroscopy, 1967, 23, 1745-1750.

[241] I. Suzuki, Bulletin of the Chemical Society of Japan, 1962, 35, 540-551.

[242] H. E. Hallam und C. M. Jones, Transactions of the Faraday Society, 1969, 65, 2607.

[243] M. Kitano und K. Kuchitsu, Bulletin of the Chemical Society of Japan, 1974, 47, 67-72.

[244] H. Ohtaki, S. Itoh und B. M. Rode, Bulletin of the Chemical Society of Japan, 1986, 59, 271-276.

[245] J. Neuefeind, P. Chieux und M. D. Zeidler, Molecular Physics, 1992, 76, 143-156.

[246] M. J. Aroney, R. J. W. Le Fèvre und A. N. Singh, Journal of the Chemical Society (Resumed), 1965, 0, 3179-3184.

[247] J. Dorie, J. P. Gouesnard, B. Mechin, N. Naulet und G. J. Martin, Organic Magnetic Resonance, 1980, 13, 126-131.

[248] D. M. Pawar, A. A. Khalil, D. R. Hooks, K. Collins, T. Elliott, J. Stafford, L. Smith und E. A. Noe, Journal of the American Chemical Society, 1998, 120, 2108-2112.

[249] S. Shin, A. Kurawaki, Y. Hamada, K. Shinya, K. Ohno, A. Tohara und M. Sato, Journal of Molecular Structure, 2006, 791, 30-40. 
[250] T. Miyazawa, Journal of Molecular Spectroscopy, 1960, 4, 155-167.

[251] A. C. Fantoni und W. Caminati, Journal of the Chemical Society, Faraday Transactions, 1996, 92, 343.

[252] Y. Kawashima, T. Usami, N. Ohashi, R. D. Suenram, J. T. Hougen und E. Hirota, Accounts of Chemical Research, 2006, 39, 216-220.

[253] Y. Kawashima, T. Usami, R. D. Suenram, G. Y. Golubiatnikov und E. Hirota, Journal of Molecular Spectroscopy, 2010, 263, 11-20.

[254] M. Sałdyka, Z. Mielke, K. Mierzwicki, S. Coussan und P. Roubin, Physical Chemistry Chemical Physics, 2011, 13, 13992-14002.

[255] R. Crespo-Otero, A. Mardyukov, E. Sanchez-Garcia, M. Barbatti und W. Sander, ChemPhysChem, 2013, 14, 827-836.

[256] J. Lee, Adaptive Aggregation über starke Wasserstoffbrücken in der Gasphase, Sierke, [Göttingen], 1. Aufl., 2012.

[257] D. E. DeGraaf und G. B. B. M. Sutherland, The Journal of Chemical Physics, 1957, 26, 716-717.

[258] G. Nandini und D. N. Sathyanarayana, Journal of Molecular Structure: THEOCHEM, 2002, 579, 1-9.

[259] N. V. Tukachev, V. Bataev und I. Godunov, Journal of Quantitative Spectroscopy and Radiative Transfer, 2016, 177, 291-300.

[260] G. Eaton, M. C. R. Symons und P. P. Rastogi, Journal of the Chemical Society, Faraday Transactions 1: Physical Chemistry in Condensed Phases, 1989, 85, 3257.

[261] Y. Sugawara, Y. Hamada, A. Y. Hirakawa und M. Tsuboi, Chemical Physics Letters, 1979, 67, 186-188.

[262] F. A. L. Anet und M. Squillacote, Journal of the American Chemical Society, 1975, 97, 3243-3244.

[263] A. N. Troganis, E. Sicilia, K. Barbarossou, I. P. Gerothanassis und N. Russo, The Journal of Physical Chemistry A, 2005, 109, 11878-11884.

[264] A. García Martínez, E. Teso Vilar, A. García Fraile und P. Martínez-Ruiz, The Journal of Chemical Physics, 2006, 124, 234305.

[265] Bunkan, Arne Joakim C, J. Hetzler, T. Mikoviny, A. Wisthaler, C. J. Nielsen und M. Olzmann, Physical Chemistry Chemical Physics, 2015, 17, 7046-7059.

[266] K. B. Wiberg und D. J. Rush, The Journal of Organic Chemistry, 2002, 67, 826-830.

[267] R. Crespo-Otero, A. Mardykov, E. Sanchez-Garcia, W. Sander und M. Barbatti, Physical Chemistry Chemical Physics, 2014, 16, 18877-18887.

[268] H. Tan, W. Qu, G. Chen und R. Liu, The Journal of Physical Chemistry A, 2005, 109, 6303-6308. 
[269] J. Neuefeind, M. D. Zeidler und H. F. Poulsen, Molecular Physics, 1996, 87, 189-201.

[270] F. Hammami, M. Bahri, S. Nasr, N. Jaidane, M. Oummezzine und R. Cortes, The Journal of Chemical Physics, 2003, 119, 4419-4427.

[271] C. Emmeluth, M. A. Suhm und D. Luckhaus, The Journal of Chemical Physics, 2003, 118, 2242-2255.

[272] A. Bourn, D. G. Gillies und E. W. Randall, Tetrahedron, 1964, 20, 1811-1818.

[273] R. E. Carter, R. J. V. Niklasson, B. Olofsson und P. H. Nielsen, Acta Chemica Scandinavica, 1968, 22, 2643-2652.

[274] M. J. Deetz, J. E. Fahey und B. D. Smith, Journal of Physical Organic Chemistry, 2001, 14, 463-467.

[275] I. Suzuki, M. Tsuboi, T. Shimanouchi und S. Mizushima, Spectrochimica Acta, 1960, 16, 471-478.

[276] S. Blanco, J. C. López, A. Lesarri, W. Caminati und J. L. Alonso, Molecular Physics, 2005, 103, 1473-1479.

[277] J.-R. Aviles Moreno, D. Petitprez und T. R. Huet, Chemical Physics Letters, 2006, 419, 411-416.

[278] B. Omondi, M. A. Fernandes, M. Layh und D. C. Levendis, Acta Crystallographica Section C: Crystal Structure Communications, 2008, 64, o137-o138.

[279] T. Nagata, K. Masuda, S. Maeno und I. Miura, Pest Management Science, 2004, 60, 399407.

[280] I. I. Marochkin und O. V. Dorofeeva, Structural Chemistry, 2013, 24, 233-242.

[281] J. A. Dickinson, M. R. Hockridge, E. G. Robertson und J. P. Simons, The Journal of Physical Chemistry A, 1999, 103, 6938-6949.

[282] D. Lauvergnat und P. C. Hiberty, Journal of the American Chemical Society, 1997, 119, 9478-9482.

[283] J.-R. Aviles Moreno, T. R. Huet und D. Petitprez, Journal of Molecular Structure, 2006, 780-781, 234-237.

[284] M. Miyazaki, J. Saikawa, H. Ishizuki, T. Taira und M. Fujii, Physical Chemistry Chemical Physics, 2009, 11, 6098.

[285] E. G. Robertson, Chemical Physics Letters, 2000, 325, 299-307.

[286] J. Klyne, M. Schmies und O. Dopfer, The Journal of Physical Chemistry B, 2014, 118, 3005-3017.

[287] F. D. S. Grasel, T. C. de Oliveira, L. A. M. Fontoura, I. J. Da Cruz Rigotti und P. A. Netz, International Journal of Quantum Chemistry, 2012, 112, 1678-1687.

[288] S. Ullrich, G. Tarczay, X. Tong, C. E. H. Dessent und K. Müller-Dethlefs, Physical Chemistry Chemical Physics, 2001, 3, 5450-5458. 
[289] S. Ullrich, G. Tarczay, X. Tong, C. E. H. Dessent und K. Müller-Dethlefs, Angewandte Chemie International Edition, 2002, 41, 166-168.

[290] Ottaviani, Melandri, Maris, Favero und Caminati, Journal of Molecular Spectroscopy, 2001, 205, 173-176.

[291] P. R. Andrews, Australian Journal of Chemistry, 1972, 25, 2243.

[292] M. Mons, I. Dimicoli, B. Tardivel, F. Piuzzi, E. G. Robertson und J. P. Simons, The Journal of Physical Chemistry A, 2001, 105, 969-973.

[293] A. V. Fedorov und J. R. Cable, The Journal of Physical Chemistry A, 2000, 104, 49434952.

[294] K. Sakota, Y. Shimazaki und H. Sekiya, Physical Chemistry Chemical Physics, 2011, 13, 6411-6415.

[295] C. Medcraft, S. Zinn, M. Schnell, A. Poblotzki, J. Altnöder, M. Heger, M. A. Suhm, D. Bernhard, A. Stamm, F. Dietrich und M. Gerhards, Physical Chemistry Chemical Physics, 2016, 18, 25975-25983.

[296] A. Poblotzki, J. Altnöder und M. A. Suhm, Physical Chemistry Chemical Physics, 2016, 18, 27265-27271.

[297] R. C. Millikan und K. S. Pitzer, Journal of the American Chemical Society, 1958, 80, 3515-3521.

[298] D. M. Hewett, S. Bocklitz, D. P. Tabor, E. L. Sibert III, M. A. Suhm und T. S. Zwier, Chemical Science, 2017, 8, 5305-5318.

[299] M. Gawrilow und M. A. Suhm, in preparation, 2019.

[300] F. Haaf, A. Sanner und F. Straub, Polymer Journal, 1985, 17, 143-152.

[301] F. Fischer und S. Bauer, Chemie in unserer Zeit, 2009, 43, 376-383.

[302] E. F. Bandle, G. Wendt, U. B. Ranalder und K.-H. Trautmann, Life Sciences, 1984, 35, 2205-2212.

[303] D. Dell, G. Wendt, F. Bucheli und K.-h. Trautmann, Journal of Chromatography B: Biomedical Sciences and Applications, 1985, 344, 125-136.

[304] R. Huisgen und H. Walz, Chemische Berichte, 1956, 89, 2616-2629.

[305] R. Huisgen, H. Brade, H. Walz und I. Glogger, Chemische Berichte, 1957, 90, 14371447.

[306] K. S. Maiti, A. Samsonyuk, C. Scheurer und T. Steinel, Physical Chemistry Chemical Physics, 2012, 14, 16294-16300.

[307] A. E. Shchavlev, A. N. Pankratov, V. B. Borodulin und O. A. Chaplygina, The Journal of Physical Chemistry A, 2005, 109, 10982-10996.

[308] P. Pandey, A. K. Samanta, B. Bandyopadhyay und T. Chakraborty, Vibrational Spectroscopy, 2011, 55, 126-131. 
[309] J. Jadzyn, J. Malecki und C. Jadzyn, The Journal of Physical Chemistry, 1978, 82, 2128 2130.

[310] S. E. Krikorian, The Journal of Physical Chemistry, 1982, 86, 1875-1881.

[311] R. Goddard, O. Heinemann, C. Krüger, I. Magdó, F. Mark und K. Schaffner, Acta Crystallographica Section C: Crystal Structure Communications, 1998, 54, 501-504.

[312] J. A. Walmsley, E. J. Jacob und H. B. Thompson, The Journal of Physical Chemistry, 1976, 80, 2745-2753.

[313] J. A. Walmsley, The Journal of Physical Chemistry, 1978, 82, 2031-2035.

[314] K. de Smet, P. Kedziora, J. Jadzyn und L. Hellemans, The Journal of Physical Chemistry, 1996, 100, 7662-7668.

[315] K. S. Maiti, Physical Chemistry Chemical Physics, 2015, 17, 24998-25003.

[316] K. S. Maiti, Chemical Physics, 2018, 515, 509-512.

[317] A. J. Thakkar, Chemical Physics Letters, 2013, 560, 71-74.

[318] A. E. Parsons, Journal of Molecular Spectroscopy, 1961, 6, 201-204.

[319] S. Oswald, M. Wallrabe und M. A. Suhm, The Journal of Physical Chemistry A, 2017, 121, 3411-3422.

[320] S. Oswald, M. A. Suhm und S. Coussan, Physical Chemistry Chemical Physics, 2019, 21, 1277-1284.

[321] K. A. E. Meyer und M. A. Suhm, The Journal of Chemical Physics, 2018, 149, 104307.

[322] C. Cox, V. G. Young und T. Lectka, Journal of the American Chemical Society, 1997, 119, 2307-2308.

[323] C. Cox, H. Wack und T. Lectka, Journal of the American Chemical Society, 1999, 121, 7963-7964.

[324] C. Cox und T. Lectka, Accounts of Chemical Research, 2000, 33, 849-858.

[325] J.-C. Micheau und J. Zhao, Journal of Physical Organic Chemistry, 2007, 20, 810-820.

[326] P. Zielke und M. A. Suhm, Physical Chemistry Chemical Physics, 2007, 9, 4528-4534.

[327] Zhifeng Xue, Diss., Georg-August Universität, Göttingen, 2010.

[328] R. J. Abraham, L. Griffiths und M. Perez, Magnetic Resonance in Chemistry, 2013, 51, 143-155.

[329] Spectral Database of Organic Compounds (SDBS), Japan, http://sdbs . db . a ist . go.jp (besucht am 24.04. 2018).

[330] V. Andrushchenko, P. Matejka, D. T. Anderson, J. Kaminsky, J. Hornicek, L. O. Paulson und P. Bour, The Journal of Physical Chemistry A, 2009, 113, 9727-9736.

[331] T. Hayashi, W. Zhuang und S. Mukamel, The Journal of Physical Chemistry A, 2005, 109, 9747-9759. 
[332] J. J. Dannenberg, The Journal of Physical Chemistry A, 2006, 110, 5798-5802.

[333] M. Avalos, R. Babiano, J. L. Barneto, J. L. Bravo, P. Cintas, J. L. Jiménez und J. C. Palacios, The Journal of Organic Chemistry, 2001, 66, 7275-7282.

[334] Y. Sugawara, A. Y. Hirakawa, M. Tsuboi, S. Kato und K. Morokuma, Chemical Physics, 1981, 62, 339-351.

[335] W. Caminati, J. C. López, S. Blanco, S. Mata und J. L. Alonso, Physical Chemistry Chemical Physics, 2010, 12, 10230-10234.

[336] M. G. Arnaudov, B. B. Ivanova, S. T. Todorov und S. I. Zareva, Spectrochimica Acta, Part A: Molecular and Biomolecular Spectroscopy, 2006, 63, 491-500. 